Göttingen Series in Social and Cultural Anthropology

Antonie Fuhse

\title{
Mobilität und Zeitgestaltung
}

Junge Wissenschaftler*innen aus Indien und ihre biographische Navigation zwischen

Gegenwart und Zukunft

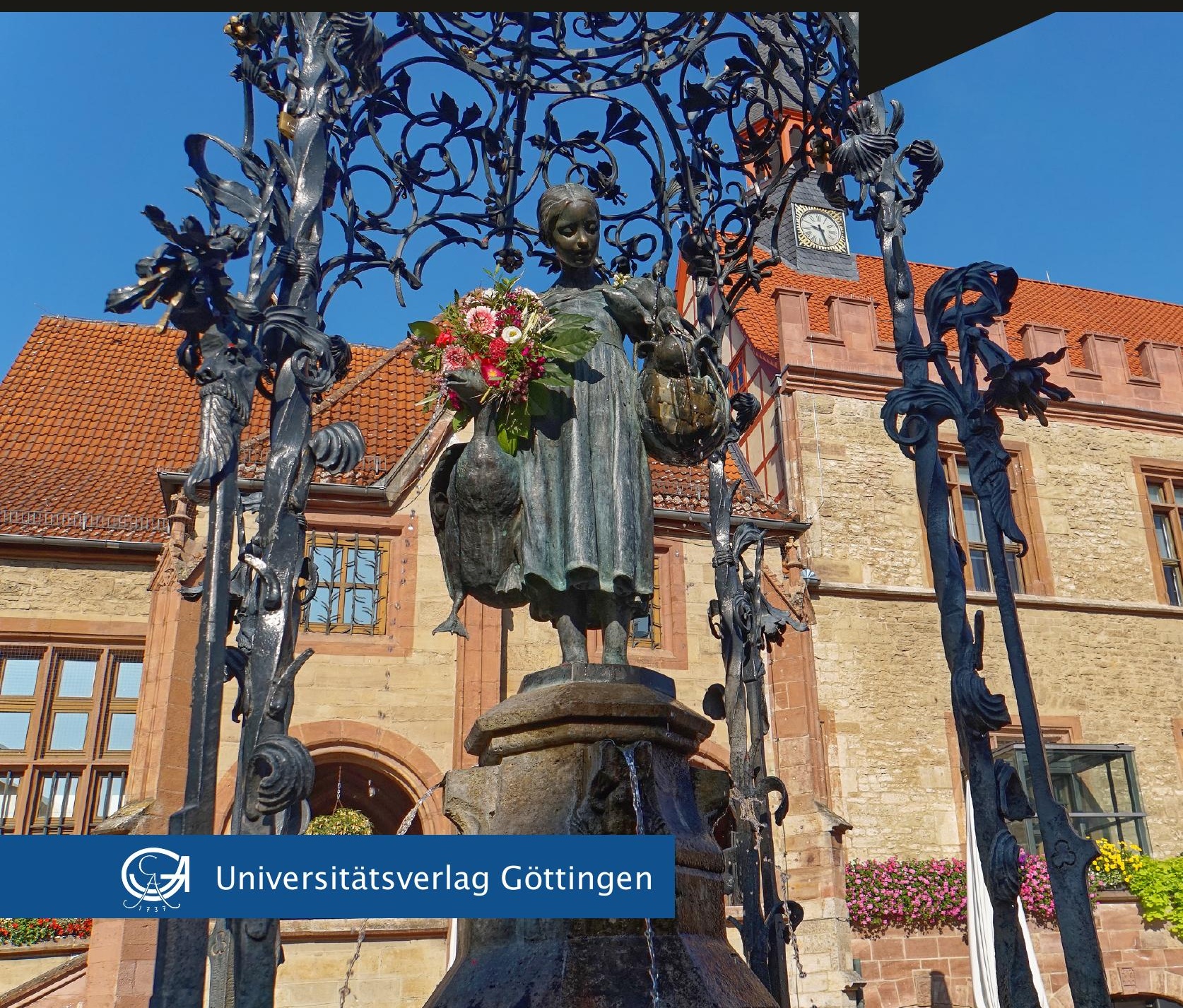



Antonie Fuhse

Mobilität und Zeitgestaltung

Dieses Werk ist lizenziert unter einer

Creative Commons

Namensnennung - Weitergabe unter gleichen Bedingungen

4.0 International Lizenz.

(c) () () 
erschienen als Band 20 in der Reihe „Göttinger Reihe zur Ethnologie Göttingen Series in Social and Cultural Anthropology“"

im Universitätsverlag Göttingen 2021

Diese Reihe ist eine Fortsetzung der „Göttinger Beiträge zur Ethnologie“ 
Antonie Fuhse

\section{Mobilität und Zeitgestaltung}

Junge Wissenschaftler*innen aus Indien und ihre biographische

Navigation zwischen Gegenwart und Zukunft

Band 20

Göttinger Reihe zur Ethnologie Göttingen Series in Social and Cultural Anthropology

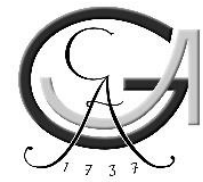

Universitätsverlag Göttingen 2021 


\title{
Bibliografische Information
}

Die Deutsche Nationalbibliothek verzeichnet diese Publikation in der Deutschen Nationalbibliografie; detaillierte bibliografische Daten sind im Internet über $<$ http://dnb.dnb.de $>$ abrufbar.

\author{
„Göttinger Reihe zur Ethnologie - Göttingen Series in Social and Cultural Anthropology" \\ berausgegeben von \\ Prof. Dr. Elfriede Hermann \\ Prof. Dr. Andrea Lauser \\ Prof. Dr. Roman Loimeier \\ Prof. Dr. Nikolaus Schareika \\ Institut für Ethnologie \\ Georg-August-Universität Göttingen \\ Theaterplatz 15 \\ D-37073 Göttingen
}

Dissertation, Georg-August-Universität Göttingen

Dieses Buch ist auch als freie Onlineversion über die Homepage des Verlags sowie über den Göttinger Universitätskatalog (GUK) bei der Niedersächsischen Staats- und Universitätsbibliothek Göttingen (https://www.sub.uni-goettingen.de) erreichbar.

Es gelten die Lizenzbestimmungen der Onlineversion.

Satz und Layout: Steffen Herrmann

Coverabbildung: Antonie Fuhse: Gänseliesel in Göttingen

(C) 2021 Universitätsverlag Göttingen

https://univerlag.uni-goettingen.de

ISBN: 978-3-86395-498-7

DOI: https://doi.org/10.17875/gup2021-1604

ISSN: $2199-5346$

eISSN: 2512-6881 


\section{Inhalt}

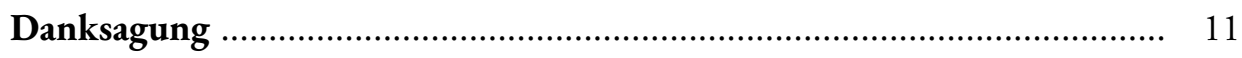

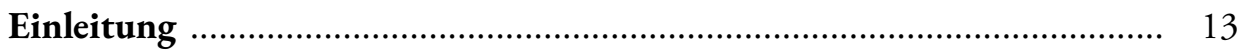

I Biographische Navigation und Zeitgestaltung .................................... 15

II Junge Menschen in Indien: Zwischen eigenen Aspirationen und gesellschaftlichen Erwartungen .............................................................. 16

Wissenschaftler*innen aus Indien und die Zeitgestaltung ...................... 17

III Zeitgestaltung: Zeit, Macht und Handlungsvermögen ........................... 18

Zeitgestaltung im Kontext ................................................................... 19

IV Mobilität und Zeitgestaltung: Die Navigation ,fremder Zeiten ' ............ 20

Gleichzeitigkeit und Zeitsouveränität .................................................... 20

Mobilität und die Gestaltung der Zukunft ........................................... 21

$\mathrm{V} \quad$ Das Regime der akademischen Mobilität ............................................ 22

VI Ethnologische Forschung mit jungen Wissenschaftler*innen aus Indien. 23

VII Aufbau des Buches ............................................................................ 25

Anmerkungen zur Schreibweise und technische Hinweise .............................. 27 
1 Theoretischer und methodischer Rahmen ...................................... 29

$1.1 \quad$ Biographische Navigation .................................................................. 30

1.1.1 Von „social navigation“ zu biographischer Navigation ............... 30

1.1.2 Biographische Navigation in sozialen Welten ............................. 32

1.1.3 Soziale Welten, Zeitlandschaften und Zeitkarten ........................ 34

1.2 Biographische Navigation und die Zeitgestaltung .................................. 37

1.2.1 Zeitgestaltung und Horizonte .................................................... 37

1.2.2 Die Gestaltung der Zukunft (Langzeithorizont) ......................... 38

1.2.3 Die Gestaltung des Alltags und besonderer Zeiten ...................... 41

1.3 Biographische Navigation und Mobilität .............................................. 43

1.3.1 Das Regime der akademischen Mobilität ..................................... 43

1.3.2 Mobilität und soziale Welten ................................................... 44

1.3.3 Mobilität, Zeitlandschaften, Zeitkarten .................................... 46

1.3.4 Mobilität und Zeitgestaltung .................................................. 47

1.4 Die Erforschung von biographischer Navigation und Zeitgestaltung .... 49

1.4.1 Wer bist du? Wer bin ich? Verortungen in der Forschung ............ 49

1.4.2 Methoden und Datenanalyse ................................................... 52

1.4.3 Göttingen als räumliches und zeitliches Feld .............................. 55

1.5 Zusammenfassung ……………………….................................... 58

2 Junge Menschen in Indien .......................................................... 61

2.1 Ein politischer und wirtschaftlicher Überblick:

Von „India Rising“ zu „India’s war on science“ .................................... 62

2.2 Person, Selbst und die Familie in der indischen Mittelschicht ............... 63

2.2.1 Person und Selbst in Indien: ,Eingebetteter Individualismus ' ...... 64

2.2.2 Die Familie: Verortung, Verantwortung und Kontrolle ............... 65

2.3 Das Aufwachsen in der indischen Mittelschicht ................................. 67

2.3.1 Die Jugend als Fundament für die Zukunft ............................. 68

2.3.2 Bildung in der middle-class: Investitionen und Erwartungsdruck 69

2.3.3 Das Bildungssystem: Gleiche Chancen für Alle? ......................... 72

2.3.4 Und nach dem Studium? Arbeitsmarktsituation in Indien .......... 74

2.3.5 Männer, Frauen und Arbeit .................................................... 75

2.4 Vorstellung vom Lebensverlauf in der indischen Mittelschicht .............. 77

2.4.1 Der genderspezifische Lebensverlauf in Indien ........................... 78

2.4.2 Das „marriagable age“: Die richtige Zeit zum Heiraten ............... 79

2.4.3 Mobilität im Lebensverlauf ...................................................... 80

2.4.4 Verbundene Lebensverläufe ..................................................... 81

2.5 Zusammenfassung ……………………………….......................... 82 
3 Das Regime der akademischen Mobilität

3.1 Zahlen und Fakten zu internationalen Studierenden und

Wissenschaftler*innen in Deutschland

3.2 Der globale Rahmen des Mobilitätsregimes ………............................... 87

3.2.1 Die Wissensökonomie ............................................................. 87

3.2.2 Die Internationalisierung der Hochschulbildung ......................... 88

3.2.3 Der akademische Lebensverlauf und Mobilitätsanforderungen ... 90

3.3 Deutschland auf der Suche nach den ,besten und schlausten Köpfen' .... 92

3.3.1 Für die Zukunft Deutschlands: Staatliche Initiativen .................. 93

3.3.2 Für die Forschung von Morgen: Institutionelle Strategien ........... 95

3.3.3 Von „IT-Indern“ und Rajesh Koothrapalli .................................. 97

3.4 Indien: Vorstellungen von Entwicklung, Bildung und Mobilität ........... 101

3.4.1 Wissen = ,Entwicklung'? .............................................................. 101

3.4.2 Bildung und Mobilität als Investitionen in die Zukunft .............. 103

3.4.3 Imaginationen von abroad ......................................................... 104

3.5 Zusammenfassung ……………..................................................... 107

$4 \quad$ Wissenschaftler*in sein und werden ............................................ 109

4.1 Alle Wege führen nach Göttingen? ......................................................... 110

4.1.1 Narrationen vom wissenschaftlichen Werdegang ......................... 110

4.1.2 Göttingen, der Ort für die wissenschaftliche Selbstverwirk-

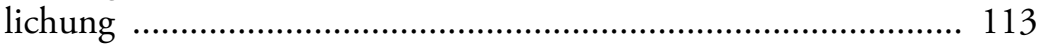

4.2 Göttingen: „A launchpad for my career“ .............................................. 115

4.2.1 Promotion auf der Überholspur .............................................. 117

4.2.2 Die Zeit in Göttingen als Zeit zum Arbeiten ............................. 118

4.2.3 Work-Life-Balance: Entschleunigung in der Beschleunigung ...... 120

4.3 Indische Wissenschaftler*innen in der „Stadt, die Wissen schafft“ ......... 123

4.3.1 Doktoreltern, Vorgesetzte und Kolleg*innen ............................... 124

4.3.2 Eine(r) von den Anderen? ....................................................... 125

4.3.3 Zwischen Religionsdiskussionen und „Delhi incident“" ............... 128

4.3.4 Indische Frauen in der Wissenschaft: „Girls with toys“ “.............. 131

4.4 Zusammenfassung .......................................................................... 133 
5 Der Alltag in Göttingen: (Gem)einsame Zeit und Gleichzeitigkeit . 135

5.1 Zeitliche Erfahrungen im Alltag .......................................................... 137

5.1.1 Vom „slow life“, neuen Freizeiten und Freiheiten ....................... 137

5.1.2 Langeweile und Einsamkeit ....................................................... 138

5.1.3 Zeitliche Horizonte im Alltag: „We know that it's temporary“ .... 140

5.2 Zeit teilen: Vielfälttige Kopräsenzen ......................................................... 143

5.2.1 Das Aufbauen neuer Freundschaften ....................................... 143

5.2.2 ,Alte` Freundschaften:

Über Raum und Zeit bestehende soziale Beziehungen ................ 150

5.2.3 Familie: Kontinuität und Kontrolle in Zeiten von ,Polymedia ' .... 152

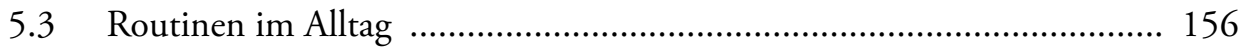

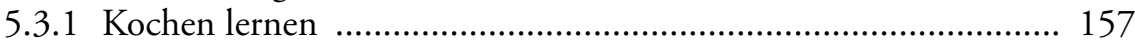

5.3.2 Kochen als alltägliche Routine: Ein bisschen Indien am Abend ... 158

5.3.3 (A)Synchronisierte Essensgewohnheiten ................................... 160

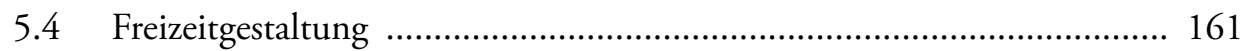

5.5 Zusammenfassung ……………………………............................. 165

6 Besondere Zeiten: Mobilität und Resynchronisation ....................... 167

6.1 „I miss home“: Die Erfahrung der Asynchronität .................................. 168

6.2 Die Indian Fraternity in Göttingen ................................................ 170

6.2.1 Die Gründung der Indian Fraternity ……................................ 170

6.2.2 Die Vereinigung und das indische „Masala“ in Göttingen ........... 171

6.2.3 Die Vereinigung und die kollektiven Zeitkarten .......................... 174

6.3 Ein Jahr in Göttingen: Von Diwali bis zum Indian Independence Day . 175

6.3.1 Diwali ................................................................................. 176

6.3.2 Saraswati-Puja: Regionale Zeitlandschaften und Zeitkarten ........ 182

6.3.3 Die „Indian Culture Night“ ...................................................... 184

6.3.4 Sportsday: Zurück in die Kindheit ......................................... 186

6.3.5 Indian Independence Day: Zeit der/für die Nation ..................... 187

6.4 Reisen nach Indien und die Zeitgestaltung .......................................... 197

6.4.1 Zeitgestaltung zwischen Resynchronisation und Hektik .............. 198

6.4.2 Reisen nach Indien und die Gestaltung des Lebensverlaufs ......... 199

6.4.3 Reisen nach Indien und das Selbst:

Kontinuitäten und Veränderungen ........................................... 200

6.5 Zusammenfassung …………........................................................... 203 
$7 \quad$ Akademische Mobilität und die Navigation von Heirat 205

7.1 Heiraten in Indien: Zwischen den Erwartungen der Familie und den eigenen Aspirationen ................................................................... 206

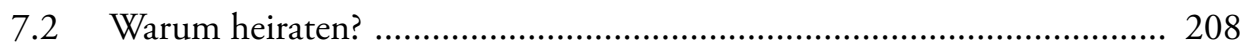

7.2.1 Von Unabhängigkeit und der Umsetzung eigener Pläne ............. 208

7.2.2 Das gute Leben: eigene und elterliche Vorstellungen ................ 210

7.2.3 Zwischen „set up marks“ und der eigenen Wahl ........................ 211

7.2.4 Alte und neue Pfade in die Zukunft .................................... 213

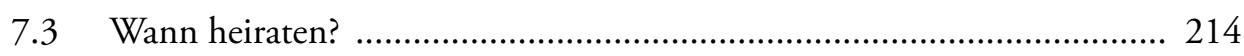

7.3.1 Die ,richtige' Zeit .............................................................. 214

7.3.2 Empowerment durch akademische Mobilität ........................ 215

7.3.3 Die richtige Reihenfolge ................................................... 217

7.3.4 Der richtige Zeitpunkt:

Zeitgestaltung und die Navigation von Zugehörigkeiten ........... 218

7.4 Wen heiraten? ............................................................................ 220

7.4.1 Von Heiratsanträgen, „Lovestories“ und dem Einfluss der Eltern 220

7.4.2 Akademische Mobilität und der Mann der eigenen Wahl ........... 221

7.4.3 Der richtige Zeitpunkt für Verhandlungen mit den Eltern ......... 222

7.4.4 Partnerwahl und Karriere ................................................... 223

7.4.5 Die Perspektive eines Mannes: unerfüllbare Erwartungen .......... 224

7.4.6 Akademische Mobilität und die Partner*innenwahl .................. 226

7.5 Auf die Heirat folgt die Bürokratie ............................................. 227

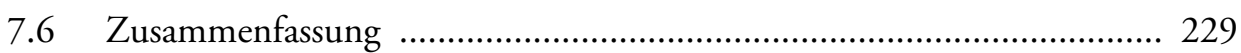

8 Die Zukunft in der Gegenwart ........................................... 231

8.1 Von Abschlüssen und Abschieden ................................................. 232

8.1.1 „PhD is not just an academic degree“ ................................... 233

8.1.2 Vom Treffen der, richtigen' Entscheidung und dualen Karrieren . 234

8.1.3 Abschluss und Abschied ..................................................... 235

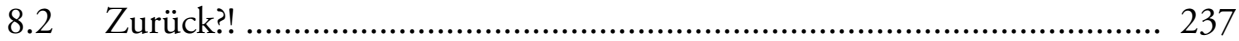

8.2.1 Rückkehr und Verantwortung für Familie und Gesellschaft ....... 237

8.2.2 Die Rückkehr: Von Vorstellungen, Wünschen und Realitäten ..... 239

8.2.3 Ein Leben hier und dort und dazwischen ................................ 243

8.3 Nächste Stationen und weitere Qualifikationen ................................ 245

8.3.1 Nächste Station: USA ......................................................... 245

8.3.2 Das Weiterentwickeln der Karriere ......................................... 246

8.3.3 „I need to do few more Postdocs“ .......................................... 247 


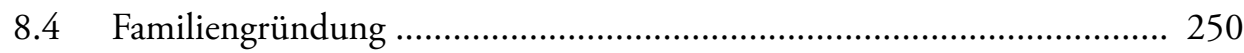

8.4.1 Karriere und/oder Kind ......................................................... 251

8.4.2 Familiengründung abroad ....................................................... 252

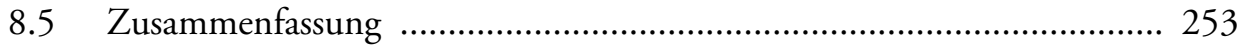

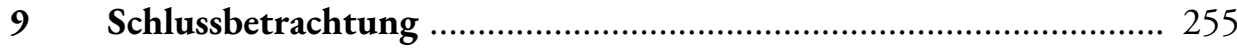

9.1 Mobile Studierende und Wissenschaftler*innen:

Mehr als ,Talente und ,Köpfe ‘.......................................................... 256

9.2 Master, $\mathrm{PhD}$, Heirat - oder:

Der ,richtige' Pfad in die ,richtige' Zukunft ......................................... 257

9.2.1 Horizonte der biographischen Navigation: Hoffnung, ,grausamer Optimismus' und ein Leben auf der Durchreise ........ 258

9.2.2 Praktiken der Zeitgestaltung:

Beschleunigen, entschleunigen, hinauszögern, ausmanövrieren ... 260

9.3 Mobilität und Zeitgestaltung ................................................................ 261

9.3.1 (Gem)einsame Zeit ................................................................... 263

9.3.2 Routinen und Resynchronisation .............................................. 264

9.4 Das Selbst in und durch die Zeit: Zwischen Kontinuität und Wandel ... 266

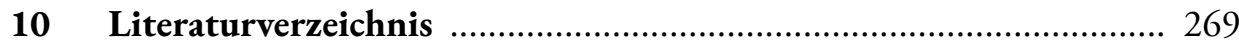

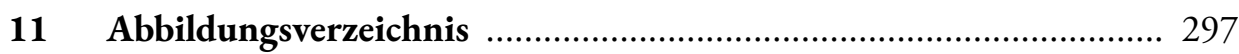

12 Kurzvorstellung der Hauptgesprächspartner*innen ........................ 299 


\section{Danksagung}

Diese Dissertation ist über einen nicht gerade unerheblichen Zeitraum von neun Jahren entstanden. In dieser Zeit haben viele Menschen zur Entstehung dieser Studie beigetragen. Zuerst gilt mein Dank Prof. Dr. Elfriede Hermann, die meine Arbeit betreut und begutachtet hat. Sie hat mir durch ihre unerschütterliche Unterstützung, ihren Glauben daran, dass ich diese Doktorarbeit schreiben kann und nicht zuletzt eine Anstellung die Promotion ermöglicht. Ich danke Dr. Julia Koch, die erst spät zu meinem Betreuungsausschuss gestoßen ist, aber eine wichtige Gesprächspartnerin in den Mittagspausen war und die mich jeden Tag durch die simple Frage „Und was machst du heute?" dazu gezwungen hat, mir Arbeitsziele zu setzen. Ich danke Prof. Dr. Steven Vertovec für seine hilfreichen Anmerkungen beim Treffen des Betreuungsausschusses. Prof. Dr. Thomas Oberlies danke ich für seine Kommentare zu den frühen Fassungen dieser Arbeit.

Ein besonderer Dank gilt den jungen Wissenschaftler*innen aus Indien, deren Veranstaltungen ich in Göttingen besuchen konnte, die mit mir über ihr Leben in Göttingen, Indien und ihre Wünsche sprachen, mir Kricket näher brachten.

Ich möchte hiermit den Menschen danken, die mit mir das Büro, Mittagspausen und die anstrengende Zeit der Promotion geteilt haben: Jelka Günther, Annika Witte, Bianca Volk, Julia Vorhölter, Paul Christensen, Jannik Schritt, Christina Schneider, Christiane Falk, Lina Klinkenborg, Valerie Liebs und Barbara Löhde. 
Außerdem danke ich meinen ,Doktorgeschwistern', die in den Kolloquien unzählige Fassungen meiner Kapitel gelesen und wertvolle Kommentare gegeben haben: Carolin Landgraf, Isabel van Manen, Wiebke Grimmig, Julia Racz, Norbert Pötzsch.

Für die Korrekturen danke ich Jovan Maud, Annika Witte, Jelka Günther, Inga Schürmann, Steffen Herrmann und Rasmus Fuhse. Meiner Familie, meinen Eltern und Geschwistern und Großeltern danke ich für ihre Geduld und ihr Verständnis.

Ich widme diese Arbeit meinem Partner und meinen beiden Kindern: Ihr macht mein Leben schöner und habt für die notwendige Ablenkung während meiner Promotion gesorgt. 


\section{Einleitung}

If you imagine your career, or your life- that includes your career, your family life - as building a house, this stage is the basement. So, your $\mathrm{PhD}$ takes roughly four years. After this, you shed your student mentality, and you start to think like a professional. Because mostly after $\mathrm{PhD}$, most of the Indians, they will get married, they will be a family person and they will have to start thinking not only for themselves but also for their life partners, and maybe they will have kids. (...). So, for that you have to have a good foundation. (Ravi $\left.{ }^{1}, 15.09 .2011\right)$

Ravi, der dreißig Jahre alt und für seine Promotion in Bioinformatik in die südniedersächsische Universitätsstadt Göttingen gekommen war, hatte eine klare Vorstellung von seiner beruflichen und privaten Zukunft. Die Realisierung seiner Zukunftsvision konzipierte er als linearen Prozess, in dem er sich vom Studenten zum „professional“ und vom Single zu einer "family person“ entwickeln würde. Die Promotion, die Phase für das Bauen des Fundaments, erhielt eine große Bedeutung - jetzt mussten wichtige Entscheidungen getroffen werden, damit das Fundament stark sein und das Haus nicht zusammenbrechen würde.

In dieser Arbeit untersuche ich anhand der spezifischen Akteursgruppe junger indischer Wissenschaftler*innen in Göttingen, wie Menschen ihre zeitlichen Erfahrungen und ihr Vermögen zur Gestaltung ihrer Zeit mit der sozialen Umwelt aushandeln und welche Rolle Mobilität in diesem Prozess spielt. Mobilität und Zeitgestaltung bilden in dieser Studie die beiden analytischen Perspektiven darauf, wie Menschen ihre multiplen Zugehörigkeiten navigieren.

\footnotetext{
${ }^{1}$ Alle Namen sind Pseudonyme.
} 
Zur Zeit meiner Forschung lebten etwa 350 indische Studierende und Wissenschaftler*innen in Göttingen, der selbst ernannten „Stadt, die Wissen schafft“2. Die meisten meiner Gesprächspartner*innen hatten in Indien bereits ein Bachelor- oder Masterstudium absolviert und waren zwischen dreiundzwanzig und dreißig Jahre alt, als sie nach Deutschland kamen. Die Bezeichnung, junge Wissenschaftler*innen habe ich gewählt, um sowohl das Alter meiner Gesprächspartner*innen als auch ihre Positionierung als angehende Wissenschaftler*innen in unterschiedlichen Phasen ihrer akademischen Laufbahn zu kennzeichnen.

Die internationale Mobilität von Studierenden und Wissenschaftler*innen ist seit einiger Zeit ein globaler Trend ${ }^{3}$ und bildet einen wichtigen Kontext für die vorliegende Studie. Die stetig anwachsende Zahl internationaler Studierender steht auch in Deutschland mit der Bedeutung im Zusammenhang, die spezifischen Formen von Wissen und den Trägern dieses Wissens - den ,Talenten' und ,schlauen Köpfen ' - für die Wissensökonomie zugeschrieben wird.

Meine Gesprächspartner*innen wie Ravi waren bzw. sind solche ,Talente', die sich selbst stark über ihre Tätigkeit in der Wissenschaft verorteten. Diese jungen Menschen waren aber nicht nur junge Wissenschaftler*innen, sondern Söhne und Töchter, Freund*innen und Ehepartner*innen. Das heißt, meine Gesprächspartner*innen hatten ein multiples Selbst, das sich durch die Zugehörigkeiten zu und die Interaktionen in verschiedenen „sozialen Welten“ (Luckmann 1970; Soeffner und Zifonun 2008: 7) konstituierte (vgl. May 2011, 2013 und 2016). Die Zugehörigkeiten zu den sozialen Welten mussten ständig (wieder-)hergestellt, bestätigt und ausgehandelt werden (vgl. Pfaff-Czarnecka 2012; Hermann, Kempf und van Meijl 2014). Genau diese Dynamik bildet den Fokus meiner Arbeit und so lauten die Hauptfragen:

- Wie handelten die jungen Wissenschaftler*innen aus Indien ihre multiplen sozialen Verortungen aus?

- Welche Rolle spielten Zeitgestaltung und Mobilität in diesen Aushandlungsprozessen?

\footnotetext{
2 Siehe u.a. Webseite der Stadt Göttingen (https://www.goettingen.de/index.php, abgerufen am 23.11.2020).

${ }^{3}$ Für das Jahr 2016 schätzt die Internationale Organisation für Migration (IOM) der UN, dass mehr als 4,8 Mio. Studierende des tertiären Bildungsbereiches international mobil waren (https://migrationdataportal.org/themes/international-students, abgerufen am 09.01.2019).
} 


\section{Biographische Navigation und Zeitgestaltung}

In meiner Studie nutze ich das Konzept der „biographischen Navigation“ (PfaffCzarnecka 2012: 11; 2013) als theoretische Perspektive auf Aushandlungsprozesse zwischen Selbst und sozialer Umwelt. Ich lege ein besonderes Augenmerk auf die Rolle von Zeit und argumentiere, dass zur biographischen Navigation in der „sozialen Umwelt“ (Vigh 2009a: 420; Vigh 2007) die Aushandlung von multiplen „timescapes“ (Adam 1998; Otto 2013: 72), also „Zeitlandschaften“ (Loimeier 2012) gehört. Die sozialen Welten, in denen Menschen verortet sind, können durch unterschiedliche Zeitlandschaften geprägt sein. Zeitlandschaften verkörpern sich in spezifischen Kontexten (Adam 2008: 1; Loimeier 2012) und bestehen unter anderem aus menschlichen und nicht-menschlichen „Rhythmen“ (Lefebvre [1992] 2007), beispielsweise von Tag und Nacht, Arbeits- und Freizeiten. Menschen navigieren Zeitlandschaften mit Hilfe von „Zeitkarten“" (Gell 1992: 242; Bear 2014: 15, 2016), das heißt Repräsentationen von Zeit, die wiederum die Erfahrung von Zeit vermitteln und Praktiken der Zeitgestaltung formen (Bear 2014: 15).

Das Konzept der Zeitkarte verwende ich in dieser Arbeit für individuelle und kollektive Vorstellungen vom ,Timing', der Dauer, der Reihenfolge, der Häufigkeit, der Geschwindigkeit und Zuteilung von Zeit sowie für abstraktere Ideen davon, wie Menschen sich durch die Zeit ,bewegen' sollten. Ich konzentriere mich in meiner Arbeit besonders darauf, wie kollektive Zeitkarten mit der individuellen Navigation interagieren und was diese Interaktion über die Beziehung zwischen Praxis und sozialer Umwelt sowie über Machtbeziehungen aussagt.

Für die biographische Navigation bieten kollektive Zeitkarten sowohl im Alltag als auch für den Lebensverlauf eine Orientierung: Sie zeigen eine gesellschaftlich sanktionierte Art und Weise der Zeitgestaltung auf und geben zeitliche Normen vor. Kollektive Repräsentationen von Zeit formen zeitliche Erfahrungen und Bewertungen, wie die der ,richtigen' und der ,falschen 'Zeit oder das Gefühl, zu wenig oder zu viel Zeit zu haben. Menschen können versuchen, auf ihre Erfahrung von Zeit einzuwirken, normative Erwartungen an ihre Zeitgestaltung auszuhandeln und zeitliche Erwartungen zu verändern. Allerdings wird das Vermögen zur Gestaltung der „Eigenzeit“ (Nowotny 1993), also die „Zeitsouveränität“ (Elchardus 1994; Garhammer 1995) von den sozialen Positionierungen geformt (Davies 1990). Ausgehend von diesen Ausführungen unterteile ich die Ausgangsfragen in folgende Teilfragen:

- Welche normativen Zeitkarten werden in der biographischen Navigation der jungen Wissenschaftler*innen sichtbar und wie werden diese ausgehandelt?

- Welche Erfahrungen von Zeit machten meine Gesprächspartner*innen in Bezug auf die Horizonte des Alltags und des Lebensverlaufs?

- Wie wirkten die jungen Menschen in ihrer biographischen Navigation auf ihre zeitlichen Erfahrungen und ihre Zeitsouveränität ein? 


\section{Junge Menschen in Indien: Zwischen eigenen Aspirationen und gesellschaftlichen Erwartungen}

Indien ist weltweit eine der Nationen mit der jüngsten Bevölkerung, fast ein Drittel der Bevölkerung ist zwischen fünfzehn und neunundzwanzig Jahre alt (Ministry for Youth Affairs and Sports 2014: 10). Diese große Anzahl junger Menschen wird in der Politik und den Medien als Problem, aber ebenso als großes Potential diskutiert. In einer guten schulischen und universitären Ausbildung der jungen Menschen, vor allem in den Natur- und Ingenieurwissenschaften, wird die Möglichkeit gesehen, Indiens ,Entwicklung' voranzutreiben (Radhakrishnan 2011; Ajantha Subramanian 2015). Für die Familien ist die Investition in die Bildung der Kinder wiederum an die Aspiration vom gesellschaftlichen Aufstieg geknüpft (Jeffery 2005: 35).

Schon in der Schulzeit werden Töchter und Söhne auf bestimmte (berufliche) Laufbahnen vorbereitet, die über ein Studium von Fächern wie Medizin, Ingenieurwissenschaften oder Jura zu Erfolg führen sollen (Säävälä 2010: 33; Gautam 2015). Vor allem junge Männer werden unter Druck gesetzt, da von ihnen erwartet wird, Hauptverdiener und Oberhaupt ihrer eigenen Familie zu werden (Donner 2015: 143; Jeffrey 2010b). Die jungen Männer versuchen, durch das Studium der ,richtigen' Fächer an den, richtigen' Universitäten den dominanten Vorstellungen eines ,richtigen' Lebensverlaufs zu folgen und so ihr Vorankommen im Leben zu sichern. Sie wollen nicht „feststecken“ und „zurückgelassen werden“, wie es beispielsweise Gesprächspartner in Craig Jeffreys Studie zur Jugend in Nordindien (2010a, 2010b) ausgedrückt haben. Gleiches befürchten viele junge Menschen weltweit, denen es nicht möglich ist, die Voraussetzungen zu schaffen, um als erwachsen zu gelten (vgl. Vigh 2007; Cole und Durham 2008a; Honwana 2012; Anne Dalsgaard et al. 2014).

In den Vorstellungen vom Lebensverlauf, die in der indischen Mittelschicht dominieren, gehört das Heiraten zum Leben dazu und ist ein wichtiger, wenn nicht der wichtigste Marker des Erwachsenseins (Osella und Osella 2000: 81; Kaur und Dhanda 2014: 271). Dies gilt sowohl für Männer als auch für Frauen, wobei es genderspezifische Vorstellungen davon gibt, in welchem Alter am besten geheiratet werden sollte (Sondhi 2013: 129). In Indien studieren mittlerweile immer mehr junge Frauen, und universitärer Erfolg, gerade in „science“, wird in hohem Maße respektiert (Mukhopadhyay 2004: 473). Allerdings sehen viele Eltern das Studium ihrer Töchter nicht unbedingt in Bezug zu einer zukünftigen Karriere, sondern in Verbindung mit Heiratsmöglichkeiten (vgl. Mukhopadhyay und Seymour 1994), da Universitätsabschlüsse ein wichtiges Kriterium auf dem Heiratsmarkt geworden sind (Donner 2015; Jeffery 2005: 29). Der Druck, den junge Frauen in Indien verspüren, bezieht sich aus diesem Grund, anders als bei den Männern, nicht auf den beruflichen Erfolg, sondern auf die Aussicht im ,richtigen 'Alter heiraten zu müssen (Sondhi 2013; Fleming 2016). Daher machen junge Frauen in Indien - und das wird in meiner Studie deutlich - weniger die Erfahrung, in der Phase der Jugend 
festzustecken, sondern haben das Gefühl, dass das Leben zu schnell auf eine Heirat und damit das Erwachsenwerden hinausläuft. Die genderspezifischen Vorstellungen des richtigen Timings interagieren unterschiedlich mit der akademischen und beruflichen Laufbahn von Männern und Frauen.

Die internationale Mobilität gehört für junge Menschen weltweit in zunehmendem Maße zur Gestaltung des Lebensverlaufs dazu und ist mit Konzepten vom Erwachsenwerden verknüpft (Robertson, Harris und Baldassar 2017). Im Fall meiner Gesprächspartner*innen verband sich diese „Mobilitätsnorm“ (Jong 2000) mit Vorstellungen der Karriereentwicklung in der Wissenschaft (Jacobs und Winslow 2004), in der internationale Mobilität ein wichtiger Faktor ist (Cohen, Duberley und Ravishankar 2015: 112; Leemann und Boes 2012: 197). Für ein Studium oder die Promotion nach Göttingen zu kommen, war aus dieser Perspektive der nächste logische Schritt auf dem Pfad, auf dem sich meine Gesprächspartner*innen bereits seit ihrer Schulzeit befanden und der sowohl von der Familie als auch dem professionellen Umfeld erwartet wurde.

Wissenschaftler*innen aus Indien und die Zeitgestaltung

Die hegemonialen Vorstellungen vom Lebensverlauf in der indischen Mittelschicht und von der Entwicklung einer Laufbahn in der Wissenschaft, wie ich sie kurz beschrieben habe, boten den jungen Menschen in meiner Studie eine wichtige Orientierung in ihrer biographischen Navigation: Sie gaben klare Koordinaten, eine spezifische Reihenfolge sowie Erwartungen vor. Allerdings setzten sie meine Gesprächspartner*innen gleichzeitig unter Druck, die richtigen Koordinaten zur richtigen Zeit anzusteuern. In dieser Situation wurde die Mobilität zu einer wichtigen Strategie, die normativen Lebensverlaufsvorstellungen zu navigieren und mit eigenen Aspirationen zu versöhnen. Die akademische Mobilität sollte dazu beitragen, die Realisierung der gesellschaftlich vorgesehenen Zukunft wahrscheinlicher zu machen und das Vorankommen im Leben zu beschleunigen. Allerdings gab es unter den jungen Wissenschaftler*innen auch einige, die die Mobilität dazu nutzten, von den eingetretenen Pfaden und den familiären Erwartungen abzuweichen und mehr Kontrolle über ihren eigenen Lebensweg zu erlangen. Ein wichtiger Faktor in diesem Prozess war die Möglichkeit, durch die Mobilität Einfluss auf den Zeitpunkt einer Heirat und die Partnerwahl auszuüben.

Der Umzug von Indien nach Göttingen bedeutete für meine Gesprächspartner*innen die Interaktion mit neuen Zeitlandschaften, Zeitkarten und sozialen Rhythmen oder, nach Cwerner (2001: 19), den „strange times“, den fremden und unvertrauten Zeiten der Universitätsstadt. Viele der jungen Wissenschaftler*innen machten die Erfahrung einer Entschleunigung ihres Alltags. Die Distanz zu ihren Familien führte zu größerer Kontrolle über die Gestaltung des Alltags, aber auch zu Gefühlen von Isolation und Langeweile. Durch unterschiedliche Strategien der Zeitgestaltung wie die Integration vertrauter Praktiken in ihr Alltagsleben, das Pflegen lokaler und transnationaler Beziehungen und das gemeinsame Begehen von Feier- 
lichkeiten konnten meine Gesprächspartner*innen diese Erfahrungen beeinflussen, sich mit Indien „resynchronisieren“ (Cwerner 2001: 23) und ihre eigene Zeitlandschaft in Göttingen reproduzieren.

Ich argumentiere in dieser Arbeit, dass die Mobilität einen großen Einfluss auf die Zeitgestaltung und die zeitlichen Erfahrungen der jungen Wissenschaftler*innen hatte. Durch die Mobilität erlangten meine Gesprächspartner*innen mehr Kontrolle über die Gestaltung ihres Lebensweges und ihres Alltags. Gleichzeitig konnten sie mit Hilfe von Strategien der Zeitgestaltung die Erfahrungen ihres mobilen Lebens im „transnationalen sozialen Raum“ (Pries 1996, 1998) navigieren. Die Navigation von Zeitlandschaften und kollektiven Zeitkarten als Teil der sozialen Umwelt ist ein wichtiger Aspekt in der Aushandlung sozialer Verortungen und der Konstruktion des Selbst.

In den folgenden Abschnitten erkläre ich die Begriffe, die den Titel dieser Arbeit bilden - Zeitgestaltung und Mobilität - und stelle die Forschungsbereiche dar, an die ich mit meiner Studie anschließe.

\section{Zeitgestaltung: Zeit, Macht und Handlungsvermögen}

Mein Verständnis von Zeit orientiert sich vor allem an Laura Bear, die Alfred Gells (1992) Konzept der Zeitkarten aufgenommen, aktualisiert und weiterentwickelt hat (Bear 2014, 2016), um Macht und Ungleichheiten in Bezug zu Zeit zu beleuchten (vgl. Nowotny 1993). Ich ziehe außerdem die feministische Perspektive von Karen Davies (Davies 1990, 2001) heran, die deutlich macht, dass Menschen ihre Zeit immer mit anderen Menschen aushandeln müssen (Davies 1990: 9). Aus diesem Fokus auf die Beziehung zwischen Macht und Zeit ergibt sich die Perspektive auf Zeit, die dieser Arbeit zugrunde liegt: Zeit verstehe ich als relational (Davies 1990, 2001: 137), als in zwischenmenschlichen Beziehungen entstehend (vgl. Nowotny 1993; James und Mills 2005a).

Ein Bereich der ethnologischen Auseinandersetzung mit der Zeit galt der Erforschung der Zeit der ,Anderen' (Evans-Pritchard 1960) und daran anschließend der Kritik der Rolle von Zeit in der Konstruktion der ,Anderen' in der ethnologischen Forschung (Fabian 1983; Adam 1988, 1995). Aufbauend auf der kritischen Betrachtung der Gegenüberstellung linearer und zyklischer Zeitvorstellungen wird mittlerweile die Pluralität von Zeiten und Zeitorientierungen betont, die nicht unbedingt zwischen, sondern innerhalb von Gesellschaften herrscht (Adam 1995: 58-60; Dilley 2005; Loimeier 2012; Otto 2013). In der Auseinandersetzung mit der Vielfalt und den unterschiedlichen Ausprägungen von Zeiten haben Ethnolog*innen und andere Sozialwissenschaftler*innen Konzepte wie „Timeshape“ (Dilley 2005: 235), „Timespace“ (May und Thrift 2001) und „Timescape“ (Adam 1998; Otto 2013) entwickelt. Ich arbeite in meiner Studie mit dem Konzept der Zeitlandschaft, da es die Verbindung der Zeiten mit konkreten Zeitorten (Loimeier 2012) und der Verkörperung in einem spezifischen Kontext (Adam 1998, 2008; Otto 2013) betont. 
Für meinen Fokus auf die Zeitgestaltung mobiler Akteure erwies sich diese Verbindung von Zeit mit Raum als besonders fruchtbar.

\section{Zeitgestaltung im Kontext}

Es existieren bereits einige Studien, die sich explizit oder implizit mit der Erforschung der Zusammenhänge zwischen Handlungs- und Wirkvermögen (agency) und Zeit beschäftigt haben. Bevor ich diese Studien kurz zusammenfasse, möchte ich an dieser Stelle festhalten, dass ich mit Sherry Ortners (1997, 1999, 2006) agency-Begriff arbeite, der aus zwei miteinander verbundenen Elementen besteht: dem Handeln in Beziehungen von Ungleichheit und Macht sowie dem Verfolgen von Projekten (2006: 139).

Wichtige Werke, die sich mit Handlungsvermögen und Zeit beschäftigen, sind Flahertys Arbeiten zur Erfahrung und Gestaltung von Zeit (Flaherty 1999, 2003, 2011, 2013), Studien zur Zeitsouveränität in der zunehmenden Flexibilisierung von Gesellschaft und Arbeitszeit (Elchardus 1994; Garhammer 1995) sowie Bears Publikationen zur „labour in and of time“ (Bear 2014, 2016). Weitere Arbeiten in diesem Bereich befassen sich mit der zeitlichen Fokussierung von Handlungsvermögen auf unterschiedliche Horizonte (Hitlin und Elder 2007), mit Praktiken, die auf die Gestaltung der Zukunft ausgerichtet sind (Ringel 2016) und Zukunftsorientierungen (Bryant und Knight 2019). Im weiteren Sinne können Appadurais Ausführungen zur Zukunft als „cultural fact“ (Appadurai 2004; 2013: 286) zu den Werken gezählt werden, die sich mit der Gestaltung der Zukunft befassen. Die diversen Schwerpunktsetzungen dieser Studien verdeutlichen die multiplen Formen, die die Interaktion von Zeit und Handlungsvermögen annimmt - ein Umstand, den ich in dieser Arbeit am Beispiel der jungen Wissenschaftler*innen zeige.

Die Zeitgestaltung, der Begriff, den ich für zeitliches Handlungs- und Wirkvermögen verwende, besteht aus zwei miteinander verbundenen Faktoren: erstens aus dem Vermögen, auf Erfahrungen von Zeit, soziale Rhythmen und andere Aspekte der Zeitlandschaft einwirken zu können, und zweitens aus der praktischen Gestaltung von zeitlichen Erfahrungen, sozialen Rhythmen und Zeitlandschaften. Ein wichtiger Ausgangspunkt für meinen Ansatz der Zeitgestaltung war Flahertys Konzept der „time work“ (Flaherty 2011: 11) und seine Beschäftigung mit der Bedeutung von zeitlichem Handlungsvermögen in der Aushandlung zwischen Selbst und Gesellschaft. Allerdings schenkt Flaherty in seiner Arbeit der Gesellschaft bzw. dem Kontext, in dem time work stattfindet, nur wenig Aufmerksamkeit. Aus diesem Grund erfasst er nicht die Ungleichheiten in den zeitlichen Erfahrungen sowie den Möglichkeiten, auf diese einzuwirken. An diesem Punkt setzte ich mit meiner Studie an und nutze die Konzepte von Zeitlandschaft und Zeitkarte, um die zeitlichen Erfahrungen, die Zeitsouveränität und Praktiken der Zeitgestaltung meiner Gesprächspartner*innen zu kontextualisieren. Die Kontextualisierung ermöglicht es, (1) den Einfluss sozialer Positionierungen und „Platzanweiser“ (Lutz 2017: 13) wie Gender und Alter auf die Zeitgestaltung zu erfassen, (2) zu verdeutlichen, mit wem die jun- 
gen Menschen ihre Zeitgestaltung aushandelten, und (3) zu verstehen, warum ihre Mobilität einen großen Einfluss auf die Zeitgestaltung hatte.

Ich beziehe, angelehnt an Hitlins und Elders Ausführungen (Hitlin und Elder 2007), den Langzeithorizont in meine Studie ein. Ich zeige, wie meine Gesprächspartner*innen versuchten, ihre Zukunft als Wissenschaftler*in, als Teil einer Familie und/oder eines Paares zu gestalten. Es wird deutlich, wie sie den richtigen Zeitpunkt für Ereignisse wie eine Heirat, den Aufbruch aus Göttingen, eine Rückkehr nach Indien oder die Familiengründung aushandelten und dabei ihre eigenen Zeitkarten entwickelten. Die jungen Menschen in meiner Studie versuchten, ganz spezifische Zukünfte herzustellen oder zu vermeiden und orientierten sich dabei an den dominanten Lebensverlaufsvorstellungen der sozialen Welten, die ihnen mögliche Pfade in die Zukunft aufzeigten (Vigh 2007: 131).

\section{Mobilität und Zeitgestaltung: Die Navigation ,fremder Zeiten'}

Saulo Cwerners Arbeiten zu den „times of migration“ (Cwerner 1999, 2001) trug dazu bei, Zeit stärker in den Mittelpunkt der Migrationsforschung zu rücken (z.B. Griffiths, Rogers und Anderson 2013; Meeus 2012; Robertson 2014; King et al. 2006; Collins und Shubin 2017; Mavroudi, Page und Chrēstu 2017). Cwerner unterscheidet acht times of migration, die daraus entstehen, dass mobile Menschen spezifische „cultures of time“ erfahren (Cwerner 2001: 17). Ich nutze das Konzept der Zeitlandschaft, um unter Hinzunahme von Cwerners Ausführungen zu den „fremden“ und „asynchronen“ Zeiten zu analysieren, welchen Einfluss die Mobilität auf die zeitlichen Erfahrungen, die Zeitsouveränität und Praktiken der Zeitgestaltung der jungen Wissenschaftler*innen hatte.

\section{Gleichzeitigkeit und Zeitsouveränität}

Ich argumentiere in dieser Arbeit, dass meine Gesprächspartner*innen sich durch ihre Mobilität in einer sozialen Umwelt mit neuer Zeitlandschaft befanden und gleichzeitig Aspekte ihrer vertrauten Zeitlandschaft ,im Gepäck' hatten. In Göttingen machten viele der jungen Wissenschaftler*innen die Erfahrung der Entschleunigung, eines langsameren und zeitlich selbstbestimmteren Lebens. Über größere Zeitsouveränität verfügten sie nicht nur durch das Leben in einer neuen Zeitlandschaft, in der andere Rhythmen und Zeitkarten vorherrschten, sondern auch durch die Entfernung zu den sozialen Beziehungen und Menschen, die ihre Zeitgestaltung in Indien kontrolliert hatten. Hier wird deutlich, dass sich die Erfahrung von Zeit und die Zeitgestaltung meiner Gesprächspartner*innen nicht ausschließlich aus ihrer Positionierung als Migrant*innen generierten, sondern ebenso aus der als junge Inder*innen, Söhne und Töchter sowie angehende Wissenschaftler*innen. Ich betone in meiner Arbeit bewusst den Einfluss multipler Positionierungen auf die zeitlichen 
Erfahrungen und die Zeitgestaltung meiner Gesprächspartner*innen. Das Konzept der Zeitkarte dient dazu, die potentiell konfliktreiche Beziehung zwischen diesen Einbindungen in der biographischen Navigation in den Blick zu nehmen.

Die Distanz zu den vertrauten sozialen Welten ging mit Gefühlen der Isolation, der Langeweile und der Asynchronität einher, auf die die jungen Wissenschaftler*innen mit Hilfe von Praktiken der Zeitgestaltung einwirken konnten. Dazu gehörten Praktiken der Resynchronisation (Cwerner 2001: 23), also der Wiederherstellung von Gleichzeitigkeit, die Integration vertrauter zeitlicher Koordinaten in das Leben in Göttingen und das Aufbauen und Aufrechterhalten lokaler und globaler sozialer Beziehungen. Diese Praktiken der Zeitgestaltung führten zu einem transnationalen Leben, das durch „simultaneity“ (Gleichzeitigkeit) (Levitt und Glick Schiller 2004) gekennzeichnet war.

Ich konzentriere mich in meiner Studie auf die Prozesse, die zu dieser Gleichzeitigkeit führten und an der Entstehung eines spezifischen transnationalen sozialen Raums (Pries 1996, 1998) mitwirkten. Meine Arbeit trägt dazu bei, die Perspektive zu erweitern, um zeitliche Erfahrungen und die Zeitgestaltung im transnationalen sozialen Raum zu berücksichtigen.

\section{Mobilität und die Gestaltung der Zukunft}

Neben Cwerners Arbeit, die sich vor allem durch die Untersuchung der multiplen Zeiten der Mobilität auszeichnet, gibt es viele Studien, die einzelne Aspekte von Zeit in den Blick nehmen. Ein Ansatz konzentriert sich auf die Interaktion von Mobilität mit dem Lebensverlauf (u.a. Findlay et al. 2015; Kirk, Bal und Janssen 2017; Kôu, Mulder und Ajay Bailey 2017). Wie in diesen Studien betont wird, hängen Entscheidungen für Mobilität, Rückkehr oder weitere Mobilitäten mit spezifischen Ereignissen im Lebensverlauf der mobilen Menschen selbst und der Menschen, mit denen sie verbunden sind, zusammen (Adrian Bailey 2009; Kóu, Mulder und Ajay Bailey 2017). Mobilität wird außerdem in Verbindung mit dem Imaginieren und Erschaffen von Zukünften analysiert (Vigh 2009b; Cole 2010; Mapril 2014; Valentin 2014; Boccagni 2017). Die Arbeiten in diesem Forschungsbereich beschäftigen sich unter anderem mit dem Einfluss zeitlicher Begrenztheit auf das Leben mobiler Menschen und deren Umgang mit dem Bewusstsein davon, dass die eigene Mobilität nur ein vorübergehender Zustand ist (Adrian Bailey et al. 2002; Griffiths 2013; Collins und Shubin 2015). Das Verständnis der eigenen Mobilität als vorübergehend kann durch die Migrationsgesetzgebung ausgelöst werden (Adrian Bailey et al. 2002) und dadurch, dass sich die mobilen Menschen selbst vorstellen, dass ihre Mobilität zu spezifischen Zielen, also „arrival points“ (Baas 2010: 6), führt. Aus dieser Dynamik entstehen Erfahrungen, die mit Konzepten wie „in-betweenness“ (Baas 2010: 7), eines Lebens im „limbo“ (Robertson und Runganaikaloo 2014) oder in „permanent temporariness" (Adrian Bailey et al. 2002; Collins und Shubin 2015) gefasst wurden. Diese Ansätze beschreiben einen Zustand der Unsicherheit, der einen Einfluss auf 
den Alltag und die sozialen Verortungen der mobilen Menschen hat (Adrian Bailey et al. 2002; Robertson und Runganaikaloo 2014; Collins und Shubin 2015).

Die jungen Menschen in meiner Studie verstanden ihre Mobilität, verbunden mit normativen Vorstellungen von der Phase der Jugend, als ein wichtiges Mittel, um ihre Zukunft zu gestalten. Die meisten meiner Gesprächspartner*innen hatten nicht vor, in Göttingen oder Deutschland zu bleiben, und verstanden ihre Zeit in der Universitätsstadt als einen Schritt auf dem Weg in die Zukunft, als ein Leben auf der Durchreise. Ich zeige, dass dieses Bewusstsein von der zeitlichen Begrenztheit und den zukünftigen Koordinaten im Alltag eine wichtige Rolle spielte: Es beeinflusste das Bilden von sozialen Beziehungen in Göttingen, Alltagspraktiken und den Umgang mit Entbehrungen und Heimweh. Ich zeige, wie sich die jungen Menschen in Göttingen ein Leben schufen und neue Vorstellungen vom Erwachsensein und -werden sowie von der Zukunft aushandelten.

\section{Das Regime der akademischen Mobilität}

Die Mobilität von Studierenden und Wissenschaftler*innen, die unter dem Begriff der "akademischen Mobilität“ (Bilecen und Van Mol 2017) zusammengefasst werden kann, hat sich zu einem wichtigen Untersuchungsgegenstand im Bereich der Migrationsforschung entwickelt (u.a. Baas 2010; Geddie 2010; Brooks und Waters 2011; Robertson 2013; Findlay et al. 2005; Findlay 2011; Findlay, King und Smith 2012; Raghuram 2013; Sondhi 2013; Hansen 2015). Die Arbeiten, die in diesem Bereich entstanden sind, zeigen die Vielzahl von Akteuren, Strukturen und Diskursen auf, die an der ,Produktion' akademischer Mobilität beteiligt sind. Dazu gehören die Wissensökonomie als größerer globaler Rahmen (Hazelkorn 2009), Strategien auf der nationalstaatlichen und institutionellen Ebene (Findlay, King und Smith 2012; Raghuram 2013; Geddie 2015) und Imaginationen, die mit Mobilität (Findlay, King und Smith 2012) und Kategorien von mobilen Menschen verbunden werden (Robertson 2015).

In meiner Studie verwende ich das Konzept des „Regimes der akademischen Mobilität", um diese multiplen Akteure, Strukturen und Diskurse in einen analytischen Rahmen zu bringen. Ich definiere das Regime der akademischen Mobilität als Geflecht von Akteuren, Strukturen, Diskursen und Imaginationen, das die Mobilität von Studierenden und Wissenschaftler*innen hervorbringt, normalisiert und reguliert (vgl. Glick Schiller und Salazar 2013; Baker-Cristales 2016). Angelehnt an Nina Glick Schillers und Noel Salazars (2013) Ausführungen über die „regimes of mobility" postuliere ich in dieser Arbeit, dass die Mobilität indischer Studierender und Wissenschaftler*innen durch ein spezifisches Regime der akademischen Mobilität als gewinnbringend für alle beteiligten Akteure (Staaten, Institutionen, Familien, Personen) und als grundsätzlich positiv konstruiert wird.

Findlay, King und Smith (2012: 121) nutzen wie viele Arbeiten zur Mobilität Studierender Bourdieus Werk (Bourdieu und Passeron 1971; Bourdieu 1982) und 
argumentieren, dass internationale Mobilität ein Mittel ist, um sich „kulturelles Kapital“ und „symbolisches Kapital“ (Bourdieu 1983) anzueignen, das von den jungen Menschen wiederum als Vorteil für ihre zukünftige Karriere verstanden wird (Findlay, King und Smith 2012: 128). Es wird deutlich, dass der Herkunftskontext internationaler Studierender und Wissenschaftler*innen in die Untersuchung einbezogen werden muss, da die Vorstellungen vom Nutzen internationaler Mobilität in lokale Imaginationen von abroad (Baas 2010) eingebettet ist und als Projekt spezifischer Klassen verstanden werden muss (Brooks und Waters 2009; 2011; Lehmann und Trower 2018). Aus diesem Grund lege ich in Kapitel 3 dar, wie der globale Wettbewerb um die ,besten Talente in Interaktion mit Aspirationen, die in Indien selbst mit Bildung und internationaler Mobilität verbunden werden, dazu führten, dass meine Gesprächspartner*innen in ihrer biographischen Navigation den Weg der internationalen Mobilität wählten.

Im Regime der akademischen Mobilität werden die jungen Inder*innen vor allem auf ihre wissenschaftliche Arbeit und Leistung reduziert und als schlaue Köpfe positioniert. Ihnen wird eine wichtige Rolle für die Zukunft der Wissenschaft und der Nation zugesprochen. Wie sie diese Verortung ausfüllten, für sich nutzten, sie ablehnten und mit anderen sozialen Verortungen kombinierten, war sehr individuell. So gab die positive Konnotation von internationaler Mobilität den jungen Menschen, die im Fokus dieser Studie stehen, ein diskursives Werkzeug für die Aushandlung eigener Aspirationen. Durch das Einbeziehen der lokalen Akteure in die ,Produktion' der akademischen Mobilität mache ich die multiplen Verortungen dieser jungen Menschen sichtbar. Meine Arbeit leistet damit einen Beitrag zu einer differenzierteren Betrachtung internationaler Studierender und Wissenschaftler*innen.

\section{Ethnologische Forschung mit jungen Wissenschaftler*innen aus Indien}

Zur Zeit meiner Forschung, die ich zwischen 2010 und 2018 mit kleineren und größeren Pausen durchführte, lebten etwa 350 indische Studierende und Wissenschaftler*innen in Göttingen. Meine Hauptgesprächspartner*innen, deren Namen ich durch Pseudonyme ersetzt habe, stelle ich im weiteren Verlauf der Arbeit vor. ${ }^{4}$ Im Folgenden gebe ich einen kurzen Eindruck davon, wer die jungen Wissenschaftler*innen waren und wie ich meine Forschung durchgeführt habe:

In Indien hatten meine Gesprächspartner*innen bereits ein Bachelor- oder Masterstudium absolviert, waren also für ein weiterführendes Studium oder eine Promotion, in einigen wenigen Fällen als Postdoktorand*innen, nach Deutschland gekommen. Als sie in Deutschland ankamen, waren sie zwischen dreiundzwanzig und

\footnotetext{
${ }^{4}$ Im Abschnitt 12 befindet sich eine Liste mit den pseudonymisierten Namen und den für diese Arbeit relevanten Daten.
} 
dreißig Jahre alt. Viele der jungen Menschen waren in einer Großstadt aufgewachsen oder für ihr Studium in eine Großstadt gezogen. Bei einem Großteil meiner Gesprächspartner*innen hatten bereits die Eltern oder zumindest häufig der Vater einen College-Abschluss gemacht. Viele hatten Schulen besucht, in denen auf Englisch unterrichtet wurde. Die jungen Wissenschaftler*innen kamen aus verschiedenen Bundesstaaten Indiens, wobei sechsundzwanzig meiner vierunddreißig Hauptgesprächspartner*innen aus vier Bundesstaaten kamen: acht aus Westbengalen, sieben aus Tamil Nadu, fünf aus Maharashtra, und fünf aus Karnataka. Die anderen kamen aus Andhra Pradesh, Delhi, Odisha, Chandigarh, Uttar Pradesh und eine Person aus Bangladesch. ${ }^{5}$ Von meinen Hauptgesprächspartner*innen waren fünfzehn Frauen und neunzehn Männer. Die jungen Wissenschaftler*innen gehörten der Mittelschicht an. Was die Zugehörigkeit zu dieser sehr vielfältigen Kategorie bedeutet, stelle ich in Kapitel 2 ausführlich dar.

Die Daten für diese Arbeit erhob ich mittels halb-strukturierter Interviews und teilnehmender Beobachtung off- und online. Ich traf meine Gesprächspartner*innen auch bei Feiern, die von der „Indian Fraternity in Göttigen“ organisiert wurden. $\mathrm{Zu}$ den Festivitäten gehörte unter anderem der Indian Independence Day, Diwali und der Indian Sportsday. In meiner Forschung wurde das soziale Online-Netzwerk Facebook zu einer wichtigen Datenquelle. Hier zeigte sich, womit sich meine Gesprächspartner*innen im Alltag beschäftigten und welche Themen ihnen wichtig waren. Und so machten mir einige Texte, die von den jungen Wissenschaftler*innen auf Facebook geschrieben und geteilt wurden, die Relevanz von Zeitgestaltung deutlich. Einer dieser Texte endet beispielsweise mit einem Aufruf, sich nach der „eigenen Geschwindigkeit“ und der ,eigenen Uhr“ zu richten:

Everything in life happens according to our time, our clock. You may look at your friends and some may seem to be ahead or behind you, but they're not, they're living according to the pace of their clock, so be patient. You are not falling behind. ${ }^{6}$

Dieser Aufruf ist ein Ausdruck der Gedanken, die sich meine Gesprächspartner*innen um ihre Lebenslaufgestaltung machten und auch in meinen Interviews artikulierten. Dabei unterschieden sich die Aspirationen, die die jungen Studierenden und Wissenschaftler*innen in unseren Gesprächen ausdrückten, in vielen Aspekten nicht von denen anderer junger Menschen: Sie wollten einen guten und (akademisch) reizvollen Arbeitsplatz, gute Beziehungen zu Freund*innen, Kolleg*innen und den

\footnotetext{
5 Zur Religionszugehörigkeit: Die Mehrzahl meiner Gesprächspartner*innen waren Hindus, zwei waren Christen und einer Buddhist. Es gab unter den indischen Wissenschaftler*innen einige wenige Muslime. Die Anzahl entspricht meines Erachtens nicht ihrem Anteil an der Gesamtbevölkerung Indiens (ca. 13\%). Eine Ursache dafür ist, dass Muslime Benachteiligungen beim Zugang zu Schul- und Hochschulbildung erfahren (vgl. Ahmad 2018; Desai und Kulkarni 2008).

${ }^{6}$ Der Text wird Julissa Loaiza zugeschrieben. Siehe Julissa Loaiza auf Lessons Learned in Life vom 15.12.2016, https://lessonslearnedinlife.com/youre-not-falling-behind/.
} 
Eltern, eine/n verständnisvolle/n Partner*in, und irgendwann eine eigene Familie. Auch die Sorgen, die die jungen Inder*innen in den Gesprächen ausdrückten, schienen sich im ersten Moment nicht von denen anderer Menschen in dieser Lebensphase zu unterscheiden. Sie überlegten, wie es nach der Promotion weitergehen sollte, wann der richtige Zeitpunkt für eine Heirat und für Kinder sei. Allerdings stellte ich im Verlauf meiner Forschung fest, dass diese Sorgen im Fall meiner Gesprächspartner*innen dadurch potenziert wurden, dass sie ganz genau wussten, was von ihnen erwartet wurde, was die, richtigen' Antworten auf die genannten Fragen waren, was der ,richtige' Pfad war, den es einzuschlagen galt. Das Bewusstsein vom richtigen Weg in die Zukunft löste in der biographischen Navigation unterschiedliche Dilemmata aus.

Für die jungen Frauen wie Smita, die 2007 für ein Promotionsstudium in Mikrobiologie nach Göttingen gekommen war, warf vor allem die Interaktion von Lebensverlaufsvorstellungen der indischen Mittelschicht und dem akademischen Lebensverlauf Fragen auf: Wie würden sie ihre wissenschaftlichen Ambitionen, ihre Leidenschaft für ihre Arbeit mit einer Ehe und den damit verbundenen Erwartungen an sie als gute Ehefrau in Einklang bringen können? Den jungen indischen Männern wie Ravi spreche ich an dieser Stelle nicht ihre Sorgen um die Zukunft ab. Sie mussten sich überlegen, wie sie den professionellen Erfolg, der von ihnen erwartet wurde, würden erreichen können. Allerdings mussten sie sich keine Sorgen darum machen, dass eine Ehe ihrer Karriere im Weg stehen könnte. Wie Smita, Ravi und die anderen jungen Menschen, die im Fokus dieses Buches stehen, mit diesen Dilemmata umgingen und sie zu lösen versuchten, werde ich in den folgenden 9 Kapiteln darlegen.

\section{Aufbau des Buches}

Ich beginne dieses Buch in Kapitel 1 mit der Vorstellung der theoretischen Konzepte und der methodischen Herangehensweise. In den ersten drei Abschnitten des Kapitels bringe ich das Konzept der biographischen Navigation als theoretische Perspektive auf die Aushandlung multipler sozialer Verortungen mit den analytischen Linsen Zeitgestaltung und Mobilität zusammen. Im vierten Abschnitt steht nach der Reflexion meiner eigenen Position während der Forschung die Datenerhebung und Auswertung im Fokus.

Kapitel 2 „Junge Menschen in Indien“ führt mich zum Kontext, in dem meine jungen Gesprächspartner*innen aufgewachsen sind. Die Lebenswelt der jungen Wissenschaftler*innen betrachte ich durch die Themen Familie, Mittelschicht und Bildung. In diesem Kapitel stelle ich eine der zentralen Zeitkarten vor, mit der meine Gesprächspartner*innen in ihrer biographischen Navigation interagierten: die Vorstellungen des Lebensverlaufs in der indischen Mittelschicht.

In Kapitel 3 „Das Regime der akademischen Mobilität“ stehen die Akteure, die an der Mobilität von Studierenden und Wissenschaftler*innen beteiligt sind, und deren Perspektive auf die Sinnhaftigkeit internationaler Mobilität im Fokus. Ich ar- 
gumentiere, dass im Regime der akademischen Mobilität drei dominante Zeitkarten wirksam werden: Vorstellungen vom akademischen Lebensverlauf, Diskurse um die lineare Entwicklung von Nationen sowie Visionen von der Zukunft, in denen internationalen Studierenden eine wichtige Rolle zugesprochen wird.

Kapitel 4 „Wissenschaftler*in sein und werden“ beginne ich mit den Interviews, in denen mir meine Gesprächspartner*innen von ihrem Werdegang und ihrer Entscheidung für ein Studium bzw. eine Promotion in Göttingen erzählten. Ich zeige nachfolgend auf, dass die jungen Wissenschaftler*innen Deutschland wählten, um auf ihrer Laufbahn ,schneller voranzukommen. Diese Strategie der Zeitgestaltung ergab sich aus der linearen Logik der Zeitkarte des akademischen Lebensverlaufs. Im dritten Abschnitt des Kapitels steht im Fokus, wie die jungen Menschen ihre Positionierung als Wissenschaftler*innen mit anderen Verortungen aushandelten.

In Kapitel 5 „Der Alltag in Göttingen: (Gem)einsame Zeit und Gleichzeitigkeit" nähere ich mich ausgehend von den zeitlichen Erfahrungen, die die jungen Wissenschaftler*innen in der Zeitlandschaft Göttingens machten, der Zeitgestaltung im Alltag. Ein Schwerpunkt liegt auf dem Aufbau und der Aufrechterhaltung lokaler und transnationaler sozialer Beziehungen und Alltagspraktiken.

Kapitel 6 „Besondere Zeiten: Mobilität und Resynchronisation“ beginnt mit der Analyse von Erfahrungen der Ungleichzeitigkeit, der Asynchronität, die meine Forschungspartner*innen machten. Viele vermissten während ihres Aufenthalts in Göttingen die Feste wie Diwali als vertraute zeitliche Koordinaten, die das Jahr strukturieren. In diesem Kapitel betrachte ich das kollektive Organisieren und Begehen der Feierlichkeiten aus der Perspektive der Resynchronisation, der Reproduktion vertrauter sozialer Rhythmen. Ein weiteres Thema des Kapitels bilden die regelmäßigen Reisen, die die jungen Wissenschaftler*innen nach Indien unternahmen und die wichtig waren, um Beziehungen zu Familie und Freund*innen aufrechtzuerhalten.

Ich zeige in Kapitel 7 „Akademische Mobilität und die Navigation von Heirat“, dass die jungen Inder*innen die Wertschätzung akademischer Bildung aus dem Ausland nutzten, um eine bessere Ausgangssituation in der Navigation verschiedener Aspekte von Heirat gegenüber den Eltern bzw. der Familie zu erreichen. Ein zentrales Argument ist, dass die internationale Mobilität ein Mittel war, um den Zeitpunkt einer Heirat bewusst hinauszuzögern.

In Kapitel 8 „Die Zukunft in der Gegenwart“ liegt der Fokus darauf, welche Vorstellungen meine Gesprächspartner*innen für ihr Leben nach dem Promotionsabschluss hatten und wie sie diese umsetzten. Drei zentrale Themen sind die mögliche Rückkehr nach Indien, das Erlangen weiterer Qualifikationen und die Familiengründung.

Im letzten Kapitel dieser Arbeit, Kapitel 9, fasse ich die zuvor gewonnenen Erkenntnisse zusammen und mache die Beiträge, die ich mit meiner Studie zur Migrationsforschung und der Untersuchung von Aushandlungsprozessen zwischen Selbst und sozialer Umwelt und der Rolle von Zeit in diesen leiste, explizit. 


\section{Anmerkungen zur Schreibweise und technische Hinweise}

Ich orientiere mich in der Schreibweise der Wörter aus dem indischen Kontext an der Schreibweise, die die jungen Wissenschaftler*innen nutzten und die sich häufig an der anglisierten Transkription ohne diakritische Zeichen anlehnte. Eine Umschrift mit diakritischen Zeichen nutze ich ausschließlich bei der Bezeichnung von Praktiken und Konzepten aus dem Hinduismus.

In dieser Arbeit verwende ich den Genderstern. Die biographische Navigation, also wie Menschen zwischen ihren Einbindungen navigieren und welche Möglichkeiten sie haben, ihre Gegenwart und ihre Zukunft zu gestalten, wird von unterschiedlichen Faktoren wie Alter und sozialer Herkunft, aber eben auch Geschlecht und Sexualität beeinflusst. In der vorliegenden Arbeit bildet die Wissenschaft als soziale Welt mit spezifischen Strukturen einen wichtigen Kontext für die Analyse. Wie meine Gesprächspartner*innen selbst thematisierten, hat Gender, also die sozial konstruierten Vorstellungen von Geschlecht und was Männlichkeit und Weiblichkeit ausmacht, einen Einfluss auf die wissenschaftliche Laufbahn - von der Fächerwahl bis zum Erreichen akademischer Positionen.

Die Zitate aus den Interviews wurden für die Publikation dieser Arbeit leicht im Sinne sprachlicher Korrektheit überarbeitet. In einzelnen, im Text markierten Fällen, habe ich die Formulierungen bewusst nicht verändert, um den spezifischen Sprachgebrauch der jungen Wissenschaftler*innen beizubehalten.

Alle Webseiten, die in dieser Arbeit angegeben werden, wurden in ihrer Abrufbarkeit überprüft und bei Archive.org gesichert. 



\section{Theoretischer und methodischer Rahmen}

In meiner Arbeit steht im Fokus, wie junge Menschen ihre zeitlichen Erfahrungen und ihr Vermögen zur Gestaltung ihrer Zeit mit der sozialen Umwelt aushandeln und welche Rolle Mobilität in diesem Prozess spielt. Mobilität und Zeitgestaltung bilden in dieser Studie die beiden analytischen Perspektiven darauf, wie Menschen ihre multiplen Zugehörigkeiten navigieren. Für meine Untersuchung verbinde ich praxistheoretische Ansätze mit Konzepten aus der Erforschung von Zugehörigkeiten, Mobilität und dem menschlichen Umgang mit Zeit. In diesem Kapitel ist es mein Ziel, die einzelnen Ansätze kurz vorzustellen, um sie dann zu verknüpfen und so einen analytischen Rahmen zu entwickeln. Ich beginne mit der Beschreibung des Konzepts der biographischen Navigation und entwickle im nächsten Schritt einen zentralen Teil meiner analytischen Perspektive, in der Zeit in den Fokus der Untersuchung von Aushandlungsprozessen zwischen Selbst und sozialer Umwelt rückt. Im darauf folgenden Abschnitt wende ich mich der zweiten analytischen Perspektive meiner Arbeit zu, der Mobilität. Die jungen Wissenschaftler*innen aus Indien verstanden internationale Mobilität als ein wichtiges Mittel, um verschiedene kurz- und längerfristige Ziele zu erreichen. Umgekehrt hatte die Mobilität einen erheblichen Einfluss auf die Navigation multipler sozialer Verortungen: Sie eröffnete neue mögliche Zugehörigkeiten und Identifikationen, um auf andere Arten und Weisen das Leben zu gestalten, und hatte einen großen Einfluss auf die Erfahrungen und die Gestaltung von Zeit. 
In der zweiten Hälfte dieses Kapitels steht meine methodische Herangehensweise im Mittelpunkt. Nach der Reflexion meiner eigenen Rolle in der Forschung gehe ich auf die Methoden der Datenanalyse ein und stelle Göttingen als räumliches und zeitliches Forschungsfeld vor.

\subsection{Biographische Navigation}

Das Konzept der biographischen Navigation verwende ich als theoretische Perspektive darauf, wie Menschen multiple soziale Verortungen aushandeln und in diesem Prozess ihr Selbst konstruieren. Das Selbst verstehe ich als multipel, fragmentiert, immer im Werden begriffen (Holland, Lachicotte jr und Skinner 1998; van Meijl 2006, 2008) und in Beziehung(en) zu anderen Menschen entstehend (May 2013: 4; Hermann 2003b).

\subsubsection{Von „social navigation“ zu biographischer Navigation}

Das Konzept der biographischen Navigation, wie es Joanna Pfaff-Czarnecka entwickelt hat, basiert auf dem Konzept der „social navigation“von Henrik Vigh (2007, 2009a). Nach Vigh bietet dieses analytische Konzept eine alternative Perspektive auf Praxis und die Intersektion zwischen Handlungsvermögen, sozialen Kräften und Wandel (Vigh 2009a: 420).

Vigh macht deutlich, dass sich nicht nur einzelne Menschen in Bewegung befinden, sondern die gesamte soziale Umwelt: „(...) we move in social environments of actors and actants, individuals and institutions, that engage and move us as we move along" (Vigh 2009a: 420). Navigation, ein Begriff aus der Luft- und Seefahrt, veranschaulicht eine besondere Form der Bewegung - jene in einer bewegten Umgebung (Vigh 2009a: 420). Durch die Benutzung des Begriffes der Navigation wird sichtbar, dass Menschen sich unbewusst oder bewusst in eine bestimmte Richtung bewegen. Das Ziel ist dabei die Verbesserung ihrer momentanen Situation und ihrer Möglichkeiten (Vigh 2009a: 425). Aber Navigation vermittelt auch, dass es Grenzen gibt, in denen Menschen ihre soziale Welt definieren und kontrollieren können. So wie das Meer die Navigation des Seefahrers beeinträchtigt, sind Menschen nie komplett frei, sich so zu bewegen, wie sie möchten (Vigh 2009a: 432). Die social navigation ist in eine Vielzahl von Machtbeziehungen eingebunden, in denen Personen bestimmende oder unterlegene Positionen einnehmen. Das Vermögen zur Bewegung ist daher von Situation zu Situation unterschiedlich (Vigh 2009a: 433). So berücksichtigt das Konzept der social navigation sowohl das Handlungsvermögen des Einzelnen als auch die Einflüsse der bewegten Umwelt, oder in Vighs Worten: „We act, adjust, attune our strategies and tactics in relation to the way we experience and imagine and anticipate the movement and influence of social forces" (Vigh 2009a: 420). Das Konzept der Navigation macht damit die Interaktivität (Jensen 1998) von Praktiken und sozialer Umwelt deutlich. So formen Handlungen die soziale Umwelt, und die 
soziale Umwelt formt Handlungen (Vigh 2009a). Das Konzept der social navigation betont die zeitliche Ebene dieser Aushandlungsprozesse zwischen sozialer Umwelt und Praktiken der Navigation:

Social navigation, in this perspective, encompasses both the assessment of the dangers and possibilities of one's present position as well as the process of plotting and attempting to actualize routes into an uncertain and changeable future. (Vigh 2009a: 425)

Gerade diese Betonung zeitlicher Dynamik in der Navigation macht Vighs Konzept für meine eigene Studie relevant. Ich werde diese Thematik in Punkt 1.2 ausführen.

Ich übernehme viele Aspekte von Henrik Vighs social navigation in meiner Studie, arbeite allerdings mit dem Begriff der biographischen Navigation (Pfaff-Czarnecka 2012, 2013). Dieser Terminus legt die Betonung stärker auf die Konstruktion des Selbst ${ }^{7}$ (Pfaff-Czarnecka 2013: 10): „Biographische Navigation besteht in fortwährenden bewussten und unbewussten Ich-Konstruktionen, Neu-Orientierungen, Aushandlungen und Positionierungen" (Pfaff-Czarnecka 2012: 58). Da Menschen gleichzeitig und nacheinander in multiplen sozialen Welten verortet sind (May 2013: 109; Pfaff-Czarnecka 2013: 9), entsteht ihre biographische Navigation, die Strategien, die sie anwenden, die Entscheidungen, die sie treffen, in der Interaktion mit diesen sozialen Welten. Genau diese Aushandlungsprozesse stehen im Fokus meiner Arbeit und so lautet die Hauptfrage:

- Wie handelten die jungen Wissenschaftler*innen ihre multiplen sozialen Verortungen aus?

Als analytische Perspektiven auf die Navigation multipler Zugehörigkeiten stehen in dieser Arbeit Mobilität und Zeitgestaltung im Fokus. Diese Perspektiven sind bei der Analyse meiner Daten, also in einem induktiven Forschungsprozess, entstanden und ermöglichen eine erste Fokussierung der Hauptfragestellung:

- Welche Rolle spielen Mobilität und Zeitgestaltung in der Aushandlung der multiplen sozialen Verortungen?

Nach einem konkreteren Blick auf die Gestalt der sozialen Welten gehe ich in den Kapitelabschnitten 1.2 und 1.3 detailliert auf die Zeitgestaltung und die Mobilität ein.

\footnotetext{
${ }^{7}$ Bei Vigh ist die Aushandlung des Selbst ein Aspekt seiner Studie, allerdings steht in seinem Fokus, wie Menschen navigieren, um überhaupt einen Platz in der Gesellschaft zu haben und um überleben zu können (Vigh 2007b; 2008).
} 


\subsubsection{Biographische Navigation in sozialen Welten}

Der Begriff der Navigation ruft durch seine Herkunft aus der Seefahrt zunächst vor allem räumliche Assoziationen hervor: Seefahrer navigieren ihr Schiff auf dem Meer, von einem Hafen zu einem anderen. Was bedeutet dies aber für das Konzept der biographischen Navigation, das sich mit der Aushandlung von Zugehörigkeiten und Identifikationen beschäftigt? Wie kann der Kontext gefasst und konzeptualisiert werden, der hier navigiert wird, und welche Rolle spielt dieser in der biographischen Navigation?

\section{Soziale Welten}

Henrik Vigh entscheidet sich in seinem Artikel Motion squared: A second look at the concept of social navigation (2009a) für den Begriff ,social environment“" (soziale Umwelt), um das Umfeld zu beschreiben, in dem Menschen navigieren. Die soziale Umwelt besteht nach Vigh aus Akteuren, Aktanten ${ }^{8}$, Individuen und Institutionen (Vigh 2009a: 420). In der vorliegenden Arbeit verwende ich diesen Begriff, wenn das Augenmerk auf dem umfassenderen Kontext der biographischen Navigation liegt. Die soziale Umwelt wird von einem Menschen aber nie vollständig erfasst. Aus diesem Grund ziehe ich zu Vighs Konzept der sozialen Umwelt jenes der „sozialen Welten“ (Luckmann 1970; Strauss 1978) hinzu. Soeffner und Zifonun definieren soziale Welten als:

(...) relatively permanent, ,institutionalized' spaces of perception and action, secured by relatively stable routines and a distribution of labor (Soeffner 1991: 363), which manifest themselves as comparatively self-sufficient fields of specialized knowledge. (Soeffner und Zifonun 2008: 7-8)

Die sozialen Positionen von Menschen entstehen in den sozialen Welten in alltäglichen Interaktionen mit verschiedenen Akteuren und Institutionen. Welche Positionen ein Mensch in einer sozialen Welt einnimmt, wird durch Faktoren wie Gender, Alter, Nationalität, gesellschaftliche Schicht und deren Intersektion beeinflusst (Yuval-Davis 2006: 199-200). Diese Faktoren, die Yuval-Davis „social locations“ (ebd.: 199) nennt, haben je nach Situation und Kontext unterschiedliche Auswirkungen auf die Machtposition eines Menschen und dessen Fähigkeit zur Navigation (ebd.; vgl. Vigh 2009a). Menschen leben gleichzeitig in verschiedenen sozialen Welten (Luckmann 1970; Strauss 1978; Soeffner und Zifonun 2008; Pfaff-Czarnecka 2012). Oder anders ausgedrückt: Die soziale Umwelt eines Menschen besteht aus einer Kombination unterschiedlicher sozialer Welten. Diese Perspektive eröffnet einen

\footnotetext{
${ }^{8}$ Der Begriff „Aktant“ kommt aus der Akteur-Netzwerk-Theorie (siehe Latour 1987, 2005). Aktanten handeln nicht allein, sondern in Netzwerken und in Verbindung mit anderen Aktanten (Bueger und Stockbruegger 2017: 50).
} 
Blick auf die vielfältigen Zugehörigkeiten meiner Gesprächspartner*innen und die Dilemmata, die durch die Eingebundenheit in multiple soziale Welten entstehen.

Die Anwendung beider Konzepte, von sozialer Umwelt und sozialen Welten, ermöglicht es mir, den Fokus in meiner Analyse unterschiedlich stark auf zwei miteinander verbundene Aspekte der biographischen Navigation zu richten: erstens auf die Navigation zwischen den multiplen Verortungen in der sozialen Umwelt; und zweitens die Aushandlung der Positionierungen innerhalb der sozialen Welten.

Im Folgenden werfe ich einen genaueren Blick darauf, was die sozialen Positionen ausmacht, wie diese entstehen und wie sie aufrechterhalten werden.

\section{Gemeinsamkeit und Gegenseitigkeit in sozialen Welten}

Soziale Welten sind mit spezifischen Wissensvorräten verbunden beispielsweise über geltende Regeln, Routinen, politische Wertesysteme oder Sprache (Soeffner und Zifonun 2008: 7; Pfaff-Czarnecka 2012: 30). Die Zugehörigkeit zu einer sozialen Welt und die mögliche Identifikation mit dieser erfordert von den Menschen die Auseinandersetzung mit diesem Wissen, das häufig implizit vorausgesetzt wird (Pfaff-Czarnecka 2012: 51). Der erfolgreiche Umgang mit dem Wissensvorrat ist ein wichtiger Aspekt von Gemeinsamkeit und Gegenseitigkeit, welche laut Pfaff-Czarnecka neben Verbindungen zur materiellen und immateriellen Welt grundlegende Dimensionen der Zugehörigkeit sind (Pfaff-Czarnecka 2012: 21).

Gemeinsamkeit definiert Pfaff-Czarnecka als „die Wahrnehmung des Teilens des gemeinsamen Schicksals, kultureller Formen (Sprache, Religion, Lebensstil), Werte, Wissensvorräte, Erfahrungen und Erinnerungskonstruktionen " (Pfaff-Czarnecka 2012: 21). Gemeinsamkeit ist wiederum multidimensional und bezeichnet auf der einen Seite implizite Vorstellungen von einem kollektiven „Wir“, auf der anderen Seite die Darstellung und Politisierung dieser Vorstellung (Pfaff-Czarnecka 2012: 21-22). Gemeinsamkeit wird häufig als eine Grenzlinie zwischen denen, die dazugehören, und denen, die nicht dazugehören, wahrgenommen. Diese soziale Grenzziehung wird durch Kategorisierungen, alltägliche Unterscheidungen und öffentliche Repräsentationen hergestellt, die wiederum eine Basis für Gemeinsamkeit bilden (Pfaff-Czarnecka 2012: 22).

Das implizite Wissen, die Sprache, Normen und Regeln sowie das Einverständnis über diese sind die Voraussetzung für Beziehungen der Gegenseitigkeit in einer sozialen Welt. Auf diese Weise wird die Gegenseitigkeit gemeinsam mit Loyalität, Verpflichtungen und Engagement (Pfaff-Czarnecka 2012; Guibernau 2013) zum ,Kleber' zwischen den Elementen in einer sozialen Welt. Kollektive versuchen, die verschiedenen Bindemittel der Gemeinsamkeit aufrechtzuerhalten und zu stabilisieren. Dieser Prozess kann ganz unterschiedlich ausfallen und unterschiedliche Typen der Gegenseitigkeit hervorbringen: formalisierte Mitgliedschaften, gemeinschaftliche Verbände oder auch freiwillige Zusammenschlüsse (Pfaff-Czarnecka 2012: 31). Gemein ist der Zusammengehörigkeit in einer sozialen Welt, sei es in einer Nation, einer Familie oder einem Freundeskreis, dass sie auf „gegenseitiger Kontrolle und der 
Aufrechnung von Leistungen" beruht (Pfaff-Czarnecka 2012: 32). Die Verortung in einer sozialen Welt gibt dadurch nicht nur ein Gefühl der Sicherheit und der Orientierung, sondern verlangt gleichermaßen Arbeit und aktive Teilnahme.

Die biographische Navigation besteht aus der Aushandlung multipler Beziehungen der Gemeinsamkeit und Gegenseitigkeit in unterschiedlichen, sich zum Teil überschneidenden sozialen Welten. Die Zeit ist in ganz unterschiedlichen Formen ein wichtiger Faktor in den Aushandlungsprozessen.

\subsubsection{Soziale Welten, Zeitlandschaften und Zeitkarten}

Es gibt unterschiedliche Ansätze in der Analyse von Zeit, die in drei Richtungen eingeteilt werden können: die phänomenologische Tradition (Gell 1992), eine praxistheoretische Herangehensweise an Zeit (Bourdieu 1976; Certeau 1984; Munn 1992) und neuere Studien, die Bezug auf die Arbeiten des Philosophen Bergson (Bergson [1869] 1988; Deleuze 1994) und sein Konzept der „la durée“ nehmen (siehe Hodges 2008; Eisenlohr 2015; Fleming 2016). Diese Ansätze sind sich darin einig, dass es nicht die eine Zeit gibt, sondern multiple Zeiten, die sich gegenseitig durchdringen und in allen Aspekten des Lebens enthalten sind (Adam 1995, 1998, 2008; Cwerner 2001: 14; James und Mills 2005b; Loimeier 2012; Shove 2013b; Bear 2014; Frederiksen und Anne Dalsgaard 2014). ${ }^{9}$ Um die Vielfältigkeit von Zeit zu fassen, arbeite ich in meiner Studie, angelehnt an die Arbeiten von Barbara Adam (1998, 2008), Roman Loimeier (2012) und Ton Otto (2013) mit dem Konzept der Zeitlandschaft. Obwohl die Autor*innen unterschiedliche Elemente von Zeitlandschaften in den Blick nehmen, ${ }^{10}$ ist den Studien gemein, dass sie die Vielfalt von Zeitlandschaften und deren mögliche konfliktreiche Beziehung betonen. In der vorliegenden Arbeit sind folgende Elemente von Zeitlandschaften besonders relevant: soziale Rhythmen (Lefebvre [1992] 2007) ${ }^{11}$ - beispielsweise von Arbeitszeiten, Freizeiten und Feiertagen - sowie zeitliche Orientierung und Wertschätzungen (vgl. Otto 2013: 72).

Gesellschaften können durch unterschiedliche Zeitlandschaften geprägt sein, und innerhalb einer Gesellschaft kann es unterschiedliche Zeitlandschaften geben, die unter Umständen miteinander konkurrieren (Otto 2013). ${ }^{12}$ Um mich der Vielfalt der Zeitlandschaften zu nähern, kopple ich das Konzept der Zeitlandschaft an jenes der sozialen Welt. Ich gehe davon aus, dass soziale Welten durch unterschied-

\footnotetext{
9 Adam (2008: 2) zählt beispielsweise folgende Elemente als essentiellen Teil von Zeitlandschaften auf: timeframe, temporality, timing, tempo, duration, sequence und temporal modalities.

${ }^{10}$ Roman Loimeier (2012: 18) untersucht die Ausprägung einer Zeitlandschaft an einem bestimmten Zeitort (Sansibar), Otto (2013: 72) koppelt seinen Begriff der Zeitlandschaft an spezifische Akteursgruppen und Adam (1998) entwickelte ihren Zeitlandschaftsbegriff in der Auseinandersetzung mit dem menschlichen Umgang mit der Umwelt.

${ }^{11}$ Lefebvre ([1992] 2007) betont, dass Rhythmen im Alltag aktiv reproduziert werden. Dieses Verständnis von Rhythmus liegt meiner Verwendung des Begriffes zugrunde.

12 Roman Loimeier (2012) verwendet Zeitlandschaft als den Oberbegriff und ordnet diesem die verschiedenen Zeitordnungen unter, die es in Sansibar gibt.
} 
liche Zeitlandschaften geprägt sein können. Diese Kombination erlaubt es mir, die Vielfältigkeit von Zeiten zu erfassen und die Rolle von sozialen Positionierungen für die Navigation dieser Zeiten herauszuarbeiten.

Im Konzept der Zeitlandschaft steht der Wortabschnitt „scape“ wie bei Appadurais Verwendung (Appadurai 1996: 33) für die Perspektivität (Otto 2013): Je nach Standort in der Landschaft ergibt sich eine andere Perspektive. Der Begriff der Zeitlandschaft verdeutlicht des Weiteren die Verbindung zwischen Zeit und Raum ${ }^{13}$. Barbara Adam postuliert: „(...) we cannot embrace time without simultaneously encompassing space and matter, that is, without embodiment in a specific and unique context" (Adam 2008: 1). Die spezifischen Kontexte, in denen sich Zeit verkörpert, sind konkrete Orte, aber ebenso andere räumliche Settings wie Schulen oder Arbeitsplätze (vgl. Nowotny 1993). Die Verbindung von Zeitlandschaften mit spezifischen geographischen Lokalitäten wird besonders für mobile Menschen spürbar, eine Thematik, die ich unter Punkt 1.3 weiter ausführe.

Der räumliche Landschaftsbegriff macht außerdem greifbar, wie Menschen Zeiten navigieren: mit Hilfe von Koordinaten und zeitlichen Karten (Otto 2013: 72; Gell 1992; Bear 2014; 2016). Die Idee zeitlicher Koordinaten findet sich bereits in Alfred Gells Konzept der „temporal maps“ (Gell 1992). Gell versteht Zeitkarten als Repräsentationen, die Menschen erschaffen, um in der Zeit zu navigieren (Gell 1992: 235-236). Laura Bear greift das Konzept auf und entwickelt es weiter, um die „collective, antagonistic and normative nature of time-maps“ in den Blick zu nehmen (Bear 2014: 17). Zeitkarten, also die Repräsentationen von Zeitlandschaften, vermitteln und formen die persönlichen Erfahrungen von Zeit (Bear 2014: 15) und sind mit Formen von Handlungsvermögen verbunden (Bear 2014).

Laura Bear postuliert, dass Zeitkarten aus der Perspektive der menschlichen Akteure mit „distinct spheres of action and aspects of personhood“ (Bear 2016: 496) verbunden sind. Die Repräsentationen von Zeit mit spezifischen sozialen Welten zusammenzubringen, ist eine wichtige Bedingung, um zu verstehen, warum Unterschiede in den Erfahrungen, in dem Vermögen zur Gestaltung der Zeit und deren Repräsentationen entstehen und warum die Multiplizität von Zeitkarten für Menschen zu Spannungen in ihrer biographischen Navigation führen kann. Zeitkarten sollten nicht nur als individuelle Mittel der Navigation von Zeit untersucht werden, sondern auch als Repräsentation, die von Kollektiven hergestellt und genutzt werden (Bear 2014: 16). Für die biographische Navigation stellt sich die Frage nach der Interaktion der beiden Ebenen: Wie beeinflussen kollektive Zeitkarten die individuelle Navigation, und wie wirkt die individuelle Navigation zurück auf kollektive Repräsentationen von Zeit?

Zeitkarten können relativ abstrakte Vorstellungen vom Modus von Zeit beinhalten. Dazu gehören das Verständnis von Zeit als „process“ (Hodges 2014), der Idee des Fortschritts (Nowotny 1993; Tsing 2015) oder, wie ich zeige, Vorstellungen von

\footnotetext{
${ }^{13}$ Die Verbindung zwischen Zeit und Raum wird auch in Konzepten wie „Timespace“ (May und Thrift 2001; Mavroudi, Page und Chrēstu 2017) betont.
} 
der ,Entwicklung' von Nationen und Personen. Diese drei Repräsentationen von Zeit sind linear und schaffen dadurch spezifische zeitliche Erfahrungen, Rhythmen und Praktiken der Zeitgestaltung. Außerdem gibt es Zeitkarten, die konkrete Koordinaten für den Einzelnen und die Gemeinschaft vorgeben.

Aufbauend auf der Analyse meiner Daten und der Beschäftigung mit Arbeiten zu Alltagspraktiken und Zeit (Davies 2001; Flaherty 2011; Shove 2013b) argumentiere ich, dass Zeitkarten folgende Faktoren beinhalten: Vorstellung vom Timing, der Dauer, der Reihenfolge, der Häufigkeit, der Geschwindigkeit und Zuteilung von Zeit und abstrakte Auffassungen davon, wie Menschen sich individuell und kollektiv durch die Zeit bewegen. Aus diesen Vorstellungen erwachsen Erfahrungen von Zeit, soziale Rhythmen und Praktiken der Zeitgestaltung und letztendlich die spezifischen Zeitlandschaften sozialer Welten.

\section{Zeitkarten und Macht}

Zeitkarten schaffen Rhythmen (Bear 2014) und wirken als Orientierung für die Gemeinschaft und den Einzelnen. Da die Repräsentationen von Zeit an soziale Welten gekoppelt sind, artikulieren sie Zugehörigkeiten und Prozesse von Exklusion und Inklusion. Hier ist das Beispiel des Kalenders besonders illustrativ (vgl. McCrone und McPherson 2009a): Welcher Tag wird zum Nationalfeiertag erklärt? Welche religiösen Feste werden zu Feiertagen? Im Fall des Kalenders sind es staatliche oder religiöse Autoritäten, die die Kontrolle über die Gestaltung und damit über soziale Rhythmen haben (vgl. Gell 1992: 312), sie legen die Zeit für die Feiertage fest und bestimmen „wann die Arbeit zu ,ruhen“ hat" (Nowotny 1993: 109). Staat und Markt, aber auch religiöse Institutionen haben einen großen Einfluss auf die Zeitlandschaft sozialer Welten, da sie Zeitkarten gestalten und verwalten, die den Alltag der Menschen strukturieren (vgl. Nowotny 1993: 110; Loimeier 2012). Darüber hinaus sind es aus der Perspektive des Einzelnen vor allem andere Menschen, die die Kontrolle über die Zeit ausüben.

In den sozialen Welten, in denen Menschen verortet sind, existieren „zeitliche Normen" (Flaherty 2011:38), die sich beispielsweise in Vorstellungen vom richtigen Timing für Praktiken wie das Essen artikulieren (Zerubavel 1981). Die zeitlichen Normen verlangen unterschiedlich stark nach ihrer Einhaltung durch die Mitglieder einer sozialen Welt (Flaherty 2011: 38). Aus dieser Perspektive wird deutlich, dass Zeit in Verbindung mit Macht gedacht werden muss (vgl. Munn 1992; Nowotny 1993: 146; Sharma 2014). Nicht alle Menschen haben die gleiche Macht darüber, wie sie ihre Zeit verbringen und wann oder wie häufig sie Praktiken durchführen. Die soziale Positionierung in der sozialen Umwelt beeinflusst, wie Menschen Zeit erfahren und wie viel Kontrolle sie über die Gestaltung von Zeit haben (Davies 1990; Davies 2001; Nowotny 1993: 108, 138). Nowotny stellt fest, dass Zeit vor allem als Konflikt erfahrbar wird (Nowotny 1993: 108). In der Analyse der Konflikte um die Zeit - zum Beispiel um den richtigen Zeitpunkt oder die richtige Dauer - werden Machtverteilung und normative Zeitkarten einer sozialen Welt erfassbar. 
Über diese Ausführungen zur Wirkung von Zeitkarten und der Kontrolle von Zeit ergibt sich das Verständnis von Zeit, das dieser Arbeit zugrunde liegt: Zeit verstehe ich als relational (Davies 1990, 2001: 137), als in zwischenmenschlichen Beziehungen entstehend: „Denn Zeit, dieses zutiefst kollektiv gestaltete und geprägte symbolische Produkt menschlicher Koordination und Bedeutungszuschreibung, behält ihren Bezug zu den anderen Menschen selbst in den Momenten ausgeprägten individuellen Empfindens" (Nowotny 1993: 9). Oder wie Wendy James und David Mills passend formulieren: „There is no such thing as a person's time“ (James und Mills 2005a: 4). Im folgenden Abschnitt beschäftige ich mich damit, was das Verständnis von Zeit als relational für das Handlungsvermögen bedeutet.

\subsection{Biographische Navigation und die Zeitgestaltung}

Die im vorherigen Abschnitt beschriebenen Eigenschaften von Zeitkarten - als wichtige Orientierung, und als möglicherweise einschränkende Norm - bringe ich im Folgenden mit Studien zum menschlichen Handlungsvermögen zusammen. Wie erfahren Menschen Zeit und wie wirken sie auf die Erfahrung ein? Welche sozialen Rhythmen navigieren sie und wie beeinflussen sie diese?

\subsubsection{Zeitgestaltung und Horizonte}

Den Ausgangspunkt für mein Konzept der Zeitgestaltung bilden Flahertys Arbeiten zum menschlichen Umgang mit Erfahrungen von Zeit (Flaherty 1999, 2003, 2011) und sein Begriff der „time work“ (Flaherty 2003, 2011). Flaherty rekurriert für sein Verständnis von Handlungsvermögen unter anderem auf das Werk von George Herbert Mead (Mead 1970) und versteht unter time work ,intrapersonal and interpersonal effort directed toward provoking or preventing various temporal experiences" (Flaherty 2011: 11). Flahertys Fokus liegt damit vor allem darauf, wie Menschen auf ihr Selbst und ihre eigenen zeitlichen Erfahrungen einwirken.

Zeitliche Erfahrungen und deren Gestaltung sind auch in meiner Studie wichtige Themen. Anders als Flaherty konzentriere ich mich aber stärker auf die sozialen Beziehungen, in die die Aushandlung und Gestaltung von Zeit eingebettet sind. Aus diesem Grund leite ich meinen Begriff der Zeitgestaltung von Sherry Ortners agency-Begriff ab (1997, 1999, 2006). Agency hat nach Ortner zwei miteinander verbundene Bedeutungen. Zum einen steht agency für intentionales Handeln und das Nachgehen von (kulturell definierten) Projekten (Ortner 2006: 139). Zum anderen bezeichnet agency das Handeln in Beziehungen von Ungleichheit und Macht (2006: 139). Ähnlich wie Ortner unterscheide ich in meiner Analyse der Interaktion kollektiver Zeitkarten mit der biographischen Navigation meiner Gesprächspartner*innen zwei miteinander verflochtene Faktoren von Zeitgestaltung: erstens das Vermögen, auf die Erfahrungen von Zeit, soziale Rhythmen und weitere Aspekte der Zeitlandschaft einwirken zu können, und zweitens die praktische Gestaltung von zeitlichen 
Erfahrungen, sozialen Rhythmen und Zeitlandschaften. Den ersten Aspekt fasse ich unter dem Begriff der „Zeitsouveränität“, der die Möglichkeit beschreibt „of recovering one's own time from all foreign-determined orders of time“" (Garhammer 1995: 176) ${ }^{14}$. Nach Garhammer umfasst Zeitsouveränität „the chance either to follow a collective rhythm or to deviate from it" (Garhammer 1995: 177, Hervorhebung im Original).

Ich untersuche die Zeitsouveränität und die Praktiken der Zeitgestaltung meiner Gesprächspartner*innen mit dem Blick auf die unterschiedlichen ,zeitlichen Horizonte' (Hitlin und Elder 2007), auf die diese ausgerichtet sind. Das Konzept der Zeitkarte benutze ich in dieser Arbeit als Werkzeug, um den von Hitlin und Elder (2007: 171) eingeführten Begriff des „time horizon“ zu konkretisieren. Ich gehe davon aus, dass die Koordinaten individueller und kollektiver Zeitkarten Horizonte schaffen und die relative Nähe bzw. Ferne der Horizonte bestimmen. Außerdem beeinflussen die Zeitkarten, welches Ereignis sich am Horizont vorgestellt wird.

Statt in meiner Analyse nach Zeitlandschaften und Zeitkarten zu ordnen, habe ich mich für die Perspektive der Horizonte entschieden, da ich auf diese Art und Weise die Wirkung erfassen kann, die zeitliche Koordinaten, die von Menschen in ihrer Zukunft lokalisiert werden, in der Gegenwart entfalten. Anders ausgedrückt, konnte ich mittels des Begriffes des Horizonts die Koexistenz von Gegenwart und Zukunft in der biographischen Navigation und damit deren zeitliche Komplexität greifen (Collins und Shubin 2017) ${ }^{15}$. Für meine Studie erwachsen die folgenden weiterführenden Fragen:

- Welche konkreten Horizonte waren in der biographischen Navigation der jungen Wissenschaftler*innen besonders relevant?

- Welche Wirkung entfalteten die Horizonte in der biographischen Navigation?

Durch die Anwendung des Konzepts der Horizonte ergeben sich unterschiedliche zeitliche Rahmen für die Analyse der biographischen Navigation und der Zeitgestaltung. In den folgenden beiden Kapitelabschnitten konzentriere ich mich auf die Zukunft und den Alltag als Horizonte der Zeitgestaltung.

\subsubsection{Die Gestaltung der Zukunft (Langzeithorizont)}

Imaginationen von der Zukunft bieten eine Orientierung und Motivation für die biographische Navigation in der Gegenwart. Sie sind mit der Hoffnung verbunden, dass sich die Zukunft gestalten lässt, und tragen damit zur Handlungsfähigkeit des

\footnotetext{
${ }_{14}$ Garhammer bezieht sich an dieser Stelle auf den Sammelband von Rainer Zoll(1988): Zerstörung und Wiederaneignung von Zeit. Frankfurt am Main: Suhrkamp.

${ }_{15}$ Francis Leo Collins und Sergei Shubin argumentieren für einen größeren Fokus auf die komplexe Beziehung zwischen Vergangenheit, Gegenwart und Zukunft (Collins und Shubin 2015, 2017). In ihrer Analyse von Zeit in der Migration greifen Collins und Shubin auf die Arbeiten des Philosophen Heidegger zurück: M. Heidegger ([1927] 2006): Sein und Zeit. Tübingen: Max Niemeyer Verlag.
} 
Menschen bei (vgl. Griffiths, Rogers und Anderson 2013; Steffen Dalsgaard und Nielsen 2016: 13). Die Möglichkeiten, auf eine Zukunft zu hoffen (Appadurai 2013: 289), und mehr noch die Fähigkeiten zur Realisierung einer erhofften Zukunft sind allerdings nicht gleich verteilt (Appadurai 2013; Bourdieu [1997] 2017).

\section{Lebensverlaufsvorstellungen als Zeitkarten}

Für die meisten Menschen gibt es nicht unendlich viele Möglichkeiten für die Zukunft, sondern nur spezifische Pfade, die von ihrer sozialen Umwelt bedingt werden (Cole 2010; Hansen 2015). Diesen Umstand macht Vigh sehr deutlich: Menschen bewegen sich „towards possible clearings and along possible routes emerging in the social options or embedded in the social ties of the given context" (Vigh 2007: 131). Die möglichen Routen in die Zukunft werden also von den eigenen Positionen in der sozialen Umwelt beeinflusst. Einen bedeutenden Effekt auf Vorstellungen von Wegen in die Zukunft haben Konzeptionen des Lebensverlaufs. In dieser Arbeit verstehe ich Vorstellungen vom Lebensverlauf als Zeitkarten, die zeitliche Koordinaten für Ereignisse im Leben wie eine Heirat oder das Erwachsenwerden festlegen. Mit Cwerners Worten sind Vorstellungen vom Lebenslauf „temporal norms referring to the sequence, duration, timing and tempo of socially expected patterns of behaviours, actions, careers and life paths" (Cwerner 2001: 14). Diese Vorstellungen können sich in einer sozialen Welt nach Faktoren wie Gender unterscheiden, wie ich in dieser Arbeit herausstelle.

Lebensverlaufsvorstellungen sind Repräsentationen von Zeit, die sozial konstruiert sind und durch menschliche Praktiken reproduziert werden (Gubrium, Holstein und Buckholdt 1994: 2-3). Die Wirkung der Vorstellungen vom Lebensverlauf auf die Möglichkeiten, sich die Zukunft vorzustellen, macht Jennifer Cole deutlich: „they make certain paths, certain trajectories, easier to imagine than others" (Cole 2010: 16). Mit dieser Perspektive auf den Lebensverlauf wird erkennbar, dass Konzepte von Entwicklungsstadien wie Kindheit, Jugend oder Erwachsensein Kategorien sind, die Menschen entwerfen und denen sie Bedeutung zuschreiben (Gubrium, Holstein und Buckholdt 1994: 2). Laut Gubrium et al. (1994) benutzen Menschen diese Kategorien und Beschreibungen, um Veränderungen im Leben zu erklären. In diesem Sinne sind der Lebensverlauf und die damit verbundenen Begriffe eine Art und Weise, über menschliche Erfahrungen in Beziehung zu Zeit zu sprechen und diese zu strukturieren (Gubrium, Holstein und Buckholdt 1994: 2-3). Welche Funktion hat die Vorstellung eines Lebensverlaufs in der biographischen Navigation?

(...) human groups, both across cultures and throughout history, seem to use various age stages or thresholds as landmarks indicating to one another where they stand and what they can expect to encounter next on life's journey. (Gubrium, Holstein und Buckholdt 1994: 5) 
Konzepte vom Lebensverlauf machen das Leben berechenbarer. Durch Vorstellungen des Lebensverlaufs können sich Menschen eine Zukunft ausmalen und erhalten eine Orientierung für ihre Handlungen in der Gegenwart.

In dieser Arbeit zeige ich, dass Vorstellungen, die sich auf den Verlauf des Lebens beziehen, nicht nur mit der sozialen Welt der Familie verknüpft sind, sondern auch mit spezifischen Berufsfeldern. In der Wissenschaft und speziell an der Universität gibt es klare Karrierewege, einen „academic-life course“ (Winslow und Davis 2016: 405), der eine recht deutliche Route durch die Zeit vorsieht.

\section{Wirkung und Gestaltung von Lebensverlaufsvorstellungen}

Konzepte des Lebensverlaufs geben nicht nur Orientierung für die biographische Navigation, sondern, wie Gubrium, Holstein und Buckholdt (1994) zeigen, werden diese normativen Repräsentationen des Lebensverlaufs als Mittel der Kontrolle genutzt und schränken das aktive Ausgestalten des eigenen Lebens ein:

Images of the life course can be used both overtly or subtly to influence, motivate, and even coerce behavior, rendering some actions, occurrences, and characteristics accountable or acceptable while casting others as inappropriate. (Gubrium, Holstein und Buckholdt 1994: 186)

In der biographischen Navigation spielen Vorstellungen vom Lebensverlauf also eine ambivalente Rolle. Normative Vorstellungen vom Lebensverlauf formen Erfahrungen von Zeit, soziale Rhythmen und Praktiken der Zeitgestaltung. Besonders Studien zu jungen Menschen (Cole und Durham 2008b; Jeffrey 2010a) zeigen, dass die inhärente Linearität von Lebensverlaufsvorstellungen spezifische zeitliche Erfahrungen hervorruft, die mit Konzepten wie „timepass“ (Jeffrey 2010a) und „waithood" (Singerman 2007; Dhillon und Yousef 2009) gefasst wurden. Beide Begriffe beschreiben Gefühle des „Steckenbleibens“ im Lebenslauf, die Erfahrung, nicht voranzukommen, zu langsam zu sein. Diese Erfahrungen und ebenso die Vorstellungen klarer Koordinaten für den Lebensverlauf (Heiraten, Kinder bekommen etc.) regen Praktiken der Zeitgestaltung an. So können Menschen versuchen, das Ankommen an den Koordinaten zu verzögern oder die Realisierung der imaginierten Zukunft wahrscheinlicher zu machen. Die Zukunft ist in diesem Sinne nicht vorbestimmt, sondern wird in der biographischen Navigation immer wieder neu hergestellt.

Menschen können sich bemühen, die Zukunft, die eine soziale Welt und ihre Zeitkarten mehr oder weniger fest vorschreiben, zu realisieren, oder den Versuch unternehmen, diese zu vermeiden oder anders zu gestalten. Diese Praxis impliziert, dass Menschen eine klare Vorstellung davon haben, was kommen wird, und versuchen, die erwartete Zukunft zu verändern (Bourdieu 2017: 302). Um zu beschreiben, wie Menschen versuchen, Zukünfte, die sie erwarten, zu verhindern oder hinauszuzögern, ziehe ich Felix Ringels Konzept des „future-tricking“ heran (Ringel 2016). Future-tricking bezeichnet Praktiken, die darauf abzielen „to subject the fu- 
ture content of the progression of time to our agency“ (Ringel 2016: 26). Zu diesen Praktiken gehören unter anderem das Planen, Hoffen, Designen, Koordinieren oder auch das Organisieren (Ringel 2016: 26).

Wie sich die Vorstellungen des Lebensverlaufs je nach sozialer Welt, aber auch anhand von Faktoren wie Gender unterscheiden, variieren die Möglichkeiten, den eigenen Lebensverlauf zu gestalten. Aus den vorangehenden Ausführungen ergeben sich folgende weiterführende Fragen für meine Arbeit:

- Welche Vorstellungen hatten meine Gesprächspartner*innen von ihrer Zukunft? Welche normativen Zeitkarten werden in diesen Imaginationen sichtbar und wie werden diese navigiert?

- Hatten die jungen Menschen ein Gefühl der Kontrolle über ihren Lebensverlauf? Durch welche Strategien versuchten sie, eine größere Zeitsouveränität in Bezug zum Verlauf ihres Lebens zu erlangen?

\subsubsection{Die Gestaltung des Alltags und besonderer Zeiten}

Der Alltag als Untersuchungshorizont ermöglicht einen Blick auf andere Zeiten und Zeitkarten als jener des Lebensverlaufs. Die Zeitlandschaft des Alltags ist weniger durch Linearität als durch Zeitkarten der Wiederholung und von Routinen gekennzeichnet.

\section{Routinen und Rhythmen}

Aus der Art und Weise, wie allägliche Praktiken koordiniert werden, entstehen Rhythmen (Shove, Trentmann und Wilk 2013: 10; Lefebvre [1992] 2007), die sich von Mensch zu Mensch, aber auch von Gesellschaft zu Gesellschaft unterscheiden (Shove, Trentmann und Wilk 2013: 4). Der vertraute Rhythmus einer Zeitlandschaft entsteht durch das Zusammenspiel kollektiver und individueller Zeitkarten und deren Interaktion mit menschlichen und nichtmenschlichen Rhythmen an einem Ort.

Routinen und Rhythmen stellen eine Ordnung her, sie reduzieren die Komplexität von Aufgaben, sparen Zeit und Energie und kreieren ein Gefühl der Sicherheit (Wilk 2013: 147). Wie bereits angesprochen, spielen Staat und Markt eine wichtige Rolle für die zeitliche Strukturierung des Alltags (Nowotny 1993: 110). Allerdings sind es die Menschen, die die entsprechenden Zeitkarten, wie die der Arbeitszeitregelung, umsetzen und reproduzieren. Die Gestaltung des Alltags liegt nicht nur in den Händen Einzelner, sondern wird von den normativen Zeitkarten der sozialen Welten beeinflusst, die zeitliche Normen und Erwartungen formulieren: Was soll im Alltag mit der Zeit angefangen werden? Wie häufig, wann, wie lang oder in welcher Reihenfolge sollten Praktiken durchgeführt werden? (Flaherty 2011; Shove 2013a: 25). Da Menschen in mehreren sozialen Welten gleichzeitig verortet sind, haben 
sie mehrere „temporal reference groups“ (Flaherty 2011: 39) und müssen deswegen unterschiedliche Ansprüche an ihre Zeit navigieren.

In jeder sozialen Welt gibt es ,besondere' Zeiten, die von der Alltagszeit abgegrenzt werden. Dazu gehören religiöse und nationale Feiertage, periodisch wiederkehrende Ereignisse wie Wahlen oder sportliche Wettkämpfe (Olympia, Fußballoder Kricketweltmeisterschaft). Diese besonderen Zeiten schaffen die zeitlichen Rhythmen einer Zeitlandschaft und sind eine „soziale Technik“ (Nowotny 1993: 32), um Gleichzeitigkeit und Verbindungen auf der diachronen Ebene herzustellen (Nowotny 1993: 32). Sie bringen die Menschen einer sozialen Welt zusammen und stärken das Zusammengehörigkeitsgefühl (Nowotny 1993: 32) - z.B. zu einer Familie oder zu einer Nation (McCrone und McPherson 2009a). Feiertage sind aber ebenso eine Gelegenheit, um sich an die Vergangenheit zu erinnern und einen Blick in die Zukunft zu werfen (McCrone und McPherson 2009b).

\section{Die Gestaltung von Routinen und Rhythmen}

Die zeitlichen Abläufe und Rhythmen des Alltags werden durch Menschen aktiv hergestellt (vgl. Lefebvre [1992] 2007). Dabei richten sie sich auch nach kollektiven zeitlichen Normen und haben unterschiedlich viel Kontrolle über die Gestaltung.

Menschen koordinieren ihre Praktiken und planen ihren Alltag unterschiedlich bewusst und reflektiert. Einige Formen der Koordination passieren einfach, andere müssen gezielt hergestellt werden (Shove 2013a: 28), vor allem dann, wenn Menschen gemeinsam Zeit verbringen wollen. Die Deinstitutionalisierung der Arbeitszeit (Garhammer 1995: 175) hin zu einer wachsenden Flexibilisierung ermöglicht beispielsweise in Form von Gleitzeit oder Vertrauensarbeitszeit größere Zeitsouveränität, erfordert von den Menschen aber viel Koordination (Garhammer 1995: 176). Auf der Basis dieser Ausführungen stellen sich folgende konkretisierte Fragen an meine Studie:

- Wie gestalteten meine Gesprächspartner*innen ihren Alltag und ihre besonderen Zeiten in Göttingen?

- Welche normativen Zeitkarten navigierten sie in der Gestaltung ihres Alltags und der besonderen Zeiten?

- Welche Erfahrungen von Zeit machten sie und wie versuchten sie auf diese Erfahrungen einzuwirken?

Da in meiner Studie mobile junge Menschen im Fokus stehen, bringe ich im folgenden Kapitelabschnitt meine Ausführungen zur biographischen Navigation und der Zeitgestaltung mit meiner zweiten analytischen Perspektive, der Mobilität, zusammen. 


\subsection{Biographische Navigation und Mobilität}

In der vorliegenden Studie arbeite ich mit dem Begriff der „Mobilität“ und damit verbundenen Konzepten (u.a. Hannam, Sheller und Urry 2006; Sheller und Urry 2006; Salazar und Smart 2011; Salazar und Jayaram 2016). Mobilität verstehe ich als Bezeichnung für räumliche Bewegungen und als eine analytische Perspektive auf diese Bewegungen (vgl. Salazar 2018). Nach Salazar und Smart sind Mobilitäten „soziokulturelle Konstrukte“ (2011: V), mit denen ganz unterschiedliche Imaginationen und Bedeutungen verbunden werden. Ich verwende in meiner Studie das Konzept des Regimes der akademischen Mobilität, um herauszustellen, wie die Mobilität von Studierenden und Wissenschaftler*innen gestaltet und mit welchen Vorstellungen sie verbunden wird. Für die Analyse der Mobilität der jungen Wissenschaftler*innen beziehe ich mich außerdem auf Studien, die sich mit unterschiedlichen Migrationsbewegungen beschäftigen und die mit Konzepten wie Transnationalismus (Glick Schiller, Basch und Blanc-Szanton 1992; Vertovec 2001, 2009) und Diaspora (Brah 1996; Brown 2006; Knott 2010) arbeiten.

\subsubsection{Das Regime der akademischen Mobilität}

Das Konzept der „regimes of mobility“ (Glick Schiller und Salazar 2013) bietet einen Rahmen, um zu verstehen, warum die Mobilität unterschiedlich kategorisierter Menschen mehr oder weniger aktiv unterstützt oder verhindert wird. Der Begriff „Regime“ lenkt dabei den Fokus einerseits auf die Einflussnahme von einzelnen Staaten und von der internationalen regulativen und überwachenden Administration auf die individuelle Mobilität. Andererseits rücken die Wechselwirkungen dieser Strukturen mit dem Handlungsvermögen der Migrant*innen in den Vordergrund (Glick Schiller und Salazar 2013: 189 ${ }^{16}$ ).

In meiner Studie habe ich ein konkretes Mobilitätsregime herausgearbeitet - das Regime der akademischen Mobilität. Mit dem Begriff des Regimes der akademischen Mobilität bezeichne ich das Geflecht von Akteuren, Strukturen, Diskursen und Imaginationen, das die Mobilität von Studierenden und Wissenschaftler*innen hervorbringt, normalisiert und reguliert (vgl. Glick Schiller und Salazar 2013; Baker 2016). Mit Hilfe dieses Konzeptes habe ich globale und lokale Prozesse und Strukturen untersucht, die zur akademischen Mobilität beitragen. Dazu gehören sowohl die Wissensökonomie als wichtiger globaler Rahmen als auch die Aspirationen, die in Indien mit der Bildung und Mobilität junger Menschen verbunden werden.

\footnotetext{
${ }_{16}$ Glick Schiller und Salazar verweisen an dieser Stelle auf M. Foucault (2000): Truth and Power (in J. Faubion (Hg.): Essential Works of Foucault 1954-1984, Vol. 3, 111-133), G. Burchel (1991): The Foucault Effect: Studies in Governmentality, Chicago: University of Chicago Press, und S. Hall (1997): Representation: Cultural Representations and Signifying Practices, London: Sage.
} 
Durch die Perspektive des Regimes der akademischen Mobilität habe ich die Diskurse analysiert, die global, aber auch lokal in Deutschland und in Indien bezüglich der Mobilität von Studierenden und Wissenschaftler*innen existieren. In diesem Prozess habe ich die Hoffnungen und Erwartungen, die von unterschiedlichen Akteuren mit diesen Personengruppen verbunden werden, herausgearbeitet. Die Geschichte der Mobilität indischer Studierender und die Stereotype, die mit indischen Studierenden und Wissenschaftler*innen verbunden werden, waren weitere Faktoren in der Analyse.

Die Ergebnisse dieser Analyse stelle ich ausführlich in Kapitel 3 dar. An dieser Stelle möchte ich nur zwei Effekte des Regimes der akademischen Mobilität beschreiben: In diesem Mobilitätsregime wird die ungleiche Behandlung mobiler Menschen mit dem Faktor Wissen oder, in der Sprache des Regimes, ,Talent' gerechtfertigt. Die Menschen, die über jene, Talente' verfügen, die in der Wissensökonomie geschätzt werden, haben einen Zugang zu Mobilität. Dem Regime der akademischen Mobilität wohnt dementsprechend eine „Nützlichkeitslogik“ (Gutekunst 2018: 130) inne, die diese Menschen auf ihren vorgesehenen Nutzen für verschiedene institutionelle und nationale Akteure reduziert - beispielsweise auf ihre Kenntnisse in bestimmten wissenschaftlichen Bereichen. Auf diese Weise werden die Studierenden und Wissenschaftler*innen durch die Diskurse des Regimes der akademischen Mobilität positioniert. ${ }^{17}$ Wie sie die Positionierung als, Talent' ausfüllen, für sich nutzen, sie ablehnen und mit anderen sozialen Verortungen kombinieren, ist sehr individuell.

Durch das Regime der akademischen Mobilität entsteht ein Umfeld, in dem die Mobilität von Studierenden und Wissenschaftler*innen als essentielle Investition in die Zukunft konstruiert wird - sowohl für die beteiligten Länder und Institutionen als auch für die Personen selbst. Dieses Verständnis von Mobilität als Mittel der Zukunftsgestaltung hatte einen großen Einfluss auf die biographische Navigation der jungen Menschen, die im Fokus dieser Studie stehen. Auf die weiteren Details des Regimes der akademischen Mobilität gehe ich in Kapitel 3 ein.

\subsubsection{Mobilität und soziale Welten}

Soziale Welten können in höchstem Maße räumlich zerstreut sein: „Decisive for their constitution is the participation of their members in a shared context of interaction, not the strict determination of territorial boundaries" (Soeffner und Zifonun 2008:

\footnotetext{
${ }_{17}$ In meiner Analyse dieser Prozesse der Positionierung beziehe ich mich auf Elfriede Hermanns Artikel Manifold identifications within differentiations, in dem sie ausführt, wie Subjekte durch Diskurse positioniert werden und es umgekehrt Diskurse ermöglichen, spezifische Positionierungen einzunehmen (Hermann 2003a: 78). Sie bezieht sich in ihrem Text auf S. Hall (1997): Introduction: Who Needs ,Identity'? In: Stuart Hall und Paul Du Gay (Hg.): Questions of Cultural Identity. London: Sage Publications, 1-17; S. Hall (1997): The Work of Representation, in: S. Hall (Hg.): Representation: Cultural Representations and Signifying Practices, London: Sage Publications, 55-56, und M. Foucault (1972): The Archaeology of Knowledge and the Discourse on Language, New York: Pantheon Books.
} 
7-8). Mit einem solchen Verständnis von sozialen Welten wird deutlich, dass sich Mobilität, Ortsgebundenheit und soziale Verortung nicht gegenseitig ausschließen (Nowicka 2007; Glick Schiller und Salazar 2013: 187).

In der Migrationsforschung wird Mobilität nicht (mehr) als einmalige Bewegung von einem Ort zu einem anderen, zwischen einem Herkunfts- und einem Ankunftsland verstanden, sondern als komplexe Bewegung in einem Feld jenseits von Ortsgebundenheit (Pries 1998: 73-74). Ludger Pries bezeichnet diese Felder als „transnationale soziale Räume" und definiert diese als

neue „soziale Verflechtungszusammenhänge“ (Elias 1986) (...), die geographisch-räumlich diffus bzw. „de-lokalisiert“ sind und gleichzeitig einen nicht nur transitorischen sozialen Raum konstituieren, der sowohl eine wichtige Referenzstruktur sozialer Positionen und Positionierungen ist, als auch die alltagsweltliche Lebenspraxis, (erwerbs-)biographischen Projekte und Identitäten der Menschen bestimmt und gleichzeitig über den Sozialzusammenhang von Nationalgesellschaften hinausweicht. (Pries 1996: 467)

Meine Gesprächspartner*innen schufen durch ihre Bewegungen, ihre Interaktionen mit verschiedenen Akteuren und Imaginationen einen spezifischen transnationalen sozialen Raum.

Transnationale soziale Räume entstehen gewissermaßen durch die Bemühungen der beteiligten mobilen und nicht-mobilen Akteure, Gemeinsamkeit und Beziehungen der Gegenseitigkeit aufrechtzuerhalten. Dies geschieht durch regelmäßige Kommunikation, Reisen, das Schicken von Geld, das Mitnehmen von Objekten (Povrzanović Frykman und Humbracht 2013), aber auch durch das Beibehalten alltäglicher Abläufe und Praktiken (Petridou 2001), die dazu beitragen, ,verbunden zu bleiben und Vertrautheit zu vermitteln (Nowicka 2007; Collins 2008). Unterschiedliche Informations- und Kommunikationstechnologien (IKT) tragen essentiell dazu bei, transnationale Beziehungen aufzubauen und zu bewahren (vgl. Baldassar 2008; Baldassar et al. 2016; Madianou 2016). Technologien wie Smartphones und soziale Netzwerke wie Facebook schaffen neue Möglichkeiten für die gegenseitige emotionale Unterstützung und Erfahrung von Kopräsenz - dem Füreinanderdasein (Baldassar et al. 2016: 134) und der Anwesenheit über die Distanz hinweg. Wie Baldassar et al. (2016: 135) betonen, verändern diese neuen Kommunikationswege nicht unbedingt die Beziehungen. Die unterschiedlichen Informations- und Kommunikationstechnologien werden vor allem dazu genutzt, die Strukturen und Bindungen aufrechtzuerhalten und die Verpflichtungen und Erwartungen, die mit der Verortung in sozialen Welten einhergehen, zu reproduzieren (Baldassar et al. 2016: 135; vgl. Wilding 2006).

Im transnationalen sozialen Raum versuchen die Akteure, nicht nur bereits bestehende soziale Beziehungen aufrechtzuerhalten, sondern ebenso neue Beziehungen zu knüpfen. Im transnationalen sozialen Raum überschneiden sich gewissermaßen die Verortungen in ,alten' und ,neuen' sozialen Welten: 
We want to emphasize that belonging is not a zero sum game where you have to decide which place or country you belong to. On the contrary it seems as if many transnational migrants are able to handle double or multiple belonging and combine new and old attachment to places with transnational ties. (Christensen und Jensen 2011: 153)

Allerdings ist es an dieser Stelle wichtig, nicht zu optimistisch mit den Möglichkeiten des Erschaffens neuer Verortungen zu argumentieren. Wie ich bereits betont habe, beruht die Zugehörigkeit zu einer sozialen Welt auf dem Teilen eines gemeinsamen Wissensvorrates. Ein ,Neuankömmling' in einer sozialen Welt, sei es in einer neuen Stadt, einem neuen Land oder einem neuen Arbeitsplatz, hat diesen Wissensvorrat nicht. Die Person kennt die Routinen und die unausgesprochenen Regeln nicht und kann somit zum Außenseiter werden (Pfaff-Czarnecka 2011: 6, 2012: 30). Das Herstellen von Gemeinsamkeit und Beziehungen der Gegenseitigkeit ist in einer solchen Situation nicht einfach und hängt stark von den Akteuren und Umständen in einer spezifischen sozialen Welt ab.

\subsubsection{Mobilität, Zeitlandschaften, Zeitkarten}

Mobilität hat, wie bereits einige Autor*innen gezeigt haben, nicht nur räumliche, sondern auch zeitliche Dimensionen (u.a. Cwerner 1999; Griffiths, Rogers und Anderson 2013; Robertson 2014; Collins und Shubin 2015; Mavroudi, Page und Chrēstu 2017). ${ }^{18}$ Mobile Menschen kommen mit neuen Orten, anderen Menschen und neuen Zeiten in Berührung. Mobilität macht Zeit erfahrbar und führt die ,eigenen' und die ,fremden' Zeiten (Cwerner 2001: 18) vor Augen:

Patterns of day and night (with the respective significance of light and darkness), work and leisure, weekdays and weekends, months, seasons, years, decades and generations, are all suffused with meanings that may appear strange to immigrants. This is the case because, despite the standardisation of clock time, the week and the year across various regions and countries in the world, these cycles retain a local degree of flexibility or elasticity. (Cwerner 2001: 20)

Mobilität führt zur Interaktion mit neuen Zeitlandschaften, mit menschlichen und nicht-menschlichen Rhythmen und mit Repräsentationen von Zeit. Diese Interaktion formt die zeitlichen Erfahrungen und die Zeitgestaltung mobiler Menschen, die wiederum ihre ganz eigenen Zeiten im Gepäck haben (Cwerner 2001: 19). Der Wechsel in eine neue soziale Umwelt bedeutet, dass die mitgebrachten Zeitkarten

\footnotetext{
${ }^{18}$ Griffiths, Rogers und Anderson (2013) geben einen sehr guten Überblick über die Fülle an Studien und die multiplen Blickwinkel auf das Thema Mobilität und Zeit.
} 
nicht funktionieren und sich die eigene Zeitgestaltung von der der ,Etablierten“ unterscheidet. Saulo Cwerner fasst die verschiedenen zeitlichen Dimensionen der Mobilität unter dem Begriff der „times of migration“ (Cwerner 2001), von denen er acht Formen unterscheidet. Jede dieser ,Zeiten' repräsentiert spezifische zeitliche Problematiken, die mobile Menschen erfahren können und die gleichzeitig wirken (Cwerner 2001: 18). Die Art und Weise der zeitlichen Erfahrung wird unter anderem von der Art der Mobilität (Arbeitsmigration, akademische Mobilität, Flucht) und der Migrationsgesetzgebung des Ziellandes beeinflusst (Robertson und Runganaikaloo 2014).

Mobile Menschen wirken aber ebenso auf ihre Erfahrung von Zeit ein, haben also Handlungsvermögen im Umgang mit den times of migration (Cwerner 2001: 22-23; Robertson und Runganaikaloo 2014: 220-21). Dem Zusammenspiel zwischen Mobilität, zeitlichen Erfahrungen und Zeitgestaltung nähere ich mich im Folgenden über die bereits vorgestellten Horizonte des Alltags und der Zukunft.

\subsubsection{Mobilität und Zeitgestaltung}

Mobilität hat einen Einfluss auf die Zeitgestaltung von Menschen (Cwerner 2001: 21). Viele mobile Menschen, vor allem jene, deren Mobilität nicht als positiv oder ,nutzbringend' eingeschätzt wird, haben wenig Kontrolle über ihre Zeit, also darüber, was wann geschieht und wie lange etwas dauern wird. Die zeitliche Unsicherheit wird besonders in Studien zu Geflüchteten und Asylsuchenden deutlich (Griffiths 2013, 2014; Ramsay 2017, 2018). Wie die Arbeiten von Elsrud (1998), Barnick (2010) und meine eigene Studie zeigen, kann Mobilität allerdings auch zu größerer Zeitsouveränität führen. Mobilität führt zur Distanz zu den sozialen Beziehungen, die Einfluss auf die Zeitsouveränität haben, und beeinflusst die soziale Positionierung in einer sozialen Welt. Eine bessere Positionierung - beispielsweise durch das Erlangen von kulturellem, symbolischem oder wirtschaftlichem Kapital mittels Mobilität kann mit größerer Zeitsouveränität einhergehen.

\section{Mobilität und die Gestaltung der Zukunft}

Viele Menschen werden mobil, um einen positiven Einfluss auf ihre Zukunft auszuüben (Vigh 2009b; Cole 2010; Mapril 2014; Valentin 2014). Mobilität wird in diesem Sinne selbst zu einem Mittel der Zukunftsgestaltung: „as means of imagining or creating futures, about hopes and aspiration" (Griffiths, Rogers und Anderson 2013: 28). Dieses Verständnis von Mobilität wird, wie in Abschnitt 1.3.1 angesprochen, auch durch Diskurse im Regime der akademischen Mobilität angeregt. Ein wichtiger Faktor in dieser Dynamik von Mobilität und Gestaltung der Zukunft ist die Vorstellung davon, dass der Migrationsprozess zu „arrival points“ (Baas 2010: 6), also ,Ankunftspunkten“ führt. Diese Ankunftspunkte sind: „,imaginary moments in the future when migrants imagine themselves as having arrived at where they 
intended to be (...). This is when they imagine themselves as having achieved what they set out to do" (Baas 2010: 6).

Die Vorstellungen vom Wert der Mobilität für die Zukunft und Imaginationen vom Ankunftspunkt orientieren sich an dominanten Vorstellungen vom Lebensverlauf. In vielen sozialen Welten ist Mobilität zu einer wichtigen Koordinate in Vorstellungen vom Lebensverlauf geworden. Mobilität wird als Teil des Erwachsenwerdens (Robertson, Harris und Baldassar 2017: 204) oder als wichtiger Faktor in der Entwicklung der beruflichen Karriere (Leemann 2010) verstanden. Durch die Mobilität soll in der linearen Logik der Zeitkarten von Lebensverlauf und „academic life course" (Jacobs und Winslow 2004; Winslow und Davis 2016) eine (bessere) Zukunft geschaffen und das Vorankommen im Lebensverlauf gesichert werden (Vigh 2009b). Die Mobilität wird in diesem Prozess zu einer Strategie, eine gesellschaftlich sanktionierte Vision von der Zukunft zu verwirklichen. Allerdings ist Mobilität auch ein Mittel des „future-tricking“ (Ringel 2016), des Versuchs, einer vorgeschriebenen Version der Zukunft zu entgehen und einen alternativen Lebensverlauf möglich zu machen. Mobilität kann eine Strategie sein, um die Ankunft an Koordinaten wie denen der Heirat oder des Erwachsenwerdens hinauszuzögern (Kóu, Mulder und Ajay Bailey 2017). Die Vorstellung eines Ankunftspunktes schafft eine zeitliche Koordinate und mit dieser einen zeitlichen Horizont, der einen großen Einfluss darauf hat, wie die Mobilität erfahren und wie mit diesen Erfahrungen umgegangen wird (Adrian Bailey et al. 2002; Baas 2010: 6; Collins und Shubin 2015).

\section{Mobilität und die Gestaltung des Alltags und besonderer Zeiten}

Die Mobilität kann sowohl zu größerer Zeitsouveränität beitragen als auch zu Brüchen im Alltag (Collins und Shubin 2015) und zu Erfahrungen von Asynchronität führen (Cwerner 2001) sowie zu Gefühlen von Isolation (Kirk, Bal und Janssen 2017) und Langeweile (Collins und Shubin 2015). Veränderungen im Alltag beeinflussen die Erfahrung von Zugehörigkeit: „When our expectations of the everyday are not met, when we cannot go about our mundane tasks as we are accustomed to, (...) we can awaken to a feeling of not belonging " (May 2013: 89). Mobile Menschen wirken durch Praktiken der Zeitgestaltung auf diese Erfahrungen ein und können sich beispielsweise resynchronisieren (Cwerner 2001: 23), indem sie vertraute Elemente ihrer Zeitlandschaft wie Feste in ihre neue soziale Umwelt integrieren. Auf einer synchronen Ebene wird so Gleichzeitigkeit (Levitt und Glick Schiller 2004) hergestellt.

Durch die Integration vertrauter Praktiken werden Brüche im Alltag, repariert und vertraute Rhythmen wiederhergestellt. Vertraute Praktiken und das Aufrechterhalten sozialer Beziehungen schaffen zudem Kontinuitäten, Verbindungen auf der diachronen Achse in Vergangenheit und Zukunft und verflechten diese mit der Gegenwart an einem neuen Ort. Im Alltag sind für die Resynchronisation und den Umgang mit Einsamkeit unterschiedliche Informations- und Kommunikationstechnologien von großer Relevanz. Sie bieten unterschiedliche Möglichkeiten, in Kon- 
takt zu bleiben und Gleichzeitigkeit herzustellen, und sind in dieser Funktion ein wichtiger Faktor in den Alltagsroutinen mobiler und nicht-mobiler Menschen (vgl. Madianou 2016).

Wie viel mobile Menschen in diese Praktiken der Zeitgestaltung investieren oder investieren können, hängt unter anderem mit dem bereits erwähnten Bewusstsein von der zeitlichen Begrenztheit des Aufenthalts zusammen (Collins und Shubin 2015). Aus diesen Ausführungen ergeben sich folgende Teilfragen:

- Welchen Einfluss hatte die Mobilität auf die zeitlichen Erfahrungen und die Zeitgestaltung der jungen Wissenschaftler*innen?

- Wie wirkten sie auf ihre zeitlichen Erfahrungen ein und gestalteten in Göttingen ihre eigene Zeitlandschaft?

\subsection{Die Erforschung von biographischer Navigation und Zeitgestaltung}

In den bisherigen Unterkapiteln habe ich die theoretischen Grundlagen gelegt, um die biographische Navigation meiner Gesprächspartner*innen aus den Perspektiven von Zeitgestaltung und Mobilität zu analysieren. Im Folgenden gehe ich genauer darauf ein, wie ich in meiner Forschung methodisch vorgegangen bin. Da meine Methodik und meine Analyse der Daten stark von meiner eigenen Person, meinem fachlichen Hintergrund, meinem Vorwissen und dem Zugang zu meinem Feld geprägt waren, beschäftige ich mich im ersten Abschnitt mit meinen Positionierungen während der Forschung.

Im zweiten Abschnitt dieses Kapitelteils gehe ich darauf ein, wie sich die Zeitgestaltung in der biographischen Navigation methodisch fassen lässt. Ich berufe mich dabei auf meine eigenen Erfahrungen in der Feldforschung und auf neue Ansätze anderer Sozialwissenschaftler*innen.

\subsubsection{Wer bist du? Wer bin ich? Verortungen in der Forschung}

Die ethnologische Forschung ist im Grunde ein Aushandlungsprozess, in dem auch die sozialen Verortungen der Forscher*innen konstruiert und produziert werden (Coffey 1999: 1). Dieser Aushandlungsprozess ist in einigen Situationen direkt und deutlich sichtbar - beispielsweise in Face-to-Face-Interviews -, in anderen Fällen passiert er indirekter, wie bei der Kommunikation über Medien. Aber immer finden Prozesse der Positionierung statt: Positionierungen zwischen Forscher*in und Gesprächspartner*in, zu (dominanten) Diskursen oder zu (anwesenden) anderen Personen. Die folgenden Ausführungen sollen dazu beitragen, den Forschungsprozess und meine Rolle bei der Entstehung der Daten transparenter zu machen. 


\section{Angehende Wissenschaftler*innen unter sich?}

Den Zugang zu meinen Gesprächspartner*innen erlangte ich über eine Vereinigung indischer Studierender in Göttingen, die sich gegründet hatte, kurz bevor ich meine Studie begann. Durch sie konnte ich erste Kontakte herstellen und erfuhr von den Veranstaltungen, die organisiert wurden. Mit der Teilnahme an der Feier des Indian Independence Day 2010 begann ich meine Forschung. Obwohl mich einige der Teilnehmer*innen fragten, was Ethnolog*innen eigentlich machen, stellte niemand infrage, dass ich Daten für meine Doktorarbeit sammelte, schließlich taten viele meiner Gesprächspartner*innen dasselbe, wenn auch häufig in einem Labor und nicht bei Festlichkeiten oder mittels Interviews. Ich wurde von vielen also zunächst als (angehende) Wissenschaftlerin wahrgenommen. In gewisser Weise handelte es sich also um ein „studying sideways“ (Hannerz 1998, 2010; Ortner 2010), um ein Forschen mit Menschen, mit denen ich die Positionierung als Wissenschaftler*in gemein hatte. Wir navigierten ähnliche akademische Strukturen und Anforderungen, die an uns als angehende Wissenschaftler*innen gestellt wurden.

Nichtsdestotrotz möchte ich an dieser Stelle nicht über Ungleichheiten und Machtverhältnisse hinwegtäuschen, in die meine Studien eingebettet waren und die mich und meine Gesprächspartner*innen positionierten. Ein Faktor, auf den ich in meiner Darstellung des Regimes der akademischen Mobilität in Kapitel 3 ausführlicher eingehe, sind die Zuschreibungen und Vorstellungen, die in der globalen Wissensökonomie mit Institutionen aus dem globalen Norden und dem globalen Süden - bzw. konkret Deutschland und Indien - verbunden werden. Ungleichheiten entstanden auch durch die dem Mobilitätsregime inhärente Nützlichkeitslogik (vgl. Gutekunst 2018), die den Aufenthaltstitel meiner Gesprächspartner*innen an deren erfolgreiche wissenschaftliche (Weiter-)Qualifizierung koppelte.

Ich führte meine Forschung in Göttingen durch, einer Stadt, in der ich selbst seit 2005 lebe und ,zuhause' bin. In der Migrationsforschung ist die Feldforschung ,zuhause' keine Seltenheit mehr (Norman 1999) und hat, wie Norman und Sökefeld herausarbeiten, unterschiedliche Konsequenzen und Herausforderungen (Norman 1999; Sökefeld 2002). Für mich bedeutete die Forschung ,zuhause‘, dass das ,Feld ohne viel Geld und Zeitaufwand zu erreichen war (vgl. Sökefeld 2002: 89). Aber vor allem ermöglichte mir die Forschung in Göttingen die Vereinbarkeit von beruflichen, akademischen und persönlichen Verpflichtungen.

Anders stellte es sich für meine Gesprächspartner*innen dar. Vor ihrer Ankunft in Göttingen hatten sie häufig mehrmonatige oder auch mehrjährige bürokratische Prozesse durchlaufen - vom Beantragen eines Visums bis hin zur Meldung bei der Ausländerbehörde. In Göttingen angekommen, lebten sie über längere Zeiträume von ihren Familien und in einigen Fällen auch Partner*innen getrennt. Anders ausgedrückt, wurde mir meine Immobilität durch die Mobilität meiner Gesprächspartner*innen ermöglicht. 


\section{Auch Ethnolog*innen verändern sich (und werden erwachsen)}

Die sozialen Verortungen, die einer Ethnologin zu Beginn der Forschung zugeschrieben werden, bleiben häufig nicht gleich (Narayan 1993; Coffey 1999: 35). Meine eigene Positionierung hat sich einige Male und je nach Gegenüber verändert. Den größten Einfluss auf meine Daten und meinen Zugang hatten aber persönliche Ereignisse, die ich auch als solche zunächst von meiner Forschung abzukoppeln versuchte. Vor allem meine erste Schwangerschaft und die Geburt meines Sohnes im Frühjahr 2012 hatten auf verschiedenen Ebenen eine beträchtliche Wirkung auf meine Forschung. Nicht zuletzt verdeutlichten sie mir, dass es in einer Feldforschung schwierig ist, zwischen privat und öffentlich sowie persönlich und beruflich zu unterscheiden. Eine ethnologische Feldforschung ist beides und damit ebenso die Beziehungen, die während der Forschung geknüpft werden (Coffey 1999: 57). Rückblickend hatte ich es mit den gleichen Problematiken zu tun, die ich erforschen wollte: der sozialen Verortung in verschiedenen sozialen Welten, die sich mal mehr und mal weniger gut voneinander trennen ließen.

Als ich sichtbar schwanger wurde, änderten sich mein Zugang, meine Rolle und meine Beziehungen im Feld (vgl. Coffey 1999: 68) und neue Gesprächsthemen wurden plötzlich relevant: Schwangerschaft, Kinder und die Vereinbarung von Familie und Beruf. Tatsächlich eröffnete mir meine neue Rolle als werdende Mutter eine neue Perspektive auf meine Forschung. Ich wurde nun gefragt, ob ich denn verheiratet sei und durch meine Verneinung ergab sich häufig ein Gespräch über die unterschiedlichen Normen diesbezüglich in Indien und Deutschland (vgl. Coffey 1999: 82-83).

\section{Sprache in der ethnologischen Forschung}

Ich entschloss mich früh in meiner Studie, Interviews und informelle Gespräche auf Englisch durchzuführen. Diese Entscheidung basierte zunächst auf Überlegungen zur praktischen Umsetzung meiner Studie. Die jungen Studierenden aus Indien kamen aus vielen unterschiedlichen Regionen Indiens und hatten demzufolge auch verschiedene Muttersprachen. Ich hatte zwar in meinem Indologie-Studium Hindi gelernt, stellte aber bald fest, dass höchstens ein Drittel meiner Gesprächspartner*innen Hindi-Muttersprachler*innen waren. Da ich meine Studie nicht auf diese eingrenzen und nicht noch weitere Sprachen erlernen wollte, entschloss ich mich dazu, bei Englisch zu bleiben. Ein weiterer Punkt, der meine Entscheidung untermauerte, war, dass Englisch von vielen der jungen Studierenden als ,neutrale' Sprache in Bezug auf Identifikationen und Zugehörigkeiten empfunden wurde. Indische Sprachen wurden dagegen mit der regionalen Herkunft und zum Teil mit aktuellen Debatten in Indien zu diesem Thema in Verbindung gebracht. ${ }^{19}$

\footnotetext{
${ }_{19}$ In Indien entstehen immer wieder Diskussionen um die Bedeutung regionaler Sprachen und den Versuch der Regierung, Hindi als gemeinsame Sprache ganz Indiens durchzusetzen. Im September
} 
Obwohl ich mich dafür entschied, Englisch zu benutzen, eine Sprache, von der ich dachte, dass ich sie recht gut beherrschen würde, ist Englisch nicht gleich Englisch (Spradley 1979: 18-19). Das Englisch der jungen Wissenschaftler*innen aus Indien unterschied sich nicht nur im Vokabular von meinem, sondern konnte auch von Person zu Person variieren. ${ }^{20}$ Das Englisch meiner Gesprächspartner*innen war außerdem stark durch ihre Einbettung in die Wissenschaft geprägt. Dieser Umstand wurde mir immer dann am deutlichsten vor Augen geführt, wenn mir Naturwissenschaftler*innen von ihren Forschungsprojekten erzählten. Im Sinne meiner vorangegangenen Ausführungen zu den sozialen Welten teilte ich in diesen Situationen nicht den gleichen Wissensvorrat und musste mir das Vokabular daher erst aneignen.

\subsubsection{Methoden und Datenanalyse}

Im folgenden Teil des Kapitels steht meine methodische Herangehensweise, die Analyse der Daten und Göttingen als Ort meiner Forschung im Mittelpunkt der Ausführungen.

\section{Interviews und teilnehmende Beobachtungen}

In meiner Forschung verwendete ich eine Mischung ethnographischer Methoden, allen voran Interviews und teilnehmende Beobachtung. Wie Shanthi Robertson (2014: 8) betont, wird bei einem Fokus auf Zeit das Wann, die Dauer und die Häufigkeit von großer Bedeutung. Robertson plädiert dafür, teilnehmende Beobachtungen und Interviews zu unterschiedlichen Zeitpunkten durchzuführen. Als geeignete Zeitpunkte versteht sie jene, die besondere Momente kennzeichnen, im Fall von mobilen Menschen beispielsweise die Ankunft, Abreise oder der Erhalt eines Visums (Robertson 2014: 8). Ich habe in meiner Studie weitere wichtige Koordinaten eingeschlossen, die sich auf die Interaktion normativer Zeitkarten mit der biographischen Navigation der jungen Wissenschaftler*innen bezogen. Dazu gehörten beispielsweise die Heirat, der Abschluss eines Studiums oder der Promotion. Das Durchführen mehrerer Interviews mit einem/einer Gesprächspartner*in zu unterschiedlichen Zeitpunkten eröffnet einen Blick auf die Veränderung von Erfahrungen und $\mathrm{Zu}$ kunftsvisionen und wird damit dem prozesshaften Charakter der biographischen Navigation gerechter.

\footnotetext{
2019 wurde beispielsweise der Innenminister Indiens, Amit Shah, für seinen Vorschlag kritisiert, Hindi zur „national language“ Indiens zu erklären. Siehe Uday Mahurkar: „Why Amit Shah thinks Hindi is the language of national integration ", India Today vom 16.09.2016, https://www.indiatoday.in/ india-today-insight/story/why-amit-shah-thinks-hindi-is-the-language-of-national-integration-1599 506-2019-09-16.

${ }^{20}$ Diese Unterschiede erklären sich meiner Ansicht nach aus der regionalen Herkunft der Gesprächspartner*innen, aber auch aus deren unterschiedlichen Schullaufbahnen und ihrer Herkunft vom Land oder aus der Stadt.
} 
Um gute Zeitpunkte herauszufinden, war es zunächst wichtig, gemeinsame Zeit mit den Studierenden und Wissenschaftler*innen aus Indien zu verbringen und so von wichtigen Ereignissen zu erfahren. Eine weitere Strategie war die Benutzung von Facebook. Hier informierte die Vereinigung der indischen Studierenden in Göttingen darüber, wann das nächste Fest oder eine andere gemeinsame Aktivität stattfinden sollte. Facebook war für die meisten meiner Gesprächspartner*innen ein integraler Bestandteil ihres Alltagslebens. Aus diesem Grund machte ich keine strenge Unterscheidung zwischen meinem, Offline'- und ,Online'-Feld (Miller 2011; Steffen Dalsgaard 2015: 96). Auf Facebook wurde ich Teil der Gruppe „Indians in Göttingen“ und wurde, um im Facebook-Jargon zu bleiben, die Freundin von Personen, mit denen ich auch offline interagierte. Viele meiner Gesprächspartner*innen teilten auf Facebook, was gerade in ihrem Leben passierte, für was sie sich interessierten, was oder wen sie vermissten, und pflegten Kontakte zu Familie und Freund*innen. Für meine Forschung war Facebook auf drei Ebenen relevant: als Quelle von Informationen, als Mittel der Zeitgestaltung, z.B. der Resynchronisation (Robertson 2014: 10), und durch das Format, in dem es existiert, als Darstellung von Zeitkarten und sozialen Rhythmen. Soziale Medien „often exist as linear constructions of personal experience, posted in 'real-time' and chronologically archived and displayed. They can, therefore, expose both daily practices and rhythms and key moments and milestones (...)“ (Robertson 2014: 10).

Offline lag mein Fokus auf der Teilnahme an den kollektiven Aktivitäten der jungen Wissenschaftler*innen in Göttingen. Seit August 2010 nahm ich bis kurz vor der Vollendung dieser Arbeit, wenn es möglich war, jedes Jahr an Feierlichkeiten wie Diwali oder dem Indian Independence Day teil. Zwischen 2010 und 2013 ging ich außerdem regelmäßig zu den Treffen der Vereinigung indischer Studierender in Göttingen. Mit einigen meiner Gesprächspartner*innen verbrachte ich Zeit in weniger offiziellem Rahmen: in ihren Wohnungen, zu einem gemeinsamen Essen in der Mensa oder bei Kricketspielen.

\section{Analyse der Daten: „Positioning analysis“}

Ich verstehe informelle und formelle Gespräche (z.B. Interviews) und die Interaktion auf Facebook als wichtige Praktiken der biographischen Navigation, da Menschen durch Kommunikation miteinander (direkt oder indirekt) nicht nur Informationen austauschen, sondern sich selbst und ihr Gegenüber positionieren (Holland, Lachicotte jr und Skinner 1998: 26; Anthias 2002: 499) und ihr Selbst konstruieren (Keupp et al. 1999: 216; vgl. Hermann und Röttger-Rössler 2003; May 2016). Der Großteil meiner Daten ist durch die Kommunikation zwischen den jungen Wissenschaftler*innen und mir oder anderen (imaginierten) Akteuren entstanden. Die Transkripte der Interviews und Screenshots von Facebook-Konversationen verstehe ich als ,Narrationen“ (Bamberg 2004, 2012; Bamberg, de Fina und Schiffrin 2007, 2011), die ich auf die Praktik der biographischen Navigation analysiere. Eine wichtige Ausgangslage für die Analyse ist dabei die Annahme, dass 
meine Gesprächspartner*innen diese Narrationen aus bestimmten Gründen vortrugen. Im Allgemeinen ist es das Ziel, etwas aus den Narrationen (Erfahrungen, Werte, Moral) für den aktuellen Sprechakt relevant zu machen (Bamberg 2012: 101-102). Für meine Untersuchung ist es zudem von großer Bedeutung, dass Akteure durch ihre Wahl des Erzählten Aspekte ihres Selbst erkennen lassen:

(...) they engage in identity claims with regard to how they would like to come across as well as in terms of potential answers to the who-am-I question. It is at this point where the relational work that is accomplished in storytelling practices is opening up glimpses into how narrators accomplish this type of relationship management and at the same time engage in identity practices that result in what we have called a sense of self - probably even a kind of sense that endures across interactive storytelling practices. (Bamberg 2012: 102)

Was Bamberg in diesem Zitat als „identity practices“ bezeichnet, verstehe ich als die Prozesse der biographischen Navigation. Im Sinne meiner vorherigen Ausführungen zur biographischen Navigation in der sozialen Umwelt wird in den Aussagen Einzelner aber nicht nur die Meinung dieser einen Person deutlich, sondern, wie van Meijl mit Verweis auf Bakhtin ${ }^{21}$ beschreibt: „what we hear in the utterances of individual speakers is not simply the talk of individuals, but also the voices of their surrounding groups and institutions" (van Meijl 2006: 930).

Laut Bamberg geht es in Narrationen unter anderem darum, das Selbst auf einer zeitlichen Ebene auszuhandeln, was er als „diachronic identity navigation“ bezeichnet (Bamberg 2012: 103; May 2016: 5). In den Narrationen verhandelten meine Gesprächspartner*innen u.a. Aspekte von Kontinuität und Wandel (Rapport 1999: 88). Dabei mussten sie die Extreme von Unveränderlichkeit und ständigem Wandel ausbalancieren. Dies machen Sprechende, indem sie ihr Selbst in irgendeiner Form von Kontinuität positionieren (Bamberg 2012: 104).

Zwei weitere wichtige Dimensionen in den Narrationen sind die Aushandlung von Gleichheit vs. Differenz (vgl. Hermann 2001: 243-246, Hermann 2003b: 96) und die Frage nach Handlungsvermögen (Bamberg 2012: 103-06). Menschen signalisieren durch die Wahl diskursiver Mittel im Gespräch ihre Positionierung gegenüber anderen. Menschen können damit sowohl ein Selbst definieren, das sich von anderen unterscheidet, als auch ihre Zugehörigkeit zu einer bestimmten sozialen Welt darstellen (Bamberg 2012: 105). Durch die diskursiven Werkzeuge, die Menschen in ihren Geschichten wählen, können sie sich selbst als mit viel oder wenig Handlungsvermögen ausgestattet konstruieren, sich beispielsweise als Opfer oder Held*in der eigenen Erzählung darstellen (Keupp et al. 1999: 209; Bamberg 2012: 106).

Obwohl Bamberg die Positionsanalyse vor allem in der Auswertung von Narrationen benutzt, kann sie meiner Ansicht nach ebenso auf andere Praktiken der

\footnotetext{
${ }^{21}$ Siehe M. Bakhtin (1984 [1929]): Problems of Dostoevsky's Poetics. (C. Emerson Hg. \& Übers.) Manchester: University Press.
} 
biographischen Navigation angewendet werden, die durch die Methodik der teilnehmenden Beobachtung erfasst werden. Meine teilnehmenden Beobachtungen führte ich vor allem bei Festivitäten oder anderen kollektiven Aktivitäten durch, also in „Situationen von Ko-Präsenz“ (Pfaff-Czarnecka 2012: 29). Diese Ereignisse analysiere ich als kollektive Praktiken der Artikulation, des Sich-Ausdrückens und SichVerbindens mit spezifischen Positionierungen (Hermann 2003a; Hall 2004: 173; Pfaff-Czarnecka 2012: 28-29). Die gemeinsamen Aktivitäten verstehe ich außerdem als einen Rahmen, in dem Praktiken der individuellen biographischen Navigation stattfinden und - beispielsweise durch das Halten von Reden, dem Singen, Tanzen, Dekorieren, Kochen und Einkleiden -Gemeinsamkeiten zum Ausdruck gebracht werden (Pfaff-Czarnecka 2012: 29). Im Sinne von Butlers Konzept der Performativität (Butler 1993) werden in diesen Situationen Normen und Konventionen bestätigt. Dies gilt zum Beispiel für die Rollenverteilung bei der Durchführung der Feste. So führten stets Männer aus Brahmanen-Familien die $p \bar{u} j \bar{a}^{22}$ durch.

Für meinen Fokus auf die Rolle der Zeitgestaltung in der biographischen Navigation ergaben sich u.a. folgende Fragen an meine Daten:

- Welche (dominanten) Repräsentationen von Zeit werden in den Narrationen und Handlungen der jungen Wissenschaftler*innen sichtbar?

- Wie werden diese in den Narrationen und Handlungen reproduziert, kritisiert und navigiert?

- Wie werden die Repräsentationen von Zeit mit sozialen Verortungen und damit mit den verschiedenen Aspekten des Selbst in Verbindung gebracht?

\subsubsection{Göttingen als räumliches und zeitliches Feld}

In meiner Forschung gab es keine räumlichen Grenzen zwischen dem, was häufig als ,Feld' bezeichnet wird, und meinem eigenen Lebensmittelpunkt. Allerdings war das Göttingen meiner Gesprächspartner*innen mit ganz spezifischen Orten und Zeiten verbunden, die ich im Folgenden darlegen werde.

\section{Die Zeitlandschaft indischer Wissenschaftler*innen in Göttingen}

In der ethnologischen Forschung wurde lange das, Wo' der Forschung thematisiert (Amit 1999; Coleman und Collins 2006). Die Zeit spielte in Darlegungen zur ethnologischen Methodik oft nur insofern eine Rolle, als dass die Dauer der Feldforschung als wichtiges Qualitätsmerkmal verstanden wurde (Steffen Dalsgaard und Nielsen 2016: 3). Ausgehend von den Arbeiten Ton Ottos, Steffen Dalsgaards und Morten

\footnotetext{
${ }^{22}$ pūjā (Sanskrit) „Verehrung“ ist ein religiöses Ritual, bei dem einer Gottheit Gaben dargebracht werden. Wird sowohl im eigenen Zuhause durchgeführt als auch in Tempeln (Oberlies 2008: 23-24).
} 
Nielsens (Steffen Dalsgaard 2013; Otto 2013; Steffen Dalsgaard und Nielsen 2016) zu den Zeiten des ethnologischen Feldes, stelle ich im Folgenden die Zeitlandschaft meiner Gesprächspartner*innen dar.

Ausgangpunkt meiner Darstellung sind die Zeiten, zu denen ich mit meinen Gesprächspartner*innen interagierte, und die in der Interaktion relevant gemachten Zeitkarten und sozialen Rhythmen. Dabei wird deutlich, dass gleichzeitig multiple Repräsentationen von Zeit und soziale Rhythmen wirkten, die sich aus Zeitlandschaften der lokalen Einbindungen in Göttingen und Indien speisten. So entstand eine transnationale Zeitlandschaft, in der die jungen Wissenschaftler*innen ihre Zeitgestaltung aushandelten.

Am ersten Tag meiner Feldforschung, dem 15.08.2010, nahm ich an der Feier des indischen Unabhängigkeitstages in Göttingen teil. Dieser Tag ist eine wichtige Koordinate in der Zeitkarte der indischen Nation: Er markiert die Geschichte und schafft eine Verbindung zwischen der Gegenwart und der Zukunft der Nation sowie deren Mitgliedern (McCrone und McPherson 2009c). Für meine Gesprächspartner*innen war er einer der wenigen Feiertage, die sie aus Indien nach Göttingen transferiert hatten, und eine Gelegenheit, Zeit zusammen zu verbringen. Weitere wichtige Zeiten für gemeinsame Aktivitäten waren: die Indian Cultural Night im März, die von Studierenden im Masterstudiengang eines Max-Planck-Instituts organisiert wurde; der Sportsday im Sommer; Diwali im Oktober/November und Zusammenkünfte an regional-spezifischen Feiertagen. In der Zeitlandschaft meiner Gesprächspartner*innen bildeten diese Ereignisse wichtige Koordinaten im Jahr und begründeten einen Rhythmus, in dem sich die jungen Inder*innen in Göttingen in größerer Anzahl trafen und mit der Zeitlandschaft Indiens resynchronisieren konnten.

Wie am Beispiel des Indian Independence Day bereits angedeutet, bilden diese Ereignisse nicht nur wichtige Koordinaten der Zeitlandschaft, sondern sind Zeitpunkte, zu denen weitere Repräsentationen von Zeit relevant gemacht wurden in der Form kollektiver und individueller Vergangenheiten, Gegenwarten und Zukünfte (Kapitel 6). Die jungen Wissenschaftler*innen wurden in ihrer Zeitgestaltung neben dem Festkalender auch durch Wahlzyklen (2014), regelmäßig wiederkehrende sportliche Ereignisse (Kricketweltmeisterschaft, z.B. 2011) und gesellschaftliche Bewegungen wie die für eine stärkere Anti-Korruptionsgesetzgebung (2011) in Indien geprägt.

In den Interviews wurden verschiedene Zeitkarten und soziale Rhythmen sichtbar, an denen sich meine Gesprächspartner*innen orientierten: an den Lebensverlaufsvorstellungen der indischen Mittelschicht (Kapitel 2) und der Wissenschaft (Kapitel 3). Die Zeitkarten waren wiederum mit den Visionen und Plänen, die die jungen Menschen von ihrer Zukunft hatten, verbunden. Je nach Zeitpunkt meiner Interviews und der teilnehmenden Beobachtung änderten sich die Fokussierungen auf bestimmte Koordinaten dieser Zeitkarten (Ende der Promotion, Heirat, Rückkehr).

Im Alltag wurde die Zeitgestaltung der Studierenden und Wissenschaftler*innen aus Indien durch die Zeitkarte der, Work-Life-Balance', wie sie meine Gesprächs- 
partner*innen nannten, beeinflusst. Sie hatten, verglichen mit den Erfahrungen, die sie in Indien gemacht hatten, in Göttingen größere Zeitsouveränität. Sie sahen die Möglichkeit, in hohem Maße selbst zu entscheiden, wann sie arbeiten würden und wie viel. Allerdings hatte das Bewusstsein von der zeitlichen Begrenztheit des Aufenthalts, das durch die Interaktion von Aufenthaltsgesetzgebung, Finanzierung der Promotion und ihren Vorstellungen vom akademischen und persönlichen Lebensverlauf entstand, einen großen Einfluss auf die Gestaltung der Arbeitszeit. So arbeiteten viele meiner Gesprächspartner*innen zwischen acht bis zehn Stunden am Tag, sechs Tage die Woche. Samstag entwickelte sich zum „Shopping-Tag“ der jungen Inder*innen. An diesem Tag wurden die nötigen Lebensmittel besorgt und Ausflüge in die Innenstadt Göttingens gemacht. Sonntag war die Zeit für Freund*innen, für die Pflege der Kontakte mit den Eltern, für sportliche Aktivitäten wie Kricket, für Ausflüge in das Umland und für die Treffen der indischen Vereinigung. Die Alltagsroutinen waren geprägt durch die Arbeit an der eigenen Forschung, dem Schreiben der Doktorarbeit sowie mit vertrauten Praktiken, die Kontinuitäten herstellten, wie dem Kochen und Essen indischer Speisen, dem Telefonieren oder Skypen mit den Eltern oder Freund*innen in Indien, dem Verfolgen von Nachrichten aus Indien und dem Schauen von Filmen.

Da meine Gesprächspartner*innen Studierende, Promovierende oder Postdoktorand*innen waren, bestand ihre Zeitlandschaft außerdem aus den Rhythmen von Vorlesungszeit und vorlesungsfreier Zeit, zeitlich befristeten Arbeitsverträgen oder Stipendien. Die Mehrzahl der jungen Wissenschaftler*innen kam im Spätsommer oder Herbst nach Göttingen. Dieser Ankunftszeitpunkt wurde unter anderem durch den Beginn von Promotionsprogrammen und dem Wintersemester in Göttingen geprägt. Diese Zeitlandschaft verkörperte sich an spezifischen Orten Göttingens und wurde in großem Maße durch die Lokalität Göttingen geformt.

\section{Die Orte indischer Wissenschaftler*innen in Göttingen}

Göttingen liegt im Süden Niedersachsens und damit in der Mitte Deutschlands. Die selbst ernannte „Stadt, die Wissen schafft“ hatte im Jahr 2017 134.824 Einwohner, wovon 31.650 Studierende waren (Referat Statistik und Wahlen der Stadt Göttingen 2018). Die große Anzahl Studierender prägt das Stadtbild und die Rhythmen der Stadt. In der vorlesungsfreien Zeit im Frühjahr und im Sommer ist die Stadt wesentlich leerer als während der Semester. Der Oktober ist wiederum von einem Ansturm von neuen Studierenden gekennzeichnet, der sich unter anderem durch äußerste Knappheit auf dem Wohnungsmarkt manifestiert.

Neben der 1737 gegründeten Georg-August-Universität befinden sich derzeit zwanzig Forschungseinrichtungen in Göttingen. Dazu zählen unter anderem fünf Max-Planck-Institute (Referat Statistik und Wahlen der Stadt Göttingen 2018). Die Universität war 2015 mit 15.215 wissenschaftlichen und nichtwissenschaftlichen Beschäftigten (einschließlich Medizin) der größte Arbeitgeber in Göttingen. Die Geschichte Göttingens ist stark mit der Geschichte der Universität verbunden. Die 
Antonie Fuhse: Mobilität und Zeitgestaltung

Bedeutung der Universität drückt sich im Motto Göttingens und im Stadtbild durch Statuen von Göttingens ,klugen Köpfen ' wie Lichtenberg, Gauß und Weber aus. An vielen Häusern befinden sich Plaketten, die auf berühmte ehemalige Bewohner*innen hinweisen. Zu den wichtigsten (historischen) Persönlichkeiten für Göttingen und die Universität zählen die Nobelpreisträger. Die Stadt ist recht klein und so kann praktisch alles mit dem Fahrrad erreicht werden.

Die Gebäude der Universität sind, wie auf der Karte zu sehen ist, in der Nähe der Innenstadt und in einem großen Nordcampus angesiedelt. Die Max-PlanckInstitute finden sich ähnlich verteilt vor allem im Norden Göttingens, im Umkreis der Universitätsmedizin und am Fassberg (auf der Karte rechts vom Nordcampus). Einige der jungen Wissenschaftler*innen wohnten in Studentenwohnheimen, die im Zentrum und dem nördlichen Teil Göttingens verortet sind. Viele lebten in Wohnungen, die sich in der Nähe ihrer Institute befanden, also rund um die Universitätsmedizin und den Nordcampus, beispielsweise in der Hermann-Rein-Straße, Annastraße, Philipp-Reis-Straße und Theodor-Heuss-Straße. Aus diesem Grund ergab sich innerhalb Göttingens ein kleineres Ballungsgebiet indischer Studierender, das von zwei meiner Gesprächspartner*innen scherzhaft „little India“ bzw. „Indian colony" genannt wurde.

Auf der Karte habe ich die Orte mit roten Zahlen markiert, an denen regelmäßig gemeinsame Aktivitäten der indischen Wissenschaftler*innen veranstaltet wurden. Auf dem Sportgelände der Universität (1) trafen sich einige meiner Gesprächspartner*innen an den Wochenenden für das Krickettraining. Auf diesem Gelände gab es außerdem einen Flaggenmast, der jedes Jahr am 15. August, dem Indian Independence Day, für das Hissen der Flagge benutzt werden durfte. In einigen Jahren wurde auf dem Sportgelände ein Indian Sportsday von der indischen Vereinigung durchgeführt. Im Max-Planck-Institut für experimentelle Medizin (2) wurde seit 2012 jedes Jahr die Feier von Diwali und ab 2013 die Feier des Indian Independence Day durchgeführt. Das Max-Planck-Institut für biophysikalische Chemie (3) war jeweils im März der Veranstaltungsort für die Indian Culture Night. Ein weiterer wichtiger Ort für Göttingen und die jungen Wissenschaftler*innen ist der Markt mit dem Gänseliesel (4) im Zentrum der Stadt. Hier wurden die Samstagnachmittage mit dem Einkaufen verbracht und nach der Verteidigung der Doktorarbeit wurde hier das Gänseliesel geküsst.

\subsection{Zusammenfassung}

In diesem Kapitel habe ich das theoretische und methodische Fundament gelegt, um in den folgenden Kapiteln die Rolle von Mobilität und Zeitgestaltung in der biographischen Navigation der jungen Wissenschaftler*innen aus Indien zu analysieren. An dieser Stelle möchte ich erneut betonen, dass ich die Zeitgestaltung als eine Perspektive darauf verstehe, wie Menschen ihre sozialen Verortungen, also ihre Einbindungen in die soziale Umwelt, aushandeln. Indem ich Zeitgestaltung 


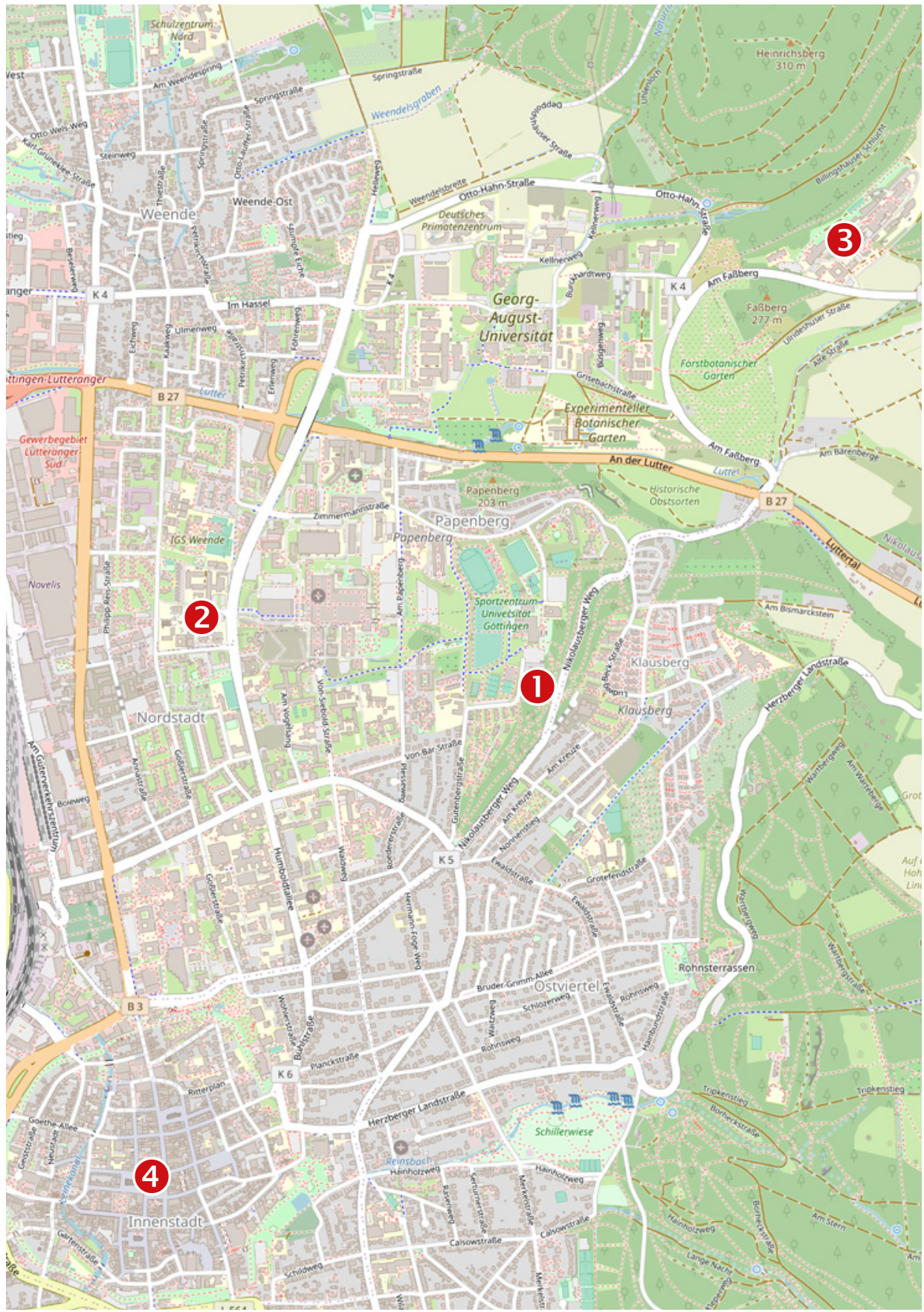

Abb. 1 Das Zentrum und der nördliche Teil Göttingens. (Karte (C) OpenStreetMapMitwirkende, CC BY-SA 2.0, siehe https://www.openstreetmap.org/copyright) 
als analytische Linse verwende, werden Machtbeziehungen und Formen von Handlungs- und Wirkvermögen wie die des Beschleunigens, des Hinauszögerns oder des Änderns der Reihenfolge sichtbar. Die vorgestellten Konzepte der Zeitlandschaften und Zeitkarten dienen der Kontextualisierung von Erfahrungen von Zeit, Zeitsouveränität und Praktiken der Zeitgestaltung. 


\section{Junge Menschen in Indien}

In Indien leben 1,35 Milliarden Menschen, ${ }^{23}$ davon sind ungefähr 27\% zwischen fünfzehn und neunundzwanzig Jahre alt (Ministry for Youth Affairs and Sports 2014: 10). Indien ist damit eine der Nationen mit der jüngsten Bevölkerung weltweit. ${ }^{24}$ In Politik und Medien wird die indische Jugend vor allem im Zusammenhang mit zwei Themen diskutiert. Zunächst wird in der großen Anzahl junger Menschen ein großes Potential für das wirtschaftliche Wachstum Indiens gesehen. Indien hat eine sogenannte ,demographische Dividende', das heißt, es leben dort mehr Menschen, die arbeiten können, als Menschen, die von der arbeitenden Bevölkerung abhängig sind (Karsch und Münz 2011). Allerdings wird die schiere Anzahl junger Menschen in Indien gleichermaßen als Problem verstanden, das es in den kommenden Jahren zu bewältigen gilt.

Für die Analyse der biographischen Navigation der jungen Wissenschaftler*innen ist es wichtig, den Kontext, also die soziale Umwelt, zu verstehen, in der sie eingebettet waren. Die Lebenswelten junger Menschen in Indien sind sehr verschieden

\footnotetext{
${ }^{23}$ Siehe Angaben auf der Seite von UN-Data (http://data.un.org/en/iso/in.html, abgerufen am 07.03. 2019).

${ }^{24}$ Siehe Central Statistics Office, Ministry of Statistics and Programme Implementation Government of India: „Youth in India“, 2017, http://mospi.nic.in/sites/default/files/publication_reports/Youth_in_ India-2017.pdf.
} 
und abhängig von ihrer Position in der sozialen Umwelt (Jeffery 2005). Die jungen Menschen, die im Fokus meiner Studie stehen, verfolgten aufgrund ihrer Herkunft aus der indischen Mittelschicht bestimmte Aspirationen und sahen sich spezifischen Erwartungen und Dilemmata ausgesetzt. Um diese Besonderheiten darzulegen, orientiere ich mich in meinen Ausführungen sowohl an aktueller ethnologischer Literatur zu jungen Menschen in Indien als auch an den Aussagen meiner Gesprächspartner*innen.

\subsection{Ein politischer und wirtschaftlicher Überblick: Von „India Rising“ $\mathrm{zu}$ „India's war on science“25}

Die jungen Menschen, die im Fokus meiner Studie stehen, wuchsen zur beziehungsweise nach der Zeit der wirtschaftlichen Liberalisierung Indiens in den frühen 1990er Jahren auf. Die Reformpolitiken von 1991, angeleitet durch den damaligen Finanzminister Manmohan Singh, und die darauffolgende wirtschaftlichen Öffnung Indiens für den globalen Markt hatten einen großen Einfluss auf die Politik, die Wirtschaft und die Gesellschaft Indiens (Lukose 2009: 2). Die jungen Menschen Indiens, vor allem jene, die in den Großstädten aufwachsen, finden sich heute in großen Einkaufszentren zwischen internationalen Marken sowie Bildern aus Bollywood und Hollywood wieder. Die Jugend ist durch Facebook und verschiedene andere Internetdienste global vernetzt, wodurch immer mehr Möglichkeiten für potentielle Lebenswege und Zugehörigkeiten in ihre Reichweite kommen. Vor allem die indische Mittelschicht hat von der wirtschaftlichen Öffnung Indiens profitiert und mehr Möglichkeiten, die Imaginationen von einem wirtschaftlich erfolgreichen, globalen Lebensstil zu erfüllen. Wie Bansal argumentiert, wurde dagegen das ,andere Indien auf Kosten der ,Entwicklung' des Landes und neoliberalen Reformen marginalisiert (Bansal 2013: 36; Lukose 2009; Derné 2008).

Die indische Politik wurde zwischen 2004 und 2014 von der Koalitionsregierung unter Manmohan Singh geprägt. In dieser Zeit entstanden neue Konzeptionen von Indiens Stellung in der Welt: weg von einem Land, das durch Armut geprägt ist, hin zu einem ,aufgehenden Stern" ${ }^{26}$ am globalen Himmel (Lukose 2009: 2). Gleichzeitig zur wirtschaftlichen Öffnung entwickelten sich hindunationalistische politische Strömungen in Indien. Die hindunationalistische Bharata Janata Party (BJP) hatte ihren Ursprung zwar schon in den 1950er Jahren, gewann aber erst in den 1990er Jahren an politischer Macht. Nach den Wahlen 1998 war die BJP das erste Mal Teil einer Koalitionsregierung und gewann seitdem an politischem Einfluss, der 2014 in der Wahl Narendra Modis zum Premierminister gipfelte. Seit der Wahl Modis, der

\footnotetext{
25 Siehe Shashi Tharoor: „India’s war on science“, Project Syndicate vom 20.02.2018, https://www. project-syndicate.org/commentary/india-modi-war-on-science-by-shashi-tharoor-2018-02.

${ }_{26}$ Diese Formulierung wurde unter anderem geprägt durch Fareed Zakaria: „India Rising“, Newsweek vom 03.05.2005, http://www.newsweek.com/india-rising-106259.
} 
in seinen Kampagnen „Sabka Saath, Sabka Vikaas“ („Entwicklung für alle“) (Kaul 2017: 528) versprach, hat Indien einige Veränderungen durchlaufen:

In the three years since then India has seen a slew of changes that have their roots in the Modi-led BJP effort to reshape society and economy in line with the right-wing politics of the Sangh Parivar (family of Hindu right-wing organizations that form the backbone of Hindu supremacism in the country) and the business interests of the corporate backers of the Modi-BJP campaign. Their efforts to fundamentally change the nature of Indian society and economy began immediately upon assuming power, continue apace, and have resulted in a targeting of constitutionally guaranteed principles such as secularism, freedom of expression, democracy, minority rights. (Kaul 2017: 528-29)

Die Wahlen von 2014 und darauf folgende Entwicklungen in Indien, wie der Versuch, ein Gesetz durchzubringen, nach dem das Schlachten von Rindern in ganz Indien verboten worden wäre ${ }^{27}$, waren und sind unter den jungen Menschen in meiner Studie Themen kritischer Auseinandersetzung. In den Gesprächen und ebenso auf Facebook drückten viele ihre Unzufriedenheit mit der Politik aus, wobei dies nicht unbedingt nur der BJP galt, sondern (indische) Politiker*innen generell als nicht vertrauenswürdig und korrupt eingeschätzt wurden. Als junge Wissenschaftler*innen setzten sich viele kritisch mit der Einstellung der Modi-Regierung gegenüber der Wissenschaft und wissenschaftlicher Fakten auseinander, die von dem indischen Politiker und Schriftsteller Shahshi Tharoor 2018 als „India's war on science“ beschrieben wurde. ${ }^{28}$

\subsection{Person, Selbst und die Familie in der indischen Mittelschicht}

Im Fokus des vorliegenden Kapitelabschnittes steht die familiäre Einbettung meiner Gesprächspartner*innen, in Intersektion mit ihrer Herkunft aus der ,middle-class', die einen Einfluss auf die Beziehungen innerhalb der Familie und die Positionierung des Einzelnen im Familienverband hat. Wer in Indien zur middle-class gehört, ist umstritten und wird je nach Forscher*in an unterschiedlichen Faktoren festgemacht, zum Beispiel am Einkommen, der eigenen Identifikation mit dieser Kategorie oder

\footnotetext{
${ }^{27}$ Einige Bundesstaaten wie Gujarat haben bereits ein Schlachtverbot von Rindern verhängt. Siehe BBC News: „India Surpreme Court suspends cattle slaughter ban“" vom 11.07.2017, https://www.bbc. com/news/world-asia-india-40565457.

${ }^{28}$ In seinem Artikel „India’s war on science“ beschreibt Shashi Tharoor eingängig, wie verschiedene Minister der Modi-Regierung und Modi selbst die Wissenschaft und die Rationalität angreifen. Siehe Shashi Tharoor: „India's war on science“, Project Syndicate vom 20.02.2018, https://www.project-syn dicate.org/commentary/india-modi-war-on-science-by-shashi-tharoor-2018-02.
} 
dem Konsumverhalten (Jaffrelot und van der Veer 2008; Lukose 2009; Säävälä 2010; Donner 2011). Für meine Studie ist Mazzarellas Perspektive auf die ,Realität` der Mittelschicht am fruchtbarsten. Er argumentiert, dass - egal wie streitbar der Begriff ist - „the category itself has become an important marker of identification, aspiration and critique in contemporary Indian public culture" (Mazzarella 2005: 3).

\subsubsection{Person und Selbst in Indien: ,Eingebetteter Individualismus}

Die Literatur zu Person und Selbst in Indien wurde lange Zeit von der dichotomen Konzeptualisierung des ,individualistischen' Selbst im ,Westen' und des ,kollektiven Selbst im ,Osten' geprägt (z.B. Daniel 1984, Roland 1988, Marriott 1976). Marriott und Inden (1977) beispielsweise sprechen vom indischen ,Dividuum', einer ungebundenen, offenen Person, die immer in Beziehung zu anderen Personen, Orten oder Dingen steht. ${ }^{29}$ Dieses Konzept wird dann wiederum mit der Person als ungebundenes und unabhängiges Individuum, also mit dem, was als das euro-amerikanische Konzept des Selbst verstanden wird, kontrastiert (Marriott 1976; Marriott und Inden 1977). Wie Sarah Lamb herausstellt, zeigen einige neuere Studien allerdings, dass die Bedeutung von Beziehungen nicht ausschließt, dass Personen die Erfahrung von Individualität machen (Lamb 1997: 282; vgl. Ewing 1990; Hermann 2003a: 85). So verdeutlichen unter anderem ethnologische Untersuchungen, dass es in Indien eine Vielzahl von Auffassungen von Person und Selbst gibt, in denen Vorstellungen vom relationalen und autonomen Selbst nebeneinander existieren und sich gegenseitig beeinflussen (Lamb 1997; Ewing 1990). Mattison Mines (1994: 2) spricht zum Beispiel vom „Tamil Individualism“, und verdeutlicht, dass es auch in Indien Vorstellungen vom Individuum und Individualität gibt, die sich aber vom westlichen Verständnis vom Individuum unterscheiden.

Neben der Literatur, die sich direkt mit Person und Selbst in Indien beschäftigt, gibt es eine wachsende Anzahl von Werken, die sich mit der Entwicklung des Selbst in der Globalisierung auseinandersetzen. Grundtenor in diesen Studien ist die Feststellung, dass sich als Antwort auf neue globale politische und wirtschaftliche Umstände ein neues Konzept des Individuums entwickelt habe. Das Individuum wird in diesem Konzept als unabhängiger Experte verstanden, der Risiken selbst managen und einen eigenen Pfad in der Welt finden müsse (Radhakrishnan 2011: 118). Beck (1994; Beck und Beck-Gernsheim 1994) nennt den Prozess, der mit der Loslösung des Menschen von traditionellen Klassen- und Familienbindungen einhergeht, Individualisierung. Auf ähnliche Weise argumentiert Anthony Giddens (1994), dass Individuen von ihren lokalen Kontexten und Beziehungen „disembedded“, also von diesen losgelöst seien.

Radhakrishnan weist daraufhin, dass diese Theorien des entbetteten und entwurzelten Selbst nicht aus Untersuchungen spezifischer Kontexte erwachsen sind (Rad-

\footnotetext{
29 Das Konzept des „Dividuums“ wurde von Marilyn Strathern (1988) benutzt, um zwischen Konzepten von Person in Melanesien und dem ,Westen` zu unterscheiden.
} 
hakrishnan 2011: 118). Genau aus diesem Grund übersehen diese Konzepte, dass das Selbst mobiler Personen von Gender, Klassenzugehörigkeit sowie familiärem und nationalem Kontext beeinflusst wird und so ganz unterschiedliche Individuen entstehen, die durch ihren jeweiligen Kontext sehr verschiedene Möglichkeiten haben (Radhakrishnan 2011: 118). Smitha Radhakrishnan spricht von „embedded individualism“ (Radhakrishnan 2011: 117-20) und argumentiert, dass sich Vorstellungen von Individualität, die im Kontext der Wissensökonomie entstehen, im Zusammenspiel mit bereits bestehenden lokalen Konzepten von Person, Identität und Relationalität entfalten (Radhakrishnan 2011: 119). Diese Entfaltung muss nicht ohne Spannungen ablaufen, so erklärte Henrike Donner (2012) in einem Interview zum Familienleben in der indischen Mittelschicht:

(...) you sense moral tensions between being an individual and being part of a family; between traditional outlooks on gender roles and the desire to be part of a hip, modern youth culture; and practices and moralities promoted in the media and local discourse (...). (Donner 2012)

Die beschriebene Spannung zwischen dem Verlangen nach individueller Freiheit und dem Wunsch nach der Sicherheit der Herkunftsgemeinschaft war auch Teil des Lebens vieler meiner Gesprächspartner*innen und musste von diesen immer wieder ausgehandelt werden. Zu einer Gemeinschaft zu gehören, von dieser unterstützt zu werden und sich dadurch sicher und aufgehoben zu fühlen, kommt in diesem Sinne mit einem Preis daher: der Loyalität zu der Gemeinschaft, der Einhaltung der Regeln dieser und damit manchmal ebenso der Einschränkung der eigenen Freiheiten (Pfaff-Czarnecka 2012: 32).

\subsubsection{Die Familie: Verortung, Verantwortung und Kontrolle}

It provides you with linkage to your home and you always have your family, you never have the feeling that you are alone in the world. It always has these two sides of the coin: on one side, as you said, it's always sacrifice and compromise but on the other side (...), keeping in link with my family. And also preserving my family tradition: that also motivates one to stay in the way of life their family wants them to. (Āmāl, 20.02.2013)

Wie Āmāl, die mit dem Erasmus-Programm für ein Semester nach Göttingen gekommen war, betonte, vermitteln familiäre Einbindungen ein Gefühl der Sicherheit und verlangen gleichzeitig das Einhalten von Regeln und Erfüllen von Pflichten. Die Familie agiert in diesem Sinne als Konstellation, die mit Pfaff-Czarnecka (2012: 79) als „Regime der Zugehörigkeit“ bezeichnet werden kann. Āmāl erklärte, wenn sie sich nicht an die Regeln ihrer Eltern halten würde, hätte sie Angst, dass diese enttäuscht sein könnten. Allerdings betonte sie auch, dass, egal was Söhne oder Töchter 
anstellen würden, die Eltern ihre Kinder nie verlassen könnten, dies sei der „weak point" von Eltern.

Das Familienleben meiner Gesprächspartner*innen wurde stark von ihrer Herkunft aus der indischen Mittelschicht und spezifischen Familienstrukturen, die von Seymour und Mukhopadhyay als „patrifocal“ (Mukhopadhyay und Seymour 1994) bezeichnet werden, geprägt. Die patrifokale Familienstruktur und Ideologie zeichnet sich aus durch

(...) an emphasis upon family generally and the extended family specifically; a complex of structural features (patrilocal residence (...)) that emphasise and reinforce the centrality of males; gender differentiated family roles and responsibilities (...), emphasis on family control and regulation of female sexuality and reproduction (...) (Seymour 1995: 71)

Die Ausprägung dieser Art der Familienstruktur ist in Indien sehr heterogen (Sondhi 2013: 92) und hat sich in den vergangenen Jahrzehnten verändert. Allerdings wird in vielen aktuellen Studien deutlich, dass der Fokus auf die Familie, die Zentralität des Mannes und die genderspezifischen Rollen und Hierarchien in den Familien weiter präsent sind (Derné 2008; Radhakrishnan 2011; Sondhi 2013; Fleming 2016). Die anhaltende Bedeutung dieser Vorstellung vom Familienleben steht unter anderem in Verbindung mit der Aushandlung des Indischseins im Kontext der Globalisierung (Derné 2008; Radhakrishnan 2011). Besonders für jene Menschen, die sich selbst als zur Mittelschicht zugehörig verstehen, ist die Betonung von Familienwerten und ,traditionellen' Geschlechterrollen- und Beziehungen ein wichtiges Mittel, um ihren Status zu kennzeichnen (Donner 2015: 135) und sich selbst vom , Westen' abzugrenzen (Derné 2008; Radhakrishnan 2011).

Die beschriebenen familiären Strukturen und Ideologien wirken sich auf Männer und Frauen unterschiedlich aus. Traditionell sollten die Männer wie in vielen anderen Gesellschaften die Hauptverdiener und das Oberhaupt der Familie sein (Donner 2015: 143; Jeffrey 2010b). Für die Männer bedeutet dies, früh in familiäre Verantwortungen eingebunden zu sein, wie auch Kundan, der für seine Promotion in Neurowissenschaften nach Göttingen gekommen war, betonte:

If you have a sister, you are responsible for her to get married; you are responsible to get the money, gold; you need to take care of your parents. So that means you need to find a job. And this makes a guy already do extra work (...), always this is there in the mind. And it stays every day that: „I have to find a job, I have to study. "And then your parents remind you this every time you speak. So it puts a lot of pressure on the guy during his development, which kind of destroys his early happy life. (Kundan, 14.08.2013) $)^{30}$

\footnotetext{
${ }^{30}$ In diesem Abschnitt des Interviews wird nicht ganz klar, ob er von sich selbst spricht. Später bestätigt er aber auf Nachfrage, dass es ihm während seines Masterstudiums so erging.
} 
Die jungen Männer müssen darauf hinarbeiten, ihre Rolle als „breadwinner“ (Jeffrey 2010b: 467) erfüllen zu können. Wie sich im Verlauf dieser Arbeit zeigen wird, wird die Erfüllung dieser vorgesehenen Rolle immer problematischer - unter anderem, weil es in Indien immer schwieriger wird, einen sicheren bzw. überhaupt einen $\mathrm{Ar}$ beitsplatz zu erhalten (Jeffrey 2010b).

Für junge Frauen bedeutet die Betonung ,traditioneller ${ }^{`}$ Familienwerte und Geschlechterrollen unter anderem, kontrolliert zu werden. Nabhi erzählte, dass sie in einer Familie aufgewachsen ist, in der Mädchen stark unterdrückt wurden. „I mean not on daily basis but it's like a broad suppression that they are just not allowed to talk to men, not allowed to interact with people." Alle Männer in der Familie lebten mit der Angst, dass die Mädchen mit einem Mann reden könnten, sich verlieben und darauf drängen könnten, ihn zu heiraten. „They just think so far away that they stop the present to grow." Ihr Vater habe sie wirklich geliebt, betonte sie, er habe nie versucht, sie zu irgendetwas zu zwingen. Als sie älter wurde, sei er aber von der Familie und dem weiteren Umfeld unter Druck gesetzt worden: „He is a dad of a daughter, and there is a certain way of raising a daughter; he cannot give all the freedom to me, so he was exerting that social pressure on me" (Nabhi, 17.03.2016).

Kontrolle nicht nur von Familienmitgliedern, sondern über das Leben generell versteht Säävälä als ein zentrales Merkmal der indischen Mittelschicht (Säävälä 2010). Ein wichtiges Element in diesem Prozess ist die Kontrolle der Reproduktion. Die Familien in Indien, die sich selbst als der Mittelschicht zugehörig definieren, haben häufig nicht mehr als zwei Kinder (Säävälä 2010: 30). Dadurch grenzen sich diese Familien von den Armen und den unteren Schichten ab, die tendenziell mehr Kinder haben. Außerdem steht die Anzahl von Kindern mit finanziellen Überlegungen im Zusammenhang. So wäre es für die meisten Familien schwierig, für mehr als zwei Kinder die angestrebte schulische Ausbildung zu finanzieren (Säävälä 2010: 31). Von meinen Gesprächspartner*innen hatten achtzehn von vierunddreißig ein Geschwisterkind, sechs mehr als ein Geschwisterkind, fünf waren Einzelkinder und von den sechs weiteren habe ich keine Angaben. Zusammen mit Gender haben Faktoren wie Alter und die Geburtenfolge einen Einfluss auf die schulische Laufbahn und damit auch die spätere universitäre und berufliche Laufbahn (Chopra 2005: 299). Außerdem beeinflussen diese beiden Aspekte, wie ich zeige, die Aushandlung von Heirat und der Rückkehr nach Indien.

\subsection{Das Aufwachsen in der indischen Mittelschicht}

Für ein besseres Verständnis der biographischen Navigation und der Rolle, die internationale Mobilität in dieser spielt, beschäftige ich mich im Folgenden damit, was die Zugehörigkeit zur indischen Mittelschicht ausmacht. Im Mittelpunkt meiner Ausführungen stehen Vorstellungen von der Jugend, die Bedeutung, die guter Bildung zugeschrieben wird, und die Erwartungen, die an die jungen Menschen aus der Mittelschicht gestellt werden. 


\subsubsection{Die Jugend als Fundament für die Zukunft}

Gender und die Lebensphase, in der sich Menschen befinden, spielen eine wichtige Rolle für Konzepte des Selbst (Lamb 1997). Meine Gesprächspartner*innen befanden sich zur Zeit meiner Studie in einer Lebensphase zwischen Kindheit und Erwachsensein, die als Jugend bezeichnet werden kann (Bansal 2013: 2). Es gibt mittlerweile einige ethnologische Studien, die sich explizit mit jungen Menschen auseinandersetzen (u.a. Cole und Durham 2007; Vigh 2007; Cole 2010; Jeffrey 2010a, 2011; Anne Dalsgaard et al. 2014). In den genannten Werken wird deutlich, dass: „(...) the transition from childhood to adulthood should not be considered fixed and stable. .... Youth is differently constituted and configured in different times and places" (Christiansen, Utas und Vigh 2006: 10). Jugend ist keine natürlich gegebene Kategorie, sondern ein Konstrukt. Aus diesem Grund muss analysiert werden, wie Jugend in einem bestimmten Kontext, zu einer bestimmten Zeit konstruiert wird und wie die jungen Menschen selbst dieses Konstrukt navigieren und definieren (Christiansen, Utas und Vigh 2006: 11).

Auf meine Frage hin, wie sie denn ihre momentane Lebensphase charakterisieren würden, antworteten zwei meiner Gesprächspartner*innen:

I think this is a really important phase of my life which will determine how my life is going to shape up further. So I just hope that after two years I will get some good position in India and settle down. (Nandita, 03.09.2011)

This is the basement; this is the foundation for the building that you want to erect for yourself. (Ravi, 15.09.2011)

Nandita und Ravi verstanden ihr gegenwärtiges Leben als wichtige Basis für ihre Zukunft. Sie wollten jetzt das Fundament für ihr zukünftiges Leben aufbauen und sahen das Erreichen weiterer akademischer Grade als wichtige Strategie dafür. Wie Ravi und Nandita waren die meisten meiner Gesprächspartner*innen sehr hoffnungsvoll, dass sie tatsächlich durch ihre eigenen Bemühungen in der Gegenwart ihre zukünftigen Chancen auf einen Arbeitsplatz würden verbessern können. Die optimistische Einstellung zur eigenen Zukunft findet sich laut Amit und Dyck (2012: 16-17) in vielen Studien zu jungen Menschen weltweit und wird auch in DeSouzas Studie Indian Youth in a Transforming World von 2009 deutlich. Bildung wird dabei von den jungen Menschen und ihren Familien als wichtiges Mittel gesehen, um sich eine (bessere) Zukunft zu schaffen, und hat einen großen Einfluss darauf, was sich für die Zukunft erhofft wird.

Ravi und Nandita schienen die Phase der Jugend nicht als Zeit des Rebellierens verstanden zu haben, einer stereotypen Interpretation von Jugend, die laut Amit und Dyck (2012: 10) in ,westlichen' Kontexten vorherrscht. Die Entscheidung zur Mobilität war für die meisten der jungen Wissenschaftler*innen nicht vorrangig mit dem Gedanken des Entkommens aus Strukturen in Indien verbunden, sondern eher 
im Gegenteil mit dem Versuch, die Anforderungen zu erfüllen, die durch die Gesellschaft an sie gestellt wurden. Allerdings bedeutete dies nicht, dass sie nicht einigen Elementen der Lebenswelt in Indien kritisch gegenübergestanden hätten oder weniger Handlungsvermögen ausüben würden. Handlungsvermögen liegt sowohl in der bewussten Entscheidung dafür, die vorgesehenen Pfade einzuschlagen, als auch im Versuch, neue Wege zu finden.

\subsubsection{Bildung in der middle-class: Investitionen und Erwartungsdruck}

Henrike Donner schreibt: „Education (...) makes a middle-class person, shaping a youngster by instilling virtues such as discipline and self-control (...)" (Donner 2015: 141). Einige meiner Gesprächspartner*innen machten deutlich, dass sie sich bewusst waren, wie sie von ihrer ,middle-classness' und damit verbundenen Werten geformt wurden. Am eindrucksvollsten führte mir dies Rohini in einem FacebookEintrag vor Augen. Sie teilte einen Text, der besagte: „I'm so middle class that I actually had to study hard“ und vermerkte dazu: „And the saga continues. But thankfully that's the value instilled in my brain quite young, and I hope to ripe [sic] the benefits...". ${ }^{31}$ Rohini schien sich auf der einen Seite fast darüber lustig zu machen, was es bedeutet, tatsächlich zur middle class zu gehören, andererseits verstand sie ihre harte Arbeit als Investition in ihre zukünftige Karriere.

Familien verstehen die schulische Bildung ihrer Kinder als Eintrittskarte zu einer besseren Zukunft, zu einer besseren Anstellung, besseren Heiratsmöglichkeiten, zu verbesserten sozialen Kompetenzen und kulturellem Kapital (Jeffery 2005: 35). Damit verbunden besteht die Hoffnung der Eltern, die Situation der Familie insgesamt zu beeinflussen und familiäre Aspirationen zu erfüllen (Jeffery 2005). Große finanzielle und persönliche Opfer für die Ausbildung der Kinder zu bringen, scheint aus dieser Perspektive eine gute Investition zu sein. Den jungen Menschen war wiederum bewusst, dass ihre Eltern viel Geld und Zeit in ihre Ausbildung investiert hatten. Sie internalisierten unter anderem aus diesem Grund ein Verantwortungsgefühl gegenüber ihren Eltern: „When your family is putting all input into you, you already realize that there is nothing else to give back, that you have to give them because they do everything to develop you, and you need to give them back something" (Kundan, 14.08.2013).

Die jungen Menschen setzen sich selbst unter Druck (Säävälä 2010: 33-34), da sie das Gefühl haben, die Erwartungen ihrer Eltern erfüllen und etwas zurückgeben zu müssen. Von Seiten der Eltern wird nicht nur Druck ausgeübt, sondern auch starke Kontrolle, die auf Überlegungen basiert, wie aus den Investitionen der größte Erfolg generiert werden kann. Dies bedeutet unter anderem, dass die Eltern versuchen, auf wichtige Entscheidungen wie die Wahl des Studienfaches Einfluss zu nehmen,

\footnotetext{
31 Am 3. April 2016 auf Facebook geschrieben. Ich habe an dieser Stelle die Formulierung „ripe“ nicht geändert, da ich den von Rohini auf Facebook veröffentlichten Text nicht ändern wollte. Ich gehe allerdings davon aus, dass sie „reap“, also „ernten“ meinte.
} 
da angenommen wird, dass nur Fächer wie Medizin, Ingenieurwissenschaften oder Jura Erfolg garantieren (Säävälä 2010: 33; Gautam 2015). Im Fall von Nathin wird deutlich, wie die Eltern versuchten, auf die Fächerwahl einzuwirken:

My dad always wanted me to be a computer engineer, but then I realized I'm really not good in maths like my dad - or computers. So I told him that I want to do biotech, but they didn't accept for two months. I didn't go to home at all, when I was in India - didn't even speak to them - and I stayed with my cousin. (...) My mom was telling him to send me back home. (...) So my dad prayed, [and] finally he is fine with what I decided. (Nathin, 08.05.2013)

Das Beispiel von Nathin macht deutlich, dass durch das Verständnis von Bildung als Investition folgende Dynamik in den Familien entsteht: Die Eltern opfern viel Zeit und Geld und stellen große Erwartungen an ihre Kinder, die Kinder möchten diese Erwartungen erfüllen und setzen sich selbst unter Druck. Oder sie versuchen, wie Nathin, ihre eigenen Pläne gegen den Willen der Eltern durchzusetzen.

Dadurch, dass es in Indien sehr viele junge Menschen gibt, steigt der Druck und es entsteht das Gefühl, ständig im Wettbewerb miteinander zu stehen:

We are so many, and we are always in a very competitive environment. It starts quite early. Like, as young as fifteen you start thinking, „I have to do this and then I will do this and then I will do this." So it's almost like you planned it for next six years, although it might happen that you are completely burned out by the end of your bachelors. But still people plan that way. (Rohini, 18.08.2016)

Die Eltern nehmen in unterschiedlichem Grad Einfluss auf die Planung der schulischen und beruflichen Laufbahn (Mukhopadhyay 2004; Chopra und Jeffery 2005; Gautam 2015). Die gesellschaftliche Auslegung der Studienfächer und Disziplinen als maskulin oder feminin hat einen Einfluss auf diese Planung (Gautam 2015). ${ }^{32}$ In der elften Schulklasse, wenn die Schüler*innen zwischen drei „streams“"33 wählen müssen, ist es für die Jungen die Norm, science zu wählen. Von den Mädchen wird wiederum erwartet science zu wählen, wenn sie gute und ,hart-arbeitende` Schülerinnen sind (Gautam 2015: 37). Die Auswahl des streams in der elften Klasse beeinflusst die Fächerwahl für das Studium und ist mit Überlegungen zur zukünftigen Karriere verbunden. Mit einigen Fächern bzw. Disziplinen wird größere Arbeitsplatzsicherheit assoziiert (Gautam 2015). Ein sicherer Arbeitsplatz ist wiederum für die Männer von großer Bedeutung, weil diese, wie bereits ausgeführt, die zukünftigen Hauptverdiener ihrer eigenen Familie sein sollen. Viele Eltern sehen dagegen das Studium

\footnotetext{
32 Eine solche Bewertung von Fächern existiert auch in Deutschland (Budde 2009).

${ }_{33}$ Bei den drei streams handelt es sich um „science“, „,ommerce“ und ,arts/humanities“ (Gautam 2015).
} 
ihrer Tochter nicht unbedingt in Bezug zu einer zukünftigen Karriere, sondern in Verbindung mit Heiratsmöglichkeiten (vgl. Mukhopadhyay und Seymour 1994). Diesen Zusammenhang kommentierte Rohini in unserem Interview mit den folgenden kritischen Worten: „Nowadays more or less everybody does a graduation. (...) Because that's the basic thing that you need to show on a matrimonial site. So this is the minimum investment that the parents will be interested to do, because otherwise they will not find a groom" (Rohini, 18.08.2016).

Obwohl die Überlegungen zur Heirat auf den Studienverlauf von Frauen immer noch einen erheblichen Einfluss haben (Gautam 2015), gibt es gerade in der höheren Mittelschicht Veränderungen in der Einstellung zur Ausbildung und Karriere von Frauen. Carol Mukhopadhyay zeigt für die frühen 2000er, dass schulischer und universitärer Erfolg gerade in science in hohem Maße respektiert wird (Mukhopadhyay 2004: 473). „A brilliant daughter was a source of pride (...).“ (Mukhopadhyay 2004: 473). Diese Einstellung wird ebenso in meiner Studie deutlich, wobei dadurch sichtbar wird, dass gerade meine weiblichen Gesprächspartner*innen zu den höheren Rängen der Mittelschicht bzw. zu den „educationally advantaged“ (Thapan 2009: 29) gehörten. Damit bezeichnet Thapan jene Menschen, die sowohl Zugang zu Bildung haben als auch ihre schulischen bzw. akademischen Ziele erreichen können (Thapan 2009).

Die schulische und universitäre Ausbildung ist stark mit Aspirationen für die Zukunft verbunden (Stambach und Hall 2017). Amit und Dyck (2012) sprechen in diesem Zusammenhang von den,Versprechen', die mit Bildung assoziiert werden und die die Aspirationen formen, die junge Menschen und ihre Familien für die Zukunft hegen (Amit und Dyck 2012: 13). In vielen Fällen gehen die Versprechen nicht auf und trotzdem werden sie durch Institutionen wie die Weltbank und deren Berichte immer wieder reproduziert. ${ }^{34}$

Durch unterschiedliche Faktoren, wie Gender, gesellschaftliche Schicht und Herkunft aus der Stadt oder vom Land entstehen unterschiedliche schulische und universitäre Laufbahnen und damit unterschiedliche Möglichkeiten auf dem Arbeits- und dem Heiratsmarkt.

\footnotetext{
34 „Delivered well, education - and the human capital it creates - has many benefits for economies and for societies as a whole. For individuals, education promotes employment, earnings, and health. It raises pride and opens new horizons. For societies, it drives long-term economic growth, reduces poverty, spurs innovation, strengthens institutions, and fosters social cohesion." (World Bank Group 2018a:V).
} 


\subsubsection{Das Bildungssystem: Gleiche Chancen für Alle?}

The benefits that can be extracted from education (...) are largely - though not wholly - pre-determined by people's locations in systems of social and economic inequality. (Jeffery 2005: 34)

In Indien haben nicht alle Menschen den gleichen Zugang zum Bildungssystem. Die Ungleichheiten bestehen bereits seit der Entstehung der formellen Wissensvermittlung in Indien in der frühen vedischen Zeit (ab 1500 v. Chr.) (Prabhu 2006: 23) und ergeben sich aus der starken Verbindung der Wissensvermittlung mit gesellschaftlichen Hierarchien. In der Kolonialzeit musste neu ausgehandelt werden, welches Wissen gelehrt und damit wertgeschätzt werden sollte und wer Zugang dazu haben sollte. $\mathrm{Zu}$ den bereits existierenden Ungleichheiten in der schulischen Ausbildung kamen neue hinzu. Die englische Sprache und der Zugang zum Erlernen dieser waren das Privileg der Eliten, die eigene Schulen gründeten und andere vom Lernen der Sprache der Kolonialmacht ausschlossen (Jeffery 2005: 16-17).

Nach der Unabhängigkeit wurde die Schulbildung direkt mit dem ökonomischen Wachstum des Landes verbunden (Prabhu 2006: 26). Jawaharlal Nehru, der erste Premierminister Indiens, vertrat eine Vision Indiens als moderne, sich entwickelnde Industrienation. Die bildungspolitische Planung der 1950er und 60er hatte als Ziel, mittels Bildung die Gleichheit unter der Bevölkerung zu fördern und allen die gleichen Chancen zu ermöglichen. Bis heute gibt es allerdings gravierende Unterschiede im Zugang zu und der Qualität der Schulbildung. Die Ungleichheiten liegen auch darin begründet, dass es in Indien eine Koexistenz verschiedener Schulformen gibt: sowohl zentralstaatlich finanzierte und kontrollierte Schulen als auch vom Bundesstaat getragene Schulen, Convent Schools (aus der christlichen Missionierung entstanden) und private International Schools (Hillger 2014). Die Qualität der Schulen ist ganz unterschiedlich, wobei den privaten Schulen eine bessere Qualität zugesprochen wird. Mittlerweile besuchen zirka $40 \%$ der Grundschüler eine private Schule (Hillger 2014).

Die Regierung konnte ihr Ziel der gleichen, qualitativ guten und kostenlosen Bildung für alle Kinder in Indien bisher nicht umsetzen. Wer wirtschaftliches und soziales Kapital hat, kann sich entsprechend bessere Bildung erkaufen. Fast 55\% der Familien in der indischen Mittelschicht sparen aus diesem Grund für die Ausbildung ihrer Kinder (GATE Germany 2011: 5). Es besteht daher nur eine Illusion der Chancengleichheit (Bourdieu und Passeron 1971), die den Menschen vortäuscht, dass alle die gleichen Möglichkeiten haben und Leistung das einzige Kriterium ist.

\section{Hochschulen in Indien: Examen und Quoten}

Auf dem College-Level gibt es Mechanismen, mit denen der indische Staat versucht, gegen die Ungleichheiten anzugehen. Allerdings entstehen viele Ungleichheiten und 
daraus resultierende Nachteile für bestimmte Bevölkerungsgruppen bereits in der Grundschule (Desai 2010: 86).

Indien hat den Hochschulsektor in den vergangenen Jahren stark ausgebaut. So entstanden zwischen 2006 und 2013 zirka 12.000 neue Colleges und 270 Universitäten. 65\% davon werden von privaten Trägern finanziert. Die Zentralregierung investierte vor allem in den Ausbau von Exzellenzzentren und so wurden neun Indian Institutes of Technology (IITs), fünf Indian Institutes of Science Education and Research (IISER) und fünfzehn Central Universities neu aufgebaut. Diese in den verschiedenen Großstädten Indiens angesiedelten Hochschulen sind Forschungseinrichtungen von internationalem Rang, die Wissenschaftler*innen und Spezialist*innen hervorbringen, die auf der ganzen Welt einen guten Ruf haben (Hillger 2014; Ajantha Subramanian 2015). Auch viele meiner Gesprächspartner*innen hatten an einem Institute of Technology oder einer Universität, die in den Rankings in Indien weit oben stand, studiert. Daraus lässt sich schließen, dass die meisten meiner Gesprächspartner*innen aus Familien kommen, die aus verschiedenen Gründen einen Zugang zu guter Bildung gewährleisten konnten. Das System der Quoten, das ich im Folgenden kurz beschreiben werde, verhalf meines Wissens nach nur einem meiner Gesprächspartner*innen zu einem Studienplatz. ${ }^{35}$

Der Zugang zu den Hochschulen wird in Indien durch Eingangsexamen und Quoten für bestimmte Gesellschaftsgruppen geregelt. So muss jede/r Bewerber*in für einen Studienplatz die gleiche standardisierte Prüfung ablegen. In jeder von der Regierung definierten Kaste bzw. Gruppe (Scheduled Tribe (ST), Scheduled Caste (SC), Other Backward Caste (OBC), Upper Caste) erhalten nur diejenigen einen Platz, die einen für ihre Gruppe festgelegten Grenzwert geschafft haben (Bertrand, Mullainathan und Hanna 2008: 2). Das System der Reservierungen, das bereits 1950 in Indien eingeführt wurde, soll dafür sorgen, dass bisher benachteiligte Bevölkerungsgruppen einen besseren Zugang zu Hochschulbindung und damit auch zu wirtschaftlichen Möglichkeiten erhalten (Sekhri 2011: 1). Der Erfolg der Quoten ist umstritten. So zeigen Statistiken zwar, dass sich der Anteil der Studierenden der Scheduled Casts mit 12,5 Prozent ihrem Bevölkerungsanteil angenähert hat, aber andere Bevölkerungsgruppen weiterhin in den Hochschulen unterrepräsentiert sind (Hillger 2014). Die Wirksamkeit und der Einfluss der Quoten auf die Hochschulbildung werden immer wieder debattiert. Die Gegner*innen der Reservierungen stehen auf dem Standpunkt, dass diese die Qualität der Ausbildung mindern würden. Die Prüfungsleistungen der Studierenden, die unter die Quoten fallen, sind im Vergleich meistens schlechter als die der anderen Studierenden. Daraus schließen die Gegner*innen der Quoten, dass die Studienplätze an die STs, SCs oder OBCs ver-

\footnotetext{
35 Obwohl ich das Thema Kastenzugehörigkeit nicht explizit erforscht habe, kann ich von meinen Daten herleiten, dass etwa 80\% meiner Gesprächspartner*innen höheren Kasten angehörten. Nur einer der jungen Männer erklärte mir, dass er unter die Kategorie „Other Backward Class (OBC)“ fallen würde.
} 
schwendet seien, da eigentlich andere die Plätze durch ihre Leistungen (merit) verdient hätten. Wie Carol Upadhya feststellt, ignoriert das „merit-Argument“ aber:

(...) the social and economic factors that produce "meritorious" candidates in the first place, especially the continuing monopoly over a certain kind of cultural capital that is enjoyed by the middle class - which is composed mainly of upper castes - due to their greater access to the best educational institutions and other processes of social closure. (Upadhya 2007: 1866)

Die Reservierungen werden in Indien immer wieder zur Streitfrage. ${ }^{36}$ Auch unter meinen Gesprächspartner*innen wurde kritisch über den Nutzen der Quotenregelung reflektiert. Sie berichteten mir von frustrierenden Erlebnissen aus Eingangsexamen und erzählten von geplatzten Träumen. Ajay zum Beispiel wollte eigentlich Medizin studieren, erreichte im Eingangsexamen sehr gute Noten, erhielt aber keinen Platz an einem medical college. Gerade in diesem Bereich ist der Wettbewerb um Studienplätze sehr groß, wie nicht zuletzt Betrugs- und Bestechungsskandale immer wieder sichtbar machen. Wie für die indischen Studierenden in Sondhis Studie (2013) wurde der starke Wettbewerb um gute Studienplätze und die zum Teil fehlende Qualität der Ausbildung für einige meiner Gesprächspartner*innen zum Grund, ein Studium im Ausland anzustreben.

\subsubsection{Und nach dem Studium? Arbeitsmarktsituation in Indien}

In Indien machen immer mehr Menschen einen Universitätsabschluss. Statistiken zeigen allerdings, dass ein Universitätsabschluss kein Garant für einen Arbeitsplatz ist. So ist in Indien ein Drittel der Universitätsabsolvent*innen arbeitslos (Ministry of Labour and Employment 2014: III). Darüber hinaus steigt die Wahrscheinlichkeit, arbeitslos zu sein, mit dem Level des Bildungsabschlusses. Gleichzeitig gibt es in Indien aber in bestimmten Bereichen eine Knappheit an qualifizierten Arbeitskräften. In der Studie der Federation of Indian Chambers of Commerce and Industry (FICCI) wird von einer „skills gap“ (FICCI 2013: 68) gesprochen. Es besteht also eine Diskrepanz zwischen den Fähigkeiten der Arbeitskräfte und den tatsächlichen Arbeitsplätzen. Sehr gute Chancen auf dem indischen und dem internationalen Arbeitsmarkt haben nur sehr wenige Absolvent*innen in Indien, nämlich diejenigen, die an einer der Elite-Universitäten studiert haben (Ajantha Subramanian 2015). Eine weitere Problematik entsteht daraus, dass die Wahl des Studiums und eines Studienfaches wie dargestellt von gesellschaftlichen und familiären Erwartungen beeinflusst wird (Sondhi 2013: 117; Gautam 2015) und weniger mit tatsächlichen

\footnotetext{
${ }^{36}$ Im Sommer 2015 versuchte der Patidar Anamat Andolan Samiti (PAAS) in Gujarat den Status der OCB für die Patida-(Patel-)Gemeinschaft zu erstreiten. Siehe NDTV: „Patel Quota Stir: 500 Families in Surat Threaten to Convert for Reservation “ vom 25.10.2015, http://www.ndtv.com/india-news/ patel-quota-stir-500-families-in-surat-threaten-to-convert-for-reservation-1236255.
} 
Perspektiven auf dem Arbeitsmarkt zu tun hat (Khadria 2008: 55). So gibt es Wirtschaftszweige, in denen die Nachfrage nach gut ausgebildetem Personal steigt (Baugewerbe, Automobilindustrie und Krankenpflege), aber bisher noch zu wenige junge Menschen, die sich für eine Ausbildung in diesen Berufen entscheiden. Seit einigen Jahren versucht die indische Regierung, dieser Dynamik mit einem besseren Berufsausbildungssystem und Initiativen wie „Skill India“"37 entgegenzuwirken.

Indien hat also eine wachsende Anzahl junger Menschen mit Universitätsabschluss, die aber auf dem einheimischen Arbeitsmarkt keine Arbeit finden, weil sie entweder über- oder unterqualifiziert sind (Jeffrey 2010b: 467). Überqualifikation betrifft dabei vor allem diejenigen, die einen Abschluss von einer Elite-Universität haben, deren Fächerwahl aber nicht mit den Arbeitsplätzen in Indien übereinstimmen. Dies gilt unter anderem für die Absolvent*innen in den sogenannten basic sciences wie Physik, Mathematik und Chemie.

\subsubsection{Männer, Frauen und Arbeit}

In der indischen Mittelschicht wird von jungen Männern erwartet, in bestimmten Berufsfeldern, die zum „white collar“-Bereich gehören, einen sicheren Arbeitsplatz zu erhalten (Jeffrey 2010b: 467). Allerdings wird dies immer schwieriger, da die Arbeitsplätze in diesem Bereich nicht im entsprechenden Maße zahlreicher werden. ${ }^{38}$ Außerdem benötigt es oft nicht nur den entsprechenden Abschluss, sondern auch „capital, connections (caste, class, and kinship), and often bribes to move toward secure employment“ (Clark-Decès 2014: 145). Aus diesem Grund ist eine wachsende Anzahl junger Männer in Indien arbeitslos. Ohne einen Arbeitsplatz ist es wiederum schwierig, eine Heiratspartnerin zu finden und damit ein wichtiges soziales Kriterium eines „Erwachsenen“ zu erfüllen (Jeffrey 2010b). Ajay machte den Zusammenhang zwischen Arbeitsplatz und Heiratschancen an der Generation seiner Eltern deutlich und stellte einen Vergleich mit der Lage seiner Generation an:

In that generation, for them it was like you get a government job [and] then your life is perfect. So you know the arranged marriage system in India, so the girls' parents will say to the girl: „Oh the guy has a government job, so marry him [and] your life will be save." But today, with respect to the population there is lot of competition. (Ajay, 14.12.2011)

Die Konkurrenz um gute Arbeitsplätze wurde in meinen Interviews immer wieder thematisiert und als Grund dafür genannt, Qualifikationen im Ausland zu erwerben, um dann bessere Chancen im Wettbewerb in Indien zu haben. Clark-Decès macht

\footnotetext{
${ }_{37}$ Weitere Informationen zu dieser Initiative finden sich auf der Webseite des Ministry of Skill Development and Entrepreneurship (https://msde.gov.in/en, abgerufen am 09.03.2020).

${ }_{38}$ Diese Dynamik ist nicht einzigartig für Indien, sondern ist ein globales Problem. Es gibt weltweit eine große Arbeitslosigkeit unter jungen Leuten (Amit und Dyck 2012).
} 
in ihrer Studie deutlich, dass viele junge Männer in der Mittelschicht allerdings auch nicht gewillt sind, Arbeit anzunehmen, die sie als nicht ihrem gesellschaftlichen Stand entsprechend wahrnehmen (Clark-Decès 2014: 145). Dies steht damit im Zusammenhang, dass die jungen Menschen, wie beschrieben, schon in ihrer Schulzeit auf bestimmte berufliche Laufbahnen vorbereitet werden und nur der Erfolg in diesen als ausreichend verstanden wird, um ,respectable adulthood as a young man" (Amit und Dyck 2012: 12) zu erreichen.

Für junge Frauen der indischen Mittelschicht sind die Erwartungen, die mit ihrem Studium verbunden werden, in vielen Familien noch vorrangig mit dem Heiraten verbunden. Allerdings zeichnen sich Veränderungen ab, die damit in Verbindung stehen, dass mehr Frauen Zugang zu universitärer Bildung erhalten. Die Universitätsabschlüsse bringen den jungen Frauen neue Möglichkeiten auf dem Arbeitsmarkt (Säävälä 2010: 37; Fleming 2016). Wie Säävälä deutlich macht, kann der finanzielle Beitrag, den Töchter durch ihre Arbeit leisten, gerade für Familien mit einer oder mehreren unverheirateten Töchtern sehr verlockend sein (2010: 38-39). Für die jungen Frauen führt diese Situation zu großen Dilemmata, da sie gegensätzlichen Botschaften ausgesetzt sind (Säävälä 2010; Radhakrishnan 2011; Ganguly-Scrase 2003; Donner 2015; Fleming 2016). Auf der einen Seite sind viele Eltern stolz darauf, wenn ihre Töchter gute Universitätsabschlüsse machen und einen guten Arbeitsplatz erhalten. Auf der anderen Seite gibt es die bereits angesprochenen idealisierten Vorstellungen von der ,guten indischen Familie', ihren Werten und der Rolle der Frau in der Aufrechterhaltung dieser (Radhakrishnan 2011: 147; Gilbertson 2014). Demgemäß sollen Frauen der Familie die zentrale Rolle in ihrem Leben zusprechen (Radhakrishnan 2011: 146; Gilbertson 2014: 226). Die Priorisierung der Familie wird von vielen Menschen in Indien als Kern indischer Werte verstanden. Das Indischsein wird somit stark über Vorstellungen von „respectable femininity" (Radhakrishnan 2011: 146) definiert. Gut ausgebildete und arbeitende Frauen werden oft mit einer spezifischen Vorstellung von Modernität verbunden, nämlich Individualismus und dem Wunsch nach Selbsterfüllung. Diese Art von Modernität wird in Indien als , westlich' verstanden. Durch das Ausbalancieren eigener Wünsche mit den familiären Verpflichtungen sollen die Frauen verhindern, dass die Familie zu westlich wird. Für viele Frauen in Indien bedeutet diese Situation, dass sie nach der Heirat oder spätestens nachdem sie Kinder bekommen haben, aufhören zu arbeiten (Donner 2015: 141) ${ }^{39}$. Auch aus diesem Grund liegt der Anteil von Frauen an der berufstätigen Bevölkerung in Indien nur bei $27 \%{ }^{40}$ (Godbole und Ramaswamy 2015: 74).

\footnotetext{
39 Mehr dazu auch im Bericht der Association of Academies and Societies of Sciences in Asia (AASSA) (AASSA 2015).

${ }^{40}$ Siehe die Angaben auf der Webseite der Weltbank zum Anteil der Frauen an der arbeitenden Bevölkerung (https://data.worldbank.org/indicator/SL.TLF.CACT.FE.ZS?locations=IN, abgerufen am 11.09.2020). Siehe Divya Arya: „Why motherhood makes Indian women quit their jobs“, $B B C$ News vom 23.04.2015, http://www.bbc.com/news/world-asia-india-32377275.
} 


\subsection{Vorstellung vom Lebensverlauf in der indischen Mittelschicht}

Im Theoriekapitel habe ich ausgeführt, dass Vorstellungen vom Lebensverlauf in der biographischen Navigation eine ambivalente Rolle spielen: Sie geben einerseits Orientierung, „blueprints for directing lives“ (Gubrium, Holstein und Buckholdt 1994: 187), und auf der anderen Seite werden Repräsentationen des Lebensverlaufs genutzt, um Kontrolle auszuüben (ebd.: 186). Aus einer konstruktivistischen Perspektive stellt sich die Frage, welche dominanten Vorstellungen es in der indischen Mittelschicht vom Lebensverlauf gibt und wie diese von den jungen Männern und Frauen aus Indien, die ich interviewt habe, navigiert wurden.

Die Vorstellung von einem Lebensverlauf basiert auf der Konzeption von Zeit als linear. Der Ursprung linearer Zeitkonzepte wird in den euro-amerikanischen Gesellschaften verortet (Cole und Durham 2008b; Jeffrey 2010a: 12, 2010b: 467). Eine Dimension linearer Konzeptionen von Zeit ist die Formalisierung von Vorstellungen, wie Menschen sich während ihres Lebens entwickeln sollten. Cole und Durham (2008b) argumentieren, dass es ein euro-amerikanisches Verständnis des Lebensverlaufes gibt, das suggeriert, dass sich Menschen im Leben natürlicherweise durch die Stadien der Kindheit, Adoleszenz, Erwachsensein und des hohen Alters entwickeln (Cole und Durham 2008b: 5). Dieses Modell wurde Mitte des 20. Jahrhunderts durch Gesetzgebungen und öffentliche Institutionen festgeschrieben „and held up as natural progression to which all should aspire" (Cole und Durham 2008b: 6). Außerdem wurden die Konzepte der Schul- und Berufslaufbahn (zunächst vor allem für Männer) allgegenwärtig (Jeffrey 2010b: 468). Die lineare Vorstellung des Lebensverlaufs in aufeinanderfolgenden, abgeschlossenen Stadien wurde dann während der kolonialen und postkolonialen Zeit durch dominante Institutionen verbreitet, und ersetzte oder verstärkte häufig die Modelle, die es vorher vom Heranreifen gab (Jeffrey 2010b: 468). ${ }^{41}$

Die Vorstellung der Entwicklung des Einzelnen durch Lebensstadien ist außerdem in der hinduistischen Tradition des ,samskāra', der Lebenszyklusrituale, verortbar (McClymond 2008: 314; Malinar 2009: 202; Diane Mines und Lamb 2010: 9). Die samskāra begleiten den Menschen durch die verschiedenen Phasen des Lebens und die damit einhergehenden sozialen Rollen (Flood 1996: 201; Malinar 2009: 202). Von den meist mit sechzehn bezifferten Lebenszyklusritualen sind die wichtigsten weiterhin das Hochzeitsritual (vivāha), die Totenriten und Initiationsriten während der Pubertät (Malinar 2009: 203). Initiationsrituale unterscheiden sich nach Geschlecht und nach Kaste (McClymond 2008: 314; Malinar 2009: 204).

\footnotetext{
${ }^{41}$ Jeffrey verweist in seiner Arbeit auf die Studie von Osella und Osella (2000). Diese stellen fest, dass Vorstellungen von „progress“ in Kerala einen großen Einfluss darauf haben, wie individuelle Lebensverläufe gestaltet werden und sozialer Aufstieg für die Familiengemeinschaft erreicht werden soll (Osella und Osella 2000).
} 
Die Lebenszyklusrituale stehen in Verbindung mit dem theoretischen Modell des áśrama, der hinduistischen Lebensphasen, die ein Mann durchlaufen sollte. Er sollte vom Schüler/Student (der traditionellen Schriften) zum ,Haushälter', zum Einsiedler und letztendlich samnyyassa ${ }^{42}$ werden (Flood 1996: 202). Eine Heirat markiert den Beginn des Lebens als ,Haushälter' (grhastha) und ist für die meisten Hindus das wichtigste Lebenszyklusritual (Flood 1996: 206; Diane Mines und Lamb 2010: 10; McClymond 2008: 315). Wie einige Publikationen feststellen, findet sich die große Bedeutung, die in Indien dem Heiraten zugeschrieben wird, über religiöse und regionale Grenzen hinweg (Diane Mines und Lamb 2010: 10; Kaur und Palriwala 2014).

Die Konstruktion eines linearen Lebensverlaufs, die sich, wie dargestellt, auf unterschiedliche Quellen zurückführen lässt, wird von Jeffrey als Teil „hegemonic temporalities" (Jeffrey 2010b: 468), also hegemonialer Temporalitäten, verstanden und löst spezifische Erfahrungen in Relation zu Zeit aus. ${ }^{43}$ Diese Erfahrungen sind, wie ich im Folgenden darlegen werde, genderspezifisch.

\subsubsection{Der genderspezifische Lebensverlauf in Indien}

Craig Jeffrey beschreibt, dass junge arbeitslose Männer in Indien das Gefühl haben, zurückgelassen zu werden und zu viel Zeit zu haben (Jeffrey 2010b), da es ihnen nicht möglich ist, in die nächste Kategorie des ,männlichen Erwachsenenseins' (Jeffrey 2010b: 468) einzutreten. Wie Vered Amit und Noel Dyck (2012) zeigen, sind die Bemühungen junger Menschen, vor allem junger Männer, den dominanten Vorstellungen des Lebensverlaufs zu folgen, zweischneidig:

On the one hand, if they do not acquire any or much formal schooling, they risk being viewed as failures; on the other hand, if they do attend school but do not succeed in achieving the kind of "good jobs" that this education is assumed to enable, then they are also likely to be viewed as failing. And in contexts, such as that described by Meinert, where families are still likely to vest particular hopes for their collective futures in the success of their sons, young men are all the more likely to feel the effects of the Catch-22 that lies at the heart of this paradigm of modernity and social mobility. (Amit und Dyck 2012: 14-15)

Wie bereits dargelegt, stellt Indien einen der Kontexte dar, in dem der Fokus auf den Erfolg des Sohnes gelegt wird. Wie Amit und Dyck betont auch Jeffrey, dass die Diskrepanz zwischen Aspirationen, die mit Bildung verbunden werden, und der Realität gerade die jungen Männer betrifft (Jeffrey 2010b: 467). Der Druck, beruf-

\footnotetext{
42 Der samnnyāsa entsagt dem weltlichen Leben (Flood 1996: 202).

43 Anders Sybrandt Hansen (2015) zeigt eine sehr ähnliche Dynamik von linearen Vorstellungen vom „advancing“ und der Erfahrung von Zeit im Fall von chinesischen Studierenden in Dänemark auf.
} 
lich erfolgreich zu sein und die Angst davor, im Lebensverlauf ,steckenzubleiben', ist dementsprechend unter jungen Männern in Indien sehr hoch und wird durch die große Anzahl junger Menschen verstärkt.

In Indien machen viele Frauen einen Universitätsabschluss, allerdings ist dieser in vielen Fällen mit der Hoffnung verbunden, einen guten Heiratspartner zu finden und nicht mit der Aspiration auf eine professionelle Karriere. Die Ziele können sich jedoch durch die Ausbildung ändern. Nichtsdestotrotz ist der Druck, im Studium und auf dem Arbeitsmarkt erfolgreich zu sein, ein anderer als bei den jungen Männern. Der Druck und die Ängste, die Frauen verspüren, beziehen sich eher auf die Aussicht im ,richtigen' Alter heiraten zu müssen (vgl. Sondhi 2013; Fleming 2016). Junge Frauen in Indien machen daher, und das wird in dieser Studie deutlich, weniger die Erfahrung, zu viel Zeit zu haben und nicht erwachsen werden zu können. Sie haben eher im Gegenteil das Gefühl, dass das Leben zu schnell auf das Heiraten und damit das Erwachsensein hinausläuft. Die Genderspezifik des Lebensverlaufs drückt sich also nicht nur durch die unterschiedlichen Erwartungen an die jungen Männer und Frauen aus, sondern auch in genderspezifischen Vorstellungen vom richtigen Timing von Ereignissen wie der Heirat.

\subsubsection{Das „marriagable age“: Die richtige Zeit zum Heiraten}

The idea of a marriageable age within the Indian context is very important when considering the life-course. It is part of the expectations for daughters to leave the natal household by a ,respectable' age to per-

form the gendered social role of a homemaker. (Sondhi 2013: 129)

In den Vorstellungen vom Lebensverlauf, die in der indischen Mittelschicht bzw. generell im indischen Kontext, dominieren, gehört das Heiraten zum Leben dazu und ist ein wichtiger, wenn nicht der wichtigste Marker des Erwachsenseins (Osella und Osella 2000: 81; Malinar 2009: 207; Kaur und Dhanda 2014: 271). Im Verlauf der vergangenen Jahrzehnte ist das Heiratsalter in Indien generell gestiegen (Clark-Decès 2014). Für Frauen wird das Alter zwischen achtzehn und Mitte zwanzig als ideal zum Heiraten verstanden. Bei Männern gilt Ende zwanzig bis Anfang dreißig als gutes Alter, um den Bund fürs Leben zu schließen (Kaur und Dhanda 2014). Dass Männer später als Frauen heiraten, hat aus Clark-Decès Sicht außerdem damit zu tun, dass es für sie schwieriger ist, die Voraussetzung fürs Heiraten zu erfüllen, nämlich einen festen Arbeitsplatz zu haben (Clark-Decès 2014; Kirk, Bal und Janssen 2017: 2782). Von den Männern wird also erwartet, vor ihrem Eintritt in das Heiratsalter die entsprechenden Bedingungen für eine Heirat zu erfüllen (vgl. Fleming 2016).

Auf jungen Frauen in Indien ruht großer familiärer und gesellschaftlicher Druck zu heiraten, bevor sie ,zu alt' sind, also spätestens, bevor sie dreißig werden. Wie Rachel Fleming feststellt, ist es in Indien für eine Frau über dreißig nicht „socially acceptable" unverheiratet zu sein (Fleming 2016: 185). Diese Vorstellung vom Alter zeigte sich auch in den Aussagen meiner Gesprächspartner*innen. Nandita erklärte 
mir zum Beispiel, dass sie jetzt alt werde: Sie war gerade sechsundzwanzig und bereits Postdoktorandin (Nandita 03.09.2011). Frauen aus der urbanen Mittelschicht mit universitärer Bildung haben zwar mittlerweile mehr Handlungsvermögen in Bezug auf das Heiraten - vor allem im Vergleich mit Frauen aus anderen Bevölkerungsgruppen - stehen aber weiterhin unter großem Druck, vor allem was den Zeitpunkt angeht (vgl. Fleming 2016; Jayasree Subramanian 2016). Dabei spielt nicht nur die Familie eine wichtige Rolle, sondern auch die Gleichaltrigen. Wie ich in der vorliegenden Arbeit zeige, war Mobilität ein wichtiges Mittel in der Aushandlung dieses zeitlichen Druckes.

Die genderspezifischen Vorstellungen des richtigen Timings von Ereignissen wie der Heirat interagieren unterschiedlich mit der akademischen und beruflichen Laufbahn von Männern und Frauen. Die universitäre Ausbildung von Frauen wird häufig nur bis zu einem bestimmten Ausmaß unterstützt, weil jeder weitere Abschluss mit dem Heiratsalter in Konflikt stehen würde oder verhindert werden soll, dass die Frau ,zu hoch ausgebildet' wird (Kôu, Mulder und Ajay Bailey 2017: 2797; Jayasree Subramanian 2016: 260). So wollen gut ausgebildete Männer zwar gut ausgebildete Frauen, allerdings sollte der Bildungsgrad der Frau nicht denjenigen des Mannes übersteigen (Fuller und Narasimhan 2008, 2012). Im Fall der Männer steht das Heiraten kaum der weiteren Ausbildung oder der beruflichen Karriere im Weg (Kirk, Bal und Janssen 2017: 2782).

\subsubsection{Mobilität im Lebensverlauf}

Die internationale Mobilität gehört im zunehmenden Maße zu dominanten Vorstellungen des Lebensverlaufs dazu: „Aspirations and imaginaries of transnational mobility (...) increasingly shape ideas of transition to adulthood for both mobile and immobile youth that cut across regional and class divides" (Robertson, Harris und Baldassar 2017: 204; vgl. Baas 2010; Skrbiš, Woodward und Bean 2013; Mapril 2014; Kirk, Bal und Janssen 2017; Kandel und Massey 2002).

Wie Shanti Robertson, Anita Harris und Loretta Baldassar argumentieren, wird besonders im Umfeld der wachsenden globalen Mittelschicht angenommen, dass internationale Mobilität zur „employability“ junger Menschen beiträgt und ihr kulturelles Kapital und somit ihre sozialen und ökonomischen Zukunftsaussichten steigert (Robertson, Harris und Baldassar 2017: 204). So soll die Mobilität den jungen Menschen ermöglichen, die kosmopolitischen und interkulturellen Fähigkeiten zu entwickeln, die nötig sind, um in einer globalisierten Welt zu funktionieren (Robertson, Harris und Baldassar 2017: 204; vgl. Skrbiš, Woodward und Bean 2013).

Die internationale Mobilität junger Menschen wird sowohl durch die Migrationsgesetzgebung als auch durch die Bildungspolitik gefördert (Robertson, Harris und Baldassar 2017: 204). Ein gutes Beispiel dafür ist das Erasmus-Programm der Europäischen Union, das Auslandsaufenthalte für Studierende finanziell und strukturell unterstützt. Die internationale Mobilität ist nicht für alle jungen Menschen weltweit gleich realisierbar (vgl. Robertson, Harris und Baldassar 2017). Faktoren 
wie Gender, die nationale Herkunft oder das Aufwachsen in einer spezifischen gesellschaftlichen Schicht beeinflussen die Möglichkeiten der jungen Menschen, mobil zu werden. Die meisten meiner Gesprächspartner*innen waren in einem Umfeld aufgewachsen, in dem internationale Mobilität nichts Außergewöhnliches ist, sondern eher die Norm (Kôu, Mulder und Ajay Bailey 2017: 2795) (siehe Kapitel 3). Trotzdem mussten sie ihre Entscheidung, nach Deutschland zu kommen, in vielen Fällen mit ihren Eltern bzw. der Familie aushandeln. Gender war einer der wichtigsten Faktoren, der beeinflusste, welche Position die jungen Inder*innen in den Aushandlungen mit ihren Eltern einnahmen und einnehmen konnten. Ein zweiter wichtiger Faktor waren die Vorstellungen vom Lebensverlauf: Die jungen Wissenschaftler*innen handelten häufig nicht nur ihre gegenwärtigen, sondern auch ihre zukünftigen Rollen in den Familien aus.

Männer tragen in Indien traditionellerweise die Verantwortung für die Ehefrau und eigene Kinder und für die Versorgung der Eltern und unverheirateten Geschwister (vgl. Sondhi 2013: 95). Diese zugeschriebene Rolle des Versorgers und die Sorge der Eltern um die Einhaltung der damit verbundenen Pflichten konnten zu einem Grund werden, der Mobilität des Sohnes skeptisch gegenüber zu stehen. Bei den Aushandlungen, die die jungen Frauen mit ihren Familien führten, stand vor allem das Heiratsalter in Vordergrund. Letztendlich erhielten alle der jungen Frauen, mit denen ich für meine Studie sprach, auf die eine oder andere Weise die Unterstützung ihrer Eltern. Dieser Umstand zeigt aber nicht unbedingt die positive Einstellung der Eltern für die internationale Mobilität ihrer Töchter, sondern betont, dass die Frauen die Unterstützung ihrer Eltern bzw. Familien brauchten und nicht ohne sie mobil werden konnten. An dieser Stelle möchte ich darauf verweisen, dass Statistiken aus dem Jahr 2015 zeigen, dass nur etwa 22\% der indischen Studierenden in Deutschland Frauen sind (DAAD und DZHW 2016: 17).

In den Aushandlungen mit den Eltern war der Wert, der Abschlüssen aus dem Ausland zugeschrieben wird, ein wichtiger Faktor. Die Mehrzahl meiner Gesprächspartner*innen finanzierte das Studium oder die Promotion in Göttingen mit Hilfe eines Stipendiums oder einer Stelle an der Universität oder einem Max-Planck-Institut. Sowohl für die Männer als auch die Frauen war die finanzielle Unabhängigkeit ein sehr wichtiges Argument in den Verhandlungen mit den Eltern.

\subsubsection{Verbundene Lebensverläufe}

Lebensläufe sind (...) keine Projekte von Individuen, sondern von sozialen Beziehungsgefügen (Paare, Familien, et cetera). (Leemann und Boes 2012: 183)

Bisher habe ich die Lebensverläufe von Männern und Frauen in Indien getrennt voneinander betrachtet. Allerdings sind die Lebensverläufe von Personen miteinander verbunden. Im Zusammenhang mit Studien zum Lebensverlauf wird von „linked lives“ gesprochen (Elder, Johnson und Crosnoe 2003: 13; Kôu, Mulder und 
Ajay Bailey 2017). Das Leben der jungen Studierenden und Wissenschaftler*innen ist zunächst vor allem mit dem ihrer Eltern und dem anderer Familienmitglieder verbunden. Diese Verbindungen bleiben während ihrer Zeit im Ausland bestehen und beeinflussen sowohl die Entscheidung zur Mobilität, die Erfahrungen, die $a b$ road gemacht werden, als auch Überlegungen zur Rückkehr (Kôu, Mulder und Ajay Bailey 2017). Im Verlauf des Lebens werden neue Beziehungen eingegangen, beispielsweise mit (Ehe)-Partner*innen. Aspirationen für die Zukunft, Möglichkeiten für die Umsetzung der Aspirationen und damit der Lebensverlauf der Partner*innen beeinflussen sich gegenseitig. „Because lives are lived interdependently, transitions in one person's life often entail transitions for other people as well“ (Elder, Johnson und Crosnoe 2003: 13). Wie die Lebensverläufe aufeinander einwirken, ist wiederum genderspezifisch. So hat im indischen Kontext in vielen Fällen die Karriere des Mannes Vorrang und von Frauen wird eher erwartet, ihre eigenen Aspirationen hintanzustellen und beispielsweise im Fall von Migration dem Mann zu folgen (Kôu, Mulder und Ajay Bailey 2017). Durch eine Ehe wird das Leben von Frauen auch mit jenem der Schwiegereltern verbunden (Kôu, Mulder und Ajay Bailey 2017: 2792), die laut normativer Vorstellungen der Familie die Autorität hätten, wichtige Entscheidungen der Schwiegertochter zu beeinflussen (Fleming 2016: 10).

\subsection{Zusammenfassung}

Für das Verständnis der biographischen Navigation der jungen Wissenschaftler*innen ist es wichtig, deren familiäre und gesellschaftliche Verortung wahrzunehmen. Sie waren Teil von Familien, in denen sie Verantwortung trugen und in Abhängigkeitsbeziehungen eingebunden waren.

In diesem Kapitel habe ich gezeigt, dass meine Gesprächspartner*innen aufgrund ihrer Herkunft aus der indischen Mittelschicht ganz spezifische Hoffnungen mit ihrer weiteren akademischen Ausbildung verknüpften. Die jungen Wissenschaftler*innen verbanden mit der Zeit, in der sie sich in ihrem Leben befanden, ganz spezifische Ziele: Sie wollten sich ein starkes Fundament für die Zukunft aufbauen. Von dieser Zukunft hatten sie - ausgehend von dominanten Vorstellungen des Lebensverlaufs in der indischen Mittelschicht - schon recht klare Visionen. Meinen Gesprächspartner*innen war bewusst, was von ihnen als Teil ihrer Familien erwartet wurde und wie sie diese Ziele würden erreichen können - durch Abschlüsse aus dem Ausland. Den jungen Wissenschaftler*innen wurde dabei die Verantwortung dafür aufgelastet, die ,richtige' Entscheidung für ihre eigene Zukunft und die der Familie zu treffen.

In der biographischen Navigation der jungen Wissenschaftler*innen wurde die internationale Mobilität zu einem wichtigen Mittel, um die familiären Erwartungen zu erfüllen und gleichzeitig größere Unabhängigkeit von ihrer Familie in Indien zu erreichen. 


\section{Das Regime der akademischen Mobilität}

Bestimmte Formen des Wissens und die Menschen, die dieses Wissen haben, stehen mittlerweile im Mittelpunkt eines globalen Wettbewerbs um „brainpower“ (Hazelkorn 2009: 80). Im vorliegenden Kapitel ist es mein Ziel, darzulegen, wie der Wettbewerb um die „besten Köpfe“44 in Interaktion mit Aspirationen, die in Indien selbst mit Bildung und internationaler Mobilität verbunden werden, dazu führen, dass indische Studierende und Wissenschaftler*innen in ihrer biographischen Navigation den Weg der internationalen Mobilität wählen. Dafür entwickle ich das Konzept des Regimes der akademischen Mobilität, das auf Glick Schillers und Salazars (2013) Regimes of Mobility-Ansatz basiert. Mit dem Begriff des Regimes der akademischen Mobilität bezeichne ich das Geflecht von Akteuren, Strukturen, Diskursen und Imaginationen, das die Mobilität von Studierenden und Wissenschaftler*innen hervorbringt, normalisiert und reguliert (vgl. Glick Schiller und Salazar 2013; Baker 2016). Ich zeige, welche Akteure, Strukturen und Diskurse in diesem interagieren und wie diese Interaktion dazu führt, dass die Mobilität von Wissenschaftler*innen

\footnotetext{
${ }_{44}$ Die Formulierung „,beste Köpfe“ wird häufig in Berichten über internationale Studierende benutzt. Zum Beispiel in der folgenden Pressemitteilung auf der Webseite des Bundesministeriums für Bildung und Forschung. BMBF: „Erstmals über 300.000 ausländische Studierende in Deutschland“ vom 22.07.2015, https://www.bmbf.de/de/erstmals-ueber-300-000-auslaendische-studierende-in-deutsch land-956.html.
} 
und Studierenden als gewinnbringend für alle Beteiligten (Staaten, Institutionen, Familien, Personen) verstanden wird.

Ein Fokus des Kapitels liegt auf staatlichen und institutionellen Akteuren und deren Darstellung der Mobilität von Studierenden und Wissenschaftler*innen. Diese Perspektive eröffnet den Blick auf kollektive Repräsentationen von Zeit beispielsweise in der Form von Vorstellungen des akademischen Lebensverlaufs und Diskursen zur ,Entwicklung' von Nationen, mit denen die jungen Wissenschaftler*innen in ihrer biographischen Navigation interagieren.

An dieser Stelle nehme ich Collins und Shubins Kritik auf, dass Werke zur Mobilität Studierender häufig zeitlich linear argumentieren (Collins und Shubin 2017). Hier erscheint die internationale Mobilität als ein Mittel zur Erfüllung klar definierter Zukünfte. Die Kritik ist berechtigt, allerdings zu kurz gedacht. Mit Hilfe des Konzepts der Zeitkarte zeige ich in der vorliegenden Arbeit, dass die Dominanz eines linearen Modus von Zeit in diesen Studien durch die kollektiven Zeitkarten der Akteure hervorgerufen wird, die an der Mobilität von Studierenden und Wissenschaftler*innen maßgeblich beteiligt sind. In diesen Repräsentationen von Zeit existiert ein starker Fokus auf (un-)mögliche Zukünfte, die es mit Hilfe von Strategien in der Gegenwart zu verhindern oder zu erreichen gilt.

Das Kapitel ist in vier Abschnitte gegliedert. Im ersten Abschnitt lege ich einige Zahlen und Fakten zu internationalen Studierenden und Wissenschaftler*innen in Deutschland dar. Im zweiten Kapitelteil steht der globale Rahmen, in dem das Regime der akademischen Mobilität eingebettet ist, im Fokus. Im dritten Teil dieses Kapitels konzentriere ich mich auf die Hoffnungen und Erwartungen, die verschiedene Akteure in Deutschland an die Mobilität von Studierenden und von Wissenschaftler*innen knüpfen, und zeige, wie sie versuchen, die Mobilität von diesen zu fördern. Ich gebe einen kurzen geschichtlichen Überblick über die Mobilität von indischen Studierenden und Wissenschaftler*innen und beschäftige mich mit Stereotypen, die in Deutschland mit indischen Studierenden und Wissenschaftler*innen verbunden werden, um zu verstehen, warum Indien als ein besonders guter Partner in der Wissensökonomie angesehen wird. Im vierten Teil dieses Kapitels liegt der Fokus auf den Strukturen, Diskursen und Akteuren, die in Indien dazu beitragen, ein Studium bzw. die wissenschaftliche Arbeit im Ausland attraktiv zu machen. Dazu gehört die Vorstellung, dass Bildung zu einer besseren Zukunft verhilft, sowohl für das Individuum, die Familie als auch für die Nation. 


\subsection{Zahlen und Fakten zu internationalen Studierenden und Wissenschaftler*innen in Deutschland}

In den vergangenen Jahren ist die Anzahl internationaler Studierender in Deutschland stetig angestiegen. Im Jahr 2019 waren 393.579 Studierende aus verschiedenen Ländern an Universitäten in Deutschland eingeschrieben. ${ }^{45}$ Chinesische Studierende (36.915) bilden in Deutschland die größte Gruppe, gefolgt von Studierenden aus Indien (17.294) und Österreich (11.130). ${ }^{46}$ Die Studierendenzahlen aus Indien weisen dabei das stärkste Wachstum unter den drei Spitzenreitern auf (DAAD und DZHW 2016: 30).

In den vergangenen Jahren gab es in Deutschland ein wachsendes Interesse am Studium der Ingenieurwissenschaften. So waren 2018 die meisten internationalen Studierenden in diesem Fachgebiet eingeschrieben, gefolgt von den Rechts-, Wirtschafts- und Sozialwissenschaften, den Geisteswissenschaften sowie an letzter Stelle den Naturwissenschaften und der Mathematik (DAAD und DZHW 2019: 54). Die Fächerpräferenzen waren je nach Herkunftsland etwas unterschiedlich. So zeigen Statistiken, dass verschiedene ingenieurwissenschaftliche Fachbereiche vor allem von Studierenden aus Indien, China, Syrien, Kamerun und Iran gewählt wurden (DAAD und DZHW 2019: 64). Daran zeigt sich, dass die Fächerpräferenz der internationalen Studierenden nicht nur durch die Strukturen der Wissensökonomie und die Interessen des Ziellandes wie Deutschland geformt wird, sondern gleichermaßen durch Akteure und Strukturen in den Herkunftsländern.

Unter den internationalen Studierenden ist das Geschlechterverhältnis insgesamt ausgeglichen (51\% Männer und 49\% Frauen). Die Statistiken aus dem Jahre 2015 zeigen allerdings starke Unterschiede zwischen den Herkunftsregionen. Im Fall von China ist das Verhältnis ausgeglichen. Im Gegensatz dazu kommen aus Russland mit $77,1 \%$ mehr Studentinnen nach Deutschland, im Falle Indiens mit 77,2\% wesentlich mehr Männer als Frauen (DAAD und DZHW 2016: 17).

Deutschland ist nicht nur für internationale Studierende, sondern auch für Promovierende, Postdoktorand ${ }^{*}$ innen, Hochschullehrer*innen, ${ }^{47}$ also Wissenschaftler*innen aus dem Ausland von wachsendem Interesse. Auch bei den Promovierenden ist China das wichtigste Herkunftsland, gefolgt von Indien und Italien (DAAD und DZHW 2019: 104). 2017 lag Indien beim wissenschaftlichen Personal an deutschen Hochschulen mit 2.573 Personen an vierter Stelle (DAAD

\footnotetext{
45 Diese Angabe stammt von der Webseite „Wissenschaft Weltoffen“, die „relevante Daten zur Internationalität von Studium und Lehre" veröffentlicht. Die Daten basieren auf Angaben des Deutschen Akademischen Austauschdiensts (DAAD) und des Deutschen Zentrums für Hochschul- und Wissenschaftsforschung (DZHW) (http://www.wissenschaftweltoffen.de/, abgerufen am 24.02.2020).

46 Die Zahlen gelten für das Jahr 2018. Siehe DAAD und DZHW: „Foreigners, Bildungsauslaender, Bildungsinlaender, total and females, by countries and regions of origin, winter term 17/18“, Wissenschaft Weltoffen, http://www.wissenschaftweltoffen.de/daten/index_html?lang=en.

${ }^{47}$ Diese Kategorien gehen teilweise ineinander über.
} 
und DZHW 2019: 106). ${ }^{48}$ Viele der ausländischen Wissenschaftler*innen sind an deutschen Hochschulen oder außeruniversitären Forschungseinrichtungen tätig, entweder direkt als Mitarbeiter*innen oder durch Drittmittel finanziert. Im Jahr 2014 waren dies insgesamt 85.000, wobei die Aufenthaltsdauer ganz unterschiedlich ausfiel (DAAD und DZHW 2016: 118). Was im Vergleich mit deutschen Wissenschaftler*innen auffällt, ist, dass die internationalen Wissenschaftler*innen überdurchschnittlich häufig aus den Fachgebieten der Naturwissenschaften und der Mathematik, den Ingenieurwissenschaften und der Medizin sowie den Gesundheitswissenschaften kommen (DAAD und DZHW 2016: 118). Unter den außeruniversitären Forschungseinrichtungen ${ }^{49}$ hat die Max-Planck-Gesellschaft mit $34 \%$ den größten Anteil internationaler Wissenschaftler*innen (DAAD und DZHW 2016: 123). Die internationalen Wissenschaftler*innen sind im Durchschnitt bei gleichem akademischem Grad deutlich jünger als die deutschen. Daraus folgert eine Studie von DAAD und DZHW: „, dass unter den ausländischen Wissenschaftlern der Anteil erfolgreicher Nachwuchswissenschaftler, d.h. junger, aber bereits promovierter Wissenschaftler, deutlich höher ausfällt als unter den deutschen Wissenschaftlern“" (DAAD und DZHW 2016: 126).

Der Aufenthalt internationaler Wissenschaftler*innen wird durch verschiedene Organisationen finanziell gefördert. Der größte Anteil der geförderten Wissenschaftler*innen ist der Fächergruppe Mathematik und Naturwissenschaften zuzuordnen (39\%). Mit großem Abstand folgen mit 17\% die Sprach- und Kulturwissenschaften, die Rechts-, Wirtschafts- und Sozialwissenschaften mit 15\% sowie die Ingenieurwissenschaften mit 12\% (DAAD und DZHW 2016: 132).

Die Statistiken zeigen ein generelles Wachstum der Anzahl internationaler Studierender und Wissenschaftler*innen in Deutschland und machen dabei die insgesamt steigende Mobilität dieser Personengruppen sichtbar. Gerade die Mobilität indischer Studierender nach Deutschland hat stark zugenommen. Die Statistiken veranschaulichen außerdem die unterschiedlich ausgeprägte akademische Mobilität in einzelnen wissenschaftlichen Bereichen. Dies macht nicht nur die Verteilung der internationalen Studierenden und Wissenschaftler*innen in Bereichen wie Naturwissenschaften, Mathematik und Ingenieurwissenschaften gegenüber beispielsweise den Sozialwissenschaften deutlich, sondern auch die vergleichsweise ungleich verteilte finanzielle Förderung einzelner Personen in diesen Bereichen. Das spezifische Regime der akademischen Mobilität, das ich als Teil meiner Studie analysiere, scheint vor allem die Mobilität in bestimmten Fachbereichen, die unter MINT (MathematikNaturwissenschaften-Informatik-Technik) zusammengefasst werden, zu fördern. Dies wird auch durch eine Pressemitteilung des Bundesministeriums für Bildung

\footnotetext{
${ }^{48}$ Nach wissenschaftlichem Personal aus Italien, China, Österreich, den USA und Russland (DAAD und DZHW 2016: 120).

${ }^{49}$ Die vier größten außeruniversitären Forschungseinrichtungen in Deutschland sind die MaxPlanck-Gesellschaft, die Fraunhofer-Gesellschaft, die Leibniz-Gemeinschaft und die Helmholtz-Gemeinschaft (DAAD und DZHW 2016).
} 
und Forschung (BMBF) mit der Überschrift „Deutsches Bildungssystem nützt dem Technologiestandort “50 deutlich. In der Pressemitteilung wird dargestellt, dass 46\% der internationalen Doktorand*innen ihren Abschluss in den Naturwissenschaften absolvieren und insgesamt 21\% der Doktorand*innen in diesem Bereich stellen.

\subsection{Der globale Rahmen des Mobilitätsregimes}

Im folgenden Abschnitt stelle ich den größeren globalen Rahmen dar, in den das Regime der akademischen Mobilität eingebettet ist. Dafür werfe ich einen Blick auf die sogenannte Wissensökonomie, die Internationalisierung der Hochschulen und die Vorstellungen vom akademischen Lebensverlauf.

\subsubsection{Die Wissensökonomie}

Laut Kujath und Schmidt (2007: 3) bezeichnen „Wissensökonomie“ und verwandte Begriffe die Anerkennung von Wissen „erstens als wichtigsten Produktionsfaktor, zweitens als immaterielles wirtschaftlich handelbares Gut bzw. als Dienstleistung und drittens als wichtige Komponente materieller / physischer Güter" (Hervorhebungen im Original). Allerdings gilt dies nur für eine bestimmte Form des Wissens, die oftmals mit den Begriffen „Science and Technology“51 (Galama und Hosek 2009: 95; Radhakrishnan 2011: 38) beschrieben wird. Gerade jenem Wissen wird eine transformierende Kraft zugesprochen, die nicht nur die Entwicklung von Personen beeinflusst, sondern die ganzer Nationen (Radhakrishnan 2011). Science and Technology scheinen der Schlüssel zu sein, um die wirtschaftliche Entwicklung einer Nation zu sichern, das Wohlergehen der Bevölkerung zu verbessern und die nationale Sicherheit zu stärken (Galama und Hosek 2009: 95). Wer in der Wissensökonomie erfolgreich sein will, muss, wie im folgenden Zitat deutlich wird, Zugang zu dieser Form des Wissens haben:

As more research and innovation is performed outside Europe, the EU will need to access this knowledge. And to remain a major global player, the EU must promote itself as an attractive location for carrying out research and innovation and be successful in the global competition for talent, while at the same time preserving its economic interests, notably as regards intellectual property rights and standards. (European Commission 2016a: 60) ${ }^{52}$

\footnotetext{
50 Die Angaben aus der Pressemitteilung stammen aus der OECD-Studie „Education at a Glance“ vom 24.11.2015, http://www.oecd-ilibrary.org/education/education-at-a-glance-2015_eag-2015-en. ${ }^{51}$ Im Folgenden werde ich diese Begriffe im Englischen belassen, da im Deutschen vor allem der Begriff der Wissenschaft ganz anders gefasst ist als ,science'. So ist das englische Wort sehr stark mit den Naturwissenschaften verbunden. Im Deutschen beinhaltet Wissenschaft aber auch Geistes- und Sozialwissenschaften.

52 Siehe Europäische Kommission: „Open Innovation, Open Science, Open to the World“, 2016,
} 
In der Aussage scheint eine gewisse Angst mitzuschwingen, dass die Europäische Union und ihre Mitgliedsstaaten in der Zukunft durch andere Nationen abgehängt werden könnten. Um weiterhin wettbewerbsfähig zu bleiben, gilt es Strategien zu entwickeln, um sowohl die eigene Leistungsfähigkeit in Science and Technology zu steigern als auch den Zugang zu Wissen zu erhalten, das andernorts generiert wird.

In der Wissensökonomie ist, wie dargestellt, die Mobilität der Wissenden, der ,Talente" oder ,schlauen Köpfe', wie sie oft bezeichnet werden, zentral (Williams und Baláž 2008). Um die Mobilität zu steigern, wird Geld in Stipendien und internationale Kooperationen investiert und die Einwanderungs- und Aufenthaltsgesetzgebung geändert. Viele Länder, die ein Interesse an der Einwanderung Hochausgebildeter oder Studierender als potentielle Fachkräfte haben, wie beispielsweise Kanada, die USA, Australien und Deutschland, vereinfachten deren Einreise und Übergang in den Arbeitsmarkt durch verschiedene Gesetzesänderungen (vgl. Geddie 2010; Shachar 2006). Studierende und Wissenschaftler*innen sind damit eine von wenigen Personengruppen, deren Mobilität als positiv wahrgenommen und aktiv unterstützt wird. Da die Herkunftsländer ein Interesse daran haben, ihre ,Talente' zu halten oder mit neuem Wissen zurückzugewinnen, versuchen die beteiligten Staaten als Partner zu agieren und eine „brain circulation“" (Saxenian 2005: 35) und somit eine Zirkulation des Wissens zu erreichen. ${ }^{53}$

Die Wissensökonomie sowie deren Interesse an der Mobilität Studierender und Wissenschaftler*innen bilden eine wichtige Komponente des Regimes der akademischen Mobilität. Sie bildet den größeren strukturellen und diskursiven Rahmen, in dem die Mobilität von Studierenden und Wissenschaftler*innen stattfindet und als positiv bewertet wird. Institutionen wie die Europäische Kommission äußern die Befürchtung, dass die EU und ihre Mitgliedsstaaten verbunden mit Faktoren wie dem demographischen Wandel (vgl. 3.3.) ihre Stellung in der Welt verlieren könnten. Diese Befürchtungen leiten die Strategien der entsprechenden Institutionen in der Gegenwart und verbinden die internationalen Studierenden und Wissenschaftler*innen mit der Zukunft der Wissenschaft und Wirtschaft in der EU.

\subsubsection{Die Internationalisierung der Hochschulbildung}

Die Internationalisierung der Hochschulen ist ein wichtiges strategisches Mittel, um das zu erreichen, was in der Wissensökonomie gefordert wird: Zirkulation und Zugang zu Wissenden und Wissen. Im folgenden Zitat wird deutlich, was sich das Bundesministerium für Bildung und Forschung (BMBF) unter Internationalisierung vorstellt und was die Ziele dieser sind:

\footnotetext{
https://ec.europa.eu/digital-single-market/en/news/open-innovation-open-science-open-world-visioneurope.

${ }^{53}$ Die partnerschaftliche Zusammenarbeit wird zum Beispiel in der Strategie der Bundesregierung zur Internationalisierung der Wissenschaft und Forschung betont (BMBF 2016: 5).
} 
Die internationale Ausrichtung deutscher Hochschulen ist wichtiger denn je. Der Austausch ist Voraussetzung für Spitzenforschung und Innovation. (...) Die Bundesregierung unterstützt die deutschen Hochschulen, sich international zu behaupten. (...) (Bundesministerium für Bildung und Forschung) ${ }^{54}$

Des Weiteren sind es finanzielle Vorteile, die die akademische Mobilität für Staaten und Institutionen interessant macht (Münch und Hoch 2013). So zahlen internationale Studierende, die in Ländern wie Großbritannien oder Australien studieren wollen, hohe Gebühren. Aber selbst wenn es keine Studiengebühren gibt, haben internationale Studierende vor allem durch ihre Konsumausgaben einen positiven Einfluss auf die Wirtschaft des Gastlandes (Münch und Hoch 2013).

Damit sich Studierende und Wissenschaftler*innen für die Mobilität entscheiden, müssen Länder und Institutionen für sich werben und für die Sinnhaftigkeit von Auslandsaufenthalten argumentieren (vgl. Raghuram 2013). In diesem Prozess entsteht ein globaler Wettbewerb zwischen den Institutionen, die mit verschiedenen Strategien versuchen, die ,schlauesten Köpfe‘ anzuwerben. In diesem Wettbewerb haben allerdings nicht alle Institutionen die gleichen Chancen, da es Hochschulen und Forschungseinrichtungen gibt, denen aus unterschiedlichen Gründen eine bessere Qualität zugeschrieben wird als anderen. Findlay, King und Smith (2012: 120) sprechen in diesem Zusammenhang von einer wachsenden globalen Differenzierung des Universitätssystems, die wiederum dazu führt, dass Abschlüssen bestimmter Universitäten, vor allem jenen im globalen Norden, ein größerer Wert zugeschrieben wird (vgl. Bilecen und Van Mol 2017). ${ }^{55}$ Angetrieben wird der nationale und internationale Wettbewerb der Universitäten nicht zuletzt von der Reduzierung staatlicher Finanzierung der Hochschulbildung und der damit entstehenden Notwendigkeit, um andere Geldquellen zu konkurrieren (Findlay, King und Smith 2012). Internationale Studierende sind dabei nicht nur willkommene Geldquellen, sondern dienen bei der Bewerbung um finanzielle Mittel als wichtiges Qualitätskriterium.

Die Internationalisierung der Hochschulen fördert den Austausch von Studierenden und Wissenschaftler*innen, ist aber in sich ein sehr ungleicher und differenzierender Prozess (Valentin 2012: 58; Brooks und Waters 2011; Findlay, King und Smith 2012; Bilecen und Van Mol 2017)). Die Ungleichheiten drücken sich nicht nur in den oben beschriebenen Hierarchisierungen der Institutionen aus, sondern auch in der ungleichen Behandlung verschiedener Kategorien von Menschen. Wie Karen Valentin deutlich macht, privilegiert die Internationalisierung bestimmte Gruppen von Menschen und benachteiligt andere - wobei dies nicht unbedingt beabsichtigt ist, aber durch Einwanderungsgesetze verstärkt wird (Valentin 2012: 58).

\footnotetext{
${ }^{4}$ Siehe Webseite des Ministeriums für Bildung und Forschung (https://www.bmbf.de/de/internatio nalisierung-der-hochschulen-924.html, abgerufen am 08.09.2020).

55 Den deutlichsten Ausdruck findet die Hierarchie der Hochschulinstitutionen in nationalen und internationalen Rankings, siehe z.B. Times Higher Education: „World University Rankings 2020“, (https://www.timeshighereducation.com/world-university-rankings/2020/world-ranking\#!/page/0/ length/25/sort_by/rank/sort_order/asc/cols/stats, abgerufen am 30.11.2020).
} 
Ich verstehe die Internationalisierungsstrategien, die unter anderem in Deutschland auf der Hochschulebene eingesetzt werden, als wichtigen Aspekt des Regimes der akademischen Mobilität. Diese Strategien haben einen großen Einfluss darauf, warum Studierende und Wissenschaftler*innen mobil werden und unterstützen die Umsetzung der Mobilität auf struktureller und finanzieller Ebene. Die an der Internationalisierung der Universitäten beteiligten Akteure schaffen durch ihre Strategien und die damit einhergehenden Diskurse rund um die Mobilität Studierender ein Bild von den ,guten internationalen Studierenden' (vgl. Sondhi 2013: 11-12) und von internationalen/mobilen Wissenschaftler*innen, die nicht nur ihre eigene $\mathrm{Zu}$ kunft, sondern auch die der Wissenschaft in den Händen halten.

\subsubsection{Der akademische Lebensverlauf und Mobilitätsanforderungen}

In der Wissenschaft und an der Universität gibt es klare Abfolgen von Abschlüssen, die (angehende) Wissenschaftler*innen absolvieren müssen, wenn sie es bis an die Spitze schaffen wollen (Winslow und Davis 2016: 405). Als Metapher für diese lineare Vorstellung von Karriereschritten wird von einigen Autor*innen der Begriff der „Pipeline“ herangezogen (Wolfinger, Mason und Goulden 2009). Winslow und Davis (2016) entscheiden sich dagegen für eine life-course-Perspektive und verstehen akademische Karrieren als "trajectories that vary in form and pace yet exist against the backdrop of a rigid and gendered lockstep ideal norm (...)" (Winslow und Davis 2016: 413). Demzufolge gibt es eine Idealvorstellung vom akademischen Lebensverlauf, die von angehenden Wissenschaftler*innen navigiert werden muss. Menschen, die wie meine Gesprächspartner*innen eine berufliche Laufbahn in der Wissenschaft anstreben, erhalten durch diese Vorstellungen eine klare Orientierung für ihre Karriereplanung, die zeitliche Koordinaten zur Reihenfolge, der Dauer und des Timings der Abschlüsse vorgeben (vgl. Jacobs und Winslow 2004; Wolfinger, Mason und Goulden 2009; Winslow und Davis 2016). Die Norm des akademischen Lebensverlaufs orientiert sich aufgrund der Geschichte des Berufsfeldes stark am männlichen Lebensverlauf (Wolfinger, Mason und Goulden 2009: 1614). Daraus erwachsen unterschiedliche Herausforderungen in der Einhaltung der vorgesehenen Route und der Koordinaten für Männer und Frauen; dies betrifft auch die Anforderung, (international) mobil zu sein, die mittlerweile zu einer Norm im wissenschaftlichen Lebensverlauf geworden ist:

Internationalität ist in den letzten Jahren in allen Disziplinen zu einem Orientierungsschema für Laufbahnen und wissenschaftliche Tätigkeiten herangewachsen, dem sich weder Wissenschaftsorganisationen noch die mit der Gestaltung ihrer Laufbahnen beschäftigten Wissenschaftlerinnen und Wissenschaftler entziehen können, (...). (Leemann und Boes 2012: 197, Hervorhebung im Original) 
Dieses Zitat betont, was die in Punkt 3.2.1 und 3.2.2 angesprochenen Entwicklungen für Menschen bedeuten, die in der Wissenschaft tätig sind: Sie müssen mobil und flexibel sein. Es entsteht laut Regula Leemann und Stefan Boes eine „Mobilitätsanforderung" (Leemann und Boes 2012: 174), die in den verschiedenen nationalen Wissenschaftssystemen und in den unterschiedlichen Fächern ungleich ausgeprägt ist (Leemann und Boes 2012: 174; vgl. Carling und Collins 2017: 9). ${ }^{56}$ Für die Schweiz stellen Leemann und Boes fest, dass „(...) die normative Erwartung, geografisch mobil zu sein, insbesondere in den Naturwissenschaften hoch ist, dass aber auch in den anderen Fachbereichen Mobilität zu den erwarteten Merkmalen einer wissenschaftlichen Laufbahn gehört" (Leemann und Boes 2012: 193). Diese Aussage gilt ebenso für Deutschland. Auch hier wird mit der Internationalisierung Exzellenz und Innovation verbunden: „Internationale Mobilität fördert eine stärkere Vernetzung und Bündelung von Know-How innerhalb Europas ebenso wie die Einbindung in weltweite Forschungsnetzwerke und schafft dadurch wissenschaftlichen Mehrwert" (BMBF 2014: 16). Was genau dieser Mehrwert eigentlich ist, wird im zitierten Strategiepapier nicht spezifiziert. Leemann und Boes sprechen in diesem Zusammenhang von Internationalität als „religiösem Glaubenssatz“, der größtenteils nicht hinterfragt wird (Leemann und Boes 2012: 198), obwohl es keine empirischen Nachweise dafür gibt, dass Erfahrungen aus dem Ausland oder internationale Kooperationen automatisch zu besseren und innovativeren Forschungsergebnissen führen (Leemann und Boes 2012: 198).

Die Institutionalisierung von Mobilität als Norm im wissenschaftlichen Werdegang ist nicht nur kritisch zu betrachten, weil sie nicht hinterfragt wird, sondern auch, weil diese Norm Ungleichheiten schafft. Dies zeigen die Studien von Leemann (2010), Leemann und Boes (2012) sowie Jöns (2011) in Bezug auf Gender. Die Autor*innen machen deutlich, dass die Mobilitätsanforderung ein neues Hindernis in der Entwicklung einer wissenschaftlichen Laufbahn ist, das für Frauen größer ist als für Männer (Leemann und Boes 2012: 198):

Im Ergebnis fällt es weiblichen Nachwuchsforschenden schwerer, sich ein internationales Profil zu erarbeiten. Es fehlt ihnen in der Folge an internationalem kulturellem und sozialem Kapital und sie können sich weniger gut als unabhängige, flexible und von sozialen Bedingungen unberührte Wissenschaftsunternehmerinnen präsentieren. (Leemann und Boes 2012: 199, Hervorhebungen im Original)

Laut Leeman werde ein Idealtyp des ,academic entrepreneur" konstruiert (Leemann 2010: 619). Dieser ,akademische Unternehmer 'ist mobil, flexibel und, wie Kenway

\footnotetext{
${ }^{56}$ Für den Fall Großbritanniens gibt es eine aktuelle Studie, siehe Susan Guthrie und Catie Lichten: "Success in academia: the pressure to be international“, Times Higher Education vom 04.05.2017, https://www.timeshighereducation.com/blog/success-academia-pressure-be-international\#surveyanswer.
} 
und Fahey ausführen, „,deterritorialised, disembodied and disembedded (...) “ (2007: 168). Dem Idealtyp können nicht alle Wissenschaftler*innen gleichermaßen gerecht werden und so werden insbesondere Frauen, ältere Akademiker*innen, Personen in einer Partnerschaft, ohne akademischen Familienhintergrund oder mit Kindern benachteiligt (Leemann 2010: 623).

Meine Gesprächspartner*innen waren sich in ihrem Selbstverständnis als angehende Wissenschaftler*innen der Bedeutung von internationaler akademischer Mobilität für ihre Karriere bewusst. In den nächsten Kapiteln zeige ich, wie sie diese Mobilitätsanforderung navigierten, nicht als entwurzelte Wissenschaftsnomaden, sondern als Teil von Familien, Partnerschaften und im Zusammenhang mit Aspirationen für die Gegenwart und Zukunft. So stellt sich die Frage, wie meine Gesprächspartner*innen die Anforderungen des akademischen Lebensverlaufs mit denen der Lebensverlaufsvorstellung der indischen Mittelschicht (Kapitel 2) zusammenbrachten.

Normative Vorstellungen vom akademischen Lebensverlauf schaffen als Repräsentation von Zeit zeitliche Erfahrungen wie beispielsweise das Gefühl, nicht oder nicht schnell genug voranzukommen. Auf diese Erfahrungen wirken Menschen mit Strategien der Zeitgestaltung ein.

\subsection{Deutschland auf der Suche nach den ,besten und schlausten Köpfen'}

Im folgenden Teil des Kapitels werde ich zeigen, wie in Deutschland von verschiedenen Akteuren für die akademische Mobilität argumentiert wird, warum gerade Indien als ein guter Partner zur Verwirklichung der eigenen Ziele angesehen wird und mit welchen Strategien Deutschland als Zielland für indische Studierende und Wissenschaftler*innen attraktiv gemacht werden soll.

Die Analyse der Diskurse über internationale Studierende und Wissenschaftler*innen und der Initiativen zur Steigerung ihrer Mobilität zeigen, mit welchen Repräsentationen von möglichen Zukünften diese in Verbindung stehen. Von Institutionen wie dem BMBF wird die Befürchtung artikuliert, dass Deutschland in der Zukunft wissenschaftlich und wirtschaftlich abgehängt werden könnte. Damit diese Version der Zukunft nicht eintritt, sollen - so die Strategien - internationale ,Talente' für Deutschland begeistert werden. Auf eine abstraktere Art und Weise drückt sich die Zukunftsorientierung im Begriff der ,Innovation“ aus, der immer wieder in den Strategiepapieren staatlicher und wissenschaftlicher Akteure benutzt wird (vgl. Nowotny 2006). 


\subsubsection{Für die Zukunft Deutschlands: Staatliche Initiativen}

Der demographische Wandel, das heißt die niedrige Geburtenrate und die große Anzahl von Fachkräften, die bald in Rente gehen, werden in Deutschland als die Ursache eines Mangels an 'Talenten' gesehen (Hazelkorn 2009: 80). Dieser von Robertson als „demographic temporality“ (Robertson 2014: 7) bezeichnete Faktor begünstigt die Mobilität junger Wissenschaftler*innen. So argumentierte 2015 beispielsweise die damalige Bundesbildungsministerin Johanna Wanka:

Die weltweite Mobilität fördert nicht nur den wissenschaftlichen und kulturellen Austausch, wir möchten auch gute Fachkräfte für uns gewinnen. Wir brauchen die besten Köpfe von überall her, um unsere Innovationskraft zu erhalten und den demografischen Wandel zu meistern. ${ }^{57}$

Bei Studien zum Fachkräftemangel liegt der Fokus auf dem Fehlen bestimmter Berufsgruppen wie Ingenieuren, also jenen Fachkräften, die als „Motor für Wachstum und Wohlstand" (Bundesagentur für Arbeit 2011: 3) ${ }^{58}$ gesehen werden. Der Fokus auf den erwarteten Mangel hochqualifizierter Fachkräfte beeinflusst wiederum die Strategien, die Deutschland einschlägt, um diesem entgegenzuwirken. Im Zentrum der Strategien steht neben der Erhöhung der Anzahl qualifizierter Fachkräfte innerhalb Deutschlands durch Ausbildung und Qualifizierung, die Anwerbung qualifizierter Fachkräfte und Studierender, dem „semi-finished human capital“ (Khadria 2008: 30) entsprechender Fachrichtungen wie Physik, Mathematik, Biologie etc. (Bundesagentur für Arbeit 2011). Diese Strategien machen wiederum das Studium zu einem der wichtigsten Zugangswege für Migrant*innen zum deutschen Arbeitsmarkt (OECD 2013a: 23).

Für Deutschland ist Indien ein wichtiger wirtschaftlicher Partner, in dem noch weiteres großes Potential vermutet wird (Mauer 2011: 28). Für eine erfolgreiche Zusammenarbeit ist es für deutsche Unternehmen und Institutionen wichtig, Kenntnisse über die indische Gesellschaft und deren Wandel zu erlangen. Dementsprechend wächst mittlerweile die Nachfrage nach Akademiker*innen mit „IndienKompetenz"59, die unter anderem mit Hilfe indischer Wissenschaftler*innen und Studierender aus den Bereichen der Sozialwissenschaft, der Geisteswissenschaft und der Wirtschaftswissenschaft gedeckt werden soll.

\footnotetext{
57 Zitat von Johanna Wanka aus der Pressemittelung des BMBF zur Anzahl ausländischer Studierender in Deutschland. Siehe BMBF: „Erstmals über 300.000 ausländische Studierende in Deutschland“, Pressemitteilung vom 22.07.2015, https://www.bmbf.de/de/erstmals-ueber-300-000-auslaendischestudierende-in-deutschland-956.html.

${ }^{58}$ Dabei wird übersehen, dass der akute Arbeitskräftemangel vor allem Ausbildungsberufe im Handwerk, die also ein „mittleres Qualifikationsniveau“ erfordern, betrifft (OECD 2013b: 44).

59 Der DAAD nennt die Erhöhung der „Indienkompetenz“ als Ziel des Programmes „A New Passage to India“. Siehe Webseite des DAAD (https://www.daad.de/de/infos-services-fuer-hochschulen/wei terfuehrende-infos-zu-daad-foerderprogrammen/a-new-passage-to-india/, abgerufen am 09.03.2021).
} 


\section{Änderungen des gesetzlichen Rahmens: Die Aufenthaltsgesetzgebung}

Mit dem Zuwanderungsgesetz, das in Deutschland am 1.1.2005 in Kraft trat, wurde ein Gesetzesrahmen entworfen, der die Zuwanderung qualifizierter Arbeitnehmer*innen und Studierender einfacher machen sollte. Studierende und Promovierende aus Ländern außerhalb der EU fallen unter die Reglungen des $\$ 16$ Abs.1 Aufenth.G. Dieser legt fest, dass die Gesamtaufenthaltsdauer internationaler Studierender für das Studium inklusive Master und Promotion insgesamt nicht länger als zehn Jahre dauern darf. Vor Beginn des Studiums müssen internationale Studierende aus NichtEU-Ländern einen Krankenversicherungsschutz und genug finanzielle Mittel für die Sicherung des Lebensunterhaltes (orientiert sich am Bafög-Höchstsatz von 670 Euro monatlich) nachweisen (Barthelt et al. 2015: 6). Eine befristete Aufenthaltserlaubnis wird für den Zeitraum ausgestellt, für den die Sicherung des Lebensunterhaltes nachgewiesen werden kann. Die Gesetzgebung regelt, wie viele Stunden die internationalen Studierenden neben dem Studium arbeiten dürfen (120 ganze bzw. 240 halbe Tage pro Jahr) (Barthelt et al. 2015: 6).

Internationale Studierende, die ihr Studium in Deutschland abgeschlossen haben, dürfen 18 Monate bleiben, um eine ihrem Abschluss ,angemessene“ Tätigkeit zu finden. Als angemessen sind ,solche Tätigkeiten zu verstehen, die üblicherweise einen akademischen Abschluss voraussetzen und bei denen die mit der Hochschulausbildung erworbenen Kenntnisse zumindest teilweise oder mittelbar benötigt werden" (Mayer et al. 2012: 23). Während der 18 Monate dürfen sie uneingeschränkt arbeiten (Barthelt et al. 2015: 6).

Obwohl es einige Vereinfachungen in der Gesetzeslage und des Ablaufes der Visabeantragung gegeben hat, ist die Situation nach wie vor relativ komplex (mehr dazu in OECD 2013a). Neben den Einschränkungen, die diese rechtlichen Regelungen für die Mobilität bedeuten, machen sie sichtbar, welche Stratifikationen anhand unterschiedlicher Kategorien von Migrant*innen vorgenommen werden. So entsteht eine Hierarchie zwischen verschiedenen Migrant*innengruppen, die auf Herkunft (EU oder Drittstaaten), Qualifikation und Berufsgruppe beruht. In Intersektion mit Gender können die unterschiedlichen Regelungen für verschiedene Berufsgruppen wiederum dazu führen, dass Männer oder auch Frauen einen besseren Zugang zur internationalen Mobilität haben (Kofman und Raghuram 2005).

Die Aufenthaltsgesetzgebung ist ein wichtiger Aspekt in der Erfahrung von akademischer Mobilität. Durch Fristen schafft sie festgelegte Zeiträume und damit zeitliche Horizonte und Rhythmen von der Beantragung eines Visums, über dessen Bewilligung, seiner potentiellen Verlängerung bis zum Ablaufen des Visums.

\section{Deutschland, das "Land der Ideen“}

Die Änderung rechtlicher Bedingungen allein reicht nicht, um internationale Studierende und Wissenschaftler*innen für Deutschland zu interessieren. Dafür braucht es außerdem direkte Kooperationen auf staatlicher und institutioneller Ebene sowie 
Maßnahmen, die auf Deutschland aufmerksam machen und über die Möglichkeiten in Deutschland informieren. Solche Maßnahmen sind unter anderem Teil der Standortinitiative „Deutschland - Land der Ideen“, die von der Bundesregierung und der deutschen Wirtschaft 2005 eingeführt wurde. Im Rahmen dieser Initiative entstand die Kampagne „Study in Germany - Land of Ideas" ${ }^{60}$. Hier werden die verschiedenen internationalen Aktionen des Deutschen Akademischen Austauschdienstes (DAAD) und anderer Akteure gebündelt, um im Auftrag des BMBF mehr junge Menschen für ein Studium in Deutschland zu begeistern.

Ein weiteres Mittel, um die Sichtbarkeit Deutschlands und der deutschen Wissenschaft zu erhöhen, sind die sogenannten „Deutschen Wissenschafts- und Innovationshäuser“ (DWIH), die zur Standortinitiative „Land der Ideen“ gehören. Seit 2009 fördert das Auswärtige Amt den Aufbau dieser Häuser an sechs internationalen Standorten - unter anderem in Neu-Delhi. Die Aufgabe der DWIH ist der gemeinschaftliche Auftritt der im Ausland vertretenen deutschen Forschungs- und Wissenschaftsorganisationen. Die DWIH werden als wichtiger Teil der „Außenwissenschaftspolitik"61 des Auswärtigen Amtes verstanden.

In den Initiativen wird Deutschland als Ziel für innovative Studierende und Wissenschaftler*innen dargestellt. In der Kombination mit Diskursen um den Fachkräftemangel werden die internationalen Studierenden und Wissenschaftler*innen zu Trägern der Zukunft der Wissenschaft und Wirtschaft Deutschlands.

\subsubsection{Für die Forschung von Morgen: Institutionelle Strategien}

Zwischen Deutschland und Indien gibt es bereits seit geraumer Zeit verschiedene Kooperationen auf der Ebene der Hochschulen und der Forschung. Dabei wird Indien als wachsender Bildungsmarkt verstanden, der Spitzenforschung an einzelnen Instituten und vor allem eine „exzellent ausgebildete Elite junger Wissenschaftlerinnen und Wissenschaftler“ (Titz 2011: 15) vorweisen kann. Die wissenschaftliche Zusammenarbeit der beiden Länder, die überdies politisch unterstützt wird, begann in den 1970er Jahren mit der gemeinsamen Kernenergie- und Weltraumforschung (Titz 2011: 15). Die Zusammenarbeit hat sich mittlerweile auf weitere Gebiete ausgedehnt (Biowissenschaften, Medizin, Chemie, IT-Bereich etc.). Konkrete Zusammenarbeiten in den Geistes- und Sozialwissenschaften gibt es dagegen sehr wenige. ${ }^{62}$

\footnotetext{
${ }^{60}$ Weitere Angaben zur Kampagne finden sich auf der folgenden Webseite, die vom DAAD herausgegeben wird https://www.study-in-germany.de/de/ueber-uns/die-study-in-germany-kampagne_71049. php, abgerufen am 07.03.2019).

${ }_{61}$ Siehe Webseite des Auswärtigen Amts (https://www.auswaertiges-amt.de/de/aussenpolitik/themen/ aussenwirtschaft/forschungtechnologie/-/213142, abgerufen am 31.08.2020).

62 Das Centre for Modern Indian Studies in Göttingen, dass 2010 gegründet wurde, versucht in seiner engen Kooperation mit der Jawaharlal Nehru University, diesem Defizit entgegenzuwirken.
} 
In meiner Betrachtung institutioneller Initiativen konzentriere ich mich auf Institutionen in Göttingen, da meine Gesprächspartner*innen alle in Göttingen studiert bzw. promoviert haben.

\section{Die Max-Planck-Gesellschaft}

Für die Max-Planck-Gesellschaft (MPG) ist Indien ein wichtiger Partner in der wissenschaftlichen Zusammenarbeit (Titz 2011: 16). Eine Basis für die Zusammenarbeit wurde 2004 mit einem memorandum of understanding gelegt, in dem verschiedene Initiativen vorgesehen sind, die die wissenschaftliche Zusammenarbeit zwischen MPG und Forschungsinstituten in Indien intensivieren sollen.

Mit 830 Personen im Jahr 2015 ist Indien zu einem der größten Partnerländer der MPG für den Austausch internationaler junger Wissenschaftler*innen geworden. Im Jahr 2015 stammte fast jeder zehnte ausländische Doktorand an MPIs aus Indien. Viele der indischen Doktorand*innen forschen im Rahmen einer International Max-Planck-Research School (IMPRS), die als Maßnahme entwickelt wurden, um für internationale ,Talente' attraktiver zu werden. Die IMPRS bestehen je zur Hälfte aus deutschen und internationalen Doktorand*innen, wobei indische Promovierende die größte Gruppe der internationalen Doktorand ${ }^{*}$ innen an den IMPRS stellen ${ }^{63}$. Viele der indischen Studierenden und Doktoranden, die ich für meine Studie interviewt habe, studierten bzw. promovierten an einer dieser Research Schools.

\section{Universität Göttingen: „Committed to Excellence“}

Seitdem Deutschland 1959 bei dem Aufbau des Indian Institute of Technology in Madras half (Matussek 2011: 11), gibt es immer wieder Abkommen zwischen deutschen und indischen Universitäten. Neben vielen verschiedenen Kooperationen mit Universitäten in der ganzen Welt hat die Georg-August-Universität Göttingen weltweit zwei Auslandsrepräsentanzen in „strategically important countries“ (President of the Georg-August-Universität Göttingen 2011: 98): eine in Nanjing, China, und eine in Pune, Indien.

Die Universität arbeitet mit verschiedenen Maßnahmen, um in Indien auf sich aufmerksam zu machen. So ist sie unter anderem Mitglied des DWIH in Neu Delhi und hat memorandums of understanding mit verschiedenen Institutionen in Indien unterzeichnet. ${ }^{64}$ In der Eigendarstellung wirbt die Universität Göttingen mit ihrer Forschungsgeschichte, beispielsweise mittels der Nobelpreisträger, die an der Univer-

\footnotetext{
${ }_{63}$ Diese Angabe stammt von der Webseite der Max-Planck-Gesellschaft (https://www.mpg.de/de/ international/indien, abgerufen am 07.03.2019).

${ }^{64}$ Genaue Angaben zur Vernetzung mit indischen Universitäten finden sich auf der folgenden Webseite der Universität Göttingen (https://www.uni-goettingen.de/de/200092.html, abgerufen am 31.08.2020).
} 
sität Göttingen tätig waren, und mit internationalen Universitätsrankings. ${ }^{65} \mathrm{Wie}$ in der Überschrift zu diesem Abschnitt angedeutet, empfiehlt sich die Universität Göttingen außerdem mit ihrer „Exzellenz“ (President of the Georg-August-Universität Göttingen 2011: 3): 2007 konnte sich die Universität mit ihrem Zukunftskonzept unter dem Motto „Göttingen. Tradition - Innovation - Autonomie“ Fördergelder unter der sogenannten Exzellenzinitiative des Bundes und der Länder sichern. Die Exzellenzinitiative, die 2005 beschlossen wurde und von der DFG und dem Wissenschaftsrat betreut wird, ist auch Teil der Strategien, die Deutschland als „Wissenschaftsstandort" stärken und seine internationale Wettbewerbsfähigkeit verbessern sollen. ${ }^{66}$ Die institutionelle Strategie der Universität Göttingen unter der Exzellenzinitiative (2007-2012) sah unter anderem folgende drei Maßnahmen vor: „Brain Gain (recruiting the best)“, „Brain Sustain (retaining the best)“, und die Etablierung der Abteilung „Göttingen International“ ${ }^{67}$ Die Universität Göttingen ist außerdem Teil verschiedener Mobilitätsprogramme, wie „A New Passage to India“, das vom DAAD gefördert wird.

Die hier dargestellten Initiativen zeichnen ein spezifisches Bild von Deutschland und der deutschen Wissenschaftslandschaft: Deutschland ist das Land für innovative Wissenschaft für die ,schlauesten Köpfe‘ und bietet Möglichkeiten für eine wissenschaftliche Karriere.

\subsubsection{Von „IT-Indern“ und Rajesh Koothrapalli}

Auf der Suche nach ,Talenten' scheint Indien der perfekte Partner für Deutschland zu sein - vor allem als scheinbar unversiegbare Quelle von sogenanntem Humankapital. Neben diesem recht offensichtlichen Grund für die Attraktivität Indiens für Deutschland gibt es aber weitere Ursachen für das Interesse Deutschlands an einer Zusammenarbeit. Eine Grundlage bildet die historische Tiefe der Migration indischer Studierender und Wissenschaftler*innen nach Deutschland. Mit der folgenden Darstellung der Geschichte mache ich deutlich, auf welcher historischen Entwicklung das spezifische Regime der akademischen Mobilität basiert, mit dem meine Gesprächspartner*innen interagierten. Außerdem zeige ich im Folgenden, welche Stereotype in Deutschland dazu beitragen, dass indische Studierende und Wissenschaftler*innen als besonders geeignetes Humankapital wahrgenommen werden.

\footnotetext{
${ }^{65}$ Auf der folgenden Webseite der Universität wird beispielsweise mit dem Ranking geworben (http://www.uni-goettingen.de/en/study--research-in-g\%C3\%B6ttingen/517595.html, abgerufen am 07.03.2019).

${ }^{66}$ Die Erklärung zur Exzellenzinitiative befindet sich auf der Webseite des DFG. (http://www.dfg. de/foerderung/programme/exzellenzinitiative/allgemeine_informationen/index.html, abgerufen am 07.03.2019).

${ }^{67}$ Die Broschüre zur Exzellenzinitiative kann auf der Webseite der Universität heruntergeladen werden (https://www.uni-goettingen.de/de/document/download/03e2b34f28caf4de5076d8c319e4a38e. pdf/brosch_zuk_web.pdf, abgerufen am 31.08.2020).
} 


\section{Die Mobilität von Studierenden und Wissenschaftler*innen aus Indien}

Indien ist bereits seit geraumer Zeit eine wichtige Quelle von Fachkräften, Hochausgebildeten und Studierenden für viele Länder des globalen Nordens (Khadria 2002: 5). Aufgrund der kolonialen Geschichte war Großbritannien zunächst das beliebteste Ziel indischer Migrant*innen. Ab den 1970er Jahren wurden allerdings andere Zielländer sowohl für hoch- als auch unausgebildete indische Migrant*innen interessant. So führte die Lockerung der Einwanderungsgesetze in den USA, Kanada und Neuseeland zu einer verstärkten Einwanderung in diese Länder (Khadria 2006: 66, Brown 2006: 45). Vor allem die USA hatte eine große Anziehungskraft auf gut ausgebildete Arbeitskräfte (Khadria 2006: 66). Die selektive Einwanderungspolitik bevorzugte ganz klar die Einwanderung Hochausgebildeter und führte zu dem, was allgemein als „Brain Drain“ aus Indien verstanden wird (vgl. Khadria 1999, 2002, 2006; Varma 2006)

Tatsächlich sind die USA immer noch eines der beliebtesten Zielländer indischer Studierender und Wissenschaftler*innen. Dazu tragen ganz unterschiedliche Faktoren bei: die Erfolgsgeschichten indischer Migrant*innen in den USA (vor allem in Bezug auf das Silicon Valley), der Ruf amerikanischer Universitäten, der immer wieder in globalen Rankings bestätigt wird, familiäre Beziehungen sowie die große Wertschätzung für amerikanische Abschlüsse in Indien. Statistiken und meine eigene Studie zeigen aber, dass Deutschland und andere europäische Länder für indische Studierende und Wissenschaftler*innen an Attraktivität gewinnen.

\section{Deutschland als Zielland indischer Studierender und Wissenschaftler*innen: Eine kurze Geschichte}

Anfang des 20. Jahrhunderts zog es einige wenige Menschen von Indien nach Deutschland (Goel 2006; Brosius 2006; Gottschlich 2012: 1). Zum einen kamen indische Nationalisten nach Deutschland, um hier Unterstützung für ihren Kampf für die Unabhängigkeit von der britischen Kolonialherrschaft zu suchen. Zum anderen kamen indische Studierende, die vom guten Ruf der deutschen Universitäten angezogen wurden (Goel 2006: 358; Gottschlich 2012: 1). Während der Regierung der Nationalsozialisten verließen die meisten indischen Studierenden Deutschland. Nach dem Zweiten Weltkrieg waren es wiederum Studierende, die die erste kleine Welle indischer Mobilität nach Deutschland anführten. Die meisten der Studierenden waren Techniker und Ingenieure, die für ihre Ausbildung Stipendien erhielten (Gottschlich 2012: 2). Viele dieser Studierenden kehrten nach Indien zurück. Einige wenige blieben in Deutschland, gründeten Familien mit deutschen Frauen und bekamen dank ihrer guten Ausbildung gute Arbeitsplätze (Goel 2006: 358-359). Waren diese ersten Migrationsströme von Indien nach Deutschland ausschließlich von Männern geprägt, begann in den späten 1960er Jahren eine Phase indischer Migration, die vor allem von Frauen getragen wurde. So kamen zwischen den späten 1960er und 1970er Jahren circa 6000 indische Krankenschwestern in die BRD, um 
in Krankenhäusern zu arbeiten. Viele dieser Frauen kehrten nach Ablauf ihrer Arbeitsverträge nach Indien zurück (Goel 2002).

Das Jahr 2000 bildet einen wichtigen Wendepunkt in der Mobilität von Indien nach Deutschland. Auf der Suche nach Fachkräften für den IT-Bereich wurde auf Initiative der deutschen Regierung die Greencard eingeführt (bis 2004). Daraufhin kamen einige Tausend indische Techniker, Ingenieure und IT-Spezialisten nach Deutschland - die meisten davon Männer. Die Greencard beschränkte die Zeit des Aufenthaltes von vorneherein auf fünf Jahre und richtete sich am „Gebot der Nützlichkeit“ aus, wie Ronneberger und Tsianos (2009: 141) herausstellen. Viele der indischen Fachkräfte planten ihren Aufenthalt von Beginn an als Durchreise auf dem Weg zu anderen Zielen oder zurück nach Indien (Goel 2006: 359). Obwohl nur einige tausend indische IT-Spezialisten nach Deutschland kamen, hatte diese Entwicklung einen großen Einfluss auf das Image Indiens in Deutschland. Vor der Ankündigung der Greencard-Initiative und der tatsächlichen Ankunft der Fachkräfte wurde Indien vor allem mit Armut, der Unterdrückung von Frauen und Religiosität in Verbindung gebracht (Goel 2006). Die Anwerbung hochausgebildeter Spezialisten aus Indien veränderte die Perspektive. So werden Inder*innen in Deutschland heute noch vor allem mit dem IT-Bereich assoziiert und als „Computer-Inder“ kategorisiert (Gottschlich 2012: 8). ${ }^{68}$

\section{Stereotype: ,Computer-Inder', ,Nerds' und, model minorities}

Die Kampagne zur Anwerbung von IT-Fachkräften aus Indien rückte Indiens technologischen Fortschritt in den Vordergrund und die indischen Fachkräfte wurden als die Lösung des IT-Problems in Deutschland gesehen (Gottschlich 2012: 8). Das Bild des ,Computer-Inders' hat auch heute, zwanzig Jahre nach Einführung der Greencard bzw. ab 2012 der Bluecard, noch einen großen Einfluss. Indische Migrant*innen werden aber nicht nur mit bestimmten Arbeitsbereichen verknüpft, sondern auch mit bestimmten Eigenschaften. In Deutschland, ebenso wie in Amerika, gelten indische Studierende und Wissenschaftler*innen als fleißig und motiviert (Khadria 2002: 17, Lall 2006: 20). Andere Klischees von Inder*innen beinhalten bestimmte Charaktereigenschaften, die mit der ,asiatischen Natur' verbunden werden. Dazu gehören zum Beispiel Passivität, Schüchternheit, Bescheidenheit und Gehorsamkeit (Gottschlich 2012: 8). Die Medien tragen einen großen Teil dazu bei, solche Stereotype zu erhalten und zu bestätigen. Ein gutes Beispiel dafür ist die Figur des Rajesh Ramayan Koothrapalli aus der in Deutschland sehr beliebten US-Serie The Big Bang Theory. ${ }^{69}$ In der Darstellung von Raj, der in sozialen Situationen unbeholfen ist und

\footnotetext{
${ }^{68}$ Diese Bezeichnung findet sich zum Beispiel in einem Artikel von Spiegel-Online. Siehe Markus Deggerich: „Hi Potentials!“, Spiegel Online vom 31.10.2001, http://www.spiegel.de/wirtschaft/schroe der-bei-den-computer-indern-hi-potentials-a-165375.html.

69 Siehe Statista: „Ranking der beliebtesten in Deutschland im Jahr 2016“ vom Juli 2016, https:// de.statista.com/statistik/daten/studie/581038/umfrage/ranking-der-beliebtesten-fernseh serien-indeutschland/.
} 
teilweise unangemessen handelt, wird in The Big Bang Theory aber nicht nur mit Klischees des ,Inders' gearbeitet, sondern auch mit denen des, Nerds' oder ,Geeks'. Raj und die anderen drei männlichen Hauptcharaktere der Serie sind alle Naturwissenschaftler bzw. Ingenieure. Sie kennen sich bestens im Marvel-Universum ${ }^{70}$ aus und sprechen Klingonisch, ${ }^{71}$ haben aber Probleme in alltäglichen Situationen und in der Kommunikation mit Personen, die nicht den gleichen akademischen Hintergrund haben. The Big Bang Theory bestätigt auf diese Weise nicht nur die Stereotype vom schüchternen Inder, sondern ebenso die vom unsozialen meist männlichen Naturwissenschaftler.

Ein weiteres Stereotyp, das vor allem in den USA und Kanada in Verbindungen mit indischen, aber genauso chinesischen Migrant*innen auftritt, ist das der ,model minority'. Dieser Diskurs besteht im Prinzip aus der Annahme, dass ,Asiaten“ eine homogene Gruppe sind, die in all ihren Bestrebungen Erfolg haben, sei es in der Schule oder am Arbeitsplatz (Shankar 2008; Ho 2014: 80), und verortet die Schuld für ein Scheitern beim Individuum und nicht bei möglichen institutionellen Grenzen, die den Erfolg verhindern (Navaratnam 2011: 24).

Obwohl meines Wissens in Deutschland in Bezug auf indische Migrant*innen nicht von einer model minority gesprochen wird, weisen die oben genannten Klischees des wissenschaftlich begabten, schüchternen Inders in eine ähnliche Richtung. Vor allem im Vergleich zur Berichterstattung und öffentlichen Meinung von anderen Migrant*innengruppen in Deutschland, werden indische Migrant ${ }^{*}$ innen in Deutschland eher positiv porträtiert. Wie bereits dargelegt, wird das positive Image sowohl von der Migrationsgeschichte indischer Studierender und Fachkräfte beeinflusst als auch von der medialen Darstellung des fleißigen, schüchternen indischen Wissenschaftlers.

Indischen Studierenden und Wissenschaftler*innen werden positive Eigenschaften zugeschrieben, die ihnen, wie es scheint, vor allem in einer Wissensökonomie sehr zum Vorteil gereichen. Sie gelten als fleißig, als hart arbeitend, als diszipliniert, als intelligent und innovativ, aber auch als unauffällig und angepasst. In diesem Sinne scheinen gerade indische Studierende und Wissenschaftler*innen in der Wissensökonomie und der Suche Deutschlands nach ,Humankapital' im Bereich der Science and Technology die beste Wahl zu sein. Doch obwohl die zugeschriebenen Eigenschaften die jungen Inder*innen bei ihren Mobilitätsbestrebungen zunächst unterstützen, können sie negative Auswirkungen haben. Denn auch positive Stereotype reduzieren Personen auf bestimmte Eigenschaften und Vorstellungen, auf Dinge, die sie angeblich gut können oder eben nicht können. Im Fall der indischen Wissenschaftler*innen erzeugt das Stereotyp ein sehr eindimensionales Bild von Menschen, die auf ihre Arbeit bzw. das Studium fixiert sind. Dies spiegelt sich auch in den Begrifflichkeiten wider, die in der Wissensökonomie im Zusammenhang mit den

\footnotetext{
${ }^{70}$ In den Comics der Marvel Reihe, wie Thor, Ironman oder Captain America wird ein eigenes Universum erschaffen, in dem die verschiedenen Helden auftreten.

${ }^{71}$ Klingonisch ist eine Sprache aus der Science-Fiction-Serie Star Trek.
} 
Studierenden und Hochausgebildeten verwendet werden: ,Humankapital', ,Talent', skill und ,Köpfe'. Alle diese Termini reduzieren Hochausgebildete auf eine Facette ihres Selbst, nämlich auf ihre wissenschaftliche Arbeit und Leistung und haben damit einen dehumanisierenden Effekt. Prozesse der Entmenschlichung wurden bisher vor allem im Kontext der Mobilität Geflüchteter diskutiert, die häufig als „less than human" (Esses, Medianu und Lawson 2012: 522) dargestellt werden. Im Fall der internationalen Studierenden und Wissenschaftler*innen verläuft die Entmenschlichung in die andere Richtung - sie werden als ,mehr als menschlich', als über-rationale Individuen dargestellt. Dabei verschleiert die scheinbar objektive Sprache des Regimes der akademischen Mobilität den Umstand, dass nicht jeder die Möglichkeit hat, ein ,Talent' zu werden, und dass die Wahrnehmung als schlauer Kopf mit exotisierenden und stereotypen Repräsentationen in Verbindung steht.

Anhand der Analyse der staatlichen und institutionellen Initiativen zur Förderung der akademischen Mobilität habe ich gezeigt, mit welchen Befürchtungen für die Zukunft diese im Zusammenhang stehen. Die jungen Wissenschaftler*innen aus Indien werden zu Hoffnungsträgern für die Wissenschaft und Wirtschaft Deutschlands. Diese Assoziation schafft Erwartungen auf Seiten der Institutionen und der jungen Menschen: Die Institutionen erwarten, entsprechend innovative und disziplinierte ,Talente ${ }^{6}$ zu gewinnen, und die Studierenden selbst die Umsetzung der versprochenen (besseren) Zukunft.

\subsection{Indien: Vorstellungen von Entwicklung, Bildung und Mobilität}

Im vierten Teil des vorliegenden Kapitels steht die ,indische' Seite des Regimes der akademischen Mobilität im Zentrum meiner Ausführungen. Dabei wird deutlich, dass die Mobilität von Studierenden und Wissenschaftler*innen in Indien von verschiedenen Akteuren als wichtiger Faktor für die nationale und individuelle ,Entwicklung' verstanden wird.

\subsubsection{Wissen $=$,Entwicklung?}

Lange Zeit wurde die Mobilität von Hochausgebildeten vor allem unter dem Schlagwort ,Brain-Drain' diskutiert, also dem Verlust der Wissenden für das Senderland. Der Diskurs hat sich mittlerweile eher in das Gegenteil gewandelt, so dass mittlerweile von ,brain gain' und ,brain circulation' die Rede ist (Khadria 2008: 42; Bhandari und Blumenthal 2009: 8, Saxenian 2005). Diese Veränderung in der Art und Weise, wie über die Migration Hochausgebildeter aus Indien gesprochen wird, steht unter anderem mit einem sehr einflussreichen Diskurs in Verbindung, der Wissen mit ,Entwicklung' verknüpft (Radhakrishnan 2011: 36). Smita Radhakrishnan verortet den Ursprung dieses Diskurses im World Development Report der Weltbank 
1998/99, der das Wort Wissen in eine Art Mantra der development policies verwandelt habe (Radhakrishnan 2011: 37). ${ }^{72}$

Der Diskurs von ,Entwicklung 'oder ,Fortschritt' (Hansen 2015) beinhaltet die Vorstellung von Linearität. Wie Radhakrishnan darlegt (Radhakrishnan 2011: 37), wird Wissen in dieser linearen Logik als Strategie des Überspringens der Industrialisierung, die eigentlich als nächster Schritt in Entwicklungsmodellen folgen sollte, konzipiert (Radhakrishnan 2011: 37). Wissen - vor allem jenes, das mit Science and Technology umschrieben wird - soll dazu beitragen, dass jene Länder, denen in der Entwicklungslogik Nachholbedarf zugeschrieben wird, ,aufholen' können (Radhakrishnan 2011: 37).

Bereits der erste Premierminister Indiens, Jawaharlal Nehru, verknüpfte Wissenschaft und Technologie mit der ,Entwicklung' Indiens. So formulierte er 1947 in seiner Rede vor dem Indian Science Congress: „I believe firmly that it is through the method and spirit of science that we can ultimately solve our problems" (J. Nehru zitiert in Abraham 1998: 46-47). Nehru verstand die Wissenschaft als Instrument für die Transformation der Nation. Dieses Verständnis von Wissenschaft schlug sich unter anderem in den Institutionen nieder, die während seiner Regierungszeit gegründet wurden, darunter die ersten Indian Institutes of Technology (IIT) ${ }^{73}$

Manmohan Sing, der von 2004-14 Indiens Premierminister war und davor bereits als Finanzminister großen Einfluss auf die wirtschaftliche Öffnung Indiens hatte, betonte immer wieder die Bedeutung von Investitionen in die Wissensökonomie. In seiner Rede bei der 16. Versammlung der Blockfreien Staaten 2013 erklärte er: „Perhaps most relevant for us is to focus on investing in the knowledge economy and building our human resources. " ${ }^{74}$

In diesen Diskursen von ,Entwicklung ' werden zwei grundlegende Ressourcen benötigt: ,Humankapital' und Wissen. Vor allem ,Humankapital' in Form der sogenannten demographischen Dividende ist in Indien in großem Maße vorhanden. Die Ausbildung dieser wird nun als das Mittel gesehen, um die große Masse an arbeitsfähigen Menschen für die ,Entwicklung' der Nation nutzbar zu machen. Obwohl in den letzten Jahren verschiedene Initiativen ergriffen wurden, um eine bessere Ausbildung für mehr Menschen in Indien zu gewährleisten, sind Studien- und Ausbildungsplätze in Indien weiterhin knapp (Bhandari und Blumenthal 2009: 7). Die Mobilität indischer Studierender scheint deswegen doppelt sinnvoll: Zum einen

\footnotetext{
72 Dass diese Verknüpfung weiterhin in Reporten der Welt Bank aufrechterhalten wird, zeigt sich in einer Ausgabe des Development Digest von 2018, die den Titel Maximizing knowledge for development trägt (World Bank Group 2018b).

${ }^{73}$ In den folgenden Städten wurde die ersten IITs gegründet: Kharagpur 1951, Mumbai 1958, Chennai 1959, Kanpur 1959, Neu-Delhi 1963.

${ }^{74}$ Siehe: The Hindu: „Statement by Prime Minister Manmohan Singh at the XVI Summit of the Non-Aligned Movement" vom 30.08.2012, http://www.thehindu.com/news/resources/statement-byprime-minister-manmohan-singh-at-the-xvi-summit-of-the-nonaligned-movement/article3839792. ece.
} 
erhalten Personen, die in Indien keinen Studienplatz bekommen haben, diesen im Ausland, zum anderen bringen diejenigen, die bereits im Ausland waren und nach Indien zurückkehren, wichtiges Wissen mit, um die ,Entwicklung' Indiens voranzutreiben. Dabei ist allerdings zu beachten, dass Wissen hier zwar zur ,Entwicklung der gesamten Nation beitragen soll, tatsächlich aber eher das Entstehen einer gut ausgebildeten Elite begünstigt (Radhakrishnan 2011: 36). Die Mitglieder der Elite, die in den Brain-Drain-Diskursen zunächst als Verräter*innen dargestellt wurden, die ihre Nation mit ihren Problemen alleine lassen würden, werden heute als Held*innen gefeiert, als Motoren der ,Entwicklung' Indiens (Khadria 2002: 30-31; vgl. Radhakrishnan 2011; Ajantha Subramanian 2015) zu einer „knowledge power“75.

\subsubsection{Bildung und Mobilität als Investitionen in die Zukunft}

Wie ich in Kapitel zwei dargelegt habe, ist der Fokus auf Bildung ein Merkmal der indischen middle class (vgl. Mazzarella 2005, Säävälä 2006). Die Mittelschicht hofft mittels der ,richtigen' Bildung an den ,richtigen' Schulen soziales und kulturelles Kapital zu erhalten, das wiederum für die spätere Karriere, aber auch für die Anbahnung von Heiratsbeziehungen wichtig ist (Säävälä 2010: 57-58). Bildung ist damit gerade für Menschen, die sich als middle class verstehen, eine wichtige Ressource, um eine bestimmte gesellschaftliche Position zu erreichen und diese zu festigen (Säävälä 2006: 393). Dementsprechend investieren Familien viel Zeit und Geld in die Ausbildung ihrer Kinder:

The young person's progress is now being carefully and ,prudentially' engineered through strategies of choice and investment of capital by ,anxious middle-class parents intent on reproducing their class advantages in the face of risky global markets that make such status less secure. (P. Singh und Doherty 2008: 119)

Bildung soll nicht nur zu Wissen verhelfen, sondern zu wirtschaftlichem Erfolg und sozialem Aufstieg (Bansal 2013: 123; Gilbertson 2016). Bildung wird in dieser Dynamik zu einer ,technology of the self (Foucault et al. 1988) und das Selbst der Gebildeten zu einem ,Unternehmen“ (Gershon 2011: 539), einer „collection of assets that must be continually invested in, nurtured, managed, and developed" (Martin 2000: 582). Im Fall der jungen Wissenschaftler*innen stand allerdings nicht unbedingt das individuelle Unternehmen im Fokus, sondern das Familienunternehmen.

Der Markt, an dem sich die Investition in dieses Unternehmen orientiert, wird immer internationaler und erfordert spezielle Eigenschaften, die nur bestimmte Bevölkerungsgruppen besitzen oder sich aneignen können: Geld, englische Sprache,

\footnotetext{
75 Manmohan Singh formulierte 2008 in einer Rede bei einer IIT-Alumni-Konferenz: „I believe it is India’s destiny to become a knowledge power“(zitiert in: Ajantha Subramanian 2015: 85).
} 
Computerkenntnisse, Privilegien, soziale Kontakte (Jeffery 2005: 20). Mit diesem neoliberalen Verständnis des Selbst als Unternehmen (Gershon 2011: 539) interagierten meine Gesprächspartner*innen in ihrer biographischen Navigation. Dabei hatte die Vorstellung von Bildung als Investition eine wichtige Funktion im Regime der akademischen Mobilität: Internationale Mobilität zur Aneignung weiterer Qualifikationen und Kenntnisse wird zu einem ganz logischen Schritt zur Optimierung des Selbst und zur Wettbewerbsfähigkeit mit anderen Unternehmen. Durch die akademische Mobilität soll weiteres soziales, kulturelles und symbolisches Kapital erlangt werden (Findlay 2011; Findlay, King, und Smith 2012) und der Auslandsaufenthalt kann als Praxis der Differenzierung von anderen jungen Menschen verstanden werden (Findlay, King und Smith 2012: 121). Mein Gesprächspartner Ajay erklärte mir beispielsweise, dass er Deutschland gewählt habe, um sich bewusst von den vielen jungen Inder*innen, die in die USA gehen würden, abzuheben. In diesem Prozess der Differenzierung spielt die bereits angesprochene Hierarchisierung von Institutionen und Lokalitäten eine wichtige Rolle.

\subsubsection{Imaginationen von abroad}

Die meisten meiner Gesprächspartner*innen waren in einem Umfeld aufgewachsen, in dem internationale Mobilität nicht als etwas Besonderes galt, sondern eher als etwas, das „alle“ irgendwie machen. Sie erzählten mir von Schulkamerad*innen, die in verschiedenen Teilen der Welt studierten, oder von Geschwistern, die gerade einen Arbeitsplatz im Ausland erhalten hatten. Smita erinnerte sich: „Always when I was in India I used to hear, ,Oh she is there, he is there.' And I kind of think everybody is gone everywhere" (Smita, 05.08.2010). Das Thema abroad bildete somit, wie es auch von Baas (2013: 9) beschrieben wird, eine Konstante im Leben der indischen Mittelschicht.

Imaginationen von Mobilität haben schon vor der eigentlichen körperlichen Bewegung eine Wirkung auf die Entscheidung und die Erfahrung von Mobilität (vgl. Appadurai 1996: 6). Obwohl solche Imaginationen ganz persönlich und individuell sind, zeigen die Aussagen meiner Gesprächspartner*innen gewisse Gemeinsamkeiten. Diese betreffen zwei Themen: die Qualität der wissenschaftlichen Arbeit und die Möglichkeit zur persönlichen Entfaltung. Die meisten meiner Gesprächspartner*innen berichteten mir, dass sie vor ihrer Abreise nicht besonders viel über Deutschland wussten. So beschränkten sich die Informationen, die viele meiner Gesprächspartner*innen hatten, zumeist auf den Stand der Wissenschaft in Deutschland und auf das Klischee des verschlossenen und pünktlichen Deutschen. Oder im Fall von Sangita auf das kalte deutsche Wetter: „I just knew that this place is good for science. So when I came here I was only expecting good science and cold weather." Sangitas Aussage macht sichtbar, wie wenig sie tatsächlich über Deutschland wusste und worauf sie sich in ihren Erwartungen konzentrierte: Auf Deutschland, das Land der Wissenschaft. 
I never had a time to think, and the lab was good. I never thought of this: that I have to compromise with the language or you know culture. That never came. So for me the lab was good, and I got a position - and in the field that I want - and I just came without even thinking about any other factors. (Smita, 05.08.2010)

In Sangitas und Smitas Aussagen spiegelt sich nicht nur die Fokussierung auf die Wissenschaft wider, sondern zeigt sich auch die Bedeutung von Imaginationen für die Wahl des Zielortes: Deutschland hat in Indien einen guten Ruf in den Naturund Ingenieurwissenschaften und wird dadurch $\mathrm{zu}$ einem attraktiven Land für Studierende und Wissenschaftler*innen in diesem Bereich. Das Zitat aus meinem Gespräch mit Smita macht die Konzentration auf die Bedeutung von Bildung und Mobilität für die Zukunft sichtbar. Wie Smita es formulierte, verlor sie kaum einen Gedanken an Konsequenzen ihrer Entscheidung für ihre Gegenwart in Deutschland. Sie maß den Wert ihrer Mobilität vor allem an deren Rolle für die Umsetzung ihrer Zukunftsvisionen. Wie ich in Kapitel 2 gezeigt habe, wird dieses Denken in Investitionen für die Zukunft und das Opfern der Gegenwart zugunsten einer (besseren) Zukunft bereits in der Schulzeit forciert.

Deutschland ist aber nicht nur als Land der Wissenschaft attraktiv, sondern wird als ,westliches' Land ebenso mit bestimmten persönlichen Freiheiten verbunden.

So many of the people will have a very, very kind of romanticized notion of [the] west - where you go and you have a very good sexual life, and where things are liberated, where things are emancipated. So there is this notion. (Anay 25.07.2015)

Angeregt durch Darstellungen in Hollywood- und Bollywood-Filmen und durch Erzählungen von Freund*innen und Familienmitgliedern im Ausland und deren Fotos auf Facebook stellten sich viele das Leben in Deutschland freier und unabhängiger vor als in Indien - eine Vorstellung, die zur Attraktivität eines Studiums in Deutschland beitragen konnte. Die Motivationen zur Mobilität waren unter den jungen Wissenschaftler*innen aus Indien sehr vielfältig und nicht zuletzt mit der Hoffnung auf eine von den Eltern unabhängigere Gegenwart verbunden. In den Aushandlungen, die meine Gesprächspartner*innen mit ihren Eltern um die Möglichkeit der Mobilität häufig führen mussten, stand allerdings immer die Bedeutung der Mobilität für die Zukunft im Vordergrund. 


\section{Wissenschaft als Pfad abroad}

Trotz der Imaginationen, die von abroad kursierten, hatten die jungen Studierenden kaum konkrete Netzwerke, die ihre Mobilität gefördert hätten. Wie die jungen Menschen in Baas' Studie (2013) mussten auch meine Gesprächspartner*innen ihren eigenen Weg aus Indien finden. Für sie boten Studium und Wissenschaft einen solchen Pfad ins Ausland, der außerdem gesellschaftlich sanktioniert wurde:

(...) conforming to social norms, our respondents explained that as students embarking on scientific careers they had seen international mobility as important because their peer groups, families, communities and even future employers saw it as essential to the pursuit of career success. Amongst our respondents, this was uncontested. Going abroad was quite simply „what you did": a key step on the socially ratified career path that the brightest young scientists followed. (Cohen, Duberley und Ravishankar 2015: 112)

Cohen et al. verdeutlichen, dass es in Indien ein starkes Bewusstsein von der Bedeutung von Mobilität für eine wissenschaftliche Karriere gibt. In den Aussagen meiner Gesprächspartner*innen zeigt sich dieses Bewusstsein sehr deutlich: „the impression for any student of science in general in India is that you do your higher education especially $\mathrm{PhD}$ in the United States or elsewhere" (Vendan, 26.03.2013).

In Kombination mit meinen Ausführungen zu Vorstellungen vom wissenschaftlichen Lebensverlauf (3.1.3) zeigt sich, dass die jungen Inder*innen in einer sozialen Umwelt navigierten, in der es klare Vorstellungen von einem "career path“ (Cohen, Duberley und Ravishankar 2015: 112) gibt. Unterschiedliche Akteure tragen dazu bei, normative Vorstellungen davon zu entwickeln und weiterzutragen, wie sich ein Mensch zu einem erfolgreichen Wissenschaftler oder einer erfolgreichen Wissenschaftlerin entwickelt. Die Vorstellungen vom Karrierepfad beinhalten räumliche und zeitliche Koordinaten: Orte, an denen die beste Wissenschaft vermutet wird (laut Ranking), und Zeiten, die sich am besten für die Mobilität eignen - so wie die Jugend, in der wichtige Grundlagen für die Zukunft geschaffen werden sollen und die Menschen sich, so wird angenommen, unabhängig von familiären Verantwortlichkeiten bewegen können. Die dargelegten Vorstellungen des Karrierepfades geben den jungen Wissenschaftler*innen aus Indien in ihrer biographischen Navigation eine wichtige Orientierung für die Zukunft, wirken aber wie andere normative Konzeptionen von der Entwicklung des Menschen durch die Zeit hinweg einschränkend. Sie setzten die (angehenden) Wissenschaftler*innen unter Druck, den richtigen Weg im richtigen Tempo einzuschlagen und die vorgesehenen Koordinaten in der richtigen Reihenfolge abzugehen.

Der Blick auf die Diskurse und Umstände, die in Indien zur Mobilität von Studierenden und Wissenschaftler*innen vorherrschen, eröffnet eine weitere Perspektive auf die Verknüpfung der individuellen Zukünfte der jungen Inder*innen mit kollektiven Zukünften von Familien und der Nation Indien. Der starke Fokus 
auf die Zukunft, der durch Entwicklungsdiskurse und dem Verständnis von Bildung und Mobilität als Investitionen begründet wird, führt dazu, dass die Gegenwart zugunsten der Zukunft ,geopfert', das heißt, immer in Bezug zur Zukunft verstanden wird.

\subsection{Zusammenfassung}

Im Regime der akademischen Mobilität wird die Mobilität von Menschen gefördert, denen eine wichtige Rolle für die Sicherung nationaler und institutioneller Zukünfte zugesprochen wird. Internationale Studierende werden in Deutschland als zukünftige Fachkräfte gesehen, als Entdecker innovativer Lösungen für die Herausforderungen der Zukunft, in Indien als Wegbereiter von ,Entwicklung'. Die Familien der jungen Wissenschaftler*innen sehen in der akademischen Mobilität eine wichtige Investition für die zukünftige Karriere. Die Repräsentationen von Zeit, die eine starke Orientierung in die Zukunft beinhalten, hatten einen großen Einfluss auf die biographische Navigation meiner Gesprächspartner*innen. Als Zeitkarten boten diese Repräsentationen eine klare Orientierung für Entscheidungen, die die jungen Menschen für ihr Leben treffen mussten und die stark mit ihrem Selbstverständnis als (angehende) Wissenschaftler*innen verbunden waren.

Die verschiedenen Repräsentationen von Zeit, die ich in diesem Kapitel thematisiert habe - Zukunftsvisionen, Entwicklungsdiskurs, akademischer Lebensverlauf schaffen zeitliche Erfahrungen und vermitteln unterschiedliche Möglichkeiten der Gestaltung dieser. Auf diese Thematik gehe ich in den folgenden Kapiteln ein.

Im Regime der akademischen Mobilität wird die Mobilität von Studierenden und Wissenschaftler*innen als grundlegend positiv konstruiert. Sie bringen Wissen und Innovation nach Deutschland und ,Entwicklung' für Indien. Sie erwerben für sich selbst und ihre Familien soziales, kulturelles und symbolisches Kapital, das für die berufliche und persönliche Zukunft wichtig ist. Sie sammeln Erfahrungen und genießen Freiheiten. Diese sehr positive Darstellung von Mobilität lässt aber aus, welche Unsicherheiten für mobile Menschen entstehen (siehe Robertson und Runganaikaloo 2014). In der Werbung für Mobilität wird vergessen zu erwähnen, was es im Alltag bedeutet, weit weg von der eigenen Familie und in einem neuen Umfeld zu leben.

Die positive Konnotation von internationaler Mobilität gab meinen Gesprächspartner*innen ein diskursives Werkzeug für die Aushandlung eigener Aspirationen. So konnten Eltern beispielsweise durch den positiven Wert, der internationaler Mobilität und akademischer Bildung zugeschrieben wird, schneller von der Sinnhaftigkeit eines Auslandsaufenthaltes überzeugt werden.

Im Regime der akademischen Mobilität werden die jungen Menschen vor allem auf ihre wissenschaftliche Arbeit und Leistung reduziert und als ,brains' und ,Talente', positioniert. Wie meine Gesprächspartner*innen diese Verortung ausfüllten, für sich nutzten, sie ablehnten und mit anderen sozialen Verortungen kombinierten, 
war sehr individuell. Das positive Bild, das von der Mobilität Studierender und Wissenschaftler*innen und dieser Personengruppe gezeichnet wird, geht mit großen Erwartungen an diese einher. Die Studierenden und Wissenschaftler*innen geraten dadurch unter großen Druck, diese Erwartungen, die sie nicht zuletzt auch an sich selbst stellen, zu erfüllen. Aus diesem Grund investieren sie viel Zeit und Energie in ihre Arbeit. Das bereits ausführlich beschriebene Stereotyp des hart arbeitenden, disziplinierten Inders verhält sich in diesem Fall wie eine selbsterfüllende Prophezeiung. 


\section{Wissenschaftler*in sein und werden}

Durch die Akteure im Regime der akademischen Mobilität wurden die jungen Wissenschaftler*innen aus Indien als wichtige ,Humanressource für die lokale und globale Wissensökonomie positioniert. Im vorliegenden Kapitel steht im Fokus, wie sie diese Positionierung navigierten: nicht als die losgelösten akademischen Unternehmer*innen, als die sie im Mobilitätsregime repräsentiert und positioniert wurden, sondern als eingebettete junge Menschen mit multiplen Zugehörigkeiten.

Im ersten Teil des Kapitels lege ich dar, wie meine Gesprächspartner*innen von ihrem Werdegang und ihrer Entscheidung für ein Studium bzw. eine Promotion in Göttingen erzählten. In den Narrationen, die ich als eine Praxis der biographischen Navigation analysiere, wurde die Mobilität nicht als Bruch im Lebensverlauf konstruiert. Meine Gesprächspartner*innen stellten den Umzug nach Göttingen vielmehr als rationalen, natürlichen nächsten Schritt auf einem Pfad dar, auf dem sie sich schon seit ihrer Schulzeit befanden. Ich argumentiere, dass die jungen Menschen auf diese Art und Weise ihre eigene Biographie mit dem Ort Göttingen verbinden konnten und damit artikulierten, wer sie in Göttingen waren und sein wollten: junge (angehende) Wissenschaftler*innen auf der Suche nach Selbsterfüllung.

In Deutschland werden in den meisten Fächern für die Promotion etwa drei Jahre vorgesehen. Viele meiner Gesprächspartner*innen begründeten ihre Entscheidung für Deutschland mit dieser im Vergleich zu den USA und Indien geringen Dauer der Promotion. Im zweiten Teil des Kapitels zeige ich, dass die Wahl Deutschlands 
eine Strategie der Beschleunigung ist. Sie ist ein Versuch, eine konkrete Koordinate im akademischen Lebensverlauf, den Promotionsabschluss, schneller zu erreichen. Diese Strategie des future tricking (Ringel 2016: 24) entwickelten die jungen Wissenschaftler*innen in ihrer biographischen Navigation in Interaktion mit dominanten linearen Zeitkarten.

Das Studium bzw. die Arbeit an Instituten in Göttingen bedeutete für meine Gesprächspartner*innen, mit neuen Zeitlandschaften und Zeitkarten in Berührung zu kommen. Im Alltag, und dies ist der zweite Fokus im Kapitelabschnitt 4.2, trafen sie auf die Zeitkarte der Work-Life-Balance. Die Vorstellung von der Work-LifeBalance ist eine Repräsentation von Zeit, die für die jungen Wissenschaftler*innen nicht nur weniger Zeitdruck bedeutete, sondern mehr Kontrolle über die Allokation ihrer Zeit.

Im dritten Kapitelabschnitt liegt das Augenmerk auf den sozialen Beziehungen und Positionierungen der jungen Menschen an ihren Instituten. Ich zeige, dass die Positionierung als (angehende) Wissenschaftler*innen immer mit weiteren Verortungen interagierte und je nach Situation unterschiedlich relevant gemacht wurde. Durch meine Ausführungen in diesem Kapitel wird die Wissenschaft als eine soziale Welt mit einer eigenen Sprache, Regeln für den Umgang miteinander (Pickering 1992: 2-3) und eigenen Zeitkarten greifbar.

\subsection{Alle Wege führen nach Göttingen?}

Im Folgenden stehen die Narrationen meiner Gesprächspartner*innen im Fokus, in denen sie die Entscheidung für ein Studium bzw. eine Promotion in Göttingen und ihren bisherigen Lebensverlauf thematisierten. Für die Analyse der biographischen Navigation sind die Narrationen auf zwei Ebenen relevant. Erstens gaben sie mir Aufschluss über wichtige Eckdaten meiner Gesprächspartner*innen und Motivationen für die internationale Mobilität. Zweitens verstehe ich diese Narrationen selbst als Praxis der biographischen Navigation, als Aushandlung der Frage danach, wer die jungen Menschen zum Zeitpunkt meiner Forschung waren und sein wollten.

\subsubsection{Narrationen vom wissenschaftlichen Werdegang}

In den Interviews stellten meine Gesprächspartner*innen ihren bisherigen Lebensverlauf dar. In der Darstellung wählten sie unterschiedliche Strategien, um die Vergangenheit mit der Gegenwart und der Zukunft zu verbinden.

\section{Der Weg nach Göttingen als lineare Entwicklung}

Der Großteil der jungen Wissenschaftler*innen orientierte sich in der Darstellung ihres Werdegangs an einem Skript, das in ihrem professionellen Umfeld, der Wissenschaft, etabliert ist, und zählten die Abschlüsse auf, die sie absolviert hatten. 
A: Maybe we can just start with you telling me a little bit about your background.

R: It's confusing actually. So my parents are from the eastern part of India. But because of my fathers' job we were moving all across India all the time, and I have changed, like, eleven schools in my entire fourteen years of schooling. So we have been moving a lot, but currently they are settled in Delhi. And bachelors - I did it in southern part of India. My bachelors was in engineering and I was studying biotechnology. Then I decided to quit engineering and go to pure science, and then I started studying biology and specialized in neurobiology and structural biology. So that's how I started. And I came for my PhD here in 2012, December. (Rohini, 18.08.2016)

Rohini stellte durch die Erzählung ihres Werdegangs wie auf einem Zeitstrahl ein kohärentes Bild von sich selbst dar. In ihrer Narration gab es keine Lücken oder Unsicherheiten, sondern nur eine kontinuierliche Entwicklung bis zum Zeitpunkt des Interviews. Dadurch verwob sie ihre Vergangenheit mit ihrer Gegenwart zu einer stimmigen Einheit (vgl. Bamberg 2012: 104). Den Beginn ihres Promotionsstudiums in Göttingen konstruierte Rohini nicht als Wendepunkt, sondern als logischen nächsten Schritt in ihrem Lebensverlauf. Damit vermittelte sie mir als ihrem Gegenüber und sich selbst das Gefühl, ihr Leben und ihr Selbst habe sich ganz kontinuierlich entwickelt, obwohl sich vieles in ihrem Leben verändert hatte wie beispielsweise ihr Wohnsitz und ihr soziales Umfeld.

Einige meiner Gesprächspartner*innen orientierten sich in den Narrationen ihres Werdegangs nicht nur an ihren Abschlüssen, sondern auch an den Institutionen, an denen sie diese erworben hatten - beispielsweise Sahil, der Postdoktorand in Soziologie war und in seiner „academic journey“, wie er es nannte (Interview vom 01.09.2015), bereits an der Jawaharlal Nehru University in Delhi, den Universitäten in Oxford und Stanford und am Max-Planck-Institut in Göttingen Station gemacht hatte. All diese Namen sind mit Imaginationen verbunden, die vor allem mit dem Stand der Institutionen in der internationalen Wissenschaft assoziiert werden. Ein Abschluss von einer solchen Eliteinstitution dient als Marker, um sich von anderen Studierenden und Wissenschaftler*innen abzuheben. In dieser Funktion gehörten diese Orte auch zu Sahils Narration. Sie signalisierten die geographischen Ausmaße und die akademischen Dimensionen seiner Mobilität.

\section{Von A nach Z: Zielstrebige akademische Unternehmer*innen}

Ajit, zur Zeit unseres Gesprächs vierundzwanzig Jahre alt, wählte in unserem Interview einen anderen Weg als Rohini und Sahil, um Göttingen, den Ort, an dem er einen Master in developmental biology machte, als logische Wahl für die Erfüllung 
seiner Ziele dazustellen. Ajit erklärte mir, er habe schon sehr früh gewusst, was er einmal studieren wollen würde, und erzählte mir die folgende Geschichte:

I knew from the very beginning, since I was in school, that I want to study biology. I was in fifth, sixth standard, and we had free tickets for all the school kids for watching the Spiderman movie. I was sitting in the first row and there was a spider coming from the roof and biting Peter Parker, and then there was a DNA combining and I was like, „Oh that's super cool.“ I went to my teacher and asked him what exactly that is. He gave me some book and I just read about it, and then I thought okay now I know what I will study. (Ajit, $04.07 .2015)^{76}$

Zur Zeit unseres Interviews hatte Ajit bereits selbst mit Spinnen gearbeitet. „That was how I got interested in studying biology. I literally worked with spiders in Göttingen last week." Mit diesem Satz verknüpfte er die Vergangenheit direkt mit seiner Gegenwart.

An anderer Stelle erklärte er mir wie viele der jungen Wissenschaftler*innen, dass die Entscheidung für ein Studium abroad seine eigene gewesen sei, die er gegen den Widerstand seiner Eltern durchgesetzt habe. Er betonte außerdem, dass er der Einzige in seiner Familie sei, der Biologie studiert habe (beide Schwestern studierten Ingenieurwissenschaften), ein Fach, das sonst in Indien nicht so beliebt sei. Damit positionierte er sich als jemand, der nicht unbedingt die gesellschaftlichen und elterlichen Erwartungen erfüllt, sondern seine Ziele als Einzelkämpfer durchboxt. Ajit finanzierte sich sein Studium durch seine Arbeit als studentische Hilfskraft und war damit von seiner Familie finanziell unabhängig.

Ajits Positionierung als zielstrebiger Einzelkämpfer muss im Kontext der Strukturen verstanden werden, die ich in Kapitel 2 und 3 dargelegt habe und die die Verantwortung für Erfolg in die Hände des Individuums legen. Seit der Schulzeit wird vermittelt, dass der Erfolg in der Zukunft von der harten Arbeit des Einzelnen in der Gegenwart abhängig ist. Ein zweiter Faktor, der laut dominantem Diskurs für den Erfolg wichtig ist, ist das Herausstechen aus der Masse (Gilbertson 2017: 31). Ajits Abgrenzungen beispielsweise von seinen Schwestern, die wie alle anderen Ingenieurwissenschaften studierten, erfüllen diesen Zweck der Distinktion.

\section{Alte und neue Ziele}

Ajay, der in angewandter Physik promovierte, erklärte mir, dass er nicht wie alle anderen Inder*innen in die USA gehen wollte und sich für Deutschland entschied, „um etwas anders zu sein“ (Ajay, 14.12.2011). Seine Wahl fiel außerdem auf Deutsch-

\footnotetext{
${ }^{76}$ Der Film kam 2002 weltweit in die Kinos. Die Filmszene findet sich auch auf Youtube unter https://www.youtube.com/watch?v=zNNJwC_5RLI, abgerufen am 06.02.2017.
} 
land, weil er während seines Bachelorstudiums der Biomedizintechnik festgestellt habe, dass die meisten Geräte, mit denen er arbeitete, aus Deutschland kamen.

Eigentlich hegte Ajay seit seiner Schulzeit den Wunsch, Arzt zu werden. Wie viele junge Menschen mit diesem Wunsch scheiterte er an dem großen Andrang auf ein Medizinstudium in Indien. Er nahm an der Aufnahmeprüfung eines medical colleges teil, erzielte sehr gute Ergebnisse, erhielt aber keinen Platz. Ajay führte dies auf den großen Wettbewerb um die Plätze und den Umstand zurück, dass er zu einer „forward caste“ gehört und damit nicht unter die Quotenregelung fällt. Durch seinen Bruder wurde Ajay auf den Studiengang der Biomedizintechnik aufmerksam, der ihm ermöglichen würde, seine Interessen für Medizin und Chemie zusammenzubringen und damit zumindest teilweise seinen Wunsch zu erfüllen, oder wie er es ausdrückte: „partly fulfilling my thoughts“ (Ajay, 14.12.2011). Für seinen Masterabschluss wollte er ins Ausland gehen. Seine Eltern unterstützten ihn in seinem Plan und ermutigten ihn dazu, Deutschland zu wählen, da sein Onkel mit seiner Familie bereits in Deutschland lebte.

Ajays Weg nach Göttingen ist nicht nur ein Beispiel dafür, welche Rolle der Gedanke der Distinktion in der Entscheidung für Deutschland spielte, sondern auch dafür, dass das Leben der jungen Wissenschaftler*innen nicht immer so geradlinig verlief, wie es viele in ihren Narrationen darstellten. Wie in Ajays Fall beinhalteten die Routen nach Göttingen ebenso Sackgassen, die eine Neuorientierung verlangten.

Die vorgestellten Narrationen von Rohini, Sahil, Ajit und Ajay waren ein wichtiges Mittel in der diachronen Aushandlung des Selbst (vgl. Bamberg 2012: 103). In ihnen machten die jungen Menschen ihre Vergangenheit für ihre Gegenwart in Göttingen relevant und vermittelten mir als Interaktionspartnerin im Interview und sich selbst ihre Entwicklung durch die Zeit hinweg, in der nach Göttingen zu kommen der nächste logische Schritt war. Die lineare Logik, die den Darstellungen von Rohini und Sahil innewohnte, ist eine Konstruktion, die sich an normativen Vorstellungen davon orientiert, wie sich junge Wissenschaftler*innen entwickeln und die eigene Veränderung durch die Zeit präsentieren sollten (vgl. Dausien 2000). Die Linearität ist eine Strategie, um in den Narrationen Aspekte von Wandel und Kontinuität auszuhandeln.

\subsubsection{Göttingen, der Ort für die wissenschaftliche Selbstverwirklichung}

You know, modern physics started in Germany: Max Planck, Heisenberg, Einstein. All are from Germany. And Germany has this rich tradition of producing wonderful physicists.

And I'm a physicist, so it was quite natural to select Germany. (Pravin, 10.07.2011)

Die jungen indischen Studierenden und Wissenschaftler*innen begründeten ihre Wahl Deutschlands und Göttingens mit den wissenschaftlichen Möglichkeiten, die sie dort erhalten würden. Pravin betonte die Geschichte Deutschlands als Land der 
Physiker, andere hoben spezifische Arbeitsgruppen hervor, die sich genau mit den Themen beschäftigten, an denen sie selbst arbeiten wollten. Kundan erklärte beispielsweise: „I want to do only the work which I want to do. So I think there are only two labs ${ }^{77}$ in the world who are working [on this]" (Kundan, 14.08.2013).

Indien wurde in den Erklärungen häufig als Vergleich hinzugezogen, auch von Kundan, der betonte, dass es in Indien gute Wissenschaft gebe, aber: ,„till for research, basic research the government doesn't support as much as in the US." In ihren Narrationen positionierten sich meine Gesprächspartner*innen als Wissenschaftler*innen auf der Suche nach akademischer Selbsterfüllung. Sie wollten nicht an ,irgendeiner Universität an ,irgendeinem 'Thema arbeiten, sondern Wissenschaftler*innen in ganz spezifischen Feldern werden. In ihren Narrationen folgten sie einem etablierten Diskurs, nach dem Wissenschaft eine „Berufung“ (Richter 2016: 59) ist und von der eigenen Leidenschaft motiviert wird. Durch den Bezug darauf, was es heißt, Wissenschaftler*in zu sein, artikulierten meine Gesprächspartner*innen ihre eigene Zugehörigkeit zu dieser sozialen Welt (vgl. Richter 2016: 61).

Wie Savage, Bagnall und Longhurst (2005: 29) feststellen, können sich Menschen, die an einem neuen Ort wohnen, zu diesem zugehörig fühlen, wenn sie ihre eigene Biographie mit diesem Ort verbinden. Viele meiner Gesprächspartner*innen betonten, dass sie sich selbst aus verschiedenen Gründen dafür entschieden hatten, für ihr Studium oder ihre Promotion ins Ausland zu gehen. Obwohl die Wahl Göttingens nicht nur in ihren Händen lag und manchmal vielleicht mehr mit dem Zufall zu tun hatte als mit einem klaren Plan, konstruierten die meisten diese Entscheidung im Rückblick als richtig bzw. als richtiger als es andere gewesen wären. Diese Logik entstand auch durch die vorherrschenden Diskurse in dem spezifischen Regime der akademischen Mobilität, das ich in Kapitel 3 ausführlich analysiert habe. In diesem wird internationale Mobilität generell als notwendig für eine Karriere und Deutschland als guter Ort für Wissenschaftler*innen und als Land, das innovative, junge Menschen schätzt, dargestellt. Mit der Erzählung ihres akademischen Werdeganges erklärten mir meine Gesprächspartner*innen gleichermaßen, warum sie in Göttingen gelandet waren: weil sie hier ihre wissenschaftlichen Ziele erfüllen können. Göttingen wurde in den Geschichten der jungen Studierenden zu einer Stadt, in der vor allem gut wissenschaftlich gearbeitet werden kann. Die Narrationen waren deswegen auch eine Antwort auf die Frage: Warum bin ich hier (und nicht woanders)? Natürlich beantworteten sie diese Frage im Rückblick auf eine bereits getroffene Entscheidung. Diese Retrospektive beeinflusste wiederum die Bewertung der Entscheidung. Ähnlich wie in Robert Frosts bekanntem Gedicht The Road Not Taken (Frost 2010: 66) ${ }^{78}$ übergingen meine Gesprächspartner*innen im Rückblick auf den getroffenen Entschluss den Zweifel, den sie zunächst vielleicht hatten. Die

\footnotetext{
77 Abkürzung für „laboratory“ und ein Beispiel für die Sprache, die die soziale Welt der wissenschaftlichen Felder meiner Gesprächspartner*innen ausmachte.

${ }^{78}$ Dieses Gedicht wurde 1916 veröffentlicht.
} 
Entscheidung für die Route nach Göttingen war die richtige, führte sie doch dahin, wo die jungen Menschen in der Gegenwart waren.

In den Ausführungen dieses Kapitelabschnitts habe ich gezeigt, dass viele der jungen Wissenschaftler*innen Deutschland und speziell Göttingen als den ,richtigen ‘ Ort für ihre wissenschaftliche Arbeit verstanden. Im Sinne Zygmunt Baumans (2011) verstanden sie Göttingen als den richtigen Hafen, um zumindest zeitweise vor Anker zu gehen. Diese Auffassung wurde wiederum davon beeinflusst, was Bauman (2011: 434) die „Schiffsladung“ nennt. Im Fall meiner Gesprächspartner*innen wurde die Ladung zunächst vor allem durch ihren Fokus auf ihre wissenschaftliche Ausbildung und das Erreichen akademischer Ziele bestimmt. So wie sich diese Ladung ändern kann, kann sich dann auch wieder die Einstellung zum Hafen Göttingen ändern.

In den Erzählungen meiner Interviewpartner*innen kamen immer wieder die gleichen Institutionen vor: die Schule, das College und die Universität. Diese Darstellung eines linearen Verlaufs durch diese Institutionen, die der schriftlichen Konvention für einen Lebenslauf entsprechen, zeigen die Stationen auf, die den akademischen Lebensverlauf bis zum Promotionsabschluss ausmachen. Wie ich bereits in Kapitel 3 angerissen habe, gibt es in der Wissenschaft normative Vorstellungen davon, wie sich ein Mensch von einem Studierenden zu einem ,ausgewachsenen' Wissenschaftler, dem Professor bzw. der Professorin, entwickelt. Diese Konzeption eines akademischen Lebensverlaufs (Jacobs und Winslow 2004) gibt eine wichtige Orientierung in der Planung der beruflichen Laufbahn, allerdings ist diese Vorstellung von akademischem Lebensverlauf genauso ein Kontroll- und Vergleichsinstrument und als solches ruft sie zeitliche Erfahrungen wie Zeitdruck hervor. In Abschnitt 4.2 zeige ich, wie die jungen Wissenschaftler*innen die zeitlichen Normen des akademischen Lebensverlaufes navigierten und versuchten, durch die Entscheidung für Deutschland einen Vorsprung in ihrer Karriere zu erreichen.

\subsection{Göttingen: „A launchpad for my career“}

In Deutschland zu promovieren bedeutete für meine Gesprächspartner*innen ,schneller' sein zu können, weniger Zeit für die Promotion aufwenden zu müssen. Diese Idee des Schnellerseins und der möglichen Beeinflussung der Dauer der Promotion durch die Wahl des richtigen Ortes setze ich im Folgenden in den Kontext linearer Vorstellungen vom Verlauf des Lebens und von Karrieren.

Wie Abbildung 2 illustriert, gibt es die Vorstellung, dass der Weg zum Doktortitel geradlinig, hindernislos und linear ist. Dass die Realität anders aussieht, steht an dieser Stelle nicht im Fokus meiner Ausführungen. Die Vorstellung eines linearen Pfades zum Promotionsabschluss schafft gemeinsam mit anderen Elementen der Zeitlandschaft der Wissenschaft zeitliche Erfahrungen und Rhythmen. Zum Beispiel legen dreijährige Promotionsprogramme oder Promotionsstellen klare Normen zur Dauer der Promotion fest und schaffen damit eine Orientierung für die jungen Wissenschaftler*innen in Form relativ klarer Anfangs- und Endpunkte. Damit gehen 

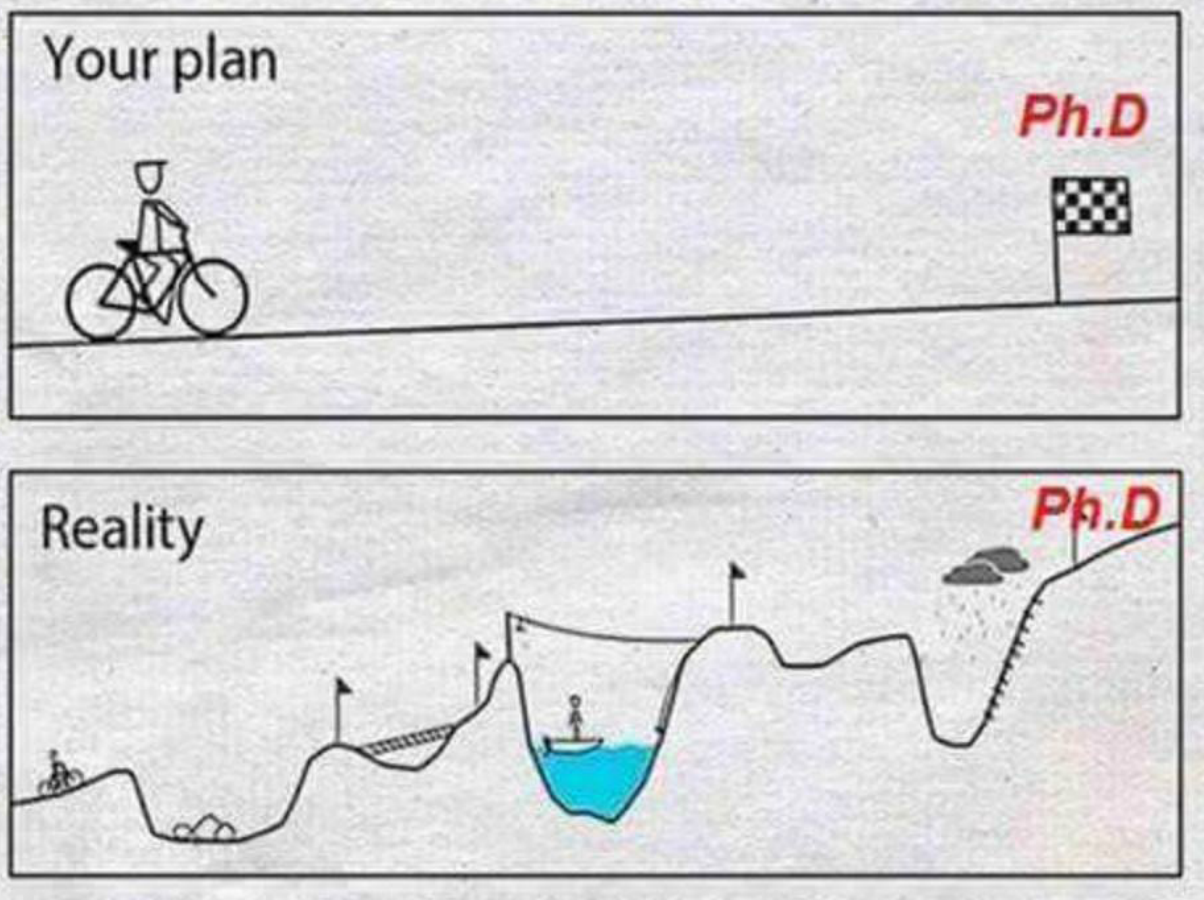

Abb. 2 „PhD plan and reality“ von Sarjana auf Facebook geteilt, Mai 2015

zeitliche Erfahrungen einher wie Zeitdruck und die Möglichkeit, sich mit anderen Menschen, die sich auf dem gleichen Pfad befinden, zu vergleichen.

In Form einer Zeitkarte vermittelt die Linearität des akademischen Lebensverlaufs eine spezifische Gestaltung von Zeit, insbesondere in Bezug zur Allokation von Zeit während dieser Lebensphase. In Analogie zu einem Rennen suggeriert ein linearer Kurs, dass der Einzelne versuchen kann, das Ziel schneller zu erreichen, schneller als vorgesehen und schneller als die anderen, die sich im Rennen befinden. Die Entscheidung meiner Gesprächspartner*innen für Deutschland verstehe ich als eine solche Strategie der Beschleunigung und als Versuch, die Zukunft, von der sie bereits ein klares Bild hatten, schneller zu erreichen.

Die Dynamik zwischen dominanten Zeitkarten, Erfahrungen von Zeit und Strategien der Zeitgestaltung lege ich im folgenden Kapitelabschnitt anhand dreier zeitlicher Horizonte dar: dem Horizont des Lebensverlaufs, dem des Endes der Zeit in Göttingen bzw. der Promotion und dem des Alltags. 


\subsubsection{Promotion auf der Überholspur}

Our reason for coming here in the first place was very simple: that we wanted to do our PhDs, and that you can do it in three years here. Whereas if you go to somewhere in North America it's five years, and since we already finished our Masters it didn't make sense to spend five years doing it. (Sadhvi, 22.09.2011)

Die Entscheidung für eine Promotion in Deutschland begründeten viele meiner Interviewpartner*innen wie Sadhvi und ihr Ehemann mit der Dauer des PhD-Programmes. Für Sangita bedeutete die Wahl zwischen den USA und Deutschland, über die Dauer ihrer Promotion und darüber, wie lange sie dafür an einem Ort leben würde, bestimmen zu können: „I wanted to do neuroscience and I wanted to do it in Europe. I did not want to go to the US because a PhD takes long there, and I wasn't sure if I would like to be at a place for at least six years" (Sangita, 08.08.2013). Tanvir zog in seinem Vergleich mit dem deutschen System die Dauer von Promotionen in Indien heran, die seinen Angaben nach zwischen acht und neun Jahren liege: „See, you are stuck in your life for eight years, and you don't even know if you are going to get a PhD after eight years“ (Tanvir, 08.06.2011). Meine Gesprächspartner*innen wollten nicht in ihrem Leben ,feststecken', sei es in Indien oder den USA, sondern möglichst schnell vorankommen.

Auch Rohini betonte, dass Zeit ein wichtiger Faktor in ihrer Entscheidung für Deutschland gewesen sei: „The duration of your $\mathrm{PhD}$ here is quite shorter. So let's say [after] three years or four years you are done with it. (...) For me, when I started applying I was 22, and for me it was ,okay you know I really want to finish it as fast as possible“" (Rohini, 18.08.2016).

Kundan verstand seine Promotion in Deutschland als Chance, die drei Jahre, die er in Indien damit verbracht hatte, Geld für die Hochzeit seiner Schwester zu verdienen, aufzuholen: „If I go to US it will be six more years minimum to finish [my] PhD. (...) Then I realized okay, maybe I'll go to Europe. I'll finish in three years so that I can save the time which I spend there" (Kundan, 14.08.2013).

An all diesen Aussagen wirkte auf mich am stärksten die Betonung der Geschwindigkeit oder, wie Amanda Gilbertson in ihrer Studie schreibt, die „language of fast-ness" (Gilbertson 2017: 31). Die Sprache des Schnell-Seins artikuliert die Idee eines Rennens, in dem es wichtig ist, schnell und schneller zu sein. Durch die Wahl Deutschlands konnten die jungen Wissenschaftler*innen aktiv auf die Dauer ihres Promotionsstudiums einwirken. Sie konnten versuchen, schneller als die Konkurrenz zu sein und schneller den Zeitpunkt zu erreichen, den viele als einen Wendepunkt in ihrem professionellen und persönlichen Leben konstruierten: den PhD-Abschluss. Eine wichtige Rolle spielte in dieser Dynamik, dass viele meiner Gesprächspartner*innen die nächste Koordinate, die nach ihrem Abschluss folgen sollte, bereits im Blick hatten: eine Heirat. 
Im Vergleich mit ihren Kommiliton*innen stellten einige der indischen Studierenden und Wissenschaftler*innen fest, dass das Leben in Deutschland scheinbar in einem anderen Tempo ablaufe. Rohini bemerkte beispielsweise: „I think you guys till your bachelors are more relaxed than we are. It's a great shock for us when we come at the beginning - because as you said, you are twenty-five and you are starting your $\mathrm{PhD}$ “ (Rohini, 18.08.2016). Rohini verwies an dieser Stelle auf meine Aussage, in der ich die Altersunterschiede zwischen indischen und deutschen Promovierenden ansprach. Viele meiner Gesprächspartner*innen waren zu Beginn ihrer Promotion zwischen dreiundzwanzig und fünfundzwanzig Jahren alt, was im Schnitt etwas jünger war als ihre Kolleg*innen aus Deutschland (vgl. DAAD und DZHW 2016: 126). Rohini erklärte diese Diskrepanz mit dem starken Wettbewerb in Indien und dem Umstand, dass in Indien kaum Jemand ein „gap-year" mache, wie es mittlerweile in Deutschland üblich sei. Die Altersunterschiede und, wie ich später zeigen werde, der Arbeitsalltag vermittelten ihnen das Gefühl, dass das Leben in Deutschland in einem anderen Tempo ablaufen würde als in Indien.

\subsubsection{Die Zeit in Göttingen als Zeit zum Arbeiten}

Der Fokus auf das Abschließen der Promotion, das Erreichen dieser Koordinate, hatte einen großen Einfluss auf die Positionierungen und den Alltag der jungen Inder*innen in Göttingen. In den Erzählungen meiner Gesprächspartner*innen wurde deutlich, dass sie sehr viel Zeit an ihrem Arbeitsplatz bzw. mit ihren Forschungsprojekten verbrachten. Dieser Umstand an sich ist nicht verwunderlich. So wenden wohl die meisten Erwachsenen einen großen Teil ihrer Wachzeit für die Arbeit auf, besonders, wenn sie sich in einer Phase wie die der Promotion befinden. Im Fokus meiner weiteren Ausführungen steht, wie meine Gesprächspartner*innen von ihrer Arbeitszeit sprachen, nicht nur auf der alltäglichen Basis, sondern auch bezogen auf ihre Zeit in Göttingen.

Sachin erklärte seinen und Sadhvis Fokus auf die Arbeit mit dem Gefühl, dass sie nach Göttingen gekommen seien, um etwas zu erreichen: „In the weekdays we just go to work, come home, eat, sleep, go to work, come home, eat, sleep. It was really like this, because we also come with the feeling that we are here for four years. We are here to do something, to achieve [something]" (Sachin, 22.09.2011).

Ravi betonte, dass seine Prioritäten ganz klar bei seiner Karriere lagen, als er sich für eine Promotion in Göttingen entschieden hat: „I had to find a job, I had to find a place to settle. So my first thing is to find a place where I can get some money and some career going - get a launch pad for my career" (Ravi, 15.09.2011). In Göttingen eine „Startrampe“ für die Karriere zu sehen, beeinflusste den Arbeitsalltag. Kundan erzählte beispielsweise, dass er etwa zehn Stunden am Tag arbeite - oft auch an den Wochenenden und in der Nacht. Er erklärte mir, dass er dies bereits während seines Masterstudiums in Indien gemacht habe und fügte hinzu: „It's not good. I wanted to stop but it's really difficult". Die Zeit in der Nacht nutze er vor allem für die Arbeit an einem Mikroskop, das den Mitarbeiter*innen des Instituts eigentlich 
nur je sechs Stunden in der Woche zur Verfügung stand. Durch das Arbeiten in der Nacht und am Wochenende umging er diese Regelung. Kundan hatte es sich zum Ziel gesetzt, schnell mit seiner Promotion fertig zu werden, um bessere Chancen im Wettbewerb um Arbeitsplätze zu haben: „I want to finish sooner then only you have the something extra. (...) But it's not pressure right now. Somehow I wanted to finish soon. It's not the family pressure anymore. (...) Here that was fine because you don't have the responsibility too much - you can choose" (Kundan, 14.08.2013). Allerdings betonte er, dass dies seine eigene Wahl sei. Damit drückte er ein Gefühl der Kontrolle über seine Zeit und die Aktivitäten, mit der er diese füllte, aus.

Das Studium bzw. die Arbeit in Göttingen wurde von meinen Gesprächspartner*innen als wichtige Basis für die berufliche und persönliche Zukunft wahrgenommen. Deswegen entschieden sich viele dazu, viel Zeit für ihre Arbeit aufzuwenden. Persönliche Beziehungen stellten viele zunächst hinten an. Diese Priorisierung der Promotion und was dies für die sozialen Beziehungen bedeutete, kommentierte Rehan im April 2014 mit Hilfe einer Abbildung auf Facebook, die ich im Folgenden kurz beschreibe. Mit Verweis auf Facebooks Kategorie „Beziehungsstatus“ fanden sich unter der Überschrift „Current relationship status“ folgende Antwortmöglichkeiten: „single“, „married“, „it's complicated“, „in a relationship“ und „in Phd“. In der Abbildung war letztere Angabe markiert.

Diese Art der Zeitgestaltung, der Zuteilung von Zeit für die Arbeit und die Fokussierung auf den Wert der Gegenwart für die Zukunft, führe ich auf den Einfluss der dominanten Zeitkarten zurück, die ich Kapitel 2 und 3 thematisiert habe. In den Vorstellungen des Lebensverlaufs, die in der indischen Mittelschicht vorherrschen, wird die Jugend als Phase verstanden, in der wichtige Grundlagen für die Zukunft gelegt werden müssen. Ähnlich verhält es sich mit Konstruktionen des akademischen Lebensverlaufs: Die Promotion ist die Zeit, sich eine professionelle Zukunft aufzubauen. Diese Logik ist mit dem Versprechen verknüpft, dass diese Phase irgendwann vorbei sein und sich die Arbeit in Form eines guten Arbeitsplatzes, mehr Freizeit und - im Fall der jungen Inder*innen - einer besseren Position auf dem Heiratsmarkt auszahlen wird.

Sarjana beschrieb diese Einstellung zum Leben und zur Gegenwart in Göttingen mit dem folgenden Satz, den sie auf Facebook teilte: „We worry about tomorrow like it's promised!“. Dazu kommentierte sie: „Que sera, sera!“, also „Es kommt, wie es kommt“ oder anders ausgedrückt: „Denk nicht so viel über die Zukunft nach“. Die Beziehung zwischen dem Versprechen eines guten Lebens in der Zukunft und den Entbehrungen, die dafür in der Gegenwart in Kauf genommen werden, lässt sich mit dem Konzept des „cruel optimism“ von Lauren Berlant (2011) fassen. ${ }^{79}$ Ein Verhältnis von cruel optimism existiert, wenn „something you desire is actually an obstacle to your flourishing" (Berlant 2011: 1). Wie Marie-Alix Thouaille (2018) beschreibt, wirkt im akademischen Bereich die Fantasie des „academic ,good life““ als „enabling

\footnotetext{
79 "Cruel optimism“ wurde bereits von anderen Autor*innen benutzt, um die Situation von Akademiker*innen zu beschreiben, u.a. von Briony Lipton (2017) und Nina Lykke (2017).
} 
object which is also disabling" (Berlant 2011: 25). Diese Fantasie beinhaltet eine unbefristete Stelle und die Möglichkeit, die eigenen wissenschaftlichen Interessen ohne Einschränkungen (zeitlich oder finanziell) verfolgen zu können. Der ,grausame Optimismus' wird dadurch angetrieben, dass nur eine Karriere in der Wissenschaft als Erfolg gesehen wird (vgl. Thouaille 2018; Richter 2016) und immer wieder die Idee betont wird, dass Akademiker*innen von der Passion für die Wissenschaft und nicht durch finanzielle Honorierung angetrieben werden (vgl. Thouaille 2018). Diese beiden Aspekte finden sich ebenso in den Narrationen meiner Gesprächspartner*innen, in denen die Betonung immer wieder auf die akademische Selbsterfüllung gelegt wurde und beispielsweise nicht bessere Gehaltsaussichten als Grund für die Mobilität angegeben wurde.

Die lineare Vorstellung vom akademischen Lebensverlauf, die recht feste Koordinaten beinhaltet, trägt dazu bei, den ,grausamen Optimismus' aufrechtzuerhalten, da immer wieder die Hoffnung geschürt wird, dass das Erreichen des nächsten akademischen Abschlusses zur Erfüllung der Fantasie vom akademischen good life führt. In Kapitel 8 gehe ich auf diese Dynamik genauer ein.

\subsubsection{Work-Life-Balance: Entschleunigung in der Beschleunigung}

Im Arbeitsalltag an den Instituten realisierten viele meiner Gesprächspartner*innen, dass der Umgang mit der Arbeitszeit ein anderer war, als sie es aus Indien oder den USA gewohnt waren. Dieser Umgang wurde häufig mit den Worten Work-Life-Balance beschrieben. Ich argumentiere im Folgenden, dass die Idee der Work-Life-Balance eine spezifische Zeitkarte darstellt, die den Umgang mit der Arbeitszeit festlegt. Für die jungen Wissenschaftler*innen aus Indien vermittelte sie neue Möglichkeiten der Zeitgestaltung, andere zeitliche Erfahrung und größere Zeitsouveränität. Als Master- bzw. Promotionsstudierende oder Postdoktoranden nahmen sie in der universitären Hierarchie eine relativ untergeordnete Stellung ein. Diese Positionierung hatte aber in Deutschland nicht den gleichen Effekt wie in Indien, wo, wie viele berichteten, die Betreuer*innen die alleinige Kontrolle über die Arbeitszeit besitzen würden.

Sachin bezeichnete die „very nice balance between work and life" als Kennzeichen für das Leben in Göttingen. Er und seine Frau Sadhvi hatten zum Zeitpunkt unseres Interviews gerade ihre Promotion abgeschlossen und würden bald für Anstellungen als Postdoktoranden in die USA gehen. In unserem Gespräch erklärte Sadhvi, dass selbst das Arbeiten in Deutschland „more relaxed“ sei als in den USA.

For example, my boss here, my $\mathrm{PhD}$ supervisor, if I would tell him that I went - I mean, PhD students work all the time, right? It doesn't matter if it's weekend or not. But if I would tell him „I went on Sunday and did an experiment, " he will almost be like laughing at me like, „Why don't you have a life?“, „Do something else“, you know? And this [is something] you never get anywhere else in the world; it's really amazing to have this. (...) And the thing 
is, it's not like Germans are any less productive than any other people, right? So it's like why can't the rest of the world live like this? It just didn't make sense to me. (Sadhvi, 22.09.2011)

Obwohl sie die Work-Life-Balance in Deutschland schätzte, wird in ihrer Aussage erkennbar, dass sie diese Möglichkeit für mehr Freizeit selbst nicht unbedingt nutzte.

Nandita war bereits als Doktorandin in Deutschland gewesen und hatte sich entschieden, für eine Stelle als Postdoktorandin erneut nach Deutschland zu kommen. Diese Entscheidung erklärte sie unter anderem mit ihrem Wunsch, neben der Arbeit auch ein „Leben“ haben zu wollen. Sie würde besonders die Arbeitskultur an den Max-Planck-Instituten schätzen und erzählte von Bekannten, die in den USA Anstellungen als Postdoktoranden hätten: „If I compare my experience with them, then I find out that it's much more calm here to work. There they have a lot of pressure, and the boss forces them to come in weekends also" (Nandita, 03.09.2011). An der Formulierung „der Chef zwingt sie“ wird erkennbar, dass Nandita davon ausging, dass ihre Bekannten in den USA gar keine Wahl hätten: Sie müssten die ganze Zeit arbeiten und hätten keine Kontrolle über die Einteilung ihrer Zeit. Ähnlich wie Nandita berichtete Ajit, der sich an ein Gespräch mit einem Doktoranden aus Indien erinnerte:

And he told me they have to work everywhere from Monday to Sunday - every day - eight hours in India in the lab. And here he had from Monday to Friday. He was really happy to do that, at least, last part of his life as a $\mathrm{PhD}$ student. In India you have to work every day in a lab. That's hard and you have no personal life, and I don't want to live like this - I need [the] weekend free. (Ajit, 14.04.2015)

Obwohl die jungen Menschen, die im Fokus meiner Studie stehen, einen starken Fokus auf ihre Arbeit legten, machten sie die Erfahrung, im Alltag weniger unter Zeitdruck zu stehen und mehr Kontrolle über ihre Zeit zu haben. Aus diesem Grund beschrieb Anay die Zeit, die er in Göttingen verbrachte, als die „leisure period“ seines Lebens: „So whenever I wake up, I wake up and I go to the center study till 6, $7,8,9,10$ - whatever. Or five come back, or eat and come back to the room, or go back to the center and then study till one or two in the night." Mit „Freizeit-Periode“ meinte er nicht, dass er weniger arbeiten würde, sondern betonte noch, dass er in Göttingen sehr produktiv sei: „It really makes you work“ (Anay, 15.07.2015). Seine Produktivität führte er nicht nur auf das entspannte Arbeitsumfeld zurück, sondern auch auf die fehlenden sozialen Beziehungen.

Pravin, Postdoktorand an einem Max-Planck-Institut, beschrieb die Erfahrung größerer Zeitsouveränität mit dem Gefühl, dass die Arbeit keine „Belastung“ (burden) sei. „Technically I'm maybe working but I'm enjoying it, there is no burden“ (Pravin, 10.07.2011). Er bezog diese Erfahrung auf die „workculture“ und die gute finanzielle Ausstattung der Max-Planck-Institute. 


\section{Frei von familiären Verpflichtungen}

Für Amala bedeutete der Aufenthalt in Göttingen sich der Notwendigkeit, eine Balance zwischen Arbeit und Familie herstellen zu müssen, entziehen zu können. Amala war zum Zeitpunkt unseres Interviews vierunddreißig Jahre alt und eine der wenigen Promovierenden, die bereits verheiratet war. Sie kam im August 2012 nach Göttingen, um Material für ihre Dissertation zu sammeln. Mit der Unterstützung eines Erasmus-Mundus-Stipendiums blieb sie etwas mehr als ein Jahr in Göttingen. Als ich sie fragte, ob ihr Leben in Deutschland anders sei als in Indien, erklärte sie mir, dass es für sie einen großen Unterschied mache. In Mumbai habe sie eine Stelle gehabt und gleichzeitig an ihrem PhD-Abschluss gearbeitet und außerdem „family responsibilities" gehabt: „every responsibility related to a house and then you have relatives (...). "Dementsprechend sei es in Indien für sie sehr hektisch gewesen und sehr schwer, Zeit und Ruhe für ihre Forschung zu finden (,to find time and peace of mind“). „It was really difficult in India, not only because of family responsibilities, but also I lived in a big city where I used to travel almost twenty kilometer every day and drive a motorcar" (Amala, 11.07.2013). In Indien folgte Amala den zeitlichen Normen, die von ihr als verheirateter Frau verlangten, sich neben der Arbeit außerdem um die Familie und den Haushalt zu kümmern. In Göttingen hatte Amala keine familiären und häuslichen Verpflichtungen. Das hieß auch, dass sie sich ihre Zeit selbst einteilen konnte und nicht versuchen musste, eine Balance zwischen Arbeits- und Familienleben zu finden. Eine solche Balance zu schaffen, liegt, wie ich in Kapitel 2 gezeigt habe, in der Verantwortung der Frau und ist ein wichtiger Aspekt in der Aushandlung davon, was eine gute Frau in der indischen Mittelschicht und damit eine gute Familie ausmacht. Amala hatte in Göttingen mehr Kontrolle über die Einteilung ihrer Zeit und weniger soziale Einbindungen, die diese Kontrolle hätten einschränken können.

In den Aussagen von Sadhvi, Nandita, Ajit, Anay, Pravin und Amala wird deutlich, dass sie das Gefühl hatten, in Göttingen mehr Zeit zu ihrer freien Verfügung zu haben und in der Gestaltung ihrer Zeit weniger fremdbestimmt zu sein. Diese Situation führe ich auf zwei mit der Mobilität meiner Gesprächspartner*innen verbundene Faktoren zurück. Erstens führte die Mobilität dazu, dass sie sich in einer neuen sozialen Welt befanden, in der andere kollektive und individuelle Zeitkarten gültig waren. Die Zeitkarte der Work-Life-Balance sieht dezidiert Freizeit vor und übt dadurch weniger Druck aus. Zweitens hatte die Positionierung meiner Gesprächspartner*innen als ,Junior-Wissenschaftler*innen' an ihren Instituten in Deutschland eine andere Wirkung auf die Kontrolle über ihre Zeit als in Indien. In Deutschland bedeutete dies nicht, dass ihre Betreuer*innen über ihre Arbeitszeit bestimmten zumindest nicht in dem Maße, in denen sie dies in Indien erlebt hatten bzw. aus Berichten von Freund*innen aus Indien kannten.

Die große Flexibilität der Arbeitszeit, die von den jungen Wissenschaftler*innen hervorgehoben wurde, hatte allerdings eine Kehrseite: Menschen mit flexibleren Arbeitszeitregelungen wie z.B. der Vertrauensarbeitszeit, die an der Universität Göt- 
tingen für das wissenschaftliche Personal gilt, arbeiten eher mehr als jene mit festen Arbeitsstunden ${ }^{80}$ und müssen sich selbst strukturieren und Freizeiten schaffen (vgl. Garhammer 1995). Gerade in Kombination mit dem Diskurs von den von ihrer Leidenschaft angetriebenen Wissenschaftler*innen führte die Flexibilität dazu, dass meine Gesprächspartner*innen wie viele angehende Wissenschaftler*innen viel Zeit in ihre Arbeit investierten. ${ }^{81}$

Wie Saulo Cwerner (2001) in seinem Artikel The Times of Migration zeigt, hat Mobilität einen großen Einfluss darauf, wie Menschen Zeit erfahren und wie viel Macht sie über die Erfahrung und die Gestaltung von Zeit haben. In seiner Arbeit beschäftigte er sich vor allem damit, dass Menschen diese Macht durch Migration verlieren. ${ }^{82}$ In meiner Studie zeige ich, dass Menschen durch Mobilität größere Zeitsouveränität erlangen können. Obwohl sich meine Gesprächspartner*innen durch das Mobilitätsregime, in dem sie navigierten, auch (neuen) zeitlichen Strukturen ausgeliefert sahen wie zum Beispiel der zeitlichen Begrenztheit ihres Aufenthalts, erreichten sie durch ihre Mobilität in spezifischen Lebensbereichen größere Kontrolle über ihre Zeit. Im Vergleich zu anderen mobilen Menschen gehörten sie damit wiederum zu einer kleinen privilegierten Schicht. Die meisten mobilen Menschen werden in ihrer Zeitsouveränität entschieden beschnitten. Am eindrücklichsten wird dies im Fall von Geflüchteten, die nicht nur in ihrer räumlichen Bewegung eingeschränkt werden, sondern gleichermaßen zeitlicher Fremdbestimmtheit unterliegen (Cwerner 2001: 19-21; vgl. Andersson 2014; Ramsay 2017).

\subsection{Indische Wissenschaftler*innen in der „Stadt, die Wissen schafft"}

Die jungen Wissenschaftler*innen studierten bzw. arbeiteten in Göttingen an naturwissenschaftlichen und mathematischen Instituten an der sogenannten Nord-Uni, an Max-Planck-Instituten am Faßberg und einige an Instituten oder Zentren der sozialwissenschaftlichen oder philosophischen Fächer am Zentralcampus (siehe Karte S.59). An diesen Orten interagierten sie mit Kommiliton*innen in ihren Masteroder Promotionsprogrammen, mit Lehrpersonen, Betreuer*innen und Kolleg*innen in den Forschungsgruppen. Im Fokus dieses Kapitelabschnittes stehen die sozialen

\footnotetext{
${ }^{80}$ Siehe Yvonne Lott: „Weniger Arbeit, Mehr Freizeit? Wofür Mütter und Väter flexible Arbeitsarrangements nutzen“, WSI Report vom März 2019, https://www.boeckler.de/pdf/p_wsi_report_47_2019. pdf.

${ }^{81}$ Siehe Gewerkschaft Erziehung und Wissenschaft: „Zwölf Überstunden die Woche: WiMis arbeiten \#unbezahlt" vom 07.02.2019, https://www.gew.de/aktuelles/detailseite/neuigkeiten/zwoelf-ueberstun den-die-woche-wimis-arbeiten-unbezahlt/. Siehe auch Forschung und Lehre: „Knapp zwölf Überstunden pro Woche sind üblich“"vom 28.01.2019, https://www.forschung-und-lehre.de/karriere/kna pp-zwoelf-ueberstunden-pro-woche-sind-ueblich-1455/.

${ }_{82}$ Cwerners Fokus enstand auch durch seine Forschung zu Brasilianer*innen in London. Viele dieser Menschen haben einen rechtlich sehr unsicheren Status.
} 
Beziehungen im Kontext der Studien- und Arbeitsorte. Der Blick auf die konkreten sozialen Beziehungen an den Instituten zeigt, dass die Positionierungen an den Instituten in den meisten Fällen über jene Verortung als bloße ,schlaue Köpfe ' hinausgingen. Allerdings hatten dominante Repräsentationen von Indien, die beispielsweise in den Medien verbreitet wurden - Bilder von Indien als technologische Supermacht oder eines Landes, das von Armut und Ungleichheit getrieben wird - einen Einfluss auf die sozialen Interaktionen und Positionierungen.

Wissenschaftlerin zu sein und dem vorgesehenen Karrierepfad folgen zu wollen, stellte die Frauen im Vergleich zu den Männern vor besondere Herausforderungen. Im letzten Abschnitt dieses Kapitels widme ich mich deswegen dem Einfluss, den das Frausein auf die Verortung meiner Gesprächspartnerinnen als (angehende) Wissenschaftlerinnen hatte.

\subsubsection{Doktoreltern, Vorgesetzte und Kolleg*innen}

Viele meiner Interviewpartner*innen hoben die positiven Beziehungen zu ihren Betreuer*innen und Vorgesetzten hervor, die, so wurde häufig betont, wesentlich weniger hierarchisch seien als in Indien. ${ }^{83}$ Gilon beispielsweise erzählte: „Professor F.'s institute is very beautiful because there is no boss and workers relationship. He is really a, I like this word, doctoral father - Doktorvater - and it's really a kind of fatherly relation" (Gilon, 19.01.2013). Gilon beschrieb die Beziehung zu seinem Promotionsbetreuer als sehr familiär und die Kontakte zu den Kolleg*innen als freundschaftlich. Ähnlich berichtete Smita von ihrem Arbeitsplatz. Sie erzählte, dass die Atmosphäre in ihrem Labor gleich von Beginn an sehr nett gewesen sei: „I never felt like that I'm out of place over here. I could easily fit in with the people and everybody and so. I was even invited a couple of times to my boss's apartment, and his wife is equally good." Sie sei nie auf Probleme gestoßen und hätte deswegen auch nicht das Gefühl gehabt, an einem neuen Ort zu sein: „So they made me very [at] home. (...) Means maybe for a month I was in cultural shock, but after that I didn't take too much time to settle in (...), and so major credit goes to my lab" (Smita, 26.01.2013).

Die Vorgesetzten, die Doktorväter und -mütter, sowie die Kolleg*innen hatten einen großen Anteil an der sozialen Verortung meiner Gesprächspartner*innen. Sie konnten ihnen das Gefühl geben, in Göttingen angekommen zu sein und einen Platz zu haben. Allerdings waren die Erfahrungen individuell und nicht alle so uneingeschränkt positiv, wie im Fall von Gilon und Smita. Die Interaktion am Arbeitsplatz war von den beteiligten Persönlichkeiten und den Strukturen an dem jeweiligen Institut abhängig.

Rohini erklärte, dass besonders die Kommunikation zwischen Studierenden bzw. Promovierenden und Betreuer*innen in Deutschland anders sei als in Indien. Diese sei viel direkter: „I mean for us if A wants to talk to B you have to go through C, or

\footnotetext{
${ }^{83}$ Im deutschen Wissenschaftssystem sind diejenigen, die die Doktorarbeit betreuen, meist auch der oder die Vorgesetzte am Arbeitsplatz.
} 
you have to find another way of saying things in India." Auch in Deutschland gebe es in der Wissenschaft Druck, beispielsweise Artikel zu veröffentlichen und Gelder einzuwerben, diese Faktoren seien universell. Allerdings bestehe in Deutschland „the freedom of work - you do what you want to do" (Rohini, 18.08.2016).

\subsubsection{Eine(r) von den Anderen?}

Viele meiner Gesprächspartner*innen nahmen Deutschland generell als gutes Land für die Wissenschaft und Wissenschaftler*innen war. Diese Einstellung hatte einen großen Einfluss auf das Gefühl in Göttingen und Deutschland am richtigen Platz zu sein. Anisha beispielsweise erzählte mir, sie habe sich in verschiedenen Ländern Europas beworben, überall, nur nicht in den USA, was sie damit erklärte, dass sie das Gefühl habe, dass indische Wissenschaftler*innen dort nicht respektiert würden: „I think because I've been in Germany now for many years somehow it has become my first preference. Because I know here your talent is respected“ (Anisha, 01.05.2011). Auch Ajay drückte dieses Gefühl der Anerkennung aus: „Germans are very good in research and ideas, which makes us learn from them. Germans are also good at recognizing and appreciating talents and brains" (Ajay, 30.01.2012). Anisha und Ajay benutzten mit talents und brains Begriffe, die genauso von verschiedenen Akteuren im Regime der akademischen Mobilität benutzt werden, um die internationalen Studierenden und Wissenschaftler*innen zu beschreiben. Durch diese Wortwahl zeigten sie nicht nur, dass sie sich dieser Diskurse bewusst waren, sondern sie benutzten sie für die eigene Verortung innerhalb Deutschlands. Da diese Begriffe im Mobilitätsregime sehr positiv konnotiert sind, ist diese Wahl für die eigene Positionierung verständlich. ${ }^{84}$ Bezeichnungen wie ,Talente erlauben außerdem die Positionierung als eine(r) von den anderen Wissenschaftler*innen an den Instituten in Deutschland. ,Schlaue Köpfe' und ,Talente' haben keine Nationalitäten, kein Gender und keine Religion und so sind gewissermaßen alle gleich.

Die Wahrnehmung der Gemeinsamkeit als (angehende) Wissenschaftler*innen beschrieb auch Gilon: „If I think in terms of my work within the university, I would say it doesn't matter; I'm one among the other PhD students. Yes, my Indian identity plays a very major role to understand situations, people, culture, which I don't have to think of when I'm within my working atmosphere" (Gilon, 19.01.2013). Sandeep erklärte auf eine ähnliche Weise, dass sein Indischsein für seine Arbeit keine Rolle spielen würde: „I'd say personally I feel I'm Indian always. But for daily life, for work life - no, because it's science, so it doesn't [matter]. But when I go back home and whenever I get time, I don't restrict myself to be in the science (...)" (Sandeep, 09.04.2013). In diesem Interviewausschnitt beschrieb Sandeep sehr eingängig den Versuch, unterschiedlichen Zugehörigkeiten oder Facetten des Selbst bestimmte

\footnotetext{
${ }^{84}$ Diese positive Konnotation wird besonders deutlich, wenn man sich die Diskurse zu anderen mobilen Menschen in Deutschland und weltweit vor Augen führt. Dazu zählen beispielsweise diejenigen um die sogenannten ,Flüchtlinge' und ,Asylbewerber' etc.
} 
Zeiträume zuzuweisen. Die Trennung zwischen den Positionierungen lag allerdings nicht immer in den Händen meiner Gesprächspartner*innen. Sowohl die Interaktionspartner*innen an den Instituten als auch spezifische Ereignisse in Indien bzw. weltweit konnten andere Positionierungen beispielsweise als junge indische Männer oder Frauen in den Fokus rücken.

\section{Eine $(r)$ von den anderen Inder*innen}

Als ich Tanvir fragte, ob er das Gefühl habe, dass seine Herkunft aus Indien in Deutschland eine Rolle spielen würde, antwortete er spontan, dass er nie auf „Probleme" gestoßen sei. Er würde sich kaum dessen bewusst werden, dass er Inder sei. In seinem „Matheleben“ (math life) sei es kein Thema. Allerdings erzählte er dann, dass seine deutschen Kolleg*innen ihn als eine „Erweiterung“ (extension) der indischen Gruppe, die es bereits in seiner Forschungsabteilung gebe, wahrnehmen würden: „I can understand that. I mean I'm not at their level. That's also one reason that maybe they just see me as an extension of the other Indians." Tanvir meinte, er meide seine deutschen Kolleg*innen und verbringe seine Zeit eher mit seinen zwei indischen Kollegen und einem Doktoranden aus Syrien: „We are kind of not accepted into the German [group], maybe because we are not as good as them. That is the reason, I found." Tanvir fügte hinzu, dass die Wissenschaftler*innen aus Kanada und Frankreich mit den deutschen Kolleg*innen durchaus Zeit verbringen und akzeptiert seien. Dies führte er darauf zurück, dass der Unterschied zwischen diesen und den Deutschen nicht so groß sei wie zwischen Deutschen und den „Asians“:

We would talk much more and that would be disturbing ((lacht)). I mean that I miss [that] I didn't get a good group to discuss maths and life simultaneously, which I would have got if I had stayed in India - I mean surely. That is a very important part of growing up in maths - I mean discuss it in a group. I feel here very much obliged to only discuss maths; maybe that is the thing that pushes me away from these discussions. (Tanvir, 17.02.2013)

Tanvir führte die Distanz zu den deutschen Kolleg*innen auf wahrgenommene Differenzen bzw. Gemeinsamkeiten zwischen ,Indern' und ,Asiaten', ,Deutschen“, ,Kanadiern' und ,Franzosen' zurück. An dieser Stelle wird deutlich, welche Rolle Gemeinsamkeiten für die Bildung sozialer Beziehungen generell und besonders für freundschaftliche Beziehungen zugesprochen wird (McKenzie und Baldassar 2017). Das Arbeiten am gleichen Institut ist eine Basis für Gemeinsamkeit, die aber von angenommenen Differenzen, die mit spezifischen Positionierungen verbunden werden, unterlaufen werden kann. Tanvir schrieb seiner Positionierung als Inder in den Interaktionen bzw. Nicht-Interaktionen große Bedeutung zu und fühlte sich ausgeschlossen. 


\section{Eine(r) von den,Ausländern`}

Rohini reduzierte die Erfahrung von Exklusion nicht auf das Arbeitsumfeld, sondern erklärte, dass sie generell das Gefühl habe, dass man in Deutschland immer „Ausländer" bleiben würde. Sie verwendete an dieser Stelle das deutsche Wort und zeigte, dass sie sich dieses Begriffes und der Konnotation, der dieser hat, bewusst war. „I mean, [in the] back of our mind we are still Ausländer." In Amerika sei das anders, Amerika sei eine „immigrant society“, dort sei es einfach, Teil der Gesellschaft zu werden und in der Masse aufzugehen (,be lost in that crowd“). Deswegen würden viele Inder*innen dorthin gehen wollen. Auf meine Frage, ob denn die Sprache ein Teil dieser Dynamik sei, bejahte Rohini und fügte hinzu: „I think you Germans take a bit of time to open up. "Zur Illustration dieser Aussage erzählte sie von Erfahrungen, die sie kurz nach der Ankunft in Göttingen gemacht hatte: „I was like ,bla, bla, bla and talking to people, and I'm calling them, let's go out for a drink' and ,let's go on, have a coffee. “ Für ihre deutschen Kolleg*innen sei dies „too much“ gewesen, das hatten sie Rohini deutlich kommuniziert. Jetzt seien viele der gleichen Kolleg*innen sehr gute Freund*innen. Rohini führte das Verhalten ihrer Kolleg*innen nicht auf ihre Positionierung als Inderin zurück, sondern auf den Umstand, dass sie neu am Institut war. In Rohinis Fall erleichterte die Struktur des Promotionsprogrammes, vor allem die gemeinsamen retreats, den Aufbau freundschaftlicher Beziehungen: „that's the time you meet other people and you go out and you talk to them, and so you become friends." Das zeigt, wie wichtig Gelegenheiten zur Interaktion und das Verbringen von Zeit miteinander für den Aufbau von sozialen Beziehungen sind (mehr dazu in Kapitel 5).

Wie dargelegt, kann die gemeinsame Arbeit an einem Institut bzw. in einer Forschungsgruppe zu einer wichtigen Basis für soziale Beziehungen werden. Viele der jungen Wissenschaftler*innen machten die Erfahrung, dass das Aufbauen von freundschaftlichen Beziehungen zu Kolleg*innen oder Kommiliton*innen etwas Zeit brauchte. Andere hatten zwar gute Beziehungen zu ihren Kolleg*innen, kamen aber über eine bestimmte Grenze nicht hinweg. Kirk et al. sprechen in ihrer Studie von einer "friendly distance“ (Kirk, Bal und Janssen 2017: 2775). Diese freundliche Distanz bemerkte auch Ravi, der daraus schloss, dass in Deutschland stärker zwischen Kolleg*innen und Freund*innen getrennt werden würde. Obwohl die Erfahrungen, die meine Gesprächspartner*innen machten, stark von den Situationen und den beteiligten Personen abhingen, kann die wahrgenommene Trennung zwischen den Lebensbereichen Arbeit und Leben auf die Zeitkarte der Work-Life-Balance zurückgeführt werden. Diese Zeitkarte baut auf einer recht klaren Trennung dieser Bereiche auf, um dann eine entsprechende Zuteilung von Zeit vorzunehmen, die als ausgeglichen bezeichnet wird.

Die hier beschriebenen Erfahrungen von Ausgrenzung werden meines Erachtens dadurch verkompliziert, dass sie nicht mit den Diskursen zusammengebracht werden können, die im Regime der akademischen Mobilität vorherrschen. In diesen wird die Mobilität von Studierenden und Wissenschaftler*innen ausschließlich positiv dar- 
gestellt und in einer Sprache der ,schlauen Köpfe` und "Talente' formuliert, die die jungen Inder*innen überrationalisiert. Wenn diese jungen Menschen ,nur ' Humanressourcen in der Wissensökonomie sind, sollten sie nach dieser Vorstellung kein Problem damit haben, sich in Deutschland, dem „Land der Ideen“, zu verorten.

\subsubsection{Zwischen Religionsdiskussionen und „Delhi incident“}

Im folgenden Kapitelabschnitt zeige ich anhand zweier spezifischer Situationen, wie sich meine Gesprächspartner*innen ihrer Positionierung als Inder*innen in ihrem Arbeitsumfeld in Göttingen bewusst wurden bzw. ihnen diese Verortung bewusst gemacht wurde.

\section{Religion versus Wissenschaft?}

In meinem Interview mit Kiran, der für seinen Masterabschluss in den USA gewesen war, sprachen wir über den Umgang mit Religion in den USA und Deutschland und er erzählte mir, dass er ein Foto von einem hinduistischen Heiligen auf seinem Schreibtisch am Institut habe. Zu seinen alltäglichen Routinen gehöre ein kurzes Gebet, dass er vor Arbeitsbeginn spreche. Dieses Foto hatte eine Diskussion mit seinen Kolleg*innen ausgelöst: „People said: ,How can you bring religion to our department? This is ridiculous. Keep everything to your house. Don't bring all that stuff " (Kiran, 02.10.2010). Kirans Kolleg*innen waren der Meinung, dass der Arbeitsplatz nicht der richtige Ort für religiöse Praktiken sei.

Kirans Praxis, vor dem Essen kurz zu beten, war bei einer gemeinsamen Mittagspause ein weiterer Punkt, der bei einigen seiner Kolleg*innen Kritik aufwarf. Nachdem Kiran auf die Frage, warum er beten würde, geantwortet hatte: „I just thank God for feeding me. I just thank God that he is giving me the opportunity to study here and everything ", hätten seine Kolleg*innen erwidert: „,that's bullshit, there is no God"“ (Kiran, 02.10.2010).

Er meinte, in Indien habe er nicht über Religion nachgedacht. Außerhalb Indiens werde er aber häufiger auf das Thema Religion angesprochen. Indien wurde von Kirans Interaktionspartner*innen vor allem mit dem Thema Religion verbunden. Kiran störte sich an dieser Assoziation an sich nicht, aber: „At the end, whenever you talk to these guys you know they try to change me." Er erklärte, dass er wohl an seinem Max-Planck-Institut auf sehr spezielle Menschen getroffen sein muss, die „social stuff“ hassen würden und gegen das Heiraten seien. „In our Max Planck people are more highly overdeveloped. Their mindset is more towards science, and they don't care much about this kind of religious practices and they think everything is bullshit" (Kiran, 02.10.2010).

Praktiken, die bereits in Indien und den USA zu Kirans alltäglicher Routine gehörten und Vertrautheit und Sicherheit vermittelten (siehe Kapitel 5), stießen in seinem Arbeitsumfeld in Göttingen auf Kritik und warfen größere Fragen auf: Was ist privat, was beruflich? Wie werden die Grenzen zwischen diesen Bereichen gezo- 
gen und was macht eine(n) gute(n) Naturwissenschaftler*in aus? Kiran ließ sich von der Einstellung seiner Kolleg*innen zur Religion nicht beeinflussen und behielt das Bild an seinem Arbeitsplatz. Er entschied sich aber dafür, mit seinen Kolleg*innen nicht mehr über Religion zu diskutieren.

\section{Das Delhi incident: Wenn das Indischsein in den Fokus rückt}

Die Positionierung meiner Forschungspartner*innen als ,indisch' konnte durch direkte Ereignisse am Institut und durch Vorfälle in Indien in das Zentrum der Aufmerksamkeit geraten. Letzteres passierte, nachdem eine junge Studentin im Dezember 2012 in Delhi von mehreren Männern vergewaltigt wurde und daraufhin an ihren Verletzungen starb. ${ }^{85}$ Auf diesen Fall wurde in Indien und weltweit mit großer Entrüstung und Protesten gegen die Gewalt an Frauen reagiert. Dieser Vergewaltigungsfall, aber vor allem die Berichterstattung und der Fokus auf Gewalt gegen Frauen in Indien, hatte einen Einfluss auf das Indienbild in Deutschland (und weltweit). Erkennbar wurde dies nicht zuletzt 2015, als ein kleinerer Skandal von Berichten in sozialen Medien ausgelöst wurde, die besagten, dass die Leipziger Professorin Annette Beck-Sickinger einen indischen Studierenden für ein Praktikum abgelehnt habe, weil Indien ein „Vergewaltigungsproblem“ habe. Die deutsche und internationale Presse berichtete über diesen Fall. ${ }^{86}$

In meinen Interviews schilderten mir einige meiner Gesprächspartner*innen, dass sie auf die Gewalt an Frauen in Indien angesprochen wurden. So erzählte beispielsweise Gilon, der von einer besorgten ehemaligen Studentin aus Frankreich eine lange E-Mail mit ihren Gedanken zu dem Vergewaltigungsfall bekommen hatte:

I wrote that I'm myself ashamed of this incident. But unfortunately, Europe has found another rationalisation for its representation of India - the orientalist representation. Because now, I mean it's how Nicholas Dirks argues about the caste: he says that it has helped the colonizers to evidentially prove that India is a savage country because of the caste system. This kind of incident is also kind of used as [a] possibility to rationalize such understandings. (Gilon, 19.01.2013)

In Gilons Aussage wird das Dilemma, in dem er steckte, sehr greifbar. Einerseits war er von dem Vorfall sehr ergriffen und verdammte, was passiert war. Anderer-

\footnotetext{
${ }^{85}$ Ein Bericht über den Fall findet sich auf der Webseite der New York Times. Siehe Gardiner Harris: "Charges Filed Against 5 Over Rape in New Delhi“, New York Times vom 03.01.2013, http:// www.nytimes.com/2013/01/04/world/asia/murder-charges-filed-against-5-men-in-india-gang-rape. html?hp\&_r=0.

${ }^{86}$ Im folgenden Artikel der Süddeutschen Zeitung können Details zur Diskussion um die Professorin nachgelesen werden. Siehe Süddeutsche Zeitung: „Rektorin der Uni Leipzig hält an Professorin fest“ vom 13.03.2015, https://www.sueddeutsche.de/bildung/nach-ablehnung-von-indischem-bewerberpraesidentin-der-uni-leipzig-haelt-zu-professorin-1.2391846.
} 
seits fühlte er ein Unbehagen, das er auf die Repräsentation Indiens in der Berichterstattung zurückführte.

Viele meiner Gesprächspartner*innen wurden an ihren Arbeitsplätzen von Kolleg*innen und Kommiliton*innen auf das Thema Vergewaltigung angesprochen. Für die indischen Männer und Frauen war dies oft unangenehm, da durch den Fokus der Medien auf dieses Thema alle indischen Männer als Täter und alle indischen Frauen als Opfer repräsentiert wurden. Dass auch meine weiblichen Gesprächspartner*innen ein Problem mit dieser Repräsentation hatten, wird durch die folgende Broschüre sichtbar, die Radha auf Facebook teilte. Der Titel der Broschüre lautet (Auch) wir sind Indiens Töchter und spielt damit auf den Titel der BBC Dokumentation Indias Daughter über den angesprochenen Vergewaltigungsfall in Delhi an. In dem kurzen Heft präsentiert die Autorin Uma Muthuraaman Profile und Bilder von elf Frauen, die aus verschiedenen Regionen Indiens kommen und mittlerweile überall auf der Welt leben. Ihr Ziel ist es, ein differenzierteres Bild indischer Frauen zu zeigen. Auf der letzten Seite des Heftes formuliert sie in einigen wenigen Sätzen, was diese Frauen gemeinsam haben. Unter anderem schreibt sie:

Wir haben unsere Träume und Ambitionen mithilfe von verantwortungsvollen und unterstützenden Vätern, Brüdern, Ehemännern und sogar Söhnen verfolgt! Nein, wir sind keine Vergewaltigungsopfer. Nein, unsere Väter, Brüder, Ehemänner und Söhne sind keine Vergewaltiger! ${ }^{17}$

Radha kommentierte die Broschüre auf Facebook mit den Worten: „Hier ist die Antwort für alle, die ständig darüber Fragen stellen!!!“ Offensichtlich wurde Radha oft auf das Thema Vergewaltigung angesprochen und wollte nun mit diesem Artikel eine Antwort geben, um zukünftige Fragen zu vermeiden.

Wer die jungen Wissenschaftler*innen in ihrem Alltag an den Instituten und in Göttingen generell waren, wer sie sein konnten, lag nicht nur in ihren Händen. Die Definitionsmacht darüber lag zu einem Teil immer bei ihren Interaktionspartner*innen, die meine Gesprächspartner*innen, beeinflusst von medialen Repräsentationen, positionierten. Für die jungen Menschen, die im Zentrum meiner Ausführungen stehen, war es nicht möglich, einfach zu vergessen, wer sie waren, woher sie kamen, da es ihnen immer wieder vor Augen geführt wurde (vgl. Butcher 2011: 9). Wie Gilon analysiert hat, ist die Darstellung Indiens in globale Machtbeziehungen eingebettet. Die Repräsentation Indiens entweder als wachsende technologische Superkraft oder als das exotisierte Andere mit einem „Vergewaltigungsproblem“ beeinflusste, wie die indischen Wissenschaftler*innen wahrgenommen wurden: als wertvolles Humankapital oder potentielle Bedrohung.

\footnotetext{
${ }^{87}$ Siehe Uma Muthuraaman: „We (too) are India’s Daughters - German version“, ISSUU vom 14.03.2015, https://issuu.com/umamuthuraaman/docs/final___german_version_indias_daugh.
} 


\subsubsection{Indische Frauen in der Wissenschaft: „Girls with toys“}

In diesem Teil des Kapitels befasse ich mich mit dem Einfluss, den das Frausein auf die Verortung meiner Gesprächspartnerinnen als (angehende) Wissenschaftlerinnen hatte. Zur Zeit meiner Forschung waren 22,8\% der indischen Studierenden in Deutschland Frauen (DAAD und DZHW 2016: 17). Viele meiner Gesprächspartner*innen machten die Verhältnisse in Indien für diesen geringen Anteil von Frauen verantwortlich. Rohini erklärte, dass es generell eine ungleiche Anzahl von Männern und Frauen in Indien gebe und, dass die Leute ihre Töchter nicht ausbilden wollen würden. Ich erwiderte, dass laut Statistiken 44\% der Studierenden in Indien Frauen seien (Government of India 2013: i). Diesen recht hohen Anteil erklärte sie damit, dass es nötig sei, die Töchter bis zu einem bestimmten Grad auszubilden, um einen Ehemann für diese finden zu können. Aber früher oder später würden die jungen Frauen aufhören zu studieren, weil sie es nicht mehr brauchen würden und ihr weiteres Studium nicht unterstützt werden würde. Rohini erklärte, dass die indischen Frauen, die es nach Deutschland geschafft haben, sicherlich sehr hart dafür gearbeitet und gekämpft hatten:

(...) if you are an educated women, you have to be tough (...). This 22 percent you are talking [about]; they are really absolute fighting people. They fought against many things: they fought against discrimination - maybe among the siblings - or let's say even poverty for many of them. (Rohini, 18.08.2016)

Der geringe Anteil von Frauen muss allerdings ebenso im Kontext der Wissenschaft als sozialer Welt mit spezifischen Normen und Strukturen verstanden werden, die für Männer und Frauen unterschiedliche Hürden bereithalten.

\section{Frauen in der Wissenschaft: boys versus girls with toys}

Durch die folgende Formulierung des Astronomen Shrinivas Kulkarni in einem Interview wurde eine Debatte zur Position von Frauen in den (Natur-)Wissenschaften ausgelöst: „Many scientists, I think, secretly are what I call ,boys with toys". " 88 Als Reaktion auf diese unbedachte Äußerung veröffentlichten Wissenschaftlerinnen aus aller Welt Bilder von sich selbst mit ihren ,Spielzeugen“ unter dem Twitter-Hashtag ${ }^{89}$

\footnotetext{
88 Siehe Joe Palca: „,Playing Around With Telescopes 'To Explore Secrets Of The Universe“, NPR vom 16.05.2015, http://www.npr.org/2015/05/16/406995846/playing-around-with-telescopes-to-exploresecrets -of-the-universe.

${ }^{89}$ Twitter ist ein Mikrobloggingdienst, auf dem Nutzer Nachrichten im Telegrammstil (max. 140 Zeichen) verbreiten können. Mit Hilfe des Hashtags (\#) können Teile der Nachricht als Schlagwort gekennzeichnet werden und damit thematisch zugewiesen werden. Siehe Martin Maciej: „Was ist ein Hashtag \#? Beispiele, Funktion und Bedeutung“, GIGA vom 15.05.2018, http://www.giga.de/extra/ social-media/specials/was-ist-ein-hashtag-sinn-bedeutung-und-anwendung/.
} 
„\#girlswithtoys “90. Die veröffentlichten Fotos der Wissenschaftlerinnen mit ihren technischen Geräten wie Mikroskopen, Teleskopen oder einem Mars-Rover teilten auch einige meiner Gesprächspartnerinnen auf Facebook, wie zum Beispiel Sarjana. Die Aussage von Shrinivas Kulkarni macht deutlich, dass bestimmte wissenschaftliche Felder immer noch vor allem mit männlichen Wissenschaftlern verbunden werden. Es ist weltweit so, dass mehr Männer in allen Wissenschaften, die unter die MINT-Bereiche (oder STEM auf Englisch) fallen, vertreten sind. ${ }^{91}$ Obwohl in vielen Ländern die Zahl weiblicher Studierender in diesem Bereich ${ }^{92}$ ansteigt - auch in Indien - ist sie immer noch vergleichsweise gering. 2017 waren in Deutschland 29,1\% der MINT-Absolvent*innen Frauen (Bundesagentur für Arbeit 2019: 21). Außerdem zeigen Statistiken aus Indien und den OECD-Ländern, dass weniger Frauen nach dem Abschluss in der Wissenschaft bleiben. In Indien sind 25-30\% der Doktorand*innen in den Naturwissenschaften weiblich, aber nur 15-20\% der Lehrenden an den Universitäten, und je höher die Position, desto geringer wird der Anteil an Frauen (Godbole und Ramaswamy 2015: 68). In den OECD-Ländern gibt es eine ähnliche Dynamik, denn selbst wenn Frauen einen Abschluss im MINT-Bereich machen, ist die Wahrscheinlichkeit, dass sie in diesem weiterarbeiten, wesentlich geringer als bei den Männern (43\% Frauen, 71\% Männer). ${ }^{93}$ Dass der Frauenanteil nach der Promotion kleiner wird, gilt nicht nur für die Naturwissenschaften. Dieser Trend ist fächerübergreifend und betrifft die meisten Länder der EU genauso wie Indien und andere Länder Asiens (European Commission 2016b; AASSA2015: III).

Ich argumentiere, angelehnt an Jacobs und Winslow (2004) sowie Winslow und Davis (2016), dass die beschriebene Situation entsteht, weil die Norm des akademischen Lebensverlaufs für Frauen andere Herausforderungen bereithält als für Männer. Anders ausgedrückt können Frauen der linearen Norm des akademischen Lebensverlaufs, die ein Voranschreiten von einer Stufe zur nächsten unbelastet durch familiäre Verpflichtungen vorsieht, nicht so einfach folgen wie Männer (Winslow und Davis 2016: 405). Die sich am männlichen Lebensverlauf orientierende Norm der akademischen Karriere erschwert es den Frauen, die Zeitkarte des akademischen Lebensverlaufs mit den Koordinaten ihres persönlichen Lebensverlaufs zusammenzubringen.

\footnotetext{
90 Siehe Lizzie Plaugic: „Astronomer calls scientists ,boys with toys, “ \#girlswithtoys proves him wrong“, The Verge vom 17.05.2015, http://www.theverge.com/2015/5/17/8617151/girls-with-toysnpr-interview-shrinivas-kulkarni.

${ }_{91}$ Diesen Umstand verdeutlichen beispielsweise Statistiken der OECD (https://www.oecd.org/gen der/data/wherearetomorrowsfemalescientists.htm, abgerufen am 31.08.2020).

92 Die Statistiken zeigen diesen Anstieg beispielsweise für Deutschland. Siehe Statista: „Anzahl der MINT-Studienanfänger* an deutschen Hochschulen nach Geschlecht in den Studienjahren von 2007/2008 bis 2019/2020" vom September 2020, https://de.statista.com/statistik/daten/studie/ 28346/umfrage/anzahl-der-mint-studienanfaenger/.

93 Siehe OECD: „Where are tomorrows female scientists ?“ (https://www.oecd.org/gender/data/ wherearetomorrowsfemalescientists.htm, abgerufen am 31.08.2020).
} 
Die berufliche Laufbahn von Männern und Frauen wird unterschiedlich vom Familienleben beeinflusst. Eine Frau und Kinder zu haben, kann für die Karriere von Männern durchaus zuträglich sein (Duberley und Cohen 2010: 190). Für Frauen gilt eher das Gegenteil. Vor allem, wenn Frauen Kinder bekommen, ändert sich oft, wie sie am Arbeitsplatz wahrgenommen werden und welche beruflichen Chancen sie haben (Duberley und Cohen 2010: 195).

Viele meiner Gesprächspartner*innen waren sich bewusst, welchen Einfluss eine Ehe und Kinder auf ihre Karriere haben können. Dieses Bewusstsein wird in ihren Strategien der biographischen Navigation - beispielsweise dem aktiven Hinauszögern einer Heirat und der strategischen Partnerwahl - deutlich. Auf diese Thematiken gehe ich in Kapitel 7 näher ein.

Der Einfluss von Gender auf die wissenschaftliche Laufbahn und die Wahrnehmung in der Wissenschaft wurden durch Shrinivas Kulkarnis Aussage wieder in das weltweite Bewusstsein gerufen. Für Frauen in der Wissenschaft und auch für meine Gesprächspartnerinnen bedeutete dieses Bewusstsein nun, das fortzuführen, was sie laut Rohini bereits seit geraumer Zeit getan hatten: Zu kämpfen und sich gegen die verschiedenen Widerstände durchzusetzen. Kampagnen wie diejenige unter dem Hashtag „\#girlswithtoys“ oder „\#distractinglysexy“94 und Initiativen von Universitäten und Forschungsinstituten ${ }^{95}$ unterstützen die Frauen auf ihrem Weg. Die Nobelpreisträgerin Marie Curie ist dabei für viele ein Symbol und ein Vorbild im Kampf gegen die ungleiche Stellung von Frauen in der Wissenschaft geworden. Sarjana teilte das Titelblatt von Curies Dissertation auf Facebook und kommentierte dazu: „What a fine example of a woman, paving the path for millions more to realise their worth beyond shackles of societal norms!“

\subsection{Zusammenfassung}

Die Mobilität war für die jungen Wissenschaftler*innen ein Mittel der Beschleunigung - eine Strategie, um (bessere) Chancen für die berufliche und persönliche Zukunft zu schaffen und den Erfolg zu erreichen, der von ihnen erwartet wurde, den sie selbst erwarteten und erhofften. Diese Strategie des future tricking als spezifische Art der Zeitgestaltung ergab sich aus der linearen Logik der Zeitkarten, mit der die jungen Menschen in ihrer biographischen Navigation interagierten.

Göttingen wurde von meinen Gesprächspartner*innen als die richtige Station auf dem Weg zur (akademischen) Selbsterfüllung wahrgenommen. Hier erhielten sie

\footnotetext{
${ }^{4}$ Der Hashtag entstand als Reaktion auf die Aussage des Mathematikers und Nobelpreisträgers Tim Hunt, der erklärte, drei Dinge würden passieren, wenn Frauen im Labor anwesend seien: „you fall in love with them, they fall in love with you, and when you criticise them they cry." Hier mehr zur Debatte um Hunts Aussage und den Hashtag. Siehe BBC News: „Female scientists post ,distractingly sexy" photos" vom 11.06.2015, http://www.bbc.com/news/blogs-trending-33099289.

${ }^{5}$ Zum Beispiel Initiativen wie der MINT-Pakt. Siehe BMBF: „MINT-Pakt und „Erfolg mit MINT“, https://www.bmbf.de/de/mint-pakt-und-girls-day-214.html, abgerufen am 24.11.2020.
} 
die wissenschaftlichen Möglichkeiten, die sie sich erhofften, fühlten sich als ,Talent wertgeschätzt und verfügten über größere Zeitsouveränität im Arbeitsalltag.

Nichtsdestotrotz bedeutete das Leben in Göttingen für die jungen Menschen auch, Erfahrungen von Exklusion zu machen, sich der eigenen Positionierung als Inder*innen und den Repräsentationen, die damit verbunden werden, bewusst zu werden, und dies navigieren zu müssen. Im Umgang mit diesen Erfahrungen spielte die Verortung als (angehende) Wissenschaftler*in eine wichtige Rolle als Mittel der diachronen Navigation des Selbst: Anhand dieser Verortung erzählten sie von sich selbst, ihrer Entwicklung über die Zeit hinweg, warum Göttingen der richtige Ort für sie war und konnten damit die Kontinuität ihres Selbst her- und darstellen. 


\section{Der Alltag in Göttingen: (Gem)einsame Zeit und Gleichzeitigkeit}

Die niedersächsische Stadt Göttingen wurde für meine Gesprächspartner*innen eine Zeit lang zu einer Art Heimat. Eines von zwei „homes“, wie Sangita bemerkte, das neben dem Zuhause in Indien bestand. Die jungen Wissenschaftler*innen waren die Verbindung zwischen diesen homes und führten ein Leben, das lokale und transnationale Verortungen einschloss. Der Alltag meiner Gesprächspartner*innen war durch „simultaneity“, also Gleichzeitigkeit gekennzeichnet: „living lives that incorporate daily activities, routines, and institutions located both in a destination country and transnationally (...)" (Levitt und Glick Schiller 2004: 1003). In diesem Kapitel konzentriere ich mich auf die Prozesse, die zu dieser Gleichzeitigkeit führten, und auf die Strategien der Zeitgestaltung, die sie möglich machten. Obwohl Levitt und Glick Schiller mit ihrem Konzept der simultaneity einen Begriff nutzen, der sich auf eine zeitliche Eigenschaft bezieht, gehen sie nicht explizit auf Zeit ein. Ich werde die zeitlichen Erfahrungen als eine wichtige Facette des Lebens im transnationalen sozialen Raum hervorheben. Dieser Fokus bedeutet nicht, dass ich diese Erfahrungen als wichtiger erachte als andere Elemente des Alltags, sondern dass ich den Umgang mit Zeit im transnationalen sozialen Raum als Thema identifiziert habe, das bisher in der ethnologischen Literatur wenig Aufmerksamkeit erhalten hat. Ich trage mit 
meiner Arbeit dazu bei, die Perspektive auf zeitliche Erfahrungen und Zeitgestaltung im transnationalen sozialen Raum auszubauen.

Den Ausgangspunkt meiner Ausführungen bildet die Beschäftigung mit dem Einfluss von Mobilität auf die Erfahrung und Gestaltung von Zeit. Der Umzug von Indien nach Göttingen bedeutete für meine Gesprächspartner*innen die Interaktion mit neuen Zeitlandschaften, Zeitkarten und sozialen Rhythmen. Wie Cwerners Studie (2001) zeigt, kann durch Mobilität das Gefühl entstehen, asynchron zu sein und in fremden Zeiten zu leben. Ich argumentiere in diesem Kapitel, dass die jungen Wissenschaftler*innen aus Indien diese zeitlichen Erfahrungen durch unterschiedliche Strategien der Zeitgestaltung beeinflussen konnten. Zu diesen Strategien gehörte das Etablieren von Freundschaften sowie Komfortzonen und -zeiten mit Menschen, durch deren Gegenwart sie sich wohl und weniger einsam fühlten. Ich zeige, wie unterschiedliche Informations- und Kommunikationstechnologien genutzt wurden, um soziale Beziehungen aufrechtzuerhalten und Gleichzeitigkeit herzustellen. Dabei steht im Fokus, wie meine Gesprächspartner*innen in diesen Prozessen ihre zeitlichen Erfahrungen und ihre Zeitgestaltung aushandelten.

Des Weiteren bauten die jungen Menschen durch vertraute und neu erlernte Praktiken Alltagsroutinen auf, die ihr lokales und transnationales Leben in den Alltag integrierten. Der Schwerpunkt dieses Kapitels liegt auf den individuellen Alltagsbeziehungen und -praktiken und rückt damit meine Gesprächspartner*innen als Erbauer*innen von Aspekten ihrer eigenen Zeitlandschaft in den Vordergrund.

Der Einfluss zeitlicher Horizonte auf die biographische Navigation im Alltag ist ein zweites wichtiges Thema dieses Kapitels. Die jungen Wissenschaftler*innen planten, nicht in Göttingen zu bleiben, und lebten demzufolge „auf der Durchreise“, wie es Rohini ausdrückte. Ich zeige, dass das Bewusstsein von der zeitlichen Begrenztheit und den zukünftigen Koordinaten im Alltag eine wichtige Rolle spielte: Es beeinflusste sowohl die Bildung sozialer Beziehungen in Göttingen und Alltagspraktiken als auch den Umgang mit Entbehrungen und Heimweh.

Der Alltag als Untersuchungshorizont ermöglicht einen Blick auf andere Zeiten und Zeitkarten als jener des Lebensverlaufs. Die Zeitlandschaft des Alltags ist durch Zeitkarten und soziale Rhythmen der Wiederholung gekennzeichnet. Mit Bezug zur Mobilität eröffnet der Fokus auf den Alltag eine Perspektive auf die Unsicherheiten, unerwarteten Herausforderungen und Brüche (vgl. Collins und Shubin 2015), die meine Gesprächspartner*innen navigierten, und auf die „Reparaturarbeiten“ (Trentmann 2013: 69), die sie in den Alltag investierten. 


\subsection{Zeitliche Erfahrungen im Alltag}

Göttingen hat als Stadt mit einer großen Population von Studierenden (ca. 134.000 Einwohner*innen und davon ca. 30.000 Studierende) eine ganz eigene Zeitlandschaft mit einem eigenen Tempo.

\subsubsection{Vom „slow life“, neuen Freizeiten und Freiheiten}

In meinen Gesprächen mit den jungen Wissenschaftler*innen erinnerten sich einige daran, dass sie bei ihrer Ankunft in Deutschland den Eindruck hatten, dass es „leer" sei, ohne Menschen, und sehr ruhig. Ähnlich beschrieben mir viele meiner Gesprächspartner*innen das Leben in Göttingen mit den Worten „quiet“, „relaxed“, "calm" oder "could be more lively“. Diese Begriffe drücken sowohl räumliche als auch zeitliche Erfahrungen aus. Auf der zeitlichen Ebene beschreiben die Worte das Gefühl, dass das Leben langsamer sei. Die jungen Menschen kontrastierten diese Erfahrungen häufig mit jenen, die sie in Indien oder in anderen Kontexten, vor allem in den USA, gemacht hatten, wobei hier Beschreibungen genutzt wurden wie "there are so many" und „we are so many“.

Sangita drückte ihre Wahrnehmung des Lebens in Göttingen sehr prägnant mit dem Begriff „slow life“ aus: „I have lived in Bombay all my life which is huge and you are always in a crowd and everybody is in a rush. I like the quietness of Göttingen and the slow life in Göttingen." In Bombay habe sie jeden Tag sehr viel Zeit damit verbracht, von einem Ort zum anderen zu kommen. Deswegen sei sie immer müde gewesen und habe kaum Zeit für sich selbst gehabt. „But here given that everything is so nearby and everything is so much slower, I think I have time for everything that I feel like doing" (Sangita, 08.08.2013).

Für Sangita bedeutete der Umzug nach Göttingen, das erste Mal alleine ohne Eltern und Schwester zu wohnen - eine Erfahrung, die sie mir begeistert beschrieb: „[It feels] awesome. That feels really, really good.“ In Göttingen fühle sie sich unabhängig. Sie müsse zwar nun alles im Alltag alleine organisieren und erledigen, habe aber dadurch gelernt, dass sie dies auch könne ,and doing them or not doing them is all about my laziness."

Viele meiner Gesprächspartner*innen hoben die positiven Effekte hervor, die das Leben in Deutschland und speziell Göttingen auf ihren Alltag hatte. Ähnlich wie Sangita betonten sie dabei das Gefühl, mehr Zeit zur freien Verfügung zu haben und weniger unter Druck zu stehen als in Indien. Dieses Gefühl ging außerdem mit der Wahrnehmung einher, dass es weniger Menschen in Göttingen gebe (als in Indien) und sich diese nicht für das Privatleben der anderen interessierten. Tanvir erzählte mir beispielsweise:

I really love this place a lot; it gives so much of opportunity to [be] yourself. I really enjoy a lot this running around the city wall - enjoy the mood, so much 
silence, nobody is caring. I mean, in India if you start running ten people will ask you what happened? Why did you start? ... You have to answer so many questions [about] anything you do in India. Here, no one is there, no one is interested - you just carry on with your life. (Tanvir, 17.02.2013)

Einige der jungen Wissenschaftler*innen genossen das Gefühl, ihre Zeit nach eigenen Vorstellungen füllen zu können und sich dabei weniger nach gesellschaftlichen Konventionen richten zu müssen. Diese Möglichkeit eröffnete sich ihnen nicht nur dadurch, dass sie gefühlt mehr Zeit hatten, sondern ebenso durch das Leben in einer neuen sozialen Umwelt: „Where a person is unknown when they arrive in a new cultural context there are no boundaries created by the expectations by other's (e.g. family and peers) familiarity" (Butcher 2011: 9). Dies bezieht sich auch auf die Erwartungen, die andere davon haben, mit was und wem Zeit verbracht werden sollte. Zugleich hat die gewonnene Freiheit, sich selbst zu definieren und die eigene Zeit zu gestalten, Grenzen, die durch die anhaltenden transnationalen Beziehungen und Selbst- und Fremdzuschreibungen entstehen (vgl. Butcher 2011: 9).

Neben der Erfahrung der Entschleunigung war es vor allem das Gefühl der Kontrolle über die eigene Zeit, das meine Gesprächspartner*innen hervorhoben. In Göttingen hatten sie, anders als sie es über Indien berichteten, die Kontrolle darüber, was sie mit ihrer Zeit anfangen wollten, und waren zeitlich weniger fremdbestimmt. Wie ich bereits für den Arbeitskontext in Kapitel 4 gezeigt habe, entstand diese Erfahrung sowohl durch die neue Zeitlandschaft und neue Zeitkarten als auch durch die veränderte Positionierung der jungen Wissenschaftler*innen. In ihrem neuen Kontext hatte ihr Alter und Gender nicht den gleichen Einfluss auf die Kontrolle über ihre Zeit, wie es in ihrem Herkunftskontext der Fall war.

Das Leben in der Zeitlandschaft Göttingens hatte nicht nur positive Auswirkungen, sondern löste Gefühle der Entfremdung aus. Es entstand die Frage, was eigentlich mit der ,gewonnenen' Zeit gemacht und mit wem sie geteilt werden kann. Ähnlich verhielt es sich mit der Privatsphäre, die Tanvir so positiv hervorhob. Dass sich, wie Tanvir es ausdrückte, keiner dafür interessiert, was der andere macht, kann zu einem Gefühl der Einsamkeit und Isolation führen (vgl. Sawir et al. 2007; Hansen 2015: 67; Kirk, Bal und Janssen 2017: 2776).

\subsubsection{Langeweile und Einsamkeit}

Bei einigen meiner Gesprächspartner*innen führte die neu gewonnene Freiheit zu Unsicherheit und Gefühlen von Einsamkeit. Anay beispielsweise empfand die Entschleunigung des Lebens in Göttingen als Langeweile:

Sometimes it's boring - because I don't have friends, I don't have that sort of social circle. It's a nice place if you have a girlfriend. If you have friends it's a nice place to be. It's a very peaceful life: you study, you are with them, there is someone [with] whom you can share. (Anay, 15.07.2015) 
Einige wenige meiner Interviewpartner*innen wünschten sich, dass Göttingen etwas lebendiger sein sollte, oder wie Anay es etwas drastischer ausdrückte: „social life is fucked up“. Anay war als Doktorand Teil einer transnationalen Forschungsgruppe, die Institutionen in Deutschland, England und Indien umfasste. Aus diesem Grund verbrachte er immer nur einige Monate am Stück in Göttingen und lebte zwischendurch in London oder Delhi. Im Vergleich zum Leben in diesen Großstädten kam ihm das Leben in Göttingen eher öde vor. Am Institut, an dem er tätig war, erklärte er mir, seien die anderen Mitarbeiter*innen wesentlich älter als er, nämlich in den Dreißigern. Er sah sich als Teil einer anderen Generation, obwohl ihn nur fünf Jahre von den „wesentlich älteren“ trennten: „since we are living in a very changing time, your parents or your five years senior would have very different kind of education or childhood. So [they have] two different kinds of anxieties, different kinds of concerns." Anay fehlte es an Menschen, mit denen er seine Zeit und seine Erfahrungen in Göttingen teilen konnte.

Ravi, der 2005 für eine Promotion in Biophysik nach Göttingen gekommen war, machte zu Beginn seines Aufenthaltes ähnliche Erfahrungen wie Anay. Als Ravi nach Göttingen kam, lebten etwa dreißig Studierende und Wissenschaftler*innen aus Indien dort. Er habe kaum andere Inder*innen angetroffen und „many foreigners ... were willing to share a professional relationship with you, but not beyond that." Es sei sehr schwierig, soziale Beziehungen oder social circle, wie er es nannte, in einem fremden Land aufzubauen - in jedem fremden Land, betonte er. Allerdings war er der Meinung, dass es in Deutschland etwas schwieriger sei: „because they are very possessive of their own friend circle. So in such a phase it is difficult for a new person coming from abroad to start building a comradeship. That's the only thing the main thing I would say - that I missed the most." Ravi erklärte sich das Fehlen von Freundschaften mit „locals“ in Göttingen unter anderem damit, dass diese bereits bestehende Beziehungen hatten. Diese Erklärung entspricht den Ergebnissen von McKenzies und Baldassars Studie (2017: 706) zu den fehlenden Freundschaften zwischen internationalen und lokalen Studierenden in Australien.

Viele meiner Gesprächspartner*innen hatten gute Beziehungen zu ihren Kolleg*innen, die aber in vielen Fällen nicht über eine gewisse Grenze hinausgingen (vgl. Kirk, Bal und Janssen 2017: 2775). Diese Dynamik macht deutlich, dass sie ihre Freund*innen nicht einfach wählen konnten. Freundschaft braucht nicht nur eine Gelegenheit, wie die gemeinsame Arbeit an einem Institut, sondern ebenso Gegenseitigkeit und die Bereitschaft von beiden Seiten (vgl. Ryan 2015: 1669). Auf diesen Punkt gehe ich in Abschnitt 5.2 „Zeit teilen“ konkreter ein.

Da Ravis Versuche, Freundschaften aufzubauen, zunächst scheiterten, arbeitete er sehr viel, denn: „I don't have anything to do in my apartment while coming back. So, as I said, the lack of [a] social circle is also a problem - and the lack of entertainment." Der Arbeit viel Zeit zu widmen, war also nicht immer von der Arbeit an sich motiviert, sondern zudem vom Fehlen sozialer Kontakte und anderer Aktivitäten. Nach dieser schwierigen Anfangszeit hatte sich Ravi erfolgreich einen Freundeskreis in Göttingen aufgebaut. Dieser bestand vor allem aus anderen in- 
dischen Wissenschaftler*innen. Gemeinsam mit einigen von ihnen gründete er 2010 die Vereinigung indischer Studierender. Ravis Erfahrungen inspirierten die Gründung der Organisation, die den Ankommenden aus Indien den Start mit Hilfe von Informationen erleichtern soll (Kapitel 6).

\subsubsection{Zeitliche Horizonte im Alltag: „We know that it's temporary“}

Meinen Gesprächspartner*innen war bewusst, dass sie nur für einen bestimmten Zeitraum in Göttingen sein würden. Das Wissen um die Begrenztheit ihres Aufenthaltes hatte einen großen Einfluss auf ihr Leben dort, darauf, mit was und wem sie Zeit verbrachten und wie sie wohnten. Diesen letzten Aspekt betonte Sadhvi in unserem Gespräch: „In our apartments, it's our apartment and we feel like it's home, but then we also don't want to for example buy a lot of things for it or decorate it really nicely." Sie wüssten, dass sie Göttingen verlassen würden und dafür sei es einfach zu viel Arbeit und Geld, die in die Wohnung gesteckt werden müssten. „So, everything, our thinking is also different. Everything that we do is also guided by this - because we know that it is temporary." Die Kleidung, die sie kaufen würden, ihre Einkäufe, alles würde von diesem Gedanken beeinflusst werden: „It's only for three years, it's only for three years" (Sadhvi 22.09.2011).

Im Vergleich zu den Wohnungen deutscher Studierender und Promovierender waren jene meiner Gesprächspartner*innen häufig nur spärlich eingerichtet. Gegenstände, die angeschafft werden mussten, wie ein Regal, ein Stuhl oder das typische Fortbewegungsmittel in Göttingen, das Fahrrad, wurden, wie es unter Studierenden üblich ist, gebraucht gekauft. Diese Dinge wurden vor dem Verlassen Göttingens wieder verkauft, in einigen Fällen direkt über die Facebook-Gruppe Indians in Göttingen und somit an die nächste Generation junger Wissenschaftler*innen weitergegeben. Wie Sadhvi ansprach, gab es in den Wohnungen kaum Dekorationen. Fotos von der Familie oder von Reisen schmückten häufig nicht die Wände der Wohnungen, sondern wurden auf Handys oder Laptops aufbewahrt oder hochgeladen und auf virtuellen Wänden ausgestellt, wie beispielsweise auf der Facebook-Chronik. Auf diese Weise konnten „tröstliche Dinge“ (vgl. Miller 2008) wie Familienfotos einfach zum nächsten Wohnort transportiert werden. Andere Dekorationen wie Rangolis wurden von einigen meiner Gesprächspartner*innen in ihren Wohnungen für bestimmte Festlichkeiten beispielsweise Diwali oder Durga Puja hergestellt. Rangolis werden aus eingefärbtem Sand gemacht, der in filigranen Mustern auf den Boden gestreut wird. Diese Dekorationen sind von Natur aus vorübergehend und werden nach den Festlichkeiten einfach weggekehrt.

Was sich anhören mag wie eine sehr asketische Lebensweise, kann als Ausdruck der Ambivalenz verstanden werden, die sich im Fall der jungen Wissenschaftler*innen aus dem Bewusstsein entwickelte, dass sie nicht in Göttingen bzw. Deutschland bleiben würden. Rohini erklärte, dass die Konzeption des Lebens als auf der Durchreise spezifisch für Deutschland als Zielland indischer Studierender und Wissenschaftler*innen sei. Indische Studierende, die nach Australien, Großbritannien 
oder die USA gehen würden, hätten zum Ziel, dort zu bleiben: „Because it’s a better life. Because it's easier.“ Deutschland dagegen sei für die meisten eine „transit phase": „you come here - you will spend, let's say seven years here - but you will move out from here, eventually." Sie führt dies unter anderem darauf zurück, dass Deutschland kein „Einwanderungsland“ sei und man immer das Gefühl habe, „Ausländer" zu sein. Als ich sie fragte, ob das Wissen, dass sie nicht in Deutschland bleiben wird, einen Einfluss auf ihren Alltag in Göttingen habe, antwortete sie: „For me, it didn't matter if I'm staying here for two years or seven years or eight years or whatever." Für andere spiele es aber eine große Rolle, vor allem, und hier bezog sie meine Frage sofort auf die sozialen Beziehungen, blieben einige deswegen nur unter anderen Inder*innen: „For many people, they like to be in that close proximity." Rohini erklärte, für sie sei es auch schwer, allein zu sein, und sie habe deswegen eine Mitbewohnerin. ${ }^{96}$ Allerdings würde sie Leute aus Indien kennen, die seit ihrem Bachelorabschluss befreundet und gemeinsam nach Göttingen gekommen seien, um hier ihren Masterabschluss zu machen. Rohini gestand ein, dass eine solche enge und kontinuierliche Beziehung in bestimmten Situationen sehr wertvoll sei: „It's absolutely excellent because then you have somebody. As I said, friends always help you." Gleichwohl sah sie die auf die eigene Gemeinschaft (own community) eingeschränkte Interaktion kritisch: „You never interact with anybody, so this is something which I don't like so much" (Rohini, 18.08.2016).

Das Bewusstsein vom zeitlich begrenzten Charakter des Aufenthalts hatte von Fall zu Fall unterschiedliche Auswirkungen auf die sozialen Beziehungen der jungen Wissenschaftler*innen. Für einige der jungen Menschen war das Wissen, dass ihr Aufenthalt in Göttingen in absehbarer Zeit enden würde, ein wichtiger Faktor im Umgang mit schwierigen Situationen wie Heimweh oder Isolation und Problemen mit ihrer Doktorarbeit. Gilon erklärte mir: „(...) there were times that I wanted to leave, but I always had the hope that I'm going anyway. For me, this surety of going back was very important" (Gilon, 19.01.2013). In Nanditas Aussage wird das Bewusstsein um die Rückkehr ähnlich deutlich: „Sometimes I miss my family and sometimes I miss food as well. But it's okay. I know that I will be [going] back to India, so these things are not that important" (Nandita, 03.09.2011). Gilon und Nandita schienen sich die Begrenztheit ihrer Zeit in Göttingen bewusst in schwierigen Situationen in Erinnerung zu rufen und damit einen Einfluss darauf zu nehmen, wie sie ihren Aufenthalt wahrnahmen und erfuhren. Damit konnten die beiden die zeitliche Begrenztheit ihres Aufenthalts, etwas, das häufig als negativ verstanden wird, neu rahmen und positiv konnotieren (vgl. Robertson 2014).

Wie ich in diesem Abschnitt gezeigt habe und im weiteren Verlauf dieses Kapitels an verschiedenen Stellen wieder aufnehmen werde, hat das Verständnis des Lebens in Göttingen als eines auf der Durchreise einen großen Einfluss auf den Alltag der jungen Wissenschaftler*innen. Francis Collins und Sergei Shubin (2015) beschreiben die Art und Weise, wie Menschen ihr Leben in einer solchen Situation organisieren,

${ }^{6}$ Rohinis Mitbewohnerin war Doktorandin im gleichen Promotionsprogramm. 
als die Normalisierung von Zeit in eine „extended or ,permanent temporariness““ (Collins und Shubin 2015: 100) und verstehen diese wie Adrian Bailey et al. (2002) als Zustand, der eine „disciplining power over bodies, families, and social fields“ (Adrian Bailey et al. 2002: 139) hat. Den Ursprung der permanent temporariness verorten Collins und Shubin (2015) und Adrian Bailey et al. (2002) in Visaregelungen, die für bestimmte Kategorien mobiler Menschen nur zeitliche begrenzte Aufenthalte möglich machen. Im Fall der jungen Wissenschaftler*innen ist die Aufenthaltsgesetzgebung ein Aspekt, der dazu beiträgt, das Ende des Aufenthaltes in Göttingen als Horizont zu erzeugen. Die Dauer ihrer Visa ist an die Dauer ihres angestrebten Abschlusses und an die Finanzierung durch ein Stipendium oder einen Arbeitsplatz gekoppelt. Ein weiterer Faktor ist die Zeitkarte des akademischen Lebensverlaufs, die die Promotion als eine wichtige Koordinate festlegt, nach der es den nächsten Schritt für den weiteren Verlauf des Lebens zu unternehmen gilt. Außerdem wussten viele meiner Gesprächspartner*innen, dass ihre Eltern von ihnen erwarteten, früher oder später nach Indien zurückzukehren und dort ihre Verantwortlichkeiten als Teil der Familie zu übernehmen (Pflege der Eltern etc.).

All diese Faktoren trugen dazu bei, das Ende der Zeit in Göttingen als Koordinate herzustellen und zu konkretisieren. Als Repräsentation von Zeit formte sie die Erfahrungen in Göttingen und Praktiken der Zeitgestaltung. Sie schuf einen zeitlichen Horizont, der je nach Fall entweder als Silberstreifen wahrgenommen wurde oder als herannahendes Unheil, dem ausgewichen werden musste.

Die Wirkung des „permanenten vorübergehenden Zustands“ liegt allerdings nicht nur in den Restriktionen, beispielsweise durch die Aufenthaltsgesetzgebung, sondern in den Antworten der Menschen auf diese. Dazu gehörte im Fall der jungen Wissenschaftler*innen das bewusste Leben auf der Durchreise, in dem keine (neuen) Möbel gekauft wurden, keine Zeit für das Lernen der deutschen Sprache aufgewandt wurde und in dem Einschränkungen hingenommen wurden. Allerdings konnte diese Einstellung zum Leben in Deutschland zu einer Art selbsterfüllender Prophezeiung werden: Weil der Horizont des Aufbruchs aus Deutschland immer präsent war, investierten einige meiner Gesprächspartner*innen nicht in ihre Gegenwart in Göttingen und weil sie kein Deutsch konnten und kaum soziale Netzwerke mit „locals" aufgebaut hatten, konnten sie sich gegen Ende ihres Aufenthalts nicht vorstellen, länger in Göttingen zu bleiben. Im Rückblick nach dem Abschluss ihrer Promotion bedauerten einige, dass sie sich nicht mehr auf das Leben in Deutschland eingelassen hatten. 


\subsection{Zeit teilen: Vielfältige Kopräsenzen}

Die Zeit des Einsamen ist nur Mangel an gemeinsam verbrachter, an geteilter Zeit. (Nowotny 1993: 9)

Für den Umgang mit Gefühlen von Einsamkeit und Isolation, wie ich sie anhand der Beispiele von Anay und Ravi dargestellt habe, war es für die jungen Wissenschaftler*innen aus Indien wichtig, Menschen zu haben, mit denen sie ihre Zeit in Göttingen teilen konnten. In diesem Abschnitt liegt mein Fokus auf den lokalen und transnationalen sozialen Beziehungen meiner Gesprächspartner*innen. Ich beziehe beide, die ,nahen' (siehe Ryan 2015) und ,fernen'sozialen Beziehungen in meine Analyse ein, um ihre Gleichzeitigkeit besser zu verstehen. Durch diese Perspektive verdeutliche ich, dass meine jungen Gesprächspartner*innen gleichzeitig in mehreren sozialen Welten verortet waren, ihre Zeit mit unterschiedlichen Menschen teilten und deswegen ihre Zeit aufteilen mussten.

Eine Repräsentation von Zeit, die in vielen Narrationen über soziale Beziehungen vorkam, war Zeit als eine Ressource, die mit anderen geteilt werden kann, die investiert werden muss und die begrenzt ist. In einigen Beziehungen, nämlich in denen $\mathrm{zu}$,alten' Freund*innen, scheint Zeit wiederum keine Rolle zu spielen. Sie werden, wie ich in Punkt 5.2.2 zeige, als „out of time“ (May 2016: 11) wahrgenommen und dargestellt.

Wie bereits mehrfach in dieser Studie angeklungen ist und in den nachfolgenden Kapiteln verstärkt thematisiert wird, waren Medien wie Facebook für die jungen Menschen wichtige Werkzeuge, um in Kontakt zu bleiben, sich mit dem Herkunftskontext zu resynchronisieren und Kopräsenz (vgl. Baldassar 2008) herzustellen. Informations- und Kommunikationstechnologien bieten aber auch das Potential zur Kontrolle und Überwachung (vgl. Madianou 2016: 195; Svašek 2018). Im zweiten und dritten Abschnitt des vorliegenden Unterkapitels zeige ich, wie die jungen Wissenschaftler*innen in der Aushandlung ihrer sozialen Beziehungen im Alltag unterschiedliche Medien einsetzten und wie die Mediennutzung mit den zeitlichen Erfahrungen und der Zeitgestaltung meiner Gesprächspartner*innen interagierte.

\subsubsection{Das Aufbauen neuer Freundschaften}

You are so far away from your family. If you don't have a bunch of good friends who are supportive to you physically, emotionally, it's tough it's really tough. You need a circle of friends. (Rohini, 18.08.2016)

In diesem Abschnitt verdeutliche ich an verschiedenen Fallbeispielen, wie die jungen Wissenschaftler*innen freundschaftliche Beziehungen aufzubauen versuchten und welchen Faktoren sie in diesen Prozessen eine Bedeutung zuschrieben. Die Ausführungen zeigen, was auch Louise Ryan (2015) in ihrer Studie feststellt: „(...) migrants 
cannot simply make friends with whomever they choose; unanticipated obstacles to accessing new networks may be encountered“ (Ryan 2015: 1673).

\section{Freundschaft in Deutschland braucht Zeit, Gelegenheit und "effort"}

Ajay kam 2006 nach Deutschland, um ein Masterstudium in Biomedizintechnik zu beginnen. Er entschied sich dafür, in Hannover zu studieren, weil einer seiner Onkel in der Nähe lebte. Ajay fiel es zu Beginn seines Aufenthaltes schwer, „die Grenzen zu durchbrechen“ (break that boundaries) (Ajay, 14.12.2011) und Freundschaften mit deutschen Studierenden zu schließen. Die gleiche Dynamik wird von Paul Kennedy (2008) beschrieben: Dessen Gesprächspartner*innen berichteten von der „relative social indifference foreigners may encounter from natives who are already locked into their own established family and friendship relationships" (Kennedy 2008: 124; vgl. Sovic 2009). Ajay zog für sich aus diesen ersten Erfahrungen den Schluss, dass er Deutsch brauchen würde, um wirklich „Teil der Gesellschaft“ zu werden - einen Entschluss, den er in den folgenden Jahren tatkräftig umsetzte.

Ajay erklärte mir, er kenne mittlerweile viele Deutsche und wisse, „that people are reserved, and they take their time to get to know each other and to get free with you, and so on." Laut Ajay läge deswegen die Verantwortung für eine erfolgreiche(re) Interaktion bei den indischen Studierenden und Wissenschaftler*innen und den deutschen Interaktionspartner*innen. Er betonte immer wieder während unseres Gesprächs, dass beide Seiten „effort“ in die Interaktionen investieren müssten: „I mean the effort has to be both ways. I mean, Germans - they should recognize that when someone is taking effort, you have to encourage them. And Indians and any foreign people should recognize that they have to take an effort to mix."

In unserem Gespräch fragte ich, ob denn das Wissen um die Rückkehr nach Indien einen Einfluss auf das Interesse habe, mit den Menschen vor Ort zu interagieren. Recht kritisch antwortete er:

That is a bad part of [the] Indian community, and I feel angry about it because some students over here - PhD students - they say, „Ah we are coming here, we do a $\mathrm{PhD}$. Anyway, we are going out. Why learn German? We have the Indian community - we talk to them. Why learn German?" (Ajay, 14.12.2011)

Er meinte, solche Menschen gebe es eben und dagegen könne man nichts unternehmen, allerdings sollten jene, die länger bleiben wollen, ermutigt werden, die Sprache zu lernen und zu interagieren. Allerdings war das die Krux: Mit Abstand die meisten meiner Gesprächspartner*innen wollten nicht in Göttingen, nicht in Deutschland bleiben. Dass sie ihre Zukunft nicht hier sahen, beeinflusste wiederum die Bemühungen, die manche von ihnen willens waren zu machen. Die gleiche Dynamik findet sich auf der Seite der „locals“, die ihre Zeit und Energie nicht unbedingt 
in soziale Beziehungen mit Menschen investieren, die nur für eine gewisse Zeit in Göttingen sein werden (Ryan 2015: 1669).

In Sahils Fall hatte das Bewusstsein von der zeitlichen Begrenztheit seines Aufenthalts eine andere Wirkung. Aus seiner Perspektive sollte die Zeit im Ausland dafür genutzt werden, neue Menschen kennenzulernen und neue Erfahrungen zu machen. „When I go back, there are lots of Indians back home. I don't need to spend time [with Indians] here. Since I'm in this country I want to meet people from Germany I want to see how their society works."

Das Aufbauen von Freundschaften braucht allerdings nicht nur Zeit und effort, sondern auch Gelegenheit (vgl. Ryan 2015). Für Ajay bot sich eine solche, als er 2009 in das Mahatma-Gandhi-Haus in Göttingen einzog. Das Mahatma-Gandhi-Haus wurde 1967 als „Afro-Asiatisches Studentenwohnheim“ gebaut. Die Bewohner*innen sind immer etwa zur Hälfte Deutsche und zur Hälfte internationale Studierende. ${ }^{97}$ Ajay schätzte die internationale Zusammensetzung des Wohnheims und die Interaktionen mit deutschen Studierenden, die dadurch angeregt wurden:

The best part is like when people are from different regions - from different countries - you get the feel of the culture. And they also get the feel of your culture. Maybe for them some things might not be impolite, but for us it might be impolite. These kind of issues you get to understand. (Ajay, 14.12.2011)

In Ajays Aussage wird deutlich, wie wichtig es ist, Orte für die Interaktion zu haben, um die Menschen in der neuen sozialen Umwelt kennenzulernen. Erst durch die Interaktion und das Verbringen von Zeit miteinander (vgl. Flaherty 2011: 110) eignen sich Menschen das Wissen an, das eine wichtige Voraussetzung für Gemeinsamkeit, Beziehungen der Gegenseitigkeit und letztendlich Freundschaft ist.

\section{„Komfortzonen“: Freundschaften unter Studierenden und Wissenschaftler*innen aus Indien}

Viele meiner Gesprächspartner*innen fanden in anderen indischen Wissenschaftler*innen gute Freund*innen - Āmāl beispielsweise, die sich in unserem Interview an die schwierige Anfangszeit erinnerte, in der sie sich selbst, ihre Identität und Handlungen infrage stellte. Die Phase kurz nach der Ankunft in Göttingen beschrieb sie als "phase of conflict within myself“. Nachdem Āmāl bei der Feier der Durga Puja ${ }^{98}$ in Göttingen einige Bengalis getroffen hatte, änderte sich die Situation, und für sie entwickelte sich eine „comfort zone“, wie sie es nannte.

\footnotetext{
${ }^{97}$ Die Idee beim Bau war „ausländischen und deutschen Studierenden eine preiswerte Unterkunft zu bieten und die Kontakte zwischen deutschen und ausländischen Studierenden zu verbessern. "Siehe u.a. die Webseite der Afrikanisch-Asiatischen Studienförderung e.V. (https://www.aasf.de/mahatmagandhi-haus/leben-im-mg-haus/, abgerufen am11.03.2019).

${ }_{98}$ Durga Puja ist ein Fest zu Ehren der Göttin Durga (Flood 1996: 181).
} 
I know that people from my land are here - my own people are here. (...) Nowadays, I feel like I'm not alone here. If I feel bored, I just message any one of my friends and they will come to my place and console me, and now it's a fun time and I want to stay here. The friends circle is something which Germany gave to me. (Āmāl, 20.02.2013)

Das Bewusstsein, eine Komfortzone zu haben, wirkte sich darauf aus, wie Āmāl das Leben in Göttingen erfuhr. Das, was sie als comfort zone bezeichnete, war eine Art Erweiterung der vertrauten sozialen Welt (vgl. Butcher 2010: 27). Hier passte ihr Wissen mit dem sozialen Kontext zusammen und es enstand ein Gefühl von „synchronicity", wie Butcher es nennt (2011: 5). So definieren sich Komfortzonen darüber, dass die Art und Weise, wie miteinander umgegangen wird, vertraut ist, die Verhaltensregeln klar sind (Butcher 2011: 3). Im Gegensatz dazu kann sich Unbehagen einstellen, wenn der Umgang miteinander und die Verhaltensregeln nicht vertraut sind. So erinnerte sich Tanvir an die Feier eines deutschen Freundes und stellte fest: „Their parties are so different from our parties. Dancing with girls, more drinking games, getting drunk, getting to know girls: this attitude is not so easy for me, even now." Indische Parties seien dagegen „share much of useless stuff among each other, take each other's cases, talk nonsense."

Die beschriebenen Komfortzonen bildeten sich aber nicht unbedingt aufgrund einer gemeinsamen Identifikation als ,indisch', sondern aufgrund gemeinsamer Erfahrungen als internationale Studierende in Göttingen oder auf der Basis einer gemeinsamen regionalen Herkunft. Wie einige meiner Gesprächspartner*innen stand Tanvir der Bedeutung einer gemeinsamen Herkunftsregion für die Gruppenbildung ambivalent gegenüber: „I hate this thing a lot.“ Allerdings rechtfertigte er dieses Verhalten als „natürlich“, da die gemeinsame regionale Herkunft eine gemeinsame Sprache und geteilte Erfahrungen bedeuten würden. Wie Tanvirs Aussage deutlich macht, ging er davon aus, dass sich eine comfort zone, und er benutzt hier, ohne dass ich es angesprochen hätte, den gleichen Begriff wie Āmāl, mit denjenigen am „natürlichsten" bildet, die man am besten kennt. Auch Ajay betonte, wie leicht es sei, zu anderen Inder*innen Kontakt aufzubauen (vgl. Jones 2013: 426). Er illustrierte diese Leichtigkeit, indem er mir von einer Begegnung mit einem jungen Inder auf dem Weihnachtsmarkt in Göttingen berichtete. Er sei mit einer Kollegin auf dem Markt gewesen, als er plötzlich von einem Mann angesprochen wurde und mit diesem ins Gespräch kam. Seine Kollegin sei etwas irritiert gewesen und habe gefragt, ob sich die beiden schon vorher gekannt hätten. Tatsächlich hätten sich die beiden dort zum ersten Mal gesehen. Ajay erklärte mir: „Indians have this good quality actually: they never feel ashamed to go and approach their own people. Especially when they are in a foreign land, they know that they are alone, [that] they need many people." Die Leichtigkeit, mit der Beziehungen zu anderen Inder*innen entstanden, führte Ajay auf „indische“ Eigenschaften und Bedürfnisse zurück. Die wahrgenommenen bzw. angenommenen Unterschiede zu den lokalen Studierenden beeinflussten die Bildung freundschaftlicher Beziehungen, was darauf hinweist, dass Freundschaft als 
etwas verstanden wurde, das auf Gemeinsamkeit aufbaut. McKenzie und Baldassar kommen in ihrer Studie zu internationalen Studierenden in Australien zu einer ähnlichen Erkenntnis (McKenzie und Baldassar 2017: 709-10).

\section{"Little India" oder die „magnetische“ Wirkung der indischen Gemeinschaft}

Das Entstehen von Komfortzonen indischer Studierender und Wissenschaftler*innen drückte sich in Göttingen nicht zuletzt räumlich aus. Nachdem ab $2010 \mathrm{im}$ mer mehr junge Inder*innen ihren Weg nach Göttingen fanden, entwickelte sich ein kleines Ballungsgebiet, das zwei meiner Gesprächspartner*innen „little India“ bzw. „Indian colony“ nannten. Dieses befand sich rund um die Philipp-Reis-Straße. Aus meinen Interviews und informellen Gesprächen erfuhr ich, dass viele in diese Gegend Göttingens gezogen waren, weil die Institute, an denen sie arbeiteten oder studierten, in der Nähe waren. Ein anderer wichtiger Grund dafür war, dass sie ihre Wohnungen über die Facebook-Gruppe Indians in Göttingen gesucht und gefunden hatten. Auf dieser Facebook-Seite gab es sowohl Wohnungsangebote von jenen Inder*innen, deren Zeit in Göttingen bald enden würde oder die umzogen, als auch Wohnungsgesuche durch jene, die noch nicht in Göttingen angekommen waren. So war es nicht selten, dass ein ,Neuankömmling aus Indien in eine Wohnung zog, die bereits zuvor von einer oder einem indischen Wissenschaftler*in bewohnt worden war. Einige meiner Gesprächspartner*innen lebten in Studentenwohnheimen, von denen die meisten im Zentrum bzw. im Norden Göttingens lagen. Dass so viele meiner Gesprächspartner*innen recht nah beieinander wohnten, ergab sich also in einigen Fällen dadurch, dass die Wohnungen über transnationale Netzwerke gesucht und gefunden wurden. Allerdings trafen einige bewusst die Entscheidung, in die Nähe anderer junger Inder*innen zu ziehen.

Lali hatte sich zu Beginn ihrer Zeit in Göttingen dazu entschieden, in eine Wohngemeinschaft mit drei deutschen Studentinnen zu ziehen, auch um besser Deutsch zu lernen. Sie hatte bereits einen Masterabschluss im Süden Deutschlands gemacht und während ihres Studiums viel Zeit mit anderen Studierenden aus Indien verbracht. Das wollte sie in Göttingen ändern. Nun hatte sie Anschluss an deutsche Studierende, wünschte sich aber gleichzeitig einen besseren Kontakt zu den anderen jungen Inder*innen. Sie trat bald nach ihrer Ankunft in Göttingen der Vereinigung indischer Studierender und Wissenschaftler*innen bei und war damit formell in die indische Gemeinschaft eingebunden. Nachdem sie zirka ein Jahr in der Wohngemeinschaft mit den drei deutschen Studentinnen gelebt hatte, beschloss Lali umzuziehen. Diese Entscheidung begründete sie mit zwei Faktoren. Zum einen wollte sie gerne näher bei den anderen Inder*innen wohnen. Ihre Wohngemeinschaft lag im Süden Göttingens und sie empfand diese Entfernung zu den indischen Studierenden im nördlichen Teil Göttingens als zu weit. Der zweite Grund für ihre Suche nach einer neuen Bleibe waren ihre Mitbewohnerinnen bzw. der fehlende Kontakt zu diesen. Sie erzählte, dass eine der Mitbewohnerinnen praktisch nie da gewesen sei 
und sie auch die anderen nicht oft gesehen habe, da jede ihren eigenen Tagesablauf und ihren eigenen „Stress" habe.

Lali zog mit einer indischen Freundin zusammen in den Norden Göttingens. Für Lali war es zu diesem Zeitpunkt wichtig, mit jemandem zusammenzuwohnen, mit dem sie den Tagesablauf teilte, so dass sie beispielsweise gemeinsam essen konnten. Mit Navita teilte sie außerdem die indische Herkunft, die Erfahrungen als indische Studierende in Deutschland, alltägliche Schwierigkeiten und persönliche Probleme wie die Einmischung der Eltern in die Partnerwahl. ${ }^{99}$

Dadurch, dass viele der jungen Wissenschaftler*innen ihren Lebensfokus in Göttingen auf ihre Arbeit legten, hatten sie wenig Zeit, um sozial aktiv zu sein. Das Zusammenleben in einer Wohnung oder in einem Häuserblock, einer Straße oder eben einer Gegend, vereinfachte es in dieser Situation, sich zu treffen, gemeinsam zu essen oder einen Film zu schauen, also Gemeinsamkeit zu leben und gemeinsame Zeit zu verbringen, ohne vorher komplizierte Absprachen treffen zu müssen oder längere Anfahrten zu organisieren.

Die „magnetische“ Wirkung der indischen Gemeinschaft, wie Ajay es nannte, war nicht für alle jungen Inder*innen so anziehend wie für Lali. Aditi erklärte, sie habe sich bewusst dafür entschieden, etwas abseits zu wohnen: „It is close to my workplace. It is a little farther from other Indians I know as I am not as social as them and need some privacy" (Aditi, 02.03.2013). Das Leben in Göttingen bot den jungen Inder*innen ein gewisses $\mathrm{Ma}$ an neuen Freiheiten weit weg vom kontrollierenden Blick der indischen Gesellschaft. Aditi wollte die gewonnene Privatsphäre bewusst nicht durch zu starke Nähe zu indischen Studierenden aufs Spiel setzen. Die oben beschriebenen Komfortzonen wirken nicht immer nur positiv, sondern tragen dazu bei, Aspekte des Herkunftskontextes aufrechtzuerhalten, die einige gerne hinter sich lassen würden (vgl. Butcher 2009: 1362).

\section{Freundschaften mit internationalen Studierenden und Wissenschaftler*innen}

Internationale Studierende entwickeln, wie viele Studien festgestellt haben, häufig Freundschaften untereinander (Jones 2013; Ryan 2015; McKenzie und Baldassar 2017). Dies deutet darauf hin, dass das Teilen von Mobilitätserfahrungen eine Rolle bei der Formierung von Freundschaften spielt (Jones 2013: 430). Weitere Gemeinsamkeiten der internationalen Studierenden sind das Bewusstsein von der zeitlichen Begrenztheit ihres Aufenthaltes (Ryan und Mulholland 2014) und die Erfahrung von Differenz und Exklusion (Butcher 2009: 1360).

Kiran erinnerte sich in unserem Gespräch an die Erfahrungen, die er in den ersten Wochen in Deutschland gemacht hatte: „So I came here - I didn't see any Indian." Nach etwa einer Woche nahm er an einem Ausflug in den Harz mit seinen Kolleg*innen teil. „I didn't talk to anybody. There were no Indians inside and everybody is different, and at least in USA everybody talks English." Sie seien ins-

${ }_{99}$ Gesprächsprotokoll nach einem Treffen am 16.01.2013. 
gesamt etwa zehn Personen gewesen und nach und nach hätten sich immer kleinere Gruppen abgespalten, die sich dann in ihrer Muttersprache unterhalten hätten. Nur die wirklich internationalen Studierenden, damit meinte er jene aus Ländern außerhalb Europas, hätten keinerlei Verbindungen (connection) in Deutschland „so they try to mingle." Die Mehrzahl seiner Freund*innen seien internationale Studierende „because they have no access, they have nobody to look, so they have to look for outside people. (...) Whenever I go to any club or pub, I see all international students." Diese Erfahrung in Kombination mit den Schwierigkeiten, die er bei der Wohnungssuche hatte, habe ihn erschreckt (terrified) und er habe bereits geplant, Deutschland zu verlassen und wieder in die USA zurückzukehren (Kiran, 02.10.2010). Eine Freundin hatte ihm geraten, noch etwas zu warten, und so blieb Kiran letztendlich bis zur Fertigstellung seiner Doktorarbeit in Göttingen.

Ajits Freundeskreis bestand ebenfalls vor allem aus internationalen Studierenden. Diese hatte er unter anderem in seinem Deutsch-Sprachkurs, den er bereits während seines Bachelorstudiums in München absolviert hatte, kennengelernt. Darunter war ein Freund, der aus dem Iran kam und dem er nach seinem Bachelor-Abschluss nach Göttingen gefolgt war. In München habe er mit seinem Freund viel Zeit verbracht, viele Partys gefeiert; in Göttingen „setze sich diese Geschichte nun fort“ (story continued). Ajit betonte, dass es ihm wichtig sei, Beziehungen über Grenzen der nationalen Zugehörigkeit hinaus zu haben: „When you go to Germany, I think it's really nice to get to know some people from around. I know a lot of - a million people from India already by now and two more will not bring anything more" (Ajit, 14.07.2015). Was das „Mehr“ sein soll, das soziale Beziehungen mit sich bringen, führt er an dieser Stelle nicht aus.

Ajit grenzte sich bewusst von den indischen Studierenden ab, die aus seiner Sicht ihre Zeit vor allem mit anderen Inder*innen verbrachten. Was er dabei ausklammerte, war, dass diese nicht unbedingt bewusst eine Entscheidung für oder gegen indische oder deutsche/internationale Freund*innen getroffen hatten, sondern, wie oben beschrieben, auf unerwartete Hindernisse getroffen waren. Ajit sprach von Zeit als einer Ressource, die bestimmten Aktivitäten und sozialen Beziehungen zugeteilt werden muss (spend time) und von der nicht unendlich viel zur Verfügung steht (need to have time). Er betonte, dass er sich bewusst dazu entschieden habe, seine Zeit mit ,Nicht-Indern' zu verbringen, eine Betonung, die mit seinem Selbstverständnis als ,globaler Inder“ einherging, das er mit der folgenden Aussage ausdrückte: „The whole planet is home“ (Ajit, 14.07.2015).

Die jungen Wissenschaftler*innen etablierten in Göttingen Freundschaften mit Menschen, durch deren Präsenz sie sich wohlfühlten, durch die sie weniger einsam waren, die sie in ihrem Selbstverständnis bestärkten (vgl. Butcher 2009). Die Basis der Beziehungen waren häufig wahrgenommene Gemeinsamkeiten wie eine gemeinsame regionale Herkunft, geteilte Mobilitätserfahrungen oder das Wohnen im gleichen Wohnheim. Die Bedeutung der Freundschaften in Göttingen lag vor allem in ihrer Präsenz, sowohl räumlich als auch zeitlich, sie waren für meine Gesprächspartner*innen nah und da. 
In allen Narrationen von (fehlenden) Freundschaften wurde deren Relevanz für das Selbst deutlich (vgl. Smart et al. 2012; May 2013). Besonders in den Erzählungen von Situationen, in denen sich die jungen Inder*innen ausgeschlossen fühlten, offenbarte sich die Wirkung, die diese auf das Selbstverständnis hatte: Die jungen Menschen stellten sich und ihr Verhalten infrage und suchten bei sich selbst und den ,Anderen' eine Erklärung für missglückte Interaktionen.

Aus unterschiedlichen Gründen fanden es einige meiner Gesprächspartner*innen schwer, lokale Beziehungen aufzubauen und konzentrierten sich auf ihre bestehenden transnationalen Beziehungen und darauf, dass ihr Aufenthalt in Göttingen begrenzt ist. Die sozialen Beziehungen veränderten sich über die Zeit hinweg, wie das Beispiel von Ajay gezeigt hat.

\subsubsection{Alte` Freundschaften: Über Raum und Zeit bestehende soziale Beziehungen}

Die Wissenschaftler*innen aus Indien erhielten durch unterschiedliche Praktiken und mit Hilfe verschiedener Medien ihre, alten' Freundschaften aus der Zeit vor der Ankunft in Göttingen aufrecht. Im Folgenden stehen zwei Aspekte dieser Beziehungen im Fokus: ihre mögliche Allgegenwärtigkeit und ihre Kontinuität über Raum und Zeit.

\section{Die Allgegenwärtigkeit Anderer}

Viele der jungen Wissenschaftler*innen nutzten Facebook, um mit einer größeren Gruppe an Bekannten in Kontakt zu bleiben. Facebook sei sehr praktisch, um sich nicht aus den Augen zu verlieren, erläuterte Anisha. Durch Facebook könne sie verfolgen, was im Leben der anderen passiere. Lachend erzählte sie: „I got to know that almost all my friends got married. They update their Facebook status and some got pregnant and they got babies." Auch Anay benutzte Facebook, um verbunden zu bleiben (being connected) und grinsend verwies er auf eine weitere Funktion Facebooks: „You get to know what people are doing, and you can also show off yourself - what you are doing, where your've been" (Anay, 15.07.2015). Tanvir benutzte Facebook vor allem, wenn er sich einsam fühlte: „You stalk some people on Facebook and send them a friend request. It's so interesting to know about someone elses life especially when you are lonely" (Tanvir, 17.02.2013). Tanvir hatte zum Zeitpunkt des Verfassens dieser Arbeit etwas mehr als 1.400 Freund*innen auf Facebook.

Wie Mirca Madianou (2016) verstehe ich die durch Anisha, Anay und Tanvir beschriebene Nutzung von Facebook nicht als eine passive Aufnahme von Inhalten, sondern als einen aktiven Prozess, der zu Erfahrungen von „ambient co-presence“ beiträgt. Unter ambient co-presence versteht Madianou „the peripheral, yet intense, awareness of distant others (...)" (Madianou 2016: 198). Soziale Medien wie Facebook haben Funktionen wie den Newsfeed, der als Startseite fungiert und auf dem Nutzer*innen in Echtzeit über die Aktivitäten anderer informiert werden. Der Newsfeed ist eine sich ständig aktualisierende Liste von Fotos, Artikeln, Links zu 
anderen Seiten, Videos, Likes etc. (Madianou 2016: 188) und wird von den Facebookkontakten der Nutzer*innen und deren Kontakten befüllt. Gemeinsam mit den anderen Funktionen, wie der integrierten Intant Messaging-Plattform, die anzeigt, wer gerade online ist und wann wer zuletzt online war, stellt Facebook eine Allgegenwärtigkeit der Aktivitäten anderer her (Madianou 2016: 188). Besonders in Kombination mit der Nutzung von Smartphones, auf denen Facebook aufgerufen werden kann, und der Verbreitung von drahtlosen Netzwerken entsteht ein „always on' lifestyle" (Madianou 2016: 188). Viele meiner Gesprächspartner*innen nutzten Facebook auf diese beschriebene Art und Weise und konnten so ein anhaltendes Gefühl der Kopräsenz von Freund*innen und Familie in Indien und anderen Teilen der Welt herstellen. Praktiken, wie das „Checken“ von Facebook gehörten bei einigen zur täglichen Routine. Andere vermieden entweder die Nutzung von Facebook generell oder schränkten diese ein. Viele der jungen Wissenschaftler*innen filterten ganz bewusst, was sie auf Facebook preisgaben. Diese Praxis stand mit einer Eigenschaft Facebooks im Zusammenhang, die ich besonders in Verbindung mit der Kommunikation mit den Eltern thematisieren werde - die Möglichkeit, Kontrolle auszuüben.

Neben dieser ambient co-presence durch indirekte Kommunikation, wählten die jungen Wissenschaftler*innen direktere Wege, um mit ihren Freund*innen in Kontakt zu bleiben. Rohini telefonierte mit ihren Freund*innen über die Instant Messaging-Dienste WhatsApp und Viber. Diese Medien, so erzählte sie mir enthusiastisch, hätten die Welt geschrumpft (shrank the world): „you can call whenever you want to call; you can write a text if something happens." Instant Messaging-Dienste wie WhatsApp und Viber ermöglichen sowohl synchrone Kommunikation über (Video-) Telefonate als auch asynchrone Kommunikation über Text- und Sprachnachrichten. Durch die Verwendung dieser Nachrichtendienste auf Smartphones kann praktisch jederzeit kommuniziert werden.

\section{Gleichbleibende Beziehungen, gleichbleibendes Selbst}

In den Gesprächen mit ihren Freund*innen würde sie sich nicht nur über ihre momentane Lebenssituation austauschen, sondern Erinnerungen teilen (share a memory), erzählte Rohini. Das Teilen von Erinnerungen ist ein wichtiger Aspekt dieser sozialen Beziehungen, die sich durch ihre Dauer und die gemeinsame(n) Geschichte(n) auszeichnen. Wie Vanessa May aufzeigt, gibt es Beziehungen, die Menschen als „out of time“ (May 2016: 5) wahrnehmen. Diese Wahrnehmung wurde in meiner Studie in der Art und Weise sichtbar, wie meine Gesprächspartner*innen von diesen Beziehungen sprachen bzw. wie sie über diese auf Facebook schrieben. Rohini schrieb in einem Beitrag über ihre beiden besten Freundinnen: „It's even more strange how some best friends stay forever even though we are miles apart." Das „für immer" in Rohinis Aussage wirkt wie eine „Prophezeiung“ (May 2016: 9), ein Versprechen, dass die Freundschaft auch in Zukunft halten wird. Rohini erklärte aber ebenso, dass es anders sei, wenn diese Freund*innen tatsächlich körper- 
lich anwesend sind: „It's a different feeling when you have them next to you. “ Sie traf sich wenn möglich einmal im Jahr mit ihren Freund*innen zu „reunions“ in Indien. Face-to-Face-Beziehungen verlieren durch die transnationalen Beziehungen mittels Informations- und Kommunikationstechnologien nicht ihre Relevanz (Baldassar et al. 2016). Beide Praktiken - die tatsächlichen Treffen als auch die Kommunikation über verschiedene Medien - können, wie Baldassar ausführt, als unterschiedliche Formen von Kopräsenz verstanden werden (Baldassar 2008).

Akhil betonte wie Rohini, dass manche Beziehungen sich nie ändern würden: „Meeting after 6 years, and some things actually never change with time." Diese Freundschaften bewegen sich außerhalb der linearen Logik von ,Entwicklung' und Veränderung und artikulieren das Gleichbleiben der Beziehungen und damit das Gleichbleiben des Selbst. Solche Freundschaften waren signifikant für ein Gefühl der Kontinuität und Sicherheit, der Vertrautheit in Zeiten von Veränderungen durch Mobilität. Damit erfüllten sie für das Selbst der jungen Wissenschaftler*innen einen anderen Zweck als die lokalen Beziehungen in Göttingen. Für die meisten meiner Gesprächspartner*innen war es wichtig, beides zu haben: lokale, nahe Freundschaften und alte, anhaltende Beziehungen.

\subsubsection{Familie: Kontinuität und Kontrolle in Zeiten von ,Polymedia}

Wie Kaur und Shruti unter anderem mit Verweis auf Arbeiten von Osella und Osella (2008) für den ,asiatischen Kontext' argumentieren, bleiben Familienmitglieder durch Beziehungen verbunden, die in ein System gegenseitiger Verpflichtungen und Verantwortungen eingebettet sind (Kaur und Shruti 2016: 81; vgl. Nedelcu und Wyss 2016). In Kapitel 2 habe ich besonders die Verpflichtungen zwischen den Generationen beschrieben und gezeigt, welche Dynamik durch diese in den Familien entsteht. Dazu gehörte die starke Kontrolle, die von der Familie über die jüngere Generation ausgeübt wird, beispielsweise in Bezug auf die Auswahl von Studienfächern.

Das Aufrechterhalten familiärer Beziehungen stellten meine Gesprächspartner*innen als selbstverständlich dar. In einer Situation, die durch das Bewusstsein der zeitlichen Begrenztheit geprägt war, waren die anhaltenden familiären Kontakte meines Erachtens besonders wichtig und eine Quelle von Zugehörigkeit und Sicherheit. Für das Aufrechterhalten der Beziehungen zu den Eltern spielten unterschiedliche Kommunikationstechnologien eine wichtige Rolle. Die Kommunikationstechnologien sind Teil einer "polymedialen Umwelt“ (Madianou und Miller 2012; Madianou 2016) in der die Nutzer*innen unterschiedliche Plattformen aus einer Reihe von Möglichkeiten aussuchen können. Die Auswahl des Mediums wurde unterschiedlich begründet. Einige meiner Gesprächspartner*innen erzählten, dass ihre Eltern kein Internet hätten oder nicht mit der Nutzung der entsprechenden technischen Geräte vertraut wären. Allerdings kann die Auswahl der Kommunikationsmedien im Sinne von Madianou und Miller (2012) bereits als ein Schritt in der Aushandlung der Beziehungen verstanden werden. Medien haben in ihrer Nutzung unterschiedliche so- 
ziale und emotionale Konsequenzen (Madianou und Miller 2012: 170; Svašek 2018) und Einfluss auf die Erfahrung von (A)-Synchronität. Im folgenden Kapitelabschnitt steht im Fokus, wie meine Gesprächspartner*innen sich mit ihren Familien über die Häufigkeit und das Timing von Kontakten verständigten, ihre Zeitsouveränität und Erfahrungen von Zeit aushandelten und in diesem Prozess ihre Zugehörigkeiten navigierten.

\section{Alltägliche Kopräsenz und Resynchronisation}

Anay telefonierte jede Woche mehrfach mit seiner Mutter und seinen Großeltern: „I normally call them twice or thrice a week - like maybe two times with my mother and one time to my grandparents. So thats how I divide - but ja, I make sure that I do call them at least twice or thrice or four times in a week" (Anay 15.07.2015). Die Gespräche würden aber nur zwischen zwei und vier Minuten dauern. Er melde sich einfach kurz und sie würden fragen: „What did you eat?“ Als er in London gewesen sei, habe er jeden Tag angerufen, weil es billiger gewesen sei als in Deutschland.

Wie Maruška Svašek thematisiert (2018: 37), beeinflussen die Kosten für Telefonate, Kurznachrichten etc. die Häufigkeit der Kommunikation. Wobei günstige Preise die Möglichkeit bieten, häufiger zu kommunizieren, aber ebenso die Erwartungen an die Frequenz der Kontakte erhöhen können (Svašek 2018: 37). Sarjana telefonierte jeden Tag mit ihrer Mutter: „Ich fühle mich auch gut, wenn ich einmal mit ihr jeden Tag spreche, sie ist wie meine Freundin." Sie sprachen über alltägliche Ereignisse wie das Essen und die Arbeit. Es fühle sich fast so an, als sei sie nur in einer anderen Wohnung und nicht in einem anderen Land. Allerdings bemerkte sie, dass sie manchmal müde sei und nicht unbedingt telefonieren wolle: „Aber ja, ich bin ein Einzelkind, ich verstehe meine Eltern vermissen mich auch, das ist normal" (Sarjana, 12.08.2013). Wie die Aussagen von Anay und Sarjana zeigen, war die Kommunikation eine Routine, mit der sie ein Gefühl der Kontinuität und der Gleichzeitigkeit mit der Familie herstellen konnten (vgl. Nedelcu und Wyss 2016). Ähnlich wie für die freundschaftlichen Beziehungen beschrieben, entstand durch diese alltägliche Kommunikation ein Gefühl von ambient co-presence.

Die Medien, die meine Gesprächspartner*innen nutzten, boten unterschiedliche Möglichkeiten und Herausforderungen beispielsweise für das Verschweigen von Informationen oder auch von Emotionen (vgl. Svašek 2018; Madianou und Miller 2012). Sarjana entschied sich bewusst dafür, am Telefon mit ihren Eltern nicht über schwierige Themen, die längere Erklärungen verlangten oder in ihren Eltern Ängste auslösen könnten, zu reden.

\section{Informations- und Kommunikationstechnologien und Kontrolle}

Die Beziehungen zwischen Familienmitgliedern werden stärker als beispielsweise Freundschaften von Verantwortlichkeiten und Verpflichtungen füreinander geprägt. Demzufolge hat die familiäre Dynamik, die ich in Kapitel 2 beschrieben habe, auch 
einen Einfluss auf die Kommunikation über die Distanz hinweg. Ein wichtiger Aspekt ist die Kontrolle, die viele Eltern auch über die Entfernung hinweg versuchen auszuüben - Kontrolle über die alltäglichen Aktivitäten und wichtige Entscheidungen für den weiteren Verlauf des Lebens.

Sangita kommunizierte mit ihrer Familie fast täglich auf die eine oder andere Weise: „I usually call them. It's quite frequent sometimes - it could be daily. I either talk to my sister - I stay in touch with my sister via Facebook - or I call my parents. So either one of the two happens at least every day, or at least every alternate day" (Sangita, 08.08.2013). Als ich in meinem Gespräch mit Sangita feststellte, dass es wohl für sie sehr wichtig sei, mit ihren Eltern in Kontakt zu bleiben, antwortete sie lachend: „And it's very important for them to stay in touch with me. The thing is that they are so used to being in touch with me, if I'm not in touch for a few days they get very worried: ,What happened? How come you were not seen online?"“ (Sangita, 08.08.2013). An dieser Stelle wird deutlich, dass die Möglichkeit zur ständigen Kommunikation die Verpflichtung zu eben dieser mit sich bringen kann (vgl. Nedelcu und Wyss 2016). Sangitas und auch Sarjanas Eltern erwarteten von ihren Töchtern den täglichen Kontakt und in Sangitas Fall nutzten die Eltern Facebook, um ihre Online-Aktivität zu überprüfen. Informations- und Kommunikationstechnologien können zu einem Mittel der Kontrolle werden. Gerade Facebook spielt eine sehr ambivalente Rolle. Einerseits ermöglicht es, mit vielen Menschen gleichzeitig in Kontakt zu bleiben, indem Nachrichten ausgetauscht werden oder indem kurze Texte zu alltäglichen und besonderen Ereignissen erstellt und veröffentlicht werden. Andererseits erlaubt es den anderen Menschen, die auf Facebook sind, die eigenen Aktivitäten zu verfolgen. Einige meiner Gesprächspartner*innen waren gerade beim Hochladen von Fotos auf Facebook sehr vorsichtig, um zu verhindern, dass die Eltern zu viel von ihrer Freizeitgestaltung und sozialen Beziehungen in Göttingen mitbekamen.

Die Kommunikationstechnologien können genutzt werden, um ständig an Verpflichtungen und elterliche Erwartungen erinnert zu werden (vgl. Svašek 2018: 37), wie beispielsweise in Kirans Fall. Kiran erzählte mir, dass seine Eltern ihn an Rama Navami, dem Feiertag, an dem Ramas Geburtstag gefeiert wird, angerufen und gefragt hätten: „Did you take the shower, and do you have new clothes?" Dieser Feiertag wird unter anderem durch das Kaufen neuer Kleidung zelebriert, einem Ritual, das Kiran in Deutschland mit der Begründung, dass Kleidung hier zu teuer sei, nicht durchführte. Kiran schilderte mir in unserem Gespräch, dass seine Eltern ihn bei Telefonaten fragen würden, ob er denn die indischen Nachrichten schaue und indische Zeitungen lese. Seine Eltern würden verhindern wollen, dass er den Kontakt verliert (disconnect), und hätten Angst, dass er möglicherweise nicht mehr nach Indien zurückkehren könnte (vgl. Sondhi 2013: 152).

Die jungen Wissenschaftler*innen nahmen sich bewusst Zeit für das Aufrechterhalten der familiären Beziehungen, für Telefonate, Gespräche über Skype oder die Kommunikation per Facebook oder E-Mail. Die Familien ihrerseits - vor allem die Eltern - pochten unterschiedlich intensiv auf den regelmäßigen Kontakt und 
forderten ihre Söhne und Töchter dazu auf, ihre Zeit in Praktiken zu investieren, die dazu beitragen sollten, die Verbindung zu Indien nicht zu verlieren. Meine Gesprächspartner*innen mussten somit ihre Zeitsouveränität und Vorstellungen von der angemessenen Zuteilung von Zeit über die Distanz hinweg aushandeln.

Ein Mittel, um eine stärkere Kontrolle über die Kommunikation mit den Eltern zu bewahren, war die bewusste Wahl von Telefonaten und festen Zeiten für die Gespräche. Gilon wählte diese Strategie: „Every Saturday I make phone calls, every Saturday, once in a week. Sometimes when there is a birthday or something I also call during week ja but Saturday is my chatting tag with my mother and father" (Gilon 19.01.2013).

Telefonate und andere Mittel der synchronen Kommunikation erfordern die zeitliche Koordination. Dieser Umstand führt, neben möglichen Problemen mit der Verbindung oder der Technik, die Grenzen der Technologie vor Augen (vgl. Svašek 2018: 42-43).

\section{Informations- und Kommunikationstechnologien und Asynchronität}

Saulo Cwerner sagte 2001 voraus, dass das Internet mit seinen Möglichkeiten einen großen Einfluss auf die Erfahrung von Asynchronität haben wird (Cwerner 2001: 23). Tatsächlich können Informations- und Kommunikationstechnologien das Gefühl vermitteln, Raum und Zeit zu teilen (Wilding 2006: 133). Allerdings weisen unterschiedliche Studien auch auf die Grenzen der Technologie hin (Wilding 2006; Svašek 2018).

Ajay telefonierte möglichst jeden Tag mit seinen Eltern, eine Routine, die allerdings im Winter durch die Zeitumstellung schwieriger beizubehalten war: „Because it's like four and a half hours time difference; so when I come back home it's late night over there." Je nachdem, welches Medium für die Kommunikation bevorzugt wurde, konnte das Leben in unterschiedlichen Zeitzonen die Kommunikation erschweren bzw. eine genaue Koordination erfordern, um einen günstigen Zeitpunkt für alle Beteiligten zu finden. Besonders bei Telefonaten oder Videochats wurde die Asynchronität spürbar. Viele meiner Gesprächspartner*innen standen mit ihren Eltern über Telefonate in Kontakt. Vendan vermisste in der Kommunikation mit seinen Eltern vor allem die Unmittelbarkeit. Er verwies wie Ajay auf die Zeitzonen und die damit einhergehende Notwendigkeit, Telefonate auf den nächsten Tag oder das Wochenende zu verschieben, auch wenn gerade etwas passiert war, über das er gerne mit den Eltern gesprochen hätte. Für Sandeep war genau dieses Bewusstsein der Distanz, die die Kommunikation hervorrufen würde, ein Grund, nicht so häufig mit den Eltern zu telefonieren: „It's very bad thinking of mine - but what I feel [is] that it's good that, you can contact them, you can be in touch. But I don't think it should be very overly done“" (Sandeep, 09.04.2013).

Laut Sandeep würde die Kommunikation mit den Eltern das Gefühl der Distanz nicht verringern, sondern dieses eher in das Bewusstsein rufen. Das heißt, Informations- und Kommunikationstechnologien eliminieren nicht unbedingt die Wirkung 
der räumlichen und zeitlichen Distanz (vgl. Wilding 2006: 138). Im Gegenteil können sie das Gefühl der Asynchronität auch verstärken.

Für die jungen Wissenschaftler*innen waren die multiplen sozialen Beziehungen, die im Fokus dieses Kapitelabschnitts standen, essentiell für die Gestaltung ihres Alltags. Sie halfen ihnen, mit Gefühlen der Isolation und der Einsamkeit umzugehen und Komfortzonen und Komfortzeiten in der neuen sozialen Umwelt zu etablieren. Für meine Gesprächspartner*innen existierten die Beziehungen zu den Eltern, zu Personen in Göttingen und zu alten Freund*innen in Indien und auf der ganzen Welt gleichzeitig. Für die biographische Navigation meiner Gesprächspartner*innen erfüllten alle diese Beziehungen unterschiedliche Zwecke. Durch die andauernden Kontakte zu den Familien und den alten Freunden konnten sie ein Gefühl der Kontinuität und der anhaltenden Zugehörigkeit herstellen (vgl. May 2016). Diese Kontakte verkörperten Verbindungen in die eigene Vergangenheit und in die Zukunft und verankerten das Selbst der jungen Menschen auf der diachronen Ebene. Die Freundschaften in Göttingen zeichneten sich wiederum durch ihre Präsenz aus. Mit den Freunden dort teilten sie ihre Erfahrungen und ihre Zeit vor Ort.

Unterschiedliche Informations- und Kommunikationstechnologien spielten für das Herstellen vielfältiger Formen von Kopräsenz und für die Resynchronisation eine entscheidende Rolle. Die verwendeten Medien hatten unterschiedliche Konsequenzen für die zeitlichen Erfahrungen, die Zeitgestaltung und die Zeitsouveränität. Die Auswahl unterschiedlicher Medien für die Kommunikation wurde unter anderem von der bestehenden Beziehungsdynamik beeinflusst und wirkte wiederum auf die Beziehungen ein.

\subsection{Routinen im Alltag}

[I] go to work, come home, eat, sleep, go to work, come home, eat, sleep. (Sachin, 22.09.2011)

Der menschliche Alltag sieht in einigen wesentlichen Elementen vermutlich überall so ähnlich aus wie der von Sachin. Die konkreten Praktiken und Abläufe im Alltag unterscheiden sich allerdings und bergen in sich das Potential, Sicherheit zu vermitteln (Wilk 2013), Konflikte anzustoßen (Ehn und Löfgren 2013) und Erfahrungen des Nicht-Dazugehörens auszulösen (May 2013: 89). Wie bereits dargelegt, hatten die jungen Wissenschaftler*innen durch ihr Leben in Göttingen mehr Kontrolle über die Gestaltung ihres Alltags und darüber, wann sie welcher Tätigkeit nachgehen wollten. Viele meiner Gesprächspartner*innen entschieden sich dazu, vertraute Praktiken aus Indien in ihren Alltag zu integrieren und damit Kontinuitäten zu ihrem Leben vor der Ankunft in Deutschland herzustellen (vgl. Butcher 2011). Für einige gehörten religiöse Praktiken zur alltäglichen Routine - beispielsweise für Smita, die erzählte, dass sie jeden Morgen eine Kerze anzünde und ein kurzes Gebet spreche: „It gives me a positive energy. When I light the candle, it gives a very nice 
feeling" (Smita, 05.08.2010). Für Gilon und Ajay war es der sonntägliche Kirchenbesuch, der einen Rhythmus in die Woche brachte und eine kontinuierliche Verortung generierte.

Ich konzentriere mich in der folgenden Analyse der alltäglichen Routinen auf die Themen Kochen und Essen. Essen verstehe ich in Anlehnung an Francis Leo Collins als Praxis, um Vertrautheit im transnationalen sozialen Raum herzustellen (Collins 2008; vgl. Hage 1997) und weniger als Ausdruck von Identifikationen. Im Zentrum meiner Ausführungen stehen die ,zeitlichen' Eigenschaften des Essens - also die Häufigkeit und das Timing - und die Bedeutung, die diesen zugeschrieben wurde.

\subsubsection{Kochen lernen}

Das Kochen und Essen indischer Gerichte wurde von vielen meiner Gesprächspartner*innen als wichtige alltägliche Praxis hervorgehoben. Im Gegensatz zu den jungen Frauen aus Indien mussten die meisten Männer allerdings erst lernen, zu kochen (vgl. Ajay Bailey 2017). In Indien wird es vor allem für Frauen als wichtig erachtet, kochen zu können. Deswegen wird in vielen Familien nur den Töchtern das Kochen beigebracht. Während des Studiums wohnen viele junge Inder*innen in Studentenwohnheimen, die in Indien nicht mit Küchen, sondern mit Kantinen ausgestattet sind, so dass es dort nicht die Gelegenheit gibt, zu kochen. Mit diesem Umstand begründete mir Anay, der das Kochen mittlerweile zu einem seiner favorisierten Zeitvertreibe (favorite pastime) erklärt hatte, warum er erst außerhalb Indiens gelernt hat, zu kochen. In Göttingen gehörte das Kochen zu Anays Alltag, zu den Praktiken, die er nach der Arbeit und manchmal zusammen mit seinen Freunden vom Institut durchführte. „When I leave the center, I cook. That's my one other pastime: I love to cook and it's refreshing for me." Auf meine Frage, was er denn koche, antwortete Anay: „I try to also cook non-Indian food, but it's always very bad. I know a bit of Italian, but with that Indian style, I would love to try lasagna." Allerdings kenne er sich mit den Lebensmitteln in Deutschland nicht aus. Lachend erklärte er: „I try to get also some German food like, bread, cheese, but I really don't like it to be very frank. It's just sometimes I'm hungry. But mostly it tends to be Indian or Italian - sort of like pizza" (Anay, 15.07.2015). Wie viele meiner Gesprächspartner*innen konnte sich Anay nicht für die deutsche Küche erwärmen, wobei er diese vor allem mit Brot und Käse assoziierte - eine Assoziation, die von vielen der jungen Inder*innen geteilt wurde. Anay lernte das Kochen von Freunden, mit denen er in London zusammengewohnt hatte. Meine Gesprächspartner*innen konsultierten für die Zubereitung spezifischer Gerichte neben ihren Freunden in Göttingen auch ihre Mütter über das Telefon oder zogen Youtube-Tutorials heran.

Ajit erinnerte sich in unserem Gespräch an die erste Zeit in Deutschland und seine anfänglichen Kochversuche. Um zu demonstrieren, wie wenig er vom Kochen wusste, erzählte er amüsiert, dass er noch nicht einmal ein Ei habe kochen können: „I take out this egg tray and ... I was confused. So, should I just put it in the cold 
water? Or should I boil the water and then put it in? And then the second question was if I should open the egg and then put it in?" (Ajit, 14.07.2015).

Anay und Ajit lernten, indische Gerichte zu kochen, und konnten dadurch Vertrautheit wiederherstellen und dem deutschen Essen ,entkommen'. Praktisches Wissen - das zeigen die Fallbeispiele - ist von großer Bedeutung, wenn es darum geht, den Alltag in einer neuen sozialen Welt zu reproduzieren (vgl. Collins 2008). In ihrem Alltag in Indien gehörte für die meisten meiner Gesprächspartner*innen das Kochen nicht dazu. Dort gab es häufig Mütter, Großmütter oder Hausangestellte, die das Kochen übernahmen. Was zu ihrem Alltag gehörte, war das Essen, je nach Region und kulinarischen Vorlieben, von Dosas, Pani Puri, Biryanis, Dal, Rotis, Reis etc. Wenn sie diese vertrauten Speisen in Deutschland haben wollten, mussten sie lernen, diese zuzubereiten. Im Zuge dessen erweiterten sie nicht nur ihre Kenntnisse, sondern entdeckten in einigen Fällen ihre Leidenschaft für das Kochen und etablierten neue Routinen in ihren Alltag.

Nicht alle meine Gesprächspartner*innen legten so großen Wert auf indisches Essen, dass sie ihre Freizeit dem Kochen gewidmet hätten. Einige waren mit dem Essen in der Mensa zufrieden, freuten sich dann aber umso mehr auf die Feierlichkeiten und Treffen mit Freunden, bei denen gemeinsam gekocht oder indisches Essen bestellt wurde.

\subsubsection{Kochen als alltägliche Routine: Ein bisschen Indien am Abend}

Als ich Ajay nach dem Ablauf seines Alltags befragte, erklärte er mir, dieser sei eigentlich ganz langweilig:

I get up, go to the institute, and you don't know when you come back: you might come back early or late - it depends on your work and your motivation - and then come back home, cooking. Indian food mostly because in the afternoon I eat in the Mensa, but in the evening I come back home. I cook fast food but in an Indian way. But I want little bit Indian in the evening - like some rice and all these stuffs. (Ajay, 14.12.2011)

„Indian“ steht in der Aussage von Ajay für das Vertraute, Bekannte und nicht unbedingt für die Identifikationen, die mit diesem Wort artikuliert werden. Reis wurde von einigen meiner Gesprächspartner*innen als Beispiel genannt, um zu kennzeichnen, was indisches Essen sei, dagegen wurde Brot paradigmatisch für die deutsche Küche herangezogen. Als ich Nandita auf die offensichtlich so große Bedeutung von Essen ansprach, lachte sie und erzählte, dass ihre Kolleg*innen erstaunt seien, dass sie jeden Tag koche. Für sie sei es aber für das Überleben (survival) unverzichtbar: „I cannot have bread and cheese twice in a day." Ajay und Nandita stellten über das Kochen und Essen indischer Speisen Vertrautheit in ihrem Alltag her. Beide betonten die Häufigkeit und Alltäglichkeit ihrer Routinen und die große Bedeutung, die diese für sie haben, um sich wohlzufühlen bzw. um zu „überleben“. 
Die große Bedeutung des Essens drückte sich auch in den Gegenständen aus, die aus Indien mitgebracht wurden. Die Vereinigung indischer Studierender listet auf ihrer Webseite unter der Frage "What to bring along with you and what to avoid“ der Kategorie „Frequently Asked Questions“ zweiundzwanzig Dinge auf. Als erster Gegenstand wurde ein Gerät aufgelistet, das sich in den meisten Küchen wiederfand, die ich während meiner Forschung betrat: ein Dampfkochtopf. Auch die folgenden neun Dinge, die aufgezählt wurden, waren entweder Lebensmittel oder Gegenstände, die zur Zubereitung von Essen gebraucht werden, wie beispielsweise eine Kokosnussreibe.

Alltägliche Praktiken bieten nicht nur die Möglichkeit, Vertrautheit herzustellen, sondern bergen in sich auch Konfliktpotential (Ehn und Löfgren 2013: 106). In Gilons Fall wurde dies besonders deutlich. Gilon kochte jeden Tag für sich selbst indisches Essen und war durchaus stolz auf seine Kochkünste: „Food I don't miss, because I cook. I'm a good cook, I can proudly say (...). " Er kochte häufig für sich und für Freunde. Dafür brauchte er durchaus einige Zeit, was, wie er mir beschrieb, für ihn selbstverständlich war, aber unter seinen nicht-indischen Freunden Erstaunen hervorrief: „(...) there are so many friends they even made fun of me. I didn't realize in the beginning that I spend a lot of time in the kitchen."

Gilons Alltagspraxis fand allerdings in dem Wohnheim, in das er nach seiner Ankunft in Göttingen im Oktober 2009 gezogen war, nicht immer Zustimmung. Er erzählte mir, dass er gemeinsam mit einem Freund sehr häufig in der Wohnheimküche gekocht und sie viel Knoblauch beim Kochen benutzt hatten. Daraufhin beschwerte sich eine seiner Mitbewohner*innen, dass ihre Kleidung, die sie im Flur zum Trocknen aufgehängt hatte, ständig nach Knoblauch rieche.

I mean, even for me as an Indian I even thought, „Okay, no, I think this is not the issue with garlic - because garlic cannot make an issue. I think she has some issue with me personally." You know, something like that. But it took such a long time to understand: so, yes it can be also a point for some people. So, things like that, very small but still there - the Indian identity is very important. (Gilon, 19.01.2013)

Das Leben in einem Studentenwohnheim, einer Wohngemeinschaft oder einer Partnerschaft bedeutet, dass Routinen ausgehandelt werden müssen. In Gilons Fall führten sie zu Konflikten, die ihm seine eigenen Praktiken bewusst machten. Praktiken, die zum eigenen Alltag gehören und Vertrautheit vermitteln, können Fragen der Zugehörigkeit und der Identifikation aufwerfen. Das Gefühl, nicht dazuzugehören, kann entstehen, wenn die eigenen Routinen immer wieder auf Widerstand stoßen. Gilon erklärte mir in unserem Interview, dass er durchaus eine schöne Zeit im Wohnheim gehabt habe „but it was something that I could not identify myself with this community. And at home I had always the possibilities of being to myself."

Gilon zog nach einem halben Jahr aus dem Wohnheim aus und entschied sich dazu, alleine zu wohnen. Durch diesen Schritt verminderte er die Aushandlungs- 
prozesse um seine Routinen. Anisha und Smita gingen, wie ich im Folgenden zeige, einen anderen Weg, um mit dem Konfliktpotential alltäglicher Routinen umzugehen.

\subsection{3 (A)Synchronisierte Essensgewohnheiten}

Im Alltag kochen und essen Menschen häufig nicht allein, sondern gemeinsam mit Freund*innen oder der Familie: „(...) food also provides a clear focus for practices of communality, especially in collective eating whether in private or in public spaces" (Hage 1997: 109). Kiran berichtete, dass in seiner Abteilung alle gemeinsam zum Mittagessen gehen würden, und verstand dies als bewussten Versuch, die internationalen Mitarbeiter*innen einzubinden (integrate) (Kiran, 02.10.2010).

Allerdings können sich die ,Essenszeiten' unterscheiden, so dass die Menschen sich synchronisieren und ihre Praktiken koordinieren müssen (Shove 2013a: 28). Diese Dynamik verdeutliche ich im Folgenden an Anishas Fall. Ich fragte Anisha, ob sie denn zum Mittagessen in die Mensa gehe, und sie erklärte mir, dass sie das nur selten tue, weil sie sonst danach zu müde sei, außerdem:

I don't prefer to go to Mensa anymore ((lacht kurz)). And also, because when my labmates go there they spend really an hour there, which for me is hard because I just want some quick thing - like in fifteen minutes I'm done. So, normally I cook more dinner and bring it as a lunch. (Anisha, 01.05.2011)

Anisha hatte einen anderen Tagesablauf als ihre Kolleg*innen. Sie aß am Abend gemeinsam mit ihrer indischen Mitbewohnerin Smita. Mit dem Kochen wechselten sich die beiden ab. Anisha erklärte mir lachend, Smita habe sich an ihre Routine angepasst: „Our eating habits are now synchronized with me, because I'm always cooking on my own what I like, and now we both are synchronized with my routine." Ähnlich wie Nandita und Ajay legten die beiden Wert darauf, regelmäßig indisches Essen zuzubereiten: „In the evening we want to have something like rice or chappati, because we are used to that thing. But if we are too tired or we come back home late, then we just cook pasta. But not two, three days in a row - second or third day definitely it has to be Indian." Anisha und Smita kochten bewusst Essen, das sie ,gewohnt ' waren und stellten so gemeinsam Vertrautheit in ihrem Alltag her.

Die Möglichkeit, gemeinsam zu essen, kann nicht nur durch unterschiedliche Routinen erschwert werden, sondern auch durch unterschiedliche Essgewohnheiten. Sadhvi und Sachin führten beispielsweise die Gruppenbildung unter den jungen Inder*innen in Göttingen auf die unterschiedlichen kulinarischen Vorlieben zurück. Es gebe zum einen, so erklärte Sachin, den Faktor des Essens von Fleisch: „There is this vegetarian - non-vegetarian thing. I think that also plays a role in how the groups are forming." Ein weiterer Aspekt sei laut Sadhvi das Trinken von Alkohol: „Many of us don't drink alcohol on a regular basis or at all. So ja, many of us tend to stick together, because it is easier for us to have our own kind of parties." Personen, 
die Fleisch äßen und Alkohol tränken, hätten es viel einfacher, abends auszugehen und mit den „locals“ Kontakte zu knüpfen (socialize): „You can go to a pub and sit and order some burger and have a beer." Für die anderen bedeute es, dass sie am Abend nicht wirklich viel unternehmen können, erklärte Sadhvi lachend: „That's why you end up staying home, and that's why you end up cooking, and that's why food is so important."

Essen und Trinken vermitteln Vertrautheit und Zugehörigkeit, aber ebenso schließen sie aus (vgl. Ajay Bailey 2017). Diese Wirkung kulinarischer Vorlieben zeigte sich in meiner Studie an Grenzziehungen zu ,Nicht-Inder*innen' und anhand der regionalen Herkunft. Tanvir, der aus Westbengal stammt, erklärte mir: „The Pune guys will try to hang out among themselves. One important problem is when the Bengali guys hang out, they will always have some whiskey or something, and Pune guys won't have." Die unterschiedlichen Ess- und Trinkgewohnheiten tragen so zur Bildung beziehungsweise Aufrechterhaltung von Gemeinschaften bei, die auf einer gemeinsamen regionalen Herkunft oder auch Religions- bzw. Kastenzugehörigkeit basieren.

Für die jungen Wissenschaftler*innen aus Indien bedeutete der Umzug nach Göttingen zunächst einen Bruch mit ihren alltäglichen Routinen und die Notwendigkeit, den Alltag an einem neuen Ort wiederherzustellen. Praktiken wie das Kochen und Essen vertrauter Speisen stellen Routinen her und sind Verbindungen $\mathrm{zu}$ anderen sozialen Welten, Orten und Zeiten. Kochen und Essen sind multisensorische Erfahrungen, die verkörperte Erinnerungen wachrufen (vgl. Rodaway 1994). Sie geben ein Gefühl von Zuhause und Vertrautheit, lösen aber auch Erfahrungen von Exklusion aus, wenn, wie in Gilons Fall, der Geruch des Essens zu einem Konfliktpunkt wird.

\subsection{Freizeitgestaltung}

Das Leben in der Zeitlandschaft Göttingens bot für meine Gesprächspartner*innen ein Mehr an freier Zeit, die sie gestalten konnten. Gerade den Frauen eröffneten sich neue Zeiten, um Hobbys nachzugehen oder sich mit Freunden außerhalb der eigenen vier Wände zu treffen. Der öffentliche Raum wird in Indien stark von Männern dominiert, Frauen dagegen wird der häusliche Bereich als Ort zugeschrieben (Donner 2015). Sich nach einer bestimmten Uhrzeit in der Öffentlichkeit aufzuhalten, gilt aus diesem Grund für Frauen als nicht sicher. Smita erinnerte sich in unserem Gespräch daran, wie stark sie noch zu Beginn ihres Aufenthalts von dieser Einstellung geprägt war und wie sich in Göttingen ein Gefühl der Sicherheit einstellte: „I remember when I came over here, I was very scared because I was going back home and it was eleven in the night and the roads were totally deserted, and I got so scared that at eleven I'm walking alone." Wenn sie in Indien gewesen wäre, überlegte sie, hätten ihre Eltern mit ihr „geschimpft“ (scolded), und so entschied sie sich, in Göttingen nie wieder so spät allein unterwegs zu sein. Allerdings fand sie sich 
dann eines Nachts um zwei Uhr allein auf dem gleichen Weg und stellte fest: „I don't bother actually. I don't care. So, I'm very relaxed when I'm walking in the street. It's a very kind of safer feeling when you walk [at] whatever time. So, it's very nice to have a city like this where it can be totally relaxing [to] not bother about the time you are walking" (Smita, 26.01.2013).

Ebenso wie die jungen Frauen nutzten die Männer die neuen freien Zeiten und die Distanz zur elterlichen und gesellschaftlichen Kontrolle, um ihren Freizeitaktivitäten nachzugehen. Die indischen Wissenschaftler*innen gestalteten ihre freie Zeit mit unterschiedlichen Aktivitäten: Kiran schaute fast jeden Tag einen Film in seiner Muttersprache an, Akhil sang - vor allem Lieder, die als ,traditionell ' bezeichnet werden können - und Rohini ruderte. Viele meiner Gesprächspartner*innen führten in Göttingen Hobbys fort, die sie bereits in Indien hatten. Allerdings können nicht alle Hobbys gleich einfach an einen neuen Ort transferiert werden. Rohini hatte in Indien Sitar gespielt, konnte aber ihr Instrument nicht mit nach Deutschland bringen:

You sometimes miss a concert - a Sitar concert or something - and then you feel that, „Oh I cannot play." So you go to an orchestra, and then you are listening to the western orchestra, and then you feel like, „Ah I can play Sitar, I have learned it for so many years, but I can't play here." So that happens sometimes. (Rohini 18.08.2016)

Im Folgenden konzentriere ich mich auf die Freizeitgestaltung von Kundan und Hritik. Diese drehte sich vor allem um Kricket, einen Sport, der in den vergangenen vier Jahren einen großen Aufschwung in Deutschland erlebt hat. ${ }^{100}$

\section{Kricket in Göttingen}

In Göttingen gibt es seit den 1990er Jahren eine Kricketmannschaft, die lange Zeit vor allem aus internationalen Studierenden und Wissenschaftler*innen bestand und deswegen an den Hochschulsport der Universität Göttingen angegliedert ist. Obwohl sich langsam ein Wandel einstellt, ist Kricket in Deutschland ebenso wie in Indien ein Sport, der vor allem von Männern praktiziert wird (Appadurai 1996: 111). In Göttingen gab es ein Frauenteam, das aus ungefähr zwölf Frauen aus Sri Lanka, Pakistan und Deutschland bestand.

Ähnlich wie das Göttinger Team haben die meisten anderen Kricketteams in Deutschland viele Spieler, die aus Pakistan, Bangladesch, Sri Lanka oder Indien kommen. Seit dem „langen Sommer der Migration“ (Hess et al. 2017) im Jahr 2015 finden immer mehr junge Männer aus Afghanistan ihren Weg in die Kricketmann-

\footnotetext{
100 Siehe Martin Einsiedler: „Mehr Plätze würden dem Spiel guttun“, Der Tagesspiegel vom 06.07.2017, https://www.tagesspiegel.de/sport/cricket-boom-in-deutschland-mehr-plaetze-wuerdendem-spiel-guttun/20029426.html.
} 
schaften - so viele, dass Kricket seit ungefähr vier Jahren einen „Boom“ in Deutschland erlebt. ${ }^{101}$ In den Zeitungsartikeln, die sich mit dieser Entwicklung beschäftigen, wird dem Kricket eine wichtige Rolle für die Integration der Geflüchteten zugesprochen und es wird häufig als ein "Stück Heimat “102 beschrieben. Eine ähnliche Wirkung entfaltete der Sport für die meine Gesprächspartner*innen: Kricket vermittelte Vertrautheit und Kontinuität und die Spiele bildeten eine Komfortzone mit bekannten Regeln und Abläufen.

Kundan verbrachte seine gesamte Freizeit mit unterschiedlichen sportlichen Aktivitäten:

I do two things: work and play. (...) Saturday practice and Sunday - every Sunday I have a match in Hannover or Hamburg or Bremen or something, so it's really difficult. So, Monday I will be free, Tuesday evening we will have badminton practice, Wednesday free, Thursday evening we will have badminton practice. (Kundan, 14.08.2013)

Bereits bevor er im Juni 2011 in Göttingen ankam, um seine Promotion zu beginnen, hatte Kundan im Internet nach einer Möglichkeit gesucht, in Deutschland Kricket zu spielen. Er fand bei seiner Online-Suche den Göttinger Kricket-Verein und registrierte sich. In unserem Gespräch erinnert er sich, dass er vom Verein bereits gefragt wurde, ob er an einem Spiel teilnehmen wolle, als er noch in Indien war. Nach seiner Ankunft, noch während er am Goethe-Institut seinen Sprachkurs absolvierte, begann er, in Göttingen Kricket zu spielen.

Kundan spielte außerdem für zwei Vereine in Göttingen Badminton. Im Interview erzählte er, dass er im Alter von sechzehn Jahren für die Jugendnationalmannschaft Indiens gespielt und eigentlich gerne eine Karriere als Sportler gemacht hätte. Allerdings unterstützten seine Eltern diese Idee nicht. Dass er in Göttingen viel Sport trieb, erzählte er seinen Eltern nicht, da sie sich Sorgen machen würden, dass er sich verletzten könnte. „Most of the time - mostly they don't know what I'm doing except that I study." 2013, zwei Jahre nach seiner Ankunft, war Kundan der Kapitän der Göttinger Kricketmannschaft. In Göttingen hatte Kundan die Möglichkeit, seine Leidenschaft für Sport, vor allem Kricket und Badminton, auszuleben, ohne dass seine Eltern davon erfuhren. Er hatte die Kontrolle über die Nutzung seiner freien Zeit.

\footnotetext{
101 Siehe The Telegraph: „German interest in Cricket surges thanks to talented refugee players“ vom 06.05.2016, https://www.telegraph.co.uk/news/2016/05/06/german-interest-in-cricket-surges-thanksto-talented-refugee-pla/. Siehe Alex Raack: „Flüchtlinge sorgen für Cricket-Boom in Deutschland“ Spiegel Online vom 21.06.2017, http://www.spiegel.de/sport/sonst/fluechtlinge-sorgen-fuer-cricketboom-in-deutschland-a-1152646.html.

102 Siehe Hilke Lorenz: „Cricket ist auch ein Stück Heimat“, Stuttgarter Zeitung vom 02.05.2016, http://www.stuttgarter-zeitung.de/inhalt.sport-und-fluechtlinge-cricket-ist-auch-ein-stueck-heimat.93 f31db6-f140-4160-ba06-0a9549cd55a1.html.
} 
Als Hritik 2005 für seine Promotion nach Deutschland kam, war Kricket zumindest in der breiten Öffentlichkeit noch ein kaum beachteter Sport. Er erklärte, Kricket sei in Göttingen zu ihm gekommen. Er habe in seiner Bewerbung erwähnt, dass er Kricket spiele. Sein Vorgesetzter hätte daraufhin einem Kollegen, der selber Kricket spielte, erzählt: „You are going to get someone who is also going to join you in your cricket team." Hritik spielte bereits den ersten Sonntag nach seiner Ankunft in Göttingen Kricket und verbrachte fast seine gesamte Freizeit mit Kricket, entweder als Spieler oder als Zuschauer. Er brauchte den Sport als eine Art „Meditation“ und Entspannung vom Alltag:

In my leisure time you can take me as a different person than other Indians. I follow cricket madly. So before I found anything else to do then, I used to follow all the matches, all the highlights - everything about cricket. So participating in online discussions about cricket - and I play cricket here - so that is how I spend my leisure time: cricket takes most of my leisure time. (Hritik, 15.09.2011)

Hritik war ein großer Fan von Sachin Tendulkar ${ }^{103}$, den er als „Inspiration“ für sein Kricketspiel bezeichnete. Hritiks Begeisterung für Tendulkar wurde auf seiner Facebook-Seite und im Interview sehr deutlich. Im Interview sprach er von Tendulkars erstaunlicher Karriere: „He is now a legend. He has been playing now more than twenty-two years, and I have been fortunate enough to follow his career right from his early ages too." Tendulkars Karriere begleitete Hritiks eigenen Weg und wie Tendulkar wurde Hritik zum Kapitän ,seines 'Teams in Göttingen. Zum Zeitpunkt des Verfassens dieser Arbeit lebte Hritik mit seiner Frau Vedika in den USA, wo er Teil einer lokalen Kricketmannschaft ist. Dem Göttinger Team blieb er auch aus der Ferne treu und kommentierte über Facebook dessen Erfolge.

Meine Gesprächspartner*innen nutzten ihre ,freie` Zeit, um vertraute Praktiken in ihren Alltag zu integrieren. Ähnlich wie das Kochen und Essen bekannter Speisen bieten Hobbys die Möglichkeit, Vertrautes in den Alltag an einem neuen Ort, in einer neuen Zeitlandschaft zu integrieren und so Kontinuitäten in und zu ihrem Leben in Indien herzustellen.

\footnotetext{
${ }_{103}$ Sachin Tendulkar ist einer der bekanntesten und am meisten verehrten Kricketspieler Indiens. Er ist mittlerweile nicht mehr als Spieler aktiv. Siehe Dileep Premachandran: „Sachin Tendulkar: retiring little master who broke almost every record“, The Guardian vom 12.11.2013, https://www.theguardi an.com/sport/blog/2013/nov/12/sachin-tendulkar-india-retiring.
} 


\subsection{Zusammenfassung}

In diesem Kapitel waren die alltäglichen zeitlichen Erfahrungen der jungen Wissenschaftler*innen der Ausgangpunkt für die Untersuchung von Praktiken der Zeitgestaltung. Ich habe gezeigt, dass das Leben in einer neuen Zeitlandschaft und die Distanz zu den vertrauten sozialen Welten zu größerer Zeitsouveränität führte, zu Erfahrungen von Entschleunigung, aber auch von Isolation und Langeweile.

In ihrer biographischen Navigation gestalteten meine Gesprächspartner*innen in Interaktion mit lokalen und transnationalen sozialen Rhythmen und Zeitkarten ihre eigene alltägliche Zeitlandschaft. Diese bestand aus neuen und alten Praktiken, die Routinen herstellten, Sicherheit boten und Vertrautheit vermittelten. Die Alltagsgestaltung gab den jungen Menschen ein Gefühl der Kontrolle (vgl. Butcher 2011: 12) über die Veränderungen in ihrem Leben und erlaubte es, auf der synchronen und diachronen Ebene Verbindungen zu verschiedenen sozialen Welten, Orten und Zeiten herzustellen.

Im Alltag teilten die indischen Wissenschaftler*innen ihre Zeit mit Freunden in Göttingen und mittels Informations- und Kommunikationstechnologien mit Familie und Freunden in Indien und der Welt. Sie gestalteten Komfortzeiten, in denen sie sich mit Menschen trafen und kommunizierten, durch deren Kopräsenz (offline oder online) sie sich wohl und weniger einsam fühlten. In diesen Situationen handelten sie auch über die Distanz hinweg und unter Nutzung unterschiedlicher Medien ihre Zeitgestaltung und Zeitsouveränität aus. Die Praktiken der Zeitgestaltung wie das Kochen, Essen und das Aufrechterhalten von sozialen Beziehungen artikulierten Verbindungen mit der Vergangenheit und der Zukunft und vermittelten damit das Gefühl der Kontinuität. Dieses Leben in Gleichzeitigkeit musste von den jungen Wissenschaftler*innen aktiv hergestellt werden.

Ein weiteres Element der Alltagszeitlandschaft war die Koordinate des mehr oder weniger nahen Endes der Zeit in Göttingen. Die Zeitgestaltung meiner Gesprächspartner*innen wurde durch das Bewusstsein von der zeitlichen Begrenztheit des Aufenthaltes beeinflusst, und so faltete sich die Zukunft in die Gegenwart. Obwohl die Alltagsgestaltung Einzelner im Fokus meiner Ausführungen stand, habe ich gezeigt, dass zeitliche Erfahrungen und die Zeitgestaltung immer in der Aushandlung mit den Anderen - mögen sie sich durch Nähe, Ferne oder ihr Fehlen auszeichnen - entstehen. 



\section{Besondere Zeiten: Mobilität und Resynchronisation}

Die Organisation der indischen Wissenschaftler*innen in Göttingen veranstaltete jedes Jahr Diwali, den Indian Independence Day, einen Sportsday und andere kollektive Aktivitäten. Für meine Gesprächspartner*innen waren Feiertage spezielle Zeiten der biographischen Navigation, da sie ihnen vor Augen führten, dass sie sich nicht in Indien bei ihrer Familie und den Freunden befanden, sondern an einem neuen Ort mit einer anderen Zeitlandschaft, anderen Zeitkarten und sozialen Rhythmen. Viele hatten gerade an solchen Tagen großes Heimweh und suchten die Nähe anderer Menschen, vor allem die Nähe jener, die ihre Erfahrungen teilten. Ein wichtiges Mittel, um mit der Erfahrung der Asynchronität, also der Ungleichzeitigkeit sowohl mit der vertrauten als auch der neuen Zeitlandschaft umzugehen, war die individuelle und kollektive Reproduktion der vertrauten Zeiten in Göttingen. In diesem Kapitel untersuche ich das Feiern vertrauter Feste und die Organisation gemeinsamer Aktivitäten durch die jungen Wissenschaftler*innen aus dieser Perspektive der Resynchronisation. Ich stelle dar, welche Feste in Göttingen begangen wurden und wie sie dazu beitrugen, Erfahrungen der fremden Zeiten (Cwerner 2001) zu navigieren und unterschiedliche soziale Verortungen zu artikulieren und auszuhandeln.

Die gemeinsamen Feste gaben die Gelegenheit zur Resynchronisation (Cwerner 2001: 23) und waren Momente, in denen sich die jungen Wissenschaftler*innen an 
ihre eigene Vergangenheit, ihre Kindheit in Indien sowie die kollektive Vergangenheit Indiens erinnern konnten (vgl. „remembered times“ Cwerner 2001: 24) und die Zukunft in den Blick nahmen. Durch die Feste wurden Aspekte der indischen Zeitlandschaft in Göttingen reproduziert und damit transnational erweitert.

Da Feste wie Diwali und Holi in Deutschland nicht zur Zeitlandschaft gehören, also nicht in der kollektiven Zeitkarte des Kalenders verankert sind, mussten meine Gesprächspartner*innen die Organisation der Feste selbst in die Hand nehmen und damit die vertraute Zeitlandschaft aktiv reproduzieren. Die „Indian Fraternity in Göttingen "104 nahm für die Organisation der gemeinsamen Aktivitäten in Göttingen eine besondere Rolle ein und wurde praktisch zur Gestalterin der kollektiven Zeitkarte. Das Komitee der Vereinigung hatte in einem gewissen Maße die Kontrolle darüber, wann und wie gemeinsam Zeit verbracht wurde. Die Ausgestaltung der kollektiven Zeiten richtete sich nach normativen Vorstellungen und vertrauten Abläufen, die Konventionen aus Indien reproduzierten. Vielen meiner Gesprächspartner*innen gab diese Reproduktion ein Gefühl von Sicherheit und Kontinuität, für andere stellte sie die Fortführung genau jener Aspekte ihres Herkunftskontextes dar, denen sie in Göttingen gerne entgehen wollten. Aus diesem Grund nahmen einige ganz bewusst nicht an den kollektiven Aktivitäten teil, die von der Vereinigung organisiert wurden, und schlossen sich damit von der Gemeinschaft aus.

Weitere besondere Zeiten der biographischen Navigation waren die regelmäßigen Aufenthalte meiner Gesprächspartner*innen in Indien. Sie waren ein wichtiges Mittel, um mit Heimweh umzugehen und soziale Beziehungen und Verortungen aufrechtzuerhalten. Allerdings waren die Reisen zur Familie und Freund*innen auch Momente, in denen die jungen Wissenschaftler*innen verstärkt ihre Zeitgestaltung aushandeln mussten und sich Veränderungen des Selbst bewusst wurden.

\section{1 „I miss home“: Die Erfahrung der Asynchronität}

Der Ausgangspunkt für Praktiken der Resynchronisation waren Erfahrungen der Ungleichzeitigkeit. Viele der jungen Wissenschaftler*innen vermissten in Göttingen neben ihrer Familie und dem indischen Essen vor allem die Feste, die vertrauten zeitlichen Koordinaten, die das Jahr in Indien strukturierten. Insbesondere zu Beginn des Aufenthaltes in Göttingen entstand eine doppelte Asynchronität, weil die jungen Menschen nicht mehr in ihrer gewohnten Zeitlandschaft lebten und mit der Zeitlandschaft vor Ort noch nicht vertraut waren.

Wie sich die Asynchronität anfühlte, beschrieb Rohini zu Diwali 2015 auf Facebook sehr eindringlich:

I MISS HOME! Damned! I miss the amazing food and the fire crackers (...) the diyas and the rangoli (...). It reminds me of my mother making rangoli

${ }^{104}$ Für die Wahrung größerer Anonymität verwende ich nicht den konkreten Namen der Vereinigung. 
and my sister overdoing it (Smiley mit herausgestreckter Zunge). I miss the smell of the food cooked in earthen pots and cooked so long that the entire house smells like a dream land. (Smiley) and you start getting hungry right from the noon (...). I miss my childhood friends (...). ...not just that I came back home at (...) $10 \mathrm{pm}$.

PS its pin drop silent and dark outside from $4 \mathrm{pm}$, with practically no LIGHT! Thats making me even more cranky. (Rohini, November 2015)

Die Festivitäten machten meinen Gesprächspartner*innen sehr bewusst, dass sie sich in einer anderen Zeitlandschaft mit anderen Zeitkarten befanden. Die Asynchronität wurde besonders spürbar, wenn sie sich wie in Rohinis Fall zu Diwali, dem Fest des Lichts, im winterlich kalten und dunklen Göttingen aufhielten. Für Rohini war es nicht nur Diwali, sondern, bedingt durch ihre Herkunft aus West Bengal, das Fest Durga Puja, das sie jedes Jahr sehr vermisste. 2017 erklärte sie auf Facebook: „I think this pujo ${ }^{105}$ what I miss the most is not the pujo by itself but the sense of belonging to somewhere."

An der Aufzählung der Dinge, die Rohini vermisste - den Geruch des Essens, die Dekoration etc. - wird erkennbar, dass es bei der Resynchronisation nicht unbedingt um das Zelebrieren einer nationalen Identität geht, sondern um das Herstellen von Vertrautheit und Zugehörigkeit. Rohini vermisste die sinnlichen Erfahrungen, die ,sinnliche Geographie‘ (Rodaway 1994), die sie mit Diwali assoziierte und das Gefühl, dazuzugehören. Die Prozesse der Resynchronisation zielten als Strategien der Zeitgestaltung darauf $\mathrm{ab}$, zeitliche Erfahrungen zu beeinflussen und mit Gefühlen wie Heimweh umzugehen. Das Integrieren der eigenen „highlights“ (Cwerner 2001: 23) in den Kalender der neuen Zeitlandschaft stellt vertraute soziale Rhythmen und Zeiten der Gemeinsamkeit her. Diese Funktion der Feste beschrieb Bhanu, der bereits zuvor, während seiner Zeit in Paderborn, Feste wie Diwali oder Holi organisiert hatte: "So that everyone comes together, cooks something, eats and meets friends. You share experiences, and that although you are in abroad, you don't feel like you are in abroad when you meet your country people." Ähnlich schilderte Sadhvi die Wirkung des gemeinsamen Feierns in Göttingen: „When you are in abroad you miss your country, and at least if you organize once in a while some activities, and then you feel happier." Bei den gemeinsamen Festen stand das Glücklichsein im Vordergrund, das Zusammensein mit Menschen, die sich in der gleichen oder einer ähnlichen Lebenssituation befanden.

Die Vereinigung der Inder*innen in Göttingen hatte eine wichtige Funktion für die Praktiken der Resynchronisation und steht aus diesem Grund zunächst im Zentrum meiner Ausführungen.

105 „pujo“ ist die Bengali-Version von pūjā. 


\subsection{Die Indian Fraternity in Göttingen}

Fast überall auf der Welt, wo es indische Studierende und Wissenschaftler*innen gibt, existieren Vereinigungen wie die Indian Fraternity in Göttingen. ${ }^{106}$ Diese wurde 2010 von einer kleinen Gruppe aus zirka acht indischen Wissenschaftler*innen gegründet und war seitdem für die Organisation von Festen und gemeinsamen Aktivitäten verantwortlich.

\subsubsection{Die Gründung der Indian Fraternity}

Im Juli 2010 fragte ich in einem Gruppeninterview mit dem Vorstand und einigen Mitgliedern, wie es zu der Gründung der Vereinigung gekommen sei. Nalika und Ravi, die beide Gründungsmitglieder waren, erklärten, dass sie über Orkut ${ }^{107}$ und Facebook häufig Nachrichten bekommen hätten, in denen Personen aus Indien um Hilfe gebeten hätten: „They started writing to us: ,I'm coming, so what do I do, what will I need.“' Daraufhin überlegten sie sich, dass es effektiver sei, eine Organisation zu gründen und Informationen zusammenzustellen, als auf individuelle Nachrichten zu antworten. Inspiration für diese Art der Organisation bezogen sie von ähnlichen Vereinigungen in den USA. Obwohl schon seit 2006 Feste wie Diwali in Göttingen organisiert wurden und immer mehr junge Inder*innen in Göttingen lebten, kam es erst 2010 zur Gründung der Vereinigung. Zu diesem Zeitpunkt gab es unter den indischen Wissenschaftler*innen in Göttingen einige, die sehr engagiert und motiviert waren, und, wie Ravi darlegte, den nötigen „push“ von der Universität, die ihrerseits ein Interesse an der Gründung einer Vereinigung hatte. Dieses Interesse stand im Zusammenhang mit der Internationalisierungsstrategie der Universität, im Zuge derer 2008 eine Repräsentanz der Universität Göttingen in Pune aufgebaut worden war. Der Aufbau von Verbindungen mit Kooperationspartnern in Indien weckte das Interesse der Universität an einer besseren Vernetzung mit indischen Studierenden und Wissenschaftler*innen vor Ort. Den Gründern der Organisation war dieser Zusammenhang durchaus bewusst: „It was also fortunate that this was a year where they [the university] wanted to extend contacts with India. So that fortunately again favored us" (Nalika, 31.07.2010).

Der Präsident der Universität Göttingen sowie der Leiter der Alumni-Abteilung waren bei der offiziellen Gründung der Vereinigung am 26.01.2010 zugegen. Dieses Datum ist wiederum signifikant, weil es den Indian Republic Day markiert. Deswegen beinhaltete das Programm für das Treffen das Singen der indischen Na-

\footnotetext{
106 Ähnliche Vereinigungen existieren z.B. in Heidelberg (http://hisaheidelberg.com/index.php, abgerufen am 18.03.2019); oder auch in den USA, an der Caltech in Pasadena (http://oasis.caltech.edu/, abgerufen am 18.03.2019).

${ }_{107}$ Orkut war bis 2014 ein soziales Netzwerk, ähnlich wie Facebook, das vor allem in Brasilien und Indien beliebt war. Es wurde von Google betrieben (http://www.orkut.com/index.html, abgerufen am 18.03.2019).
} 
tionalhymne und des Nationalliedes Vande Mãtaram. Das Treffen endete mit einem informellen Zusammenkommen, bei dem sich die jungen Wissenschaftler*innen, ähnlich wie beim Frühlingsfest Holi, gegenseitig die Gesichter mit Farbe einrieben.

Beim ersten offiziellen Treffen der neu gegründeten Organisation wurde der Name und das Logo durch den damaligen Präsidenten der Vereinigung, Bhanu, vorgestellt. In einer PowerPoint-Präsentation erklärte er die Bedeutung des Namens und des Logos unter der Überschrift „Our Identity“: „(...) (sanskrit) means ,creation: The logo signifies creation of an environment of mutual support, social interaction and a blend of Indian-German cultures." 108

Der Name und das Logo der Organisation führen seit der Gründung immer wieder zu mehr oder weniger offener Kritik, die sich auf die hinduistischen Anleihen des Namens oder auf den sprachlichen Ursprung, der mit Sanskrit vor allem in Nordindien zu verorten ist, bezog. Im folgenden Kapitelabschnitt beschäftige ich mich ausgehend von kritischen Aussagen gegenüber der Vereinigung mit der Heterogenität unter den indischen Wissenschaftler*innen in Göttingen und den Versuchen, mit dieser umzugehen.

\subsubsection{Die Vereinigung und das indische „Masala“ in Göttingen}

Kundan, der zum Zeitpunkt unseres Interviews Teil des Komitees war, sah die Probleme des Namens der Organisation in seiner Unbekanntheit. Als ich ihn fragte, ob er vor seiner Ankunft in Göttingen von der Vereinigung gewusst hätte, antwortete er frei heraus: „No, that was a terrible name that nobody knows.“ Weil aus ihrer Sicht niemand den Namen kannte, hatten sich Kundan und die anderen Mitglieder des Komitees überlegt, diesen zu ändern. Er erklärte, keiner wisse, was der Name bedeute, und deswegen würden sie eine englische Bezeichnung präferieren. Allerdings wollten Kundan und die anderen Mitglieder des neu gewählten Komitees diese Änderung nicht gleich beim ersten Treffen vornehmen, da sie befürchteten, dass die Umbenennung auf ihre regionale Herkunft und eine generelle Ablehnung von Hindi bezogen werden würde. Das Komitee der Vereinigung bestand 2013 zur Hälfte aus Wissenschaftler*innen, deren Herkunftsregion Tamil Nadu war.

The main purpose [is that] we want to bring the English as the main source. I mean, we are in Germany - we want to advertise our things. If you do it in Hindi, how are you going to attract the people? And that was another problem: even in the function, in the last time Diwali, we had the gameshow - [it] was in Hindi, and we have the German colleague people sitting, [and] even we don't understand. (Kundan, 14.08.2013)

\footnotetext{
108 Das Logo zeigt eine geöffnete Hand mit einem kleinen Häufchen Erde, aus dem eine junge Pflanze sprießt. Der Name der Vereinigung ist in den Farben der indischen und deutschen Flagge geschrieben.
} 
Bis zum Zeitpunkt des Verfassens dieser Arbeit wurde der Name auf der Webseite der Organisation, die an die Universität angeschlossen ist, nicht geändert. Für die Bezeichnung der Facebook-Gruppe der Vereinigung wurde der englische Untertitel nach vorne gesetzt. Dass es gerade meine Gesprächspartner*innen aus Tamil Nadu waren, die das Thema der Bezeichnung und generell der Sprache aufnahmen, muss im Kontext von Debatten gesehen werden, die in Indien immer wieder um Sprache(n) geführt werden. ${ }^{109}$ In ihnen steht die Dominanz von Hindi im Vordergrund, das heißt konkret die unterschiedlichen Versuche, Hindi als nationale Sprache Indiens zu etablieren, obwohl es offiziell zweiundzwanzig anerkannte Sprachen und inoffiziell noch viele mehr gibt. Seit 2014 wurden die Diskussionen durch Maßnahmen der Modi-Regierung, wie beispielsweise die ausschließliche Nutzung von Hindi auf den Webseiten und social-media-accounts der Regierung, angeheizt. Besonders in Tamil Nadu stießen diese Aktionen auf großen Widerstand. Kundan war durch die Betonung, dass es ihm und seinen Mitstreiter*innen um die Einführung eines englischen Namens gehe, darum bemüht, die Diskussion um die Namensgebung von der regionalen Herkunft zu entkoppeln. Englisch wurde in diesen Prozessen als Sprache verstanden, die nicht mit einer regionalen oder nationalen Herkunft verbunden ist.

\section{„Unity in Diversity“?}

Die jungen Wissenschaftler*innen kamen aus unterschiedlichen indischen Bundesstaaten - ein Faktor, der sich in der angesprochenen sprachlichen Vielfalt niederschlug. Je mehr junge Menschen aus Indien nach Göttingen kamen, desto mehr differenzierten sich Untergruppen heraus, die sich über eine gemeinsame regionale Herkunft definierten (Fuhse 2018). Es entstanden Freundeskreise und transnationale Netzwerke unter den jungen Inder*innen aus der gleichen Region und manchmal aus der gleichen Stadt. Diese Netzwerke führten dazu, dass es zum Beispiel zeitweise ein little Pune in der Philipp-Reis-Straße in Göttingen gab. Wie ich in Kapitel 5 gezeigt habe, boten die Freundeskreise wichtige Komfortzonen und Sicherheit. Allerdings formierten sich ebenso einige kritische Stimmen, die die Gruppenbildung anhand regionaler Herkunft als Fortführung von Grenzziehungen aus Indien verstanden. Die aktiven Mitglieder der Organisation sahen in der Etablierung regionaler Gruppen eine Herausforderung für die Vereinigung. Ajay, der ein aktives Mitglied der Organisation war, meinte, dass die jungen Inder*innen nun die Möglichkeit hätten, sich in ihren kleinen Gruppen zu treffen und gemeinsam Aktivitäten zu organisieren, und dass deswegen einige nicht mehr zu den vom Verein organisierten Events kämen. Er kritisierte vor allem die aus seiner Sicht fehlende Reziprozität. Viele der Neuankömmlinge würden sich vor und nach ihrer Ankunft für Hilfe - beispielsweise

\footnotetext{
109 Die Debatten werden zum Beispiel im folgenden Artikel aufgezeigt: Maseeh Rahman: „Narendra Modi's Hindi-only-tweets order stirs fears of India language shift“, The Guardian vom 12.06.2014, https://www.theguardian.com/world/2014/jun/20/india-narendra-modi-hindi-priority-language-fears.
} 
bei der Wohnungssuche - an die Organisation wenden und sich dann aber nicht bei der Vereinigung als Mitglieder registrieren und nicht an den Aktivitäten teilnehmen. An Ajays Kritik zeigt sich, welche Bedeutung Beziehungen der Gegenseitigkeit und die „Norm der Reziprozität“ (Nowotny 1993: 133), in der nur Zeit gegen Zeit getauscht werden kann, für soziale Beziehungen und deren Aufrechterhaltung haben. Ajay wünschte sich, dass die Zeit, die die Mitglieder in das Beantworten von Anfragen und die Organisation von Aktivitäten investierten, wertgeschätzt und durch eine aktive Mitgliedschaft und Teilnahme an den Festen erwidert werden würde.

Vendan, der nach Ajay Präsident der Vereinigung wurde, stellte nicht den Faktor der Gegenseitigkeit in den Fokus, sondern die Bedeutung von Interaktion zwischen den unterschiedlichen cultures:

There have been instances where people confined from a particular region did not respect the differences from the other region, and they somehow tried to either dominate or suppress others. So that's one thing we wanted to promote: that India is of different values and different diversity, and this needs to be respected and accepted as a fact. And that's where it brings in more spice, I guess. Because if you are expecting your Indian food to be spicy, it's not just one spice that brings in, but it's a lot of things that brings in that taste - so I believe this. [S]imilarly, [the] Indian community is also a masala of something. (Vendan, 26.03.2013)

Vendan verwendete den Begriff des „Masala“110, einer Bezeichnung für eine Gewürzmischung, um die indische Gemeinschaft zu beschreiben. Er illustrierte damit, dass er Indisch-Sein als Divers-Sein verstand. Mit diesem Verständnis knüpfte er an Diskurse zu Indiens ,Unity in Diversity an, denen ich während meiner Feldforschung immer wieder begegnete. Vor allem in Kontexten, in denen es galt, Indien oder die ,indische Kultur ${ }^{6} \mathrm{zu}$ (re)präsentieren, wurde Indiens Einheit in der Vielfalt betont. Das Ziel dieser Art der Darstellung ist, die Vielfalt Indiens bei den Festen sichtbar zu machen und damit alle jungen Inder*innen in ein großes indisches Masala zu integrieren. Obwohl viele meiner Gesprächspartner*innen die Bemühungen, die Diversität Indiens darzustellen, lobend kommentierten, war für andere gerade diese eher apolitische Haltung zu Indiens Vielfalt ein Grund für Kritik an der Vereinigung. Besonders die Gesprächspartner*innen, die politisch aktiv waren oder sich für ihre Forschung mit der Geschichte und Gesellschaft Indiens auseinandersetzten, kritisierten die Repräsentation Indiens durch die Organisation als idealisierend und essentialisierend. Die Kritiker*innen hielten bewusst Abstand von der Vereinigung und den Veranstaltungen, die von dieser organisiert wurden.

\footnotetext{
${ }^{110}$ Für die Darstellung von Diversität wird häufig zu Essens-Metaphern gegriffen, z.B. in der „Salad bowl"-Metapher für die amerikanische Gesellschaft oder auch in Munasinghes Buch (2001) zu Trinidad mit dem Titel Callaloo or tossed salad? East Indians and the cultural politics of identity in Trinidad.
} 


\subsubsection{Die Vereinigung und die kollektiven Zeitkarten}

Für Ravi, einen der Mitbegründer, hatte die Organisation zwei Aufgaben. Sie sollte als Informationsplattform dienen und Zeiten für das Zusammenkommen organisieren. Ravi war stolz auf die Regelmäßigkeit, mit der die Vereinigung es schaffte, die Feste zu organisieren. „After (the Indian fraternity) started to take this over, we started to organize celebrations in more Indian way, so (that) every little thing started to remind us of our home. So, we wanted to celebrate Diwali at the closest weekend possible. “ Sie hätten ihre „indischen Feste“ in einem fremden Land wiederherstellen (recreate) wollen. Dieses Ziel hätten sie zu Beginn nicht gehabt: „We just wanted to get everybody together and have fun." Mittlerweile zögen die Feierlichkeiten viele Leute an: „They can find more Indians here and it brings back the Indian celebration, the whole mood, everything" (Ravi, 15.09.2011).

Ravi betonte die Faktoren, die dazu beitrugen, dass sich die Feste wie in Indien anfühlten. Dazu gehörte, die Diwali-Feier möglichst nah an den Zeitpunkt des Feiertages in Indien zu legen. Allerdings mussten sich die jungen Wissenschaftler*innen bei der Organisation der Feste gleichzeitig nach ihrem Arbeitsalltag und der Zeitlandschaft in Göttingen richten. Das bedeutete, die gemeinsamen Feste auf die Wochenenden zu legen.

Für Ajay sollte die Organisation ein Gefühl von „home“ vermitteln:

I mean these guys, everyone are coming out of India - let them have a home away from home. Whatever way possible, let them interact, organize events, whatever. It's just one day for something, but it's fun - let them enjoy, let them have interaction among Indians first of all (Ajay, 14.12.2011).

Die Vereinigung spielte bei der Auswahl der gemeinsamen Zeiten eine bedeutende Rolle und schuf damit gewissermaßen eine kollektive Zeitkarte. Die Kontrolle über die gemeinsam verbrachte Zeit lag bei der Organisation: Der Zeitpunkt der gemeinsamen Aktivität, die Dauer, die Häufigkeit und die Art und Weise der kollektiven Zeit wurde durch die Mitglieder des Komitees entschieden.

Die Auswahl der gemeinsamen Aktivitäten wurde mir in einem Gruppeninterview, das ich 2010 durchführte, durch die Komitee-Mitglieder und einige andere Anwesende folgendermaßen geschildert: „Diwali is one of the biggest festivals that happens all across India, I would say more or less. So that's why Diwali is being celebrated. Independence day (...) all India.“ Zu Diwali würden alle auf der „höchsten Stufe“ (highest level) feiern, meinte Ravi. Außerdem würde es in allen Regionen Indiens am gleichen Tag begangen. Andere Feste wie Ganesh Chathurti hätten sie zwar schon in Göttingen organisiert, seien aber zu regional. In den Aussagen wird deutlich, dass vor allem Feste gewählt wurden, die als ,indisch' galten und von denen angenommen wurde, dass sie von allen in Indien - unabhängig von regionalen oder religiösen Grenzen - gefeiert werden. Wie Dhara, die sich bewusst von Veranstaltungen der Vereinigung fernhielt, analysierte, wurde dadurch die ,indische Kultur 
essentialisiert (Dhara, 24.04.2013). Dies bedeutet, es werden spezifische Traditionen - im Fall Indiens häufig solche des brahmanischen Hinduismus - als die aller normalisiert und legitimiert (vgl. Thomas und Geraci 2018).

Durch die Auswahl, die die Organisation anhand der genannten Kriterien traf und die zunächst inklusiv gemeint war, bestimmte sie, welche Ereignisse zur Wiederherstellung der indischen Zeitlandschaft in Göttingen gewählt wurden. Dadurch entstanden Inklusions- und Exklusionsprozesse, die ich in den Ausführungen zu den einzelnen gemeinsamen Aktivitäten konkreter analysiere. Die kollektiven Aktivitäten, vor allem jene, die mit dem Indisch-Sein assoziiert wurden, übten einen „sozialen Anschlusszwang" (Nowotny 1993: 33) aus: Es wurde wichtig, teilzunehmen und die eigene Zeit in die Organisation oder zumindest in die Anwesenheit zu investieren, wenn man dazugehörte oder dazugehören wollte.

\section{Zeitliche Diversität und die Schwierigkeit der Koordination}

Für die jungen Wissenschaftler*innen, die zum Komitee der Vereinigung gehörten oder sich aktiv engagierten, gab es neben den Festen noch weitere Termine. Das Komitee traf sich regelmäßig an den Wochenenden. Wenn Feste anstanden, wurden die Treffen häufiger und es kamen einige weitere Menschen hinzu, um sich an der Organisation zu beteiligen. Die Treffen wurden zunächst immer auf einen Samstag gelegt. Da der Samstag sich für die meisten als shopping-day etablierte, wurden die Treffen bald auf Sonntag verlegt. Für einen meiner Gesprächspartner, Gilon, bedeutete diese Umstellung, dass er nicht mehr teilnehmen konnte:

Initially, I used to go to (the) meetings, but then they changed their meeting time from Saturday to Sunday. And Sunday was not possible for me because it was church day, and that's how I got no contact with any Indian friends - lost all the contacts. (Gilon 19.01.2013)

Praktiken, die gemeinsam ausgeübt werden, verlangen die Koordination untereinander (Shove 2013a: 28). Im Fall der Vereinigung wurde zugunsten der hinduistischen Mehrheit entschieden und Gilon damit ausgeschlossen.

\subsection{Ein Jahr in Göttingen: Von Diwali bis zum Indian Independence Day}

Viele der jungen Wissenschaftler*innen kamen zwischen August und Oktober in Göttingen an. Diese Ankunftszeit wurde durch den Semesterrhythmus der Universität bestimmt und konfrontierte sie mit kaltem Wetter, kürzer werdenden Tagen und Dunkelheit. Diwali, das Fest der Lichter, fällt je nach Jahr auf Ende Oktober oder Anfang November und war für viele die erste Gelegenheit, um eine größere Anzahl der anderen jungen Inder*innen in Göttingen kennenzulernen. Ich beginne 
meine Ausführungen in diesem Abschnitt mit Diwali und bewege mich chronologisch weiter durch das Jahr, so wie es auch meine Gesprächspartner*innen erlebten.

\subsubsection{Diwali}

2006 wurde in Göttingen das erste Diwali-Fest organisiert, erzählten mir einige Mitglieder der Vereinigung in einem Gruppeninterview. Bevor es die formelle Organisation gab, waren es die „senior PhD students“ oder Postdoktoranden, die die Verantwortung dafür übernahmen. Die anschließenden Ausführungen sind in drei Aspekte aufgegliedert: die Organisation Diwalis, die Feier an sich und Stimmen einzelner Gesprächspartner*innen zur Feierlichkeit.

\section{Organisation von Diwali}

Im Folgenden beziehe ich mich auf die Daten meiner teilnehmenden Beobachtungen an den drei Planungstreffen für die Diwali-Feier 2012. Das Komitee begann im September mit der Planung der Diwali-Feier. Alle Mitglieder der Vereinigung oder jene, die gerne bei der Organisation helfen wollten, konnten zu den Planungstreffen, die in der Cafeteria des Max-Planck-Instituts für experimentelle Medizin stattfanden, kommen. Zuerst wurde ein Datum für die Feierlichkeit festgelegt, das so nah wie möglich an dem Zeitpunkt des Festes ${ }^{111}$ in Indien liegen sollte. In Indien wird Diwali über fünf Tage hinweg gefeiert. Da die Feier in Göttingen mit dem Arbeitsalltag vereinbart werden musste, fand sie jedes Jahr an einem Samstagabend statt. Als Örtlichkeit wurde das Max-Planck-Institut für experimentelle Medizin gewählt, das seine Räumlichkeiten auch in den folgenden Jahren kostenfrei für die Diwali-Feier zur Verfügung stellte. Die Feier, so wurde beschlossen, sollte um 18:00 Uhr anfangen, damit genug Zeit für das Programm und das Essen zur Verfügung stehen würde. Allerdings wurde nach kurzer Beratung über den ,indischen' Umgang mit Pünktlichkeit beschlossen, auf der Einladung 17:30 Uhr als Anfangszeit zu kommunizieren, um sicherzustellen, dass um $18 \mathrm{Uhr}$ die meisten da sein würden.

Bei den Planungstreffen galt es, Freiwillige für folgende Aufgaben zu finden: das Einsammeln des Geldes für die Teilnahme, das Koordinieren des cultural programme, das Durchführen der $p \bar{u} j a \bar{a}$, die Dekoration des Raumes, die Moderation des Abends, das Durchführen von Spielen, das Erklären von Diwali für ,Nicht-Inder*innen', die Auswahl von Musik für den dance-floor. Die ersten beiden Aufgaben übernahmen Mitglieder des Komitees. Die püjā sollte von Harendra und Kahal, zwei jungen Männern, die aus Brahmanen-Familien stammen und über die entsprechenden Kenntnisse verfügten, durchgeführt werden. Für die Planung der Dekoration und der Gestaltung des Rangolis ${ }^{112}$ meldeten sich Prani und Asvi.

\footnotetext{
${ }^{111}$ Diwali ist ein mehrtägiges Fest, das aber einen Höhepunkt an einem Tag hat, der je nach Region abweichen kann (Malinar 2009: 164).

${ }^{112}$ In Indien ist die Gestaltung des Rangoli, eines bunten Musters auf dem Boden, Aufgabe der
} 
Nachdem die Rollenverteilung geklärt war, entzündete sich beim Planungstreffen am 4. November eine kurze Debatte darüber, wo das Essen für die Feier bestellt werden sollte. Ajay, der zu diesem Zeitpunkt der Präsident der Vereinigung war, kommentierte dazu: „People are very particular with food“ (Ajay, 04.11.2012). Und tatsächlich hingen die Kritiken an der Feierlichkeit häufig mit der Qualität des Essens zusammen. Es wurde sich darauf geeinigt, vegetarische Speisen beim Restaurant Maharadscha zu bestellen. Das nächste, ähnlich sensible Thema, das aufgenommen wurde, war die Sprache. Im Vorjahr gab es, so wurde bemerkt, einige Beschwerden von Studierenden aus Tamil Nadu, weil die Person, die den Abend moderiert hatte, häufig zu Hindi gewechselt war. Kahal kommentierte, dass Diwali ja auch hauptsächlich in Nordindien gefeiert werde. Die meisten anderen Anwesenden bemühten sich, Kahals Aussage schnell zu widersprechen.

Zuletzt wurde das cultural programme für den Abend besprochen. Die Vereinigung hatte im Vorfeld über Facebook dazu aufgerufen, sich Gedanken über mögliche eigene Beiträge zum Kulturprogramm zu machen. Sandeep war gekommen und schlug vor, einen Programmpunkt anlässlich des 150. Geburtstags von Rabindranath Tagore ${ }^{113}$ in die Diwali-Feier zu integrieren: Er würde gerne eine 30-minütige Präsentation halten mit Film, Gesang, Gemälden und Gedichten von Tagore und zu dessen Aktualität. Ajay versuchte diplomatisch zu bleiben und fragte nach der Relevanz dieser Ausführungen für Diwali. Bei Diwali stehe der Spaß im Vordergrund: „All Indians get together and have fun, that's the main idea." Nach einer kurzen Diskussion wurden Sandeep zwanzig Minuten für seine Präsentation zugesprochen.

Für die Teilnahme an der Diwali-Feier war es erforderlich, sich online zu registrieren und einen Betrag von zwölf Euro für das Essen zu überweisen. Beim letzten Organisationstreffen vor Diwali, das an dem Tag stattfand, bis zu dem sich registriert werden sollte, hatten sich erst fünfundvierzig Menschen angemeldet. Ajay erklärte, dass es für die Organisatoren problematisch sei, dass sich die Leute nicht bzw. nicht rechtzeitig registrieren würden. Dazu kommentierte einer der Anwesenden: „It's the normal tendency of our community to extend for two, three days" (11.11.2012). Ajay war von diesem Verhalten spürbar verärgert und verstand es als Mangel an Wertschätzung gegenüber der Arbeit der Vereinigung und monierte: „No one is thinking about us. That's the sad part" (Ajay 11.11.2012).

\footnotetext{
Frauen im Haushalt. Für diese bunten Muster gibt es je nach Region verschiedene Bezeichnungen. Ich benutze im Folgenden die anglisierte Schreibweise, die auch meine Gesprächspartner*innen verwendeten.

113 Rabindranath Tagore war ein bengalischer Dichter, Philosoph, Maler, Komponist und Musiker und erhielt 1913 den Nobelpreis für Literatur. Er wird vor allem in seiner Herkunftsregion Bengal verehrt. Siddhart organisierte 2013 am 100. Jahrestag der Nobelpreis-Verleihung an Tagore eine „Tagore Night“ in Göttingen und lud dafür internationale Gäste ein.
} 


\section{Die Diwali-Feier 2012}

Ich nahm von 2010 bis 2018 jedes Jahr mit Ausnahme von 2013 und 2017 an den Diwali-Feiern teil. Die folgenden Ausführungen basieren auf meiner teilnehmenden Beobachtung bei der Diwali-Feier am 17.11.2012.

Gegen 10 Uhr morgens machte ich mich auf den Weg zum Max-Planck-Institut für experimentelle Medizin in der Hermann-Rein-Straße. Im Vorfeld der Feierlichkeiten hatte ich mich über Facebook dazu gemeldet, bei der Dekoration des Hörsaals zu helfen. Prani, die sich in diesem Jahr für das Schmücken verantwortlich zeigte, hatte sich gemeinsam mit Asvi Gedanken über die Gestaltung des Rangoli gemacht. Wie jedes Jahr sollte ein solches Muster, das aus buntem Sand gefertigt wird, den Eingang zum Hörsaal zieren (siehe Abb 3).

Prani breitete eine Papiertischdecke aus, auf der sie das Muster vorgezeichnet hatte, und wir begannen mit den improvisierten Materialien ${ }^{114}$, die sie in einem Geschäft für Dekorationsbedarf in Göttingen besorgt hatte, das Bild zu ,legen'. Asvi hatte sich für den unteren Teil des Bildes ein Motiv ausgedacht, das sie aus traditionellen Ornamenten ihres Heimatstaates Maharashtra abgeleitet hatte. Nach kurzer Zeit gesellten sich noch zwei weitere junge Wissenschaftlerinnen aus Indien zu uns. Während wir den bunten Sand auf der Unterlage in den vorgesehenen Formen verteilten, stellten sich die jungen Frauen, die sich untereinander zum Teil noch nicht kannten, vor, unterhielten sich über die Diwali-Traditionen ihrer Herkunftsregionen, über ihr Studium sowie die Arbeit in Göttingen und scherzten über das Heiraten.

Die jungen Männer, unter anderem Ajay und Vendan, begannen nebenan im Hörsaal mit dem Schmücken und dem Arrangieren der Möbel. Prani hatte Materialien für die Dekoration der Räume mitgebracht: Bunte Ausdrucke von Kerzenhaltern (dìya) und Schriftzügen sowie Stoffe, die in Form eines dìya a gefaltet werden sollten. Auf einem Zettel hatte sie sich „Frohes Diwali“ auf verschiedenen indischen Sprachen notiert, um die Tafel im Hörsaal damit beschreiben zu können. Die Räumlichkeiten des Max-Planck-Instituts für experimentelle Medizin wurden langsam visuell transformiert und veränderten ihren Zweck: Statt für die Produktion und Vermittlung von wissenschaftlichem Wissen genutzt zu werden, wurde der Raum zu einem Ort, an dem die Anwesenden je nach Herkunft etwas über Indien lernen konnten, an ihr Leben in Indien erinnert wurden oder sich nach Indien versetzt fühlen konnten: zu einem ein Ort des gemeinsamen Feierns und Essens.

Nachdem die Vorbereitungen für die Feier abgeschlossen waren, gingen die Organisatoren und die freiwilligen Helfer*innen nach Hause, um sich für die Feier am Abend umzuziehen, um sich also mit Hilfe von Kleidung selbst zu transformieren. Die Frauen zogen Saris oder Salwar Kameez an, viele der Männer eine Jeans und eine

\footnotetext{
114 Prani und Asvi hatten nicht den ,echten' Sand für das Rangoli in Göttingen zur Verfügung und besorgten deswegen Deko-Sand und -Kieselsteine bei „Nanu Nana“. Beides war zwar etwas gröber, erfüllte aber seinen Zweck.
} 


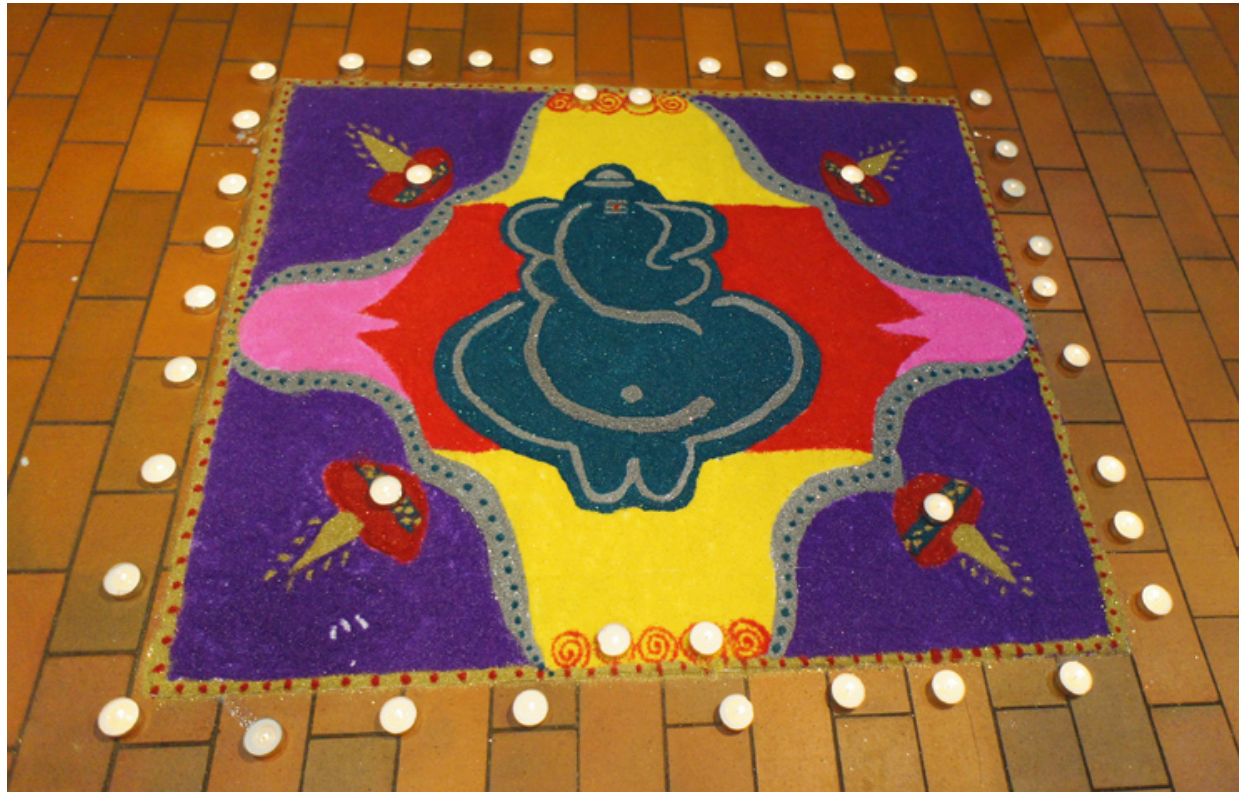

Abb. 3: Rangoli zu Diwali 2014: In der Mitte des Kreises ist Ganesha abgebildet. (Foto: Antonie Fuhse)

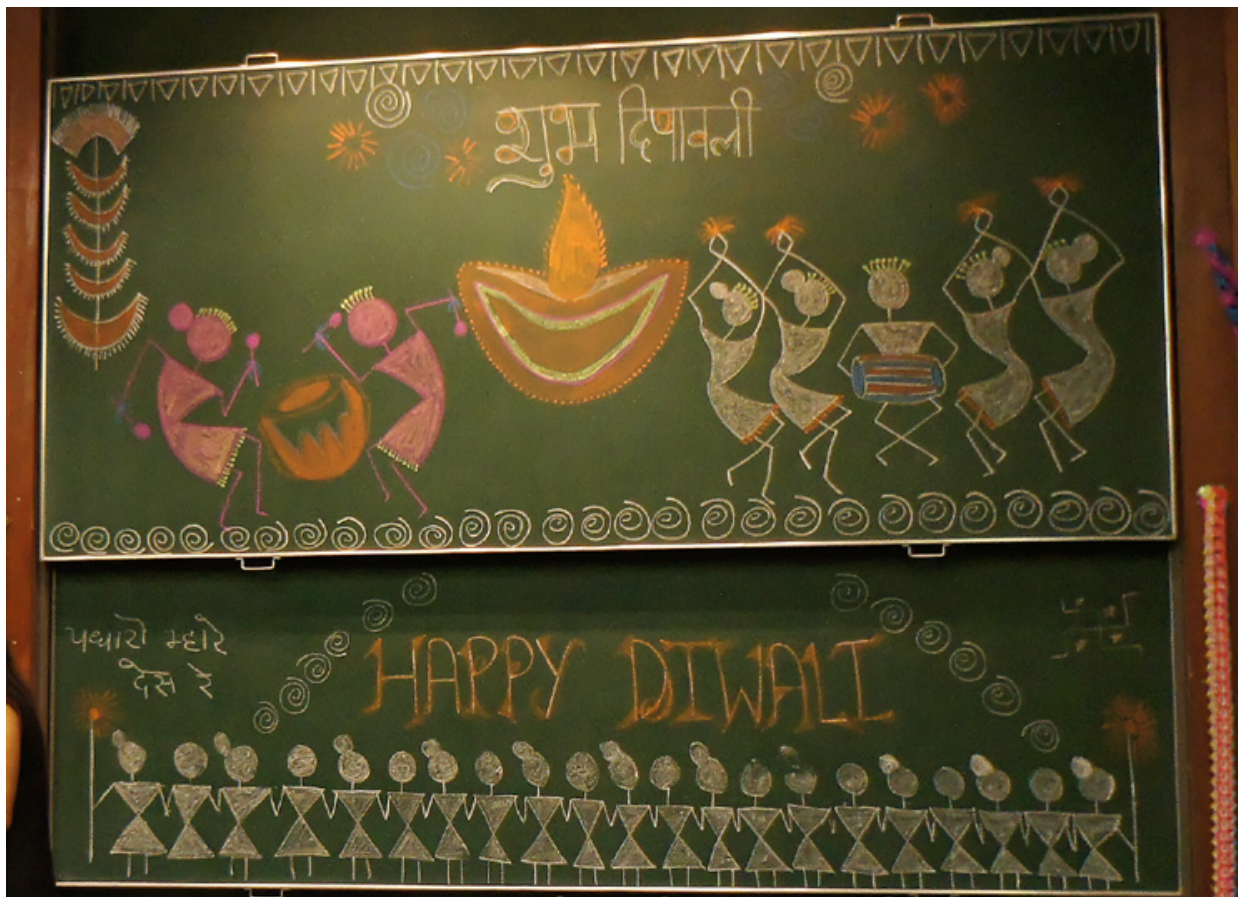

Abb. 4: Der dekorierte Hörsaal des MPI für experimentelle Medizin. (Foto: Antonie Fuhse) 
Kurta. Da kaum einer der jungen Wissenschaftler*innen diese Kleidung im Alltag trug, wurde die besondere Zeit der Feste auch durch diese Praktik markiert.

Am Nachmittag kam ich gegen 17:30 Uhr am Max-Planck-Institut an. Es waren noch nicht besonders viele von den etwa 100 Personen, die sich angekündigt hatten, anwesend. Wir gingen in den Hörsaal, wo gegen $18 \mathrm{Uhr}$ in der linken unteren Ecke mit der $p \bar{u} j \bar{a}$ begonnen wurde. Durch die Räucherstäbchen, die für dieses hinduistische Ritual angezündet wurden, wurde der Hörsaal nun zusätzlich olfaktorisch verändert. Im Publikum wurde ein Zettel, auf dem das Śri Mabișāsuramardini Stotram ${ }^{115}$ in Devanagari geschrieben war, verteilt. Harendra, der aus einer Familie von Tempelpriestern (püjäri) kommt, führte die püjā gemeinsam mit Kahal, der sich seine Haare abrasiert hatte, durch und sagte in großer Geschwindigkeit die entsprechenden Sanskritverse auf. Der Hörsaal war noch recht leer und das Interesse an der $p \bar{u} j \bar{a}$ hielt sich wie in den vorangegangenen Jahren in Grenzen. Es kam der Moment in der $p \bar{u} j \bar{a}$, in dem zur gemeinsamen Rezitation aufgerufen wird. Nur wenige beteiligten sich und die meisten mussten nach einem ersten Versuch abbrechen, da Harendra sehr schnell rezitierte und viele die Sanskritverse nicht lesen konnten.

Ich ging in den Vorraum des Hörsaals, in dem das Rangoli den Boden schmückte. Hier hatten sich einige Menschen versammelt und fotografierten sich gegenseitig entweder in kleineren Gruppen oder einzeln. Es wurde gescherzt, dass diese Fotos optimal für Heiratsanzeigen (matrimony) seien. Viele der Fotos wurden später auf Facebook gestellt.

Mittlerweile hatten sich die Räumlichkeiten des Max-Planck-Instituts mit indischen Wissenschaftler*innen und deren Freund*innen und Kolleg*innen unterschiedlicher Herkunft gefüllt. Wir gingen zurück in den Hörsaal, da nun das cultural programme mit Gesang und Tanz begann. Die vorgetragenen bzw. vorgetanzten Stücke waren häufig eine Mischung aus ,traditionellen` und eher ,modernen' Bollywood-Elementen und wurden als solche angekündigt. Die Stimmung im Hörsaal stieg und war bei den sehr beliebten Gesangs- und Tanzvorstellungen besonders enthusiastisch: Es wurde im Takt mitgeklatscht, gepfiffen und gejubelt. Das Programm dauerte länger als geplant, da sich einige spontan dazu entschlossen, etwas vorzutragen. Nach Sandeeps Präsentation zu Tagore wurde das Publikum dazu eingeladen, sich an einigen Spielen zu beteiligen.

Gegen 20:15 Uhr war das Programm zu Ende und alle stellten sich in der an den Hörsaal grenzenden Cafeteria an, um sich etwas zu essen und zu trinken zu holen. An den Tischen der Cafeteria wurde gemeinsam gegessen und geredet, so dass der Raum bald vom Gemurmel der verschiedenen Gespräche erfüllt war. Nach dem Essen war die Zeit gekommen die Wunderkerzen, die als Ersatz für die in Indien an Diwali ausgiebig abgefeuerten Böller und Raketen dienten, im Außenbereich des Max-Planck-Instituts anzuzünden. Nachdem der offizielle Teil des Abends damit geendet hatte, wurde über eine Anlage die Musik aufgedreht, das Licht ausgemacht und zum Tanzen aufgefordert. Die Tische und Stühle wurden aus der hinteren Hälf-

115 Der Name des Gebetes bedeutet aus dem Sanskrit übersetzt: „Gebet an die Göttin“. 
te der Cafeteria entfernt, um Platz für die stetig wachsende Menge Tanzender zu machen. Männer und Frauen tanzten in kleineren und größeren Gruppen zusammen zu Musik aus den verschiedenen Regionen Indiens. Bei Liedern aus bekannten Filmen wurden Teile der Choreographie übernommen und teilweise lautstark mitgesungen. Viele meiner Gesprächspartner*innen wirkten in diesem Umfeld unter Freund*innen und Kolleg*innen unbefangen und unbeschwert. Sie befanden sich in einer Komfortzone, in der die Regeln und das angemessene Verhalten vertraut und bekannt waren, anders als beispielsweise bei Feierlichkeiten ihrer deutschen Freunde oder bei abendlichen Discobesuchen.

\section{Diwali: Gleichzeitigkeit, gemeinsame Zeit}

Vanika erklärte mir bei der Diwali-Feier 2014, für sie komme es nicht auf die pījā an, sondern auf das feeling. Später fügte sie noch hinzu, dass es wirklich gut sei, in Göttingen zu feiern, weil sie die Feste sehr vermissen würde und traurig sei, zu Diwali im Büro zu sitzen und zu wissen, dass die Familie zuhause feiere.

Sadhvi und Sachin betonten, dass es bei der Diwali-Feier in Göttingen darum gehe, nicht allein zu sein: „if you are a couple they have each other, but if you come here as a student alone then you are just completely alone at this festival." Diwali sei außerdem als Event so beliebt geworden, weil zu dieser Zeit im Jahr viele neue Studierende und Wissenschaftler*innen aus Indien in Göttingen ankämen: „There are a lot of fresh people, new people. They are first time away from home, and their first Diwali away from home, and they are looking to do something" (22.09.2011). Sangita betonte ebenso, dass Diwali eine wichtige Gelegenheit biete, sich zu treffen, und dass die Leute „kind of really depressed“ (08.08.2013) wären, wenn die Feste nicht existierten. Im Zusammenhang mit Gefühlen von Einsamkeit und Heimweh wurde oftmals auf mögliche Depressionen hingewiesen.

Diwali, so zeigen die Aussagen, war eine besondere Zeit für die jungen Wissenschaftler*innen: Sie vermissten ihre Familien, Freunde und die Stimmung zu dieser Zeit des Jahres in Indien. Das gemeinsame Feiern von Diwali vermittelte Vertrautheit, trug zum Wohlbefinden bei und war ein Ereignis, auf das sie sich freuen konnten. Die Feier von Diwali in Göttingen bot die Möglichkeit, sich mit der Zeitlandschaft Indiens zu synchronisieren und als „kollektive Praktik des Erinnerns“ (Cwerner 2001: 24) Kontinuitäten herzustellen.

Die Diwali-Feier in Göttingen war eine Plattform, um die Zugehörigkeit zu einem diversen Indien und zur eigenen Herkunftsregion in einer Umgebung zu artikulieren, die durch visuelle, auditive und olfaktorische Mittel in einen vertrauten Ort transformiert wurde. Die Diversität Indiens wurde während der Planungsphase und während der Feierlichkeit immer wieder thematisiert. Gleichzeitig gab es Grenzen der Diversität bzw. Aussagen, die in ihrer Pauschalität über die Vielfältigkeit Indiens hinwegsahen. Bei der Diwali-Feier 2018 war es beispielsweise folgende Bemerkung des Moderators: „Indians have this tradition of praying to Ganesh before they start an important event" (03.11.2018). Hier findet eine Gleichsetzung von Hindus mit 
Inder*innen statt, die unter der Regierung der Bharatiya Janata Party (BJP), die seit 2014 an der Macht ist, einen stetigen Aufschwung erfahren hat.

Als kollektive Aktivitäten waren die Diwali-Feiern Performanzen, in denen Konventionen und Normen bestätigt wurden (vgl. Butler 1993: 12) und die wenig Platz zur Umgestaltung dieser boten. Dies betraf beispielsweise das kulinarische Angebot es wurde stets vegetarisches Essen bestellt und bewusst kein Alkohol ausgeschenkt. ${ }^{116}$ Ebenso wurde die Rollenverteilung bei Praktiken wie der püjā beibehalten und diese stets von Männern aus Brahmanenfamilien abgehalten. Die Beschreibung Diwalis als Ereignis, das in ganz Indien gefeiert wird, und als Indian cultural event auf Facebook stellt eine „culturalization“ (Thomas und Geraci 2018) des religiösen Festes dar. Die Bezeichnung als cultural verschleiert die religiöse Natur der Feier (vgl. ebd.: 108) und normalisiert den Hinduismus als die Religion der indischen Wissenschaftler*innen in Göttingen und Indiens (vgl. Thomas und Geraci 2018). ${ }^{117}$

\subsubsection{Saraswati-Puja: Regionale Zeitlandschaften und Zeitkarten}

Neben Diwali, das als ,indisches' Fest verstanden und gefeiert wurde, wurden in Göttingen Feierlichkeiten organisiert, die besonders in bestimmten Regionen Indiens begangen werden. Die Saraswati-Puja war eines dieser Feste, das von Mera, einer indischen Wissenschaftlerin, die 1994 mit einem DAAD-Stipendium nach Deutschland gekommen war und sich nach der Heirat mit einem deutschen Mann in Göttingen niedergelassen hatte, seit zwanzig Jahren in unterschiedlich großem Rahmen organisiert wurde. Ihre Kontakte zur Vereinigung waren mal mehr und mal weniger stark, je nach Besetzung des Vorstands und Meras Beziehungen zu diesem. 2013 war Mera im Komitee der Organisation. Aus diesem Grund waren in jenem Jahr besonders viele der jungen Wissenschaftler*innen an der Planung und der Durchführung der Saraswati-Puja beteiligt.

2013 wurde die Saraswati-Puja über Facebook als Bengali Spring Festival angekündigt. Mit folgendem Text wurde dazu eingeladen, teilzunehmen:

We are now named as Bangladesh and West Bengal (part of India). Politically and officially we are two different countries. Somebody had drawn a fractal boundary and we are divided. But, we still share the same culture. Our cultural authenticity, our language transcend the political boundaries. One of our cultural events to welcome the spring is Saraswati pujo. It is a Bengali

\footnotetext{
116 Ab 2016 wurde noch expliziter kommuniziert, dass der Verzehr von Alkohol an Diwali nicht „angemessen“ sei. So wurde auf Facebook geschrieben: „As Diwali is an Indian cultural event, so alcohol is not recommended. Alcohol will not be served and kindly do not bring with you."

117 Thomas und Geraci (2018) finden ähnliche Prozesse der Normalisierung des Hinduismus in ihrer Analyse der Ayudha-Puja, eines Festes, das sie als „worship of the machines“ bezeichnen. Dieses fest wird vor allem in Karnataka von Wissenschaftler*innen und Ingenieur*innen an ihren Arbeitsplätzen durchgeführt und von vielen als „kulturell“ bezeichnet (Thomas und Geraci 2018).
} 
Hindu religious custom of worshipping the goddess of education and culture. But, we celebrate all Bengali cultural events without maintaining any barrier of religious and political boundaries. We carry our culture beyond the geography, surpassing the religious view. So, come and join us in this joyous moment to welcome the spring.

Die Autor*innen der Einladung benutzten Ausdrücke wie „wir“ und „unsere kulturelle Authentizität“, „unsere Sprache“, „gleiche Kultur“ und betonten, dass die „Bengali-Kultur“ religiöse und politische Grenzen überwinden würde. Dadurch unterstrichen sie die Zusammengehörigkeit, die gemeinsame Kultur und Sprache von Bengalis, sei es in Indien oder Bangladesch. Die Hervorhebung der gemeinsamen Herkunft findet sich in dem auf der Einladung angekündigten Programm für die Feierlichkeit wieder. Folgende Aktivitäten waren geplant: „Breakfast with typical Saraswati pujo food; games that will remind your childhood with some gifts; all homemade typical Saraswati pujo food made with the spirit of Charuibhati (picnic): Khichuri, Aloor dum, Payesh, Pantooa.“ Das Essen für die Feier wurde gemeinsam in der Küche des Internationalen Besucherzentrums (IBZ), in dem die SaraswatiPuja 2013 stattfand, gekocht.

Die $p \bar{u} j \bar{a}$ wurde von Āmāl durchgeführt. Āmāl, die Soziologie studierte und für ein Semester in Göttingen lebte, hatte beim Treffen, bei dem die Organisation der $p \bar{u} j \bar{a}$ besprochen wurde, vorgeschlagen, dass ganz entgegen der Tradition und Konvention eine nicht-brahmanische, nicht-hinduistische Frau die $p \bar{u} j \bar{a}$ machen solle. Sie erzählte mir, dass eine solche Person aber nicht auffindbar gewesen sei bzw. die $p \bar{u} j \bar{a}$ nicht habe machen wollen. Aus diesem Grund habe sie sich gemeldet, obwohl sie leider Brahmanin sei, aber wenigstens eine Frau. In Indien führen Frauen die Rituale in ihrem eigenen Haushalt durch, aber nicht unbedingt außerhalb desselben. Besonders Tempelrituale werden nur sehr selten von Frauen abgehalten (Malinar 2009: 217). In einem gewissen Rahmen konnten, wie das Beispiel der püjā zeigt, die Konventionen gebrochen oder verändert werden. Im Fall der Saraswati-Puja geht das Brechen und Infragestellen von Traditionen mit der Fremd- und Eigenpositionierung von Bengalis als weniger traditionell und konservativ als Inder*innen aus anderen Regionen einher, der ich in meiner Feldforschung mehrfach begegnet bin.

Nach der $p \bar{u} j \bar{a}$ wurde teilweise in kleineren Gruppen auf dem Boden sitzend zusammen gegessen. Anschließend wurde gemeinsam gesungen. Obwohl die Einladung nicht auf Bengalis beschränkt war, hatten mindestens $80 \%$ der Teilnehmenden bengalische Wurzeln - entweder in Indien oder in Bangladesch. Die Saraswati-Puja bot die Möglichkeit, vertraute Speisen zu essen, Bengali zu sprechen, auf Bengali zu singen, Spiele zu spielen und hinduistische Rituale durchzuführen. Für Bengalis schuf die Feierlichkeit einen Raum, um ihre Zugehörigkeit zu dieser Region, ihrer Geschichte, ihrer ,Kultur' und ihre Zusammengehörigkeit zu artikulieren. Besonders die Möglichkeit, sich auf Bengali unterhalten zu können, war ein wichtiger Aspekt dieser Feierlichkeit und stellte Gefühle der Vertrautheit und Zusammengehörigkeit 
her. ${ }^{118}$ Gleichzeitig wirkte die Dominanz von Bengali gegenüber den Personen, die es nicht sprechen konnten, ausschließend.

In den folgenden Jahren organisierte Mera die Saraswati-Puja abgekoppelt von der Vereinigung. Vor allem für die Bengalis in Göttingen bildete die Feier der Saraswati-Puja eine wichtige zeitliche Koordinate. Durch die Feier konnten sich die Anwesenden mit der regionalen Zeitlandschaft Bengalens resynchronisieren. Die Feierlichkeit rief Erinnerungen an die eigene Kindheit und vergangene SaraswatiPujas wach und stellte somit diachrone Verbindungen mit dem Zuhause meiner Gesprächspartner*innen her.

\subsubsection{Die „Indian Culture Night“}

Im Rahmen des integrierten Master- und PhD-Programms der Molekularbiologie und den Neurowissenschaften finden seit 2002 jedes Jahr verschiedene "Culture Nights" statt. ${ }^{119}$ Für die Organisation und die Durchführung der Culture Nights sind die Masterstudierenden dieser Programme verantwortlich. Je nach Herkunft der insgesamt vierzig Masterstudierenden gibt es über das Jahr verteilt u.a. eine Indian Culture Night (ICN), eine Slavic Night, eine German Culture Night, TurkishMediterranean Culture Night oder The Americas Night. Die Indian Culture Night ist nach Aussagen des Koordinators des Programmes die beliebteste - es nahmen jedes Jahr etwa 100 Menschen teil - und erhält deswegen für die Durchführung etwas mehr Geld als die anderen. Das Geld wird für alkoholfreie Getränke und die Zutaten, die für die Zubereitung des Essens benötigt werden, eingesetzt. Der Termin der Indian Culture Night liegt jedes Jahr im März und wird gezielt in die Zeit gelegt, in der die Bewerber*innen für die nächste Master-Runde ihre Vorstellungsgespräche haben. So würden die Kanditat*innen, nach Aussagen des Koordinators, einen Eindruck vom Programm und von dem Sozialleben am Max-Planck-Institut erhalten. Jedes Jahr kamen einige der Alumni des Programms zur Indian Culture Night, auch wenn sie bereits nicht mehr in Göttingen lebten.

Die Verantwortung für die Gestaltung des Abends, das Thema und das Essen lag in den Händen der indischen Studierenden des Master-Programms. ${ }^{120}$ Das Essen wurde für die Indian Culture Night von den Studierenden selbst gekocht. Alle insgesamt vierzig Masterstudierenden waren in die Umsetzung am Abend der Indian Culture Night eingebunden. So wurden gemeinsam Choreographien und Musik-

\footnotetext{
${ }_{118} \mathrm{Zu}$ der Bedeutung von Sprache für Zugehörigkeiten und Erfahrungen von Gemeinsamkeit siehe auch Marco Antonsich (2010).

11 Auf folgender Webseite des Studiengangs kann nachgelesen werden, welche Cultural Nights und weiteren Events für die nächsten Monate geplant sind: http://www.gpmolbio.uni-goettingen.de/con tent/c_events.php, abgerufen am 21.11.2018.

${ }^{120}$ Die Vereinigung war bei der Indian Culture Night nicht als Organisation beteiligt. Allerdings waren häufig viele der Mitglieder anwesend und halfen bei der Durchführung aus. Einige der Mitglieder waren selbst Teil des Master- oder PhD Programmes.
} 
stücke eingeübt und aufgeführt sowie kleine Sketche ausgearbeitet, einstudiert und vorgetragen. Der Koordinator des Programms erklärte mir, dass die Studierenden nur für dieses eine Masterjahr zusammen sein und sich dann für ihren PhD-Abschluss auf die verschiedenen Forschungsgruppen aufteilen würden. Die Culture Nights sollten die Studierenden des Programms, die jedes Jahr sehr international seien, zusammenbringen. Ausgehend von meinen Beobachtungen 2013 gehe ich im nächsten Abschnitt auf den Ablauf der Indian Culture Night und die Themen, die für die Repräsentation Indiens gewählt wurden, ein.

Dass die Indian Culture Night unter den Studierenden und Wissenschaftler*innen der beteiligten MPI-Programme besonders beliebt war, wurde mir bei meiner ersten Teilnahme 2013 bereits auf dem Weg in das Max-Planck-Institut für biophysikalische Chemie, das am Faßberg liegt, bewusst. Der Bus, in den ich in der Innenstadt gestiegen war, füllte sich an jeder Station mit jungen Menschen ganz unterschiedlicher Herkunft. Statt der Viertelstunde, die der Bus für die Strecke zum Faßberg brauchen sollte, benötigte er dreißig Minuten, weil die Fahrerin an einigen Stationen aussteigen musste, um den Mitfahrenden zu erklären, dass sie sich von den Türen fernhalten müssten, damit diese schließen könnten. Am Faßberg angekommen, leerte sich der Bus plötzlich, ein Strom Menschen bewegte sich in Richtung Max-Planck-Institut und steuerte auf den Eingang des Gebäudes zu.

In der Eingangshalle, in der ein Rangoli den Boden zierte, traf ich auf Ajay und andere Bekannte. Wir gingen in den Manfred-Eigen-Hörsaal, an dessen Eingang uns Zucker angeboten wurde („,for a sweet beginning“). Der Hörsaal war geschmückt. An der Tafel war in verschiedenen (indischen) Sprachen „Willkommen“ zu lesen. „Indian Culture Night" stand in den Farben der indischen Flagge an die Tafel geschrieben. An der Tafel und den Wänden hingen Ornamente, an der Eingangstür zum Hörsaal ein Fadenvorhang. Der Abend begann mit einer PowerPoint-Präsentation und der Vorstellung der Masterstudierenden aus Indien, die 2013 im Programm angefangen hatten. Nur einer von den fünf neuen Masterstudierenden aus Indien war ein Mann. Auf einer Karte wurde gezeigt, aus welcher Gegend in Indien die jungen Studierenden stammten. Die Moderatorin merkte an, dass alle bis auf eine Person aus Südindien kamen. Für das Programm der Indian Culture Night wurde 2013 bewusst, wie die Moderatorin betonte, ein Thema gewählt, das die Diversität Indiens zeigen sollte: „Festivals of India“. Während des Abends stellten die Masterstudierenden je ein Fest vor und illustrierten mit Hilfe von Gesang oder Tanz, wie es begangen wird. Als Erstes wurde ein Arti for Lord Ganesh gesungen, da dieser als Remover of Obstacles immer zu Beginn einer Veranstaltung angerufen wird. Folgende Feste wurden im weiteren Verlauf vorgestellt: Holi, Durga Puja, Dusshera/Navarathri, Onam, Ganesh Chaturthi, Eid al Fitr, Thai Pongal und Diwali. Bei der Präsentation Diwalis wurden Bilder von der Feier in Göttingen gezeigt. $\mathrm{Zu}$ jedem Fest wurde entweder ein Lied oder ein Tanz aufgeführt. Gerade die Tänze waren bei den Zuschauenden sehr beliebt und ernteten anerkennenden Jubel und Applaus - beispielsweise beim Tanz zum Erntefest Pongal, der zu einer von Trommeln dominierten Musik vorgeführt wurde, 
die durch den Manfred-Eigen-Hörsaal schallte. Die Aufführung wurde gefilmt und von einem Anwesenden auf Youtube hochgeladen. ${ }^{121}$

Zum Programm der Indian Culture Night gehörten das gemeinsame Essen und Spiele wie ein Wettessen, bei dem Gol Gappas (indischer Snack) so schnell wie möglich aufgegessen werden sollten oder ein Sari draping contest, in dem die teilnehmenden Frauen und Männer so schnell wie möglich einen Sari anlegen sollten.

Die Themen, die für die Indian Culture Night gewählt wurden, waren bewusst darauf ausgelegt, einen gewissen Grad an Diversität zu zeigen. Die Poster und das cultural programme stellten Indien als bunt und vielfältig, aber trotzdem als Einheit - eben als ,Unity in Diversity - dar. Die Darstellung griff häufig Imaginationen auf, die es in Deutschland von Indien gibt: eine von Bollywood inspirierte Mischung aus Gesang, Tanz und Essen.

Das Publikum bei den Indian Culture Night war jedes Jahr sehr international und setzte sich vor allem aus Masterstudierenden und Promovierenden der beteiligten Max-Planck-Institute zusammen. Von meinen Gesprächspartner*innen versuchten einige, jedes Jahr dabei zu sein. Dabei stand der Spaß im Vordergrund, das Verbringen eines Abends mit Kolleg*innen und Freunden nach dem Motto, mit dem z.B. 2014 auf Facebook für die Indian Culture Night geworben wurde: „Come Discover India with us on March 8th. A Night Full of Dances, Drama and Romance and a lot of Spicy food."

\subsubsection{Sportsday: Zurück in die Kindheit}

Die indische Vereinigung führte ab 2010 fast jedes Jahr im Sommer einen „Sportsday" auf dem Sportgelände der Universität Göttingen durch. Die Organisator*innen erhielten vom Hochschulsport die Zusage, die Außenfläche des Geländes nutzen zu dürfen, und Material, um beispielsweise die Spielfelder zu markieren. Für den Sportsday wurden bewusst Spiele oder Sportarten ausgewählt, die im Hochschulsport nicht vertreten waren und auch sonst nicht in Göttingen durchgeführt werden konnten. $\mathrm{Zu}$ diesen Sportarten und Spielen gehörten Lagori, Kho Kho und Kabaddi. Viele der Sportarten, die ausgewählt wurden, kannten meine Gesprächspartner*innen aus ihrer Kindheit in Indien. Einige erzählten, dass es in ihren Schulen jedes Jahr einen Sportsday gegeben habe. Andere assoziierten die Spiele nicht unbedingt mit ihrer Schulzeit, sondern mit Picknicks mit der Familie. In einigen Jahren wurden Sportarten integriert, die in Indien besonders beliebt sind, wie beispielsweise Badminton.

Jedes Jahr gab es beim Sportsday Spiele für kleinere Kinder, z. B. Eierlaufen. 2012 nahmen beim Sportsday etwa vierzig Menschen teil, darunter auch einige jüngere Kinder. Der Sportsday, so erklärten mir einige der Anwesenden, würde sie in ihre Kindheit zurückversetzten. „I felt like I'm a kid again“, fasste eine junge Frau dieses Gefühl in Worte. Nicht alle der Anwesenden hatten die Assoziation mit ihrer Kindheit, da einige der Spiele wie beispielsweise Kho Kho regionalspezifisch sind.

${ }^{121}$ Siehe auf Youtube: https://www.youtube.com/watch?v=Qmn72kPdZ4o, abgerufen am19.3.2019. 
Trotzdem schätzten sie den Sportsday als Gelegenheit „to play some common childish Indian games, enjoy the weekend, and meet more Indians“. Die Stimmung beim Sportsday war jedes Jahr sehr ausgelassen und bei manchen Spielen ziemlich kompetitiv. 2011 brach sich ein Teilnehmer des Sportsday beim Spielen von Kabaddi die Nase, so dass das Spiel in den folgenden Jahren wegen zu hoher Verletzungsgefahr nicht mehr ins Programm genommen wurde. Nach der gemeinsamen sportlichen Betätigung wurde zusammen auf dem Unisport-Gelände gegrillt.

Beim Sportsday standen das Zusammensein und der gemeinsame Spaß im Vordergrund. Die lockere, ausgelassene Stimmung und die vertrauten Spiele riefen Erinnerungen an die eigene Vergangenheit wach.

\subsubsection{Indian Independence Day: Zeit der/für die Nation}

Nationalfeiertage wie der Indian Independence Day (IID) unterscheiden sich von anderen Feiertagen durch ihre Fokussierung auf die Nation und ihr Potential, nationale Identität zu formen und nicht nur widerzuspiegeln (McCrone und McPherson 2009c: 219). Nationalfeiertage sind nach McCrone ein „,moment' frozen in time“, der die Anerkennung der Nation in das Bewusstsein ruft (McCrone und McPherson 2009c: 215) und bekräftigt, wer das „wir“ der nationalen Gemeinschaft ist (McCrone und McPherson 2009b: 1). Er markiert die Zeit (McCrone und McPherson 2009c: 220) und ist eine wichtige Koordinate in der Zeitkarte der Nation, an der jedes Jahr aufs Neue über die Vergangenheit, Gegenwart und Zukunft Indiens reflektiert wird.

Ravi erzählte mir in unserem Interview, dass er gemeinsam mit einigen Freund*innen 2007 das erste Mal die indische Flagge in Göttingen gehisst habe. ${ }^{122}$ Die Organisation der Feier des Indian Independence Day wurde seit der Vereinsgründung 2010 vom Komitee zusammen mit Freiwilligen übernommen. In den meisten Jahren wurden die Feierlichkeiten auf zwei Tage aufgeteilt, da der 15. August auf einen Wochentag fiel. Am eigentlichen Datum des Indian Independence Day wurde am Morgen das Hissen der Flagge durchgeführt und an dem Wochenende danach gab es eine ausgedehntere Feier mit cultural programme und gemeinsamem Essen. Auf diese Art und Weise konnte der indische Unabhängigkeitstag angemessen und am tatsächlichen Datum begangen und gleichzeitig in den Arbeitsalltag integriert werden.

Zum kurzen Programm am 15. August gehörte stets eine Rede, für die während der Organisationsphase ein senior gefunden werden musste. In manchen Jahren erklärten sich indische Gastwissenschaftler ${ }^{123}$ bereit, eine kurze Ansprache zu halten. In anderen Jahren waren es junge Studierende oder Wissenschaftler*innen, die eine Rede hielten.

\footnotetext{
122 Seit der Entscheidung des obersten indischen Gerichtshofes 2002 ist es indischen Staatsbürgern (nicht nur Staatsbeamten) an nationalen Ereignissen erlaubt unter Einhaltung der Regeln, die unter dem Flag Code of India festgehalten sind, die indische Flagge zu hissen.

${ }^{123}$ Die Gastwissenschaftler, die Reden hielten, waren stets Männer.
} 


\section{Indian Independence Day: Ein ,kulturelles' oder, politisches' Ereignis?}

Im Vorlauf des Indian Independence Day 2011 schlug Sudhi auf Facebook vor, den Tag zu nutzen, um Solidarität mit der Anti-Korruptionsbewegung zu zeigen. Er schrieb in einem Beitrag in der Facebook-Gruppe der Vereinigung: „Maybe we are thousands miles away from our country ... though I think we also can raise some voice against corruption.....". Sudhi rief dazu auf, das Treffen anlässlich des Indian Independence Day für eine friedliche Demonstration zu nutzen. 2011 gab es in Indien, angeheizt durch mehrere Skandale, eine starke Anti-Korruptionsbewegung, in der Anna Hazare, damals fünfundsiebzig Jahre alt, die Gallionsfigur war (Khandekar und Reddy 2013: 222). Das Ziel der Bewegung „India Against Corruption“ war unter anderem die Einführung eines starken Jan Lokpal-Gesetzes ${ }^{124}$, das es ermöglichen würde, Korruptionsvorwürfe von einem unabhängigen Gremium untersuchen zu lassen (Khandekar und Reddy 2013).

Die jungen Wissenschaftler*innen informierten sich bewusst über politische, soziale oder auch sportliche Ereignisse in Indien. Diese Praktiken sind ein wichtiger Aspekt der Resynchronisation und „helps to maintain the sense of belonging to the homeland's flowing present" (Cwerner 2001: 23). Das Wissen um die politischen Geschehnisse in Indien löste in Sudhis Fall das Bedürfnis aus, sich an der Bewegung für die Jan Lokpal Bill von Deutschland aus zu beteiligen. Allerdings teilten nicht alle der jungen Wissenschaftler*innen diesen Aktionismus und so löste sein Aufruf eine Diskussion in der Facebook-Gruppe aus. Sudhi wurde darauf hingewiesen, dass Demonstrationen in Deutschland angemeldet werden müssen. Er antwortete, dass er geplant habe, einige Poster zu gestalten, auf denen er seine Unterstützung für die Jan Lokpal Bill ausdrücken wolle: „hang the poster frm ur neck... n join the Independence day event... atleast the people in parliament shld knw evryone wants this fight.. evn the people whoe $\mathrm{r} n \mathrm{nw}$ in foreign soil“". ${ }^{125} \mathrm{Er}$ wurde von einer weiteren Person darauf hingewiesen, dass man in Deutschland nicht unangemeldet demonstrieren dürfe, und wurde gebeten, von jeglicher Demonstration bei der Feier des indischen Unabhängigkeitstages abzusehen. Der letzte Kommentator begründete diese Entscheidung mit möglichen Konsequenzen für weitere Aktivitäten von der Vereinigung:

(The) members have taken lots of efforts to organize the event and get a place to celebrate the Independence Day. And, when they have promised the university that this get-together at social place like Uni-Sport centre is meant to celebrate a national event, and does not involve any religious/ political mo-

\footnotetext{
1242010 hatte die indische Regierung eine Version für die Lokpal Bill entworfen, die aber von vielen als zu schwach angesehen wurde, weil der Premierminister, Mitglieder des Parlaments und des Kabinetts davon ausgenommen waren (M. Singh und Sohoni 2016: 113).

${ }^{125}$ Ich habe die Sprache an dieser Stelle absichtlich nicht korrigiert. Sudhi schrieb, wie viele Menschen in SMS, WhatsApp-Nachrichten oder auf Facebook, mit Kürzeln.
} 
tive, it is really a bad notion that we have any sort of demonstrations, this will definitely affect future gatherings.

Bei dem Organisationstreffen wurde kurz über den Vorschlag von Sudhi gesprochen und Ajay, der damalige Präsident, betonte, dass die Feier purely cultural sei und nicht political.

Sudhis Vorschlag zeigt den Einfluss politischer Bewegungen in Indien auf die jungen Wissenschaftler*innen in Göttingen, die in einigen das Bedürfnis auslösten, sich von Deutschland aus zu engagieren. Er zeigt außerdem, dass der Termin des Indian Independence Day zwar eine feste zeitliche Koordinate war, aber seine Ausgestaltung durchaus zur Debatte stand und die Frage aufwarf, was dieser Tag eigentlich ist: Ein Tag, an dem sich politisch engagiert wird oder der „rein kulturell“ ist. Die Verortung des Indian Independence Day als „kulturell“ artikuliert eine normative Vorstellung davon, wie er begangen werden sollte, wie diese Koordinate der indischen Nation gestaltet wird und die eigene Zugehörigkeit zu Indien angemessen ausgedrückt wird. Dadurch wurden jene von den Feierlichkeiten ausgeschlossen, die sich eine kritischere Auseinandersetzung mit Indien gewünscht hätten.

\section{Indian Independence Day und das ,Indisch-Sein“}

Für die Mitglieder der Vereinigung bedeutete die Organisation der verschiedenen Feste und gemeinsamen Aktivitäten viel Arbeit. Wie bereits mehrfach angeklungen ist, wünschten sie sich im Gegenzug eine rege Teilnahme an den kollektiven Ereignissen. Jyoti, die 2012 cultural secretary der Organisation war, machte drei Tage vor der Feier ihrem Frust über das anscheinend fehlende Interesse an der Teilnahme am Indian Independence Day in einem Facebook-Text Luft:

On this occasion, many questions come to my mind about being Indian. What is the true essence of being an Indian? Is wearing Indian dress a sign of being Indian? Or is it food? Or is it speaking our own language and interacting with people? I know ,unity in diversity' is the trademark or common way to describe ourselves as Indians. But an important question remains in my mind ,Are we really united enough to show our diversity?' Do we really respect and love our nation to the extent we have to? It is hard for me to convince myself with an answer ,yes', for example, when I see (from the events list on FB) the amount of people who are coming for the Flag Hoisting ceremony of the Independence Day event.

Einige Mitglieder der Gruppe Indians in Göttingen, in der Jyoti diesen Text veröffentlicht hatte, fühlten sich durch ihre Aussagen angegriffen und dazu aufgerufen, zu antworten. Eine junge Doktorandin schrieb, sie begrüße die Bemühungen vollkommen. Allerdings werde sie beim Hissen der Flagge nicht dabei sein: „Personally it is a highly symbolic event for me and serves no practical purpose other than social- 
izing. I don't feel more patriotic suddenly on this day by joining a group of fellowIndians to hoist the national flag and sing the anthem." Sie glaube an praktischere Mittel, mit denen sie „die Liebe für ihr Land“ ausdrücken könne, z.B. indem sie die Schulbildung eines Kindes finanziere. Sie fände es unfair den Patriotismus einer Person an der Teilnahme an einem Event zu messen. Nachdem sie ihre Einstellung zum Indian Independence Day deutlich gemacht hatte, betonte sie erneut, dass sie die Bemühungen der Vereinigung respektiere und Jyotis Enttäuschung verstehe. Diese antwortete im gleichen, um Höflichkeit bemühten Ton, dass die Organisation den Indian Independence Day als Gelegenheit für das socializing verstehe, und betonte: „I never intended to make anyone patriotic for a day." Allerdings ist laut McCrone und McPherson genau dies die Wirkung nationaler Feiertage: Sie üben einen kalendarischen Zwang aus, exakt an diesem einen Tag patriotisch zu sein (McCrone und McPherson 2009b: 17).

In Göttingen bestand viel eher als in Indien die Möglichkeit, sich den Feierlichkeiten zu entziehen, da der Indian Independence Day nicht zur offiziellen Zeitkarte Deutschlands gehört. Dhara entschied sich bewusst dafür, nicht an der Feier des Indian Independence Day teilzunehmen. Sie erklärte mir, sie störe das „India shining image“, das ihrer Meinung nach an diesem Tag zelebriert werden würde. Allerdings, und das zeigt die Debatte um die Anwesenheit beim Flaggehissen, konnte das Fernbleiben von der kollektiven Aktivität dazu führen, dass nicht nur die ,Liebe für das Land', sondern auch das eigene ,Indisch-Sein' durch andere infrage gestellt wurde. Zusammengehörigkeit basiert, wie diese Auseinandersetzung um die Teilnahme deutlich macht, auf gegenseitiger Kontrolle und Erwartungen an die Investition der eigenen Zeit (vgl. Pfaff-Czarnecka 2012: 32). Es entstand ein „kollektiver Erwartungsdruck“ (ebd.), der die Vereinigung als ein Regime der Zugehörigkeit (ebd.) kennzeichnet. Wer dazugehören wollte, von den Angeboten der Vereinigung profitiert hatte oder profitieren wollte, sollte, so die implizite Aussage, tunlichst bei den kollektiven Aktivitäten anwesend sein. In diesen Situationen, den Konflikten um die Zeit und was mit ihr anzufangen sei, äußert sich die Macht der Organisation über die kollektive Zeitgestaltung der indischen Wissenschaftler*innen in Göttingen.

In einem weiteren Kommentar erklärte eine junge Frau mit Verweis auf das Thema Diversität, das ebenso von Jyoti aufgegriffen wurde: „Diversity' of our nation is not merely limited to diversity in language, food or culture but also in our thoughts, opinions and perspectives, and respecting these differences symbolizes true ,Unity in diversity." Die beiden dargestellten Debatten, die in zwei unterschiedlichen Jahren dem Indian Independence Day in Göttingen vorausgingen, zeigen, dass dieses Ereignis dazu anregte, zu hinterfragen, was es eigentlich heißt, Inder*in zu sein, und wie diese Positionierung artikuliert werden sollte. Die Vereinigung nahm dabei eine dominante Rolle ein, da das Komitee die Kontrolle darüber hatte, wie die jungen Inder*innen in Göttingen repräsentiert wurden und welche Artikulationen des Indischseins in den gemeinsamen Aktivitäten Raum hatten. 


\section{Das Hissen der Flagge}

Seit 2011 fand das Hissen der Flagge auf dem Gelände des Hochschulsports statt, weil es dort einen Fahnenmast gab, der benutzt werden und an dem die Flagge den ganzen Tag bis zum Sonnenuntergang bleiben durfte, wie es vom Flag Code of India verlangt ist. Im Folgenden beschreibe ich den Ablauf der Veranstaltung, der jedes Jahr sehr ähnlich war, exemplarisch anhand meiner Beobachtungen im Jahr 2011.

Ich fuhr gemeinsam mit Lali, die in der gleichen Straße wie ich wohnte, zum Gelände des Unisports. Als wir um kurz vor acht Uhr ankamen, wurde gerade die Fahne präpariert: Ajay und Ravi legten Blütenblätter auf die Fahne und falteten sie in einer Art und Weise, die im Flag Code of India vermerkt ist. Es kamen nach und nach mehr Menschen am Veranstaltungsort an, unter anderem der Ehrengast, ein Professor von der Jawaharlal Nehru University, der eine Rede halten würde. Um 8:20 Uhr begann das Programm damit, dass Ravi und der Ehrengast die Fahne gemeinsam hissten. Das Aufspannen funktionierte nicht sofort, allerdings entfaltete sich die Flagge nach einem weiteren kurzen Ruck an der Schnur und die Blütenblätter schwebten effektvoll gen Boden. Die Anwesenden sangen gemeinsam die indische Nationalhymne Jana Gana Mana. Ich hatte das Gefühl, dass einige vom gemeinsamen Singen der Hymne sehr berührt waren. Diese Einschätzung bestätigte sich in den kurzen Gesprächen, die ich mit einigen Anwesenden führte. Lali erklärte: „I think, it's more emotional here. In a foreign land, hoisting your own national flag - it's something really touchy." Es fühle sich gut an, betonte Lali. Der Ehrengast, ein Geschichtsprofessor, hielt seine Rede. Danach folgten zwei weitere Reden - eine auf Hindi und eine auf Englisch, die von zwei Promovierenden gehalten wurden. Zum Schluss wurden Süßigkeiten verteilt und ein Gruppenfoto mit dem Fahnenmast im Hintergrund gemacht.

Die Stimmung war während der Veranstaltung recht ernst, vor allem im Vergleich zu der ausgelassenen Stimmung an Diwali oder dem Sportsday. Nach etwa dreißig Minuten war das Ereignis zu Ende und die ersten Personen machten sich auf den Weg in ihre Büros, um ihren Arbeitsalltag zu beginnen. Hierin sah Tanvir den größten Unterschied zwischen dem Feiern des Indian Independence Day in Indien und in Göttingen:

In India, you are with people and felicitating a holiday. So probably you will spend the day with your family and watch some patriotic movies, listen to some songs, get the feeling arising. But today, here we go into the institute, talking with other friends who are not Indians probably. (Tanvir, 15.08.2011)

Trotzdem, so betonte Tanvir auf meine Nachfrage, sei es für ihn wichtig, den Indian Independence Day in Göttingen zu feiern. Er erinnerte sich daran, dass in seiner Kindheit die Schule am Indian Independence Day geschlossen gewesen sei und er zuhause die Flagge gehisst habe: „It's like a tradition you keep with you.“ 
Die Feier des Indian Independence Day war für die jungen indischen Wissenschaftler*innen eine wichtige Kontinuität, die sie, wie ich im Folgenden zeige, auf der synchronen und diachronen Ebene mit ihrem Leben in Indien verband. Das Ereignis schuf außerdem eine wichtige zeitliche Koordinate im Jahr, an der meine Gesprächspartner*innen gemeinsame Zeit verbringen konnten.

\section{Die Indian-Independence-Day-Reden: Vergangenheit und Zukunft Indiens in der Gegenwart in Göttingen}

Am 15. August 2011 begann der Ehrengast seine Rede, die er mit Conscripts and Volunteers betitelt hatte, mit der Frage: „Would you be at an Independence Day gathering if you were in India at this moment?" Und äußerte seine Vermutung, dass es wohl einige der Anwesenden nicht sein würden. Er ging kurz auf die Geschichte des Indian Independence Day ein und stellte fest, dass: „Nowadays, Independence Day audiences are like a collection of conscripts. What was once a festival of the people has become a ritual of the state." Als Wissenschaftler*innen an einer Universität müssten sie Ereignisse und Ideologien kritisch betrachten. Der indische Nationalismus, so seine Aussage, konnte trotz der politischen Errungenschaften nicht den Versprechen seiner Anführer, wie denen von Nehru, gerecht werden. So gebe es zum Beispiel immer noch Kommunalismus ${ }^{126}$. Weltweit habe der Nationalismus positive und negative Aspekte gehabt. Positiv, wenn er eine Kraft der Vereinigung und Befreiung gewesen sei wie in Indien unter der kolonialen Herrschaft; negativ, wenn er in Krieg, Teilung und Zerstörung gemündet sei.

Der Professor warnte davor, Nationalismus immer oder unkritisch zu feiern: „We must ask more carefully what it is that we wish to celebrate." Damit kam er zurück zu seiner Anfangsfrage und zum Grund dafür, warum die Anwesenden an diesem Tag versammelt seien. Er vermutete, dass das Vermissen des Zuhauses ein Grund für die Teilnahme sein könnte: „And by that we miss something which is not easy to define, but to which we owe allegiance. "Das Zuhause, überlegte er, sei eine komplizierte Mischung aus Essen, Filmen, Freundschaften, Sprachen, Humor und Geographie. Es gebe zwar eine politische Idee und eine Karte im Hintergrund, aber es sei weit mehr als dies: „In short, it is mainly cultural, in the broadest and most generous sense, and it has brought us here today in a voluntary act of affiliation and affection." Er schloss seine Rede mit folgendem Gedanken: „If gatherings in India on 15 August become as truly voluntary as ours, then our nationalism will be healthier still, and we will have reason to be truly happy." Der Professor aus Delhi nutzte seine Rede, um die Praxis des Indian Independence Day kritisch zu reflektieren und die Anwesenden dazu aufzurufen, dies auch zu tun.

\footnotetext{
126 Als Kommunalismus werden mit Bezug auf Indien die Konflikte zwischen Bevölkerungsgruppen, die sich auf der Basis von Religion voneinander abgrenzen, bezeichnet (Lukose 2010: 208). Besonders die Konflikte zwischen Hindus und Muslime flammen in Indien immer wieder auf.
} 
Einen anderen Ton hatte die Rede, die 2012 von einem indischen Promovierenden gehalten wurde. Er begann seine Rede damit, dass er allen Anwesenden und „every Indian across the world“ einen frohen Unabhängigkeitstag wünschte. Damit rückte er in den Fokus, dass die jungen Inder*innen zu einer größeren Gemeinschaft von Menschen gehörten, die diesen Tag auf der ganzen Welt gleichzeitig feierte.

Er drückte einen kollektiven Dank an die Freiheitskämpfer aus: „All freedom fighters that have fought for our independence and providing us an opportunity to live life independently - a life where we can define our future and progress of our country, and moreover contribute to a better world." Das Thema des Fortschritts, das er bereits in diesem Satz anschnitt, zog sich durch die gesamte Rede und fand seinen Ausdruck im Begriff der „Entwicklung“ (development). Er betonte, Indien befinde sich auf dem „Pfad der Entwicklung“. Allerdings müsse sichergestellt werden, dass die Vorteile der Entwicklung jeden bedürftigen Staatsbürger erreichten und „the light of development light every household in India. Taking India to the next level and maintaining Indian development is our next challenge." Die Verantwortung für die „Entwicklung“ liege auf den Schultern der jungen Generation. Aus diesem Grund beendete er die Rede mit der folgenden Aufforderung: „I request you today to make a promise to always contribute in all means possible to service and interest of our motherland India. Vande Mātaram!“127 (15.08.2012)

In den Reden wurden die Geschichte, die gegenwärtige Situation und die Zukunft Indiens thematisiert. Sie waren ein Mittel, um zu reflektieren, was es bedeutet, Inder*in zu sein, was an diesem Tag gefeiert wird, und um sich zur Situation in Indien zu positionieren. Die Redner drückten durchaus ihre persönliche Haltung aus, allerdings stand die Verortung als „Wir“, als Kollektiv, im Mittelpunkt. Der Fokus auf das Kollektiv artikulierte sich unter anderem in der Sprache, die in den Reden genutzt wurde.

Besonders in der zweiten Rede wurde der Indian Independence Day als Koordinate markiert, an der über die Veränderung Indiens durch die Zeit reflektiert und gefragt wurde, ob es sich ,richtig' entwickelt habe. Der Redner brachte die Vorstellung von einer ,entwickelten' Zukunft Indiens direkt mit der Zukunft der jungen Wissenschaftler*innen zusammen, die er in der Verantwortung sah, ihr „Mutterland" zu unterstützen.

\section{Indian Independence Day Teil zwei: Potluck Party}

Am Wochenende nach dem 15.08. wurde der Indische Unabhängigkeitstag in einem informelleren Rahmen gefeiert. 2011 und 2012 fanden die Feierlichkeiten im Internationalen Besucherzentrum (IBZ) des Studentenwohnheims im Kellnerweg statt. Seit 2013 war das Max-Planck-Institut für experimentelle Medizin Veranstaltungsort. Die Feierlichkeit wurde damit eingeleitet, dass die Reden vom 15.08. wiederholt wurden oder eine andere Person kurz ein paar Worte zur Relevanz des Indian

${ }^{127}$ Auf den folgenden Seiten erkläre ich diesen Ausruf näher. 
Independence Day vortrug. 2013 fiel diese Aufgabe an Vendan, den damaligen Präsidenten der Vereinigung. In seiner kurzen Ansprache erklärte er, dass der Indian Independence Day, bis er zwanzig Jahre alt war, kaum etwas für ihn bedeutet habe. Seine Einstellung habe sich aber nun geändert: „When I recently turned twenty-five, now I realize that this is a day that marks something really great." Der Kampf um die Unabhängigkeit Indiens habe viel früher begonnen als er durch den Indian Independence Day datiert werde, bereits vor der Revolte von 1857. Das bedeute, dass es viele unsung heroes geben würde. Er habe sich in den vergangenen Jahren am Indian Independence Day bewusst vorgenommen, sich dieser unbekannten Held*innen zu erinnern und außerdem den Blick in die Zukunft zu richten. Der Indian Independence Day sei ein Tag, der „Entwicklung“ (progression) markiere. Tatsächlich, betonte Vendan, habe er für einige Zeit gedacht, dass sich Indien gut und in eine positive Richtung entwickeln würde, aber: „On the sad side, we have to also accept this truth that the equality that we all enjoy is not for everyone in the country." Er verwies auf die Rede, die von einem indischen Postdoktoranden am 15.08. gehalten worden war und auf dessen Aufruf zur Reflexion „what we can contribute to our country and to bring up equality in terms of caste, religion, creed and political power in position". Vendan wollte erneut daran erinnern, dass „we need to think forward in future in order to create the equality in our country." Sie würden alle planen, irgendwann in „ihr Land“ zurückzugehen und für das Land „zu arbeiten“.

Nach den einführenden Worten folgte, ähnlich wie bei der Feier von Diwali, ein cultural programme mit Gesang und Tanz. Der Ton war allerdings etwas weniger ausgelassen, sondern ernsthafter und nachdenklicher. Diese Stimmung fand ihren Ausdruck unter anderem in den Liedern, die für das kulturelle Programm gewählt wurden. Ein Lied, das jedes Jahr in irgendeiner Form präsent war, war der national song „Vande Mātaram“. ${ }^{128}$ Der Titel des Liedes kann in etwa mit „Ich verehre die Mutter" übersetzt werden (Lipner 2008: 27). Vande Mãtaram wurde von Bankimcandra Cattopadhyay (1838-94), einem bengalischen Autor, geschrieben und in seinem Roman Änandamath veröffentlicht (ebd.: 28). Der „national song“ besteht aus den ersten beiden Strophen (siehe Textfeld), des aus neun Strophen bestehenden Liedes. Das Lied wurde vom Roman abgekoppelt bekannt, unter anderem weil Rabindranath Tagore 1896 die ersten beiden Strophen unterlegt mit einer selbstgeschriebenen Melodie vor dem National-Kongress in Kolkata sang (ebd.: 38). Der Titel des Liedes wurde zu einer Art Slogan und erhielt seine politisierte Bedeutung während der Proteste gegen die erste Teilung Bengalens 1905. Obwohl der Ausruf „Vande Mātaram" bei diesem Ereignis auch von Muslimen genutzt wurde, wurde er ab 1906/07 vor allem mit der nationalistischen Bewegung der Hindus assoziiert (ebd.: 39). 1950 wurden die ersten beiden Strophen zum national song erklärt (ebd.:

\footnotetext{
${ }^{128}$ Das Lied wurde in verschiedenen Versionen musikalisch umgesetzt. Eine der beliebtesten ist die des indischen Komponisten und Sängers A. R. Rahman. Ein Beispiel findet sich auf Youtube unter https://www.youtube.com/watch?v=Ss-kLGW2pHQ, abgerufen am 29.11.2018.
} 
42). Seitdem gab es wiederholt Kontroversen um das Lied, z.B. $2006^{129}$ (ebd.). In Göttingen wurden die Debatten um das Lied nicht sichtbar - es wurde jedes Jahr aufs Neue gesungen oder dazu getanzt.

Für das Programm wurden außerdem Lieder aus Bollywood-Filmen oder Gedichte indischer Autoren gewählt, die häufig die Liebe für die Heimat oder Heimweh ausdrückten, wie im Fall des Liedes Aye mere pyare watan, dessen Titel „Oh, mein geliebtes Heimatland“ bedeutet. Bevor Aditi dieses Lied 2012 gemeinsam mit Tanvir zu singen begann, leitete sie die Performance mit einigen Worten ein. Sie erklärte, dass sie, Tanvir, Ajay und Harendra sich zum Einstudieren der Lieder getroffen und viel Spaß dabei gehabt hätten. Aditi erzählte, Tanvir habe die Beobachtung gemacht, dass alle vier aus unterschiedlichen Regionen Indiens kämen: „So, I think it's quite nice that four music enthusiasts from four corners of India come together in Germany and celebrate this unifying fact that we all are Indians and that you rejoice India's Independence with us." Sie und Tanvir würden nun das erste Lied gemeinsam singen, das Lied Aye mere pyare watan aus dem Film Kabuliwala ${ }^{130}$.

Der Text des Liedes sei „very, very sweet and soulful and a portrayal of what we Indians feel currently here living miles away from our motherland, from our parents" (Aditi, 18.08.2012). Aditi drückte ihre Hoffnung aus, dass sich viele der Anwesenden mit diesem Lied würden identifizieren können. Das Lied ist langsam und hat einen traurigen, melancholischen Ton, so dass das Vermissen der Heimat, das im Text ausgedrückt wird, gleichermaßen durch den Klang spürbar wird.

Akhil, der während seiner Zeit in Göttingen bei jeder Festlichkeit Lieder vortrug, wählte 2013 für seinen Auftritt beim Indian Independence Day zwei Texte des tamilischen Dichters Mahakavi Bharathi ${ }^{131}$. Bharathi habe seine Gedichte vor etwa einhundert Jahren verfasst, allerdings seien sie heute noch sehr relevant: „Because, I mean, in some sense nothing has really changed." So meinte Akhil, er habe das Gefühl, dass noch nicht die wahre Unabhängigkeit erreicht worden sei. Es gebe weiterhin so viel Ungleichheit, Armut, Frauen seien nicht frei und viele Menschen hätten nicht die Freiheit, sich nach ihren Wünschen zu verlieben. „But still we hope that all these problems will one day cease and we'll actually truly get freedom." Die Erläuterungen zu der Auswahl der Lieder wiederholte er auf Tamil.

\footnotetext{
${ }_{129}$ Die Kulturministerin Indiens, Ambika Soni, schlug vor, zum hundertsten Jubiläum der Entstehung des Liedes ein simultanes landesweites Singen zu veranstalten. Dieser Vorschlag traf auf großen Widerstand unter anderem von Muslimen, die das Lied als „idolatrous, anti-Muslim, and anti-secular" bezeichneten (Lipner 2008: 28).

130 „Kabuliwala“ bedeutet „Mensch aus Kabul“. Es handelt sich um den Titel einer Kurzgeschichte von Rabindranath Tagore über einen afghanischen Mann, der jedes Jahr nach Kolkata kommt, um dort Trockenfrüchte zu verkaufen. Er hat großes Heimweh und vermisst seine Tochter. Das Lied stammt aus der gleichnamigen Verfilmung der Kurzgeschichte von 1961 (Lal 2010).

131 Subramanya Bharathi (1882-1921), genannt „Mahakavi“, was „großer Dichter“ bedeutet. Er wurde verehrt für seinen modernisierenden Einfluss auf die tamilische Dichtung und für seine reformerischen Ideen z.B. bezüglich der Emanzipation der Frauen und die Abschaffung des Kastensystems (Sri 1996: 290-92).
} 
In den ausgewählten Liedern und Texten fanden sich häufig wie im national song der Vergleich oder die Gleichsetzung Indiens mit einer Mutter. Dieses Thema griffen auch Dhana und Yagav für ihren Beitrag am Indian Independence Day 2013 auf: „Our performance is dedicated to the relationship of a mother and a child, which we would like to compare with the relationship of citizen and the motherland." Die Staatsbürger eines Landes würden zu ihrem Mutterland aufschauen wie ein Kind, das die Zuneigung seiner Mutter sucht. So kostbar sei das Band der Liebe, so tief das Gefühl der Zugehörigkeit, dass selbst die kleinste Entfernung von der Mutter dem Kind Angst mache: „Like a man far away from his native soil, teary eyed and reluctant to be sent away from the mother, the child sings out his last grief." An dieser Stelle begann Yagav das Lied Meri Maa, also „Meine Mutter“ aus dem Film Taare Zameen Par (2007) zu singen. Dhana begleitete ihn dazu auf der Gitarre. Nachdem der Applaus für den Gesang abgeebbt war, erklärte Yagav, das Kind werde dann bald erwachsen werden, die Mutter nicht mehr nach den Details jeder Mahlzeit fragen und "he is ready to stand up as a protector, defender, and fight for her and enjoys the life in her service." Nach der Überleitung folgte das Lied Lukka Chuppi aus dem Film Rang de Basanti (2005). Dhana und Yagav sangen zusammen, Dhana spielte dazu die Gitarre. Im Film wird dieses Lied in der Szene gespielt, in der ein junger Mann, der in der indischen Armee gedient hat, beerdigt wird. Dhana kommentierte zum letzten Lied:

However, no tear is ever shed in vain. Another sacrifice is not just another obscure tragedy; our motherland, our nation, continues to flourish and burst with life. As teardrops shine into stars on cloudless evenings (?) of laughter echo as children run back home to the aroma of freshly prepared night meals. In the mother's arms peace endures, the river of life flows secure, and our motherland embraces us in all her glory. (Dhana, 17.08.2013)

Danach sangen die beiden Aisa Des Hai Mera aus dem Film Veer-Zaara ${ }^{132}$ (2004). Der Titel des Liedes bedeutet übersetzt „Dies ist mein Land“. Den Bezug zwischen „Mutter Indien“, Müttern generell und der Heimat, den Dhana und Yagav durch die Auswahl der Lieder betonten, nutzte Vendan 2013 in seiner Überleitung zum nächsten Programmpunkt, dem Essen, und erklärte: „Once we relate with mother and motherland, then we always remember delicious food. It's already time for dinner, the most awaited event of this event."

Die Feier des indischen Unabhängigkeitstages war jedes Jahr eine Mischung aus Nostalgie, Heimweh, kritischer Reflexion der Ungleichheit in Indien und der eigenen Beziehung zur Nation. In der biographischen Navigation war der Indian Independence Day eine Koordinate, die den Fokus auf die Zeitkarte der Nation lenkte. An diesem Tag wurde jedes Jahr aufs Neue die ,Entwicklung' Indiens reflektiert. Was

${ }_{132}$ Im Zentrum dieses Films steht die Liebesgeschichte zwischen Veer, einem Piloten der indischen Air Force, und Zaara, einer jungen Frau aus Pakistan. 
als ,Entwicklung ' und ,Fortschritt' galt, wurde in den Reden und kurzen Ansprachen deutlich: ein Wandel weg vom Kastensystem, das häufig als Symbol für ,Tradition gilt (Fleming 2016: 117), hin zu Freiheit und Gleichheit für alle.

Als „soziale Technik, um Gleichzeitigkeit herzustellen“ (Nowotny 1993: 33) war der Indian Independence Day für die indischen Wissenschaftler*innen eine Möglichkeit, sich mit der Zeitlandschaft in Indien zu synchronisieren. An diesem Tag wurden Vergangenheit, Gegenwart und Zukunft zusammengebracht und damit Kontinuitäten und Vertrautheit hergestellt.

\subsection{Reisen nach Indien und die Zeitgestaltung}

In wissenschaftlichen Studien zu Reisen von Migrant*innen in das Herkunftsland wird deren Bedeutung für die Fortdauer und Aushandlung von Zugehörigkeiten betont (Marschall 2017; Baldassar 2001). Die Reisen werden außerdem als wichtiger Aspekt des Lebens im transnationalen sozialen Raum verstanden (Oeppen 2013; Skrbiš 2008; Földes und Savu 2018). Allerdings wird auch deutlich, dass sich die Motivationen für die Reisen und die Erfahrungen, die während der Aufenthalte gemacht werden, unterscheiden und beispielsweise von den Gründen für die eigentliche Migration oder auch dem sozio-ökonomischen Status beeinflusst werden (Marschall 2017: 216). Die jungen Wissenschaftler*innen unternahmen mehr oder weniger regelmäßig Reisen nach Indien - zu ihren Eltern, Familien und Freund*innen. Einige reisten möglichst jedes Jahr nach Indien, andere schränkten ihre Reisen nach Indien ein, was häufig mit der Arbeit an Forschungsprojekten oder der Fertigstellung der Promotion begründet wurde. Die Reisen nach Indien bildeten, neben den zuvor beschriebenen Festen, weitere besondere Zeiten der biographischen Navigation. Sie boten die Möglichkeit für physische Kopräsenz, für das Verbringen von Zeit mit der Familie und Freund*innen, waren aber auch mit der Erfüllung familiärer Verpflichtungen verbunden. Wieder bei der Familie zu sein, bedeutete deswegen für die jungen Wissenschaftler*innen, Erwartungen an ihre Zeitgestaltung aushandeln zu müssen. Auf diese Aushandlungsprozesse - auf der Ebene des Alltags und bezogen auf den Lebensverlauf - gehe ich auf den folgenden Seiten ein. Durch die Beschäftigung mit den Reisen nach Indien wird die Interaktion zwischen Mobilität und Zeitgestaltung besonders sichtbar. So zeigt sich, wie die Zeitgestaltung während des Lebens in Deutschland die Zeitpunkte und die Dauer von Reisen nach Indien beeinflusste, welche Wirkung dominante Zeitkarten auf das Timing von Reisen hatten und wie, umgekehrt, während der Aufenthalte in Indien die Zeitgestaltung ausgehandelt werden musste.

Wieder in Indien zu sein, wenn auch nur vorübergehend, gab einigen meiner Gesprächspartner*innen das Gefühl von Kontinuität und Zugehörigkeit. Für andere war die Erfahrung ambivalenter und konnte Brüche mit der Vergangenheit und Veränderungen des Selbst ins Bewusstsein rufen (vgl. Marschall 2017). Mit dem Ein- 
fluss der Reisen nach Indien auf die Aushandlungen des Selbst beschäftige ich mich im letzten Abschnitt.

\subsubsection{Zeitgestaltung zwischen Resynchronisation und Hektik}

Meine Gesprächspartner*innen freuten sich darauf, ihre Familien und Freund*innen zu besuchen und ihre Lieblingsspeisen zu essen. In Indien machten viele die Erfahrung, dass sie sich zunächst wieder an die klimatischen Bedingungen und die soziale Umwelt gewöhnen mussten, eine Erfahrung, die auch als „reverse culture shock“ bezeichnet wird (Pritchard 2011; Sondhi 2013: 44). Vendan betonte in einem Vergleich mit Deutschland vor allem die Menge an Menschen, auf die man in Indien ständig stoße: „When I go to India there are more people, it's more crowded and lively and vibrant." Obwohl er sich manchmal über die Menschenmengen und die Hektik (rush) beschwere, berichtete er, sei dies ein Aspekt, den er liebe: „In India it's unorganized but I still love it, thats one part which I love there" (Vendan, 26.03.2013). Die Hektik und das Gedränge waren für Vendan vertraute Erfahrungen, die er mit seinem Leben in Indien verband.

Wie Sabine Marschall in ihrer Studie betont, können die Aktivitäten von Migrant*innen während ihrer Reisen als Suche nach „familiar routines, networks and ,physical props', the traces of their former lives" verstanden werden (Marschall 2017: 220). Die jungen Wissenschaftler*innen überlegten und planten bereits vor der Abreise, mit wem und mit welchen Aktivitäten sie ihre Zeit in Indien verbringen würden. Besonders das Treffen von alten Schulfreund*innen konnte einigen Planungsaufwand bedeuten, wie mir Rohini berichtete: „Nowadays it's a bit tough to catch up because we are all from different professions" (Rohini, 18.08.2016). Es sei schwer, einen Zeitraum zu finden, in dem alle Zeit hätten und Urlaub nehmen könnten. Außerdem lebten ihre Freund*innen aus der Schulzeit mittlerweile in unterschiedlichen Teilen Indiens und der Welt. Diese Treffen setzten damit ein hohes Maß an räumlicher und zeitlicher Koordination voraus.

Von den Treffen mit Freund*innen abgesehen, berichtete mir Rohini, dass sie die ersten zwei Wochen in Indien stets mit ihren Eltern verbringe: „For me when I go home the first two weeks I'm just at home, because I really want to stay with my parents and spend some time with my family." Sie gingen gemeinsam essen oder machten gemeinsam einen kurzen Urlaub. Lachend fügte Rohini noch hinzu: „So for me India is like, I basically go there to sleep. This is what my aunt told me because I didn't go to Calcutta last year" (Rohini, 18.08.2016). Wie Rohinis Ergänzung zu ihrer Tante verdeutlicht, achteten die Familienmitglieder darauf, wer besucht wurde und wer nicht. Im folgenden Abschnitt gehe ich auf die Aushandlung der Zuteilung von Zeit während der Aufenthalte in Indien ein. 


\section{Allokation und Erfahrung von Zeit in Indien}

Für die Aufenthalte in Indien planten meine Gesprächspartner*innen häufig eine Dauer von drei bis vier Wochen ein. Wie Smita mir erklärte: „It's long distance and you have to do so many things over there." Bei jeder Reise nach Indien gilt es, viele Verwandte zu besuchen, fährt Smita fort: „If you don't visit, they feel bad and get angry that ,you haven't come to' and this and that." Das Besuchen der Familienmitglieder sei eine Pflicht, die erfüllt werden müsse. Smita empfand die Reisen nach Indien aus diesem Grund auch nicht als „Ferien“, sondern als eine Art Arbeit: „It's like another kind of job that you do over there" (Smita, 26.01.2013).

Als ich Sangita fragte, wie sich ihre Reisen nach Indien gestalten würden, fasste sie diese mit den folgenden Worten zusammen: „Travelling a lot, meeting a lot of people, eating a lot, falling sick“ (Sangita, 08.08.2013). Der Versuch die Aufenthalte in Indien mit möglichst vielen Verwandtschaftbesuchen und Treffen mit Freund*innen zu füllen, mache die Zeit in Indien laut Sangita sehr stressig und ermüdend: „It's way more tiring. I have been in India for a month and I have never had the straight eight hours sleep for almost throughout the month." Während ihrer Besuche würde sie sich „strapazieren“ (strain) so sehr sie könne „and then I come back to Göttingen and I take rest" (Sangita, 08.08.2013). Trotz des Stresses, den die vielen Besuche auslösten, betonte Smita, dass sie die Zeit in Indien genieße: „But it's a nice time to enjoy because you meet all your relatives and old friends and for one month you will live your old life actually. So it's like a more hectic form of old life but definitely it's nice" (Smita, 26.01.2013).

Die Aussagen von Sangita und Smita verdeutlichen, dass die jungen Wissenschaftler*innen während ihrer Aufenthalte in Indien ihre Zeitgestaltung, besonders die Zuteilung von Zeit mit ihrer sozialen Umwelt, aushandeln mussten. In Indien machten sie spezifische Erfahrungen von Zeit, die mit Hektik oder Stress beschrieben wurden, die aber auch eine gewisse Vertrautheit vermittelten, wie aus Vendans Aussage deutlich wird. Anders ausgedrückt, befanden sich Smita, Sangita und Vendan in einer Zeitlandschaft, mit der sie sich wieder arrangieren mussten, in der sie aber auch vertraute zeitliche Erfahrungen machten.

\subsubsection{Reisen nach Indien und die Gestaltung des Lebensverlaufs}

Die Zeitpunkte von Reisen nach Indien und auch die Häufigkeit dieser wurden durch die Koordinaten dominanter Zeitkarten und von der Zeitgestaltung meiner Gesprächspartner*innen in Deutschland beeinflusst. Die Konzentration auf die Fertigstellung der akademischen Abschlüsse, wie ich sie in Kapitel 4 beschrieben habe, konnte dazu führen, dass Reisen auf die Zeit nach den erreichten Abschlüssen vertagt wurden. Nathin erzählte mir beispielsweise, dass er während seines Bachelorstudiums in Schweden, das etwa zweieinhalb Jahre andauerte, nicht nach Indien gereist sei. In den Ferien, die er während seines Studiums hatte, absolvierte er unterschiedliche Praktika, unter anderem an der Universität Stuttgart. Der Fokus auf den Abschluss 
seiner Promotion sorgte in Kundans Fall dafür, dass er drei Jahre lang nicht nach Indien reiste. Als ich mit Kundan sprach, plante er gerade seine Reise zur Familie und erklärte mir lachend, dass er vor hätte, alle Mahlzeiten, die er während der drei Jahre verpasst hatte, nachzuholen: „Everyday I'll eat different Indian dishes for the whole three years together" (Kundan 14.08.2013).

Wichtige Ereignisse im Lebensverlauf meiner Gesprächspartner*innen oder ihrer Familienmitglieder boten Anlässe für Reisen nach Indien und beeinflussten das Timing dieser. Einige flogen nach Indien, um potentielle Heiratspartner*innen zu treffen, wie beispielsweise Tanvir und Bhanu. Andere begaben sich zu ihren Familien, um mit diesen ihre Partner*innenwahl auszuhandeln, so zum Beispiel Ravi. Die Hochzeit von Familienmitgliedern und Freund*innen waren weitere Zeitpunkte, an denen meine Gesprächspartner*innen nach Indien reisten. Da Hochzeiten in Indien häufig in den Monaten Dezember, Januar, Februar und März stattfinden, was ich in Kapitel 7 genauer erläutere, bildeten diese Monate die Hauptreisezeit.

Vendan war im Januar 2013 zu seiner Familie nach Indien gereist und meinte, dass der Besuch auch wegen des Zeitpunkts sehr schön gewesen sei: „It was really very great because I went in a time when there was this harvest festival that usual happens in January in my region." Nach diesem Fest fänden außerdem traditionellerweise Hochzeitfeiern statt: "This is considered to be one of the luckiest times for people to get married so one of my cousins got married and another cousin got enganged.“ Somit habe es eine großes Familientreffen gegeben: „I got to meet a lot of my family, extended family whom I would otherwise not have met" (Vendan, 26.03.2013). Die Zusammenkunft der Familie anlässlich der Heirat hatte Vendan einige weitere Reisen innerhalb Indiens erspart.

Die Assoziation von Indienreisen mit Lebensverlaufsereignissen konnte allerdings auch dazu führen, dass die jungen Menschen Reisen zu ihren Familien vermieden oder hinauszögerten. So berichtete mir Āmāl von einer Freundin, die Angst davor hatte, nach Indien zu reisen, da sie befürchtete, dem Druck zu heiraten nicht standhalten zu können und während ihres Aufenthaltes verlobt zu werden.

Während der Aufenthalte bei den Familien mussten meine jungen Gesprächspartner*innen häufig erneut die Gestaltung ihres Lebensverlaufs aushandeln - sei es den Zeitpunkt einer Heirat, die Partner*innenwahl oder später auch die Familiengründung (siehe Kapitel 8).

\subsubsection{Reisen nach Indien und das Selbst: Kontinuitäten und Veränderungen}

Reisen von Migrant*innen in das Herkunftsland werden in der Literatur unter anderem im Zusammenhang mit der Suche nach „home“ (Baldassar 2001) und als integraler Aspekt transnationaler Lebensweisen und der Aushandlung von Zugehörigkeiten und Identifikationen (Chang, Sam und Jackson 2015; Marschall 2017; Tie, Holden und Park 2015) analysiert. Saulo Cwerner postuliert mit Blick auf Prozesse der Resynchronisation: „Ultimately, however, only the occasional trip back home can re-install the wholeness of the feeling of belonging and, thence, renew the tem- 
poral synchronization of one's life path to that of the homeland" (Cwerner 2001: 23). Wie oben bereits dargelegt, konnten die jungen Wissenschaftler*innen während ihrer Aufenthalte in Indien vertraute Erfahrungen machen, für ein paar Wochen eine Version ihres „alten Lebens" leben und Verpflichtungen gegenüber der Familie erfüllen. In den Gesprächen wurde allerdings deutlich, dass die Zeit in Indien auch Veränderungen des Selbst und in der Beziehung zu den Eltern sichtbar machen konnte. Beide Zusammenhänge werde ich auf den folgenden Seiten thematisieren.

\section{Beziehungen zu den Eltern}

Tanvir berichtete mir, als wir auf seine Reisen nach Indien zu sprechen kamen, dass er sich zuhause ständig mit seiner Mutter streite: „Everything I decide, she has to oppose it and make a different decision, and everything she decides I have to oppose it and make different decision. "Sein Vater scherze immer, dass sie sich wie Geschwister verhalten würden und nicht wie Mutter und Sohn. Diese Beziehungsdynamik habe es schon vor seiner Entscheidung für eine Promotion in Deutschland gegeben und würde sich nun bei den Aufenthalten in Indien fortsetzen. Andere Gesprächspartner*innen schienen eher eine gewisse Distanz zu ihren Eltern zu spüren (vgl. Ghosh und Wang 2003; Marschall 2017), die sich nicht nur aus der geographischen Entfernung ergab, sondern auch durch das Studium und die Arbeit in der Wissenschaft. ${ }^{133}$ Besonders die jungen Wissenschaftler*innen, die auf dem Land aufgewachsen waren und deren Eltern nicht studiert hatten, drückten Gefühle von Entfremdung zum Leben der Eltern aus bzw. spürten den Kontrast zum eigenen Leben am deutlichsten.

Andere merkten bei ihren Aufenthalten in Indien, dass sich ihre Positionierung innerhalb der Familie durch das Studium im Ausland verändert hatte. Nabhi stellte fest, dass sich ihr Status innerhalb der Familie verbessert hatte und ihr dies neue Möglichkeiten für die Durchsetzung ihrer eigenen Vorstellungen vom Leben verschaffte (vgl. Ghosh und Wang 2003: 275). Durch ihre Promotion in Deutschland hatte sie sich kulturelles Kapital angeeignet, dass ihr nun zu größerem Handlungsvermögen verhalf. In Kapitel 7 werde ich noch spezifischer auf Nabhis Fall eingehen.

Die jungen Wissenschaftler*innen wie beispielsweise Ajay vermissten ihr Leben in Indien: „mainly family, the mood, the warmth“ (Ajay, 14.12.2011). Heimweh und das Vermissen spezifischer Aspekte des Lebens in Indien und die Erinnerungen an dieses beeinflussten die Erfahrungen, die während der Reisen gemacht wurden (vgl. Marschall 2017: 220). Sabine Marschall spricht in diesem Zusammenhang vom „memory gaze“ (Marschall 2017: 220; Marschall 2015) und definiert diesen als "the constant search for the most ordinary, familiar traces of their remembered past" (Marschall 2017: 220). Die Suche nach dem Vertrauten kann allerdings dazu führen, dass Veränderungen deutlich werden - unter anderem Veränderungen des Selbst (Marschall 2017: 220).

\footnotetext{
133 Soziale Mobilität durch Bildung kann in Entfremdung resultieren (Pfaff-Czarnecka 2012: 41; Marschall 2017: 220).
} 


\section{Veränderungen des Selbst und multiple Zugehörigkeiten}

In den Gesprächen über die Aufenthalte in Indien zogen die jungen Wissenschaftler*innen häufig Vergleiche zwischen den Erfahrungen, die sie früher und bei ihren Aufenthalten in Indien gemacht hatten sowie zwischen ihrem Leben in Deutschland und in Indien. Ein Vergleichspunkt, der in vielen Interviews angesprochen wurde, war der eigene Umgang mit dem Wetter in Indien. Ajay begründete beispielweise seine Entscheidung dafür, im Dezember nach Indien zu reisen, mit den milden Temperaturen, die dann in seiner Herkunftsregion vorherrschen würden: „My city during the summer it's like 45 degrees 43, 42 43. Now if I go there I can't handle it. So I go there during the winter" (Ajay, 14.12.2011). Jetzt, nachdem er bereits sechs Jahre in Deutschland gelebt hatte, halte er das heiße Wetter nicht mehr aus. Neben Ajay erwähnten auch viele der anderen jungen Wissenschaftler*innen, dass sie es nicht mehr so einfach fänden, mit dem Wetter in Indien zurechtzukommen. Ajit erklärte lachend, es sei so warm, vierzig Grad Celsius: „I was in India in May and it was a nightmare for me. Oh god, my parents are really happy with the forty degrees, and I died there" (Ajit, 14.07.2015).

In anderen Aussagen wurde das Leben in Deutschland bzw. Göttingen und das Leben in Indien verglichen. Vendan erklärte beispielsweise nach seiner Aussage zur „Unorganisiertheit“ Indiens, die er liebe: „The other part that I love here is that it's very calm. Göttingen being a very small city, it's calm and peaceful, that's something which I really love“ (Vendan, 26.03.2013). Ajay erklärte auf ähnliche Weise, dass das Leben in Indien „chaotisch“ sei und berichtete lachend von seiner eigenen Reaktion darauf: „I mean right know if I go back to India I'll get mad because it will be a very chaotic situation" (Ajay, 14.12.2011).

In diesen Vergleichen thematisierten die jungen Wissenschaftler*innen ihre eigenen Reaktionen auf Faktoren wie das Wetter in Indien. Ich verstehe diese Vergleiche in den Interviews als die Artikulation des Bewusstwerdens von Veränderungen des Selbst (vgl. Marschall 2017).

Nach Indien zu reisen bedeutete für die Wissenschaftler*innen, auch wieder aufbrechen und sich von der Familie verabschieden zu müssen. Diese Momente waren nicht einfach: „Every time, before I take my flight in Bombay airport I feel terrible. Every time, even now, I feel terrible, like ,ohm my god, why do I have to leave “". Sangita betonte, dass besonders die Phase des Fluges schrecklich sei. „But then as soon as I reach Göttingen it feels home again. It's only the middle phase where you're neither in Bombay nor in Göttingen that makes you feel terrible about it" (Sangita, 08.08.2013). Wie diese Aussage von Sangita verdeutlicht, wurden sich die jungen Wissenschaftler*innen bei ihren Indienreisen besonders bewusst, dass sie mehr als ein Zuhause und multiple Zugehörigkeiten hatten (vgl. Ghosh und Wang 2003). Diese Perspektive rückt die Reisen als transnationale Praktik in den Fokus, als Mittel, um multiple Verortungen und Verbindungen über Raum und Zeit hinweg aufrechtzuerhalten. 
Wie in den Ausführungen in diesem Kapitelabschnitt deutlich wurde, schärft die Analyse der Reisen nach Indien den Blick für die Interaktion zwischen Mobilität und Zeitgestaltung. Die Zeitgestaltung während der Zeit in Göttingen, der Fokus auf spezifische Koordinaten wie jene der Fertigstellung der Doktorarbeit, beeinflusste die Häufigkeit von Reisen und die Zeitpunkte dieser. Wann die jungen Wissenschaftler*innen nach Indien reisten, hing allerdings auch mit Koordinaten dominanter Zeitkarten, und zwar besonders jener der Lebensverlaufsvorstellungen, zusammen. Während der Aufenthalte in Indien lebten sie in einer Zeitlandschaft, die durch bekannte Erfahrungen Vertrautheit vermittelte. Allerdings bedeutete das Leben bei der Familie - auch wenn es nur ein paar Wochen andauerte - eine erneute Aushandlung von Zeitgestaltung und Zeitsouveränität. Während der Aufenthalte in Indien konnten sich die jungen Wissenschaftler*innen durch unterschiedliche Praktiken Resynchronisieren und Kontinuitäten herstellen. Die Aufenthalte in Indien führten aber auch Veränderungen vor Augen: Veränderungen in den Beziehungen zu den Eltern, der eigenen Positionierung innerhalb der Familie und Veränderungen des Selbst. Das Bewusstwerden von den Veränderungen des Selbst hatte wiederum einen Einfluss auf Überlegungen zu einer permanenten Rückkehr nach Indien und offenbarte einigen der jungen Wissenschaftler*innen, was eine solche Rückkehr bedeuten würde (siehe Kapitel 8).

\subsection{Zusammenfassung}

Die Erfahrungen von Asynchronität, die durch das Leben in der Zeitlandschaft Göttingens ausgelöst wurden, waren der Ausgangspunkt für meine Ausführungen zu Praktiken der Resynchronisation. Die kollektiven Aktivitäten bildeten wichtige Koordinaten in der Zeitlandschaft meiner Gesprächspartner*innen: Sie strukturierten das Jahr auf eine vertraute Weise und waren etwas, auf das sie sich freuen konnten. Für die biographische Navigation der jungen Wissenschaftler*innen gaben die gemeinsamen Feierlichkeiten die Möglichkeit, sich mit der Zeitlandschaft Indiens zu resynchronisieren und Kontinuitäten des Selbst herzustellen.

In Göttingen lag die Kontrolle über die Ausgestaltung der gemeinsamen Aktivitäten bei den jungen Wissenschaftler*innen und dabei besonders bei der indischen Vereinigung, die eine Anpassung an die lokale Zeitlandschaft vornahm. Die Gestaltung der Feierlichkeiten - besonders von Diwali und dem Indian Independence Day - richtete sich nach den Abläufen, die aus dem Herkunftskontext vertraut waren. So wurden Normen und Konventionen aufrechterhalten, die für die einen Sicherheit und Vertrautheit boten und für die anderen Grund waren, sich von den kollektiven Aktivitäten fernzuhalten. In den Konflikten, die mehr oder weniger offen über die Gestaltung der Feste ausgetragen wurden, wurde die Diversität unter den indischen Wissenschaftler*innen sichtbar, die sich, wie im Zusammenhang mit der Feier des Unabhängigkeitstages betont wurde, eben nicht nur auf sprachliche oder 
religiöse Diversität bezog, sondern auch auf die Vielfältigkeit von Sichtweisen und Meinungen.

Die Vereinigung versuchte in den Veranstaltungen, die Diversität - vor allem die sprachliche und regionale - anzuerkennen und ihr einen Platz einzuräumen, indem sie als wichtiger Aspekt des Indisch-Seins artikuliert wurde. Allerdings gab es immer wieder Situationen, in denen deutlich wurde, dass die Aktivitäten, die bei den Festen als ,indisch' dargestellt wurden, eigentlich die Praktiken einer spezifischen Bevölkerungsgruppe war: die von Hindus aus den oberen Kasten. Der Einfluss dieser Bevölkerungsgruppe unter den indischen Wissenschaftler*innen macht zwei Faktoren sichtbar - erstens die relativ große Anzahl von Hindus oberer Kasten unter den jungen Inder*innen in Göttingen und zweitens die dominante Rolle, die diese bei der Organisation der gemeinsamen Zeiten spielten.

Die Indienreisen meiner Gesprächspartner*innen waren weitere besondere Zeiten, die im Fokus dieses Kapitels standen. Sie ermöglichten die Resynchronisation mit der Zeitlandschaft des Herkunftskontextes, mit dem ,alten' Leben, und das Aufrechterhalten von familiären und freundschaftlichen Beziehungen. Während der Aufenthalte in Indien musste die Zeitgestaltung im Alltag und bezogen auf den Lebensverlauf erneut ausgehandelt werden. Außerdem wurden den jungen Wissenschaftler*innen bei ihren Indienreisen die Veränderungen ihres Selbst und die eigene Verortung in multiplen sozialen Welten besonders bewusst. 


\section{Akademische Mobilität und die Navigation von Heirat}

Everytime I log into Facebook, someone gets married.

(Facebookeintrag, Januar 2016)

Mit diesem kurzen Kommentar auf Facebook drückte eine der jungen Wissenschaftler*innen sehr treffend aus, wie allgegenwärtig das Thema Heiraten für meine Gesprächspartner*innen war. Wie ich in Kapitel 2 dargelegt habe, ist das Heiraten in Indien ein fester Bestandteil der Lebensverlaufsvorstellungen und wichtiger Marker des Erwachsenseins. Die jungen indischen Wissenschaftler*innen befanden sich zur Zeit meiner Forschung genau in der Altersspanne, die als „marriagable age“ bezeichnet wird, und waren in unterschiedlicher Art und Weise mit der Aushandlung der gesellschaftlichen Erwartungen beschäftigt, die aus diesem Grund an sie gestellt wurden.

Zwischen akademischer Bildung, internationaler Mobilität und Heirat gibt es eine starke Verbindung. Für ein Studium ins Ausland zu gehen, war für die jungen Menschen in der vorliegenden Studie ein wichtiges Mittel, um sich soziales und symbolisches Kapital für sich selbst und ihre Familie anzueignen. Dieses Kapital konnte wiederum bei der Anbahnung von Partnerbeziehungen und Ehen eine wichtige Rolle spielen. Ich zeige in diesem Kapitel, dass die jungen Wissenschaftler*innen die Wertschätzung akademischer Bildung aus dem Ausland überdies nutzten, um eine bessere Ausgangssituation in der Navigation verschiedener Aspekte von Heirat (wie Zeitpunkt und Partnersuche) gegenüber den Eltern bzw. der Familie zu erreichen. Ein zentrales Argument dieses Kapitels ist, dass die Mobilität für die biographische Navigation der jungen Wissenschaftler*innen ein wichtiges Mittel in der Aushandlung normativer Zeitkarten war und ihnen größere Zeitsouveränität verlieh. Durch 
die Mobilität konnten meine Gesprächspartner*innen den Zeitpunkt einer Heirat hinauszögern und für sich selbst den richtigen Zeitpunkt schaffen. Damit trug die Mobilität dazu bei, dass sie ihre eigenen Wünsche und Aspirationen leichter mit denen ihrer Familie vereinbaren konnten. In diesem Kapitel wird daher nicht nur erkennbar, mit welchen Dilemmata die jungen Menschen konfrontiert waren, sondern auch, welche kreativen Lösungen sie für diese fanden.

Wie die jungen Wissenschaftler*innen das Thema Partnersuche und Heirat aushandelten, werde ich im Folgenden anhand dreier Fragen darstellen: Warum heiraten? Wann heiraten? Wen heiraten? Ich beginne das Kapitel mit einem kurzen Einblick in das Thema Heiraten in Indien, um die Strategien meiner Gesprächspartner*innen zu kontextualisieren. Dabei liegt das Augenmerk besonders auf der indischen Mittelschicht.

\subsection{Heiraten in Indien: Zwischen den Erwartungen der Familie und den eigenen Aspirationen}

Michiel Baas stellt fest: „Almost everybody in India grows up with the knowledge that he or she will one day get married“" (Baas 2009: 292). Das Heiraten gehört zur Norm des Lebensverlaufs und ist besonders für erwachsene Frauen der einzige „,normal' way to belong to a family" (Lamb 2018: 64). Diese Heiratsnorm führt mit dem Blick auf Gender und Sexualität zu unterschiedlichen Dilemmata. In Indien ist Homosexualität seit 2018 entkriminalisiert. Da aber Ehen von gleichgeschlechtlichen Paaren nicht anerkannt werden, können diese die Heiratsnorm nicht erfüllen.

In Indien, erklärte mir Rohini, entscheide nicht das Paar über eine Heirat, sondern dessen Familien, da eine Ehe das Zusammenkommen zweier Familien bedeute. „In India, that is very prevalent, and no matter how educated you get there is a time point when people start bugging you with that." In Indien gebe es das System der arrangierten Heirat, erläuterte Rohini weiter. Die Familie würde also die Braut bzw. den Bräutigam auswählen und dabei versuchen, jemanden aus der eigenen „community "zu finden. „If you have to do that process, trust me it's a ridiculous process." Die Optionen seien sehr eingeschränkt: „You want to find a person from that community, speaking the same language, well educated." Für die Eltern, deren Aufgabe das Finden eines Partners, einer Partnerin sei, sei dieser Prozess eine wahnsinnig schwere Aufgabe. Lachend rief Rohini aus: „Just imagine! It's worse than finding a job!“ (Rohini, 18.08.2016).

Wer bei der Auswahl eines Heiratspartners ein Mitspracherecht hat, wird in Indien ganz unterschiedlich ausgehandelt. In manchen Familien sind es vor allem die Eltern, die die Partnersuche und die Wahl vorantreiben, in anderen legen die Eltern die Entscheidung in die Hände ihrer jungen Söhne oder Töchter (Clark-Decès 2014; Fleming 2016). Bei der Suche nach einem Heiratspartner bzw. einer Heiratspartnerin ist die Kaste immer noch ein wichtiges Kriterium, doch steigt die Bedeutung akademischer und beruflicher Qualifikationen (Jeffery 2005). Abschlüsse von den 
Elite Colleges genießen das höchste Ansehen, dies gilt sowohl für Männer als auch für Frauen. So möchten gut ausgebildete Männer gut ausgebildete Frauen heiraten und umgekehrt (Fuller und Narasimhan 2012) ${ }^{134}$.

Clark-Decès (2014) und viele andere Ethnolog*innen (Donner 2012; Gilbertson 2014), die sich mit der Heirat in Indien beschäftigen, betonen, wie wichtig dieses Thema im Alltag der Familien ist: „(...) marriage is too important to be left to chance individual attraction - in fact, a child's marriage is the most important and often the most expensive decision a South Asian family ever has to make" (Clark-Decès 2014: 143-144). Auch aufgrund dieses finanziellen Aspekts spielen die Eltern und andere Familienmitglieder bei der Suche nach einem Partner oder einer Partnerin oft eine entscheidende Rolle. Allerdings steht bei der Auswahl nicht mehr nur die familiäre Kompatibilität im Fokus, sondern ebenfalls, ob die jungen Menschen zueinander passen (Palriwala und Kaur 2014: 14; Donner 2012; Fuller und Narasimhan 2008). Deswegen konsultieren Eltern ihre jungen Söhne und Töchter im Prozess der Partnersuche und ermutigen diese zur Kommunikation mit möglichen Kandidat*innen (Fuller und Narasimhan 2008: 748). So steht in der indischen Mittelschicht, gleichgültig ob die Eltern bei der Partnerwahl eine Rolle spielen oder nicht, mittlerweile die Beziehung zwischen zwei „intimate selves“ (Fuller und Narasimhan 2008: 751; Parry 2001: 816) im Vordergrund. Diese Art der Beziehung wird als „companionate“, also als „kameradschaftlich“, bezeichnet (Fuller und Narasimhan 2008; Gilbertson 2014). Laut Fuller und Narasimhan hat sich in der indischen Mittelschicht eine spezifische Form der companionate marriage entwickelt - eine Form, bei der Eltern und Kinder gemeinsam einen Partner bzw. eine Partnerin wählen (2008: 751). Diese Entwicklung verweist darauf, dass eine klare Unterscheidung zwischen , arrangierter Ehe ' und ,Liebesehe', wie sie in Indien von vielen Akteuren vorgenommen wird, gar nicht möglich und in den Augen einiger Forscher*innen gar nicht sinnvoll ist (Fuller und Narasimhan 2008: 737; Baas 2009).

Von meinen Gesprächspartner*innen richteten sich viele bewusst oder unbewusst nach den Wünschen der Eltern bzw. der Gesellschaft, rahmten ihre Entscheidung aber als ihre Wahl (choice) (vgl. Baas 2009: 301). Wie Fleming (2016) in ihrer Studie zeigt, ist diese Rahmung Teil der Rhetorik, nach der junge Menschen in Indien seit der ökonomischen Liberalisierung mehr Entscheidungsfreiheit hätten (Fleming 2016: 153). Wie Fleming betont, sind die „choices“ allerdings eingeschränkter, als die Akteure selbst wahrhaben wollen (Fleming 2016: 153). ${ }^{135}$ Die Bezeichnung der Entscheidung als die eigene Wahl steht in Zusammenhang mit Vorstellungen von „Modernität“ (Palriwala und Kaur 2014: 9; Fleming 2016) in Opposition zu arrangierten Ehen, die als „traditionell“ verstanden werden. Die Betonung der eigenen

${ }^{134}$ Der Artikel von Fuller und Narasimhan ist auf der Webseite der London School of Economics zu finden: http://blogs.lse.ac.uk/southasia/2012/10/05/companionate-marriage-among-a-middle-classbrahman-subcaste/ (letzter Zugriff 20.03.2019).

135 Obwohl Fleming sich in ihrer Argumentation auf junge Frauen in Bangalore konzentriert, gilt diese Feststellung meiner Meinung nach auch für junge Männer, die ganz andere Vorstellungen von einem guten Leben haben als ihre Eltern. 
Wahl durch die jungen Menschen in meiner Studie ist damit eine Positionierung als ,modern', nicht nur im Kontext Indiens, sondern auch Deutschlands.

\subsection{Warum heiraten?}

In meiner Forschung traf ich nur auf sehr wenige Menschen, die eine Heirat grundsätzlich infrage stellten. Im folgenden Kapitelabschnitt gehe ich darauf ein, wie drei meiner Gesprächspartner*innen ihre eigenen Vorstellungen vom Leben mit den familiären und gesellschaftlichen Auffassungen des Lebensverlaufs aushandelten.

\subsubsection{Von Unabhängigkeit und der Umsetzung eigener Pläne}

Diesen Kapitelabschnitt möchte ich mit Radhas Fall einleiten. Radha kam aus einem kleinen Dorf in West Bengal und war die Erste aus ihrer Familie, die die Möglichkeit hatte, zu studieren und ins Ausland zu gehen. In unserem Interview wirkte Radha auf mich wie eine sehr starke und fokussierte junge Frau. Diesen Effekt erzielte sie dadurch, dass sie immer wieder explizit und implizit ihren Wunsch nach Unabhängigkeit (von den Eltern, einem Stipendium, einem Mann etc.) darstellte.

In Indien hatte Radha Interkulturelle Germanistik studiert, so dass wir unser Interview auf Deutsch durchführten. Sie musste anders als viele meiner Gesprächspartner*innen, die in den MINT-Fächern tätig waren, ihren Aufenthalt in Deutschland größtenteils selbst finanzieren. Dafür arbeitete sie zunächst in einem Baumarkt und später als studentische Hilfskraft an der Universität Göttingen. Einen Arbeitsplatz zu haben und ihr eigenes Geld zu verdienen, verlieh ihr, wie sie es ausdrückte, „ein gutes Gefühl“ und Selbstvertrauen. Auf Radhas Auseinandersetzung mit dem Thema Heirat wurde ich durch einige ihrer Aussagen auf Facebook aufmerksam. Im Juli 2016 teilte sie auf Facebook ein Bild, auf der folgende Botschaft zu lesen war: „It's funny when people discuss Love marriage vs Arranged marriage. It's like asking someone if suicide is better or being murdered.“ Radha kommentierte dazu: „No offense!!!!"“ Wenige Zeit später teilte sie einen Artikel mit der Überschrift 15 reasons why marriage should be the last thing on your mind right now. Because you will know when you are ready! und schrieb dazu: „This is for my parent." In der ersten Nachricht knüpfte Radha an die bereits thematisierte Debatte um „Liebes- oder arrangierte Ehe" an und positionierte sich auf humorvolle Weise außerhalb dieser Diskussion, indem sie deutlich machte, dass beides zum gleichen Ergebnis führt. Wen Radha ansprechen wollte, machte sie in ihrem zweiten Facebook-Eintrag explizit, indem sie den Kommentar an ihre Eltern adressierte. ${ }^{136}$ Allerdings kann ihr zweiter Eintrag ebenso als Aufruf an Gleichaltrige auf Facebook verstanden werden, sich über die Einmischung der Eltern in das eigene Leben auszutauschen.

\footnotetext{
${ }^{136}$ Aus der öffentlichen Kommunikation auf Facebook konnte ich nicht schließen, ob ihre Eltern auf Radhas Eintrag reagiert haben.
} 
In beiden Einträgen drückte Radha eine negative Einstellung zum Heiraten aus und schien eine Eheschließung zumindest für diesen Zeitpunkt in ihrem Leben - sie war gerade sechsundzwanzig Jahre geworden - auszuschließen. In meinem Interview mit ihr stellte sich mir jedoch ein komplexeres Bild dar. Radha erklärte mir, dass sie mit ihren Eltern bereits über das Heiraten gesprochen habe, auch weil ihre Verwandtschaft Druck ausübe:

Meine Eltern verstehen das jetzt schon und ich habe das auch irgendwie klargemacht, dass „okay, ich kann gerne heiraten, ihr müsst dann einen Mann finden" und ich glaube, das ist eine sehr schwere Aufgabe für meine Eltern, weil sie verstehen jetzt, dass ich keinen Bauern heirate, der aus einem Dorf kommt. Das verstehen sie gut. Ja, das ist gut, dass es für sie eine sehr schwere Aufgabe ist, jemanden zu finden, der im Ausland und der in Deutschland lebt. (Radha, 13.08.2015)

Radha lachte kurz, als sie mir von den Schwierigkeiten erzählte, die ihre Eltern auf der Suche nach einem geeigneten Partner hätten.

Radha konnte mit Hilfe ihrer Mobilität ihre Position in den Verhandlungen mit den Eltern verbessern. Außerdem gab ihr der Aufenthalt in Deutschland die Möglichkeit, sich mit Männern zu verabreden, ohne gleich an das Heiraten denken zu müssen. In Indien wäre diese Art von ,Dating' für sie nicht so einfach gewesen. Dies machen nicht nur die Aussagen einiger meiner Gesprächspartner*innen deutlich, sondern ebenso Zeitungsartikel, in denen thematisiert wird, dass die Polizei in Indien Hotels räumt, weil sich dort unverheiratete Pärchen treffen. ${ }^{137}$ In Indien gibt es in vielen Lebensbereichen eine Trennung zwischen Männern und Frauen (vgl. Costanzo Kapur 2010; Fleming 2016). Die gesellschaftlichen Strukturen machen es dementsprechend schwer, einen Raum für eine Verabredung zu finden, in dem sich die jungen Menschen nicht beobachtet und moralisch verurteilt fühlen. In Deutschland war es für Radha weniger kompliziert, Beziehungen einzugehen und mit Hilfe dieser auszuloten, welche Eigenschaften für sie an einem potentiellen Partner wichtig sind. Allerdings stellte sie fest, dass es für sie nicht einfach sein würde, den ,richtigen Partner' zu finden.

Wenn ich einen Inder heirate, dann wäre seine erste Vorstellung, ob ich zuhause bleibe, ob ich eine gute Mutter sein kann. Für mich ist das ein Teil des Lebens, aber das kann nicht mein Leben sein. Da bin ich ganz, ganz klar. Deswegen weiß ich nicht, ob ich so einen liberalen Inder treffe oder nicht. Und dann bin ich in Deutschland jetzt und die deutschen [Männer] weiß ich nicht. Also ich hatte schon Freunde, aber ich finde sie zu liberal. Das ist

\footnotetext{
137 Siehe Adam Taylor: „The latest target of India’s morality police: Unmarried couples in hotel rooms", Washington Post vom 10.08.2015, https:/www.washingtonpost.com/news/worldviews/ $\mathrm{wp} / 2015 / 08 / 10 /$ the-latest-target-of-indias-morality-police-unmarried-couples-in-hotel-rooms/.
} 
auch ein Problem. (...) Wenn du zusammen bist, dann ist alles ganz toll, aber wenn du ein bisschen weiterdenken möchtest, dann kriegen sie sofort Angst. (...) Das Problem für mich ist, dass die meisten Männer nicht wissen, was sie wollen. Ich weiß, was ich vom Leben möchte, und dann ist es besser, ich mache mein eigenes Ding. (Radha, 13.08.2015)

Radhas Aussage macht zwei Faktoren deutlich, die auf ihre Einstellung zum Heiraten und zu Beziehungen generell einwirkten. Erstens war sich Radha sehr bewusst, welchen Einfluss ein Mann auf die Umsetzung ihrer Pläne für die Zukunft haben könnte. Wie Ritty A. Lukose in ihrer Studie (2009: 127-28) herausstellt, wird die Zukunft von Frauen in Indien stark von ihrem Ehemann beeinflusst. Er und zum Teil seine Familie wird einen großen Einfluss darauf haben, ob sie arbeiten und wie viele Kinder sie haben wird (vgl. Lukose 2009; Fleming 2016). Ähnlich wie Radha waren sich viele meiner Gesprächspartnerinnen dieser Abhängigkeit bewusst und versuchten, entsprechend auf die Partnerwahl einzuwirken (mehr dazu unter 7.4).

Zweitens ging es Radha nicht nur darum, dass ihr Partner ihre Aspirationen akzeptieren sollte. Sie wollte einen Mann haben, der ihre Einstellung zum Leben teilt und weiß, was er will. Ein passender Partner war für Radha demzufolge ein Partner, der sich überhaupt eine Zukunft mit ihr vorstellen konnte. Bis sie einen solchen Partner fand, wollte Radha zunächst ihre eigenen Pläne umsetzen und ihre Unabhängigkeit genießen: „Ich bin nicht frustriert, dass ich keinen Freund habe. Es ist besser, dass ich keinen Freund habe, kein Kind habe, oder Mann habe; so habe ich auch keine Verantwortung. Ich kann diese Zeit für mich selber nutzen."

\subsubsection{Das gute Leben: eigene und elterliche Vorstellungen}

Natürlich machten sich nicht nur die jungen Frauen aus Indien, sondern gleichermaßen die Männer Gedanken um das Heiraten - so zum Beispiel Gilon. Ich traf Gilon, der in Geschichte promoviert hatte, kurz nach der Abgabe seiner Dissertation. Er hatte schon konkrete Pläne für seine Rückkehr nach Indien, und so fragte ich ihn, ob er denn bald heiraten werde. Gilon antwortete, dass diese Frage für ihn nicht einfach zu beantworten sei und einen „personal struggle“ darstelle. Er schätze eigentlich einen "monastical way of life“. Nach der Abgabe seiner Dissertation sei er für drei Tage in ein Kloster gegangen: „I always see that this is the place and these are all the people with whom I identify myself more than others." Allerdings sei es sehr schwer, seine Eltern davon zu überzeugen, dass er keine Familie gründen wolle. Sie würden schon seit einer Weile versuchen, eine passende Partnerin (match) zu finden. Gilon habe ihnen gesagt, dass sie die Suche beenden sollen, „but I think it's kind of hurting to my mother especially. (...) I think for her it is not important that I get a $\mathrm{PhD}$. I think for her it is important that I get settled in life. That is to have a wife, have a family and some earnings - to have a good life" (Gilon, 19.01.2013).

In Gilons Aussage wird deutlich, dass die Entscheidung für oder gegen eine Ehe für ihn nicht so einfach war, da er nicht nur seine eigenen Wünsche und Zukunfts- 
pläne in Betracht zog. Außerdem werden hier die unterschiedlichen Vorstellungen vom ,good life' sichtbar. Für seine Mutter gehörten eine Frau und Kinder dazu. Gilon dagegen konnte sich eine andere Version des guten Lebens ohne Familie vorstellen.

Ähnlich wie andere jungen Wissenschaftler*innen aus Indien hatte Gilon seinen Aufenthalt in Deutschland genutzt, um eine endgültige Entscheidung zu diesem Thema hinauszuzögern. Noch sichtbarer wird diese Strategie, wenn wir einen Blick auf die Zeit nach der Rückkehr Gilons nach Indien werfen: Einige Wochen nach unserem Interview verließ Gilon Göttingen und ging zurück nach Indien, um dort eine Stelle als Professor anzutreten. Ich traf Gilon erneut im Juni 2015, als er für einen Vortrag nach Göttingen gekommen war. Gilon war mittlerweile seit einem halben Jahr verheiratet. Er erzählte mir, dass diese Ehe „selbst-arrangiert" sei und rahmte damit die Heirat als seine Entscheidung und in Opposition zu einer traditionellen arrangierten Ehe. Seine nun Angetraute hatte er auf einer kleinen Konferenz in Indien getroffen. Beide Familien zeigten sich einverstanden mit dieser Verbindung. So wie Gilon ist seine Frau christlichen Glaubens, ein Umstand, der auch zur raschen Zustimmung der Eltern beigetragen habe, erklärte Gilon in unserem Gespräch.

Obwohl Gilon noch im Januar 2013 sehr unsicher war, ob er überhaupt jemals heiraten wollen würde, war er bereits zwei Jahre später tatsächlich verheiratet. Dass er seine Ehe „selbst arrangiert" hatte, seine Partnerin selbst wählen konnte, hatte sicherlich einen Einfluss darauf, dass er letztlich dem Wunsch seiner Mutter nachgekommen war. Wie er es ausdrückte: „Now everyone is happy.“

\subsubsection{Zwischen „set up marks“ und der eigenen Wahl}

Rohini erzählte mir in unserem Interview, dass ihre Eltern kein Interesse an der schwierigen Aufgabe hätten, einen Ehemann für sie zu finden, und sie nicht unter Druck setzen würden, „rechtzeitig“ zu heiraten. Trotzdem schien sich Rohini mit dem Thema Heirat auseinanderzusetzen. Sie teilte z.B. den Satz, der dieses Kapitel einleitet: „Everytime I log into Facebook, someone gets married“.

Seiten wie Facebook leben davon, dass Menschen von Geschehnissen in ihrem Leben berichten und diese mit Bildern illustrieren. Die eigene Hochzeit ist dabei eines der wichtigsten Ereignisse, das ausgiebig auf Facebook geteilt wird. Obwohl das Teilen der Ereignisse als Informationsaustausch interpretiert werden könnte, kann es ebenso als Aufruf zu einem Wettbewerb verstanden werden. Auf Facebook zeigen Menschen, was sie bereits erreicht haben, und rufen damit indirekt ihre Freunde und Bekannten auf, es ihnen gleichzutun. Durch das Format von Facebook, in dem Informationen chronologisch auf der sogenannten „Chronik“ abgebildet werden, entsteht eine lineare Repräsentation von Zeit, in der alltägliche Praktiken und wichtige Meilensteine wie eine Heirat sichtbar werden. Auf diese Art und Weise werden soziale Rhythmen deutlich, wie es sich in Rohinis Gefühl widerspiegelte, dass auf einmal alle in ihrer Generation heiraten würden. Durch Facebook werden dominante Lebensverlaufsvorstellungen sichtbar: Anhand der Profile sehen die Nutzer*innen, 
wer welche Koordinate bereits genommen hat, wer wo im Leben steht, und stellen Vergleiche zum eigenen Leben an.

Maya lotete wie viele meiner Gesprächspartner*innen die dominanten Vorstellungen vom Heiraten und den Vergleich mit den Anderen in Form von Scherzen aus. Allerdings war es Maya nicht immer möglich, die Aussagen und Artikel, die ihre Freund*innen auf Facebook teilten, mit Humor zu nehmen. Besonders deutlich wurde dies, als einige ihrer Freund*innen an der sogenannten „motherhood challenge" auf Facebook teilnahmen. Bei der motherhood challenge waren Mütter dazu aufgerufen, fünf Bilder auf Facebook zu veröffentlichen, die sie glücklich machen, Mutter zu sein, zehn Frauen zu markieren, von denen frau denkt, dass sie großartige Mütter sind, die wiederum fünf Bilder hochladen sollen, die sie glücklich machen, dass sie Mütter sind. ${ }^{138}$ Maya schrieb dazu auf Facebook:

I can't express what I felt when I saw a few of my recently wed friends with babies started taking up this challenge. I was uncomfortable and at one point even got panicked that my biological clock is ticking as well, and the career path I am in will delay every bit of it. (...) So many women came to my mind whose life has been a hell because they didn't stand up to the set up marks (...) Why it has to be a race to show off your fertility. Please don't get me wrong being a mother must be amazing. But motherhood is a choice. Choice for my body and mind. (Facebookeintrag, April 2016)

Maya kritisierte in ihrer Aussage ganz deutlich die Tendenz ihrer Altersgenoss*innen, alles zu einem Wettrennen zu machen. In ihrem nachdrücklichen Kommentar zeigt sich, welchen Einfluss die Facebook-Aktivitäten anderer auf die Wahrnehmung der eigenen Entscheidungen haben. Maya stellte - wenn auch nur für einen Moment ihr eigenes Leben und ihre Karriere infrage. Dieser Umstand rückt Facebook als Kontrollinstrument in den Fokus, als Mittel, die Einhaltung normativer Praktiken der Zeitgestaltung zu kontrollieren.

Durch die Kritik an der motherhood challenge positionierte sich Maya als selbstbewusste Frau, die ihre eigenen Entscheidungen trifft, die das Frauenbild solcher challenges reflektiert und kritisch hinterfragt. Biographische Navigation hieß für die jungen Menschen in meiner Studie unter anderem die Aushandlung bestehender Rollenbilder, die Auseinandersetzung mit den „set up marks“, also den Zielen und Koordinaten, die von der Gesellschaft vorgegeben werden. Maya plädierte für das Hinterfragen der Normen und das Finden alternativer Routen.

\footnotetext{
${ }^{138}$ Die Challenge wurde in verschiedenen Artikeln und Blogs kritisiert. Siehe Flic Everett: „Facebook's motherhood challenge makes me want to punch my computer screen", The Guardian vom 02.02.2016, https://www.theguardian.com/commentisfree/2016/feb/02/facebook-motherhood-chal lenge. Siehe Alice Judge-Talbot: „Why are women falling for the Facebook motherhood challenge“, The Telegraph vom 04.02.2016, https://www.telegraph.co.uk/family/parenting/why-are-womenfalling-for-the-facebook-motherhood-challenge/.
} 
Maya kritisierte die challenge, das Frauenbild dieser und die Personen, die an dieser Aktion teilnehmen, ohne wie sie darüber nachzudenken. Sie veröffentlichte ihren Kommentar auf ihrer Facebook-Seite, so dass ihre Freund*innen, von denen sie in ihrer Aussage spricht, diesen lesen konnten. Ihr Kommentar war damit relativ deutlich adressiert und ein Mittel, um sich gegenüber ihren Freund*innen und Bekannten auf Facebook zu positionieren. Maya rief dazu auf, sich dem ständigen Vergleich und damit gewissen normativen Vorstellungen von Frau- und Mutter-Sein zu entziehen und eigene Entscheidungen zu treffen. Allerdings ist es nicht immer einfach, die eigene Wahl gegen bestehende dominante Vorstellungen und Diskurse durchzusetzen. Dies sah auch Maya und erklärte mir: „I know a lot of people have that and that's how it is. I mean, it's a societal thing - it's again a communal thing - try to fit into the thing" (Maya, 18.08.2016). Zu heiraten verstand sie dementsprechend als Versuch ihrer Altersgenoss*innen, sich in gesellschaftliche Strukturen einzupassen.

\subsubsection{Alte und neue Pfade in die Zukunft}

Die jungen indischen Wissenschaftler*innen waren sich bewusst, was von ihnen gesellschaftlich erwartet wurde - nämlich eine Heirat. Mit dieser Erwartung mussten sie sich immer wieder auseinandersetzen: am Telefon, per Skype, auf Facebook. Das Heiraten war bei Treffen unter Freund*innen und bei den Festen immer wieder ein Thema, das häufig scherzhaft aufgegriffen und womit sich gegenseitig geärgert wurde.

Die Mobilität war für meine Gesprächspartner*innen ein Mittel, um sich mehr Zeit in den Aushandlungen mit der Familie zu verschaffen und herauszufinden, was sie sich selbst vom Leben erhofften. Durch die Distanz zu den Eltern und den gesellschaftlichen Strukturen Indiens konnten sie andere mögliche Lebenswege ausloten. Die Mobilität führte auf diese Weise zu größerer Kontrolle über die Gestaltung des Lebensverlaufs und damit zusammenhängender Konzepte von Jugend und Erwachsensein. Die meisten meiner Gesprächspartner*innen nutzten ihre Mobilität, um einen Mittelweg zwischen dem, was sie sich selbst für ihr Leben erhofften, und den familiären und gesellschaftlichen Erwartungen zu finden. Sie versuchten nicht, den normativen Vorstellungen vom Lebensverlauf komplett zu entkommen. Die Zugehörigkeit zu einer Familie gab den jungen Menschen ein Gefühl der Sicherheit, eine wichtige Kontinuität in ihrem mobilen Leben (siehe Kapitel 4). Diese Verortung in der Familie wollten meine Gesprächspartner*innen nicht durch einen kompletten Widerstand gegen die Erwartungen aufs Spiel setzen. 


\subsection{Wann heiraten?}

Die meisten Kommentare und Aussagen zum Thema Heirat, auf die ich in meiner Studie stieß, bezogen sich auf den Zeitpunkt. Diesen Umstand führe ich auf das Alter meiner Gesprächspartner*innen zurück, das mit Mitte bis Ende zwanzig dem „Heiratsalter" entsprach (Sondhi 2013: 129).

\subsubsection{Die ,richtige Zeit}

Ich traf mich mit Smita in einem Café, in dem wir bereits unser erstes Interview geführt hatten. Seit unserem ersten Treffen waren drei Jahre vergangen und es hatten sich einige Dinge verändert: Smita hatte ihre Promotion in Mikrobiologie abgeschlossen und geheiratet. Nachdem wir uns bereits eine halbe Stunde unterhalten hatten, kamen wir auf das Thema Heirat zu sprechen, und ich erkundigte mich bei ihr, wie ihre Ehe zustande gekommen war. „Mine is not at all a love marriage, as I would say," meinte sie. Sie und ihr jetziger Ehemann seien gute Freunde gewesen und er habe bereits vor einigen Jahren um ihre Hand angehalten. Zu jener Zeit, so erzählte Smita, war sie aber generell nicht an einer Heirat interessiert gewesen: „I was more interested in doing a PhD and really research and all this. I think I was never at all romantic in my life - I'm still not, believe me." Sie sei eine sehr praktische Person und eine Ehe sei eigentlich nicht unbedingt Teil ihrer Lebenseinstellung (attitude). Das Heiraten sei für sie nie eine Priorität gewesen. „But it is still there that parents rule your life, and for them it's a very important thing. Since I was the eldest in the family, I have to be married first so that everybody else after me gets married" (Smita, 26.01.2013).

Bereits bei unserem ersten Gespräch im August 2010 erzählte sie mir, dass sie nichts dagegen habe, eine arrangierte Ehe einzugehen. Einen Mann zu heiraten, den ihre Eltern wählen würden, schien für Smita kein Problem darzustellen. Allerdings wollte sie den Zeitpunkt einer Heirat selbst festlegen. Eigentlich sollte sie bereits nach ihrem Masterabschluss heiraten: „So it was a big pressure on me, but then I really told them ,you know this is not the time for me"“. Smita wollte zu diesem Zeitpunkt nicht heiraten und erklärte dies ihrer Familie. Sie hatte sich bereits um Promotionsstellen außerhalb Indiens beworben. Die Zusage, die sie aus Göttingen erhalten hatte, konnte sie nun in der Aushandlung mit der Familie in die Waagschale werfen und mit Unterstützung ihrer Eltern ihren Wunsch durchsetzen, zunächst einen PhD-Abschluss zu machen. Der große Wert, der universitärer Bildung und internationaler Mobilität in der indischen Mittelschicht zugeschrieben wird, wirkte in diesem Aushandlungsprozess zu Smitas Gunsten. Selbst der Onkel, der zunächst gegen ihr Promotionsstudium in Göttingen war, sei mittlerweile stolz auf sie, erzählte mir Smita.

Smita begann 2007 ihr Promotionsstudium in Göttingen. Im November 2012 schloss sie dieses ab und heiratete dann im Dezember 2012. In der Zeit ihres Stu- 
diums in Deutschland hielten Smitas Eltern Ausschau nach einem geeigneten Ehepartner für sie. Smita erzählte, dass sie ihre Eltern auch dazu aufgefordert habe. Bei den Treffen mit den potentiellen Ehemännern stellte sich aber jeweils recht schnell heraus, dass keine Ehe zustande kommen würde. Ihre Eltern waren dann laut Smita an einen Punkt gekommen, an dem sie akzeptierten, dass ihre Promotion etwas länger dauern würde, und waren bereit, zunächst die jüngeren Geschwister heiraten zu lassen. So verlängerte sich mit der Dauer ihres Promotionsstudiums Smitas Zeit als Single.

2011 traf sie dann auf den Mann, der ihr bereits einige Jahre zuvor einen Heiratsantrag gemacht hatte. Sie begegnete ihm bei einer Konferenz und sie verstanden sich auf Anhieb wieder sehr gut und beschlossen, zu heiraten. Smitas Fall macht auf diese Weise besonders deutlich, welche Rolle Zeit bei der Aushandlung von Heirat spielt. Sie hatte den ersten Antrag ihres jetzigen Ehemannes nicht abgelehnt, weil er der falsche Mann war, sondern weil es der falsche Zeitpunkt war. Durch ihr Promotionsstudium in Deutschland konnte sie sich den Raum und die Zeit schaffen, um selbst den richtigen Zeitpunkt zu finden und festzulegen.

\subsubsection{Empowerment durch akademische Mobilität}

Nabhi kam 2011 nach Göttingen, um an einer International Max Planck Research School ein Masterstudium zu beginnen. Wir begegneten uns das erste Mal im Juli 2012 beim Indian Sportsday. Als ich mich für das Ereignis registrierte, kamen wir ins Gespräch. An dieser Stelle zitiere ich aus meinem Beobachtungsprotokoll:

Nabhi stellt sich kurz vor und fragt mich, wer ich bin. Ich deute auf meine Fragebögen zum Sportsday, die Lali netterweise auf dem Anmeldetisch platziert hat und meine: „That's me“. Nabhi beginnt einen der Fragebögen auszufüllen und fragt mich: „Are you married?" Ich verneine und gebe die Frage zurück. Sie sagt nein und meint, dass dies wohl auch nicht so schnell passieren wird, da sie viel arbeiten würde und sie kaum Zeit habe. Ich antworte: „You have some time.“ „Ja, three years." erwidert Nabhi. (Beobachtungsprotokoll 21.07.2012)

Das Heiraten schien Nabhi bereits beim Sportsday sehr zu beschäftigen und ihre Aussage wirkt im Rückblick fast wie eine Prophezeiung: 2015, also ziemlich genau drei Jahre nach unserem Gespräch beim Sportsday, heiratete Nabhi.

In unserem Interview im März 2016 erinnerte sich Nabhi an die Zeit, bevor sie nach Deutschland gekommen war. Sie hatte gerade ihren Master an einer Universität in Mumbai abgeschlossen:

I really feel that I was lucky that I came to Germany, because it is a bitter truth of my life: if at that particular year I wouldn't have come to Germany, then 
it was sure that I would have been so much pressurized by my family that I would have [had] to marry a stranger. (Nabhi, 17.03.2016)

Nabhi erinnerte sich, dass ihr in jenem Jahr von ihrer Familie ständig gesagt wurde, dass sie bestimmte Männer treffen und über diese nachdenken solle. Der Druck sei so hoch gewesen, dass ihr klar geworden sei: „Okay, I will graduate and then I will get married." Sie habe es nicht geschafft, ihre Eltern davon zu überzeugen, dass sie zu jenem Zeitpunkt für eine Heirat nicht bereit war. Nabhi erklärte, es sei sehr anstrengend gewesen, sich jeden Tag mit ihren Eltern auseinanderzusetzen. „When I came here it was just relax: like, ,Okay now if someone asks me to get married, I'm not going back home.' I felt empowered." In Indien sei es sehr schwierig gewesen, Abstand von den Eltern zu gewinnen, sie habe nicht einfach sagen können, dass sie sie nicht sehen wolle. „But here there is no one around who knows me; there is no one constantly bothering me or asking me, ,Why are you not married?"“ Nabhi erinnerte sich, dass ihre Tante sie jedes Jahr an ihrem Geburtstag angerufen und ihr ihr Alter gesagt habe, um dann zu fragen, wann sie denn heiraten werde. „Seriously, and this was so annoying, and all these tiny botherings were simply deleted from my daily life, and I was so happy and relaxed here." In Deutschland habe sie sich dann endlich auf ihre Karriere konzentrieren können: „I could really think that what I want to do instead of thinking about marriage. In India it was more like, $\mathrm{Oh}$ my god I have to get married. Otherwise everyone will constantly keep bothering me.' It was such a daily fear in my mind" (Nabhi, 17.03.2016).

Nabhis Darstellung zeigt, wie der Druck zu heiraten ihren ganzen Alltag in Indien durchdrang und dabei jegliche anderen Gedanken beiseite schob. Ähnlich wie Smita konnte Nabhi durch ihr Studium in Deutschland einer Ehe zu einem Zeitpunkt entgehen, an dem sie nicht heiraten wollte. Da einem Studienabschluss aus dem Ausland in Indien ein großer Wert beigemessen wird und die International Max Planck Research Schools in Indien einen guten Ruf genießen, hatten Nabhis Eltern keine Einwände gegen ihren Plan, nach Göttingen zu kommen. Ein Abschluss aus dem Ausland könnte schließlich bessere berufliche Chancen in der Zukunft und noch bessere Optionen bei der Partnerwahl bedeuten.

Für Nabhi führte der Umzug nach Göttingen zu einem Gefühl von „empowerment". Sie war weit weg von den ständigen Fragen nach einer Hochzeit und hatte ihren Kopf frei, um über ihre eigenen Ambitionen und Wünsche nachzudenken. Nabhi entging in diesem Sinne einer Zukunft, von der sie das Gefühl hatte, dass sie eigentlich schon feststehen würde, und eröffnete sich neue mögliche Pfade in die Zukunft.

Smita und Nabhi konnten in ihrer biographischen Navigation die Strukturen und Diskurse des Regimes der akademischen Mobilität strategisch nutzen, um den Zeitpunkt ihrer Heirat um einige Jahre nach hinten zu verschieben und diesen selbst zu bestimmen. ${ }^{139}$ So fanden sie eine kreative Lösung für ihr Dilemma zwischen dem

139 Um eine Heirat hinauszuzögern, gibt es auch andere Strategien. Ritty A. Lukose beschreibt bei- 
Wunsch nach der Erfüllung eigener Aspirationen und dem Verlangen nach der Sicherheit familiärer Einbindung. Denn keine von beiden wollte die Beziehung zu den Eltern zugunsten ihrer eigenen Wünsche und Pläne aufs Spiel setzen.

\subsubsection{Die richtige Reihenfolge}

Anisha, die mit ihrem Freund gemeinsam nach Deutschland gekommen war, erklärte mir, dass ihre Eltern sie nicht drängen würden, bald zu heiraten. Gerade, erklärte sie, sei nicht der richtige Zeitpunkt für eine Heirat: „It's my last year probably. So, we decided that first we both will complete our $\mathrm{PhD}$, and then we [will] decide to get together and [get married]. It's a wise decision, I think, and my parents understand this" (Anisha, 01.05.2011). Während des Studiums und der Promotion wollten sich die jungen Wissenschaftler*innen zunächst auf ihre Arbeit konzentrieren und nicht auf die Organisation einer Hochzeit - auch wenn der Prozess der Partnersuche für viele bereits früher begann.

Anisha führte das Verständnis ihrer Mutter für ihre Entscheidung darauf zurück, dass diese selbst nicht die Möglichkeit gehabt hatte, ihren Wunsch nach einer Promotion zu erfüllen, sondern heiraten musste. Die Eheschließung war dabei gleichbedeutend mit dem Ende einer akademischen Laufbahn gewesen: In der Elterngeneration meiner Gesprächspartner*innen bedeutete eine Ehe für viele der Frauen, dass sie, wenn sie einer Arbeit nachgingen, diese aufgeben mussten. Andere arbeiteten zwar weiter, aber meist in Berufen, die als familienkompatibel angesehen wurden und als respektabel für eine Ehefrau und Mutter (z.B. Lehrerin).

Eine Heirat hatte und hat noch immer einen großen Einfluss auf die Karriere von indischen Frauen und Frauen generell, wie ich in Kapitel 4 thematisiert habe. Die Interviews, die ich führte, und die Aussagen und Artikel, die auf Facebook geteilt wurden, machten deutlich, dass sich gerade die jungen indischen Frauen darüber Gedanken machten, wie sie diesen Einfluss auf Karriere und Berufsleben würden aushandeln können. Ein wichtiger Schritt war für viele, vor einer Heirat die angestrebten akademischen Abschlüsse zu erreichen.

Der Zeitpunkt der Heirat konnte mit der Rückkehr nach Indien in Zusammenhang stehen. Anisha erklärte mir, dass sie nach Ansicht ihrer Eltern möglichst vor ihrer Rückkehr nach Indien heiraten sollte:

According to Indian customs, a girl cannot live her own life with her parents. That will be like societal pressure on the parents that „why your daughter is not married and why she is living with you?" and you know that's Indian stuff.

spielsweise, wie eine junge muslimische Frau in Indien Heiratsanträge abwendete, indem sie ihrem Vater ausgedachte Geschichten über die potentiellen Heiratspartner erzählte (im Sinne von: Er ist ein Störenfried in der Schule etc.). Da die junge Frau die Einzige war, die regelmäßig ihr Dorf verließ, konnte ihre Familie ihre Geschichten nicht prüfen und glaubte ihr zunächst (Lukose 2009: 123). 
(...) My parents are even more worried that I should get married first. They are more worried about this rather than that I come back. (Anisha, 01.05.2011)

Anisha und ihr Freund hätten nach ihrem Abschluss in Deutschland also nicht einfach zurück nach Indien gehen können, um dort als unverheiratetes Paar zusammenzuleben. Im Mai 2014 heirateten die beiden in Indien, zogen aber nicht nach Indien zurück. Anfang 2019 lebten sie weiterhin in Deutschland, arbeiteten beide an einer Universität bzw. einem Forschungsinstitut und hatten ein Kind.

Anisha machte in ihrer Aussage mir gegenüber deutlich, dass sie die beschriebene Praxis für „Indian stuff“ hielt. Viele meiner Gesprächspartner*innen verglichen die unterschiedlichen „indischen“ und „deutschen/westlichen " 140 Praktiken beim Thema Heirat. Dies deutet darauf hin, dass die Aushandlung des Heiratens die Navigation größerer Fragen von Zugehörigkeit beinhaltet (Hermann und Fuhse 2018). Durch den Vergleich von dem, was sie als, indische " und was sie als ,deutsche 'Praktiken empfanden, konnten sich den Studierenden neue Handlungsmöglichkeiten eröffnen, aber ebenso Dilemmata der Zugehörigkeit entstehen. So konnten folgende Fragen aufgeworfen werden: Bin ich indisch, wenn ich keine arrangierte Ehe haben möchte? Kann ich eine moderne Frau/ein moderner Mann sein, wenn ich eine arrangierte Ehe eingehe?

In einem internationalen Umfeld wie der Universität und den Max-Planck-Instituten kamen die indischen Wissenschaftler*innen mit vielen Menschen anderer Herkunft in Kontakt und mussten nicht selten Fragen nach ihren kulturellen Praktiken und dem, was beispielsweise Deutsche als ,indische' Konzepte verstanden, beantworten. Ihre Mobilität führte dazu, dass meine Gesprächspartner*innen Praktiken, die in Indien selbstverständlich sind, überdachten und diese zum Teil verteidigten (vor sich selbst und anderen).

\subsubsection{Der richtige Zeitpunkt: Zeitgestaltung und die Navigation von Zugehörigkeiten}

Die Ausführungen zu Smita, Nabhi und Anisha zeigen, dass die jungen Wissenschaftler*innen aus Indien in ihrer biographischen Navigation nicht nur dominante Vorstellungen vom perfekten Zeitpunkt einer Ehe aushandelten, sondern eigene Konzepte des richtigen Timings und der Reihenfolge wichtiger Meilensteine entwickelten. Viele wollten vor einer Heirat zunächst ihre akademischen Ziele erreichen. Dies bedeutete wiederum, dass vor allem die Frauen nicht in dem Alter heiraten wollten, das in Indien weitläufig als das marriagable age verstanden wird.

Mittlerweile sind etwas mehr als die Hälfte der jungen Menschen, mit denen ich in meiner Forschung gearbeitet habe, verheiratet. Der Großteil heiratete kurz vor oder nach dem Abschluss der Promotion. Jayasree Subramanian zeigt in ihrem Artikel Perceiving and Producing Merit: Gender and Doing Science in India einen

${ }^{140}$,Deutsch', ,westlich` und ,europäisch ' wurden teilweise synonym verwendet. 
ähnlichen Trend auf (vgl. Jayasree Subramanian 2016: 269). In Indien gibt es nicht nur eine Vorstellung davon, wann im Lebensverlauf die beste Zeit zum Heiraten ist, sondern auch, wann im Jahr - besonders für jene, die sich als Hindus verorten. So gelten Dezember bis März aus klimatischen Gründen (es ist kühler) und auf Grund von Angaben in religiösen Regelwerken (z.B. in den Gụhyasūtras [Malinar 2009]) als die besten Monate für eine Hochzeit. Von meinen Gesprächspartner*innen schloss die Mehrzahl genau in dieser Zeitspanne den Bund fürs Leben. Diese Praxis und das mittlerweile gängige Teilen von Hochzeitsfotos auf Facebook machten die sozialen Rhythmen und die dominanten Zeitkarten sehr sichtbar. Und so ist es kein Zufall, dass Rohini gerade im Januar feststellte, dass jedes Mal, wenn sie sich auf Facebook einloggte, wieder jemand geheiratet hatte.

Doch warum ist die Wahl des Zeitpunkts eigentlich so wichtig? Auf diese Frage gibt Helga Nowotny eine eindeutige Antwort: „Den richtigen Augenblick zu kennen nützt; ihn zu bestimmen verleiht Macht und verheißt Kontrolle“ (Nowotny 1993: 154). Demzufolge argumentiere ich, dass die jungen indischen Wissenschaftler*innen durch das Bestimmen des Zeitpunkts ihrer Heirat das Gefühl erlangten, ihr eigenes Leben in der Hand zu haben. Sie wollten ihr Leben selbst gestalten und nicht das Gefühl haben, dass ihr Leben von anderen Personen gesteuert wird: „One seeks to direct and control the movement of ones [sic] life rather than having it directed and moved by the shifting of the unstable social environment it is immersed in" (Vigh 2007: 130).

Meiner Ansicht nach ist die Wahl von Zeitpunkten und der Reihenfolge wichtiger Ereignisse im Leben, also das Ausüben von Zeitsouveränität, ein relativ subtiles Instrument, um dieses Gefühl der Kontrolle zu erreichen. So wehrten sich viele meiner Gesprächspartner*innen nicht explizit gegen eine (arrangierte) Heirat, sondern versuchten, den Zeitpunkt dieser zu beeinflussen. Die internationale Mobilität wurde dabei zu einem Mittel, um mehr Einfluss auf das Timing einer Heirat auszuüben. Durch die Mobilität konnten die meisten meiner Gesprächspartner*innen den Zeitpunkt ihrer Hochzeit um mehrere Jahre hinauszögern. Dadurch hatten sie Zeit, ihre akademische Karriere auf den Weg zu bringen und herauszufinden, was sie wollen und wer sie sind, ohne die enge Einbindung in die Familie. Letztendlich heirateten die meisten irgendwann, selbst wenn sie, wie zum Beispiel Gilon, ursprünglich gar nicht heiraten wollten. Und somit waren auch die Wünsche und Vorstellungen der Familien erfüllt.

Verschiedene Studien weisen bereits daraufhin, dass sich die Jugendphase (global) verlängert (Cole und Durham 2007; Jeffrey und Dyson 2008). In meiner Studie wird deutlich, dass diese Verlängerung nicht nur ein Nebenprodukt längerer Ausbildungszeiten und der schwierigen Arbeitsmarktsituation ist, sondern von jungen Menschen aktiv hergestellt wird, um verschiedenste Ziele zu erreichen. Dazu gehören die Erfüllung beruflicher Aspirationen und das Umgehen gesellschaftlicher Konventionen.

Des Weiteren ergänzt die vorliegende Studie Cwerners Ausführungen zu den „times of migration“ (Cwerner 2001). Sie zeigt, dass Mobilität die zeitlichen Erfah- 
rungen in unterschiedlichen Lebensbereichen nicht in gleichem Maße beeinflusst. Wie in diesem Kapitelabschnitt dargestellt, erreichten die jungen Wissenschaftler*innen durch ihre Mobilität mehr Kontrolle über den Zeitpunkt einer Heirat. Das bedeutet aber nicht, dass sie in anderen Lebensbereichen gleichermaßen an Zeitsouveränität gewannen.

\subsection{Wen heiraten?}

Die Wahl eines Heiratspartners ist in Indien, aber sicherlich nicht nur dort, eine sehr wichtige Entscheidung, die einen Einfluss auf verschiedene Zugehörigkeiten und die Umsetzung persönlicher Zukunftspläne hat. Die Partnerwahl kann als Ausdruck der Zugehörigkeit zu einer bestimmten gesellschaftlichen Schicht oder gar einer Nation und ihrer Werte verstanden werden (Fuller und Narasimhan 2008; Gilbertson 2014). Sie beeinflusst die Karriere und, ob und wie viele Kinder eine Person haben wird.

Im Folgenden ist es mein Ziel, zu zeigen, wie meine Gesprächspartner*innen das schwierige Terrain der Partnerwahl navigierten und welche Funktion die akademische Mobilität in dieser Navigation einnahm.

\subsubsection{Von Heiratsanträgen, „Lovestories“ und dem Einfluss der Eltern}

Lali, die älteste von drei Geschwistern (jüngerer Bruder, jüngere Schwester) hatte ihren Master an einer Universität in Deutschland abgeschlossen und zog im Sommer 2011 für ihr Promotionsstudium nach Göttingen. Zu diesem Zeitpunkt war sie vierundzwanzig Jahre alt und ihre Eltern waren bereits auf der Suche nach einem Ehemann für sie. Als wir uns für ein Gespräch trafen, berichtete sie aufgeregt, dass ihre Eltern einen Mann in Großbritannien gefunden hätten. Dieser kam wie sie aus Andhra Pradesh, so dass sie sich in ihrer Muttersprache unterhalten konnten, was Lali positiv hervorhob. Lali war mit ihm über Facebook in Kontakt getreten und sie hatten bereits über Skype telefoniert. Der Mann wolle sie gerne treffen, aber Lali habe ihm erklärt, dass sie erst einmal reden wolle. Der Mann, den Lalis Eltern vorgeschlagen hatten, war aber nicht der einzige, der ein Interesse an ihr bekundete. So erzählte sie mir von einem guten Freund, den sie bereits in Indien kennengelernt hatte und der mittlerweile in Deutschland lebte. Er hatte ihr mehrmals einen Heiratsantrag gemacht, den Lali nach einigen Überlegungen abgelehnt hatte. Sie erklärte mir, dass sie sich wünsche, niemand völlig Fremdes zu heiraten, sondern mit ihrem Zukünftigen erst einmal lange Gespräche führen und sich treffen wolle, damit sie sich besser kennenlernen könnten (Beobachtungsprotokoll 20.08.2011).

Etwas mehr als drei Jahre nach unserem Gespräch, also im Frühjahr 2014, heiratete Lali einen Mann, der aus Andhra Pradesh stammte und Hindu war. Außerdem promovierte er in Österreich, so dass sie sich vor ihrer Eheschließung persönlich treffen konnten. 
Lalis Eltern hatten einen großen Einfluss auf ihre Partnerwahl: Sie initiierten die Suche nach einem Heiratspartner, lehnten einen Mann ab, den Lali vorschlug und wählten ihren zukünftigen Ehemann aus. Allerdings hatte Lali in diesem Prozess ein großes Mitspracherecht und entschied sich erst nach längerer Kommunikation und einem persönlichen Treffen mit dem Kandidaten ihrer Eltern für eine Heirat mit diesem. Lali und ihre Eltern wählten also gemeinsam einen Ehemann aus. In diesem Prozess wendeten sie ,traditionelle` und ,moderne' Kriterien an, die gewährleisten sollten, dass die beiden zueinander passen. Zum einen spielten Religionszugehörigkeit und die Herkunftsregion eine bedeutende Rolle - vor allem für Lalis Eltern. Zum anderen legten die Eltern Wert darauf, dass die Qualifikationen des Zukünftigen denen Lalis gleichkamen. So schlugen sie meines Wissens nur Männer vor, die gerade ein Promotionsstudium absolvierten.

Bei ihrer Suche nach einem Partner für Lali achteten die Eltern darauf, dass dieser in ihrer ,Nähe' war, so dass sie sich treffen konnten, um zu sehen, ob sie sich mögen. Lali war es selbst sehr wichtig, mit ihrem potentiellen Heiratspartner viel zu reden und sich auszutauschen. Dies drückte sie aus, als sie mir erklärte, sie wünsche sich eine „Lovestory“ vor der Ehe. Damit meinte sie weniger eine romantische oder intime Beziehung, wie man sie sich bei dem Begriff Liebesgeschichte vorstellt, sondern das gegenseitige Kennenlernen. Die Wahl eines Kandidaten, der in Europa lebte, war meiner Ansicht nach von Überlegungen zur Kompatibilität motiviert. Dadurch, dass Lali und ihr Ehemann beide in Europa promoviert hatten, teilten sie Erfahrungen, die ein solches Studium im Ausland mit sich bringt.

\subsubsection{Akademische Mobilität und der Mann der eigenen Wahl}

An dieser Stelle greife ich erneut Nabhis Geschichte auf. Unter Punkt 7.3.2 habe ich beschrieben, wie Nabhi mit Hilfe eines Masterstudiums und ihrer Promotion einer arrangierten Ehe mit Mitte zwanzig entgangen war. Mittlerweile ist Nabhi mit einem jungen Mann aus Bangladesch verheiratet, den sie in ihrem Masterprogramm in Göttingen kennengelernt hatte: „I found a guy, I got married, I’m really happy.“ Nabhi meinte, dass es für sie in Indien sicherlich nicht so einfach gewesen wäre, den Mann ihrer Wahl und dann auch noch einen aus Bangladesch, zu heiraten: „When I came here people have to raise their standards somehow, because they cannot compare me with other girls who just stay at home."Nur weil sie in Deutschland gewesen sei, seien ihre Eltern den Kompromiss eingegangen und hätten ihrer Wahl zugestimmt. Ihr Aufenthalt in Deutschland hatte aus Nabhis Sicht einen Einfluss darauf, wer zur Hochzeitsfeier in Indien erschienen war. „If I would have been in India and I would get married to a guy from Bangladesh, maybe so many people will not attend my marriage." Da sie aber in Deutschland gewesen sei und ihre Verwandten sie so lange nicht gesehen hätten, seien alle gekommen und hätten ihr ihren Segen (blessings) gegeben (Nabhi, 17.03.2016).

Dass Nabhis Eltern ihrer Ehe mit ihrem jetzigen Mann zugestimmt hatten, stellte Nabhi in direkten Zusammenhang mit ihrem Aufenthalt in Deutschland. Nabhi 
konnte durch ihre Mobilität als Studierende größere Handlungsmacht gegenüber ihren Eltern erreichen. Sie konnte den Zeitpunkt ihrer Hochzeit und ihren Heiratspartner selbst wählen.

Diese Strategie zum Erlangen größerer Unabhängigkeit von der Familie steht aber in Indien nicht allen Menschen im gleichen Maße zur Verfügung. Das in Kapitel 2 beschriebene Streben der Familien nach Kontrolle der sozialen Reproduktion wird vor allem durch Kontrolle von (jungen) Frauen ausgeübt. Dies mag auch ein Grund dafür sein, warum der Frauenanteil unter den jungen indischen Wissenschaftler*innen in Deutschland so gering war und nur bei $22,8 \%$ lag (DAAD und DZHW 2016: 17).

\subsubsection{Der richtige Zeitpunkt für Verhandlungen mit den Eltern}

Nalika und Ravi waren beide 2006 für ein Promotionsstudium nach Göttingen gekommen. Sie lernten sich dort kennen und verliebten sich ineinander. Gegen Ende ihres Promotionsstudiums beschlossen sie, zu heiraten. Ravi und Nalika kamen beide aus Karnataka und waren beide Hindus, allerdings kam Ravi aus einer Brahmanenkaste und Nalika nicht. Nalika erzählte mir, dass ihre Eltern einer Heirat bereits zugestimmt hätten, nicht zuletzt, weil sie bereits dreißig Jahre alt sei.

Bei einem Treffen im Sommer 2011 berichtete Nalika, dass Ravi sehr bald nach Indien reisen werde, um seine Eltern von einer Heirat mit ihr zu überzeugen. Ravis Schwester hatte zu Beginn des Jahres geheiratet und so sei nun ein guter Zeitpunkt, um mit den Eltern zu verhandeln. Diese Überlegung steht damit im Zusammenhang, dass sich die Eheschließungen von Geschwistern gegenseitig beeinflussen können. Wie bereits Smitas Fall gezeigt hat, gilt dies für den Zeitpunkt der Eheschließung und für die Partner*innenwahl. Wenn eine Person jemanden heiratet, der in den Augen der Familie bzw. der Gesellschaft unangemessenen ist, kann dies die möglichen Heiratspartner*innen der Geschwister ungünstig beeinflussen (vgl. Baas 2009: 303). Da Ravis Schwester nun verheiratet war und er keine weiteren Geschwister hatte, konnte seine eigene Eheschließung mit einer Frau aus einer niedrigeren Kaste keine negativen Auswirkungen mehr haben.

Ravis Eltern stimmten seiner Heirat mit Nalika allerdings nicht gleich zu. Als ich mich mit Smita, die mit Ravi und Nalika sehr gut befreundet war, darüber unterhielt, erklärte mir diese, dass es in Indien immer etwas Zeit brauche, um die Eltern von einer Liebesehe zu überzeugen (vgl. Fleming 2016: 161). Laut Smita sei dieser Prozess schon fast wie eine Zeremonie: „It's just the initial one or two months that they have to do a convincing thing. It's a ceremony that you have to convince, but once you convince everything is fine" (Smita, 26.01.2013).

In meinem Gespräch mit Nalika, das in der halb leer geräumten Wohnung stattfand, in der sie gemeinsam mit Ravi lebte, wurde die Unsicherheit ihrer Lage spürbar: „Now behind everything are question marks." Sie wusste in diesem Moment nicht, wohin ihr Leben führen würde: Sie hatte keinen Arbeitsplatz und wusste nicht, ob sie Ravi würde heiraten können. Sie wusste nur, dass sie bald zurück nach Indien 
reisen würde, um zunächst bei ihren Eltern zu leben, ihre Dissertation zu publizieren und sich auf Stellen in den USA und in Europa zu bewerben. Die Aussicht auf die Rückkehr zu den Eltern erschwerte die Situation und so prophezeite sie, dass es sicherlich nicht einfach sein werde, wieder bei ihren Eltern zu wohnen.

Ravis Aushandlungen mit seiner Familie waren u.a. dank der strategischen Wahl des Zeitpunktes von Erfolg gekrönt und so heirateten die beiden im Frühjahr 2012 in Indien. Zum Zeitpunkt ihrer Hochzeit waren Ravi und Nalika etwas mehr als vier Jahre ein Paar und hatten in Göttingen einige Zeit zusammen in einer Wohnung gelebt. Ein solches Zusammenleben als unverheiratetes Paar wäre in Indien kaum möglich gewesen und wurde durch ihre internationale Mobilität ermöglicht.

Nalika und Ravi konnten durch ihre Mobilität einen günstigen Zeitpunkt für die Aushandlung mit den Eltern herstellen und sich die Zeit nehmen, um die Eltern von einer Heirat zu überzeugen.

\subsubsection{Partnerwahl und Karriere}

Von Smitas erfolgreichem Hinauszögern einer Heirat habe ich bereits in Abschnitt 7.3.1 berichtet. In diesem Teil des Kapitels gehe ich nun näher darauf ein, warum sie sich letztendlich für ihren Partner entschieden hatte.

Smita beschrieb sich mir gegenüber als karriereorientierte und rationale Frau, die eigentlich nicht an einer Ehe interessiert sei. Da sie aber wusste, wie wichtig ihre Heirat für ihre Eltern und andere Mitglieder ihrer Familie war, gestand sie ihren Eltern zu, einen Ehemann für sie zu finden. Aus den Treffen mit potentiellen Kandidaten, die ihre Eltern ausgewählt hatten, ergab sich aber kein ,passender ' Partner. Smita erklärte dies auch damit, dass die Männer oft andere Vorstellungen von der Zukunft hatten als sie. So plante Smita, nach Indien zurückzukehren, viele der Männer wollten aber im Ausland bleiben.

Die geplante Rückkehr nach Indien schien für sie ein Grund dafür zu sein, dass sie eine Heirat mit einem deutschen Mann nicht in Betracht zog. Smita erzählte mir, dass sie ein Kollege einmal gefragt habe, ob sie denn überhaupt einen Deutschen heiraten dürfe. Darauf habe sie geantwortet: „Ja, well that's a big question, but I think I would like to marry an Indian." Einen indischen Mann würde sie viel besser kennen, sie würde sein Verhalten voraussagen können. „But if I spend more time over here maybe. (...). Because the culture is totally different from here to India and - it's not a problem. Obviously, you never know, and you might adjust and everything works out fine" (Smita, 26.01.2013). Für Smita schien eine gemeinsame nationale und damit verbunden kulturelle Herkunft eine wichtige Voraussetzung für das gegenseitige Verständnis und die Kompatibilität eines Paares zu sein.

Wie bereits erwähnt, heiratete Smita einen jungen Mann, den sie aus ihrem Studium in Indien kannte. Er promovierte mittlerweile Kanada. Sie erzählte, dass sie sich auf einer Konferenz getroffen und sich auf Anhieb wieder gut verstanden hatten. „Then I thought: Okay, at least he understands my profession, and he knows what 
my aims are in my life, and he will support me in that. He gives me the room I want" (Smita, 26.01.2013).

Gerade für Frauen, die nach einer Heirat weiter arbeiten möchten, ist es wichtig, einen Partner zu wählen, der dieses Ziel unterstützt. Dies verdeutlicht ein Artikel, den eine Gesprächspartnerin auf Facebook teilte und der den folgenden Titel trug: Pick your husband wisely: Your career depends on it. In dem Artikel wurde festgestellt, dass in Indien 48\% der arbeitenden Frauen ihren Beruf mitten in ihrer Karriere aufgäben. ${ }^{141}$ Den Partner selbst zu wählen, bedeutet gerade für die jungen Frauen, eine größere Kontrolle über ihre zukünftige Karriere auszuüben (vgl. Baas 2009: 300).

Die Ausführungen zu Smitas Navigation der Partnerwahl machen besonders sichtbar, welche wichtige Rolle Zukunftsvisionen in diesem Prozess spielen. Smita hatte recht klare Vorstellungen davon, wo ihr Leben hinführen sollte, und vom Ziel ihres Mobilitätsprozesses: Sie wollte zurück nach Indien und dort weiter in der Wissenschaft tätig sein. Sie wählte bewusst einen Mann, von dem sie annahm, dass er sie bei der Realisierung ihrer Vorstellung unterstützten würde.

\subsubsection{Die Perspektive eines Mannes: unerfüllbare Erwartungen}

In diesem Kapitel lag der Fokus bisher vor allem auf der Perspektive der Frauen. Eine Ausnahme bilden meine Ausführungen zu Gilon. Im Folgenden widme ich mich der Geschichte von Vivān. Vivān kam 2011 im Alter von dreiundzwanzig Jahren nach Göttingen, um in Physik zu promovieren. Er war einer der wenigen Männer, die mit mir über ihre Erfahrungen bei der Suche nach einer Partnerin sprachen. Als ich Vivān für ein zweites Interview (17.02.2013) traf, erzählte er, dass er bald eine Reise nach Indien zu seinen Eltern unternehmen werde. Als ich ihn fragte, was er für den Aufenthalt in Indien plane, erwiderte er, dass er sich mit einigen Frauen treffen wolle, mit denen er online Nachrichten ausgetauscht hatte. Einige seiner Freunde hätten ihn bereits damit aufgezogen, dass er auf der Suche nach einer Braut sei. „I don't accept it, but I think it’s true." Vivān war auf der Suche nach einer Partnerin und hatte bereits in Göttingen nach einer passenden Frau Ausschau gehalten. „Here it's difficult for me. I mean, at least the persons whom I had had a crush or something - I tried but it didn't work. So, now I'm left with no choices here." Er meinte, die meisten Frauen aus Indien, die er in Göttingen kennengelernt hatte, hätten bereits einen Freund, und schlussfolgerte lachend: „I don't stand a chance anymore - it's too late." In Indien habe er schon einige Frauen getroffen und „crushs" gehabt, aber „all of them went wrong; none of them were a success." Seine Eltern würden sich nicht in seine Partnerinnenwahl einmischen, versicherte er mir, aber er habe in der Vergangenheit seine Treffen immer mit seiner Mutter besprochen. Mittlerweile habe er jedoch entschieden, erst mit ihr zu sprechen, wenn tatsächlich etwas passiere, er

\footnotetext{
${ }^{141}$ Bithika Misha Rahman: „Pick Your Husband Wisely: Your Career Depends On It“, http://www. huffingtonpost.in/shenomics/the-career-decision-we-al_b_9868086.html (depubliziert, zugänglich via Archive.org).
} 
„erfolgreich“ sei. Zwei der Frauen, die er in Indien treffen wollte, kannte er noch aus seiner Schulzeit und hatte sie über Facebook kontaktiert. Ich wollte von Vivān wissen, was er plane, falls er mit einer dieser Frauen "Erfolg" habe. Lachend antwortete er „I myself will get afraid if I'm successful - so many failures in a stretch.“ Es sei heutzutage sehr schwer, Frauen in Indien zu beeindrucken. Sie würden alles haben wollen: „They want you to be tall, they want some masculine body, and then at the same time you have to be homely and not hanging out with other girls and anything." Die jungen Frauen hätten eine gewaltige Kriterienliste. „So it's difficult to fit in" (Vivān, 17.02.2013). ${ }^{142}$

Vivān war der Meinung, dass er die Art von Maskulinität, die von ihm verlangt wurde, nicht erfüllen könne, er hegte Selbstzweifel und fühlte sich unzulänglich (vgl. (Clark-Decès 2014: 154). In Vivāns Erzählung hatten die Frauen die Macht und schienen den Männern voraus zu sein, da sie häufig schon in einer Beziehung bzw. verheiratet waren (vgl. Clark-Decès 2014: 149). Aus Vivāns Aussagen spricht vor allem eine große Unsicherheit, die auch in den Konversationen ums Heiraten, die häufig in einem neckenden Ton ausgetragen wurden, spürbar war. Ich erinnere mich an eine gemeinsame Zugfahrt mit Vivān, Kiran und Pravin im Juni 2011, auf der sie immer wieder über das Heiraten sprachen und alle drei zu dem Schluss kamen, dass die Männer heutzutage häufig leiden würden, weil es weniger Frauen gebe und diese die Männer ausnutzen würden. In den Aussagen schwang Frustration, aber auch die Angst mit, nie als Ehemann ausgewählt zu werden, denn so schien es aus der Perspektive der Männer zu sein: Sie müssten von den Frauen gewählt werden. Die Geschichte von Lali, die mehrfach die Heiratsanträge eines Freundes abgelehnt hatte, und Smitas Erzählung von ihrer Partnerwahl lassen einen ähnlichen Schluss zu.

Vivāns Aufenthalt in Indien war nicht von Erfolg gekrönt. Allerdings lernte er wenig später im Sommer 2013 eine junge indische Promovierende in Göttingen kennen und begann mit ihr eine Beziehung. Sie kam wie Vivān aus Andhra Pradesh und die beiden teilten ihre Leidenschaft für die Musik. Nach etwa anderthalb Jahren beendete Vivān die Beziehung, unter anderem, so erzählte er mir, wegen des Stresses, den ihm die Fertigstellung seiner Dissertation bereitete.

Im November 2015 schloss Vivān seine Promotion erfolgreich ab. Wenige Tage nach seiner Verteidigung verließ er Göttingen und reiste nach Indien. Er zog wieder zu seinen Eltern und begann mit der Suche nach einem Arbeitsplatz und einer Partnerin. Ihm wurde recht schnell deutlich, dass es schwieriger werden würde als er gedacht hatte, eine Stelle in seinem speziellen Arbeitsfeld zu bekommen. Dies machte wiederum die Suche nach einer Partnerin nicht leichter, denn die Frauen, die er traf, suchten einen Mann mit einem sicheren Arbeitsplatz. Er hingegen wünschte sich eine unabhängige, gebildete und flexible Frau, die mit ihm ins Ausland gehen würde,

\footnotetext{
${ }_{142}$ Baas beschreibt die Entwicklung von Körperidealen in der Mittelschicht unter anderem in seinem Artikel The New Indian Male: Muscles, Masculinity and Middle Classness (Baas 2015). Laut neuerer Körperideale, die unter anderem durch Bollywood-Filme transportiert werden, soll der männliche Körper durchtrainiert sein, z.B. einen „Sixpack“ aufweisen.
} 
wenn seine Karriere dies erforderte. Vivān hatte somit seinerseits spezifische Anforderungen an eine Partnerin, die jene Frauen, die er in Indien getroffen hatte, nicht erfüllten. So erzählte er mir, dass ihm häufig schon bei der Kontaktaufnahme mit den Frauen deren Abhängigkeit von den Eltern und deren Unselbstständigkeit aufgefallen sei, weil sich in vielen Fällen zuerst die Eltern am Telefon gemeldet hätten.

In Vivāns Fall zeigt sich, wie sich gesellschaftliche und individuelle Erwartungen, sowohl an Männer als Ernährer, die eine spezifische Vorstellung von Maskulinität erfüllen sollten, aber auch an die Frauen als ,modern' aber auch ,indisch', auf die Partnerwahl auswirken, und zu welchen Dilemmata sie führen.

Im Januar 2016 kam Vivān für kurze Zeit zurück nach Göttingen, um seine Promotion mit dem Küssen des Gänseliesels zu feiern. Als wir uns trafen, wirkte er sehr niedergeschlagen und enttäuscht. Er erklärte mir, dass er geplant hatte, nach Indien zurückzukehren und sich dort „niederzulassen“ (settle down). Er betonte, er sei nun dreißig Jahre alt, also in einem Alter, in dem man einen Arbeitsplatz und eine Frau haben sollte. Der Zeitpunkt, den Vivān wählte, um nach Indien zurückzukehren, war von dominanten Lebensverlaufsvorstellungen der indischen Mittelschicht beeinflusst.

Vivān, der als einziges Kind seiner Eltern und als indischer Mann eigentlich der zukünftige Ernährer einer eigenen Familie sein sollte, hatte das Gefühl, gescheitert zu sein. Dieses Gefühl drückte er mit den folgenden Worten aus: „I was at the top of the ocean and then at the bottom of the sea. "Vivāns Beispiel führt vor Augen, dass nicht alle jungen Wissenschaftler*innen in ihren Strategien gleichermaßen erfolgreich waren. Anders ausgedrückt, konnten nicht alle meiner Gesprächspartner*innen ihre Zukunft während ihrer Zeit in Göttingen ausmanövrieren. Dies lag unter anderem daran, dass sich die Umstände, die sie ursprünglich dazu bewegt hatten, Indien zu verlassen, nicht geändert hatten und von den jungen Menschen kaum zu beeinflussen waren. Dazu gehörten beispielsweise die schlechte Arbeitsmarktsituation, aber auch die gesellschaftlichen Vorstellungen davon, was es für einen Mann heißt, erfolgreich zu sein (vgl. Clark-Decès 2014).

\subsubsection{Akademische Mobilität und die Partner*innenwahl}

Im vorangehenden Kapitelabschnitt habe ich dargestellt, wie die jungen Wissenschaftler*innen aus Indien ihre Wahl eines Heiratspartners bzw. einer Heiratspartnerin navigierten. Die internationale Mobilität war dabei in vielen Fällen ein wichtiges strategisches Mittel, das auf die Aushandlung mit den Familien einen großen Einfluss hatte. Hierfür war die Distanz zu den Eltern und den gesellschaftlichen Strukturen in Indien sowie das soziale und kulturelle Kapital, das mit Hilfe der akademischen Mobilität erlangt wurde, entscheidend. Die Mobilität machte eine strategische Wahl des Zeitpunkts für die Aushandlungen mit den Eltern möglich, was wiederum einen Einfluss auf die erfolgreiche Umsetzung eigener Aspirationen haben konnte.

Des Weiteren wurde sichtbar, dass bei der Partnerwahl verschiedene Variablen eine Rolle spielten: die Wünsche der jungen Menschen, ihre Aspirationen für die 
Zukunft sowie die Vorstellungen ihrer Eltern von einem ,passenden' Partner/ einer ,passenden“ Partnerin. Die akademische Mobilität wurde dabei in vielen Fällen zu einem wichtigen Mittel, um diese Variablen miteinander in Einklang zu bringen. Viele meiner Gesprächspartner*innen konnten durch dieses Vorgehen die Strukturen und Diskurse des Regimes der akademischen Mobilität für ihre eigenen Ziele einsetzen. Wie die Geschichte von Vivān verdeutlichte, trug die Mobilität nicht in allen Fällen zu besseren Chancen auf dem Heiratsmarkt bei.

Alle meine Gesprächspartner*innen, die bereits geheiratet haben (etwa 54\%), entschieden sich für eine Person, die ähnliche Qualifikationen hatte und bereits im Ausland studiert oder promoviert hatte. Dies lag auch daran, dass beide Faktoren wichtige Kriterien bei der Partnerwahl waren. Außerdem lernten die jungen Menschen ihre zukünftigen Partner*innen häufig an der Universität oder dem Arbeitsplatz kennen. Die jungen Inder*innen heirateten in vielen Fällen innerhalb einer Kategorie, die als international mobile indische Wissenschaftler*innen bezeichnet werden $k^{2} n^{143}$ - wobei innerhalb dieser Kategorie ,traditionelle 'Kriterien wie Religion, Herkunftsregion und Kaste eine Rolle spielten. Von meinen Gesprächspartner*innen sind bereits $72 \%$ der Frauen verheiratet, aber nur $42 \%$ der Männer. Diese Diskrepanz hat unterschiedliche Gründe. Allerdings spielen genderspezifische Vorstellungen vom Heiratsalter und von den erforderlichen Voraussetzungen für eine Eheschließung eine wichtige Rolle.

\subsection{Auf die Heirat folgt die Bürokratie}

In diesem letzten Abschnitt des Kapitels beschäftige ich mich mit Sarjana und Sunil, deren Fall eine weitere Perspektive auf das Zusammenspiel von Studierendenmobilität und Heirat eröffnet. An ihrem Beispiel wird besonders sichtbar, wessen Mobilität durch das Regime der akademischen Mobilität gefördert wird und wessen nicht.

Sarjana kam aus Indien und Sunil aus Bangladesch. Als sie sich in Göttingen im Frühjahr 2013 kennenlernten, spielte die Herkunft aus zwei verschiedenen Nationalstaaten kaum eine Rolle. Sie verstanden sich als Bengalis mit einer gemeinsamen Muttersprache, einer gemeinsamen Religion und einem großen Interesse an Musik. Sarjana war für ihr Promotionsstudium in Biologie nach Göttingen gekommen und hatte ein Stipendium. Sunil finanzierte seinen Aufenthalt in Deutschland mithilfe seiner Familie. Außerdem hatte er einen Nebenjob in einem indischen Restaurant. Er wollte in Göttingen Sozialwissenschaften studieren, musste aber zunächst ein Studienkolleg absolvieren, da sein Abschluss nicht als Hochschulzulassung anerkannt wurde. Sein Visum für Deutschland musste Sunil deswegen alle drei Monate erneuern lassen.

\footnotetext{
143 Ähnlich stellt es Michiel Baas in seiner Studie zu jungen Menschen in der IT-Branche in Indien fest (Baas 2009).
} 
Ich traf Sunil und Sarjana nach unserem Interview im August 2013 häufig bei verschiedenen Festlichkeiten, die von den indischen Studierenden organisiert wurden, oder einfach zu informellen Gesprächen. Die beiden schmiedeten schon bald Pläne für eine gemeinsame Zukunft und beschlossen, zu heiraten. Sunil musste im Frühjahr 2014 Deutschland verlassen, da er das Studienkolleg nicht erfolgreich abschließen konnte und eine Verlängerung seines Aufenthaltstitels abgelehnt wurde. Im Herbst 2014 heirateten Sunil und Sarjana zunächst in Bangladesch und feierten die Eheschließung später in Indien. Sarjana kehrte dann nach Göttingen zurück, um weiter an ihrer Promotion zu arbeiten. Sunil beantragte ein Familienzusammenführungsvisum, das an die Dauer von Sarjanas Aufenthaltsgenehmigung und damit an ihr Stipendium gekoppelt war. Dieses Visum zu erhalten, stellte sich allerdings als schwieriger heraus, als sie gedacht hatten. So wurde Sunils Antrag unter anderem mit der Begründung abgelehnt, dass die Ehe eine Scheinehe sei. Sarjana und Sunil mussten daraufhin in einem monatelangen bürokratischen Verfahren nachweisen, dass sie eine ,richtige' Ehe führten. Ihre unterschiedlichen Nationalitäten wurden auf diese Weise für ihre Beziehung relevant gemacht. Während dieses Prozesses baten sie neben Kolleg*innen und Freund*innen auch mich, einen Brief an die deutsche Botschaft in Dhaka zu formulieren, in welchem ich die ,Richtigkeit' der Ehe bestätigte. Außerdem baten sie mich um die Audiodatei unseres Interviews, mit der sie nachweisen konnten, dass sie bereits im August 2013 ein Paar waren. Um zu beweisen, dass sie ihre Ehe aus den ,richtigen ' Gründen, also aus Liebe, eingegangen waren, und nicht, damit Sunil eine Aufenthaltsgenehmigung in Deutschland erhalten konnte, reichten sie Fotos von gemeinsamen Urlauben und Protokolle von Telefonaten ein.

Für Sarjana und Sunil bedeutete dieser langwierige Prozess, dass sie mehrere Monate getrennt lebten und sich ständig mit Botschaften und Ämtern auseinandersetzen mussten. Diese Situation machte es Sarjana schwer, sich auf ihr Promotionsstudium zu konzentrieren, und durchkreuzte zunächst alle Pläne, die Sarjana und Sunil für ihre gemeinsame Zukunft hatten. Im August 2015, etwa elf Monate nach der Heirat, erhielt Sunil sein Visum für Deutschland.

Mit Blick auf den Fall von Sunil und Sarjana argumentiere ich, dass das Regime der akademischen Mobilität die Möglichkeiten zu und Erfahrungen von Mobilität ganz unterschiedlich beeinflusst. Für Sarjana war das Reisen zwischen Indien und Deutschland recht einfach - zumindest für die Zeit ihres Promotionsstipendiums, an das ihr Visum gekoppelt war. Sunil dagegen, dessen Abschluss aus Bangladesch nicht als Hochschulzulassung anerkannt wurde und der eine Prüfung des Studienkollegs nicht bestanden hatte, musste Deutschland wieder verlassen. Zugespitzt ausgedrückt: Sarjana, Promovierende in einem naturwissenschaftlichen Fach, wurde, um die Sprache der Wissensökonomie zu nutzen, als wichtige ,humane Ressource gesehen, Sunil nicht.

Obwohl Sunil im Endeffekt ein Visum erhielt, um gemeinsam mit Sarjana in Deutschland sein zu können, waren die elf Monate bis zu diesem Punkt durch große Unsicherheit und ständiges Warten gekennzeichnet. Sie konnten kaum ihre nächsten 
Schritte planen und hatten keine Kontrolle darüber, wann eine Entscheidung fallen und wie diese aussehen würde. Für Sarjana und Sunil bedeutete gerade diese Wartezeit einen Verlust von Zeitsouveränität - eine Erfahrung, die viele mobile Menschen machen (siehe Cwerner 2001; Griffiths 2013, 2014).

\subsection{Zusammenfassung}

In diesem Kapitel sind die dominanten Lebensverlaufsvorstellungen sichtbar geworden, die ein klares Timing und die Reihenfolge von Ereignissen implizierten und damit eine wichtige Zeitkarte für die biographische Navigation meiner Gesprächspartner*innen darstellten. In den Narrationen und den Handlungen der jungen Wissenschaftler*innen artikulierten sich die zeitlichen Normen, an denen sie sich in ihrer biographischen Navigation orientierten, die sie kritisch reflektierten oder reproduzierten. Da die dominanten Zeitkarten und sozialen Rhythmen an die Verortung in der Familie geknüpft waren, bedeutete die Navigation dieser die Aushandlung der eigenen Positionierung in der Familie. Aus diesem Grund wurden die zeitlichen Normen durch die Distanz und das Leben in einer neuen Zeitlandschaft nicht irrelevant und die Einhaltung durchaus weiterhin von der Familie kontrolliert.

Meinen Gesprächspartner*innen war es wichtig, einen Weg zu finden, um sowohl ihre eigenen Aspirationen als auch die gesellschaftlichen und elterlichen Erwartungen erfüllen zu können. Die Mobilität war für viele genau dieser Weg. Durch sie konnten meine Gesprächspartner*innen größere Kontrolle über den Zeitpunkt einer Heirat und die Partnerwahl erlangen.

Mit der Heirat als gesellschaftlicher Norm mussten sich alle meine Gesprächspartner*innen auseinandersetzen, wobei die konkreten Unsicherheiten und Dilemmata sich unterscheiden konnten - besonders zwischen den Männern und den Frauen. Keine der jungen Frauen schien infrage zu stellen, dass sie einen Ehepartner finden würde und dass sie würde heiraten können. Sie sorgten sich vor allem darum, welchen Einfluss eine Ehe auf die Umsetzung ihrer beruflichen Aspirationen haben würde. In den Geschichten der Frauen bot die Mobilität neue Möglichkeiten, gesellschaftliche Erwartungen mit akademischen Ambitionen zusammenzubringen, die beiden dominanten Zeitkarten der wissenschaftlichen Karriere und des Lebensverlaufs zumindest für eine Zeit zu vereinen und einen Partner zu wählen, von dem sie annahmen, dass er ihre Wünsche für die Zukunft unterstützen würde.

Auch einige der Männer nutzten die Mobilität, um etwas Zeit zu gewinnen. Allerdings verstanden sie ihr Studium oder ihre Promotion im Ausland vor allem als Möglichkeit, bessere Voraussetzungen für eine Heirat zu schaffen und damit die dominanten Vorstellungen von Männlichkeit zu erfüllen. Die Männer schien die mögliche Zurückweisung durch eine Frau mit Unsicherheit zu erfüllen. Ihre Angst davor, keine Partnerin zu finden und damit nicht ihre gesellschaftlich vorgesehene 
Rolle erfüllen zu können, wurde u.a. durch Statistiken und Artikel über den sogenannten „marriage squeeze" ${ }^{144}$ geschürt (Kaur 2013).

In der Navigation des Zeitpunkts einer Heirat und der Partnerwahl spielte der Blick auf zukünftige Koordinaten eine entscheidende Rolle: sei es die Hoffnung auf eine Rückkehr nach Indien, der Wunsch nach beruflicher Verwirklichung oder das Streben, gesellschaftlicher Erwartungen zu erfüllen.

${ }_{144}$ Als „marriage squeeze“ wird die ungleiche Anzahl von Männern und Frauen im heiratsfähigen Alter bezeichnet. Indien hat aufgrund der Präferenz von Söhnen und selektiver Abtreibungen mehr Männer als Frauen im gleichen Alter (Kaur 2013). 


\section{Die Zukunft in der Gegenwart}

Die jungen Wissenschaftler*innen aus Indien waren nach Göttingen gekommen, um unterschiedliche Aspirationen zu erfüllen: Sie wollten ihre wissenschaftliche Karriere auf den Weg bringen, einige wollten einer Heirat entgehen, andere wollten die Voraussetzung für eine Heirat schaffen, wieder andere sehnten sich nach einem unabhängigeren Leben fern von der Familie. Die jungen Menschen planten damit, dass ihre Zeit in Göttingen ein Ende haben würde. Wie ich in Kapitel 5 gezeigt habe, bildete das Erreichen des PhD-Abschlusses in Verbindung mit dem Ende des Aufenthaltes in Deutschland einen deutlichen Horizont in der biographischen Navigation. Die Imaginationen, die meine Gesprächspartner*innen für ihr Leben nach der Promotion hatten, und wie sie diese zu realisieren versuchten, stehen im Mittelpunkt dieses Kapitels.

Ich argumentiere, dass sich die Imaginationen von der Zukunft und die Praktiken der Zeitgestaltung an dominanten Vorstellungen des Karrierepfades in der Wissenschaft und normativen Lebensverlaufsvorstellungen orientierten. Die jungen indischen Wissenschaftler*innen versuchten in ihrer biographischen Navigation, die Erwartungen an sie als Zukunft Indiens, als gute Söhne und Töchter, mit eigenen Vorstellungen von einem erfüllenden Leben zusammenzubringen. In diesem Kapitel wird deutlich, dass das Leben in Göttingen einen Einfluss darauf hatte, was sich die jungen Menschen für ihre Zukunft wünschten. 
Im ersten Abschnitt dieses Kapitels zeige ich, wie meine Gesprächspartner*innen ihren Abschluss in Göttingen zelebrierten. Den Abschluss konstruierten sie als Koordinate im Lebensverlauf, an der es galt, wichtige Entscheidungen für die Zukunft zu treffen und diese mit der Familie und dem Ehepartner auszuhandeln. Im zweiten Abschnitt dieses Kapitels beschäftige ich mich mit den Imaginationen und Realitäten einer Rückkehr nach Indien. Die meisten meiner Gesprächspartner*innen erklärten, dass sie irgendwann nach Indien zurückkehren wollen würden. Ich zeige, welche Gründe sie für eine Rückkehr anbrachten, welche Vorstellungen sie von der Rückkehr hatten und welche Erfahrungen sie nach einer tatsächlichen Rückkehr machten.

Diejenigen der indischen Wissenschaftler*innen, die (noch) nicht nach Indien zurückgekehrt waren, begründeten dies, wie ich im dritten Kapitelabschnitt zeige, vor allem damit, dass ihre berufliche Laufbahn noch nicht am richtigen Punkt angekommen sei, um in Indien den ersehnten Arbeitsplatz erhalten zu können. Die meisten hofften, durch ihren Abschluss und weitere Qualifikationen die richtigen Voraussetzungen für eine erfolgreiche Zukunft in Indien zu schaffen. Allerdings war der Erwerb weiterer Qualifikationen außerhalb Indiens ebenso eine Strategie, die Ankunft an bestimmten Koordinaten wie Heirat, Rückkehr oder Familiengründung hinauszuzögern.

Im vierten Abschnitt dieses Kapitels konzentriere ich mich darauf, wie sich die verheirateten indischen Wissenschaftler*innen mit der nächsten Koordinate dominanter Lebensverlaufsvorstellungen auseinandersetzten: der Gründung einer eigenen Familie.

\subsection{Von Abschlüssen und Abschieden}

In diesem Abschnitt stehen die achtundzwanzig meiner vierunddreißig Forschungspartner*innen im Fokus, die bis zum Ende meiner Untersuchung ihre Promotion in Göttingen abgeschlossen haben. Der Weg zum PhD-Abschluss war nicht so geradlinig, wie ihn sich viele vorgestellt bzw. gewünscht hatten. Wie die Illustration Plan und Realität der Promotion (Abbildung 2) in Kapitel 4 verdeutlicht, war die Zeit bis zur fertigen Doktorarbeit durch verschiedene Herausforderungen gekennzeichnet, die meine Gesprächspartner*innen als (angehende) Wissenschaftler*innen navigieren mussten. Dazu gehörten der Alltag in einer sozialen Welt fern ab von der Familie und der vertrauten Zeitlandschaft, das Arbeiten in einem internationalen Umfeld sowie die mehr oder weniger intensiven Aushandlungsprozesse rund um das Thema Heirat. Besonders in der Abschlussphase der Doktorarbeit, die häufig vom Bewusstsein der auslaufenden Finanzierung begleitet wurde, konzentrierten sich die jungen Wissenschaftler*innen stark auf die Fertigstellung ihrer Dissertation und reduzierten ihre sozialen Kontakte. Bei einigen kam das Gefühl auf, dass sie nie fertig werden würden, oder wie Rohini es in einem Kommentar auf Facebook formulierte: „It's never an end ... ask a PhD scholar" (Rohini 09.02.2016). 
Umso mehr freuten sich meine Gesprächspartner*innen über ihren Promotionsabschluss und das Göttinger Ritual, das diesen markiert: das Küssen des Gänseliesels. Auf dieses Ereignis fieberten viele der jungen Wissenschaftler*innen hin. Einige betonten in Gesprächen, wie wichtig ein solches Ereignis sei, um sich dem Ende der Promotionszeit bewusst zu werden und damit abschließen zu können.

\section{Der Brauch des Gänseliesel-Küssens}

Das Gänseliesel ist eine bronzene Statue, die vor dem alten Rathaus in Göttingen in der Mitte eines kleinen Brunnens steht. Nach einem Verbot des Küssens dieser Statue, das von 1926 bis 2001 bestand, ist es Doktorand*innen wieder offiziell erlaubt, auf den Brunnen zu klettern und dem Gänseliesel einen Kuss zu geben. ${ }^{145}$ Begleitet wird das Spektakel von lauter Musik und feiernden Familienangehörigen, Kolleg*innen und Freund*innen. Bereits der Weg zum Gänseliesel-Brunnen wird auf einem geschmückten Wagen zurückgelegt, der im Idealfall vom Doktorvater oder der Doktormutter gezogen wird.

\subsection{1 „PhD is not just an academic degree“}

Der Abschluss der Promotion markierte nicht nur das Erreichen einer wichtigen Koordinate des akademischen Lebensverlaufs, sondern bedeutete für meine Gesprächspartner*innen gleichfalls das Ende des Studentenlebens und der Beginn eines neuen Lebensabschnitts. Folgender Text, den Sandeep zu einem Foto auf Facebook schrieb, das ihn beim Küssen des Gänseliesels zeigte, verdeutlicht, welche Relevanz diesem Moment zugeschrieben wurde:

It's been the most fantastic journey so far! PhD is not just an academic degree - it is the end of student life, it is the time when we realize the meaning of the word ,family', it is when our life got highly entangled with academia! However, last Friday was one of the best days in my life! It's a feeling which is hard to describe! (Sandeep, 31.10.2016)

Die Aussicht darauf, das Gänseliesel irgendwann küssen zu dürfen, war es, was Smita mir mit einem Augenzwinkern als ihre Motivation für eine Promotion nannte: „The motivation is not a Doctor." Smita erinnerte sich daran, dass sie im ersten Monat nach ihrer Ankunft in Göttingen beim Gänseliesel-Küssen einiger ihrer Kolleg*innen dabei gewesen war, und zog eine Parallele zu indischen Hochzeitszeremonien: „It was so nice because it gives a feeling - like in India we have marriages, when the groom

\footnotetext{
145 Diese Information stammt aus einem Artikel zum Gänseliesel im Göttinger Tageblatt. Siehe Heidi Niemann: „Nach der Promotion ein Kuss fürs Liesel“, Göttinger Tageblatt vom 01.06.2011, http:// www.goettinger-tageblatt.de/Campus/Goettingen/Nach-der-Promotion-ein-Kuss-fuers-Liesel. Die Statue vor dem alten Rathaus ist eine Replik. Das Original steht im Stadtmuseum Göttingen.
} 
is actually made to sit on the horse, and then you know we all go dancing in the road and stuff." In unserem Interview zwei Jahre später fragte ich Smita, wie sie das Ende ihrer Promotion und das Gänseliesel-Küssen tatsächlich erlebt habe. „It means lot of more things are coming. For now, I have to find something to work upon" (Smita, 26.01.2013). Sie erinnere sich immer noch an die fantastische Zeit (fantastic time), die sie an jenem Tag gehabt habe, allerdings sei ihr bewusst gewesen, dass bereits die nächsten wichtigen Entscheidungen vor der Tür stünden.

Die Mehrzahl meiner Gesprächspartner*innen verließ Göttingen kurz nachdem sie ihren angestrebten Abschluss erreicht hatten, und etwas mehr als die Hälfte war zu diesem Zeitpunkt bereits verheiratet. Harendra, einer der jungen Promotionsstudierenden, zog Ende 2017, bevor er seine Arbeit fertigstellen konnte, zurück nach Indien, da seine Finanzierung nach drei Jahren ausgelaufen war.

\subsubsection{Vom Treffen der ,richtigen` Entscheidung und dualen Karrieren}

Das Bewusstsein, dass das Ende der Promotion es erfordern würde, wichtige Entscheidungen für die Zukunft zu treffen, dämpfte Sangitas Freude auf ihren Abschluss. Sie fühle sich „anxious“ erklärte sie einen Monat vor der Abgabe ihrer Arbeit: „I have this feeling that I still have to figure out where I want to move next, and how do I want to shape my career henceforth?" Es sei nun die Zeit, um Entscheidungen zu treffen: „It's like okay ,stay cool, stay calm, just take the right decisions" (Sangita, 08.08.2013).

Smita und Sangita hatten kurz vor bzw. kurz nach der Fertigstellung ihrer Promotion geheiratet. Aus Smitas Sicht hatte die Ehe, anders als sie es erwartet hatte, vieles in ihrem Leben verändert. Vor der Ehe habe sie genau gewusst, was sie wann machen wollen würde. Nun habe sie ihre Erwartungen reduziert. Smita stellte fest, dass sie sich in einer komplett anderen Phase des Lebens (stage of life) befinden würde, wenn sie nicht geheiratet hätte. „Now I have lots of commitments and all. (...) Now it has to be wherever he goes I have to go, or either we discuss wherever I go or he comes. So, it's much more collaborative effort nowadays. So, it does change your life" (Smita, 26.01.2013).

Wie Smita mir erklärte, bedeutete eine Ehe für sie, Kompromisse eingehen zu müssen. Aus diesem Grund plante sie zum Zeitpunkt unseres Interviews, zu ihrem Mann in die USA zu gehen und nicht nach Indien. Allerdings betonte sie, dass sie dort arbeiten wollen würde: „I would definitely like to work - so that's the idea. That's why I wanted to go to the US as a student and not as a wife - so that is my aim." Smita und ihr Ehemann zogen 2013 gemeinsam zurück nach Indien. Ihr Mann hatte eine Position als Postdoktorand an einem Institut in Nordindien erhalten und Smita folgte ihm, obwohl sie in einem Gespräch die Befürchtung ausdrückte, dass sie in Indien weniger Chancen auf einen Arbeitsplatz haben werde. In einer Kommunikation über E-Mail erkundigte ich mich im August 2013 bei Smita nach ihrem Leben in Indien. Sie erklärte, dass sie zwar in Kontakt mit einem Professor stehe, aber noch keine sichere Stelle habe. „I am very relaxed but at the same time 
very much stressed, as I have not got a confirmed position as in yet ... As they say: no work and all play is equally bad as all work and no play" (Smita, Facebook-Kommunikation 08.08.2013). Tatsächlich dauerte es etwa ein halbes Jahr, bis sie eine Stelle an dem Institut erhielt, an dem auch ihr Mann arbeitete.

Sangita und ihr Mann suchten ebenfalls nach einem Ort in Indien, an dem sie beide würden arbeiten können. Als ich Sangita 2017 bei einer Absolventenfeier traf, erzählte sie mir, dass sie und ihr Mann schon einen „Plan A bis F“ gemacht hätten und nach und nach ihre Optionen ausloten würden. Seit Ende 2017 leben sie zusammen in Indien.

Wie ich bereits in Kapitel 4 und 7 angesprochen habe, waren sich vor allem die jungen Frauen sehr bewusst, welchen Einfluss eine Heirat auf ihre zukünftige Karriere haben könnte. Alle meine Gesprächspartner*innen, die mittlerweile verheiratet sind, wählten eine Person, die selbst wissenschaftlich tätig war. Studien zeigen, dass Wissenschaftlerinnen im Vergleich zu ihren männlichen Kollegen eher mit Personen verheiratet sind, die selbst in der Wissenschaft tätig sind. Dies gilt sowohl für Indien (Jayasree Subramanian 2016) als auch für Deutschland (Rusconi und Solga 2007). Dies bedeutet, wie Schaer, Dahinden und Toader feststellen, „to experience the so-called dual-body problem, in which the partners - both confronted with a high demand for mobility - have to coordinate their career plans" (2017: 1294, vgl. Rusconi und Solga 2007). Durch eine Heirat werden die Lebensverläufe von Personen miteinander verbunden und beeinflussen sich gegenseitig (Elder, Johnson und Crosnoe 2003; Ajay Bailey und Mulder 2017). Gender und die damit verbundenen Erwartungen an beruflichen Erfolg spielen für die Entscheidung, wessen Karriere den „Vorrang“ hat, eine wichtige Rolle (Ajay Bailey und Mulder 2017). Unter den jungen indischen Wissenschaftler*innen versuchten die Paare häufig einen Ort zu finden, an dem es für beide möglich war, zu arbeiten. In einigen Fällen war es aber zunächst die Karriere des Mannes, die scheinbar den Vorrang erhielt - so wie bei Smita und ihrem Mann und Ajay und seiner Frau.

\subsubsection{Abschluss und Abschied}

Der Promotionsabschluss wurde von den jungen Wissenschaftler*innen mit dem Küssen des Gänseliesels und einer Feier zelebriert. Viele nutzten dafür die Räumlichkeiten der Universität, des Studentenwerks oder der Max-Planck-Institute, an denen sie gearbeitet hatten. In vielen Fällen reisten Eltern, Großeltern oder Geschwister aus Indien an, um beim Abschluss der Promotion anwesend zu sein.

Bei den Abschlussfeiern wurde häufig zusammen gegessen, getanzt und vor allem geredet: über die gemeinsame Zeit in Göttingen und die Pläne für die Zukunft. Für den Großteil meiner Gesprächspartner*innen ging ihr Promotionsabschluss mit dem Verlassen Göttingens einher. Die Doktorandenfeiern waren deswegen häufig gleichzeitig Abschiedsfeiern und durch eine Mischung aus Ausgelassenheit, Erleichterung und einer Spur Traurigkeit gekennzeichnet. Akhil artikulierte diese Stimmung bei seiner Feier mit Hilfe zweier Lieder, wie er es während seiner Zeit in Göttingen so 
häufig bei den unterschiedlichen Feierlichkeiten getan hatte. Er sang ein „farewellLied" mit dem Titel Beete hue Lamho ki Kasak Sath toh Hogi aus dem Film Nikaah (1982). Der Titel bedeutet übersetzt in etwa: „Der nostalgische Schmerz vergangener Momente soll stets mit dir sein." Akhil würde zwei Tage später gemeinsam mit seiner Mutter, die für seinen Abschluss nach Göttingen gekommen war, zurück nach Indien fliegen. Auf meine Frage, was seine Gedanken zur Rückkehr seien, meinte er: „I'm happy to go to India, sad to leave Göttingen.“

Als ich mich bei Sadhvi nach dem Ende ihrer Promotion erkundigte, war ihr Abschluss bereits einige Monate her, und sie erinnerte sich: „I thought I would be really excited and feel different somehow. But no, I don't really feel different." Es sei eher , anticlimactic“ gewesen. Sie habe gedacht, es würde so aufregend werden, und nun habe sie ihren Abschluss, und sie und ihr Mann würden bereits ihre Wohnung ausräumen. „Now I'm feeling really sad to leave Göttingen and Germany in general." Sadhvi hatte mit ihrem Mann insgesamt vier Jahre in Göttingen gelebt. Für sie hatten diese Jahre eine besondere Bedeutung, weil sie, wie sie betonte, noch nie so lange ohne größere Unterbrechung an einem Ort gelebt habe. Sadhvi war in den USA aufgewachsen und mit ihren Eltern jedes Jahr vier Monate lang nach Indien gereist. „So four years - it's very significant for me.“

Eine meiner Gesprächspartner*innen artikulierte ihre Gedanken über das Verlassen Göttingens und lieb gewonnener Menschen auf Facebook. Sie hatte sich im Flugzeug, das sie in die USA bringen sollte, fotografiert, das Foto auf Facebook geteilt und dazu geschrieben: „I'm glad that I am taking priceless memories and lifelong relationships with me. (...) I will miss Göttingen, my lab, all my friends and my time in Germany for [the] rest of my life" (Facebookeintrag 25.05.2016).

Nicht zuletzt macht dieser Text deutlich, dass die jungen (angehenden) Wissenschaftler*innen lokal verortete Menschen mit engen sozialen Beziehungen waren und keine losgelösten ,schlauen Köpfe'. Das Leben in Deutschland, auch wenn es nur als vorübergehend verstanden wurde, ging mit neuen Beziehungen zu Menschen und Orten einher, die, wie im Text betont wurde, für den Rest des Lebens präsent sein würden.

Das Ende der Promotion war eine besondere Koordinate im Leben meiner Gesprächspartner*innen. Dieser Meilenstein markierte das Ende des Studierendendaseins und für viele das Ende ihrer Zeit in Göttingen und den Anfang eines neuen Kapitels im Leben. Für etwas mehr als die Hälfte meiner Gesprächspartner*innen begann der neue Lebensabschnitt mit der Heirat und den darauf folgenden Aushandlungen des nächsten gemeinsamen Schrittes.

Der PhD-Abschluss war ein wichtiger Zeitpunkt, um sich erneut zu orientieren und herauszufinden, wie es nun weitergehen sollte. Die Vorstellung vom akademischen Karrierepfad, familiäre Erwartungen und der Wunsch meiner Gesprächspartner*innen, beides vereinen zu können, waren in diesem Prozess richtungsweisende Faktoren. 


\subsection{Zurück?!}

Für meine Gesprächspartner*innen war der Gedanke an eine permanente Rückkehr nach Indien unterschiedlich konkret und sie hatten divergierende Vorstellungen vom besten Timing für diese (Fuhse 2018). Der Zeitpunkt einer Rückkehr wurde durch mehrere Faktoren beeinflusst. Dazu gehörten u.a. die Heirat, das Alter der Eltern, Vorstellungen von der wissenschaftlichen Laufbahn und der Arbeitsmarkt in Indien. Von meinen vierunddreißig Hauptgesprächspartner*innen waren bis zum Zeitpunkt des Verfassens der vorliegenden Arbeit fünfzehn zurück in Indien, neun lebten in Deutschland und zehn hatte ihr Pfad in einen anderen Teil der Welt geführt - fünf davon in die USA.

Die Einstellung zu einer möglicherweise permanenten Rückkehr wurde durch den Prozess der Mobilität beeinflusst. Das heißt, der Gedanke an eine Rückkehr wirkte sich nicht nur auf die Erfahrungen von Mobilität aus, sondern das Leben in Göttingen beeinflusste die Einstellung zur Rückkehr und Vorstellungen vom besten Zeitpunkt für diese.

\subsubsection{Rückkehr und Verantwortung für Familie und Gesellschaft}

Viele der jungen Wissenschaftler*innen waren sich bewusst, dass von ihnen eine Rückkehr nach Indien erwartet wurde. Einige verstanden eine Rückkehr als wichtigen Aspekt in der Erfüllung ihrer Verantwortlichkeiten gegenüber der Familie und der ,Entwicklung' der Nation. Bereits bei der Aushandlung der Entscheidung für ein Studium oder eine Promotion im Ausland spielte unter anderem die Frage nach der Rückkehr eine Rolle. Dies wird besonders in Nanditas Fall deutlich. Als ich Nandita fragte, was ihre Eltern von ihrem Aufenthalt in Deutschland halten würden, erklärte sie mir: „They are happy about it. They know that it will be good for my career. If I decide that I want to stay in Germany forever, then their reaction may be different. But I'm here only for two, three years, so it's good for them" (Nandita, 03.09.2011).

Nandita war ein Einzelkind, ein Umstand, der einen entscheidenden Einfluss darauf hatte, wie stark sie mit ihrer Rückkehr rechnete: "That is also a reason for me to go back to India, and not to think anything else. " Besonders jene meiner Gesprächspartner*innen, die keine Geschwister hatten oder die Erstgeborene waren, waren sich ihrer Verantwortung für die Pflege ihrer Eltern im Alter sehr bewusst. Vendan, der ältere von zwei Brüdern, erklärte mir, dass er zwar zunächst als Postdoktorand in Deutschland bleiben werde, aber spätestens dann zurück nach Indien gehen werde, wenn seine Eltern der Pflege bedürften. Das Leben von Personen, mit denen die jungen Wissenschaftler*innen verbunden waren - von Eltern, Partner*innen und Kindern - hatte einen großen Einfluss auf die Entscheidung für oder gegen und eine Rückkehr (Kôu, Mulder und Ajay Bailey 2017). Diese Dynamik steht mit gesellschaftlichen Erwartungen an die jungen Menschen als, gute' Söhne, Töchter oder 
Schwiegertöchter im Zusammenhang. Meine Gesprächspartner*innen navigierten diese Erwartungen und die damit verbundenen Vorstellungen von der Familie in der indischen Mittelschicht.

Ein weiterer Grund, der für eine Rückkehr nach Indien angeführt wurde, war der Wille, etwas zur ,Entwicklung' Indiens beizutragen. Nandita erklärte, Deutschland und andere europäische Länder seien ihrer Einschätzung nach „saturated from development", sie könnten nicht noch mehr entwickelt werden. In Indien dagegen gebe es noch viele „Dinge“ zu tun: „So if everyone will run away, who will develop it?" Nandita sprach zwei Diskurse an, die im Zusammenhang mit der Mobilität Hochausgebildeter immer wieder sichtbar werden: der Diskurs um den sogenannten „Brain-Drain“ und der Diskurs, der Wissen mit Entwicklung verbindet. In Kapitel 3 habe ich bereits dargelegt, dass vor allem der zweite Diskurs eine wichtige Funktion im Regime der akademischen Mobilität innehat. Durch die Verknüpfung von Wissen mit ,Entwicklung ' wird den Wissenden eine entscheidende Rolle für den ,Fortschritt' Indiens zugesprochen. Die jungen indischen Wissenschaftler*innen nahmen diese Rolle unterschiedlich an, wobei in vielen Fällen deutlich wurde, dass sie eine gewisse Verantwortung gegenüber Indien verspürten und etwas ,zurückgeben' wollten. Dies gilt zum Beispiel für Sangita, die ihren Wunsch, gemeinsam mit ihrem Mann nach Indien zurückzukehren, folgendermaßen begründete: „I would just say that as a kid I was taught that you should give back to the society that you took things from" (Sangita, 08.08.2013). Bei den gemeinsamen Festen der jungen Inder*innen in Göttingen, die ich in Kapitel 6 analysiert habe, wurde diese Verantwortung unter anderem in den offiziellen Reden betont und dieser Diskurs damit kollektiv bekräftigt.

Sahil war postdoctoral fellow in Göttingen und wollte zurück, um vor allem außerhalb der Wissenschaft tätig werden zu können. Es sei gut für ihn gewesen, einen Teil seiner wissenschaftlichen Ausbildung im Ausland absolviert zu haben, um neue Perspektiven kennenzulernen, aber das Leben in Deutschland: „would be just in the classroom. And outside the classroom, there is not much that I feel I can do here" (Sahil, 01.09.2015). Sahil hatte das Gefühl, dass er in Indien etwas beitragen könne und verspürte den Drang, seine akademische Ausbildung in der Praxis anzuwenden. Seit 2017 hat er eine Stelle als assistant professor in Indien.

\section{„Zurückgeben“ aus der Ferne}

Sandeep erklärte mir, er würde gerne nach Indien zurückgehen: „Of course my aim would be to sometimes go back and teach some of them." Allerdings sei dies wegen der Forschung, die er durchführe, nicht wirklich möglich. Nach ein oder zwei „Postdocs“ werde er aber möglicherweise, und dann ergänzte er: „,certainly“, zurückgehen: „There is no question about it." Sandeep und seine Frau lebten seit 2016 in Belgien und engagierten sich gemeinsam mit anderen indischen Wissenschaftler*innen in einer selbst gegründeten Organisation, die Schüler*innen in Indien dabei helfen soll, 
ihre eigenen akademischen Interessen zu entdecken. ${ }^{146}$ Dafür organisierten sie einen Workshop in einer Stadt im Süden Indiens. Sandeep und seine Frau waren zwar (noch) nicht endgültig nach Indien zurückgekehrt, wollten aber aktiv dazu beitragen, etwas an der akademischen Situation in Indien zu ändern. In diesem Sinne hat Sandeep seinen Plan, nach Indien zurückzukehren und dort zu lehren, umgesetzt, allerdings auf eine andere Art und Weise, als er zunächst gedacht hatte.

Nicht alle der jungen Wissenschaftler*innen verstanden es als ihre Pflicht, bewusst zur ,Entwicklung' Indiens beizutragen, und stellten sich gegen die Idee, dass die eigene Identität als Inder*in dadurch demonstriert werden müsse. Ajit erklärte mir: „I know always that I'm from India and I like it that way. But I'm not too strict with where I belong, because I don't think I have to save my country to prove I'm Indian." Er argumentierte weiter, dass der ganze Planet ein „Zuhause“ sei und nicht ein Land. Er denke, dass jeder leben könne, wo er oder sie wolle, „and if you do something good in Germany, it doesn't make you any bad or good person with respect to India." Ajit verstand das Indisch-Sein nicht als an eine Lokalität gebunden und nicht als etwas, das durch Handlungen bewiesen werden muss. Er vertrat eine eher kosmopolitische Einstellung und verband diese mit seiner wissenschaftlichen Tätigkeit als Biologe: Wenn er eine Impfung erfände, wäre diese für alle Menschen gut. Ähnlich argumentierte Kundan: „So doing science in any place is fine for the whole world“ (Kundan, 14.08.2013).

Ajit war sich, das wird in seiner Argumentation sichtbar, bewusst, welche gesellschaftlichen Erwartungen an ihn gestellt wurden, und versuchte, sich von diesen zu distanzieren. Jeder solle leben, wo er oder sie sich wohlfühle, betonte er: „If I want to live next to my parents, I would live in India. If I don't want to live in a society where you have to work all the time from Monday to Sunday and get watched by people, then I would live somewhere else“ (Ajit, 04.07.2015). Ajit schätze das Leben in Deutschland mit der größeren Zeitsouveränität und der Distanz zur Familie und wollte dies zumindest zum Zeitpunkt unseres Interviews nicht gegen eine Rückkehr eintauschen.

\subsubsection{Die Rückkehr: Von Vorstellungen, Wünschen und Realitäten}

Von meinen Gesprächspartner*innen drückten fast alle den Wunsch aus, zu gegebener Zeit nach Indien zurückkehren zu wollen. Viele waren sich allerdings bewusst, dass eine Rückkehr nicht einfach sein würde. Osella und Osella fassen diese ambivalente Einstellung treffend zusammen, indem sie schreiben: „The homeland simultaneously beckons and repels" (2008: 165).

\footnotetext{
146 Sandeep verlinkte einen kurzen Zeitungsartikel über den Workshop auf Facebook. Darüber erfuhr ich von seinem Engagement und den Zielen, die er damit verfolgte.
} 


\section{Wünsche und Vorstellungen}

Rohini war sich bewusst, dass eine Rückkehr nicht einfach sein würde: „It will be bit of a challenge to go back, but somebody has to do it, “ erklärte sie lachend. „You either make it or you don't. At least try - it's the only thing you can do." Sie kenne einige Leute, die nicht zurückkehren wollen würden. „But on my personal ground, I want to move around a bit, but at some point I would like to go back." Rohini betonte immer wieder, wie wichtig es für Indien sei, dass die gut ausgebildeten Menschen zurückkehren. Den Zeitpunkt ihrer eigenen Rückkehr verortete sie allerdings noch recht unkonkret in der Zukunft.

Noch deutlicher wurde die zwiespältige Einstellung zur Rückkehr in der Aussage Ajits, der mir erzählte: „I like the idea [of going back]. I like the idea, only it's complicated“ (Ajit, 14.07.2015). Wie viele meiner Gesprächspartner*innen mochte Ajit die Vorstellung einer Rückkehr, die Realität wurde aber oft anders empfunden. Dhara, eine meiner Gesprächspartner*innen, die seit etwa zehn Jahren in Deutschland lebte, analysierte, dass es vor allem das "desire“ sei, die Sehnsucht nach einer Rückkehr, an der die jungen Inder*innen „hängen“ würden (Dhara, 24.04.2013), weniger die Realität.

\section{Realitäten}

Gilon und Tanvir waren zwei meiner Gesprächspartner*innen, die sofort nach ihrem Abschluss nach Indien zurückkehrten. Für Gilon stand eine Rückkehr immer außer Frage, eine Einstellung, die er selbst nicht ganz erklären konnte: „I ask myself why it is like that. I have no answer, but for me it's very obvious that I would like to go back" (Gilon, 19.01.2013). Auf meine Frage, ob er denn irgendwelche Schwierigkeiten in Indien erwarte, sagte er: „Some, yes of course. I think I'm very much aware that the future in India is not easy." Gilon ging davon aus, dass seine akademischen Qualifikationen in Indien nicht die gleiche Rolle für seine Position an seinem Institut spielen würden wie in Deutschland. Er meinte, dass er, auch wenn er noch so viele wissenschaftliche Artikel produzieren würde, nie den gleichen Status erreichen würde wie die älteren und bereits etablierten Kollegen.

Seine Rückkehr nach Indien verstand Gilon als den Anfang seiner beruflichen Karriere und einer neue Phase seines Lebens: „In a way, that it is of course an end to all this freedom - of working without my responsibility as an assistant professor. I really enjoyed this time (...). In a way, it's kind of [over] and you finish a phase and enter into another phase of life."

Im Juni 2015, etwa zwei Jahre nach unserem Interview, traf ich Gilon, als er für einen kurzen Besuch in Göttingen war. Ich fragte, wie er seine Rückkehr nach Indien erlebt habe. Er erzählte, dass er sehr glücklich sei, wieder in Indien zu leben. Er habe sehr nette Kollegen am College, von denen vier in Deutschland studiert oder promoviert hätten. Er habe sich erst an die „politics“ an der Universität in Indien gewöhnen müssen, nehme sie aber nun so an, wie sie sei. 
Auch zu Tanvirs Planung gehörte, in Zukunft nach Indien zurückzukehren: „I would obviously love to return; it's your own place and you have a special attachment." Allerdings betonte er in unserem ersten Gespräch im Juni 2011, dass er dahin gehen werde, wo seine Arbeit ihn hinführe: „That's the way I have defined my life to be.“ Im Herbst 2015 hatte er seine Promotion abgeschlossen und verließ Göttingen, noch bevor er das Gänseliesel küssen konnte (dafür kam er im Dezember kurz nach Göttingen zurück). Als ich ihn in einer E-Mail nach den Gründen für seine Rückkehr nach Indien befragte, schrieb mir Tanvir eine ausführliche Antwort, in der er zu dem Schluss kam, dass er wohl nicht ganz aufrichtig gewesen sei, als er mir erklärt hatte, dass er sich immer nach seinen beruflichen Perspektiven richten werde. Mittlerweile habe er erkannt, dass es der Wunsch nach einer Partnerin sei, der seine Entscheidungen beeinflusse, und "that I believe screws up things most of the time“ (Tanvir, 25.08.2016). Da er keine Hoffnung gehabt habe, in Göttingen eine Partnerin zu finden, und für seine wissenschaftliche Karriere keine Chance in Deutschland gesehen habe, habe er gedacht, dass er zurück nach Indien gehen sollte.

Gegen Ende seiner Promotion sei das Verhältnis zu seinem Betreuer nicht besonders gut gewesen, "mainly because of what I was taught in my masters: that whenever I faced a problem, I should try it myself before asking for help." Diese Einstellung habe seine Produktivität während der Promotion eingeschränkt, so dass sein Betreuer ihm nahegelegt habe, dass es in Deutschland für ihn kaum Optionen geben würde. „So, I kind of believed that I have to try for jobs in India." Dass sich Tanvir keine Zukunft in Deutschland vorstellen konnte, führte er auch darauf zurück, dass er während seiner Zeit in Göttingen sehr auf seine Promotion fixiert gewesen sei:

I came to Germany for [my] $\mathrm{PhD}$, and that was the main task ... I should accomplish. In the process, I could not open up very much that would also help me believe [in] settling down in Germany. You know, the usual stuff: making friends, learning the language. (Tanvir, 25.08.2016)

Wie ich in Punkt 5.1.3 angemerkt habe, konnte die Einstellung zum Leben in Göttingen - der starke Fokus auf die Arbeit bzw. auf das Studium und auf die temporäre Natur des Aufenthalts - wie eine selbsterfüllende Prophezeiung wirken. Tanvir bereute im Rückblick, dass er so wenig in eine mögliche Zukunft in Deutschland investiert hatte.

Nach der Fertigstellung seiner Doktorarbeit setzte Tanvir seine beruflichen und privaten Hoffnungen auf eine Rückkehr nach Indien. Allerdings verlief seine Rückkehr nicht so erfolgreich, wie erhofft. „But India, too, was a bad experience, mainly because I was not prepared for the huge difference in work culture, climate, and so on." Tanvirs Erfahrungen bei der Rückkehr waren durch das gekennzeichnet, was als „reverse culture shock“ (Pritchard 2011; Sondhi 2013: 44) bezeichnet wird.

In Tanvirs Fall war die Strategie, mit der er sich eine erfolgreiche berufliche und persönliche Zukunft aufzubauen versucht hatte, nicht geglückt: Durch seine Mobilität hatte er weder seine Aussicht auf einen Arbeitsplatz noch auf eine Heirat 
verbessert. Tanvirs Geschichte macht sehr deutlich, dass auch Abschlüsse aus dem Ausland und Versuche, die Zukunft zu überlisten, die Umstände in Indien nicht ändern können: Die Arbeitsmarktsituation für Akademiker*innen ist gerade in der Grundlagenforschung, wie sie Tanvir betrieb, weiterhin schlecht, und ein guter Arbeitsplatz ist weiterhin eine wichtige Voraussetzung für eine Heirat. Tanvirs Plan war es im Jahr 2016, erneut in Europa nach einem Arbeitsplatz und nach einer Partnerin zu suchen. Er wählte in diesem Sinne erneut Mobilität als Mittel, um Einfluss auf seine Zukunft zu nehmen und einen Weg aus einer sehr unsicheren Position zu finden. Auf Facebook teilte er in dieser Zeit den folgenden, auf seine Situation sehr passenden Spruch: „The path isn't a straight line; it's a spiral. You continually come back to things you thought you understood and see deeper truths" (Tanvir auf Facebook, 01.08.2016).

Etwa ein Jahr später, im Mai 2017, hatte sich Tanvirs Lage geändert. Er hatte nun eine Stelle als Postdoktorand in Hyderabad in Südindien. Diese war zwar auf ein Jahr befristet, aber Tanvir schrieb mir in einem beschwingten Ton, dass ihm das Leben in Hyderabad sehr gut gefalle: „The (...) campus is on the outskirts, without much noise and lots of trees and bushes...I have already seen at least 5 scorpions and 1 cobra inside the campus since I came here. (...) Also with other PostDocs and PhD scholars in the campus, I am overall having a good time." Die Situation meiner Gesprächspartner*innen konnte sich immer wieder ändern, neue, ungeahnte Möglichkeiten konnten sich auftun und vermeintliche Umwege zum Ziel führen. Auf eine sehr poetische Art drückte Tanvir diese Dynamik mit einem Gedicht aus, das er auf Facebook teilte und in dem das Leben mit einem gewundenen Fluss verglichen wurde. In der zweiten Strophe des Gedichtes heißt es:

But it is not a single end that come,

The river distributes before a Delta.

Just as in life;

A many open ends form before it's termination.

Ähnlich wie das Flussbett dem Fluss, um bei der Metapher aus dem Gedicht zu bleiben, geben die Zeitkarten dem Leben einen scheinbar alternativlosen Verlauf vor. Die letzte Strophe des Gedichtes kann dementsprechend als die Erkenntnis interpretiert werden, dass es mehr als einen möglichen Pfad gibt. Allerdings stelle sich diese Erkenntnis, erst am Ende des Weges ein:

A single path is not therefore enough! Multiple diversions arise. To keep flowing until the end to realize (am 29.03.2018 auf Facebook gestellt). 


\subsubsection{Ein Leben hier und dort und dazwischen}

Das Leben in Deutschland hatte einen Einfluss darauf, was sich als Ankunftspunkt (vgl. Baas 2010), also als Resultat der Mobilität, vorgestellt wurde. Besonders der Fall von Ajay führt dies vor Augen. Ajay lebte zum Zeitpunkt des Verfassens dieser Arbeit bereits seit zwölf Jahren in Deutschland. Er hatte seinen Masterabschluss in Hannover erworben und hat mittlerweile eine Position als Postdoktorand an einem Max-Planck-Institut in Göttingen. Er ist mit einer Frau aus Indien verheiratet, die selbst ein Promotionsstudium in Großbritannien absolviert hat. Gemeinsam haben sie ein Kind. Schon vor der Familiengründung in Deutschland hatte Ajay in unserem Interview beim Gedanken an eine Rückkehr nach Indien eine gewisse Unsicherheit ausgedrückt. Er würde gerne noch für einige Jahre in Deutschland bleiben, um weitere Berufserfahrung zu sammeln, erklärte er. Wenn er darüber nachdenke, habe er aber ebenso das Gefühl, dass „I can go back to India also at some point of time in my life. Because I want my parents and my place and everything" (Ajay, 14.12.2011). Allerdings habe sich die Situation mittlerweile verkompliziert und er könne Deutschland nicht einfach so verlassen. „I don't know. It suits me the German culture point of view. I don't want to miss both and that is really, really confusing." Aus seiner Sicht sei die optimale Lösung für dieses Dilemma ein guter Arbeitsplatz, „where I can jump here and there, then that would be perfect." Er lachte kurz ob der Aussicht auf eine solche mehr oder weniger realisierbare Stelle.

Dass Ajay bezüglich seiner Rückkehr zwiegespalten war, wurde in unserem Gespräch in der Art und Weise deutlich, in der er immer wieder Vergleiche zwischen Deutschland und Indien und den Menschen in diesen Ländern zog. Am Leben in Deutschland und Indien fand er gute und schlechte Seiten und für beide Gesellschaften, für die deutsche ebenso wie für die indische, hatte er lobende und kritische Worte übrig. Ähnlich stellte Smita fest, dass sowohl das Leben in Indien als auch das in Deutschland - das eine sei auf die Familie ausgerichtet, das andere auf Freunde und Individualität - Vor- und Nachteile habe. Beide Arten zu leben habe sie nun kennengelernt und für ihre eigene Zukunft wünschte sie sich: „If I'm able to strike a balance when I go back home - or wherever I go in the world - at some point of my life, then it will be very nice" (Smita, 26.01.2013).

Für Ajay war die positive Verortung in Deutschland ein weiterer Aspekt seines multiplen Selbst. Er erklärte mir, dass er die Frage danach, wer er eigentlich sei, bereits in Indien nicht einfach habe beantworten können:

It's very difficult for me to answer. I just tell him that I'm an Indian. They might laugh at me, but it is the truth. In India generally, ask a person: „Who are you?" He will say „I'm a Tamilian“, „I'm a Malayali“, „I'm that region“. (Ajay, 14.12.2011)

Wenn ihn jemand fragen würde, wer er sei, könne er nicht einfach sagen, „Ich bin ein Malayali“, weil er eben nicht wirklich einer sei: „I'm born and brought up in 
Chennai. If people ask me, ,Are you a Tamilian?‘ I wouldn't say exactly I'm Tamilian because my roots are Malayali." Weder seine Wurzeln noch die Stadt, in der er aufgewachsen sei, könne er einfach hinter sich lassen. Deswegen habe er nicht nur eine Identität, genauso gehe es ihm mittlerweile mit Deutschland: „I've been here, I'm taking the culture, but I'm not a German. “ Ein „true Indian“ sei er aber auch nicht, weil er die guten Aspekte der deutschen Kultur kennengelernt und übernommen habe. Am liebsten würde er die guten Aspekte der indischen und der deutschen Kultur zusammenlegen: „And in that process, maybe people might think I'm not an Indian. I don't know. They might say, ,Oh this guy is behaving like a German' [or] ,Oh you are half German." "Er spüre, dass er manchmal wie ein Deutscher denke, und verdeutlicht dies am Thema Pünktlichkeit. Er werde mittlerweile sauer, wenn sich Menschen verspäten würden. „I'm becoming a German at that point of time, but at the same time I can't leave my roots." Seine Mutter habe ihn gefragt, als was er sich selbst verstehe, und Ajay habe geantwortet: „I'm a global Indian.“

\section{Die Navigation von Imaginationen und Realitäten der Rückkehr}

Die Rückkehr nach Indien spielte als Koordinate in der biographischen Navigation meiner Gesprächspartner*innen in unterschiedlichen Ausprägungen eine entscheidende Rolle. Für die einen war die Rückkehr nach Indien mit der Idee verbunden, etwas an die indische Gesellschaft zurückzugeben und sich an der ,Entwicklung' Indiens zu beteiligen. Diese Vorstellung wurde dadurch bestärkt, dass den jungen, gut ausgebildeten Inder*innen in staatlichen Diskursen genau diese Rolle in der Zukunft Indiens zugeschrieben und damit gewissermaßen erwartet wurde, dass sie ihren Beitrag für die Nation leisten würden. Die jungen Menschen gingen unterschiedlich mit diesen Ansprüchen um und grenzten sich teilweise - wie beispielsweise Ajit bewusst von ihnen ab. Nichtsdestotrotz bildete die Rückkehr auch in seinem Fall als Idee einen wichtigen Faktor in seiner biographischen Navigation, wenn auch nur als Koordinate, die er umschiffen wollte.

Für Gilon und Tanvir wurde die Rückkehr bereits kurz nach dem Abschluss ihres $\mathrm{PhD}$ zur Realität. Für Gilon war sie ein Schritt, den er schon seit seiner Abreise aus Indien eingeplant hatte. Tanvirs Einstellung zu einer Rückkehr hatte sich während seiner Zeit in Göttingen mehrfach geändert und wurde von Aussichten auf eine Anstellung und auf das Finden einer Partnerin beeinflusst. Vor allem in seinem Fall wurde deutlich, dass die indischen Wissenschaftler*innen das kulturelle Kapital, das sie in Deutschland in Form von Titeln und Arbeitserfahrungen gesammelt hatten, bei ihrer Rückkehr nicht immer umsetzen konnten. Auf die Anwendung des kulturellen Kapitals hatten die Strukturen des wissenschaftlichen Bereichs, Gender (vgl. Duberley und Cohen 2010) und die Herkunft (vgl. Ajay Bailey und Mulder 2017) einen Einfluss.

Die meisten meiner Gesprächspartner*innen hatten eine ambivalente Einstellung zu einer Rückkehr nach Indien. Auf der einen Seite verspürten sie ein „homing desire“ (Brah 1996: 197), einen Wunsch, sich zuhause, zugehörig und aufgehoben zu 
fühlen. Auf der anderen Seite waren sie sich bewusst, dass eine Rückkehr bedeuten würde, sich wieder an die soziale Welt Indiens anpassen zu müssen (Sondhi 2013: 196). Vor allem für jene der jungen Wissenschaftler*innen, die sich wie beispielsweise Ajay in Deutschland wohl fühlten und positiv verorteten, war eine weitere, meistens befristete Anstellung in Deutschland ein guter Grund, die Entscheidung über eine (permanente) Rückkehr hinauszuzögern. Andere fanden, wie ich im Folgenden darlegen werde, eine Alternative zu einer Rückkehr nach Indien, die aus ihrer Sicht „the best of two worlds“ (Sadhvi, 22.09.2011) zusammenbringen würde - ein Leben in den USA.

\subsection{Nächste Stationen und weitere Qualifikationen}

Viele der jungen Wissenschaftler*innen wollten (noch) nicht sofort nach ihrer Promotion nach Indien zurückkehren. Sie argumentierten, dass sie weitere akademische Qualifikationen bräuchten, bevor sie auf dem Arbeitsmarkt in Indien eine Chance hätten bzw. den Arbeitsplatz in Indien ergattern könnten, der ihren Qualifikationen entspreche.

\subsubsection{Nächste Station: USA}

Von meinen Gesprächspartner*innen lebten zum Zeitpunkt des Verfassens dieser Arbeit fünf von vierunddreißig in den USA: Ravi und Nalika, Lali und ihr Mann, Kiran, sowie Kundan und seine Partnerin. Alle hatten Anstellungen als Postdoktorand ${ }^{*}$ innen, teilweise an sehr prestigeträchtigen Institutionen.

Ravi und Nalika, die 2011 ihre Promotion in Göttingen abgeschlossen hatten, entschieden sich für die USA, weil sie sich dessen bewusst waren, welche Bedeutung Abschlüssen US-amerikanischer Universitäten in Indien zugeschrieben wird. Ravi erklärte mir in einem Gespräch Anfang 2012: „If I had to go back to India and start a research group, then I should have completed three years of postdoctoral studies after $\mathrm{PhD}$ studies." Ravi betonte, dass es in Indien in der Wissenschaft mittlerweile gute Möglichkeiten für ihn und Nalika gebe und zählte auf, dass bereits vier oder fünf seiner Freunde dieses Ziel erreicht hätten. Allerdings erklärte er, dass Nalika und er zunächst als Postdoktoranden im Ausland arbeiten müssten. Erst dann hätten sie das notwendige „Gewicht" für ihre Lebensläufe, um selbst Forschungsgruppen leiten zu können. Der konkrete Ort schien dabei weniger wichtig zu sein als das Prädikat „USA“. Ravi argumentierte weiter: „We have stayed in Germany for too long." Es sei nicht so, dass sie Deutschland nicht mögen würden, es sei aber gut für die beiden, weiterzuziehen (to move on) (Ravi, 15.09.2011).

In Kapitel 3 „Das Regime der akademischen Mobilität“ habe ich beschrieben, dass eine wachsende globale Differenzierung des Hochschulsystems existiert, die dazu führt, dass Abschlüssen spezifischer Universitäten ein größerer Wert zugeschrieben wird (vgl. Findlay, King und Smith 2012). In Ravis Aussage wird deutlich, dass 
in Indien vor allem Abschlüsse aus den USA wertgeschätzt werden und dass er und Nalika sich dieser Wertschätzung sehr bewusst waren. Sie leben seit Anfang 2012 in den USA und haben 2018 ein Kind bekommen. Aus den drei Jahren als Postdoktoranden sind also mittlerweile fast sieben geworden und der richtige Zeitpunkt für die Rückkehr scheint noch nicht gekommen zu sein.

Sadhvi erklärte mir in unserem Interview kurz vor ihrer Abreise, dass die USA für sie das Beste von „beiden Welten“ hätten: „You get the kind of developed nation and all the advantages that comes with this. But then there is also such a huge Indian community, you don't feel like you are so far away from home. (...) You don't feel out of place so much." In Deutschland sei es ein bisschen schwerer, sich zu „integrieren“. Diese Faktoren würden eine wichtige Rolle bei der Entscheidung für eine Rückkehr spielen und im Fall von Deutschland eine solche wahrscheinlicher machen. Sadhvi betonte, für sie und Sachin werde der Übergang von Deutschland in die USA gar nicht so einfach werden: „Because in the last four years we are so used to the European way of life." Die europäische Art zu leben sei etwas entspannter, was Sadhvi und Sachin sehr schätzen würden: „We just think about going to the US and being in this - like crazy all the time - being in a hurry and this kind of lifestyle that ... we know we're not gonna like that part so much" (Sadhvi, 22.09.2011).

Sadhvi verwies hier auf zeitliche Erfahrungen, die viele meiner Gesprächspartner*innen in Göttingen machten und mit den Worten langsam, entspannt und ruhig beschrieben. Wie ich in Kapitel 4 ausführlich dargelegt habe, stand diese Erfahrung von Zeit mit der Zeitkarte der Work-Life-Balance in Verbindung, die nicht nur ein anderes Tempo, sondern außerdem mehr Kontrolle über die Zuteilung der eigenen Zeit mit sich brachte. In den USA, so stellte sich Sadhvi vor, würde sie sich nicht so fremd fühlen, hätte aber weniger Kontrolle über ihre Zeit.

\subsubsection{Das Weiterentwickeln der Karriere}

Viele meiner Gesprächspartner*innen wollten weiter an ihrer wissenschaftlichen Laufbahn arbeiten, bevor sie nach Indien zurückkehrten. Ajit erläuterte mir lachend: "I'd like to go to India, but not now because it's too early. I don't know - maybe in some 100 years or something" (Ajit, 14.07.2015). Obwohl Ajit in seiner Zeitangabe übertrieb, drückte er eine Ansicht aus, die viele der jungen Wissenschaftler*innen teilten, nämlich, dass sie noch nicht an dem Punkt in ihrer Karriere angekommen seien, an dem eine Rückkehr den erhofften Erfolg bringen würde.

Die indischen Wissenschaftler*innen versuchten, ähnlich wie beim Heiraten, das richtige Timing für ihre Rückkehr herzustellen. Die Vorstellungen vom idealen Zeitpunkt für eine Rückkehr wurden in den meisten Fällen von beruflichen Überlegungen beeinflusst, wie in Ajits Aussage deutlich wird: „I intended to stay in biology. In India we have very good computer and electronic engineering, medicine - but not life science like natural sciences." Wenn er sich entschiede, nach Indien zurückzukehren, würde er für irgendeine Firma arbeiten müssen, was er aber nicht wolle. Außerdem sei das Wissenschaftssystem in Deutschland sehr gut: „We have very 
much freedom as a science student, and enough funding for running the projects in Germany." Allerdings könne man nie wissen, wie die Lage in zehn Jahren aussehen werde, und so mutmaßte Ajit lachend: „Maybe then everyone has to move to China or India because half of the world is bankrupt - possible right?" (Ajit, 14.07.2015).

Kundan, der zum Zeitpunkt des Verfassens dieser Arbeit in den USA als Postdoktorand arbeitete, erklärte: „When you start your career, it is difficult in India to develop. “ Dort könne er nicht so unabhängig arbeiten. „If you want to write a grant, you cannot write it yourself. You need to show [it] to your super[visor], then he will ask to put his name in that" (Kundan, 14.08.2013). Er räumte ein, dass es mittlerweile einige wenige Institute gebe, an denen das Klima anders sei, aber die meisten würden immer noch so funktionieren. Kundan sah in der Kontrolle, die Politiker über die Universitäten ausüben würden, ein großes Problem: „If you want to become an assistant professor, you pay them 50 lakhs or something, then you get [the position]. It doesn't matter how many papers you have." Kundan erklärte, dass die Voraussetzungen für eine gute Position in Indien weniger Qualifikationen und Publikationen seien als Verbindungen zu den richtigen Leuten und Geld. Die richtigen Verbindungen hatte Kundan allerdings nicht vorzuweisen. Er schloss daraus: „I don't want to go there when I'm developing; it will be a bit more struggle there“ (Kundan, 14.08.2013).

Ähnlich wie im Falle Gilons wird deutlich, dass die jungen Wissenschaftler*innen das kulturelle Kapital, das sie sich an den Instituten in Göttingen erarbeiteten, nicht unbedingt in Indien umsetzen konnten. Kundan und andere meiner Gesprächspartner*innen schienen sich dieses Problems durchaus bewusst zu sein. Die Antwort darauf war für viele, sich noch weiter zu qualifizieren und das richtige Timing für die Rückkehr herzustellen. Obwohl die meisten die Hoffnung hatten, dass diese Strategie aufgehen würde, sind die Aussagen durch einen „Zwang zur Aspiration“ (Gilbertson 2017) gekennzeichnet. Für meine Gesprächspartner*innen schien es keine Alternative dazu zu geben, sich weiter zu qualifizieren, weil ihnen das nötige soziale, kulturelle und wirtschaftliche Kapital fehlte, um ihre Ziele auf einem anderen Weg zu erreichen.

\subsection{3 „I need to do few more Postdocs“}

Rohini erläuterte mir, dass es in Indien zwei Arten von Universität gebe: auf der einen Seite private, die laut Rohini praktisch „money vending machines“ seien und an denen Wissenschaftler*innen nie ,job satisfaction“ erreichen würden. Auf der anderen Seite gebe es - und hier spielte sie auf die Indian Institutes of Technology (IITs) an - die großen Institute, die von der Regierung betrieben und finanziert würden, „and if you are good they will take you." Rohini erklärte, wenn sie ehrlich sein solle, kämen für sie nur die government institutes infrage, denn dort könne sie „Arbeitszufriedenheit“ erreichen. Für eine Stelle an einem dieser Institute müsse sie allerdings noch einige „Postdocs“ absolvieren. Nach der Fertigstellung ihrer Promotion nahm Rohini eine Stelle als Postdoktorandin in Göttingen an. Auf dieser 
beendete sie die Forschung, die sie während ihrer Promotion durchgeführt hatte. Sie wollte die Stelle außerdem dazu nutzen, sich auf andere Positionen zu bewerben: „I will again do the same process which I did four years back: start looking for different places and different things." In dieser Bewerbungsrunde werde sie auch die USA erwägen, da sie das Gefühl habe, dass die Sprache in Deutschland irgendwann ein Problem werden würde: „I mean, I can learn still - it's not a problem - but I will have a disadvantage of let's say a German Professor and me. So there will be a bit of a difference - it's a silent way but it is there." Tatsächlich zeigen Studien, dass die Sprache als „,inkorporiertes Kulturkapital“ (Bourdieu 1983: 187), in Interaktion mit anderen Faktoren wie Nationalität und Gender, einen großen Einfluss auf die berufliche Entwicklung hat (vgl. Grigoleit-Richter 2017).

Bhanu, der zum Zeitpunkt unseres Interviews im Oktober 2011 bereits seit zehn Jahren in Deutschland gelebt hatte, war sehr optimistisch, dass er als Chemieingenieur bei einer Rückkehr nach Indien sofort eine Stelle finden würde: „I'm sure that if I go tomorrow to India I will get a job “. Trotzdem entschied er sich gemeinsam mit seiner Frau dazu, eine Stelle in Österreich anzunehmen. Bhanu begründete dies mit dem Wunsch, vor einer Rückkehr seine Expertise in unterschiedlichen Arbeitsfeldern zu erhöhen. In Österreich würde er in einem neuen Bereich arbeiten. „That means I would like to know everything - not completely - but little bit. So, which might increase the chances for me to succeed in the career if I go back to India“" (Bhanu, Oktober 2011).

Ähnlich wie Bhanu erklärte Pravin, er müsse noch einige Voraussetzungen schaffen, bevor er sich auf eine Stelle in Indien bewerben könne. Pravin erläuterte mir: "Given the chance, I want to go back. I'm here to fulfil that requirement" (Pravin 10.07.2011). Er meinte, es sei ganz natürlich, dass er zurückgehen wolle, er habe einige soziale Verpflichtungen: „I have to go and serve my people, something like that." Einen Arbeitsplatz außerhalb Indiens würde er nur ,gezwungenermaßen“ annehmen und nicht, weil es seine Wahl wäre: „Because if I don't get any job in India - good job I mean - which can suit my qualification and my interest, then I may take up some job in Europe or US. But it is definitely not by choice. I will, given the chance, I want to go back" (Pravin 10.07.2011). Pravins passiv gehaltener Ausdruck suggeriert, dass er das Gefühl hatte, keine Kontrolle über seine Chancen auf einen guten Arbeitsplatz in Indien zu haben.

Pravin wechselte nach dem Ende seiner Stelle als Postdoktorand in Göttingen an ein Institut in Israel. Nach etwa einem Jahr ging er für eine weitere Anstellung als Postdoktorand nach Kanada. In einer E-Mail-Korrespondenz im März 2013 erläuterte er mir, dass er mindestens drei Jahre Erfahrung als Postdoktorand sammeln müsse, bevor er sich auf eine gute Stelle in Indien bewerben könne. 2019, neun Jahre nach dem Antritt seiner ersten Anstellung als Postdoktorand in Deutschland, hatte er in Indien scheinbar die Stelle gefunden, die seinen Qualifikationen entsprach: eine Stelle als assistant professor an einer Privatuniversität im Norden Indiens.

Ravi, Nandita, Ajit, Kundan, Rohini, Bhanu und Pravin waren sich zum Zeitpunkt der Interviews einig darin, dass sie noch nicht da angekommen waren, wo 
sie sein wollten: Sie waren noch nicht Forschungsgruppenleiter*innen, hatten noch nicht die Stelle an einem Indian Institute of Technology mit der erhofften job satisfaction, waren noch nicht assistant professor. Diese Arbeitsplätze befanden sich irgendwo am Horizont, in mehr oder weniger konkreten Umrissen. Allerdings war ihnen bewusst, welchen Weg sie bis dorthin einschlagen mussten, wie sie den normativen Karrierepfad weiter ablaufen mussten, um am Ende dort anzukommen. Dazu gehörten weitere Qualifikationen als Postdoktorand*innen im Ausland. Die Wünsche, die die jungen Wissenschaftler*innen für ihre berufliche Zukunft hegten, zeigt, dass eigentlich nur eine Anstellung in der Wissenschaft selbst als Erfolg verstanden wurde (vgl. Thouaille 2018; Richter 2016).

Die Aussagen meiner Gesprächspartner*innen waren in vielen Fällen von dem Optimismus gekennzeichnet, dass bei der richtigen Leistung und Qualität der eigenen Arbeit die Aspirationen auf den guten Arbeitsplatz in Erfüllung gehen würden. Sie verorteten die Verantwortung für ihre erfolgreiche Zukunft - beruflich und privat - bei sich selbst (vgl. Radhakrishnan 2011: 95). Damit stehen sie im Kontrast zu den Wissenschaftler*innen in Caroline Richters Studie, deren Verwendung des Topos „Glück“ in Bezug auf ihre Karrieren wenig Einflussmöglichkeiten und damit zusammenhängend wenig Verantwortung ausdrückt (Richter 2016: 56). Die Autorin versteht die Verwendung der Glückssemantik unter anderem als Strategie, um mit der Unsicherheit und schweren Planbarkeit von akademischen Karrieren umzugehen (ebd.: 58). Die jungen indischen Wissenschaftler*innen schienen dagegen die weitere Qualifizierung als Strategie anzuwenden, um die Unvorhersehbarkeit zu kompensieren. Die Narrationen von ihrer bisherigen und zukünftigen beruflichen Laufbahn rahmten sie in der Sprache der individuellen Leistung, wie Rohini erklärte: „Wenn du gut bist, wirst du genommen." Meiner Ansicht nach ist diese Rahmung auf den für Indien spezifischen Diskurs um "merit“ im Bildungsbereich (Ajantha Subramanian 2015; Jayasree Subramanian 2016) und in der Wissensökonomie (Radhakrishnan 2011: 88-89) zurückzuführen. Der merit-Diskurs, der Gedanke, dass jeder Mensch durch Talent und Leistung den Aufstieg, beruflich und gesellschaftlich, schaffen kann (Radhakrishnan 2011: 89-90), verschleiert den Einfluss von Faktoren wie Gender und Kaste auf die Möglichkeiten des Einzelnen. Für die jungen Wissenschaftler*innen in meiner Studie war der Fokus auf die individuelle Leistung eine Möglichkeit, in der Gegenwart handlungsfähig zu bleiben und weiter an der Umsetzung ihrer Aspirationen zu arbeiten, denn Glück kann nicht beeinflusst werden, die eigene Leistung jedoch schon.

Die Strategie der ständigen Weiterqualifizierung war mit der Hoffnung bzw. dem "grausamen Optimismus" (Berlant 2011) verbunden, dass hinter der nächsten Koordinate auf dem Karrierepfad die sichere Stelle Realität werden würde. Dadurch, dass die meisten Postdoktorandenstellen befristet sind, mussten sich die jungen indischen Wissenschaftler*innen in regelmäßigen Abständen wieder Gedanken über die nächsten Schritte machen. So entstand ein Rhythmus aus Hoffnung, Optimismus und Resignation. Dabei lag letztendlich die Macht bei den Institutionen wie der Universität und den Forschungseinrichtungen, „da sie die Zeit und die Geschwindigkeit 
beherrschen, mit denen die Erwartungen in Erfüllung gehen (...)“ (Bourdieu [1997] 2017: 294).

An dieser Stelle möchte ich betonen, dass eine Rückkehr nach Indien von den jungen Wissenschaftler*innen je nach Zeitpunkt unterschiedlich stark ersehnt wurde bzw. mit unterschiedlichen antizipierten Problematiken verbunden war: Für diejenigen, die nicht verheiratet waren, mit (noch) größerem Druck zu heiraten, für Ehepaare mit dem gesellschaftlichen Druck, Kinder zu bekommen. Eine weitere Stelle abroad anzunehmen und sich dabei noch weiter zu qualifizieren, war eine gute, gesellschaftlich sanktionierte Strategie, die Rückkehr hinauszuzögern - zumal, wenn die jungen Wissenschaftler*innen feststellten, dass sie auf dem Weg bereits bei ihrem Ziel angekommen waren. So schien es in Jyotis Fall zu sein, die auf Facebook zu dem Foto einer Straße in ihrer neuen Heimat in den USA schrieb: „The beautiful thing about the most beautiful roads is that the destination is forgotten and the journey itself becomes the destination" (Facebookeintrag, 24.10.2016).

\subsection{Familiengründung}

Die jungen Wissenschaftler*innen, die bereits verheiratet waren, wurden bald nach einer Eheschließung mit der nächsten Koordinate normativer Lebensverlaufsvorstellungen konfrontiert: dem Gründen einer eigenen Familie. Ähnlich wie die Vorstellungen vom Heiratsalter gibt es für das Bekommen von Kindern die ,richtige' Zeit: nicht zu bald nach der Eheschließung, aber nicht zu lang danach, so dass die Fruchtbarkeit der Ehepartner nicht infrage gestellt werden kann (Donner 2008: 91).

Nabhi, die mittlerweile Postdoktorandin war und mit ihrem Mann gemeinsam in Göttingen lebte, wurde, wie ich in Kapitel 7 dargelegt habe, bereits wegen des Heiratens von ihrer Familie unter großen Druck gesetzt. Etwa drei Jahre nach ihrer Heirat spürte sie erneut die Belastung familiärer Erwartungen. Bei einem Treffen im Oktober 2018 erzählte sie mir, dass ihre Mutter ihr zu Beginn des Jahres erklärt habe, dass es doch nun langsam Zeit für ein Kind sei.

Ähnlich wie bei der Heirat waren es die Familien und die Gleichaltrigen, die meine Gesprächspartner*innen mit diesem Thema konfrontierten. Dies wurde auf Facebook und bei den kollektiven Aktivitäten der jungen Inder*innen in Göttingen in zunehmendem Maße sichtbar. Auf Facebook erschienen in den Chroniken im Abstand von zwei, drei Jahren nach dem Hochzeitsfoto Bilder von Babys. Bei den Feierlichkeiten waren häufiger Kinder anwesend und es wurde aufmerksam festgestellt, welches der verheirateten Paare denn schon ein Kind hatte und welches nicht. So wurde ich beispielsweise von Mera mehrfach gefragt, ob ich denn wisse, ob ein befreundetes Paar, das mittlerweile in Österreich lebte, ein Kind bekommen habe.

Diese Praktiken artikulierten und reproduzierten zeitliche Normen der dominanten Lebensverlaufsvorstellungen und konfrontierten meine Gesprächspartner*innen mit diesen. Die jungen Menschen gingen in ihrer biographischen Navigation ganz unterschiedlich mit der Konfrontation um. Nabhi zum Beispiel stellte fest, dass die 
Kinder wie Trophäen zur Schau gestellt würden, und kritisierte dieses Verhalten (23.11.2018). Rohini wählte wie bereits beim Thema Heirat Humor als Strategie und stellte ein Foto von sich und einer Labor-Maschine auf Facebook und kommentierte dazu „my dream baby“ (11.12.2017).

\subsubsection{Karriere und/oder Kind}

Die Entscheidung für oder gegen ein Kind und die Versuche, den richtigen Zeitpunkt für eine Schwangerschaft auszuloten, wurden vor allem für die jungen Frauen, mit denen ich für meine Studie sprach, durch verschiedene Faktoren verkompliziert. Als Wissenschaftlerinnen waren sie sich bewusst, dass die Entscheidung für ein Kind einen erheblichen Einfluss auf ihre Karriere haben würde - gleichgültig, wo sie leben würden, in den USA (Winslow und Davis 2016), Deutschland (Schürmann und Sembritzki 2017) oder Indien (Gupta und Sharma 2002; Jayasree Subramanian 2016). In der Wissenschaft hat das Gründen einer Familie auf die Karriere von Frauen häufig einen negativen Einfluss, der sich bei Wissenschaftlern mit Familien nicht feststellen lässt (Duberley und Cohen 2010). Dieser Umstand erklärt sich aus genderspezifischen Rollenerwartungen (Schürmann und Sembritzki 2017) und der veränderten Wahrnehmung von Frauen nach der Geburt von Kindern. Wissenschaftlerinnen mit Kindern werden als weniger karriereorientiert und engagiert gesehen (Duberley und Cohen 2010: 195; Jayasree Subramanian 2016). Frauen machen sich aus diesem Grund viel häufiger als ihre männlichen Kollegen Gedanken über einen Karriere- oder Berufsausstieg, der durch die Familiengründung ausgelöst werden könnte (Schürmann und Sembritzki 2017: 39).

Dies wurde auch in meiner Studie deutlich. Mit zunehmendem Alter und verändertem Familienstand wurde das Thema Kinder und Karriere auf Facebook und in den Gesprächen immer relevanter. Als zweifache Mutter wurde ich dazu befragt, wie ich Arbeit und Familienleben organisieren würde und welche Regelungen es in Deutschland zu Mutterschutz und Elternzeit gebe. Anisha, die mit ihrem Mann in Süddeutschland lebte und 2017 ein Kind bekommen hatte, teilte im Juni 2018 auf Facebook den Artikel einer Wissenschaftlerin mit dem Titel „My career seemed to hit a wall. Now I see it was discrimination" ${ }^{147}$. In dem Artikel beschreibt eine amerikanische Wissenschaftlerin, wie ihre Karriere blockiert wurde und ihr nach und nach klar wurde, dass dies damit zusammenhing, dass sie Mutter war.

Wie Winslow und Davis (2016: 413) andeuten, streben Männer vor allem in der Wissenschaft in zunehmendem Maße eine gleichberechtigte Partnerschaft an und stehen dann mitunter vor dem gleichen Dilemma wie die Frauen: der Unvereinbarkeit von Wissenschaft und Familie. Ein weiterer Faktor, der sowohl die jungen Männer als auch die jungen Frauen betraf, war die schwierige Planbarkeit der Zukunft,

\footnotetext{
147 Siehe Sharon Ramos Goyette: „My career seemed to hit a wall. Now I see that it was discrimination“, Science vom 14.06.2018, http://www.sciencemag.org/careers/2018/06/my-career-seemed-hitwall-now-i-see-it-was-discrimination.
} 
die mit den häufig befristeten Stellen in der Wissenschaft einhergeht (Schürmann und Sembritzki 2017: 39). Gerade wenn beide in der Wissenschaft tätig sind, stellt sich die Frage, wann eigentlich ein guter Zeitpunkt für die Gründung einer Familie sein soll. Wie Wolfinger, Mason und Goulden feststellen: „More than most vocations, academia does not really offer any good time to have children" (2009: 1613). So sehr dieser Umstand alle Personen, die in der Wissenschaft tätig sind, betrifft, stellte er meine Gesprächspartner*innen vor eine besondere Herausforderung, da das Gründen einer Familie, wie bereits angesprochen, eine wichtige Koordinate normativer Lebensverlaufsvorstellungen der indischen Mittelschicht ist (Thapan 2009).

Women's roles as wives and mothers are valorized in India across religious and caste communities, as the ideal girl becomes the ideal woman through marriage and childbearing. Women are expected to maintain the family, their own respectability, and cultural traditions by concentrating on the domestic sphere. (Fleming 2016: 7)

Obwohl sich die gesellschaftlichen Normen ändern (Fleming 2016; Radhakrishnan 2011) und die jungen Frauen in meiner Studie durch ihre universitäre Ausbildung, ihre Arbeit und Mobilität einen größeren Spielraum in der Aushandlung der Normen hatten, mussten diese von den jungen Wissenschaftler*innen navigiert werden (vgl. Radhakrishnan 2011: 156-57). Wie ich in Kapitel 2 dargelegt habe, wird die Priorisierung der Familie von vielen Menschen in Indien als Kern indischer Werte verstanden und den Frauen die Verantwortung für die Aufrechterhaltung dieser zugeschrieben (Radhakrishnan 2011; Fleming 2016). Durch das „Ausbalancieren“ von Karriere und Familie (Radhakrishnan 2011), den eigenen Wünschen und den Erwartungen der Gesellschaft sollen sie die gute indische Familie aufrechterhalten, davor schützen, zu westlich zu werden, und die indische Kultur bewahren (Belliappa 2013; Fleming 2016: 4). Die Entscheidungen der Frauen bezüglich der Gründung einer Familie und der Rolle, die sie in dieser als Mutter einnehmen wollen, werden demzufolge mit der Verortung als, indisch` bzw. im Gegensatz dazu als, westlich assoziiert.

\subsubsection{Familiengründung abroad}

Meine Gesprächspartner*innen, die bereits Kinder bekommen haben - Ravi und Nalika, Ajay und seine Frau, Bhanu und Rukmi, Anisha und ihr Mann - lebten zum Zeitpunkt des Verfassens der vorliegenden Arbeit außerhalb Indiens. Dies hat unterschiedliche Ursachen. Zum einen hatten die jungen Wissenschaftler*innen zum Zeitpunkt der Schwangerschaft alle bzw. zumindest einer der Ehepartner eine Anstellung und so zumindest für einige Zeit noch eine berufliche Perspektive. Zum anderen konnte die Distanz zur sozialen Welt in Indien meiner Ansicht nach gerade aus der Perspektive der jungen Frauen den Druck mindern, eine spezifische Art von Mutterschaft, die mit der Verortung in der indischen Mittelschicht verbunden wird, 
zu artikulieren. Eine Rückkehr nach Indien würde für die jungen Frauen bedeuten, sich in ihren beruflichen Ambitionen (noch) stärker einschränken zu müssen (vgl. Radhakrishnan 2011). In Deutschland oder auch den USA hatten sie stärker die Möglichkeit, gemeinsam mit dem Ehepartner auszuloten, wie sie ihr Familienleben gestalten wollten. Die Mobilität verhalf hier durch die Distanz zur sozialen Welt und eine veränderte Positionierung in dieser erneut zu einer größeren Kontrolle über die Ausgestaltung des Lebens.

Das Gründen einer eigenen Familie, weit weg von den Eltern, brachte allerdings andere Herausforderungen mit sich. Die Paare mussten sich alleine, ohne familiäre und vor allem großmütterliche Unterstützung, wie es in Indien häufig üblich ist, um die Kinderbetreuung und die Erziehung kümmern. Obwohl ihnen dafür beispielsweise in Deutschland viel eher als in Indien staatliche Kinderbetreuung in Form von Krippen oder Kindergärten zur Verfügung stand, vermissten einige den Beistand, den eine in der Nähe lebende Familie bedeuten konnte.

Die jungen Frauen und Männer mussten in ihrer biographischen Navigation zwei zuweilen in Konflikt stehende Zeitkarten aushandeln: die des akademischen Karrierepfades und die der normativen Lebensverlaufsvorstellungen der indischen Mittelschicht. Vor allem die jungen Frauen wurden durch die zwei unterschiedlichen Arten und Weisen, den Pfad in die Zukunft zu gestalten, vor große Herausforderungen gestellt. Hinzu kam, dass sie für den Umgang mit diesen Dilemmata kaum Vorbilder hatten, da sie zu einer der ersten Generationen indischer Frauen gehörten, für die eine Karriere ein wichtiger Teil ihres Selbstverständnisses war (vgl. Fleming 2016). Das Terrain, das es zu navigieren galt, war noch kaum begangen und kartographiert, und so mussten sich die jungen Frauen einen eigenen Weg bahnen.

\subsection{Zusammenfassung}

In ihrer biographischen Navigation kamen die jungen indischen Wissenschaftler*innen immer wieder an Koordinaten, an denen sie sich über ihre nächsten Schritte Gedanken machen mussten. In diesem Prozess gaben dominante Vorstellungen vom Lebensverlauf und vom Karrierepfad eine wichtige Orientierung, stellten meine Gesprächspartner*innen allerdings vor die schwierige Aufgabe, diese mitunter in Konflikt stehenden Zeitkarten auszuhandeln. Die Mobilität war ein wichtiges Mittel, um die Koordinaten der einen oder der anderen Zeitkarte zu umschiffen oder die Ankunft an diesen hinauszuzögern und damit die Kombination zu vereinfachen.

Meine Gesprächspartner*innen wollten durch ihre internationale Mobilität ihre Zukunft gestalten. Ein wichtiger Aspekt war die Hoffnung, durch den Abschluss aus dem Ausland die Chancen auf dem Arbeitsmarkt (in Indien) zu vergrößern. Die Umsetzung des kulturellen Kapitals in eine gute Stelle war allerdings nicht in allen Fällen gewährleistet, und einige antizipierten unterschiedliche Schwierigkeiten auf dem Arbeitsmarkt in Indien. Aus diesem Grund qualifizierten sie sich mittels Anstellungen als Postdoktorand*innen z.B. in Deutschland oder den USA weiter 
und hofften darauf, dadurch eine Zukunft mit einer erfüllenden und sicheren Stelle herstellen zu können. Andere waren schon angekommen, vielleicht nicht unbedingt an dem Ankunftspunkt, den sie sich vor ihrer Mobilität vorgestellt hatten, aber an einem neuen Ziel, das sie auf dem Weg gefunden hatten. 


\section{Schlussbetrachtung}

Im Fokus dieser Arbeit stand die Frage, wie junge Wissenschaftler*innen aus Indien ihre multiplen Zugehörigkeiten im Prozess der Mobilität aushandelten und dabei die Zeitlandschaften und dominanten Zeitkarten ihrer sozialen Umwelt navigierten. In der vorliegenden Arbeit habe ich gezeigt, dass meine Forschungspartner*innen durch die Mobilität als Studierende größere Kontrolle über die Gestaltung ihrer Lebens- und Alltagszeit erlangten.

In meiner Studie habe ich das Konzept der biographischen Navigation als theoretische Perspektive auf die Aushandlungsprozesse zwischen Selbst und sozialer Umwelt angewandt. Mit Hilfe dieses Ansatzes war ich in der Lage, die Erfahrungen und Aspirationen meiner Forschungspartner*innen in größere gesellschaftliche Kontexte einzubetten und ihre Praktiken als Ergebnis ständiger Interaktionen zwischen ihren Vorstellungen und Wünschen sowie denen ihrer sozialen Umwelt zu analysieren. Die Konzepte der Zeitkarte und der Zeitlandschaft waren wichtige Hilfsmittel, um den Fokus der Untersuchung auf jene Faktoren zu lenken, die Erfahrungen von Zeit und die Möglichkeiten und Praktiken der Zeitgestaltung formen und vermitteln.

Wichtige Aspekte der sozialen Umwelt meiner Gesprächspartner*innen bildeten jene Akteure, Strukturen und Diskurse, die ich unter Anwendung des Konzepts des Regimes der akademischen Mobilität untersucht habe. Im ersten Abschnitt des Schlusskapitels gehe ich auf die Ergebnisse meiner Analyse des Handelns der Wissenschaftler*innen im Kontext dieses spezifischen Mobilitätsregimes ein. Danach fasse 
ich die drei Aspekte der biographischen Navigation zusammen, die sich aus meinem analytischen Fokus auf Mobilität und Zeitgestaltung ergeben haben: die Aushandlung normativer Zeitkarten, der Einfluss von Mobilität auf die Zeitsouveränität und Zeitgestaltung sowie die Aushandlung des Selbst in und durch die Zeit.

\subsection{Mobile Studierende und Wissenschaftler*innen: Mehr als ,Talente' und ,Köpfe}

Die internationale Mobilität Studierender nimmt stetig zu und ist zu einem wichtigen Forschungsgegenstand in sozialwissenschaftlichen Migrationsstudien geworden (z.B. Baas 2010; Brooks und Waters 2011; Findlay, King und Smith 2012; Robertson 2013; Raghuram 2013; Collins 2017). Ich habe in der vorliegenden Arbeit, ausgehend von Glick Schillers und Salazars Ausführungen (2013), das Konzept des Regimes der akademischen Mobilität entwickelt, um die vielfältigen Akteure, die an der Mobilität Studierender beteiligt sind, in einen analytischen Rahmen zu fassen. In Kapitel 3 habe ich ausgeführt, welche Interessen verschiedene Akteure an der Mobilität Studierender haben und wie sie diskursiv und strukturell versuchen, die Mobilität dieser zu unterstützen. Ich habe gezeigt, dass auf der Basis von ,Talent eine spezifische Kategorie mobiler Menschen geschaffen wird, die als wertvolle ,Humanressource verstanden und im Zusammenhang mit ihrer Jugend als „desirable subject" (Collins und Shubin 2017: 23) konstruiert werden. Im beschriebenen Mobilitätsregime wird die Mobilität von Studierenden und Wissenschaftler*innen als positiv für alle Beteiligten, die mobilen Menschen selbst, deren Herkunftskontext sowie das Zielland, dargestellt.

Ausgehend von der Analyse der Diskurse, die im Regime der akademischen Mobilität dominierten, habe ich argumentiert, dass die jungen Menschen, die im Zentrum meiner Studie standen, auf ihren wahrgenommenen Nutzen reduziert werden. Sie werden entmenschlicht (Esses, Medianu und Lawson 2013) im Sinne einer Darstellung als überrational und als losgelöste akademische Unternehmer*innen (Leemann 2010). Die Verortung als (angehende) Wissenschaftler*innen war wichtig für das Selbstverständnis und die Aspirationen der jungen Menschen (Kapitel 4 und 8). Allerdings interagierte diese Verortung mit weiteren Positionierungen meiner Gesprächspartner*innen, beispielsweise als junge Frauen und Männer aus Indien. Aus der Interaktion entstanden unterschiedliche Erfahrungen - von Gefühlen der Anerkennung als ,Talent' bis zu Erlebnissen von Exklusion.

Indem ich mich auf die multiplen Verortungen der jungen Wissenschaftler*innen und deren Aushandlungen konzentriert habe, trage ich mit meiner Arbeit zu einer menschlicheren Repräsentation internationaler Studierender und Wissenschaftler*innen bei. Ein wichtiger Faktor in der differenzierteren Betrachtung war das Einbeziehen des Themas der Heirat in meine Analyse. Dieses Thema wurde meines Wissens bisher nur in wenigen Studien zu mobilen Studierenden und Wissenschaftler*innen 
bzw. den „highly skilled“ betrachtet (Kirk, Bal und Janssen 2017; Ajay Bailey 2017; Fong 2011; Sondhi 2013).

Durch die Anwendung des Konzeptes des Regimes der akademischen Mobilität hinterfragte ich die rein positive Darstellung der Mobilität Studierender und junger Wissenschaftler*innen. Diese Perspektive bildete die Basis für einen Fokus auf die Erfahrung meiner Gesprächspartner*innen und auf deren Umgang mit ihrer Positionierung als wertvolle ,Humanressource'. In der Entwicklung dieses Konzepts sehe ich einen wichtigen Beitrag meiner Arbeit im Bereich der Mobilitäts- und Migrationsforschung. Für zukünftige Studien bietet das Konzept die Möglichkeit, unterschiedliche Regime der akademischen Mobilität miteinander zu vergleichen und damit die Wirkung von Faktoren wie der Aufenthaltsgesetzgebung, den Umständen in Herkunfts- und Zielland sowie den lokalen Diskursen zur Migration herauszustellen. Es wäre außerdem zu prüfen, ob es möglich ist, ähnliche Mobilitätsregime für anders kategorisierte Migrant*innen, zum Beispiel Geflüchtete, zu postulieren.

\subsection{Master, PhD, Heirat - oder: Der ,richtige ${ }^{`}$ Pfad in die ,richtige' Zukunft}

In ethnologischen Studien zu Mobilität, besonders von jungen Menschen, wird diese häufig im Zusammenhang mit Aspirationen oder Wünschen für die Zukunft analysiert (u.a. Vigh 2009b; Cole 2010; Brooks und Waters 2011; Findlay, King und Smith 2012; Mapril 2014; Valentin 2014; Carling und Collins 2017). Die Mobilität soll dazu verhelfen, einer Zukunftslosigkeit im Herkunftskontext zu entgehen (vgl. Vigh 2009b; Griffiths 2013) oder eine gesellschaftlich sanktionierte Version der Zukunft herzustellen bzw. gerade diese auszumanövrieren (vgl. Collins 2017). Die mit Mobilität verbundenen Aspirationen und Wünsche einzelner Akteure werden, wie Collins (2017: 5) zeigt, durch die soziale Umwelt geformt. Mit Hilfe des Konzepts der Zeitkarte habe ich dargelegt, wie die sozialen Welten, in denen die indischen Wissenschaftler*innen verortet waren, deren Aspirationen entlang spezifischer Pfade lenkten und damit die „capacity to aspire“ (Appadurai 2004: 67) beeinflussten.

Ich habe verdeutlicht, dass Lebensverlaufsvorstellungen in der indischen Mittelschicht und Vorstellungen des Karrierepfades in der Wissenschaft ein lineares Fortschreiten durch die Zeit über konkrete Koordinaten vorsehen (Kapitel 2 und 3). Wie ich in den Kapiteln 4, 7 und 8 dargelegt habe, boten diese Zeitkarten den jungen Wissenschaftler*innen eine wichtige Orientierung, wirkten aber auch einschränkend. Sie legten einen spezifischen Pfad fest, dessen Einhaltung an soziale Verortungen gekoppelt war, ein Umstand, der besonders am Beispiel des Heiratens deutlich wurde (Kapitel 7). Das Heiraten als Koordinate dominanter Lebensverlaufsvorstellungen und die Norm des genderspezifischen Heiratsalters wurden durch Familien und Gleichaltrige reproduziert; zugleich kontrollierten sie die Einhaltung dieser Regeln. Entscheidungen, beispielsweise für eine Familie oder für eine Karriere, waren an Vorstellungen davon verknüpft, was es bedeutet, ,indisch', ,westlich' oder 
,modern' zu sein. Demzufolge navigierten die jungen Wissenschaftler*innen in ihrer Wahl - für oder gegen eine Heirat oder eines bestimmten Partners - multiple soziale Verortungen und mögliche Zukünfte.

Diese Dynamik war gegendert (vgl. Fleming 2016: 16), weil die normativen Zeitkarten des Lebensverlaufs genderspezifische Vorstellungen von der Zukunft beinhalten. Die jungen Männer sollten die Versorger ihrer eigenen Familie werden und sich gleichzeitig im Alter um die Pflege der Eltern kümmern. Die jungen Frauen sollten heiraten, Kinder bekommen und nach der Eheschließung der Familie Vorrang vor einer Karriere einräumen. Für die jungen Männer und Frauen erwuchsen aus den Erwartungen in Kombination mit Vorstellungen von der wissenschaftlichen Laufbahn unterschiedliche Dilemmata. Vor allem die jungen Frauen sahen sich immer wieder mit der Frage konfrontiert, wie sie ihre beruflichen Ambitionen mit den Ansprüchen, die an sie als ,indische' Frau aus der Mittelschicht gestellt wurden, versöhnen können würden. Die Mobilität war eine wichtige Strategie im Umgang mit dieser schwierigen Situation: Sie schuf eine gewisse Distanz zu familiären Erwartungen und bot die Möglichkeit, eine Heirat hinauszuzögern.

Der Zwang, auf eine spezifische Zukunft hoffen zu müssen (vgl. „compulsion to aspire", Gilbertson 2017), ergab sich im Fall meiner Gesprächspartner*innen außerdem daraus, dass ihre persönliche Zukunft von unterschiedlichen Akteuren mit kollektiven Zukünften in Verbindung gebracht wurde: Die Familien erhofften sich durch die Investition in die Ausbildung der jungen Generation den sozialen Aufstieg und eine sichere Zukunft für die Familienmitglieder (Kapitel 2); Diskurse zur ,Entwicklung' Indiens (Kapitel 3 und 6) und Strategien gegen den demographischen Wandel in Deutschland (Kapitel 3) positionierten die jungen Wissenschaftler*innen als wichtige Hoffnungsträger.

All diese Zeitkarten, von Vorstellungen des Lebensverlaufs einzelner Menschen bis hin zu Diskursen von der Entwicklung ganzer Nationen, sind lineare Repräsentationen von Zeit. Die Linearität der Zeitkarten schafft Horizonte der Zeitgestaltung, spezifische Erfahrungen von Zeit, aus denen wiederum Praktiken der Zeitgestaltung erwachsen.

\subsubsection{Horizonte der biographischen Navigation:}

Hoffnung, grausamer Optimismus' und ein Leben auf der Durchreise

Das Konzept des Horizonts war in dieser Studie ein Werkzeug, um die Wirkung zu erfassen, die zeitliche Koordinaten, die von Menschen in ihrer Zukunft lokalisiert werden, in der Gegenwart entfalten. Anders ausgedrückt, konnte ich mittels des Begriffes des Horizonts die Koexistenz von Gegenwart und Zukunft in der biographischen Navigation und damit deren zeitliche Komplexität greifen.

Die Koordinaten individueller und kollektiver Zeitkarten, wie beispielsweise eine Heirat oder die Rückkehr in das Herkunftsland, schaffen Horizonte, auf die sich Menschen in ihren Aspirationen und Praktiken der Zeitgestaltung ausrichten (Appadurai 2004: 81-82; Hitlin und Elder 2007). In der biographischen Navigation 
gaben die Koordinaten am Horizont eine Orientierung, trugen zur Handlungsfähigkeit in der Gegenwart bei und beeinflussten die Zuteilung von Zeit.

In Kapitel 4 habe ich gezeigt, dass sich meine Forschungspartner*innen, motiviert von der Vorstellung, ihre Promotion in Deutschland innerhalb von drei bis vier Jahren abschließen zu können, in Göttingen auf ihre Arbeit konzentrierten. Die Investition der Zeit in das Studium bzw. die Promotion war mit der Hoffnung verbunden, dass ein Abschluss in kürzerer Zeit zu besseren Chancen auf dem Arbeitsmarkt führen würde. Die Fokussierung auf den Nutzen der Gegenwart für die Zukunft basierte auf Konzeptionen von Jugend als Fundament für das weitere Leben und auf Vorstellungen von der Promotion als Grundlage für eine Karriere in der Wissenschaft.

Wie viele junge Menschen weltweit verstanden meine Gesprächspartner*innen Bildung als eine wichtige Strategie, um für sich eine (bessere) Zukunft zu schaffen (vgl. Amit und Dyck 2012). Die hoffnungsvolle Einstellung zur Zukunft und zu den eigenen Möglichkeiten ihrer Gestaltung stand mit dem Versprechen, das mit Bildung assoziiert wird (Amit und Dyck 2012: 13), in Verbindung - insbesondere mit dem Versprechen, dass die eigene harte Arbeit und Leistung sowie Talent zu Erfolg in der vorgesehenen Karriere verhelfen (Kapitel 2 und 8). Diese Strategie wurde durch die dominanten Zeitkarten forciert, in denen Bildung sowohl mit der individuellen als auch mit der nationalen ,Entwicklung verbunden wird (vgl. Radhakrishnan 2011; Hansen 2015).

Die Beziehung zwischen dem Versprechen eines guten Lebens in der Zukunft und den Entbehrungen, die dafür in der Gegenwart in Kauf genommen werden, habe ich in den Kapiteln 4 und 8 mit dem Konzept des cruel optimism von Lauren Berlant (2011) gefasst. Ich habe gezeigt, dass die Zeitkarte des akademischen Lebensverlaufs, die eine klare Abfolge von Abschlüssen vorsieht, dazu beiträgt, den ,grausamen Optimismus' aufrechtzuerhalten. Die jungen Wissenschaftler*innen aus Indien hofften mit jeder weiteren Qualifikation an der richtigen Institution und in der richtigen Zeit, ihre Chance auf das „academic good life“ (Thouaille 2018) - eine wissenschaftlich erfüllende, unbefristete Stelle mit guter Finanzierung und Freiheit in der Forschung - zu erhöhen. Viele meiner Gesprächspartner*innen, wie Rohini, Ravi und Kundan, arbeiteten noch an der Realisierung dieser Aspiration und einige, wie beispielsweise Tanvir, hatten bereits feststellen müssen, dass diese Strategie der Zukunftsgestaltung nicht immer funktioniert (Kapitel 8). Denn obwohl unterschiedliche Akteure suggerierten, dass die jungen Wissenschaftler*innen ihre eigene Zukunft, ihren eigenen Erfolg in der Hand hätten, konnten sie die Faktoren, die ihre Möglichkeiten auf dem Arbeitsmarkt bestimmten, kaum beeinflussen.

Das Bewusstsein vom mehr oder weniger nahen Ende des Aufenthaltes in Göttingen war ein wichtiger Horizont in der biographischen Navigation der jungen Wissenschaftler*innen. Vielfältige Faktoren erzeugten und konkretisierten diesen Horizont: die Aufenthaltsgesetzgebung, die die Dauer der Visa an die Dauer der angestrebten Abschlüsse und die Finanzierung durch ein Stipendium oder einen Arbeitsplatz koppelte, die Zeitkarte des akademischen Lebensverlaufs, die Erwartungen 
der Eltern an eine Rückkehr, die Wahrnehmung Deutschlands als Station auf dem Weg und nicht als permanentes Ziel (im Gegensatz zu den USA). Dieser Horizont wirkte unterschiedlich auf die biographische Navigation ein (Kapitel 5). Für manche, wie Gilon und Nalika, wirkte er wie ein Silberstreifen am Horizont, der den Umgang mit Heimweh und Erfahrungen des Nicht-Dazugehörens vereinfachte. Das Bewusstsein von der zeitlichen Begrenztheit des Aufenthalts in Göttingen beeinflusste die Alltagsgestaltung meiner Gesprächspartner*innen. Das Leben auf der „Durchreise" regte einige dazu an, die Zeit zu nutzen, um neue Erfahrungen zu machen, Menschen aus anderen Kontexten kennenzulernen. Im Gegensatz dazu führte das Wissen um den vorübergehenden Charakter des Aufenthaltes dazu, dass manche der jungen Wissenschaftler*innen nicht in ihre Gegenwart in Göttingen investierten, beispielsweise kein Deutsch lernten und sich auf ihre existierenden sozialen Beziehungen konzentrierten. Dabei war die Zeitgestaltung im Alltag immer von den Interaktionspartner*innen, beispielsweise an den Instituten oder in den Wohnheimen abhängig. Wie ich anhand des Falls von Tanvir gezeigt habe, konnte der Fokus auf den vorübergehenden Zustand der Mobilität wie eine selbsterfüllende Prophezeiung wirken: Weil er nicht in lokale soziale Beziehungen und das Lernen von Deutsch investiert hatte, konnte sich Tanvir nach dem Ende seiner Promotion nicht vorstellen, in Deutschland zu bleiben.

\subsubsection{Praktiken der Zeitgestaltung: Beschleunigen, entschleunigen, hinauszögern, ausmanövrieren}

Der lineare Charakter der dominanten Zeitkarten formte die Erfahrungen von Zeit und die Strategien der Zeitgestaltung meiner Forschungspartner*innen. Lineare Vorstellungen der ,Entwicklung' von Menschen und Nationen erschaffen Zeitdruck und die Angst davor, auf ,der Strecke zu bleiben' und zurückgelassen zu werden (vgl. Jeffrey 2010b). Wie ich in Kapitel 4 argumentiert habe, müssen die Strategien der Zeitgestaltung der jungen Wissenschaftler*innen, ihre Versuche des "future tricking“" (Ringel 2016: 24), im Kontext dieser Linearität analysiert werden. Wie der Vergleich mit einem Wettrennen verbildlicht, suggeriert die Linearität, dass das Vorankommen des Einzelnen, aber auch das von Kollektiven, beschleunigt werden kann.

Die angehenden Wissenschaftler*innen aus Indien verbanden mit ihrer Mobilität die Möglichkeit, die Zukunft auszumanövrieren (Kapitel 4). Die Entscheidung für die Promotion in Deutschland war mit dem Gedanken des, Schnell- und SchnellerSeins verbunden (vgl. Gilbertson 2017). Durch die kürzere Dauer der Promotion wollten sie rascher im Lebensverlauf vorankommen und damit die potentielle Konkurrenz auf dem Arbeitsmarkt ausstechen.

Mit Hilfe der Mobilität war es allerdings ebenso möglich, das Vorankommen in einem anderen Lebensbereich zu entschleunigen. So konnte zum Beispiel die Ankunft an einer Koordinate, die für die Mehrheit meiner Gesprächspartner*innen unvermeidbar schien - die der Heirat - hinausgezögert werden. Dass die jungen Menschen in meiner Studie später als vorgesehen heirateten, war somit in vielen 
Fällen kein zufälliges Nebenprodukt längerer Ausbildungszeiten und der schwierigen Arbeitsplatzsituation (vgl. Cole und Durham 2007; Jeffrey und Dyson 2008), sondern mittels Mobilität bewusst herbeigeführt. Besonders im Fall von Nabhi (Kapitel 7) wurde deutlich, dass die Mobilität eine Möglichkeit war, innezuhalten und einen Blick in neue Richtungen zu werfen. Nabhi konnte durch ihre Mobilität einer arrangierten Ehe zu einem Zeitpunkt entgehen, an dem sie sich noch nicht für eine Heirat bereit fühlte. Darüber hinaus eröffnete sich Nabhi mögliche Zukünfte, die sie sich zuvor in Indien nicht hatte ausmalen können.

Die zeitlichen Erfahrungen, die meine Forschungspartner*innen machten, wie viel Zeitsouveränität sie hatten und wie sie ihre Zeit gestalteten, war genderspezifisch. Die jungen Männer machten eher die Erfahrung, im Lebensverlauf festzustecken (vgl. Jeffrey 2010b) bzw. sie befürchteten, dass es ihnen so ergehen könnte. Dagegen hatten viele der Frauen das Gefühl, zu schnell heiraten und damit,erwachsen werden zu müssen sowie zu wenig Zeit für ihre beruflichen Ambitionen zu haben. Beide Erfahrungen sollten mittels Mobilität beeinflusst werden.

Die Formen von Handlungs- und Wirkvermögen, wie die des Beschleunigens, Hinauszögerns oder Änderns der Reihenfolge, wurden erst durch die analytische Linse der Zeitgestaltung und des Konzepts der Zeitkarte sicht- und analysierbar. Durch das angewandte Konzept der Zeitkarte konnte ich die Entscheidungen und Dilemmata der jungen indischen Wissenschaftler*innen kontextualisieren oder, anders ausgedrückt, die Struktur erfassen, mit der die jungen Menschen in ihrer biographischen Navigation interagierten. Mittels der Kontextualisierung habe ich gezeigt, dass meine Forschungspartner*innen ihre Lebensverläufe nicht als losgelöste Individuen (Beck 1994; Beck und Beck-Gernsheim 1994), sondern als eingebettete Akteure gestalteten (Hermann und Röttger-Rössler 2003; Radhakrishnan 2011; Fleming 2016; Lamb 2018). Die Mobilität war in diesem Prozess ein wichtiger Faktor, um mit neuen möglichen Lebenswegen und Formen des Erwachsenseins in Berührung zu kommen und diese auszuloten - die jungen Wissenschaftler*innen konnten neue Wege zum fertigen Haus finden, um Ravis Metapher aus dem Einleitungskapitel der vorliegenden Arbeit aufzugreifen.

\subsection{Mobilität und Zeitgestaltung}

Meine Gesprächspartner*innen machten in Göttingen die Erfahrung unvertrauter zeitlicher Rhythmen und Routinen und interagierten mit neuen Zeitkarten. Ich habe in dieser Arbeit das Konzept der Zeitlandschaft (Adam 1998; Loimeier 2012; Otto 2013) genutzt, um die Erfahrungen fremder Zeiten zu analysieren. In den Kapiteln 4, 5 und 7 habe ich aufgezeigt, dass meine Forschungspartner*innen in Göttingen größere Zeitsouveränität in Bezug auf die Gestaltung ihres Alltags und ihres Lebensverlaufs hatten. Der Komparativ bezieht sich an dieser Stelle auf die von den jungen Wissenschaftler*innen häufig angestellten Vergleiche mit den Erfahrungen, die sie selbst oder Freunde in Indien und anderen Kontexten gemacht hatten. Durch die 
Verknüpfung der Konzepte der Zeitlandschaft und Zeitkarte mit dem der sozialen Welt konnte ich darlegen, dass die Mobilität sowohl zu einer Distanz zu den normativen Erwartungen an die Gestaltung der Zeit als auch zu einer besseren Lage in deren Aushandlung führte. Durch die akademische Mobilität erlangten die jungen Menschen kulturelles Kapital, das eine veränderte Positionierung in ihren sozialen Welten und damit mehr Zeitsouveränität bedeuten konnte.

Die größere Kontrolle über ihre eigene Alltagszeit schrieben die jungen indischen Wissenschaftler*innen einer Zeitkarte zu, der sie in ihrem Arbeitsumfeld in Göttingen begegneten und die einige mit dem Begriff der Work-Life-Balance fassten (Kapitel 4). Diese Repräsentation von Zeit vermittelte ihnen eine Zeitgestaltung, in der bewusst arbeitsfreie Zeit vorgesehen ist und nicht erwartet wird, dass die Arbeitnehmer*innen ,immer“ arbeiten. Die Mehrheit meiner Gesprächspartner*innen hob diese Einstellung zur Arbeitszeit als einen großen Unterschied im Vergleich zu Kontexten wie Indien und den USA hervor. Wie ich allerdings in Kapitel 4 herausgestellt habe, führte die Interaktion mit dieser neuen Zeitkarte nicht unbedingt dazu, dass sie weniger arbeiteten. Sie vermittelte aber das Gefühl der Kontrolle über die eigene Zeitgestaltung.

Dass die jungen Wissenschaftler*innen durch ihre Mobilität an Zeitsouveränität gewannen, kennzeichnet sie im Vergleich zu anderen mobilen Menschen als relativ privilegiert. Viele Migrant*innen machen eher die Erfahrung des Kontrollverlusts über ihre Zeit; dies gilt besonders für jene, deren Aufenthaltsstatus nicht geklärt ist (Griffiths 2013, 2014; Cwerner 2001: 21-22). Die Art und Weise der Mobilität als Studierender, Wissenschaftler*in, geflüchtete Person etc. beeinflusst in Interaktion mit Faktoren wie Gender und der nationalen Herkunft die Zeitsouveränität mobiler Menschen.

Die Zeitlandschaft in Göttingen zeichnete sich für die indischen Wissenschaftler*innen durch einen langsameren, im Vergleich zu Indien entspannten Rhythmus aus. Sie machten die Erfahrung der Entschleunigung des Alltags, die unter anderem durch fehlende soziale Einbindung hervorgerufen wurde (Kapitel 5). Das Leben in der neuen Zeitlandschaft hatte, wie ich in den Kapiteln 5 und 6 hervorgehoben habe, in der biographischen Navigation eine ambivalente Wirkung. Neben größerer Kontrolle über die Gestaltung der eigenen Zeit und Gefühlen der Entschleunigung konnte das Leben in der neuen Zeitlandschaft zu Erfahrungen der Isolation, Langeweile und des Heimwehs führen. Die jungen Menschen wirkten auf diese Erfahrungen durch Praktiken der Zeitgestaltung ein, die Gleichzeitigkeit herstellten, vertraute Routinen in den Alltag integrierten und sie mit der vertrauten Zeitlandschaft resynchronisierten. 


\subsection{1 (Gem)einsame Zeit}

Die Beschäftigung mit den sozialen Beziehungen und Praktiken im Alltag in den Kapiteln 4 und 5 hat Brüche, Unsicherheiten und Grenzen aufgezeigt, auf die die jungen Menschen in Göttingen trafen. Besonders die Geschichten von Interaktionen mit Kolleg*innen und gescheiterten Versuchen, Freundschaften zu etablieren, führten vor Augen, dass es für meine Gesprächspartner*innen nicht einfach war, sich in einer neuen sozialen Welt zu verorten. Das Leben in Göttingen beinhaltete immer wieder Erfahrungen von Einsamkeit und Isolation. Diese Erfahrungen wurden von den Studierenden und Wissenschaftler*innen aus Indien durch unterschiedliche Praktiken beeinflusst: Sie etablierten neue Freundschaften, hielten familiäre und freundschaftliche Beziehungen aufrecht oder konzentrierten sich bewusst auf den zeitlich begrenzten Charakter ihres Aufenthaltes.

Meine Gesprächspartner*innen etablierten in Göttingen, wie ich in Kapitel 5 ausgeführt habe, Freundschaften sowie Komfortzonen und -zeiten mit Menschen, durch deren Präsenz sie sich wohl und weniger einsam fühlten. Häufig bildeten wahrgenommene Gemeinsamkeiten die Grundlage der Beziehungen: eine gemeinsame regionale Herkunft, die geteilte Erfahrung als mobile Studierende oder Wissenschaftler*innen, das Wohnen im gleichen Wohnheim, die Arbeit am gleichen Institut. Einen zentralen Faktor bildeten gemeinsame Alltagsroutinen und gleiche zeitliche Erfahrungen, wie beispielsweise jene der zeitlichen Begrenztheit (Collins 2017: 24). Für die Aufrechterhaltung der Beziehungen zu den Familien und zu ,alten' Freund*innen wurden unterschiedliche Informations- und Kommunikationstechnologien genutzt. Diese ermöglichten verschiedene Formen der Kopräsenz und die Resynchronisation mit dem Herkunftskontext. Wie ich in Kapitel 5 dargelegt habe, führte die Nutzung von Facebook und anderer Medien, besonders in Kombination mit Smartphones, zu einem Gefühl von „ambient co-presence“ (Madianou 2016: 198) - der Allgegenwärtigkeit der Anderen. Die Informations- und Kommunikationstechnologien hatten unterschiedliche Konsequenzen für die Erfahrung von Zeit, die Zeitgestaltung und die Zeitsouveränität. Gerade die Kommunikation mit den Eltern wurde von der bestehenden Beziehungsdynamik beeinflusst und so wurde auch über die Distanz hinweg die Kontrolle über die Zeitgestaltung ausgehandelt - von der Häufigkeit von Telefonaten bis hin zu den Plänen für die Zukunft.

Wichtige Zeitpunkte, zu denen die indischen Wissenschaftler*innen in Göttingen gemeinsam Zeit verbringen konnten, waren Feierlichkeiten wie Diwali und der Indian Independence Day, die durch die indische Vereinigung organisiert wurden. Diese Feierlichkeiten boten außerdem die Gelegenheit, sich mit der vertrauten Zeitlandschaft in Indien zu resynchronisieren (vgl. Cwerner 2001) und wurden wichtige zeitliche Koordinaten für die jungen Inder*innen in Göttingen.

Die Reisen nach Indien boten den jungen Wissenschaftler*innen die Möglichkeit, Zeit mit der Familie und Freund*innen zu verbringen, waren aber auch mit der Erfüllung familiärer Verpflichtungen verbunden. Wie ich in Kapitel 6 thematisiert habe, bedeutete die temporäre Rückkehr in den Herkunftskontext für meine Ge- 
sprächspartner*innen, erneut Erwartungen an ihre Zeitgestaltung - sowohl für den Aufenthalt in Indien als auch für den Lebensverlauf - aushandeln zu müssen.

\subsubsection{Routinen und Resynchronisation}

Die Mobilität führte zum Bruch mit vertrauten Alltagsroutinen und zum Bewusstwerden der eigenen alläglichen Rhythmen. Diese Situation bot die Gelegenheit, sich neu zu orientieren (vgl. Butcher 2011: 9; May 2011), führte aber auch zu Erfahrungen des Nicht-Dazugehörens (May 2011: 370). In Göttingen mussten meine Gesprächspartner*innen ihren Alltag wiederherstellen. Sie etablierten vertraute Praktiken in den Tagesablauf, wie das Kochen und Essen indischer Gerichte, religiöse Handlungen oder Freizeitaktivitäten wie Kricket. Durch die vertrauten zeitlichen Tagesabläufe konnte ein Gefühl der Sicherheit, der Kontinuität und der Verortung in Göttingen hergestellt werden. Wie ich in Kapitel 5 argumentiert habe, mussten diese Praktiken der Zeitgestaltung mit der Zeitlandschaft Göttingens und Personen vor Ort, wie beispielsweise Mitbewohner*innen, ausgehandelt werden. Die Praktiken der jungen Wissenschaftler*innen konnten dabei, wie das Beispiel von Gilon und seinem Einsatz von Knoblauch beim Kochen gezeigt hat, zu Situationen führen, die erneut Erfahrungen von Exklusion auslösten.

Eine wichtige Routine war die regelmäßige Kommunikation mit den Eltern und Freund*innen in Indien. Die Häufigkeit unterschied sich je nach Gesprächspartner*in und reichte von täglichen Anrufen bis zur Kommunikation einmal in der Woche. Mit Hilfe von Telefonaten und über Plattformen wie Facebook resynchronisierten sich die jungen Wissenschaftler*innen mit der Zeitlandschaft Indiens und blieben über Ereignisse im Leben wichtiger Bezugspersonen informiert. Allerdings wurde deutlich, dass diese Technologien auch Grenzen in Bezug auf das Herstellen von Gleichzeitigkeit haben. Besonders die synchrone Kommunikation über Telefonate oder Videochats erforderte eine zeitliche Koordination, die die Asynchronität, die unter anderem durch das Leben in unterschiedlichen Zeitzonen hervorgerufen wurde, besonders spürbar machte.

Als Ort formte Göttingen die zeitlichen Erfahrungen der jungen Wissenschaftler*innen und die Möglichkeiten, vertraute Praktiken wieder aufzunehmen. Besonders zu Beginn des Aufenthaltes und zu spezifischen Zeitpunkten machten meine Gesprächspartner*innen die Erfahrung von Asynchronität (Kapitel 5 und 6). Dieser konnten sie mit der Einrichtung eigener zeitlicher Koordinaten begegnen. Von besonderer Bedeutung waren in dieser Hinsicht die kollektiven Aktivitäten, die organisiert wurden. Das gemeinsame Begehen von Festen wie Diwali bot die Möglichkeit der Resynchronisation und der Artikulation multipler sozialer Verortungen. Auf der diachronen Ebene waren die kollektiven Aktivitäten Koordinaten, an denen sich an die persönliche und/oder kollektive Vergangenheit erinnert werden konnte und die Zukunft in den Blick genommen wurde. Besonders meine Analyse zur Feier des Indian Independence Day in Göttingen (Kapitel 6) verdeutlicht, dass die Gestaltung dieser gemeinsamen Aktivitäten allerdings nicht konfliktfrei geschah. Die 
Diversität der indischen Wissenschaftler*innen führte zu Diskussionen über die kollektive Zeitgestaltung. Die Herkunft aus unterschiedlichen Regionen Indiens löste beispielsweise Debatten über die Sprache, die bei den gemeinsamen Aktivitäten dominieren sollte, aus. Die Organisation der indischen Studierenden und besonders das gewählte Komitee der Vereinigung nahm dabei häufig eine dominante Rolle ein und wirkte als ein „Regime der Zugehörigkeit“ (Pfaff-Czarnecka 2012: 77), das die Kriterien von Exklusion und Inklusion festlegte. Die Anwesenheit bei den gemeinsamen Aktivitäten und damit die Investition der eigenen Zeit in diese waren in diesem Prozess ein wichtiger Faktor. Den indischen Wissenschaftler*innen, die sich mit der Gestaltung durch die Vereinigung nicht einverstanden zeigten oder generell die Gegenwart anderer Inder*innen vermeiden wollten, bot das Leben in der Zeitlandschaft Göttingens die Möglichkeit, den normativen zeitlichen Koordinaten auszuweichen.

Reisen nach Indien waren ein wichtiges Mittel, um sich mit der Zeitlandschaft des Herkunftskontextes zu resynchronisieren, für ein paar Wochen eine Version des „alten Lebens“ zu leben und Verpflichtungen gegenüber der Familie zu erfüllen. Das Timing und die Häufigkeit wurden, wie ich in Kapitel 6 dargelegt habe, von der Zeitgestaltung in Göttingen beeinflusst. Die Beschäftigung mit den Reisen der jungen Wissenschaftler*innen nach Indien machte die Interaktion zwischen Mobilität und Zeitgestaltung besonders sichtbar: Die Zeitgestaltung während der Zeit in Göttingen, der Fokus auf spezifische Koordinaten wie jene der Fertigstellung der Doktorarbeit beeinflusste die Häufigkeit von Reisen und die Zeitpunkte dieser. Wann die jungen Wissenschaftler*innen nach Indien reisten, hing allerdings auch mit Koordinaten dominanter Zeitkarten zusammen, besonders jenen der Lebensverlaufsvorstellungen.

Durch die Praktiken der Resynchronisation, das Etablieren vertrauter Praktiken in den Tagesablauf, das Aufrechterhalten sozialer Beziehungen und das Herstellen lokaler Verortungen in Göttingen entstand ein transnationales „Leben in Gleichzeitigkeit“ (Levitt und Glick Schiller 2004). Die Gleichzeitigkeit bezieht sich dabei auf die räumliche und die zeitliche Ebene. Die Praktiken der Zeitgestaltung stellten Verbindungen zu vertrauten Orten, Zeiten und Menschen auf der synchronen und der diachronen Ebene her (Kapitel 5 und 6). Mit dem Augenmerk auf die zeitlichen Erfahrungen als Ursprungsfaktor für diese Praktiken konzentrierte sich meine Arbeit auf die Prozesse, die zu einem transnationalen Leben in Gleichzeitigkeit führten. Die jungen Wissenschaftler*innen wurden in diesem Sinne zu Architekten ihrer eigenen transnationalen Zeitlandschaft, die lokale Elemente aus Göttingen und ihrer sozialen Welten in Indien einschloss.

Meines Wissens wurde der Begriff der Zeitlandschaft bisher noch nicht in Studien zu Mobilität bzw. Migration angewandt. In meiner Studie erwies sich das Konzept der Zeitlandschaft als sehr geeignet, um die zeitlichen Erfahrungen, die Zeitsouveränität und die Praktiken der Zeitgestaltung mobiler Akteure in einem konkreten Kontext zu analysieren. Ich sehe in der Anwendung dieses Konzepts ein großes Potential für zukünftige Studien im Bereich der Migrationsforschung. Es kann beispielsweise 
ein Werkzeug sein, um die Wirkung konkreter Lokalitäten - von der Großstadt, über die Nachbarschaft, den Arbeitsplatz bis hin zu Flüchtlingslagern - auf die Zeitsouveränität, die zeitlichen Erfahrungen und die Praktiken der Zeitgestaltung in den Fokus zu nehmen.

\subsection{Das Selbst in und durch die Zeit: Zwischen Kontinuität und Wandel}

Ich habe in dieser Arbeit das theoretische Konzept der biographischen Navigation (Pfaff-Czarnecka 2011, 2012, 2013) genutzt, um die Aushandlungsprozesse zwischen Selbst und sozialer Umwelt zu beleuchten. In meinen Ausführungen zu diesem Konzept in Kapitel 1 habe ich mich an Henrik Vighs Begriff der „social navigation“ (Vigh 2009a) orientiert, der einen Fokus auf die Prozesshaftigkeit der Aushandlungen beinhaltet. Wie Vigh betont, umfasst die Navigation einen Blick in mögliche Zukünfte und das Erfassen möglicher Veränderungen in der sozialen Umwelt (ebd.: 420, 425). Die dominanten Zeitkarten der sozialen Welten hatten einen wichtigen Einfluss darauf, was sich die jungen Menschen in meiner Studie für ihre Zukunft vorstellen konnten. In der biographischen Navigation haben Zeitkarten eine weitere zentrale Funktion, die in den Narrationen meiner Gesprächspartner*innen sichtbar wurde: Sie sind wichtige Mittel in der diachronen Navigation des Selbst (Bamberg 2012), also in der Aushandlung des Selbst über die Zeit hinweg.

Die soziale Umwelt und das Selbst befinden sich in einem ständigen Wandel und Zugehörigkeiten müssen ständig aktiv hergestellt werden (May 2011: $372^{148}$ ). Die Zeitkarten sind in dieser Situation ein bedeutsames Instrument der Aushandlung von Veränderung und Kontinuität. Normative, meist lineare Zeitkarten bieten Menschen in ihren Narrationen und darüber hinaus eine Orientierung für die Erzählung und Darstellung eines kohärenten Selbst, das sich durch die Zeit hinweg bewegt. May postuliert, dass ,a sense of coherent self is partly achieved by reference to the linear temporality of enduring experiences of belonging with people, particularly with friends" (May 2016: 2). Ich ergänze diese Erkenntnis und argumentiere, dass lineare Zeitkarten eine ähnliche Wirkung haben: Sie machen den Wandel des Selbst erklärbar und erzählbar und stellen bestimmte Formen der Veränderung als ganz logische Entwicklung des Selbst dar (zum/zur Erwachsenen, Wissenschaftler*in etc.). Neue Aspekte des Selbst werden so in die eigene Geschichte integriert - im Fall der jungen Wissenschaftler*innen beispielsweise der Ort Göttingen als wichtige

\footnotetext{
${ }_{148}$ Vanessa May verweist nach ihren Ausführungen zur aktiven Herstellung von Zugehörigkeit auf folgende Arbeiten: V. Bell, V. (1999): Performativity and Belonging: An Introduction. In: V. Bell (Hg.): Performativity and Belonging. London: SAGE, S. 1-10. L. Miller (2003): Belonging to Country: A Philosophical Anthropology. Journal of Australian Studies 27(76): 215-23. P Scheibelhofer (2007): His-Stories of Belonging: Young Second-Generation Turkish Men in Austria. Journal of Intercultural Studies 28(3): 317-30.
} 
Station auf ihrem Pfad und die lokalen sozialen Welten als wichtiger neuer Teil des Selbst (Kapitel 4 und 8).

Die Zeit ist nicht linear, aber lineare Repräsentationen von Zeit bieten Menschen die Möglichkeit, sich selbst und anderen darzulegen, warum sie sich auf eine bestimmte Art und Weise verändert haben. Mit Hilfe linearer Zeitkarten können sie erfassen, wo sie im Vergleich zu anderen stehen, und antizipieren, was als Nächstes kommen wird (Gubrium, Holstein und Buckholdt 1994). Zeitkarten entfalten allerdings ebenso einen normativen Charakter, und zwar dann, wenn es nur noch die eine Art gibt, sich durch die Zeit zu verändern und sich richtig zu ,entwickeln'. Dies gilt für die Ebene von Personen, aber auch für die von Nationen.

In den Kapiteln 5 und 6 habe ich gezeigt, dass es noch weitere Praktiken gab, die es meinen Gesprächspartner*innen möglich machten, Veränderungen, die durch die Mobilität ausgelöst wurden, auszuhandeln. Dazu gehörten die kontinuierlichen $\mathrm{Zu}$ gehörigkeiten zur Familie und zu Freunden, die das Gefühl gaben, in der Gegenwart und der Zukunft sicher aufgehoben zu sein (May 2016). Ein weiterer Aspekt - und hier geht meine Analyse über Vanessa Mays Arbeiten hinaus - waren Praktiken der Zeitgestaltung, die gewohnte Routinen wiederherstellten und auf der synchronen und diachronen Ebene Verbindungen zu vertrauten Zeiten, Menschen und Orten herstellten. Das relationale Selbst, das durch multiple soziale Verortungen entsteht, ist somit in multiplen Zeiten verankert. Die multiplen Zeiten, mit denen sich die jungen Menschen durch diese Praktiken verbanden, verdeutlichen die Multiplizität des Selbst (May 2016), das immer im Entstehen und gleichzeitig in Vergangenheit, Gegenwart und Zukunft verortet ist. Diese Verortungen in multiplen Zeiten müssen, ebenso wie andere, bewusst hergestellt werden (May 2011; Pfaff-Czarnecka 2012), beispielsweise durch Praktiken der Zeitgestaltung.

Die Reisen nach Indien, die viele meine Gesprächspartner*innen etwa einmal im Jahr unternahmen, waren wichtig für die Herstellung von Kontinuitäten und das Aufrechterhalten von Zugehörigkeiten. Die Aufenthalte in Indien führten aber auch Veränderungen vor Augen: Veränderungen in den Beziehungen zu den Eltern, der eigenen Positionierung innerhalb der Familie und des Selbst. Des Weiteren wurde vielen der jungen Wissenschaftler*innen auf ihren Reisen bewusst, dass sie nun mehr als ein „home“ hatten. Die Ambiguitäten, die einige meiner Gesprächspartner*innen in Bezug auf ihr Selbst ausdrückten, wie etwa Ajay, der sich als "globaler Inder“ bezeichnete, waren eine Artikulation des multiplen Selbst der jungen Wissenschaftler*innen. Die Zeit in Göttingen, die Beziehungen, die sie dort aufgebaut, die Erfahrungen, die sie gemacht haben, der Moment des Gänseliesel-Küssens, das alles sind für viele der jungen Menschen, die im Mittelpunkt dieser Studie standen, Aspekte ihres multiplen Selbst geworden. Auch nach der Abreise bleibt Göttingen bei diesen ein Teil ihres Selbst, wie im Fall von Akhil, der seit 2017 wieder in Indien lebt und im März 2019 auf Facebook ein Foto von der Kirschblüte auf dem Campus der Universität Göttingen teilte und dazu kommentierte: „Meine Stadt im Frühling“.

Mit dem Fokus auf die vielfältigen Verortungen der jungen Wissenschaftler*innen bietet meine Studie nicht nur im wissenschaftlichen Sinne, sondern für alle 
Leser*innen Anknüpfungspunkte. Viele Menschen werden sich in Aspekten der biographischen Navigation meiner Gesprächspartner*innen wiederfinden. Dies gilt für Wissenschaftler*innen, die sich mit der Unsicherheit befristeter Stellen und dem ,grausamen Optimismus' des akademischen Lebensverlaufs auseinandersetzen, wie auch für Menschen, die manchmal das Gefühl haben, die Gestaltung ihrer Zeit nicht kontrollieren zu können, für Personen, die sich schon einmal gefragt haben, ob sie die Einzigen sind, die nicht alles zur ,richtigen 'Zeit machen, und für alle, die schon einmal Heimweh hatten und sich in der Küche beim Kochen ihrer Lieblingsspeise aus der Kindheit wiederfanden. Zu guter Letzt werden sich in den Aussagen der jungen Wissenschaftler*innen jene wiedererkennen, die selbst promoviert haben und zwischendurch das Gefühl hatten, dass die Doktorarbeit nie fertig werden würde. 


\section{Literaturverzeichnis}

Abraham, Itty (1998): The Making of the Indian Atomic Bomb: Science, Secrecy and the Postcolonial State. London: Zed Books.

Adam, Barbara (1988): Social Versus Natural Time: A Traditional Distinction Reexamined. In: Michael D. Young und Tom Schuller (Hg.): The Rhythms of Society, 198-226. London: Routledge.

Adam, Barbara (1995): Das Diktat der Uhr: Zeitformen, Zeitkonflikte, Zeitperspektiven. Frankfurt am Main: Suhrkamp.

— (1998): Timescapes of Modernity: The Environment and Invisible Hazards. London: Routledge.

- (2008): Of Timescapes, Futurescapes and Timeprints. http://www.cardiff. ac.uk/socsi/futures/conf_ba_lueneberg170608.pdf (depubliziert, zugänglich via Archive.org).

Ahmad, Mashkoor (2018): Access of Muslims to Education in Uttar Pradesh, India. Journal of Muslim Minority Affairs 38 (4): 551-66. Doi:10.1080/1360 2004.2018.1542179.

Amit, Vered (Hg.) (1999): Constructing the Field: Ethnographic Fieldwork in the Contemporary World. London: Taylor \& Francis.

Amit, Vered und Noel Dyck (2012): Introduction: Pursuing Respectable Adulthood: Social Reproduction in Times of Uncertainty. In: Vered Amit und 
Noel Dyck (Hg.): Young men in uncertain times, 1-34. New York, NY: Berghahn.

Andersson, Ruben (2014): Time and the Migrant Other: European Border Controls and the Temporal Economics of Illegality. American Anthropologist 116 (4): 795-809. Doi:10.1111/aman.12148.

Anthias, Floya (2002): Where Do I Belong? Narrating Collective Identity and

Translocational Positionality. Ethnicities (2(4)): 491-514. Doi:10.1177/1468 7968020020040301.

Antonsich, Marco (2010): Searching for Belonging - An Analytical Framework.

Geography Compass 4 (6): 644-59. Doi:10.1111/j.1749-8198.2009.00317.x.

Appadurai, Arjun (1996): Modernity at Large: Cultural Dimensions of Globalization.

Minneapolis: University of Minnesota Press.

- (2004): The Capacity to Aspire: Culture and the Terms of Recognition. In:

Vijendra K. Rao und Michael Walton (Hg.): Culture and Public Action,

59-84. Stanford: Stanford University Press.

- (2013): The Future as Cultural Fact: Essays on the Global Condition. London:

Verso Books.

AASSA (Association of Academies and Societies of Sciences in Asia) (2015): Women in Science and Technology in Asia. Seongnam. https://nassl.org/wp-content/ uploads/2016/01/AASSA_Report_Women_in_S_T_in_Asia.pdf

Baas, Michiel (2009): The IT Caste. Love and Arranged Marriages in the IT

Industry of Bangalore. Journal of South Asian Studies (32 (2)): 285-307.

Doi:10.1080/00856400903049531.

—- (2010): Imagined mobility: Migration and transnationalism among Indian students in Australia. London: Anthem.

- (2013): In-Betweeness: The (Dis)Connection Between Here and There. The

Case of Indian Student-Migrants in Australia. Conserveries mémorielles 13.

https://journals.openedition.org/cm/1468.

- (2015): The New Indian Male: Muscles, Masculinity and Middle Classness.

In: Knut A. Jacobsen (Hg.): Routledge Handbook of Contemporary India, 444-56. London: Routledge.

_- (2016): Becoming Trans/Nationally Mobile: The Conflation of Internal and International Migration in the Trajectories of Indian Student-Migrants in Australia and Beyond. South Asia: Journal of South Asian Studies. Doi:10.1080/00856401.2016.1109166.

Bailey, Adrian J. (2009): Population Geography: Lifecourse Matters. Progress in Human Geography 33 (3): 407-18. Doi:10.1177/0309132508096355.

Bailey, Adrian J., Richard A. Wright, Alison Mountz et al. (2002): (Re)producing

Salvadoran Transnational Geographies. Annals of the Association of American Geographers 92 (1): 125-44. Doi:10.1111/1467-8306.00283.

Bailey, Ajay (2017): The Migrant Suitcase: Food, Belonging and Commensa-

lity Among Indian Migrants in the Netherlands. Appetite 110: 51-60.

Doi:10.1016/j.appet.2016.12.013. 
Bailey, Ajay und Clara H. Mulder (2017): Highly Skilled Migration Between the Global North and South: Gender, Life Courses and Institutions. Journal of Ethnic and Migration Studies 43 (16): 2689-2703.

Doi:10.1080/1369183X.2017.1314594.

Baker-Cristales, Beth (2016): Regime. In: Noel B. Salazar und Kiran Jayaram (Hg.): Keywords of Mobility: Critical Engagements, 152-70. New York: Berghahn.

Bakhtin, M. M. und Caryl Emerson (1984): Problems of Dostoevsky's poetics. Manchester: Manchester University Press.

Baldassar, Loretta (2001): Visits Home: Migration Experiences Between Italy and Australia. San Fior to Perth - The Migrant Experience. Carlton South: Melbourne University Press.

_ (2008): Missing Kin and Longing to Be Together: Emotions and the Construction of Co-Presence in Transnational Relationships. Journal of Intercultural Studies 29 (3): 247-66. Doi:10.1080/07256860802169196.

Baldassar, Loretta, Mihaela Nedelcu, Laura Merla et al. (2016): ICT-Based CoPresence in Transnational Families and Communities: Challenging the Premise of Faceto-Face Proximity in Sustaining Relationships. Global Networks 16 (2): 133-44. Doi:10.1111/glob.12108.

Bamberg, Michael (2004): Positioning with Davie Hogan: Stories, Tellings, and Identities. In: Colette Daiute und Cynthia Lightfoot (Hg.): Narrative Analysis: Studying the Development of Individuals in Society, 135-57. Thousand Oaks: Sage.

- (2012): Narrative Practice and Identity Navigation. In: James A. Holstein und Jaber F. Gubrium (Hg.): Varieties of Narrative Analysis, 99-124. Los Angeles: Sage.

Bamberg, Michael, Anna de Fina und Deborah Schiffrin (Hg.) (2007): Selves and Identities in Narrative and Discourse. Amsterdam: Benjamins.

Bamberg, Michael, Anna de Fina und Deborah Schiffrin (2011): Discourse and Identity Construction. In: Seth J. Schwartz (Hg.): Handbook of Identity Theory and Research, 177-99. New York: Springer.

Bansal, Parul (2013): Youth in Contemporary India: Images of Identity and Social Change. New Delhi: Springer.

Barnick, Heather (2010): Managing Time and Making Space: Canadian Students' Motivations for Study in Australia. Anthropology in Action 17 (1). Doi:10.3167/aia.2010.170103.

Barthelt, Franziska, Diana Meschter, Friederike Meyer zu Schwabedissen et al. (2015): Internationale Studierende - Aktuelle Entwicklungen und Potenziale der globalen Bildungsmigration. Bundeszentrale für politische Bildung. http://www.bpb.de/gesellschaft/migration/kurzdossiers/212090/internation ale-studierende.

Bauman, Zygmunt (2011): Migration and Identities in the Globalized World. Philosophy and Social Criticism 37 (4): 425-35. Doi:10.1177/019145371039 6809. 
Bear, Laura (2014): Introduction: Doubt, Conflict, Mediation: the Anthropology of Modern Time. In: Laura Bear (Hg.): Doubt, Conflict, Mediation: The Anthropology of Modern Time, 3-30. Malden: Wiley.

- (2016): Time as Technique. Annual Review of Anthropology 45: 487-502. Doi:10.1146/annurev-anthro-102313-030159.

Beck, Ulrich (1994): Jenseits von Stand und Klasse?. In: Ulrich Beck und Elisabeth Beck-Gernsheim (Hg.): Riskante Freiheiten: Individualisierung in modernen Gesellschaften, 43-60. Frankfurt am Main: Suhrkamp.

Beck, Ulrich und Elisabeth Beck-Gernsheim (1994): Individualisierung in modernen Gesellschaften: Perspektiven und Kontroversen einer subjektorientierten Soziologie. In: Ulrich Beck und Elisabeth Beck-Gernsheim (Hg.): Riskante Freiheiten: Individualisierung in modernen Gesellschaften, 10-42. Frankfurt am Main: Suhrkamp.

Belliappa, Jyothsna (2013): Gender, Class and Reflexive Modernity in India. Basingstoke: Palgrave Macmillan.

Bergson, Henri ([1869] 1988): Matter and Memory. New York: Zone Books. Berlant, Lauren G. (2011): Cruel Optimism. Durham: Duke University Press. Bertrand, Marianne, Sendhil Mullainathan und Rema Hanna (2008): Affirmative Action in Education: Evidence from Engineering College Admissions in India. NBER working paper series 13926. Cambridge: National Bureau of Economic Research. Doi:10.3386/w13926.

Bhandari, Rajika und Peggy Blumenthal (2009): Global Student Mobility: Moving Towards Brain Exchange. In: Rajika Bhandari und Shepherd Laughlin (Hg.): Higher Education on the Move: New Developments in Global Mobility, 1-16. New York: Institute of International Education.

Bilecen, Başak und Christof van Mol (2017): Introduction: International Academic Mobility and Inequalities. Journal of Ethnic and Migration Studies 43 (8): 1241-55. Doi:10.1080/1369183X.2017.1300225.

Boccagni, Paolo (2017): Aspirations and the Subjective Future of Migration: Comparing Views and Desires of the ,Time Ahead' Through the Narratives of Immigrant Domestic Workers. Comparative Migration Studies 5 (1): 1-18. Doi:10.1186/s40878-016-0047-6.

Bourdieu, Pierre (1976): Entwurf einer Theorie der Praxis: Auf der ethnologischen Grundlage der Kabylischen Gesellschaft. Frankfurt am Main: Suhrkamp.

_- (1982): Die feinen Unterschiede: Kritik der gesellschaftlichen Urteilskraft. Frankfurt am Main: Suhrkamp.

_- (1983): Ökonomisches Kapital, kulturelles Kapital, soziales Kapital. In: Reinhard Kreckel (Hg.): „Soziale Ungleichheiten.“ Sonderheft von Soziale Welt (Sonderband 2): 183-98.

—_([1997] 2017): Meditationen: Zur Kritik der scholastischen Vernunft. Frankfurt am Main: Suhrkamp. 
Bourdieu, Pierre und Jean-Claude Passeron (1971): Die Illusion der Chancengleichheit: Untersuchungen zur Soziologie des Bildungswesens am Beispiel Frankreichs. Stuttgart: Klett.

Brah, Avtar (1996): Cartographies of Diaspora: Contesting Identities. London: Routledge.

Brooks, Rachel und Johanna Waters (2009): International Higher Education and the Mobility of UK Students. Journal of Research in International Education 8(2): 191-209. Doi:10.1177/1475240909105204.

- (2011): Student Mobilities, Migration and the Internationalization of Higher Education. Basingstoke, Hampshire: Palgrave Macmillan.

Brosius, Christiane (2006): masala.de: Menschen aus Südasien in Deutschland. Heidelberg: Draupadi.

Brown, Judith M. (2006): Global South Asians: Introducing the Modern Diaspora. Cambridge: Cambridge University Press.

Bryant, Rebecca und Daniel M. Knight (2019): The Anthropology of the Future. Cambridge: Cambridge University Press.

Bueger, Christian und Jan Stockbruegger (2017): Actor-Network Theory: Objects and Actants, Networks and Narratives. In: Daniel R. McCarthy (Hg.): Technology and World Politics: An Introduction, 42-59. London: Routledge. Bundesagentur für Arbeit (2011): Perspektive 2025: Fachkräfte für Deutschland. https://www.uni-heidelberg.de/md/journal/2013/01/perspektive2025.pdf.

- (2019): Blickpunkt Arbeitsmarkt - MINT-Berufe. https://statistik.arbeitsagentur.de/DE/Statischer-Content/Statistiken/Themen-im-Fokus/Berufe/ Generische-Publikationen/Broschuere-MINT.pdf.

BMBF (Bundesministerium für Bildung und Forschung) (2014): Strategie der Bundesregierung zum Europäischen Forschungsraum (EFR) - Leitlinien und nationale Roadmap. https://www.bmbf.de/upload_filestore/pub/Strategie_der_Bundesregierung_zum_EFR.pdf.

- (2016): Internationalisierung von Bildung, Wissenschaft und Forschung: Strategie der Bundesregierung. https://www.bmbf.de/upload_filestore/pub/ Internationalisierungsstrategie.pdf.

Butcher, Melissa (2009): Ties that Bind: The Strategic Use of Transnational Relationships in Demarcating Identity and Managing Difference. Journal of Ethnic and Migration Studies 35 (8): 1353-71. Doi:10.1080/13691830903123153.

- (2010): From ,Fish Out of Water 'to ,Fitting In': The Challenge of Re-placing Home in a Mobile World. Population, Space and Place 16: 23-36.

- (2011): Managing Cultural Change: Reclaiming Synchronicity in a Mobile World. Farnham: Ashgate.

Butler, Judith (1993): Bodies That Matter: On the Discursive Limits of, Sex'. New York: Routledge.

Carling, Jørgen und Francis Collins (2017): Aspiration, Desire and Drivers of Migration. Journal of Ethnic and Migration Studies 18 (2): 1-18. Doi:10.108 0/1369183X.2017.1384134. 
Certeau, Michel de (1984): The Practice of Everyday Life. Berkeley: University of Minnesota Press.

Chang, Ik Young, Michael P. Sam und Steven J. Jackson (2015): Transnationalism, Return Visits and Identity Negotiation: South Korean-New Zealanders and the Korean National Sports Festival. International Review for the Sociology of Sport 52 (3): 314-35. Doi:10.1177/1012690215589723.

Chopra, Radhika (2005): Sister and Brothers: Schooling, Family and Migration. In: Radhika Chopra und Patricia Jeffery (Hg.): Educational Regimes in Contemporary India, 299-315. New Delhi: Sage.

Chopra, Radhika und Patricia Jeffery (Hg.) (2005): Educational Regimes in Contemporary India. New Delhi: Sage.

Christensen, Ann-Dorte und Sune Jensen (2011): Roots and Routes: Migration, Belonging and Everyday Life. Nordic Journal of Migration Research 1 (3): 17. Doi:10.2478/v10202-011-0013-1.

Christiansen, Catrine, Mats Utas und Henrik Vigh (2006): Introduction: Navigating Youth, Generating Adulthood. In: Catrine Christiansen, Mats Utas und Henrik Vigh (Hg.): Navigating Youth, Generating Adulthood: Social Becoming in an African Context, 9-28. Uppsala: Nordic Africa Institute.

Clark-Decès, Isabelle (2014): The Right Spouse: Preferential Marriages in Tamil Nadu. Stanford: Stanford University Press.

Coffey, Amanda (1999): The Ethnographic Self: Fieldwork and the Representation of Identity. London: Sage.

Cohen, Laurie, Joanne Duberley und M. N. Ravishankar (2015): Examining the Interplay of Career, Migration and National Cultural Identity: the Case of Indian Scientists. International Migration 53 (5): 104-121. Doi:10.1111/ j. 1468-2435.2012.00768.x.

Cole, Jennifer (2010): Sex and Salvation: Imagining the Future in Madagascar. Chicago: University of Chicago Press.

Cole, Jennifer und Deborah Durham (2007): Generations and Globalization: Youth, Age, and Family in the New World Economy. Bloomington: Indiana University Press.

- (Hg.) (2008a): Figuring the Future: Globalization and the Temporalities of Children and Youth. Santa Fe: School for Advanced Research Press.

- (2008b): Introduction: Globalization and the Temporality of Children and Youth. In: Jennifer Cole und Deborah Durham (Hg.): Figuring the Future: Globalization and the Temporalities of Children and Youth, 3-24. Santa Fe: School for Advanced Research Press.

Coleman, Simon und Peter J. Collins (2006): Locating the Field: Space, Place and Context in Anthropology. Oxford: Berg.

Collins, Francis L. (2008): Of Kimchi and Coffee: Globalisation, Transnationalism and Familiarity in Culinary Consumption. Social \& Cultural Geography 9(2): 151-69. Doi:10.1080/14649360701856094. 
— (2017): Desire as a Theory for Migration Studies: Temporality, Assemblage and Becoming in the Narratives of Migrants. Journal of Ethnic and Migration Studies 18 (2): 1-17. Doi:10.1080/1369183X.2017.1384147.

Collins, Francis L. und Sergei Shubin (2015): Migrant Times Beyond the Life Course: The Temporalities of Foreign English Teachers in South Korea. Geoforum 62: 96-104. Doi:10.1016/j.geoforum.2015.04.002.

- (2017): The Temporal Complexity of International Student Mobilities. In: Elizabeth Mavroudi, Ben Page und Anastasia Chrēstu (Hg.): Timespace and International Migration, 17-30. Cheltenham: Edward Elgar Publishing. Costanzo Kapur, Cari (2010): Rethinking Courtship, Marriage and Divorce in an Indian Call Center. In: Diane P. Mines und Sarah Lamb (Hg.): Everyday Life in South Asia, Second Edition, 50-61. Bloomington: Indiana University Press.

Cwerner, Saulo B. (1999): The Times of Migration: A Study of the Temporalities of the Immigrant Experience. Dissertation, University of Lancaster.

- (2001): The Times of Migration. Journal of Ethnic and Migration Studies 27(1): 7-36. Doi:10.1080/13691830125283.

DAAD und DZHW (Deutscher Akademischer Austauschdienst und Deutsches Zentrum für Hochschul-und Wissenschaftsforschung) (2016): Wissenschaft weltoffen 2016: Daten und Fakten zur Internationalität von Studium und Forschung in Deutschland. Bielefeld. http://www.wissenschaftweltoffen.de/ publikation/wiwe_2016_verlinkt.pdf.

- (2019): Wissenschaft weltoffen 2019: Daten und Fakten zur Internationalität von Studium und Forschung in Deutschland. Bielefeld. http://www.wissen schaftweltoffen.de/publikation/wiwe_2019_verlinkt.pdf.

Dalsgaard, Anne L., Martin Frederiksen, Susanne Hojlund und Lotte Meinert (Hg.) (2014): Ethnographies of Youth and Temporality: Time Objectified. Philadelphia: Temple University Press.

Dalsgaard, Steffen (2013): The Field as a Temporal Entity and the Challenges of the Contemporary. Social Anthropology/Anthropologie Sociale 21 (2): 213-25. Doi:10.1111/1469-8676.12012.

- (2015): The Ethnographic Use of Facebook in Everyday Life. Anthropological Forum 26 (1): 96-114. Doi:10.1080/00664677.2016.1148011.

Dalsgaard, Steffen und Morten Nielsen (2016): Introduction: Time and the Field. In: Steffen Dalsgaard und Morten Nielsen (Hg.): Time and the Field, 1-19. New York: Berghahn.

Daniel, E. Valentine (1984): Fluid Signs: Being a Person the Tamil Way. Berkeley: University of California Press.

Dausien, Bettina (2000): ,Biographie' als rekonstruktiver Zugang zu ,Geschlecht'Perspektiven der Biographieforschung. In: Doris Lemmermöhle-Thüsing (Hg.): Lesarten des Geschlechts: Zur De-Konstruktionsdebatte in der erziehungswissenschaftlichen Geschlechterforschung, 96-115. Opladen: Leske + Budrich. 
Davies, Karen (1990): Women, Time and the Weaving of the Strands of Everyday Life. Aldershot: Avebury.

—- (2001): Responsibility and Daily Life: Reflections over Timespace. In: Jon May und Nigel J. Thrift (Hg.): Timespace: Geographies of Temporality, 133-48. London: Routledge.

Deleuze, Gilles (1994): Difference and Repetition. New York: Columbia University Press.

Derné, Steve (2008): Globalization on the Ground: Media and the Transformation of Culture, Class, and Gender in India. Los Angeles: Sage.

Desai, Sonalde (2010): Human Development in India: Challenges for a Society in Transition. New Delhi: Oxford University Press.

Desai, Sonalde und Veena Kulkarni (2008): Changing Educational Inequalities in India in the Context of Affirmative Action. Demography 45 (2): 245-70.

DeSouza, Peter R. (Hg.) (2009): Indian Youth in a Transforming World: Attitudes and Perceptions. Los Angeles: Sage.

Dhillon, Navtej und Tarik Yousef (Hg.) (2009): Generation in Waiting: The Unfulfilled Promise of Young People in the Middle East. Washington: Brookings Institution Press.

Dilley, Roy (2005): Time-Shapes and Cultural Agency Among West African Craft Specialists. In: David M. Wendy James (Hg.): The Qualities of Time: Anthropological Approaches, 235-50. Oxford: Berg.

Donner, Henrike (2008): Domestic Goddesses: Maternity, Globalization and MiddleClass Identity in Contemporary India. Aldershot: Ashgate.

- (Hg.) (2011): Being Middle-Class in India: A Way of Life. London: Routledge.

- (2012): Marriage in Modern India: „The Middle-Class Ideal of an Indian Marriage Has Not Changed". London School of Economics and Political Science. http://blogs.lse.ac.uk/southasia/2012/10/01/marriage-in-modernindia-the-middle-class-ideal-of-an-indian-marriage-has-not-changed-henrike-donner/.

— (2015): Making Middle-Class Families in Calcutta. In: James G. Carrier und Don Kalb (Hg.): Anthropologies of Class: Power, Practice and Inequality, 131-48. Cambridge: Cambridge University Press.

Duberley, Joanne und Laurie Cohen (2010): Gendering Career Capital: An Investigation of Scientific Careers. Journal of Vocational Behavior 76 (2): 187-97. Doi:10.1016/j.jvb.2009.09.005.

Ehn, Billy und Orvar Löfgren (2013): Routines - Made and Unmade. In: Elizabeth Shove (Hg.): Time, Consumption and Everyday Life: Practice, Materiality and Culture, 99-112. London: Bloomsbury.

Eisenlohr, Patrick (2015): Mediating Disjunctures of Time: Ancestral Chronotopes in Ritual and Media Practices. Anthropological Quarterly 88 (2): 281-304. Doi:10.1353/anq.2015.0023. 
Elchardus, Mark (1994): In Praise of Rigidity: On Temporal and Cultural Flexibility. Social Science Information/Information sur les Sciences Sociales 33 (3): 459-77. Doi:10.1177/053901894033003006.

Elder, Glen, Monica K. Johnson und R. Crosnoe (2003): The Emergence and Development of Life Course Theory. In: Jeylan T. Mortimer, Michael J. Shanahan und Monica K. Johnson (Hg.): Handbook of the Life Course, 3-19. New York: Kluwer Academic.

Elias, Norbert (1986): Was ist Soziologie? Weinheim: Juventa.

Elsrud, Torun (1998): Time Creation in Travelling: The Taking and Making of Time among Women Backpackers. Time \& Society 7(2): 309-34. Doi:10.11 77/0961463X98007002008.

Esses, Victoria M., Stelian Medianu und Andrea S. Lawson (2013): Uncertainty, Threat, and the Role of the Media in Promoting the Dehumanization of Immigrants and Refugees. Journal of Social Issues 69(3): 518-36. Doi:10.1111/ josi. 12027.

European Commission (2016a): Open Innovation, Open Science, Open to the World: A Vision for Europe. Doi:10.2777/06165.

- (2016b): She Figures 2015. Directorate-General for Research and Innovation. Doi: $10.2777 / 744106$.

Evans-Pritchard, E. E. (1960): The Nuer: a Description of the Modes of Livelihood and Political Institutions of a Nilotic People. Oxford: Clarendon Press.

Ewing, Katherine P. (1990): The Illusion of Wholeness: Culture, Self and the Experience of Inconsistency. Ethos 18: 251-78. Doi:10.1525/eth.1990.18.3.02a 00020 .

Fabian, Johannes (1983): Time and the Other: How Anthropology Makes Its Object. New York: Columbia Press.

FICCI (Federation of Indian Chambers of Commerce and Industry) (2013): Higher Education in India: Vision 2030: FICCI Higher Education Summit. https://www.teqipgoodgovernance.in/FICCI-E\%20Y\%20Report\%20Final. pdf.

Findlay, Allan M. (2011): An Assessment of Supply and Demand-Side Theorizations of International Student Mobility. International Migration 49(2): 162-90. Doi:10.1111/j.1468-2435.2010.00643.x.

Findlay, Allan, David McCollum, Rory Coulter et al. (2015): New Mobilities Across the Life Course: A Framework for Analysing Demographically Linked Drivers of Migration. Population, Space and Place 21 (4): 390-402. Doi:10.1002/psp.1956.

Findlay, Allan M., Russel King und Fiona M. Smith (2012): World Class? an Investigation of Globalisation, Difference and International Student Mobility. Transactions of the Institute of British Geographers 37: 118-31. Doi:10.1111/ j.1475-5661.2011.00454.x. 
Findlay, Allan M., Alexandra Stam, Russel King et al. (2005): International Opportunities: Searching for the Meaning of Student Migration. Geographica Helvetica 60 (3): 192-200.

Flaherty, Michael G. (1999): A Watched Pot: How We Experience Time. New York: New York University Press.

—- (2003): Time Work: Customizing Temporal Experience. Social Psychology Quarterly 66 (1): 17-33. Doi:10.2307/3090138.

- (2011): The Textures of Time: Agency and Temporal Experience. Philadelphia: Temple University Press.

- (2013): Age and Agency: Time Work Across the Life Course. Time \& Society 22 (2): 237-53. Doi:10.1177/0961463X12455598.

Fleming, Rachel C. (2016): Working for a Happy Life in Bangalore: Gender, Generation, and Temporal Liminality in India's Tech City. Dissertation, University of Colorado, Boulder. https://scholar.colorado.edu/concern/ graduate_thesis_or_dissertations/z316q158b.

Flood, Gavin D. (1996): An Introduction to Hinduism. Cambridge: Cambridge University Press.

Földes, Ionuț und Veronica Savu (2018): Family Practices Across Generations and National Borders. Studia Ubb Sociologia 63 (LXIII) (2): 143-69.

Fong, Vanessa L. (2011): Paradise Redefined: Transnational Chinese Students and the Quest for Flexible Citizenship in the Developed World. Stanford: Stanford University Press.

Foucault, Michel, Luther Martin, Huck Gutman und Patrick Hutton (1988):

Technologies of the Self: A Seminar with Michel Foucault. Amherst: University of Massachusetts Press.

Frederiksen, Martin D. und Anne L. Dalsgaard (2014): Introduction: Time Objectified. In: Anne L. Dalsgaard et al. (Hg.): Ethnographies of Youth and Temporality: Time Objectified, 1-21. Philadelphia: Temple University Press.

Frost, Robert (2010): Promises to keep: Poems - Gedichte. München: LangewiescheBrandt.

Fuhse, Antonie (2018): Navigating Belonging in a Mobile World: Young Indian Students and Researchers in Germany. In: Elfriede Hermann und Antonie Fuhse (Hg.): India Beyond India: Dilemmas of Belonging, 49-63. Göttingen: Göttingen University Press. Doi:10.17875/gup2020-1263.

Fuller, C. J. und Haripriya Narasimhan (2008): Companionate Marriage in India: The Changing Marriage System in a Middle-Class Brahman Subcaste. Journal of the Royal Anthroplogical Institute 14: 736-54. Doi:10.1111/j.146 7-9655.2008.00528.x.

_- (2012): Marriage in modern India: Companionate marriage among a middleclass Brahman subcaste. London School of Economics and Political Science. http://blogs.lse.ac.uk/southasia/2012/10/05/companionate-marriageamong-a-middle-class-brahman-subcaste/. 
Galama, Titus und James Hosek (2009): Global Competitiveness in Science and Technology and the Role of Mobility. In: Rajika Bhandari und Shepherd Laughlin (Hg.): Higher Education on the Move: New Developments in Global Mobility, 95-112. New York: Institute of International Education.

Ganguly-Scrase, Ruchira (2003): Globalization, Modernity and Gender Equality. In: Timothy J. Scrase, Todd J. Holden und Scott Baum (Hg.): Globalization, Culture and Inequality in Asia, 68-94. Melbourne: Trans Pacific Press.

Garhammer, Manfred (1995): Changes in Working Hours in Germany. Time \& Society 4 (2): 167-203. Doi:10.1177/0961463X95004002002.

GATE Germany, Konsortium Internationales Hochschulmarketing (2011): Länderprofile: Indien: Informationen für das internationale Bildungsmarketing. Bonn: DAAD. https://www.gate-germany.de/fileadmin/dokumente/Publika tionen/Laenderprofile/GATE-Germany-Laenderprofil-Indien.pdf.

Gautam, Meenakshi (2015): Gender, Subject Choice and Higher Education in India. Contemporary Education Dialogue 12 (1): 31-58. Doi:10.1177/09 73184914556865.

Geddie, Kate (2015): Policy Mobilities in the Race for Talent: Competitive State Strategies in International Student Mobility. Transactions of the Institute of British Geographers 40 (2): 235-48. Doi:10.1111/tran.12072.

Geddie, Katherine P. (2010): Transnational Landscapes of opportunity? Post-graduation settlement and career strategies of international students in Toronto, Canada and London, UK. Dissertation, University of Toronto. Toronto. https://tspace.library.utoronto.ca/bitstream/1807/26526/3/Geddie_Katheri ne_P_201011_PhD_thesis.pdf.

Gell, Alfred (1992): The Anthropology of Time: Cultural Constructions of Temporal Maps and Images. Oxford: Berg.

Gershon, Ilana (2011): „Neoliberal Agency“. Current Anthropology 52 (4): 537-56. Doi:10.1086/660866.

Ghosh, Sutama und Lu Wang (2003): Transnationalism and Identity: A Tale of Two Faces and Multiple Lives. The Canadian Geographer (47(3)): 269-82. Doi:10.1111/1541-0064.00022.

Giddens, Anthony (1994): Living in a Post-Traditional Society. In: Ulrich Beck, Anthony Giddens und Scott Lash (Hg.): Reflexive Modernization: Politics, Tradition and Aesthetics in the Modern Social Order, 56-109. Stanford: Stanford University Press.

Gilbertson, Amanda (2014): From Respect to Friendship? Companionate Marriage and Conjugal Power Negotiation in Middle-Class Hyderabad. South Asia: Journal of South Asian Studies 37 (2): 225-38. Doi:10.1080/00856401.2014 .899552 .

- (2016): Cosmopolitan Learning, Making Merit, and Reproducing Privilege in Indian Schools. Anthropology \& Education Quarterly 47 (3): 297-313. Doi:10.1111/aeq.12157. 
- (2017): Aspiration as Capacity and Compulsion: The Futures of Urban Middle-Class Youth in India. In: Amy Stambach und Kathleen D. Hall (Hg.): Anthropological Perspectives on Student Futures: Youth and the Politics of Possibility, 19-32. New York: Palgrave Macmillan.

Glick Schiller, Nina, Linda Basch und C. Blanc-Szanton (Hg.) (1992): Towards a Transnational Perspective on Migration: Race, Class, Ethnicity, and Nationalism Reconsidered. New York: New York Academy of Sciences.

Glick Schiller, Nina und Noel B. Salazar (2013): Regimes of Mobility across the Globe. Journal of Ethnic and Migration Studies 39 (2): 183-200. Doi:10.108 0/1369183X.2013.723253.

Godbole, Rohini M. und Ramakrishna Ramaswamy (2015): Women Scientists in India. In: AASSA: Women in Science and Technology in Asia, 67-84. Seongnam. https://nassl.org/wp-content/uploads/2016/01/AASSA_Report_Wo men_in_S_T_in_Asia.pdf

Goel, Urmila (2002): Von Freiheitskämpfern zu Computer-Indern: Südasiaten in Deutschland. Südasien 1: 70-73.

- (2006): Germany. In: Brij V. Lal (Hg.): The Encyclopedia of the Indian Diaspora, 358-60. Honolulu: University of Hawai'i Press.

Gottschlich, Pierre (2012): CARIM-India - Developing a Knowledge Base for Policymaking in India-EU Migration: German Case Study. Robert Schuman Centre for Advanced Studies, European University Institute. http://cadmus. eui.eu/bitstream/handle/1814/20822/Carim_India_RR_2012_03_Gott schlich.pdf.

Government of India (2013): All Indian Survey on Higher Education: 2011-12 (Provisional). New Delhi: Ministry of Human Resource Development, Department of Higher Education. http://14.139.60.153/bitstream/123456 789/3947/1/All\%20India\%20Survey\%20on\%20Higher\%20Education .\%202011-12\%20\%28Provisional\%29.pdf.

Griffiths, Melanie (2013): Frenzied, Decelerating and Suspended: The Temporal Uncertainties of Failed Asylum Seekers and Immigration Detainees. Working paper 105. Centre on Migration, Policy and Society, University of Oxford. https://www.compas.ox.ac.uk/wp-content/uploads/WP-2013-105Griffiths_Failed_Asylum_Seekers.pdf.

- (2014): Out of Time: The Temporal Uncertainties of Refused Asylum Seekers and Immigration Detainees. Journal of Ethnic and Migration Studies 40 (12): 1991-2009. Doi:10.1080/1369183x.2014.907737.

Griffiths, Melanie, Ali Rogers und Bridget Anderson (2013): Migration, Time and Temporalities: Review and Prospect. COMPAS Research Resources Paper. Oxford: COMPAS Centre on Migration, Policy and Society. https://www. compas.ox.ac.uk/wp-content/uploads/RR-2013-Migration_Time_Tempora lities.pdf.

Grigoleit-Richter, Grit (2017): Highly Skilled and Highly Mobile? Examining Gendered and Ethnicised Labour Market Conditions for Migrant Women in 
STEM-Professions in Germany. Journal of Ethnic and Migration Studies 43 (16): 2738-55. Doi:10.1080/1369183X.2017.1314597.

Gubrium, Jaber F., James A. Holstein und David R. Buckholdt (1994): Constructing the Life Course. Dix Hills: General Hall.

Guibernau, Montserrat (2013): Belonging: Solidarity and Division in Modern Societies. Cambridge: Polity Press.

Gupta, Namrata und Arun K. Sharma (2002): Women Academic Scientists in India. Social Studies of Science 32 (5-6): 901-15. Doi:10.1177/030631 270203200505.

Gutekunst, Miriam (2018): Grenzüberschreitungen - Migration, Heirat und staatliche Regulierung im europäischen Grenzregime: Eine Ethnographie. Bielefeld: transcript.

Hage, Ghassan (1997): At Home in the Entrails of the West: Multiculturalism, ,Ethnic Food', and Migrant Home-Building. In: Helen Grace et al. (Hg.): Home/World: Communality, Identity and Marginality in Sydney's West, 99153. Sydney: Pluto Press.

Hall, Stuart (2004): Wer braucht ,Identität'?. In: Stuart Hall, Juha Koivisto und Andreas Merkens (Hg.): Ideologie, Identität, Repräsentation, 167-87. Hamburg: Argument.

Hannam, Kevin, Mimi Sheller und John Urry (2006): Editorial: Mobilities, Immobilities and Moorings. Mobilities 1 (1): 1-22. Doi:10.1080/174501005004 89189.

Hannerz, Ulf (1998): Other Transnationals: Perspectives Gained from Studying Sideways. Paideuma: Mitteilungen zur Kulturkunde 44: 109-23. https:// www.jstor.org/stable/40342025.

— (2010): Field Worries: Studying Down, Up, Sideways, Through, Backward, Forward, Early or Later, Away and at Home. In: Ulf Hannerz (Hg.): Anthropology's World: Life in a Twenty-First-Century Discipline, 59-86. London: Pluto. Doi:10.2307/j.ctt183p30z.8.

Hansen, Anders S. (2015): The Temporal Experience of Chinese Students Abroad and the Present Human Condition. Journal of Current Chinese Affairs 3: 49-77.

Hazelkorn, Ellen (2009): Higher Education Rankings and the Global ,Battle for Talent'. In: Rajika Bhandari und Shepherd Laughlin (Hg.): Higher Education on the Move: New Developments in Global Mobility, 79-94. New York: Institute of International Education.

Hermann, Elfriede (2001): Kulturelle Differenz via Gender-Differenz: Reflexionen im Kontext von Diskursen der Fiji-Banabans. In: Judith Schlehe (Hg.): Interkulturelle Geschlechterforschung: Identitäten - Imaginationen - Repräsentationen, 236-50. Frankfurt am Main: Campus.

- (2003a): Manifold Identifications Within Differentiations: Shapings of Self Among the Relocated Banabans of Fiji. In: Toon van Meijl und Henk Driessen (Hg.): „Multiple Identifications and the Self.“ Sonderheft von Focaal - European Journal of Anthropology 42: 77-88. 
- (2003b): Positionierungen des ethnischen Selbst: Autobiografische Assoziationen einer Banaban-Frau in Fiji. In: Elfriede Hermann und Birgitt RöttgerRössler (Hg.): Lebenswege im Spannungsfeld lokaler und globaler Prozesse: Person, Selbst und Emotion in der ethnologischen Biografieforschung, 79-103. Münster: Lit.

Hermann, Elfriede und Antonie Fuhse (2018): Introduction: Dilemmas of Belonging in Indian Diasporas. In: Elfriede Hermann und Antonie Fuhse (Hg.): India Beyond India: Dilemmas of Belonging, 11-24. Göttingen: Göttingen University Press. Doi:10.17875/gup2020-1261.

Hermann, Elfriede, Wolfgang Kempf und Toon van Meijl (Hg.) (2014): Belonging in Oceania: Movement, Place-Making and Multiple Identifications. New York: Berghahn.

Hermann, Elfriede und Birgitt Röttger-Rössler (2003): Einleitung: Persönliche Handlungsmöglichkeiten im lokal-globalen Kontext. In: Elfriede Hermann und Birgitt Röttger-Rössler (Hg.): Lebenswege im Spannungsfeld lokaler und globaler Prozesse: Person, Selbst und Emotion in der ethnologischen Biografieforschung, 1-24. Münster: Lit.

Hess, Sabine, Bernd Kasparek, Stefanie Kron, Mathias Rodatz, Maria Schwertl und Simon Sontowski (Hg.) (2017): Der Lange Sommer Der Migration: Grenzregime III. Berlin: Assoziation A.

Hillger, Doris (2014): Expansion, Qualität, Gerechtigkeit: Herausforderungen des indischen Bildungssystems. Bundeszentrale für politische Bildung. https:// www.bpb.de/internationales/asien/indien/44534/indiens-bildungssystem.

Hitlin, Steven und Glen Elder (2007): Time, Self, and the Curiously Abstract Concept of Agency. Sociological Theory 25 (2): 170-91. Doi:10.1111/ j.1467-9558.2007.00303.x.

Ho, Rob (2014): Do all Asians look alike? Asian Canadians as Model Minorities. Studies on Asia Series IV 4 (2): 78-107. https://castle.eiu.edu/studiesonasia/ documents/seriesIV/Ho_Studies_Fall14.pdf.

Hodges, Matt (2008): Rethinking Time's Arrow: Bergson, Deleuze and the Anthropology of Time. Anthropological Theorie 8 (4). Doi:10.1177/14634996080 96646.

— (2014): Immanent Anthropology: a Comparative Study of ,Process' in Contemporary France. Journal of the Royal Anthroplogical Institute 20: 33-51. Doi:10.1111/1467-9655.12092.

Holland, Dorothy, William Lachicotte jr und Debra Skinner (1998): Identity and Agency in Cultural Worlds. Cambridge: Harvard University Press.

Honwana, Alcinda M. (2012): The Time of Youth: Work, Social Change, and Politics in Africa. Sterling: Kumarian Press.

Jacobs, Jerry A. und Sarah E. Winslow (2004): The Academic Life Course, Time Pressures and Gender Inequality. Community, Work \& Family 7 (2): 143-61. Doi:10.1080/1366880042000245443. 
Jaffrelot, Christophe und Peter van der Veer (Hg.) (2008): Patterns of Middle Class Consumption in India and China. Los Angeles: Sage.

James, Wendy und David Mills (2005a): Introduction: from Representation to Action in the Flow of Time. In: Wendy James und David Mills (Hg.):

The Qualities of Time: Anthropological Approaches, 1-15. Oxford: Berg.

— (Hg.) (2005b): The Qualities of Time: Anthropological Approaches. Oxford: Berg.

Jeffery, Patricia (2005): Introduction: Hearts, Minds and Pockets. In: Radhika Chopra und Patricia Jeffery (Hg.): Educational Regimes in Contemporary India, 13-38. New Delhi: Sage.

Jeffrey, Craig (2010a): Timepass: Youth, Class, and the Politics of Waiting in India. Palo Alto: Stanford University Press.

- (2010b): Timepass: Youth, Class, and Time Among Unemployed Young Men in India. American Ethnologist 37 (3): 465-81. Doi:10.1111/j.1548-1425.20 10.01266.x.

- (2011): Great Expectations: Youth in Contemporary India. In: Isabelle ClarkDecès (Hg.): A Companion to the Anthropology of India, 62-79. Malden: Wiley-Blackwell.

Jeffrey, Craig und Jane Dyson (Hg.) (2008): Telling Young Lives: Portraits of Global Youth. Philadelphia: Temple University Press.

Jensen, Jens F. (1998): ,Interactivity': Tracking a New Concept in Media and Communication Studies. Nordicom Review 19 (1): 185-204. https://www. nordicom.gu.se/sites/default/files/kapitel-pdf/38_jensen.pdf.

Jones, Demelza (2013): Cosmopolitans and ,Cliques': Everyday Socialisation Amongst Tamil Student and Young Professional Migrants to the UK. Ethnicities 13 (4): 420-37. Doi:10.1177/1468796813483729.

Jong, Gordon F. de (2000): Expectations, Gender, and Norms in Migration Decision-Making. Population Studies 54 (3): 307-19. Doi:10.1080/713779089.

Jöns, Heike (2011): Transnational Academic Mobility and Gender. Globalisation, Societies and Education 9 (2): 183-209. Doi:10.1080/14767724.2011.5771 99.

Kandel, William und Douglas S. Massey (2002): The Culture of Mexican Migration. Social Forces 80 (3): 981-1004. https://www.jstor.org/stable/3086463.

Karsch, Margret und Rainer Münz (2011): Demografische Dividende. OnlineHandbuch Demografie. Berlin-Institut für Bevölkerung und Entwicklung. http://www.berlin-institut.org/fileadmin/user_upload/handbuch_texte/ pdf_Karsch_Demografische_Dividende.pdf (depubliziert, zugänglich via Archive.org).

Kaul, Nitisha (2017): Rise of the Political Right in India: Hindutva-Development Mix, Modi Myth, and Dualities. Journal of Labour and Society 20: 523-48. Doi:10.1111/wusa.12318. 
Kaur, Ravinder (2013): Mapping the adverse Cosequences of Sex Selection and Gender Imbalance in India and China. Economic and Political Weekly XLVIII (35): 37-44.

Kaur, Ravinder und Priti Dhanda (2014): Surfing for Spouses: Marriage Websites and the ,New' Indian Marriage?. In: Ravinder Kaur und Rajni Palriwala (Hg.): Marrying in South Asia: Shifting Concepts, Changing Practices in a Globalising World, 271-92. New Delhi: Orient BlackSwan.

Kaur, Ravinder und Rajni Palriwala (Hg.) (2014): Marrying in South Asia: Shifting Concepts, Changing Practices in a Globalising World. New Delhi: Orient BlackSwan.

Kaur, Ravinder und Ishita Shruti (2016): Mobile Technology and ,Doing Family in a Global World: Indian Migrants in Cambodia. In: Sun S. Lim (Hg.): Mobile Communication and the Family: Asian Experiences in Technology Domestication, 73-91. Dordrecht: Springer Science+Business.

Kennedy, Paul (2008): The Construction of Trans-Social European Networks and the Neutralisation of Borders: Skilled EU Migrants in Manchester - Reconstituting Social and National Belonging. Space and Polity 12 (1): 119-33. Doi: 10.1080/13562570801969564.

Kenway, Jane und Johannah Fahey (2007): Policy Incitements to Mobility: Some Speculations and Provocations. In: Debbie Epstein et al. (Hg.): World Year Book of Education 2008: Geographies of Knowledge, Geometries of Power: Higher Education in the 21st Century, 161-79. London: Routledge.

Keupp, Heiner, Thomas Ahbe, Wolfgang Gmür et al. (1999): Identitätskonstruktionen: Das Patchwork der Identitäten in der Spätmoderne. Reinbeck: Rowohlt.

Khadria, Binod (1999): The Migration of Knowledge Workers: Second-Generation Effects of India. New Delhi: Sage.

_- (2002): Skilled Labour Migration from Developing Countries: Study on India. International Migration Papers 49. Geneva: International Labour Office. http://www.fudepa.org/Biblioteca/recursos/ficheros/BMI20060000455/ imp49e.pdf.

—— (2006): The Migration of Professionals. In: Brij V. Lal (Hg.): The Encyclopedia of the Indian Diaspora, 70-74. Honolulu: University of Hawai'i Press.

- (2008): India in the Global Labour MarketInternational Economic Relations, Mobility of the Highly Skilled and Human Capital Formation. ISAS Working Paper 32. National University of Singapore. https://www.isas.nus. edu.sg/wp-content/uploads/media/isas_papers/32_WP.pdf.

Khandekar, Aalok und Deepa S. Reddy (2013): An Indian Summer: Corruption, Class, and the Lokpal Protests. Journal of Consumer Culture 15 (2): 221-47. Doi: $10.1177 / 1469540513498614$.

King, Russel, Mark Thomson, Tony Fielding et al. (2006): Time, Generation and Gender in Migration and Settlement. In: Rinus Penninx (Hg.): The Dynamics of International Migration and Settlement in Europe: A State of the Art, 233-68. Amsterdam: Amsterdam University Press. 
Kirk, Kate, Ellen Bal und Sarah R. Janssen (2017): Migrants in Liminal Time and Space: An Exploration of the Experiences of Highly Skilled Indian Bachelors in Amsterdam. Journal of Ethnic and Migration Studies 43 (16): 2771-87. Doi:10.1080/1369183X.2017.1314600.

Knott, Kim (Hg.) (2010): Diasporas: Concepts, Intersections, Indentities. London: Zed Books.

Kofman, Eleonore und Parvati Raghuram (2005): Gender and Skilled Migrants: Into and Beyond the Work Place. Geoforum 36 (2): 149-54. Doi:10.1016/j. geoforum.2004.06.001.

Kóu, Anu, Clara H. Mulder und Ajay Bailey (2017): ,For the Sake of the Family and Future: The Linked Lives of Highly Skilled Indian Migrants. Journal of Ethnic and Migration Studies 43 (16): 2788-2805. Doi:10.1080/1369183X. 2017.1314608.

Kujath, Hans J. und Suntje Schmidt (2007): Wissensökonomie und die Entwicklung von Städtesystemen. Working paper. Erkner: IRS Institut für Regionalentwicklung und Strukturplanung. https://leibniz-irs.de/fileadmin/ user_upload/IRS_Working_Paper/wp_staedtesysteme.pdf.

Lal, Malashri (2010): Tagore, Imaging the ,Other': Reflections on The Wife's Letter o Kabuliwala. Asian and African Studies XIV (1): 1-8. Doi:10.4312/ as.2010.-14.1.1-8.

Lall, Marie (2006): Indian Students in Europe: Trends, Constraints and Prospects, Living in ,The Age of Migration'. ENCARI Briefing Paper 5. http://mariel all.com/Files/Reports/Indian\%20students\%20in\%20Europe.pdf.

Lamb, Sarah (1997): The Making and Unmaking of Persons: Notes on Aging and Gender in North India. Ethos 25 (3): 279-302. Doi:10.1525/eth.1997.25 .3.279.

- (2018): Being Single in India: Gender Identities, Class Mobilities, and Personhood Flux. Ethos 46 (1): 49-69. Doi:10.1111/etho.12193.

Latour, Bruno (1987): Science in Action: How to Follow Scientists and Engineers Through Society. Milton Keynes: Open University Press.

- (2005): Reassembling the Social: An Introduction to Actor-Network-Theory.

Oxford: Oxford University Press.

Leemann, Regula J. (2010): Gender Inequalities in Transnational Academic Mobility and the Ideal Type of an Academic Entrepreneur. Discourse: Studies in the Cultural Politics of Education 32 (5): 605-25. Doi:10.1080/01596306.2010 .516942 .

Leemann, Regula J. und Stefan Boes (2012): Institutionalisierung von ,Mobilität und ,Internationalistät ' in wissenschaftlichen Laufbahnen: Neue Barrieren für Frauen auf dem Weg an die Spitze?. In: Sandra Beaufays, Anita Engels und Heike Kahlert (Hg.): Einfach Spitze? Neue Geschlechterperspektiven auf Karrieren in der Wissenschaft, 174-203. Frankfurt am Main: Campus.

Lefebvre, Henri ([1992] 2007): Rythmanalysis: Space, Time and Everyday Life. With an Introduction by Stuart Elden. London: Continuum. 
Lehmann, Wolfgang und Holly Trower (2018): Forms of Capital and Habitus in the Decision to Go on Academic Exchange. Canadian Review of Sociology 1 Revue canadienne de sociologie 55 (1): 136-48. Doi:10.1111/cars.12182.

Levitt, Peggy und Nina Glick Schiller (2004): Conceptualizing Simultaneity: A Transnational Social Field Perspective on Society. International Migration Review 38 (3): 1002-39. Doi:10.1111/j.1747-7379.2004.tb00227.x.

Lipner, Julius (2008): ,Icon and Mother': An Inquiry into India's National Song. The Journal of Hindu Studies 1 (1-2): 26-48. Doi:10.1093/jhs/hin008.

Lipton, Briony (2017): Measures of Success: Cruel Optimism and the Paradox of Academic Women's Participation in Australian Higher Education. Higher Education Research \& Development 36 (3): 486-97. Doi:10.1080/07294360. 2017.1290053.

Loimeier, Roman (2012): Eine Zeitlandschaft in der Globalisierung: Das islamische Sansibar im 19. und 20. Jahrhundert. Bielefeld: transcript.

Luckmann, Benita (1970): The Small Life-Worlds of Modern Man. Social Research 37 (4): 580-96. https://www.jstor.org/stable/40970035.

Lukose, Ritty A. (2009): Liberalization's Children: Gender, Youth, and Consumer Citizenship in Globalizing India. Durham: Duke University Press.

- (2010): Recasting the Secular: Religion and Education in Kerala, India. In: Diane P. Mines und Sarah Lamb (Hg.): Everyday Life in South Asia, Second Edition, 206-18. Bloomington: Indiana University Press.

Lutz, Helma (2017): Geschlechterverhältnisse und Migration: EInführung in den Stand der Diskussion. In: Helma Lutz und Anna Amelina (Hg.): Gender, Migration, Transnationalisierung: Eine intersektionelle Einführung, 13-44. Bielefeld: transcript.

Madianou, Mirca (2016): Ambient Co-Presence: Transnational Family Practices in Polymedia Environments. Global Networks 16 (2): 183-201. Doi:10.1111/ glob. 12105.

Madianou, Mirca und Daniel Miller (2012): Polymedia: Towards a New Theory of Digital Media in Interpersonal Communication. International Journal of Cultural Studies 16 (2): 169-87. Doi:10.1177/1367877912452486.

Malinar, Angelika (2009): Hinduismus. Göttingen: Vandenhoeck \& Ruprecht. Mapril, José (2014): The Dreams of Middle Class: Consumption, Life-course and Migration Between Bangladesh and Portugal. Modern Asian Studies 48 (3): 693-719. Doi:10.1017/S0026749X1200025X.

Marriott, McKim (1976): Hindu Transactions: Diversity without Dualism. In: Bruce Kapferer (Hg.): Transaction and Meaning: Directions on the Anthropology of Exchange and Symbolic Behavior, 109-42. Philadelphia: Institute for the Study of Human Issues.

Marriott, McKim und Ronald Inden (1977): Toward an Ethnosociology of South Asian Caste Systems. In: Kenneth David (Hg.): The New Wind: Changing Identities in South Asia, 227-38. The Hague: Mouton. 
Marschall, Sabine (2015): ,Homesick Tourism': Memory, Identity and (Be)longing. Current Issues in Tourism 18 (9): 876-92. Doi:10.1080/13683500.2014.920 773.

- (2017): Migrants on Home Visits: Memory, Identity and a Shifting Sense of Self. International Journal of Tourism Research 19 (2): 214-22. Doi:10.1002/ jtr.2103.

Martin, Emily (2000): Mind-Body Problems. American Ethnologist 27: 569-90. Doi:10.1525/ae.2000.27.3.569.

Matussek, Thomas (2011): Partner in einer globalen Welt. Länderprofile - Informationen für das internationale Bildungsmarketing: Indien, 11. Bonn: GATE Germany. https://static.daad.de/media/daad_de/pdfs_nicht_barrierefrei/ laenderinformationen/asien/1\%C3\%A4nderprofil_indien_.pdf.

Mauer, Stefan (2011): Land der Gegensätze. Länderprofile - Informationen für das internationale Bildungsmarketing: Indien, 28-29. Bonn: GATE Germany. https://static.daad.de/media/daad_de/pdfs_nicht_barrierefrei/laenderinfor mationen/asien/1\%C3\%A4nderprofil_indien_.pdf.

Mavroudi, Elizabeth, Ben Page und Anastasia Chrēstu (Hg.) (2017): Timespace and International Migration. Cheltenham: Edward Elgar Publishing.

May, Jon und Nigel J. Thrift (Hg.) (2001): Timespace: Geographies of Temporality. London: Routledge.

May, Vanessa (2011): Self, Belonging and Social Change. Sociology 45 (3): 363-78. Doi:10.1177/0038038511399624.

- (2013): Connecting Self to Society: Belonging in a Changing World. Basingstoke: Palgrave Macmillan.

- (2016): What Does the Duration of Belonging Tell Us About the Temporal Self?. Time \& Society 25 (3): 634-51. Doi:10.1177/0961463X16656853.

Mayer, Matthias M., Sakura Yamamura, Jan Schneider et al. (2012): Zuwanderung von internationalen Studierenden aus Drittstaaten: Studie der deutschen nationalen Kontaktstelle für das europäische Migrationsnetzwerk (EMN). Working Paper der Nationalen Kontaktstelle des EMN und der Forschungsgruppe des Bundesamtes 47. Nürnberg: Bundesamt für Migration und Flüchtlinge. https:/lec.europa.eu/home-affairs/sites/homeaffairs/files/whatwe-do/networks/european_migration_network/reports/docs/emn-studies/ immigration-students/10b._germany_national_report_immigration_of_in ternational_students_final_de_version_de.pdf.

Mazzarella, William (2005): Middle Class. South Asia Keywords Project. London: School of Oriental and African Studies, University of London, Centre of South Asian Studies. https://www.soas.ac.uk/south-asia-institute/keywords/ file24808.pdf.

McClymond, Kathryn (2008): Ritual. In: Sushil Mittal (Hg.): Studying Hinduism: Key Concepts and Methods, 314-26. London: Routledge.

McCrone, David und Gayle McPherson (Hg.) (2009a): National Days: Constructing and Mobilising National Identity. Basingstoke: Palgrave Macmillan. 
—_ (2009b): Introduction. In: David McCrone und Gayle McPherson (Hg.): National Days: Constructing and Mobilising National Identity, 1-9. Basingstoke: Palgrave Macmillan.

- (2009c): Marking Time: The Significance of National Days. In: David McCrone und Gayle McPherson (Hg.): National Days: Constructing and Mobilising National Identity, 212-21. Basingstoke: Palgrave Macmillan.

McKenzie, Lara und Loretta Baldassar (2017): Missing Friendships: Understanding the Absent Relationships of Local and International Students at an Australian University. Higher Education 74 (4): 701-15. Doi:10.1007/s10734-0160073-1.

McNeil, Laurie und Marc Sher (1999): Dual-Science-Career Couples: Survey Results. http://www.physics.wm.edu/ -sher/survey.pdf.

Mead, George H. (1970): Mind, Self, and Society: From the Standpoint of a Social Behaviorist. Chicago: University of Chicago Press.

Meeus, Bruno (2012): How to ,Catch' Floating Populations? Research and the Fixing of Migration in Space and Time. Ethnic and Racial Studies 35 (10): 1775-93. Doi:10.1080/01419870.2012.659272.

Miller, Daniel (2008): The Comfort of Things. Cambridge: Polity Press.

- (2011): Tales from Facebook. Cambridge: Polity Press.

Mines, Diane P. und Sarah E. Lamb (2010): Introduction to Part One: the Family and the Life Course. In: Diane P. Mines und Sarah Lamb (Hg.): Everyday Life in South Asia, Second Edition, 9-13. Bloomington: Indiana University Press.

Mines, Mattison (1994): Public Faces, Private Voices: Community and Individuality in South India. Berkeley: University of California Press.

Ministry for Youth Affairs and Sports, Government of India (2014): National Youth Policy 2014. https://www.yas.nic.in/sites/default/files/National-YouthPolicy-Document.pdf.

Ministry of Labour and Employment, Government of India (2014): Report on Youth Employment: Unemployment scenario. Chandigarh: Ministry of Labour and Employment, Government of India. https://labour.gov.in/sites/ default/files/Report\%20Vol\%202\%20final.pdf.

Mukhopadhyay, Carol C. (2004): A Feminist Cognitive Anthropology: The Case of Women and Mathematics. Ethos 32 (4): 458-92. Doi:10.1525/eth.2004.32. 4.458 .

Mukhopadhyay, Carol C. und Susan C. Seymour (Hg.) (1994): Women, Education, and Family Structure in India. Boulder: Westview Press.

Munasinghe, Viranjini (2001): Callaloo or Tossed Salad? East Indians and the Cultural Politics of Identity in Trinidad. Ithaca: Cornell University Press.

Münch, Claudia und Markus Hoch (2013): Studentische Mobilität und ihre finanziellen Effekte auf das Gastland. Berlin: DAAD, Prognos. https://eu.daad. de/medien/eu.daad.de.2016/dokumente/service/medien-und-publikationen/ 
studien-und-auswertungen/studentische_mobilit\%C3\%A4t_und_ihre_fi nanziellen_effekte_auf_das_gastland_prognos.pdf.

Munn, Nancy D. (1992): The Cultural Anthropology of Time: A Critical Essay. Annual Review of Anthropology 21: 93-123. Doi:10.1146/annurev. an.21.100192.000521.

Narayan, Kirin (1993): How Native Is a ,Native“ Anthropologist?. American Anthropologist 95 (3): 671-86. https://www.jstor.org/stable/679656.

Navaratnam, Sangheetha (2011): Guilt, Shame and Model Minorities: How South Asian Youth in Toronto Navigate the Canadian Educational System. Masterarbeit, Ontario Institute for Studies in Education. Ontario. https://tspace. library.utoronto.ca/bitstream/1807/30100/1/Navaratnam_Sangeetha_20 1111_MA_thesis.pdf.

Nedelcu, Mihaela und Malika Wyss (2016): ,Doing Family‘ Through ICT-mediated Ordinary Co-presence: Transnational Communication Practices of Romanian Migrants in Switzerland. Global Networks 16: 202-18. Doi:10.1111/ glob.12110.

Norman, Karin (1999): Phoning the Field: Meanings of Place and Involvement in Fieldwork ,At Home'. In: Vered Amit (Hg.): Constructing the Field: Ethnographic Fieldwork in the Contemporary World, 120-46. London: Taylor \& Francis.

Nowicka, Magdalena (2007): Mobile Locations: Construction of Home in a Group of Mobile Transnational Professionals. Global Networks 7 (1): 69-86. Doi:10.1111/j.1471-0374.2006.00157.x.

Nowotny, Helga (1993): Eigenzeit: Entstehung und Strukturierung eines Zeitgefühls. Frankfurt am Main: Suhrkamp.

- (2006): Unersättliche Neugier: Innovation in einer fragilen Zukunft. Frankfurt am Main: Büchergilde Gutenberg.

Oberlies, Thomas (2008): Hinduismus. Frankfurt am Main: Fischer.

Oeppen, Ceri (2013): A Stranger at ,Home': Interactions Between Transnatinal Return Visits and Integration for Afghan-American Professionals. Global Networks 13 (2): 261-78. Doi:10.1111/glob.12008.

OECD (Organisation für wirtschaftliche Zusammenarbeit und Entwicklung) (2013a): Gesamtbeurteilung und Empfehlungen. In: OECD (Hg.): $Z u$ wanderung ausländischer Arbeitskräfte: Deutschland, 19-32. Paris: OECD Publishing. Doi:10.1787/9789264191747-4-de.

— (2013b): Kontext der Arbeitsmigration. In: OECD (Hg.): Zuwanderung ausländischer Arbeitskräfte: Deutschland, 37-50. Paris: OECD Publishing. Doi:10.1787/9789264191747-6-de.

Ortner, Sherry B. (1997): Thick Resistance: Death and the Cultural Construction of Agency in Himalayan Mountaineering. Representations (59): 135-62.

Doi:10.2307/2928818.

(1999): Life and Death on Mt. Everest: Sherpas and Himalayan Mountaineering.

Princeton: Princeton University Press. 
- (2006): Anthropology and Social Theory: Culture, Power, and the Acting Subject. Durham: Duke University Press.

- (2010): Access: Reflections on Studying up in Hollywood. Ethnography 11 (2): 211-33. Doi:10.1177/1466138110362006.

Osella, Caroline und Filippo Osella (2008): Nuancing the Migrant Experience: Perspectives from Kerala, South India. In: Susan Koshy und Rajagopalan Radhakrishnan (Hg.): Transnational South Asians: The Making of a NeoDiaspora, 146-78. New Delhi: Oxford University Press.

Osella, Filippo und Caroline Osella (2000): Social Mobility in Kerala: Modernity and Identity in Conflict. London: Pluto Press.

Otto, Ton (2013): Times of the Other: The Temporalities of Ethnographic Fieldwork. Social Analysis 57 (1). Doi:10.3167/sa.2013.570105.

Palriwala, Rajni und Ravinder Kaur (2014): Introduction: Marriage in South Asia: Continuities and Transformations. In: Ravinder Kaur und Rajni Palriwala (Hg.): Marrying in South Asia: Shifting Concepts, Changing Practices in a Globalising World, 1-27. New Delhi: Orient BlackSwan.

Parry, Jonathan P. (2001): Ankalu's Errant Wife: Sex, Marriage and Industry in Contemporary Chhattisgarh. Modern Asian Studies 35: 783-820. Doi:10.1017/S0026749X01004024.

Petridou, Elia (2001): The Taste of Home. In: Daniel Miller (Hg.): Home Possessions: Material Culture Behind Closed Doors, 87-104. Oxford: Berg.

Pfaff-Czarnecka, Joanna (2011): From ,Identity' to ,Belonging' in Social Research: Plurality, Social Boundaries, and the Politics of the Self. Working Papers in Development Sociology and Social Anthropology 368. https://nbn-resolving. org/urn:nbn:de:0168-ssoar-431029.

—_ (2012): Zugehörigkeit in der mobilen Welt: Politiken der Verortung. Göttingen: Wallstein.

- (2013): Multiple Belonging and the Challenges to Biographic Navigation. MMG Working Paper 13-05. Göttingen: Max-Planck-Institut zur Erforschung multireligiöser und multiethnischer Gesellschaften. https://pure. mpg.de/rest/items/item_1690397_2/component/file_1690396/content.

Pickering, Andrew (1992): From Science as Knowledge to Science as Practice. In: Andrew Pickering (Hg.): Science as Practice and Culture, 1-26. Chicago: University of Chicago Press.

Povrzanović Frykman, Maja und Michael Humbracht (2013): Making Palpable Connections. Objects in Migrants' Transnational Lives. Ethnologia Scandinavica 43 (2013): 47-67.

Prabhu, Joseph (2006): Educational Institutions and Philosphies, Traditionals and Modern. In: Stanley A. Wolpert (Hg.): Encyclopedia of India. Vol. 2, 23-27. Detroit: Thomson Gale.

President of the Georg-August-Universität Göttingen (2011): Tradition - Innovation - Autonomy: Institutional Strategy 2007-2012. https://www.uni-goettin 
gen.de/de/document/download/03e2b34f28caf4de5076d8c319e4a38e.pdf/ brosch_zuk_web.pdf.

Pries, Ludger (1996): Transnationale Soziale Räume: Theoretisch-empirische Skizze am Beispiel der Arbeitswanderung Mexiko-USA. Zeitschrift für Soziologie 25 (6): 456-72. Doi:10.1515/zfsoz-1996-0603.

- (1998): Transnationale Soziale Räume: Theoretisch-empirische Skizze am Beispiel der Arbeitswanderungen Mexiko - USA. In: Ulrich Beck (Hg.): Perspektiven der Weltgesellschaft, 55-86. Frankfurt am Main: Suhrkamp.

Pritchard, Rosalind (2011): Re-entry Trauma: Asian Re-integration After Study in the West. Journal of Studies in International Education 15 (1): 93-111. Doi:10.1177/1028315310365541.

Radhakrishnan, Smitha (2011): Appropriately Indian: Gender and Culture in a New Transnational Class. Durham: Duke University Press.

Raghuram, Parvati (2013): Theorising the Spaces of Student Migration. Population, Space and Place 19: 138-54. Doi:10.1002/psp.1747.

Ramsay, Georgina (2017): Incommensurable Futures and Displaced Lives: Sovereignty as Control over Time. Public Culture 29 (3 (83)): 515-38. Doi:10.1215/08992363-3869584.

- (2018): Impossible Refuge: The Control and Constraint of Refugee Futures. London: Routledge.

Rapport, Nigel (1999): The Narrative as Fieldwork Technique: Processual Ethnography for a World in Motion. In: Vered Amit (Hg.): Constructing the Field: Ethnographic Fieldwork in the Contemporary World, 71-95. London: Taylor $\&$ Francis.

Referat Statistik und Wahlen der Stadt Göttingen (2018): Daten - Fakten - Zahlen 2018 Göttingen: Stadt, die Wissen schafft. https://duva-stg-extern.kdgoe.de/ Informationsportal/Dokumente/Faltblatt2018.pdf.

Richter, Caroline (2016): Vom Glück der Berufung: ,Glück' als Topos in Berufsbiographien des wissenschaftlichen Nachwuchses. Bios: Zeitschrift für Biographieforschung, oral history und Lebensverlaufsanalysen 29 (1): 47-66. Doi:10.3224/bios.v29i1.04.

Ringel, Felix (2016): Can Time Be Tricked? A Theoretical Introduction. The Cambridge Journal of Anthropology 34 (1): 22-31. Doi:10.3167/ca.2016.340104.

Robertson, Shanthi (2013): Transnational Student-Migrants and the State: The Education-Migration Nexus. Basingstoke: Palgrave Macmillan.

- (2014): The Temporalities of International Migration: Implications for Ethnographic Research. ICS Occasional Paper Series 5-1. Sydney: Institute for Culture and Society. Doi:10.4225/35/57a969210f1a0.

- (2015): The Production of the Indian Student: Regimes and Imaginaries of Migration, Education, Labour, Citizenship and Class. CCS Journal 7 (3): 1-22. Doi:10.5130/ccs.v7i3.4508.

Robertson, Shanthi, Anita Harris und Loretta Baldassar (2017): Mobile Transitions: A Conceptual Framework for Researching a Generation on the Move. 
Journal of Youth Studies 21 (2): 203-17. Doi:10.1080/13676261.2017.1362 101.

Robertson, Shanthi und Anjena Runganaikaloo (2014): Lives in Limbo: Migration Experiences in Australia's Education-Migration Nexus. Ethnicities 14 (2): 208-26. Doi:10.1177/1468796813504552.

Rodaway, Paul (1994): Sensuous Geographies: Body, Sense, and Place. London: Routledge.

Roland, Alan (1988): In Search of Self in India and Japan: Toward a Cross-Cultural Psychology. Princeton: Princeton University Press.

Ronneberger, Klaus und Vassilis Tsianos (2009): Panische Räume: Das Ghetto und die „Parallelgesellschaft“. In: Jana Binder, Sabine Hess und Johannes Moser (Hg.): No integration?! Kulturwissenschaftliche Beiträge zur Integrationsdebatte in Europa, 137-51. Bielefeld: transcript.

Rusconi, Alessandra und Heike Solga (2007): Determinants of and Obstacles to Dual Careers in Germany. Zeitschrift für Familienforschung 19 (3): 311-36. https://nbn-resolving.org/urn:nbn:de:0168-ssoar-58150.

Ryan, Louise (2015): Friendship-Making: Exploring Network Formations Through the Narratives of Irish Highly Qualified Migrants in Britain. Journal of Ethnic and Migration Studies 41 (10): 1664-83. Doi:10.1080/1369183X.20 15.1015409.

Ryan, Louise und Jon Mulholland (2014): French Connections: the Networking Strategies of French Highly Skilled Migrants in London. Global Networks 14 (2): 148-66. Doi:10.1111/glob.12038.

Säävälä, Minna (2006): Entangled in the Imagination: New Middle-Class Apprehensions in an Indian Theme Park. Ethnos 71 (3): 390-414. Doi:10.1080/ 00141840600902729.

Säävälä, Minna (2010): Middle-Class Moralities: Everyday Struggle over Belonging and Prestige in India. New Delhi: Orient BlackSwan.

Salazar, Noel B. (2018): Theorizing Mobility Through Concepts and Figures. Tempo Social 30 (2): 153-68. Doi:10.11606/0103-2070.ts.2018.142112.

Salazar, Noel B. und Kiran Jayaram (Hg.) (2016): Keywords of Mobility: Critical Engagements. New York: Berghahn.

Salazar, Noel B. und Alan Smart (2011): Anthropological Takes on (Im)mobility. Identities 18 (6): i-ix. Doi:10.1080/1070289X.2012.683674.

Savage, Mike, Gaynor Bagnall und Brian J. Longhurst (2005): Globalization and Belonging. Thousand Oaks: Sage.

Sawir, Erlenawati, Simon Marginson, Ana Deumert et al. (2007): Loneliness and International Students: An Australian Study. Journal of Studies in International Education 12 (2): 148-80. Doi:10.1177/1028315307299699.

Saxenian, AnnaLee (2005): From Brain Drain to Brain Circulation: Transnational Communities and Regional Upgrading in India and China. Studies in Comparative International Development 40 (2): 35-61. Doi:10.1007/ BF02686293. 
Schaer, Martine, Janine Dahinden und Alina Toader (2017): Transnational Mobility Among Early-Career Academics: Gendered Aspects of Negotiations and Arrangements Within Heterosexual Couples. Journal of Ethnic and Migration Studies 43 (8): 1292-1307. Doi:10.1080/1369183X.2017.1300254.

Schürmann, Ramona und Thorben Sembritzki (2017): Wissenschaft und Familie: Analysen zur Vereinbarkeit beruflicher und familialer Anforderungen und Wünsche des wissenschaftlichen Nachwuchses. Hannover: Deutsches Zentrum für Hochschul- und Wissenschaftsforschung.

Sekhri, Sheetal (2011): Affirmative Action and Peer Effects: Evidence from Caste Based Reservation in General Education Colleges in India. University of Virginia. https://conference.nber.org/conferences/2010/EDf10/Sekhri.pdf (depubliziert, zugänglich via Archive.org).

Seymour, Susan C. (1995): Family Structure, Marriage, Caste and Class, and Women's Education: Exploring the Linkages in an Indian Town. Indian Journal of Gender Studies 2 (1): 67-86. Doi:10.1177/097152159500200104.

Shachar, Ayelet (2006): The Race for Talent: Highly Skilled Migrants and Competitive Immigration Regimes. New York University Law Review 81 (1): 148-206. https://www.nyulawreview.org/wp-content/uploads/2018/08/11. pdf.

Shankar, Shalini (2008): Desi Land: Teen Culture, Class, and Success in Silicon Valley. Durham: Duke University Press.

Sharma, Sarah (2014): In the Meantime: Temporality and Cultural Politics. Durham: Duke University Press.

Sheller, Mimi und John Urry (2006): The New Mobilities Paradigm. Environment and Planning A: Economy and Space 38 (2): 207-26. Doi:10.1068/a37268.

Shove, Elizabeth (2013a): Everyday Practice and the Production and Consumption of Time. In: Elizabeth Shove (Hg.): Time, Consumption and Everyday Life: Practice, Materiality and Culture, 17-34. London: Bloomsbury.

— (Hg.) (2013b): Time, Consumption and Everyday Life: Practice, Materiality and Culture. London: Bloomsbury.

Shove, Elizabeth, Frank Trentmann und Richard R. Wilk (2013): Introduction. In: Elizabeth Shove (Hg.): Time, Consumption and Everyday Life: Practice, Materiality and Culture, 1-16. London: Bloomsbury.

Singerman, Diane (2007): The Economic Imperatives of Marriage: Emerging Practices and Identities Among Youth in the Middle East. Washington: Wolfensohn Center for Development at the Brookings Institution und Dubai School of Government.

Singh, Megha und R. K. Sohoni (2016): The Anti-Corruption Movement in India and the Lokpal. Imperial Journal of Interdisciplinary Research 2 (4): 112-14. http://www.onlinejournal.in/IJIRV2I4/020.pdf.

Singh, Parlo und Catherine Doherty (2008): Mobile Students in Liquid Modernity: Negotiating the Politics of Transnational Identity. In: Nadine Dolby und 
Fazal Rizvi (Hg.): Youth Moves: Identities and Education in Global Perspective, 115-30. New York: Routledge.

Skrbiš, Zlatko (2008): Transnational Families: Theorising Migration, Emotions and Belonging. Journal of Intercultural Studies 29 (3): 231-46. Doi:10.1080/072 56860802169188.

Skrbiš, Zlatko, Ian Woodward und Clive Bean (2013): Seeds of Cosmopolitan Future? Young People and Their Aspirations for Future Mobility. Journal of Youth Studies 17 (5): 614-25. Doi:10.1080/13676261.2013.834314.

Smart, Carol, Katherine Davies, Brian Heaphy et al. (2012): Difficult Friendships and Ontological Insecurity. The Sociological Review 60 (1): 91-109. Doi:10.1111/j.1467-954X.2011.02048.x.

Soeffner, Hans-Georg und Dariuš Zifonun (2008): Integration - An Outline from the Perspective of the Sociology of Knowledge. Qualitative Sociology Review 4 (2): 3-23. http://www.qualitativesociologyreview.org/ENG/Volume10/ QSR_4_2_Soeffner.pdf.

Sökefeld, Martin (2002): Feld ohne Ferne: Reflexionen über ethnologische Forschung ,zu Hause، - in Hamburg, zum Beispiel. EthnoScripts 4 (1): 82-96.

Sondhi, Gunjan (2013): Gendering International Student Mobility: An Indian Case Study. Dissertation, School of Global Studies, University of Sussex. http://sro.sussex.ac.uk/id/eprint/46066.

Sovic, Silvia (2009): Hi-Bye Friends and the Herd Instinct: International and Home Students in the Creative Arts. Higher Education 58 (6): 747-61. Doi: 10.1007/s10734-009-9223-z.

Spradley, James P. (1979): The Ethnographic Interview. New York: Holt, Rinehart and Winston.

Sri, P. S. (1996): Twentieth-Century Tamil Literature. In: Nalini Natarajan (Hg.): Handbook of Twentieth-Century Literatures of India, 289-305. Westport: Greenwood Press.

Stambach, Amy und Kathleen D. Hall (Hg.) (2017): Anthropological Perspectives on Student Futures: Youth and the Politics of Possibility. New York: Palgrave Macmillan.

Strathern, Marilyn (1988): The Gender of the Gift: Problems with Women and Problems with Society in Melanesia. Berkeley: University of California Press.

Strauss, Anselm (1978): A Social World Perspective. In: Norman K. Denzin (Hg.): Studies in Symbolic Interaction: An Annual Compilation of Research, 119-28. Greenwich: Jai Press.

Subramanian, Ajantha (2015): Making Merit: The Indian Institutes of Technology and the Social Life of Caste. Comparative Studies in Society and History 57 (2): 291-322. Doi:10.1017/S0010417515000043.

Subramanian, Jayasree (2016): Perceiving and Producing Merit: Gender and Doing Science in India. Indian Journal of Gender Studies 14 (2): 259-84. Doi:10.1177/097152150701400203. 
Svašek, Maruška (2018): Ageing Kin, Proximity and Distance: Translocal Relatedness as Affective Practice and Movement. In: Birgitt Röttger-Rössler und Jan Slaby (Hg.): Affect in Relation: Families, Places, Technologies, 31-49. London: Routledge, Taylor \& Francis.

Thapan, Meenakshi (2009): Living the Body: Embodiment, Womanhood and Identity in Contemporary India. New Delhi: Sage.

Thomas, Renny und Robert M. Geraci (2018): Religious Rites and Scientific Communities: Ayudhya Puja as ,Culture' at the Indian Institute of Science. Zygon 53 (1): 95-122. Doi:10.1111/zygo.12380.

Thouaille, Marie-Alix (2018): Is Pursuing an Academic Career a Form of ,Cruel Optimism'? In: LSE Impact Blog, London School of Economics and Political Science. http://blogs.lse.ac.uk/impactofsocialsciences/2018/03/01/is-pur suing-an-academic-career-a-form-of-cruel-optimism/.

Tie, Caroline, Andrew Holden und Hyung yu Park (2015): A ,Reality of Return': The Case of the Sarawakian-Chinese Visiting China. Tourism Management 47: 206-12. Doi:10.1016/j.tourman.2014.09.021.

Titz, Sven (2011): Auf dem Weg zur Spitze. Länderprofile - Informationen für das internationale Bildungsmarketing: Indien, 14-16. Bonn: GATE Germany. https://static.daad.de/media/daad_de/pdfs_nicht_barrierefrei/laenderinfor mationen/asien/1\%C3\%A4nderprofil_indien_.pdf.

Trentmann, Frank (2013): Disruption Is Normal: Blackouts, Breakdowns and the Elasticity of Everyday Life. In: Elizabeth Shove (Hg.): Time, Consumption and Everyday Life: Practice, Materiality and Culture, 67-84. London: Bloomsbury.

Tsing, Anna L. (2015): The Mushroom at the End of the World: On the Possibility of Life in Capitalist Ruins. Princeton: Princeton University Press.

Upadhya, Carol (2007): Employment, Exclusion and ,Merit' in the Indian IT Industry. Economic and Political Weekly 42 (20): 1863-68. https://www.jstor. org/stable/4419609.

Valentin, Karen (2012): Caught Between Internationalisation and Immigration: The Case of Nepalese Students in Denmark. Learning and Teaching 5 (3): 56-76. Doi:10.3167/latiss.2012.050304.

- (2014): Certificates for the Future: Geographical Mobility and Educational Trajectories Among Nepalese Youth. In: Anne L. Dalsgaard et al. (Hg.): Ethnographies of Youth and Temporality: Time Objectified, 117-38. Philadelphia: Temple University Press.

van Meijl, Toon (2006): Mutiple Identifications and Dialogical Self: Urban Maori Youngsters and Cultural Renaissance. Journal of the Royal Anthropological Institute 12 (4): 917-33. Doi:10.1111/j.1467-9655.2006.00370.x.

- (2008): Culture and Identity in Anthropology: Reflections on ,Unity' and ,Uncertainty' in the Dialogical Self. International Journal for Dialogical Science 3 (1): 165-90. http://ijds.lemoyne.edu/journal/3_1/pdf/IJDS.3.1.13. VanMeijl.pdf. 
Varma, Roli (2006): Harbingers of Global Change: India. Lanham: Lexington Books.

Vertovec, Steven (2001): Transnationalism and Identity. Journal of Ethnic and Migration Studies 27 (4): 573-82. Doi:10.1080/13691830120090386.

- (2009): Transnationalism. London: Routledge.

Vigh, Henrik (2007): Navigating Terrains of War: Youth and Soldiering in GuineaBissau. New York: Berghahn.

- (2009a): Motion Squared: A Second Look at the Concept of Social Navigation. Anthropological Theory 9 (4): 419-38. Doi:10.1177/146349960935 6044.

— (2009b): Wayward Migration: on Imagined Futures and Technological Voids. Ethnos 74 (1): 91-109. Doi:10.1080/00141840902751220.

Wilding, Raelene (2006): ,Virtual' Intimacies? Families Communicating Across Transnational Contexts. Global Networks 6 (2): 125-42. Doi:10.1111/ j.1471-0374.2006.00137.x.

Wilk, Richard R. (2013): The Edge of Agency: Routines, Habits and Volition. In: Elizabeth Shove (Hg.): Time, Consumption and Everyday Life: Practice, Materiality and Culture, 143-54. London: Bloomsbury.

Williams, Allan M. und Vladimír Baláž (2008): International Migration and Knowledge. London: Routledge.

Winslow, Sarah und Shannon N. Davis (2016): Gender Inequality Across the Academic Life Course. Sociology Compass 10 (5): 404-16. Doi:10.1111/soc4.1 2372.

Wolfinger, Nicholas, Mary A. Mason und Marc Goulden (2009): Stay in the Game: Gender, Family Formation and Alternative Trajectories in the Academic Life Course. Social Forces 87 (3): 1591-1621. Doi:10.1353/sof.0.0 182.

World Bank Group (2018a): Learning to Realize Education's Promise: Overview. Washington. https://openknowledge.worldbank.org/bitstream/handle/10986 /28340/211096ov.pdf.

_- (2018b): Maximizing Knowledge for Development. Development Digest 4 (April 2018). Global Knowledge and Research Hub in Malaysia. http:// documents.worldbank.org/curated/en/224671523630239607/pdf/125247WP-PUBLIC-add-series-Development-digest.pdf.

Yuval-Davis, Nira (2006): Belonging and the Politics of Belonging. Patterns of Prejudice 40 (3): 197-214. Doi:10.1080/00313220600769331.

Zerubavel, Eviatar (1981): Hidden Rhythms: Schedules and Calendars in Social Life. Chicago: University of Chicago Press. 


\section{Abbildungsverzeichnis}

Abb. 1: Das Zentrum und der nördliche Teil Göttingens.................................... 59

Abb. 2: „PhD plan and reality“, von Sarjana auf Facebook geteilt..................... 116

Abb. 3: Rangoli zu Diwali 2014 ……............................................................ 179

Abb. 4: Der dekorierte Hörsaal des MPI für experimentelle Medizin................. 179 



\section{Kurzvorstellung der Hauptgesprächspartner*innen}

In der Aufzählung finden sich, sofern ich die Angaben zur Verfügung hatte, folgende Informationen zu meinen wichtigsten Gesprächspartner*innen: das Geburtsjahr, die regionale Herkunft, der Familienstand, der Beruf der Eltern und die Anzahl der Geschwister. Die Namen sind Pseudonyme und einige Details so verändert, dass sie die Wiedererkennung der Person erschweren, aber trotzdem das Datenset nicht verzerren.

Ajay (Jg. 1985). Ajay wuchs in einer Großstadt in Tamil Nadu auf. Seine Eltern stammen aus Kerala. Ajays Vater war Beamter im Finanzbereich und seine Mutter Lehrerin. Beide Eltern sind im Ruhestand. Er hat einen Bruder. Einer seiner Onkel lebt in Deutschland. Ajay war bereits für sein Masterstudium nach Deutschland gekommen. Nach Göttingen kam er 2009, um in angewandter Physik zu promovieren. Ajay war ein aktives Mitglied der Vereinigung der indischen Wissenschaftler*innen. Ende 2013 heiratete Ajay und lebt mittlerweile zusammen mit seiner Frau und einer Tochter in Göttingen.

Ajit (Jg. 1991). Ajit wuchs in einem Dorf in Maharashtra auf. Der Vater ist Staatsbeamter und die Mutter Hausfrau. Er hatte zwei Schwestern. Er war bereits für sein Bachelorstudium nach Deutschland gekommen. Für sein Masterstudium in Entwicklungsbiologie zog er 2015 nach Göttingen. 
Akhil (Jg. 1991). Akhil wuchs in einer Großstadt in Karnataka auf. Seine Eltern sind beide Naturwissenschaftler. Akhil hat einen jüngeren Bruder. 2011 kam Akhil für sein Masterstudium nach Göttingen. An dieses schloss sich direkt seine Promotion in Neurowissenschaft an. Akhil hat eine Leidenschaft für das Singen. 2017 schloss er seine Promotion ab und kehrte nach Indien zurück. Dort arbeitet er seit 2018 an einer Universität in Nordindien.

Āmāl (Jg. 1990). Āmāl stammte aus West Bengal und absolvierte ihr Masterstudium in Soziologie in Pune. Mit Hilfe eines Erasmus-Mundus-Stipendiums konnte sie 2012 für ein Semester in Göttingen studieren. Sie hat einen jüngeren Bruder und ist eine begeisterte Sängerin. Sie kehrte nach diesem einen Semester wieder nach Indien zurück, plante aber, nach ihrem Masterabschluss für eine Promotion nach Deutschland zurückzukehren. Sie lebt und studiert in einer Großstadt im Westen Indiens.

Amala (Jg. 1979). Amala stammt aus einer Großstadt in Maharashtra. Als sie nach Göttingen kam, um ihre Promotion in Geschichte fertigzustellen, war sie bereits verheiratet. Ihr Vater war zum Zeitpunkt meiner Forschung bereits in Rente. Zuvor arbeitete er im Bankwesen. Amala hat einen älteren Bruder. Mittlerweile lebt Amala wieder in Indien und ist weiterhin in der Wissenschaft tätig.

Anay (Jg. 1990). Anay kam aus einer Großstadt im Norden Indiens. Der Vater hat einen eigenen Laden und arbeitet dort als Verkäufer. Die Mutter ist Hausfrau und verdient sich Geld durch Stickereien dazu. Er hat eine jüngere Schwester und einen jüngeren Bruder. Anay war Doktorand in einer transnationalen Forschungsgruppe und promovierte in Geschichte. Seit 2019 ist er assistant professor in den USA.

Anisha (Jg. 1982). Anisha wuchs in Delhi auf. Sie kam gemeinsam mit ihrem Freund nach Deutschland und promovierte in Göttingen im Fach Biologie. Ihr Vater ist Geschäftsmann und arbeitet in der vom Großvater gegründeten Firma. Die Mutter hat einen Masterabschluss und ist als Hausfrau tätig. Sie hat einen Bruder. Anisha und ihr Freund heirateten 2014, leben seitdem gemeinsam im Süden Deutschlands und haben ein Kind.

Bhanu (Jg. 1980). Bhanu stammt aus Tamil Nadu. Er kam bereits 2001 für sein Masterstudium des Chemieingenieurwesens nach Deutschland. Für seine Promotion blieb er in Deutschland und begann 2007, in Göttingen für einen Technologiekonzern zu arbeiten. Er war einer der Mitbegründer der Vereinigung der indischen Wissenschaftler*innen. 2010 heiratete er Rukmi. 2011 zogen sie nach Österreich, wo sie zum Zeitpunkt des Verfassens dieser Arbeit mit ihrem Kind lebten.

Dhara (Jg. 1971). Dharas Familie stammt aus West Bengal. Sie wuchs in Delhi auf und studierte in Delhi und Mumbai. Sie promovierte in Geschichte und kam als Postdoktorandin 2012 nach Deutschland. Dhara distanzierte sich von der indischen 
Vereinigung. Sie lebt seit 2015 gemeinsam mit ihrem Mann in Hamburg und arbeitet dort an einem Forschungsinstitut.

Gilon (Jg. 1982). Gilon stammt aus einem Dorf in Karnataka. Seine Eltern arbeiten in der Landwirtschaft. Gilon hat eine Schwester und fünf Brüder. Er kam für seine Promotion in Geschichte, die er 2013 abschloss, nach Göttingen. 2013 kehrte Gilon nach Indien zurück, wo er eine Stelle an einer Universität innehat. Gilon heiratete etwa ein halbes Jahr nach seiner Rückkehr nach Indien.

Harendra (Jg. 1988). Harendra wuchs in einer Kleinstadt in Maharashtra auf. Er stammt aus einer Priesterfamilie, weswegen er einige Male die pūjā bei den DiwaliFeiern in Göttingen durchführte. Für seine Promotion in Geschichte kam er nach Göttingen. Da die Finanzierung seiner Promotion ausgelaufen war, verließ er Göttingen 2017, bevor er seine Doktorarbeit beenden konnte. Er lebt seitdem wieder in Indien.

Hritik (Jg. 1981). Hritik stammt aus Tamil Nadu und war zeitweise der Kapitän der Göttinger Kricketmannschaft. Er war 2005 für seine Promotion in Physik nach Göttingen gekommen. Er lebt mittlerweile gemeinsam mit seiner Frau Vedika in den USA.

Kiran (Jg. 1986). Kiran wuchs in einem kleinen Dorf in Andhra Pradesh auf. Seine Eltern waren in seiner Kindheit beide berufstätig. Er hat eine jüngere Schwester. Bevor er nach Göttingen kam, hatte er in den USA einen MA-Abschluss gemacht. Kiran promovierte in Physik. Nach einer Anstellung als Postdoktorand in Spanien zog er 2016 wieder zurück in die USA. Seit 2018 arbeitet er dort als postdoctoral researcher.

Kundan (Jg. 1984). Kundan wuchs in einem Dorf in Tamil Nadu auf. Beide Eltern waren in der Landwirtschaft tätig. Er hat eine Schwester. 2011 kam er für seine Promotion in Neurowissenschaften nach Göttingen. Seit 2015 hat er eine Anstellung als Postdoktorand in den USA.

Lali (Jg. 1987). Lali stammt aus einer Großstadt in Andhra Pradesh und hat ihren Bachelor-Abschluss in Delhi erworben. Lali hat zwei jüngere Geschwister. Sie war für ihr MA-Studium nach Deutschland gekommen, $2011 \mathrm{zog}$ sie für ihre Promotion in Biologie nach Göttingen. Lali war einige Zeit lang sehr aktiv in der Vereinigung indischer Studierender in Göttingen. Sie heiratete im Frühjahr 2014 einen jungen Mann aus Andhra Pradesh, der zuvor in Österreich promoviert hatte. Gemeinsam zogen sie 2016 in die USA.

Maya (Jg. 1989). Maya wuchs in West Bengal auf. Ihre Mutter ist Lehrerin, ihr Vater Geschäftsmann. Sie hat einen jüngeren Bruder und promovierte in Neurowissen- 
schaften. Nach einer kurzen Phase als Postdoktorandin in Göttingen zog sie 2018 nach Belgien, wo sie für einen Wissenschaftsverlag arbeitet.

Nabhi (Jg. 1987). Nabhi stammt aus einer Kleinstadt in Uttar Pradesh. Ihr Vater ist Staatsbeamter und die Mutter Hausfrau. Sie hat einen jüngeren Bruder. Sie kam für ihren Masterabschluss nach Göttingen und promovierte im Anschluss in Biologie. 2015 heiratete sie einen jungen Mann aus Bangladesch. Sie hatten sich während ihres Masterstudiums in Göttingen kennengelernt. Sie lebt gemeinsam mit ihrem Mann und dem gemeinsamen Kind in Göttingen.

Nalika (Jg. 1982). Nalika stammt aus einer Kleinstadt in Karnataka und promovierte in Neurowissenschaften. Sie hat eine jüngere Schwester, die in Kanada promovierte. Seit dem Frühjahr 2012 ist sie mit Ravi verheiratet, mit dem sie ab 2012 in den USA lebte. Sie war eine der Mitbegründer*innen der indischen Vereinigung. Seit 2017 lebt sie mit Ravi und dem gemeinsamen Kind im Süden Indiens.

Nandita (Jg. 1985). Nandita kommt aus Uttar Pradesh. Der Vater ist in Rente und die Mutter Hausfrau. Nandita hat keine Geschwister. 2011, zum Zeitpunkt des Interviews hatte sie eine Stelle als Postdoktorandin in Göttingen. Ihren Promotionsabschluss in Biologie hatte sie zuvor in Deutschland erworben. 2013 heiratete sie in Indien. 2015 war sie postdoctoral researcher in Kanada.

Nathin (Jg. 1989). Nathin stammt aus einem Dorf in Tamil Nadu. Seine Schulzeit verbrachte er auf einem Internat. Er kam für seinen Master in Molekularbiologie nach Göttingen. Zuvor hatte er seinen Bachelorabschluss in Schweden erworben. Nathins Vater hat einen Bachelorabschluss in Mathematik und arbeitet im Bereich der IT-Sicherheit. Seine Mutter hat einen Bachelorabschluss und eine Schule aufgebaut, deren Rektorin sie ist. Nathin hat einen jüngeren Bruder. Nathin promoviert an einer Universität im Süden Deutschlands.

Pravin (Jg. 1982). Pravin wuchs in einem Dorf in Karnataka auf. Er kam für eine Anstellung als Postdoktorand in Physik nach Göttingen. Danach führten ihn weitere Positionen als Postdoktorand nach Israel und Kanada. Seit 2019 ist er zurück in Indien und hat dort eine Anstellung als assistant professor an einer Privatuniversität im Norden Indiens.

Radha (Jg. 1989). Radha stammt aus einem kleinen Ort in der Nähe der Hauptstadt von Odisha. Sie absolvierte einen Master in Euroculture. Ihr Vater arbeitet als Verkäufer und ihre Mutter hat ein eigenes Geschäft. Radha hat einen jüngeren Bruder. Um sich ihr Studium zu finanzieren, arbeitete sie nebenher, u.a. als studentische Hilfskraft. Ihren Masterabschluss erhielt sie 2018. Sie lebt mittlerweile in Hamburg. 
Ravi (Jg. 1981). Ravi stammt aus einer Großstadt in Karnataka. Er hat eine Schwester. Er promovierte in Göttingen im Fach Bioinformatik. Er war einer der Mitbegründer der Vereinigung der indischen Studierenden und Wissenschaftler*innen. 2012 heiratete er Nalika, die er in Göttingen kennengelernt hatte. Seit 2017 lebt er mit Nalika und dem gemeinsamen Kind im Süden Indiens.

Rohini (Jg. 1988). Rohini stammt aus West Bengal. Während ihrer Schulzeit zog sie häufig mit der Familie um. Der Vater arbeitete zunächst als Staatsbeamter und später für eine internationale Nichtregierungsorganisation, die Mutter ist Lehrerin. Rohini hat eine jüngere Schwester und ist eine begeisterte Musikerin. Sie promovierte in Neurowissenschaften und schloss ihre Promotion 2017 ab. Bis 2019 hatte sie eine Stelle als Postdoktorandin in Göttingen. Mittlerweile hat sie eine Anstellung in Dänemark angetreten.

Sachin (Jg. 1983). Sachin stammt aus Tamil Nadu. Er hatte in den USA studiert und kam gemeinsam mit seiner Frau Sadhvi für seine Promotion in Physik nach Göttingen. Nach dem Abschluss seiner Promotion 2011 hatte er eine Anstellung als Postdoktorand in den USA. Mittlerweile ist er group leader an einem Forschungsinstitut im südindischen Bundesstaat Karnataka.

Sadhvi (Jg. 1983). Sadhvi stammt aus Tamil Nadu und wuchs in den USA auf. In den USA hatte sie ihren Masterabschluss in Biologie erworben und war dann gemeinsam mit ihrem Ehemann Sachin für ihren PhD nach Göttingen gekommen. 2011 schloss sie ihre Promotion ab und zog für eine Anstellung als Postdoktorandin in die USA. Seit 2018 ist sie Mitarbeiterin an einer Universität im südindischen Bundesstaat Karnataka.

Sahil (Jg. 1982). Sahil wuchs in einer Großstadt in Maharashtra auf. Sein Vater ist im Vorstand einer Fabrik tätig. Er hat zwei Brüder. Sahil hatte in Soziologie promoviert und kam für eine Anstellung als Postdoktorand nach Göttingen. Seit 2017 ist er assistant professor an einer Universität im Norden Indiens.

Sandeep (Jg. 1988). Sandeep wuchs in der Nähe von Kolkata auf. Sandeeps Eltern waren beide in der Landwirtschaft tätig. Nach seinen Bachelorabschluss an einem Indian Institute of Technology absolvierte er ein Masterstudium in England. Sandeep heiratete 2011. Für seine Promotion in Mathematik kam er 2012 nach Göttingen. Seinen PhD-Abschluss erlangte er 2016 und zog kurz darauf mit seiner Frau nach Belgien. Dort arbeiten beide weiterhin an ihren wissenschaftlichen Karrieren.

Sangita (Jg. 1986). Sangitas Eltern kommen aus West Bengal. Sie selbst ist in einer Großstadt in Maharashtra aufgewachsen. Ihr Vater ist Staatsbeamter und die Mutter Hausfrau. Sie hat eine jüngere Schwester. Für ihre Promotion in Neurowissenschaften kam sie 2009 nach Göttingen. Sangita schloss 2013 ihre Promotion ab und hei- 
ratete 2014. Seit 2017 lebt sie gemeinsam mit ihrem Mann im Süden Indiens. Sie ist mittlerweile im Bereich der Wissenschaftskommunikation tätig.

Sarjana (Jg. 1988). Sarjana stammt aus West Bengal. Für ihre Promotion in Biologie kam sie 2012 nach Göttingen. In Göttingen lernte sie Sunil kennen, den sie 2014 heiratete. Ihre Promotion schloss sie 2016 ab. Seitdem arbeitet sie weiter als Postdoktorandin an der Universität Göttingen. Sarjanas Vater ist Geschäftsmann. Sie hat keine Geschwister.

Smita (Jg. 1980). Smita ist in Mumbai aufgewachsen. Ihr Vater hatte nach seinem Masterabschluss begonnen zu arbeiten. Ihre Mutter ist Hausfrau. Smita hat zwei jüngere Geschwister. Smita kam 2007 nach Göttingen und erlangte 2012 ihren PhD-Abschluss in Mikrobiologie. Sie war eine der Mitbegründer*innen der indischen Vereinigung. Smita heiratete im Dezember 2012 und zog kurz darauf mit ihrem Ehemann nach Indien. Sie arbeitet mittlerweile an einem Forschungsinstitut in Nordindien.

Tanvir (Jg. 1985). Tavir wuchs in einer Stadt in der Nähe von Kolkata auf. Er kam für seine Promotion in Mathematik nach Göttingen. Sein Vater arbeitet für die „State Bank of India" und seine Mutter ist Lehrerin. Tanvir hat keine Geschwister. Auf der Suche nach einem Arbeitsplatz begab er sich Ende 2015, nach dem Abschluss seiner Promotion, zurück nach Indien. Seit 2017 ist er postdoctoral research fellow an einer Universität in Hyderabad.

Vendan (Jg. 1989). Vendan wuchs in einer Kleinstadt in Tamil Nadu auf. Ab 2011 promovierte er in Göttingen im Fach Biologie. Sein Vater ist Ingenieur und arbeitet für die staatliche Eisenbahngesellschaft. Die Mutter arbeitet als Mathematiklehrerin. Vendan hat einen jüngeren Bruder und war in der Vereinigung indischer Postgraduierter aktiv. Seit dem Abschluss seiner Promotion arbeitet er als Postdoktorand in Göttingen.

Vivān (Jg. 1987). Vivān kam 2011 für seine Promotion in Physik nach Göttingen. Er war in der Nähe von Hyderabad aufgewachsen. 2015 zog er nach seiner abgeschlossenen Promotion zurück nach Indien. 
$\mathrm{n}$ den vergangenen Jahren ist die Anzahl internationaler Studierender und Wissenschaftler*innen in Deutschland stetig angestiegen. Ausgehend von ihrer ethnologischen Forschung in der Universitätsstadt Göttingen geht Antonie Fuhse der Frage nach, wie junge Wissenschaftler*innen aus Indien ihre multiplen sozialen Verortungen während ihres Studiums beziehungsweise ihrer Promotion aushandelten. Sie setzt sich besonders damit auseinander, welche Rolle Mobilität in der Aushandlung normativer Vorstellungen von der Gestaltung des Lebensverlaufs und der Zukunft spielt.

In diesem Buch wird das Konzept des, Regimes der akademischen Mobilität‘ entwickelt und in der Analyse angewandt, um die vielfältigen Akteure, Diskurse und Strukturen herauszustellen, die an der Mobilität von Studierenden und Wissenschaftler*innen beteiligt sind und Ungleichheiten im Zugang zu internationaler Mobilität erzeugen. Die Autorin zeigt, dass Alter, die Herkunft aus der indischen Mittelschicht und Gender in ihrer Interaktion einen großen Einfluss darauf hatten, warum die jungen Wissenschaftler*innen nach Deutschland kamen, welche Erfahrungen sie in Göttingen machten und was sie sich für ihre Zukunft erhofften. Sie stellt heraus, dass die Mobilität auf vielfache Weise mit dem Heiraten verknüpft war, und rückt damit ein Thema in den Fokus, das in der Erforschung internationaler Studierender und Wissenschaftler ${ }^{\star}$ innen bisher wenig beachtet wurde.

Antonie Fuhse ist Ethnologin und arbeitet zu Migrationsprozessen.

Weitere Forschungsschwerpunkte sind Jugendforschung und die Ethnologie der Zukunft. Sie studierte Ethnologie und Indologie an der Universität Göttingen, wo sie mit der vorliegenden Arbeit ihre Promotion abschloss. Gemeinsam mit Prof. Dr. Elfriede Hermann veröffentlichte sie 2018 den Sammelband „India beyond India: Dilemmas of Belonging“.

ISBN: $\quad 978-3-86395-498-7$

ISSN: $2199-5346$ eISSN: 2512-6881 